\title{
Interventional strategies to combat muscle disuse atrophy
}

Citation for published version (APA):

Dirks, M. L. (2016). Interventional strategies to combat muscle disuse atrophy. [Doctoral Thesis, Maastricht University]. Datawyse / Universitaire Pers Maastricht. https://doi.org/10.26481/dis.20161118md

Document status and date:

Published: 01/01/2016

DOI:

10.26481/dis.20161118md

Document Version:

Publisher's PDF, also known as Version of record

\section{Please check the document version of this publication:}

- A submitted manuscript is the version of the article upon submission and before peer-review. There can be important differences between the submitted version and the official published version of record.

People interested in the research are advised to contact the author for the final version of the publication, or visit the DOI to the publisher's website.

- The final author version and the galley proof are versions of the publication after peer review.

- The final published version features the final layout of the paper including the volume, issue and page numbers.

Link to publication

\footnotetext{
General rights rights.

- You may freely distribute the URL identifying the publication in the public portal. please follow below link for the End User Agreement:

www.umlib.nl/taverne-license

Take down policy

If you believe that this document breaches copyright please contact us at:

repository@maastrichtuniversity.nl

providing details and we will investigate your claim.
}

Copyright and moral rights for the publications made accessible in the public portal are retained by the authors and/or other copyright owners and it is a condition of accessing publications that users recognise and abide by the legal requirements associated with these

- Users may download and print one copy of any publication from the public portal for the purpose of private study or research.

- You may not further distribute the material or use it for any profit-making activity or commercial gain

If the publication is distributed under the terms of Article $25 \mathrm{fa}$ of the Dutch Copyright Act, indicated by the "Taverne" license above, 


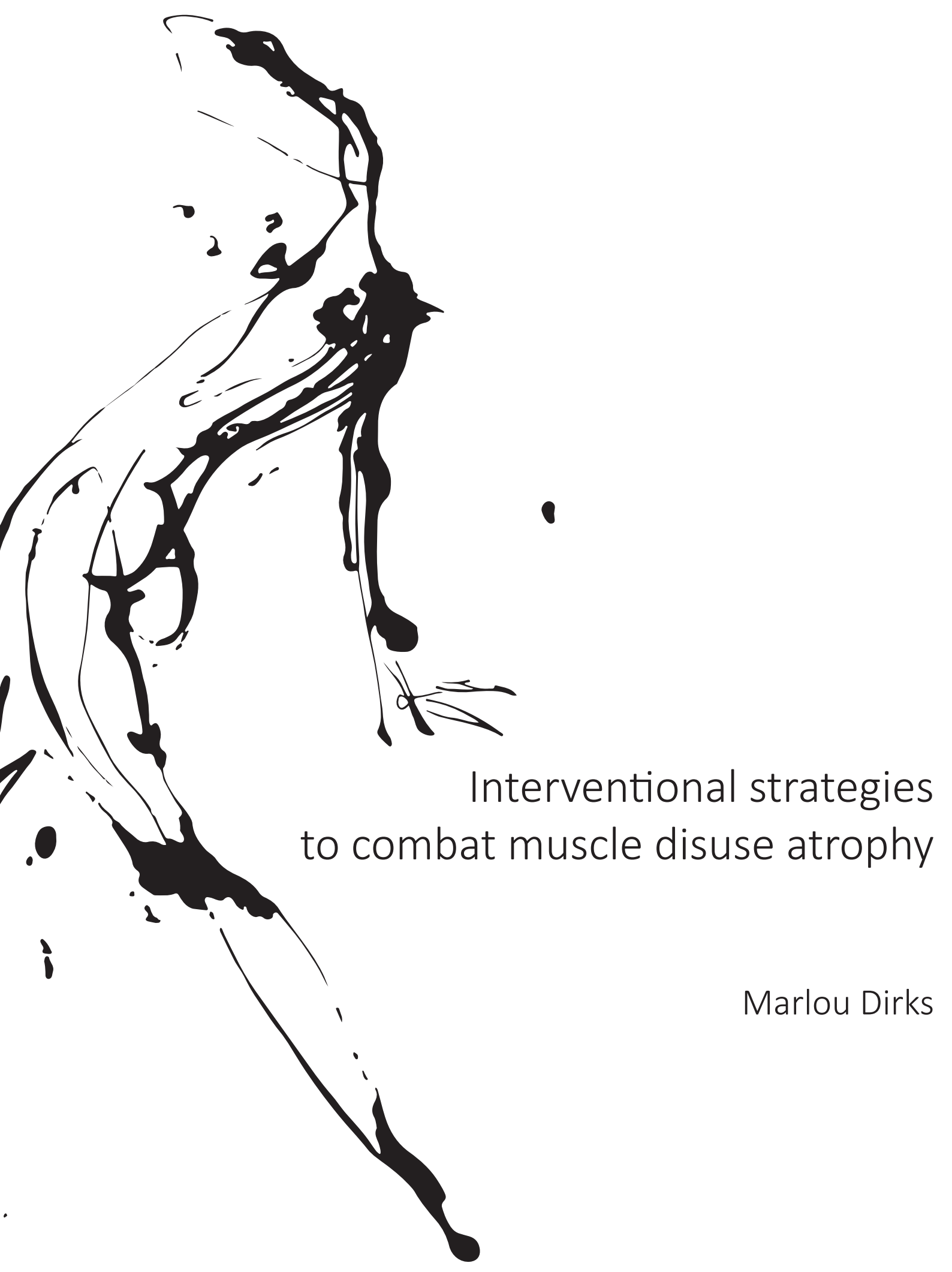



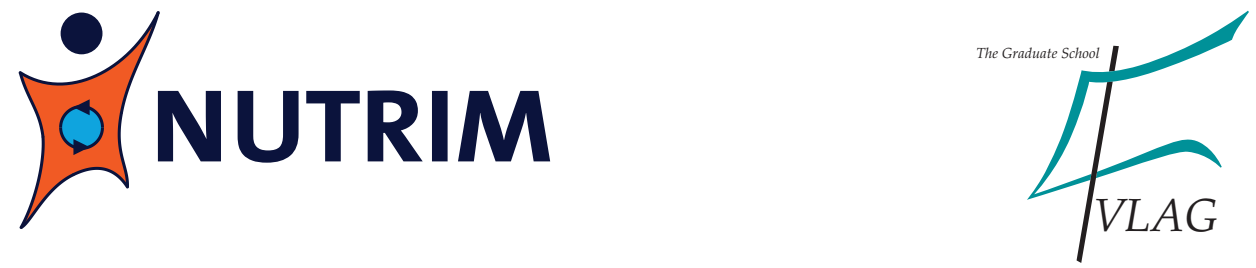

The work presented in this thesis was performed within NUTRIM, School of Nutrition and Translational Research in Metabolism, which participates in the Graduate School VLAG (Food Technology, Agrobiotechnology, Nutrition and Health Sciences), accredited by the Royal Netherlands Academy of Arts and Sciences.

(C) Copyright Marlou Dirks, Maastricht 2016

Cover image: Caleb Adams

Printing: Datawyse | Universitaire Pers Maastricht

ISBN 9789461595928

All rights reserved. No part of this thesis may be reproduced or transmitted in any form or by any means electronic, or mechanical, including photocopying, recording or any information storage or retrieval system, without permission from the author in writing, or when appropriate, from the publishers of the publications. 


\title{
Interventional strategies to combat muscle disuse atrophy
}

\author{
DISSERTATION \\ to obtain the degree of Doctor at Maastricht University, \\ on the authority of the Rector Magnificus, Prof. Dr. Rianne M. Letschert \\ in accordance with the decision of the Board of Deans, \\ to be defended in public \\ on Friday 18 November 2016, at 14:00 hours
}

by

Maria Louise Dirks 


\section{PROMOTOR}

Prof. dr. L.J.C. van Loon

\section{CO-PROMOTORS}

Dr. L.B. Verdijk

Dr. B.T. Wall (University of Exeter, UK)

\section{ASSESSMENT COMMITTEE}

Prof. dr. A.M.W.J. Schols (chair)

Prof. dr. E.E. Blaak

Prof. dr. C.H.C. Dejong

Prof. dr. C.P.G.M. de Groot (WUR, Wageningen)

Prof. dr. D. Paddon-Jones (UTMB, Galveston, TX, USA) 
Look at the patient lying alone in bed What a pathetic picture he makes.

The blood clotting in his veins.

The lime draining from his bones.

The scybola stacking up in his colon.

The flesh rotting from his seat. The urine leaking from his distended bladder and the spirit evaporating from his soul. Teach us to live that we may dread unnecessary time in bed. Get people up and we may save patients from an early grave.

Dr. Richard Asher, 1942 



\section{CONTENTS}

CHAPTER 1 General Introduction

CHAPTER 2 Substantial skeletal muscle loss occurs during only 5 days of disuse

CHAPTER 3 Short-term muscle disuse lowers myofibrillar protein synthesis rates and induces anabolic resistance to protein ingestion

CHAPTER 4 Neuromuscular electrical stimulation prevents muscle disuse atrophy during leg immobilization in humans

CHAPTER 5 Neuromuscular electrical stimulation prevents muscle wasting in critically ill, comatose patients

CHAPTER 6 Skeletal muscle disuse atrophy is not attenuated by dietary protein supplementation in healthy, older men

CHAPTER 7 A single session of neuromuscular electrical stimulation does not augment postprandial muscle protein accretion

CHAPTER 8 Neuromuscular electrical stimulation increases postprandial muscle protein accretion during overnight sleep

CHAPTER 9 The impact of bed rest versus leg immobilization on muscle disuse atrophy

CHAPTER 10 One week of bed rest leads to substantial muscle atrophy and induces whole-body insulin resistance in the absence of skeletal muscle lipid accumulation

CHAPTER 11 General Discussion

Summary

Samenvatting

Valorization

Dankwoord

Curriculum Vitae

List of publications 

CHAPTER

General Introduction 
Skeletal muscle mass is of great importance for overall health and well-being. Well known is the relevance of muscle tissue quantity to muscle strength, functional capacity and physical performance. However, the critical role of skeletal muscle mass in the metabolism of the macronutrients carbohydrate, fat, and protein is often underappreciated. Numerous situations, such as the recovery from illness or rehabilitation after injury, can necessitate a period of physical inactivity in otherwise healthy individuals. This muscle disuse is initiated to enhance the recovery process but is accompanied by muscle loss, termed muscle atrophy [1-3]. Muscle atrophy contributes to numerous negative health consequences including a loss of functional capacity and muscle strength [4-6], the development of insulin resistance [7, 8], a decline in basal metabolic rate $[9,10]$, and the accrual of body fat $[11,12]$. For these reasons, it is important to develop interventional strategies to attenuate atrophy of disused muscle. The present thesis will focus on experimentally induced muscle disuse and the underlying physiological mechanisms involved in muscle disuse atrophy. Particular emphasis will be placed on discussing potential nutritional or exercise-mimicking interventional strategies to reduce the loss of muscle mass during muscle disuse.

\section{Models of muscle disuse atrophy}

Muscle disuse is generally studied in healthy humans with various lab-based models. Models of local inactivity that have been used include arm or leg immobilization [3]. When the effect of whole-body disuse is to be assessed, researchers can choose for a head-down tilted bed rest setting, where volunteers are placed in a - 6 degrees headdown position to mimic microgravity [11]. Alternatively, a more clinically relevant bed rest setting can be inferred, during which volunteers are allowed to sit semi-upright, mimicking hospitalization [13]. While these approaches do not induce the contraindications associated with injury or illness, they provide a more controlled setting in which the impact of disuse per se can be examined. The current thesis will focus on results from leg immobilization and 'hospitalization' bed rest, as they are regarded as the most clinically relevant situations of experimental muscle disuse.

Leg immobilization is a model of local inactivity, induced by a leg cast or removable brace, which isolates muscle disuse in a single muscle or muscle group while the contralateral leg serves as a control. This model is of great practical value as participants are able to carry out their activities of daily life to a certain extent and maintain their habitual dietary intake. Due to the absence of systemic factors that could potentially influence muscle atrophy, leg immobilization represents a suitable model to study muscle loss caused by isolated disuse per se. In contrast, with the use of the bed rest model, muscle loss is possibly influenced by systemic factors that are activated due to wholebody disuse. Examples of processes that possibly impact on muscle atrophy during bed rest are increased systemic inflammation [14, 15], microvascular dysfunction [16], impairments in whole-body insulin sensitivity [8], and unfavourable hormonal changes [17, 
18], which all potentially contribute to a higher metabolic stress response. Whereas these factors influence muscle loss and consequently make it impossible to study the impact of disuse alone, the experimental bed rest model represents a more clinically relevant model as it directly translates to the hospitalized patient who is bedridden in an attempt to facilitate recovery. An experimental consideration of the bed rest model is that the decline in energy expenditure during whole-body inactivity [19] requires nutritional modification away from an individual's habitual diet [20, 21].

Due to the presence (during bed rest) or absence (during leg immobilization) of systemic factors that possibly modulate muscle atrophy, one can expect differences in the quantity of muscle loss such that bedridden participants will lose more muscle mass than immobilized individuals. Indeed, estimations based on previous studies show an approximate $0.5 \%$ daily decline in local muscle mass during limb immobilization, whereas an estimated $0.6 \%$ daily decrease in whole-body muscle mass is observed with bed rest (Figure 1; [3]). In contrast, previous work, comparing results from separate studies, has suggested that more muscle is lost with immobilization than with bed rest [22]. However, to date, no direct comparisons between both experimental models have been made.

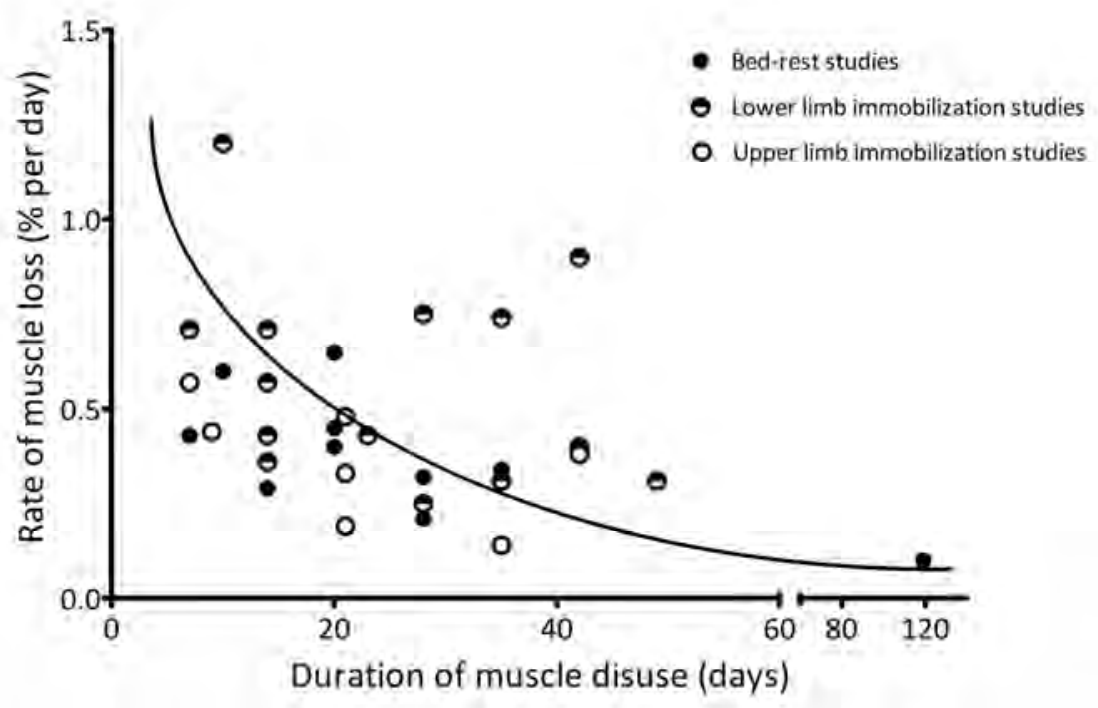

Figure 1 | Relationship between the duration of muscle disuse and the subsequent rate of muscle atrophy. The rate of muscle atrophy is expressed as percentage of muscle lost per day. Reproduced with permission [3].

Although researchers have attempted to extrapolate the impact of leg immobilization to a whole-body level, this extrapolation is difficult as different muscle groups show different rates of muscle (fibre) atrophy [23]. When choosing a model to study the impact of 
muscle disuse, all characteristics of the different models should be kept in mind in order to select the appropriate model to answer the specific research question.

\section{Regulation of muscle protein turnover during disuse}

Skeletal muscle proteins are constantly built up and broken down to maintain a good quality of skeletal muscle tissue. On a daily basis, this turnover of muscle proteins is approximately $1-2 \%$. The processes of muscle protein synthesis and muscle protein breakdown determine net muscle protein balance, and therefore dictate the quantity of muscle mass in an individual. When both processes are in equilibrium, the quantity of muscle mass will remain constant (Figure 2A).

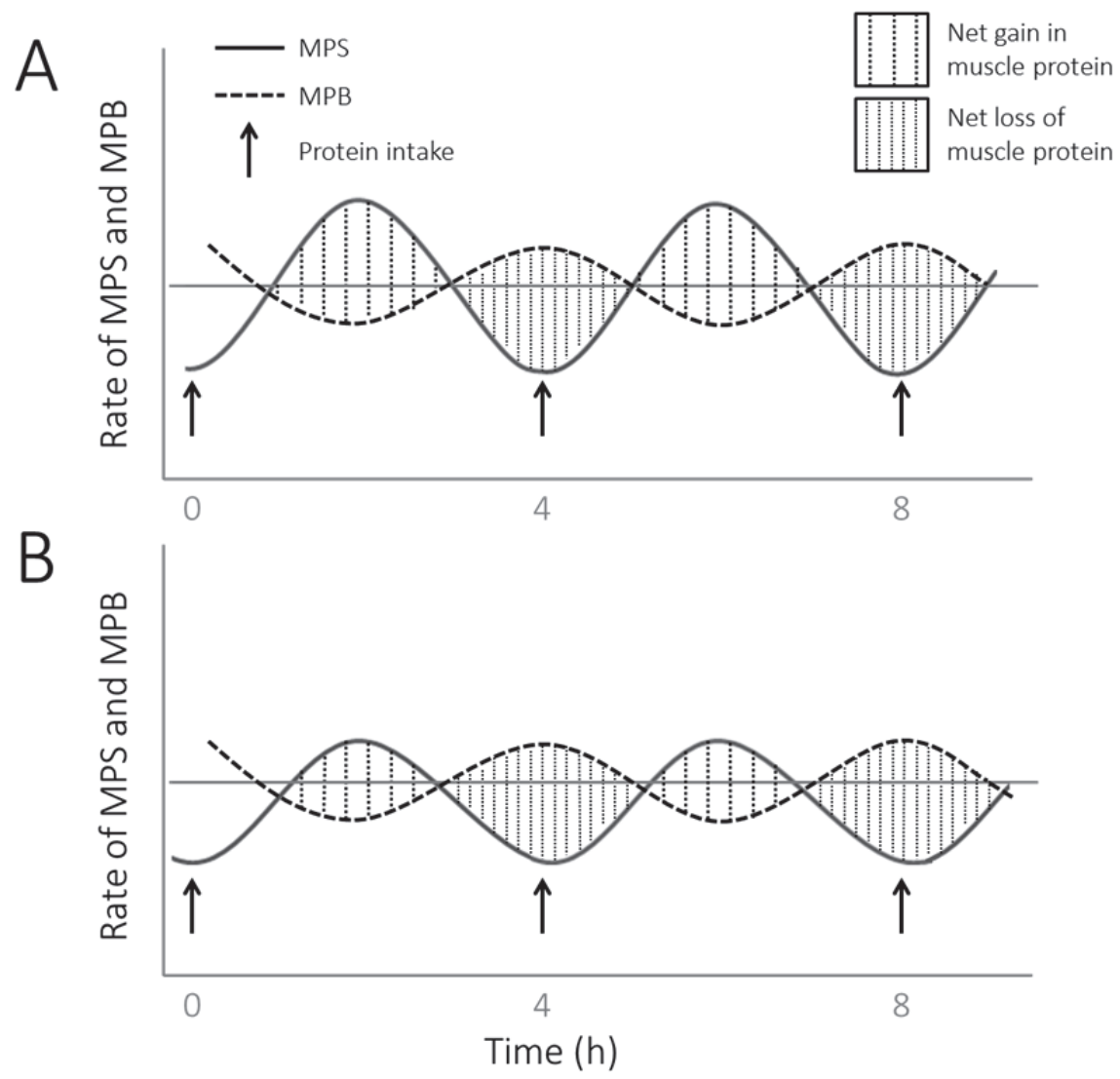

Figure 2 | Schematic representation of the processes of muscle protein synthesis (MPS, line) and muscle protein breakdown (MPB, dotted line). A: Normal state, in which both processes are in equilibrium and the net gain and loss of muscle protein are equal, resulting in muscle mass maintenance. B: During muscle disuse, the anabolic response to protein feeding is decreased while muscle protein breakdown is not significantly altered, resulting in a net loss of muscle protein. Adapted from [24]. 
Both muscle protein synthesis and muscle protein breakdown rates can be strongly modulated by food ingestion [25-27]. In a normal, rested individual in a postabsorptive state, whole-body net protein balance is negative [28-30]. Conversely, after food ingestion, muscle protein synthesis is stimulated and muscle protein breakdown is decreased, resulting in a net positive protein balance [28, 30, 31]. However, during a period of muscle disuse, there must be a (temporary) shift towards a more negative muscle protein balance, resulting in the net breakdown of muscle proteins. This change in protein net balance must occur due to a decline in muscle protein synthesis, an increase in muscle protein breakdown, or a combination of both.

Declines in basal (postabsorptive) muscle protein synthesis rates following a period of disuse have been shown multiple times. A $25-50 \%$ decrease in basal muscle protein synthesis rates has been reported following both leg immobilization [5, 32-34] and bed rest $[11,35-38]$ in young, healthy volunteers. Given the magnitude of the decline in muscle protein synthesis following muscle disuse, multiple research groups have suggested that muscle protein breakdown could not have changed substantially, thereby ruling out a major role for changes in muscle protein breakdown in the development of muscle disuse atrophy $[3,5,11,24,39,40]$. Measuring muscle protein breakdown in vivo in humans is technically challenging and rates of muscle protein breakdown are therefore relatively sparsely reported. Two studies have reported no changes in muscle protein breakdown rates following several weeks of bed rest $[11,37]$. Conversely, several studies using static markers of muscle protein breakdown suggest a rise in muscle protein breakdown immediately following the onset of muscle disuse [32, 41, 42]. Taken together, the current literature suggests that muscle disuse atrophy occurs due to a decline in basal muscle protein synthesis rates, accompanied by a concomitant decline in muscle protein breakdown [40]. Evidence suggests that muscle protein breakdown could be upregulated in the first few days of muscle disuse [40], however, this should be confirmed by in vivo human intervention studies using methods to directly measure muscle protein breakdown rates.

Muscle mass maintenance is influenced by basal muscle protein synthesis as well as by the postprandial muscle protein synthetic response to feeding [30]. More recent work has provided evidence to show that disuse reduces the sensitivity of skeletal muscle tissue to the anabolic properties of amino acid or protein administration. Specifically, an attenuated rise in whole-body protein synthesis following food intake was observed in bed-rested individuals when compared to an ambulatory group [43, 44]. In concurrence, 1-2 weeks of disuse reduced the postprandial muscle protein synthetic response to both amino acid infusion [34] and essential amino acid ingestion [45]. In agreement, we have shown comparable declines in postprandial muscle protein synthesis rates after ingestion of a bolus of intact protein following 14 days of leg immobilization [46]. These results imply that a blunted muscle protein synthetic response following dietary protein ingestion, also termed 'anabolic resistance', plays an important role in the muscle atrophy that is observed during disuse (Figure 2B). Whether or not post- 
prandial muscle protein breakdown rates are also affected following muscle disuse, and therefore contribute to the observed muscle loss, remains to be established.

Postprandial protein handling and the subsequent muscle protein synthetic response are regulated on various levels $[47,48]$. Their regulation ranges from protein digestion and amino acid absorption [49], the insulin-stimulated (postprandial) increase in muscle tissue perfusion [50, 51], the uptake of amino acids in skeletal muscle [52], intramyofibrillar anabolic signalling $[53,54]$, to the increase in muscle protein synthesis rate and inhibition of muscle protein breakdown [55]. Many of these factors may be affected during a period of disuse, resulting in altered rates of postabsorptive as well as postprandial muscle protein synthesis and breakdown. Muscle disuse, and particularly (head-down tilt) bed rest, may also modulate the digestion and absorption of ingested foods $[11,56]$. Additionally, physical inactivity could lead to impairments in insulinmediated tissue perfusion, as has been demonstrated in older men presenting anabolic resistance [50]. This would impair amino acid delivery to the muscle and attenuate the postprandial rise in muscle protein synthesis rates. Other researchers pointed out that amino acid uptake in muscle may also be affected by an attenuated postprandial rise in amino acid transporter protein content following a period of bed rest [45]. Currently, the contribution of these individual factors to disuse-associated anabolic resistance remains to be elucidated. However, irrespective of where anabolic resistance actually resides, it is clear that interventional strategies to preserve muscle mass during disuse should aim to increase both postabsorptive muscle protein synthesis rates as well as improve the muscle protein synthetic response to feeding.

\section{Interventions to prevent muscle loss during disuse}

The two key anabolic stimuli for skeletal muscle tissue are muscle contraction [57] and food intake [30]. Indeed, re-introduction of some level of physical activity can already preserve skeletal muscle mass during a period of disuse [58, 59]. However, in some cases an increase in physical activity is simply not realistic due to limb immobilization or general weakness. In those cases, neuromuscular electrical stimulation (NMES) can be used as an exercise mimetic by evoking muscle contractions. By means of placing one or more electrodes on the skin and applying an electric current, the muscle contracts involuntarily. Previous work from our group showed that NMES can be applied to increase postabsorptive muscle protein synthesis rates in vivo in humans [60]. Although previous studies have successfully applied NMES in prolonged models of disuse atrophy [33], it is currently unclear if NMES can preserve muscle mass during short periods of muscle disuse. Aside from physical activity, dietary protein consumption has been shown essential to maintain skeletal muscle mass in a disuse setting [3]. In the past, high-dose essential amino acid supplementation has been shown to attenuate the loss of muscle mass during prolonged bed rest in young [13, 61, 62] and older [38] individuals. However, it remains to be established whether an increase in dietary protein consumption can alle- 
viate muscle mass losses in the older population. Furthermore, the synergy between physical activity and food intake in driving postprandial muscle protein synthesis [63-67] indicates that interventional strategies should combine the benefits of exercise or exercise mimetics with the anabolic properties of amino acid or protein feeding to prevent or attenuate muscle disuse atrophy. 


\section{THESIS OUTLINE}

This thesis describes a collection of studies that characterize the impact of short-term muscle disuse on muscle mass and function, and examine the efficacy of various interventional strategies to attenuate the loss of muscle mass and strength. We start by assessing the impact of short-term disuse, which plays an important role in the loss of muscle mass and strength with ageing, termed sarcopenia. Chapter 2 compares the impact of 5 versus 14 days of leg immobilization on muscle mass and strength in young, healthy males, and shows that merely 5 days of muscle disuse already leads to a substantial decline in both muscle mass and strength. Therefore, in Chapter 3 we describe a study where we applied such a short period of disuse to assess its impact on postabsorptive and postprandial muscle protein synthesis rates. The subsequent chapters investigate various interventional strategies to prevent the loss of muscle mass and strength during short-term disuse. We start off by examining whether NMES can be used as an exercise mimetic to attenuate muscle loss during leg immobilization (Chapter 4). We also assessed the efficacy of NMES in a clinical model of critically ill, comatose patients, who are suffering from severe muscle wasting (Chapter 5). We demonstrate that NMES can prevent muscle atrophy in a setting of one-legged knee immobilization as well as prevent muscle wasting in comatose patients. Chapter 6 describes the efficacy of a nutritional intervention to attenuate the decline in muscle mass and strength during a short period of leg immobilization in older men. Subsequently, we combined both nutritional and exercise (mimetic) stimuli in two parallel studies (Chapter 7 and 8). Chapter 7 describes a study assessing the surplus effect of NMES prior to ingestion of a meallike amount of protein on muscle protein accretion in active, older individuals. To translate these findings to a more clinically relevant setting, the combined impact of NMES and protein ingestion was tested in in a group of healthy older men during a day of bed rest (Chapter 8). Chapter 9 discusses the differences between the two most widely used experimental models of muscle disuse (i.e. bed rest and immobilization). Though muscle atrophy seems comparable between both models, bed rest is shown to have substantial impact on metabolic health. Chapter 10 describes the last study in this thesis where we assessed the impact of one week of bed rest on both muscle mass and metabolic health. Finally, in Chapter 11, the outcomes of Chapters 2 through 10 are discussed and a general conclusion is provided. Additionally, the implications of this thesis are discussed and directions for future research are formulated. As such, this thesis forms an overview of interventional strategies that can attenuate muscle loss during disuse, and provides targets for future research to prevent and treat muscle disuse atrophy. 


\section{REFERENCES}

1. Ingemann-Hansen, T. and J. Halkjaer-Kristensen, Computerized tomographic determination of human thigh components. The effects of immobilization in plaster and subsequent physical training. Scand J Rehabil Med, 1980. 12(1): p. 27-31.

2. Wall, B.T., M.L. Dirks, T. Snijders, F.B. Stephens, J.M. Senden, M.L. Verscheijden, and L.J. van Loon, Shortterm muscle disuse atrophy is not associated with increased intramuscular lipid deposition or a decline in the maximal activity of key mitochondrial enzymes in young and older males. Exp Gerontol, 2015. 61: p. $76-83$.

3. Wall, B.T. and L.J. van Loon, Nutritional strategies to attenuate muscle disuse atrophy. Nutr Rev, 2013. 71(4): p. 195-208.

4. Deitrick, J.E., The effect of immobilization on metabolic and physiological functions of normal men. Bull $\mathrm{N}$ Y Acad Med, 1948. 24(6): p. 364-75.

5. Gibson, J.N., D. Halliday, W.L. Morrison, P.J. Stoward, G.A. Hornsby, P.W. Watt, G. Murdoch, and M.J. Rennie, Decrease in human quadriceps muscle protein turnover consequent upon leg immobilization. Clin Sci (Lond), 1987. 72(4): p. 503-9.

6. White, M.J., C.T. Davies, and P. Brooksby, The effects of short-term voluntary immobilization on the contractile properties of the human triceps surae. Q J Exp Physiol, 1984. 69(4): p. 685-91.

7. Stuart, C.A., R.E. Shangraw, M.J. Prince, E.J. Peters, and R.R. Wolfe, Bed-rest-induced insulin resistance occurs primarily in muscle. Metab Clin Exp, 1988. 37(8): p. 802-6.

8. Bergouignan, A., F. Rudwill, C. Simon, and S. Blanc, Physical inactivity as the culprit of metabolic inflexibility: evidence from bed-rest studies. J Appl Physiol, 2011. 111(4): p. 1201-10.

9. Haruna, Y., Y. Suzuki, K. Kawakubo, R. Yanagibori, and A. Gunji, Decremental reset in basal metabolism during 20-days bed rest. Acta Physiol (Scand). Supplementum, 1994. 616: p. 43-9.

10. Tzankoff, S.P. and A.H. Norris, Effect of muscle mass decrease on age-related BMR changes. J Appl Physiol Respir Environ Exerc Physiol, 1977. 43(6): p. 1001-6.

11. Ferrando, A.A., H.W. Lane, C.A. Stuart, J. Davis-Street, and R.R. Wolfe, Prolonged bed rest decreases skeletal muscle and whole body protein synthesis. Am J Physiol, 1996. 270(4 Pt 1): p. E627-33.

12. Brooks, N., G.J. Cloutier, S.M. Cadena, J.E. Layne, C.A. Nelsen, A.M. Freed, R. Roubenoff, and C. Castaneda-Sceppa, Resistance training and timed essential amino acids protect against the loss of muscle mass and strength during 28 days of bed rest and energy deficit. J Appl Physiol, 2008. 105(1): p. 241-8.

13. Paddon-Jones, D., M. Sheffield-Moore, R.J. Urban, A.P. Sanford, A. Aarsland, R.R. Wolfe, and A.A. Ferrando, Essential amino acid and carbohydrate supplementation ameliorates muscle protein loss in humans during 28 days bedrest. J Clin Endocrinol Metab, 2004. 89(9): p. 4351-8.

14. Mutin-Carnino, M., A. Carnino, S. Roffino, and A. Chopard, Effect of muscle unloading, reloading and exercise on inflammation during a head-down bed rest. Int J Sports Med, 2014. 35(1): p. 28-34.

15. Drummond, M.J., K.L. Timmerman, M.M. Markofski, D.K. Walker, J.M. Dickinson, M. J Am Med Assocluddin, A.R. Brasier, B.B. Rasmussen, and E. Volpi, Short-term bed rest increases TLR4 and IL-6 expression in skeletal muscle of older adults. Am J Physiol Regul Integr Comp Physiol, 2013. 305(3): p. R216-23.

16. Hamburg, N.M., C.J. McMackin, A.L. Huang, S.M. Shenouda, M.E. Widlansky, E. Schulz, N. Gokce, N.B. Ruderman, J.F. Keaney, Jr., and J.A. Vita, Physical inactivity rapidly induces insulin resistance and microvascular dysfunction in healthy volunteers. Arterioscler Thromb Vasc Biol, 2007. 27(12): p. 2650-6.

17. Guerra, B., J.G. Ponce-Gonzalez, D. Morales-Alamo, A. Guadalupe-Grau, K. Kiilerich, T. Fuentes, S. Ringholm, R.S. Bienso, A. Santana, C. Lundby, H. Pilegaard, and J.A. Calbet, Leptin signaling in skeletal muscle after bed rest in healthy humans. Eur J Appl Physiol, 2014. 114(2): p. 345-57.

18. Chouker, A., B. Feuerecker, S. Matzel, I. Kaufmann, C. Strewe, M. Hoerl, G. Schelling, and M. Feuerecker, Psychoneuroendocrine alterations during 5 days of head-down tilt bed rest and artificial gravity interventions. Eur J Appl Physiol, 2013. 113(8): p. 2057-65. 
19. Blanc, S., S. Normand, P. Ritz, C. Pachiaudi, L. Vico, C. Gharib, and G. Gauquelin-Koch, Energy and water metabolism, body composition, and hormonal changes induced by 42 days of enforced inactivity and simulated weightlessness. J Clin Endocrinol Metab, 1998. 83(12): p. 4289-97.

20. Weiss, E.P., S.B. Racette, D.T. Villareal, L. Fontana, K. Steger-May, K.B. Schechtman, S. Klein, A.A. Ehsani, J.O. Holloszy, and C.G. Washington University School of Medicine, Lower extremity muscle size and strength and aerobic capacity decrease with caloric restriction but not with exercise-induced weight loss. J Appl Physiol, 2007. 102(2): p. 634-40.

21. Biolo, G., B. Ciocchi, M. Stulle, A. Bosutti, R. Barazzoni, M. Zanetti, R. Antonione, M. Lebenstedt, P. Platen, M. Heer, and G. Guarnieri, Calorie restriction accelerates the catabolism of lean body mass during 2 wk of bed rest. Am J Clin Nutr, 2007. 86(2): p. 366-72.

22. Clark, B.C., In vivo alterations in skeletal muscle form and function after disuse atrophy. Med Sci Sports Exerc, 2009. 41(10): p. 1869-75.

23. LeBlanc, A.D., V.S. Schneider, H.J. Evans, C. Pientok, R. Rowe, and E. Spector, Regional changes in muscle mass following 17 weeks of bed rest. J Appl Physiol, 1992. 73(5): p. 2172-8.

24. Phillips, S.M., E.I. Glover, and M.J. Rennie, Alterations of protein turnover underlying disuse atrophy in human skeletal muscle. J Appl Physiol, 2009. 107(3): p. 645-54.

25. Volpi, E., M. Sheffield-Moore, B.B. Rasmussen, and R.R. Wolfe, Basal muscle amino acid kinetics and protein synthesis in healthy young and older men. J Am Med Assoc, 2001. 286(10): p. 1206-12.

26. Banos, G., P.M. Daniel, S.R. Moorhouse, and O.E. Pratt, The movement of amino acids between blood and skeletal muscle in the rat. J Physiol, 1973. 235(2): p. 459-75.

27. Silk, D.B., G.K. Grimble, and R.G. Rees, Protein digestion and amino acid and peptide absorption. Proc Nutr Soc, 1985. 44(1): p. 63-72.

28. Paddon-Jones, D., M. Sheffield-Moore, X.J. Zhang, E. Volpi, S.E. Wolf, A. Aarsland, A.A. Ferrando, and R.R. Wolfe, Amino acid ingestion improves muscle protein synthesis in the young and elderly. Am J Physiol Endocrinol Metab, 2004. 286(3): p. E321-8.

29. Phillips, S.M., K.D. Tipton, A. Aarsland, S.E. Wolf, and R.R. Wolfe, Mixed muscle protein synthesis and breakdown after resistance exercise in humans. Am J Physiol, 1997. 273(1 Pt 1): p. E99-107.

30. Rennie, M.J., R.H. Edwards, D. Halliday, D.E. Matthews, S.L. Wolman, and D.J. Millward, Muscle protein synthesis measured by stable isotope techniques in man: the effects of feeding and fasting. Clin Sci (Lond), 1982. 63(6): p. 519-23.

31. Gorissen, S.H., N.A. Burd, H.M. Hamer, A.P. Gijsen, B.B. Groen, and L.J. van Loon, Carbohydrate coingestion delays dietary protein digestion and absorption but does not modulate postprandial muscle protein accretion. J Clin Endocrinol Metab, 2014. 99(6): p. 2250-8.

32. de Boer, M.D., A. Selby, P. Atherton, K. Smith, O.R. Seynnes, C.N. Maganaris, N. Maffulli, T. Movin, M.V. Narici, and M.J. Rennie, The temporal responses of protein synthesis, gene expression and cell signalling in human quadriceps muscle and patellar tendon to disuse. J Physiol, 2007. 585(Pt 1): p. 241-51.

33. Gibson, J.N., K. Smith, and M.J. Rennie, Prevention of disuse muscle atrophy by means of electrical stimulation: maintenance of protein synthesis. Lancet, 1988. 2(8614): p. 767-70.

34. Glover, E.I., S.M. Phillips, B.R. Oates, J.E. Tang, M.A. Tarnopolsky, A. Selby, K. Smith, and M.J. Rennie, Immobilization induces anabolic resistance in human myofibrillar protein synthesis with low and high dose amino acid infusion. J Physiol, 2008. 586(Pt 24): p. 6049-61.

35. Ferrando, A.A., K.D. Tipton, M.M. Bamman, and R.R. Wolfe, Resistance exercise maintains skeletal muscle protein synthesis during bed rest. J Appl Physiol, 1997. 82(3): p. 807-10.

36. Kortebein, P., A. Ferrando, J. Lombeida, R. Wolfe, and W.J. Evans, Effect of 10 days of bed rest on skeletal muscle in healthy older adults. J Am Med Assoc, 2007. 297(16): p. 1772-4.

37. Symons, T.B., M. Sheffield-Moore, D.L. Chinkes, A.A. Ferrando, and D. Paddon-Jones, Artificial gravity maintains skeletal muscle protein synthesis during 21 days of simulated microgravity. J Appl Physiol, 2009. 107(1): p. 34-8.

38. Ferrando, A.A., D. Paddon-Jones, N.P. Hays, P. Kortebein, O. Ronsen, R.H. Williams, A. McComb, T.B. Symons, R.R. Wolfe, and W. Evans, EAA supplementation to increase nitrogen intake improves muscle function during bed rest in the elderly. Clin Nutr, 2010. 29(1): p. 18-23. 
39. Rennie, M.J., A. Selby, P. Atherton, K. Smith, V. Kumar, E.L. Glover, and S.M. Philips, Facts, noise and wishful thinking: muscle protein turnover in aging and human disuse atrophy. Scand J Med Sci Sports, 2010. 20(1): p. 5-9.

40. Wall, B.T., M.L. Dirks, and L.J. van Loon, Skeletal muscle atrophy during short-term disuse: implications for age-related sarcopenia. Ageing Res Rev, 2013. 12(4): p. 898-906.

41. Dirks, M.L., B.T. Wall, T. Snijders, C.L. Ottenbros, L.B. Verdijk, and L.J. van Loon, Neuromuscular electrical stimulation prevents muscle disuse atrophy during leg immobilization in humans. Acta Physiol (Oxf), 2014. 210(3): p. 628-41.

42. Bodine, S.C., E. Latres, S. Baumhueter, V.K. Lai, L. Nunez, B.A. Clarke, W.T. Poueymirou, F.J. Panaro, E. Na, K. Dharmarajan, Z.Q. Pan, D.M. Valenzuela, T.M. DeChiara, T.N. Stitt, G.D. Yancopoulos, and D.J. Glass, Identification of ubiquitin ligases required for skeletal muscle atrophy. Science, 2001. 294(5547): p. 1704-8.

43. Biolo, G., B. Ciocchi, M. Lebenstedt, R. Barazzoni, M. Zanetti, P. Platen, M. Heer, and G. Guarnieri, Shortterm bed rest impairs amino acid-induced protein anabolism in humans. J Physiol, 2004. 558(Pt 2): p. 381-8.

44. Biolo, G., B. Ciocchi, M. Lebenstedt, M. Heer, and G. Guarnieri, Sensitivity of whole body protein synthesis to amino acid administration during short-term bed rest. J Gravit Physiol, 2002. 9(1): p. P197-8.

45. Drummond, M.J., J.M. Dickinson, C.S. Fry, D.K. Walker, D.M. Gundermann, P.T. Reidy, K.L. Timmerman, M.M. Markofski, D. Paddon-Jones, B.B. Rasmussen, and E. Volpi, Bed rest impairs skeletal muscle amino acid transporter expression, mTORC1 signaling, and protein synthesis in response to essential amino acids in older adults. Am j Physiol Endocrinol Metab, 2012. 302(9): p. E1113-22.

46. Wall, B.T., T. Snijders, J.M. Senden, C.L. Ottenbros, A.P. Gijsen, L.B. Verdijk, and L.J. van Loon, Disuse impairs the muscle protein synthetic response to protein ingestion in healthy men. J Clin Endocrinol Metab, 2013. 98(12): p. 4872-81.

47. Burd, N.A., S.H. Gorissen, and L.J. van Loon, Anabolic resistance of muscle protein synthesis with aging. Exerc Sport Sci Rev, 2013. 41(3): p. 169-73.

48. Groen, B.B., A.M. Horstman, H.M. Hamer, M. de Haan, J. van Kranenburg, J. Bierau, M. Poeze, W.K. Wodzig, B.B. Rasmussen, and L.J. van Loon, Post-Prandial Protein Handling: You Are What You Just Ate. PLoS One, 2015. 10(11): p. e0141582.

49. Rodriguez, P.O., M. Setten, L.P. Maskin, I. Bonelli, S.R. Vidomlansky, S. Attie, S.L. Frosiani, S. Kozima, and R. Valentini, Muscle weakness in septic patients requiring mechanical ventilation: protective effect of transcutaneous neuromuscular electrical stimulation. J Crit Care, 2012. 27(3): p. 319 e1-8.

50. Rasmussen, B.B., S. Fujita, R.R. Wolfe, B. Mittendorfer, M. Roy, V.L. Rowe, and E. Volpi, Insulin resistance of muscle protein metabolism in aging. FASEB J, 2006. 20(6): p. 768-9.

51. Zhang, H., K.C. Dellsperger, and C. Zhang, The link between metabolic abnormalities and endothelial dysfunction in type 2 diabetes: an update. Basic Res Cardiol, 2012. 107(1): p. 237.

52. English, K.L. and D. Paddon-Jones, Protecting muscle mass and function in older adults during bed rest. Curr Opin Clin Nutr Metab Care, 2010. 13(1): p. 34-9.

53. Cuthbertson, D., K. Smith, J. Babraj, G. Leese, T. Waddell, P. Atherton, H. Wackerhage, P.M. Taylor, and M.J. Rennie, Anabolic signaling deficits underlie amino acid resistance of wasting, aging muscle. FASEB J, 2005. 19(3): p. 422-4.

54. Fry, C.S., M.J. Drummond, E.L. Glynn, J.M. Dickinson, D.M. Gundermann, K.L. Timmerman, D.K. Walker, S. Dhanani, E. Volpi, and B.B. Rasmussen, Aging impairs contraction-induced human skeletal muscle mTORC1 signaling and protein synthesis. Skelet Muscle, 2011. 1(1): p. 11.

55. Volpi, E., A.A. Ferrando, C.W. Yeckel, K.D. Tipton, and R.R. Wolfe, Exogenous amino acids stimulate net muscle protein synthesis in the elderly. J Clin Invest, 1998. 101(9): p. 2000-7.

56. Idkaidek, N. and T. Arafat, Effect of microgravity on the pharmacokinetics of Ibuprofen in humans. J Clin Pharmacol, 2011. 51(12): p. 1685-9.

57. Koopman, R. and L.J. van Loon, Aging, exercise, and muscle protein metabolism. J Appl Physiol, 2009. 106(6): p. 2040-8. 
58. Bamman, M.M., M.S. Clarke, D.L. Feeback, R.J. Talmadge, B.R. Stevens, S.A. Lieberman, and M.C. Greenisen, Impact of resistance exercise during bed rest on skeletal muscle sarcopenia and myosin isoform distribution. J Appl Physiol, 1998. 84(1): p. 157-63.

59. Oates, B.R., E.I. Glover, D.W. West, J.L. Fry, M.A. Tarnopolsky, and S.M. Phillips, Low-volume resistance exercise attenuates the decline in strength and muscle mass associated with immobilization. Muscle Nerve, 2010. 42(4): p. 539-46.

60. Wall, B.T., M.L. Dirks, L.B. Verdijk, T. Snijders, D. Hansen, P. Vranckx, N.A. Burd, P. Dendale, and L.J. van Loon, Neuromuscular electrical stimulation increases muscle protein synthesis in elderly type 2 diabetic men. Am J Physiol Endocrinol Metab, 2012. 303(5): p. E614-23.

61. Stein, T.P., M.R. Donaldson, M.J. Leskiw, M.D. Schluter, D.W. Baggett, and G. Boden, Branched-chain amino acid supplementation during bed rest: effect on recovery. J Appl Physiol, 2003. 94(4): p. 1345-52.

62. Stuart, C.A., R.E. Shangraw, E.J. Peters, and R.R. Wolfe, Effect of dietary protein on bed-rest-related changes in whole-body-protein synthesis. Am J Clin Nutr, 1990. 52(3): p. 509-14.

63. Pennings, B., R. Koopman, M. Beelen, J.M.G. Senden, W.H.M. Saris, and L.J.C. van Loon, Exercising before protein intake allows for greater use of dietary protein-derived amino acids for de novo muscle protein synthesis in both young and elderly men. Am J Clin Nutr, 2011. 93(2): p. 322-331.

64. Burd, N.A., Y. Yang, D.R. Moore, J.E. Tang, M.A. Tarnopolsky, and S.M. Phillips, Greater stimulation of myofibrillar protein synthesis with ingestion of whey protein isolate $\mathrm{v}$. micellar casein at rest and after resistance exercise in elderly men. Br J Nutr, 2012. 108(6): p. 958-62.

65. Tang, J.E., D.R. Moore, G.W. Kujbida, M.A. Tarnopolsky, and S.M. Phillips, Ingestion of whey hydrolysate, casein, or soy protein isolate: effects on mixed muscle protein synthesis at rest and following resistance exercise in young men. J Appl Physiol, 2009. 107(3): p. 987-92.

66. Witard, O.C., M. Tieland, M. Beelen, K.D. Tipton, L.J. van Loon, and R. Koopman, Resistance exercise increases postprandial muscle protein synthesis in humans. Med Sci Sports Exer, 2009. 41(1): p. 144-54.

67. Yang, Y., L. Breen, N.A. Burd, A.J. Hector, T.A. Churchward-Venne, A.R. Josse, M.A. Tarnopolsky, and S.M. Phillips, Resistance exercise enhances myofibrillar protein synthesis with graded intakes of whey protein in older men. Brit J Nutr, 2012: p. 1-9. 


\title{
CHAPTER
}

Substantial skeletal muscle loss occurs during only 5 days of disuse

\author{
Benjamin T Wall \\ Marlou L Dirks \\ Tim Snijders \\ Joan M Senden \\ John Dolmans \\ Luc JC van Loon
}

Acta Physiol 2014 March;210(3):600-11 
ABSTRACT

Background The impact of disuse on the loss of skeletal muscle mass and strength has been well documented. Given that most studies have investigated muscle atrophy after more than 2 weeks of disuse, few data are available on the impact of shorter periods of disuse. We assessed the impact of 5 and 14 days of disuse on skeletal muscle mass, strength and associated intramuscular molecular signaling responses.

Methods Twenty-four healthy, young ( $23 \pm 1 \mathrm{y}$ ) males were subjected to either 5 $(n=12)$ or $14(n=12)$ days of one-legged knee immobilization using a full leg cast. Before and immediately after the immobilization period, quadriceps muscle cross-sectional area (CSA), leg lean mass and muscle strength were assessed, and biopsies were collected from the vastus lateralis.

Results Quadriceps muscle CSA declined from baseline by $3.5 \pm 0.5(P<0.0001)$ and $8.4 \pm 2.8 \%(P<0.0001)$, leg lean mass was reduced by $1.4 \pm 0.7$ $(P=0.07)$ and $3.1 \pm 0.7 \%(P<0.01)$, and strength decreased by $9.0 \pm 2.3$ $(P<0.0001)$ and $22.9 \pm 2.6 \%(P<0.0001)$ following 5 and 14 days of immobilization, respectively. Muscle myostatin mRNA expression doubled following immobilization $(P<0.05)$ in both groups, while the myostatin precursor isoform protein content decreased after 14 days only $(P<0.05)$. Muscle MAFBx mRNA expression increased from baseline by a similar magnitude following either 5 or 14 days of disuse, whereas MuRF1 mRNA expression had increased significantly only after 5 days.

Conclusion We conclude that even short periods of muscle disuse can cause substantial loss of skeletal muscle mass and strength, and are accompanied by an early catabolic molecular signaling response. 


\section{INTRODUCTION}

The recovery from illness or injury often requires otherwise healthy humans to undergo a period of muscle disuse (e.g. bed rest or limb immobilization). A major consequence of disuse is skeletal muscle atrophy [1-3]. The ensuing impairments in muscle function [1-5], metabolic rate [6, 7] and insulin sensitivity [8], and accrual of body fat mass [9-11] following two or more weeks of muscle disuse have been well documented.

Studies investigating muscle disuse atrophy generally employ relatively long experimental periods, ranging from two to as long as 17 weeks of bed rest or limb immobilization $[2,5,12,13]$. However, over the last decade, efforts have been made within healthcare systems to reduce the duration of bed rest/immobilization that patients endure due to illness or injury. At present, the average length of hospitalization for elderly patients admitted with acute illness is only 5-6 days [14]. Moreover, periods of illness and minor injury that do not require hospitalization but necessitate inactive recovery at home generally last only a few days. Recent work has reported that limb immobilization for only four days already causes a decline in functional strength in older adults $[15,16]$. However, currently, no data exist on the impact of such short periods of disuse (i.e. $<7$ days) on skeletal muscle mass. Such information is of important clinical relevance since it has been hypothesized that successive bouts of muscle loss during short periods of disuse accumulate throughout the lifespan and may be responsible for much of the loss of muscle mass typically observed in the ageing population [17-19]. Accordingly, we hypothesize that even short periods of muscle disuse can lead to substantial loss of muscle tissue. Therefore, in the present study, we compared the impact of a short (i.e. 5 days) versus more prolonged (i.e. 14 days) period of immobilization on skeletal muscle mass and functional strength.

Skeletal muscle atrophy during more prolonged disuse (i.e. $>10$ days) is attributed to declines in both the rate of postabsorptive and postprandial muscle protein synthesis, without any apparent changes in muscle protein breakdown rates $[2,10,17,20]$. We $[17,18]$, and others $[15,21-23]$, have argued that elevated muscle protein breakdown may also contribute to muscle loss during the first few days of disuse. Myostatin has been demonstrated to be a key negative regulator of muscle mass in animals [24, 25] and humans [26]. Aside from its documented role in the regulation of muscle protein synthesis [27] and myogenesis [28, 29], myostatin has also been suggested as a regulator of muscle protein breakdown [30]. Therefore, in the present study, we also assessed the impact of 5 and 14 days of limb immobilization on the skeletal muscle mRNA and protein expression of myostatin and associated genes.

In the present study we selected 24 healthy, young men who were subjected to either $5(n=12)$ or $14(n=12)$ days of one-legged knee immobilization. Skeletal muscle mass and function were assessed before and after immobilization, and muscle biopsies were collected to assess the associated molecular signaling responses. This is the first study to report substantial muscle atrophy after merely 5 days of muscle disuse in vivo in humans. 


\section{METHODS}

\section{Subjects}

Twenty-four healthy, young $(23 \pm 1$ y) men volunteered to participate in the present study. All subjects were fully informed of the nature and possible risks of the experimental procedures, before their written informed consent was obtained. Subjects were screened to exclude any person with lower limb and/or back injuries sustained within a year prior to beginning the study, a (family) history of thrombosis/cardiovascular disease, use of anti-coagulants, musculoskeletal/orthopaedic/haemostatic disorders, or participation in any regular resistance training program within 6 months of beginning the study. During screening, all subjects were instructed and familiarized with safe lifting technique for the leg extension exercise. Maximum strength was assessed using the multiple repetitions testing procedure [31] for each leg separately. The study was approved by the Medical Ethics Committee of the Maastricht University Medical Centre, Maastricht, the Netherlands. The present study is part of a greater project investigating muscle disuse atrophy in humans.

\section{Experimental design}

In the present study a parallel design was applied with a subject cohort being assigned to an experimental treatment in which subjects underwent either 5 or 14 days of onelegged knee immobilization by means of a full leg cast. Before and after the immobilization period, computed tomography (CT) scans, dual-energy x-ray absorptiometry (DXA) scans and 1 repetition max (1RM) tests were performed to determine changes in muscle mass and strength, and muscle biopsies were collected to assess changes in muscle fibre characteristics and the mRNA and protein expression of key genes associated with the regulation of muscle mass.

\section{Diet and physical activity}

All subjects received the same standardized meal the evening prior to the experimental test days (33 $\pm 2 \mathrm{~kJ} \cdot \mathrm{kg}$ body weight ${ }^{-1}$, providing 44 energy\% (En\%) carbohydrate, $22 \mathrm{En} \%$ protein, and $34 \mathrm{En \%}$ fat). All volunteers were instructed to refrain from strenuous physical activity, avoid alcohol intake and to keep their diet as constant as possible for 2 days prior to the experimental test day.

\section{Experimental visits}

Subjects participated in two identical experimental test days, before and immediately after the immobilization period. Approximately two days prior to the immobilization period, subjects participated in the first test day. During the visit, subjects arrived at the laboratory at $08.00 \mathrm{~h}$ in the fasted state and body weight was measured with a digital 
balance with an accuracy of $0.1 \mathrm{~kg}$ (SECA GmbH, Hamburg, Germany). Thereafter, a single slice CT-scan (Philips Brilliance 64, Philips Medical Systems, Best, the Netherlands) was performed to assess upper leg muscle cross-sectional area (CSA). The scanning characteristics were as follows: $120 \mathrm{kV}, 300 \mathrm{~mA}$, rotation time of $0.75 \mathrm{~s}$, and a field of view of $500 \mathrm{~mm}$. With subjects lying supine with their legs extended and feet secured, a $3 \mathrm{~mm}$ thick axial image was taken $15 \mathrm{~cm}$ proximal to the top of the patella. The precise scan position was marked with semi-permanent ink for the duration of the experimental protocol to ensure accurate repeat measurements. Muscle area of the right leg was selected between 0 and 100 Hounsfield units [32], after which the quadriceps muscle was selected by manual tracing using ImageJ software (version 1.45d, National Institute of Health, Maryland, USA) [32, 33]. After CT scanning, body composition (fat, fat-free mass and bone mineral content) was determined by DXA scan (Hologic Inc., Bedford, USA). Lean mass and percent body fat were determined on a whole-body level and for specific regions (e.g. legs). Thereafter, a muscle biopsy was collected from the vastus lateralis muscle of the leg identified as the leg to become immobilized (or the previously immobilized leg in the case of the second visit). Muscle biopsy samples were obtained from the middle region of the vastus lateralis, $\sim 1-3 \mathrm{~cm}$ below the level that the $\mathrm{CT}$ scan was performed, and $\sim 3 \mathrm{~cm}$ below entry through the fascia, by using the percutaneous needle biopsy technique [34]. Muscle samples were dissected carefully and freed from any visible non-muscle material and were immediately frozen in liquid nitrogen and stored at $80^{\circ} \mathrm{C}$ until further analysis. Thereafter, subjects single leg one repetition maximum (1RM) was assessed [35]. After warming up, the load was set at $97.5 \%$ of the estimated 1RM from the screening visit, and increased after each successful lift until failure. Three min rest periods were allowed between lifts. A repetition was considered valid when the subject used proper form and was able to complete the entire lift in a controlled manner without assistance. Finally, subjects were instructed on, and familiarized with, the use of crutches. On the day of cast removal, all measures of muscle mass and strength were repeated in the same manner, and a muscle biopsy was collected prior to any weight bearing activity from a site $\sim 2 \mathrm{~cm}$ from the position of the first biopsy.

\section{Limb immobilization}

Approximately $48 \mathrm{~h}$ after the first test day, subjects reported at $8.00 \mathrm{~h}$ at the Casting Room at Maastricht University Medical Centre, to have a full leg cast fitted. The application of the cast signified the first day of either the $5(n=12)$ or $14(n=12)$ day immobilization period. The circular leg cast extended from $10 \mathrm{~cm}$ above the ankle to approximately $25 \mathrm{~cm}$ above the patella. The knee was casted at a $30^{\circ}$ angle of flexion to prevent subjects performing any weight bearing activities with the casted leg. Subjects were provided with crutches for proper ambulation. All subjects were instructed to perform a series of simple ankle exercises (i.e. plantar and dorsal flexion, and circular movements of the entire foot) to keep the calf muscle pump activated in the immobilized leg, 
thereby minimizing the risk of developing deep vein thrombosis. Subjects remained in the cast for the duration of the period but those in the group immobilized for 14 days were required to visit the Casting Room after 7 days to have the cast tightened. At the end of the immobilization period, subjects were collected from their home by car, and brought into the laboratory for the second test day at $08.00 \mathrm{~h}$. Prior to the start of the second test day, subjects visited the Casting Room to have the cast removed. Thereafter, subjects were taken by wheelchair to the laboratory so the muscle biopsies could be collected prior to any weight bearing exercise being performed. Following biopsy collection, subjects were able to bear weight on the immobilized leg for approximately 1-2 h prior to strength testing to minimize deficits due to initial joint stiffness.

\section{Immunohistochemistry}

Frozen muscle biopsies were cut into $5 \mu \mathrm{m}$ thick cryosections using a cryostat at $-20^{\circ} \mathrm{C}$, and thaw mounted on uncoated pre-cleaned glass slides. Samples from pre and post immobilization were mounted together on the same glass slide. Care was taken to properly align the samples for cross-sectional fibre analyses. Muscle biopsies were stained for muscle fibre typing (FT). First antibodies were directed against laminin (polyclonal rabbit anti-laminin, dilution 1:50; Sigma, Zwijndrecht, the Netherlands) and myosin heavy chain (MHC)-I (A4.840, dilution 1:25; Developmental Studies Hybridoma Bank, lowa City, IA). Appropriate secondary antibodies were applied: goat anti-rabbit IgG AlexaFluor647 and goat anti-mouse IgM AlexaFluor555 (dilution 1:400 and 1:500, respectively; Molecular Probes, Invitrogen, Breda, the Netherlands). All primary and secondary antibodies were diluted in 0.1\% Bovine Serum Albumin (BSA) in 0.1\% Tweenphosphate-buffered saline (PBS). All incubations took place at room temperature, unless otherwise stated. Staining procedures were as follows. After fixation (5 minutes acetone), slides were air dried and incubated at room temperature for 30 minutes with $3 \%$ BSA in $0.1 \%$ Tween-PBS. Slides were then washed (5 minutes PBS). Thereafter, primary antibodies against laminin and MHC-I were applied for 45 minutes. Slides were then washed and incubated with the appropriate secondary antibodies. After a final washing step, all slides were mounted with cover glasses using Mowiol (Calbiochem, Amsterdam, the Netherlands).

Images were visualized and automatically captured at 10x magnification with a fluorescent microscope equipped with an automatic stage (IX81 motorized inverted microscope, Olympus, Hamburg, Germany) EXi Aqua CCD camera (QImaging). Micromanager 1.4 software was used for image acquisition [36]. Quantitative analyses were done using Image J software package (version 1.45d, National Institute of Health, [33]). All image recordings and analyses were performed by an investigator blinded to subject coding. Mean muscle fibre size was calculated for the type I and type II muscle fibres separately. As a measure of fibre circularity, form factors were calculated by using the following 
formula: $(4 \pi \cdot \mathrm{CSA}) \cdot(\text { perimeter })^{-2}$. No differences in fibre circularity were observed over time or between groups (data not shown).

rtPCR

Total RNA was isolated from $10-20 \mathrm{mg}$ of frozen muscle tissue using TRIzol ${ }^{\circledR}$ Reagent (Life Technologies, Invitrogen), according to the manufacturer's protocol. Total RNA quantification was carried out spectrophotometrically at $260 \mathrm{~nm}$ (NanoDrop ND-1000 Spectrophotometer, Thermo Fisher Scientific, USA), and RNA purity was determined as the ratio of readings at $260 / 280 \mathrm{~nm}$. Thereafter, first strand cDNA was synthesized from $1 \mu \mathrm{g}$ RNA sample using iScriptTM cDNA synthesis kit (BioRad; cat. 170-8891) in a reaction volume of $20 \mu \mathrm{L}$. Taqman PCR was carried out using a 7300 Real Time PCR System (AppliedBiosystems, USA), with $2 \mu \mathrm{L}$ of cDNA, $12.5 \mu \mathrm{l}$ TaqmanTM master mix, $1.25 \mu \mathrm{l}$ TaqmanTM probe and $9.25 \mu \mathrm{H}_{2} \mathrm{O}$ in a $25 \mu \mathrm{L}$ final well volume. Each sample was run in duplicate, together with a serial dilution standard curve. The housekeeping gene $18 \mathrm{~S}$ was used as an internal control as this gene has been used previously in similar human immobilization studies $[12,37,38]$ and was unaffected by immobilization (i.e. mean Ct values were unaffected over time during each assay: example $C t$ values pre and post immobilization were $8.99 \pm 0.08$ and $9.04 \pm 0.05 ; P=0.53$, for the 5 day group: $9.46 \pm 0.10$ and 9.52 $\pm 0.12 ; P=0.56$ for the 14 day group). Taqman primer/probe sets were obtained from Applied Biosystems (Foster City, USA): myogenin, MyoD, myostatin, MAFBx, MuRF1, FOX01, mTOR, P70S6K, citrate synthase, PGC1 $\alpha$, FAK, PAT1, LAT1 and 18S. The thermal cycling conditions used were: $2 \mathrm{~min}$ at $50^{\circ} \mathrm{C}, 10 \mathrm{~min}$ at $950 \mathrm{C}$, followed by 40 cycles at $95^{\circ} \mathrm{C}$ for $15 \mathrm{~s}$ and $60^{\circ} \mathrm{C}$ for $1 \mathrm{~min}$. Ct values of the target genes were normalized to $\mathrm{Ct}$ values of the internal control and final results were calculated as relative expression against the standard curve. The $\mathrm{Ct}$ values of all genes of interest were always within the lower and upper boundaries of the standard curve.

\section{Western blotting}

A portion of each muscle sample frozen for biochemical analyses was homogenized in 14 volumes Tris buffer (20 mM Tris-HCL, 5 mM EDTA. 10 mM Na-pyrosphospate, 100 $\mathrm{mM} \mathrm{NaF}, 2 \mathrm{mM} \mathrm{Na}_{3} \mathrm{VO}_{4}, 1 \%$ Nonident P-40; $\mathrm{pH}$ 7.4) supplemented with the following protease and phosphatase inhibitors: Aprotinin $10 \mu \mathrm{g} \cdot \mathrm{mL}^{-1}$, Leupeptin $10 \mu \mathrm{g} \cdot \mathrm{mL}^{-1}$, Benzamidin $3 \mathrm{mM}$ and PMSF $1 \mathrm{mM}$. After homogenization, each muscle extract was centrifuged for $5 \mathrm{~min}$ at $10,000 \mathrm{~g}\left(4^{\circ} \mathrm{C}\right)$ and sample buffer was added to the supernatant to final concentrations of $60 \mathrm{mM}$ Tris, $5 \%$ glycerol, $20 \mathrm{mg} \cdot \mathrm{mL}^{-1} \mathrm{SDS}, 0.1 \mathrm{mM} \mathrm{DTT}, 20 \mu \mathrm{g} \cdot \mathrm{mL}^{-1}$ bromophenol blue. The supernatant was then heated for $5 \mathrm{~min}$ at $100^{\circ} \mathrm{C}$ and immediately placed on ice. Immediately before analyses, the muscle extraction sample was warmed to $50^{\circ} \mathrm{C}$ and centrifuged for $1 \mathrm{~min}$ at 3,000 (RT). Total amount of sample loaded on the gel was based on weight (1.0 mg per lane). Protein samples were run on a Criterion 'any kDa' gel (Biorad Order No. 567-1124) for 10 min at $50 \mathrm{~V}$ (constant volt- 
age) and $\pm 90 \mathrm{~min}$ at $150 \mathrm{~V}$ (constant voltage) and transferred onto a Trans-blot Turbo $0.2 \mu \mathrm{m}$ nitrocellulose membrane (Biorad Order No. 170-4159) in $7 \mathrm{~min}$ at 2.5A and 25V. Specific proteins were detected by overnight incubation at $4^{\circ} \mathrm{C}$ on a shaker with specific antibodies in 50\% in PBS Odessey blocking buffer (Li-Cor Biosciences Part No. 92740000) after blocking for 60 min at RT in 50\% in PBS Odessey blocking buffer. The antibodies used in this study were anti-myostatin $(52$ and $26 \mathrm{kDa}$; rabbit polyclonal IgG, Santa Cruz, sc-6885-R; dilution 1:500 in 50\% Odessey blocking buffer), anti-myoD (37 kDa; rabbit polyclonal IgG, Santa Cruz, sc-760; dilution 1:1000 in 50\% Odessey blocking buffer), anti-myogenin (34 kDa; rabbit polyclonal IgG, Santa Cruz, sc-576; dilution 1:500 in 50\% Odessey blocking buffer), and anti $\alpha$-actin (42 kDa; mouse monoclonal IgM, Sigma A2172; dilution 1:10000 in 50\% Odessey blocking buffer. Following incubation membranes were washed 3 times $10 \mathrm{~min}$ in 0.1\%PBS-Tween 20 and 1 time $10 \mathrm{~min}$. with PBS. Next, samples were incubated ( $1 \mathrm{~h}$ at RT) with Infrared secondary antibodies, donkey anti-rabbit IRDYE 680 (Li-Cor, Cat. No. 926-32223, dilution 1:10000) and donkey anti-mouse IRDYE 800CW (Li-Cor, Cat. No. 926-32212, dilution 1:10000) dissolved in $50 \%$ in PBS Odessey blocking buffer. After a final wash step ( $3 \times 5 \mathrm{~min}$ ) in $0.1 \%$ Tween20-PBS and 1 time $10 \mathrm{~min}$. with PBS protein quantification was performed by scanning on an Odyssey Infrared Imaging System (LI-COR Biotechnology, Lincoln, NE). Values for target proteins were adjusted for total $\alpha$-actin content as the protein levels of this housekeeper were not altered by either 5 (from $20.24 \pm 3.06$ to $17.30 \pm 2.18$ arbitrary units; $P=0.39$ ) or 14 days of immobilization (from $15.69 \pm 3.36$ to $15.98 \pm 3.17$ arbitrary units; $P=0.90)$.

\section{Statistics}

All data are expressed as means \pm SEM. A two-way ANOVA with time (pre and post) and treatment ( 5 and 14 days) as factors was used to compare differences in all time dependent parameters. When a significant main effect was detected, a Bonferoni posthoc test was applied to locate these differences. For non-time dependent variables, a paired t test was used to compare means. Statistical significance was set at $P<0.05$. All calculations were performed by using GraphPad Prism version 5.0 for Windows, GraphPad Software, San Diego California USA. 


\section{RESULTS}

\section{Participants}

Subjects' characteristics in the 5 day immobilization group (body mass; $76.0 \pm 4.2 \mathrm{~kg}$, $\mathrm{BMI} ; 22.5 \pm 1.2 \mathrm{~kg} \cdot \mathrm{m}^{-2}$, body fat; $15.6 \pm 1.4 \%$, lean body mass; $60.0 \pm 2.7 \mathrm{~kg}$, and blood $\mathrm{HbA1c} ; 5.1 \pm 0.1 \%$ ) did not differ from those in the 14 day immobilization group (body mass $82.5 \pm 3.1 \mathrm{~kg}, \mathrm{BMI} ; 25.0 \pm 1.1 \mathrm{~kg} \cdot \mathrm{m}^{-2}$, body fat; $20.0 \pm 2.1 \%$, lean body mass; $63.5 \pm 2.7$ $\mathrm{kg}$, and blood $\mathrm{HbA} 1 \mathrm{c} ; 5.7 \pm 0.3 \%)$. No changes in any of these parameters were observed during the experimental periods (data not shown).

\section{Muscle mass, strength and muscle fibre cross-sectional area}

Measurements of muscle mass and strength are displayed in Figure 1. Quadriceps crosssectional area (CSA) determined by CT-scan (A) did not differ between groups at baseline, but significantly declined by $3.5 \pm 0.5 \%$ (from $7504 \pm 342$ to $7238 \pm 324 \mathrm{~mm}^{2} ; P<0.001$ ) and $8.4 \pm 2.8 \%$ (from $7666 \pm 382$ to $7022 \pm 375 \mathrm{~mm}^{2} ; P<0.001$ ) following 5 and 14 days of immobilization, respectively. A significantly greater amount of muscle mass was lost following 14 days when compared with 5 days of immobilization (significant interaction; $P<0.05)$. Interestingly, when taking the $m$ rectus femoris as an individual muscle, no atrophy was present in either the 5 (from $745 \pm 55$ to $737 \pm 52 \mathrm{~mm}^{2} ; P>0.05$ ) or 14 (from $598 \pm 34$ to $659 \pm 56 \mathrm{~mm}^{2} ; P>0.05$ ) day groups. Leg lean mass, as determined by DXA (B), did not differ between groups at baseline and declined over time in both groups (effect of time; $P<0.001$ ) by $1.4 \pm 0.7$ (from $10133 \pm 425$ to $9986 \pm 411 \mathrm{~g} ; P=0.66$ ) and $3.1 \pm 0.7 \%$ (1187 \pm 452 to $10841 \pm 450 \mathrm{~g} ; P<0.01$ ) following 5 and 14 days of immobilization, respectively. Whole-body lean and fat mass did not change throughout the experiment in either group. 

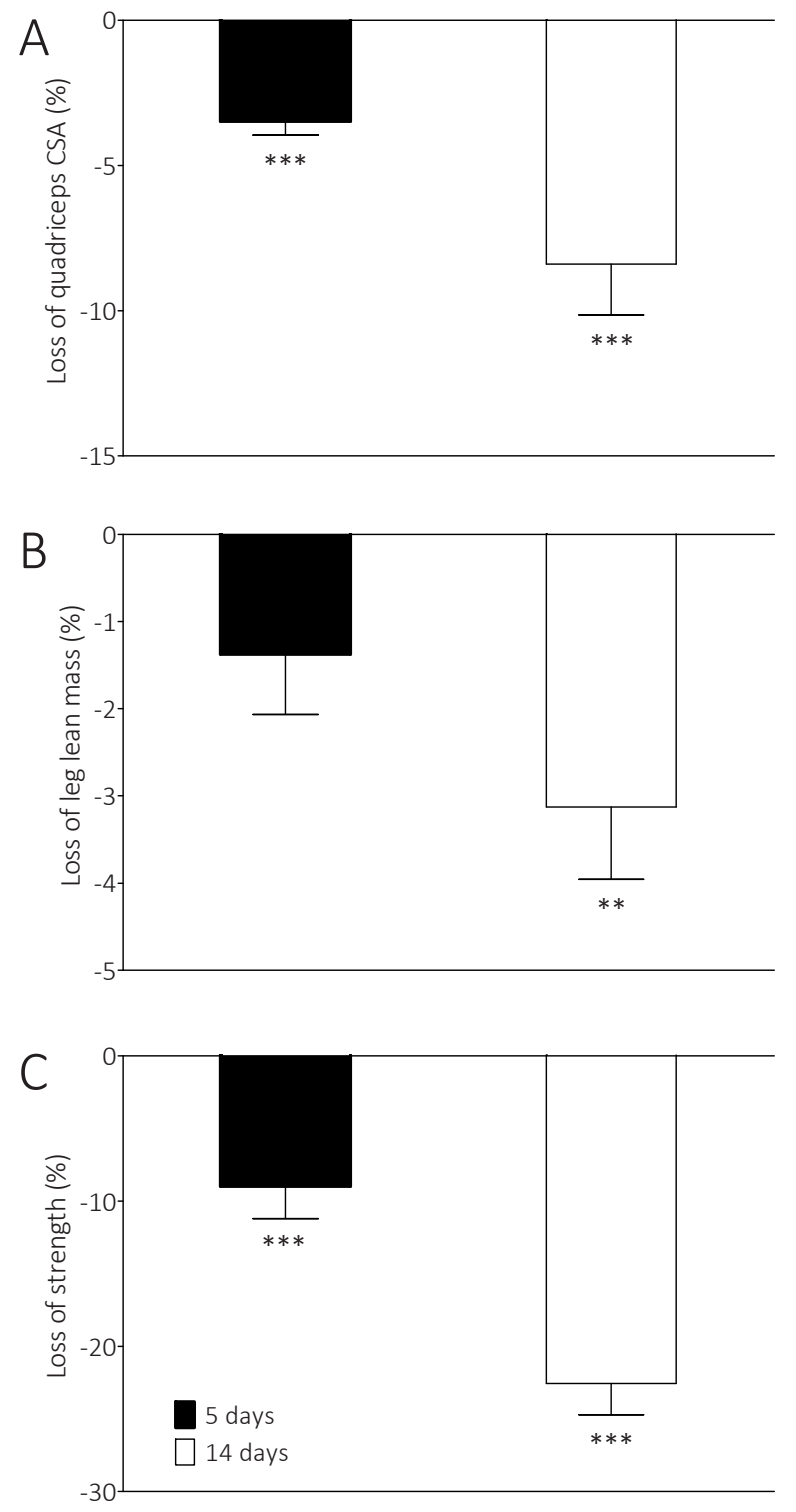

Figure 1 | Mean ( \pm SEM) percentage loss of quadriceps cross-sectional area (CSA; A), lean leg mass (B) and single leg 1 repetition maximum strength (1RM; C) following either 5 ( $n=12)$ or 14 days $(n=12)$ of one-legged knee immobilization in healthy, young men. Data were analyzed using absolute values pre and post immobilization in both groups with a two-way ANOVA (time $x$ treatment). In case of a significant interaction effect, Bonferroni post-hoc tests were applied to locate the individual differences. A: Significant time $(P<0.001)$ and interaction $(P<0.05)$ effects. B: Significant time $(P<0.001)$ effect. C: Significant time $(P<0.001)$ and interaction $(P<0.001)$ effects. ${ }^{* * *} P<0.001$ compared with pre immobilization. 
Muscle strength (C) did not did not differ between groups at baseline, but declines of $9.0 \pm 2.3$ (from $77.9 \pm 3.9$ to $71.1 \pm 4.1 \mathrm{~kg} ; P<0.001$ ) and $22.9 \pm 2.6 \%$ (from $73.8 \pm 4.2$ to $55.9 \pm 3.6 \mathrm{~kg} ; P<0.0001)$ were observed following 5 and 14 days of immobilization, respectively (effect of time; $P<0.001$ ). A significantly greater loss in muscle strength was experienced by volunteers following 14 versus 5 days of immobilization (interaction effect; $P<0.001)$.

Type I and II muscle fibre CSA of biopsies collected before and after immobilization in both groups are displayed in Figure 2. Type I muscle fibre CSA was significantly greater at baseline in the 14 day compared to 5 day immobilization group $(P<0.05)$. Limb immobilization resulted in a significant decrease in type I muscle fibre CSA after 14 days of immobilization $(7 \pm 3 \%$ decline; $P<0.05)$ but not after 5 days of muscle disuse (pre: $5259 \pm 328 \mu \mathrm{m}^{2}$, post: $\left.5404 \pm 404 \mu \mathrm{m}^{2} ; P>0.05\right)$. Type II muscle fibre CSA did not differ between groups at baseline $(P=0.11)$ and declined over time in both groups (significant effect of time; $P<0.05)$, reaching statistical significance after 14 days of immobilization only $\left(7735 \pm 463\right.$ to $6619 \pm 311 \mu \mathrm{m}^{2} ; 13 \pm 4 \%$ decline; $\left.P<0.05\right)$.

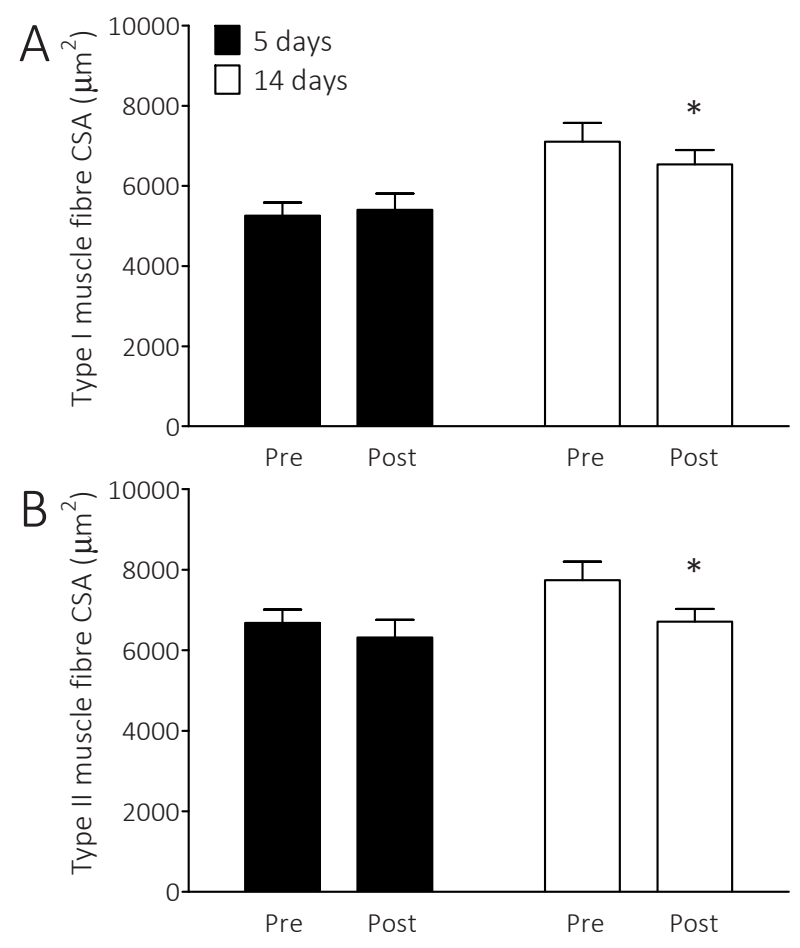

Figure 2 | Mean ( \pm SEM) type I (A) and type II (B) muscle fibre cross-sectional area (CSA) before (pre) and after (post) either $5(n=12)$ or 14 days $(n=12)$ of one-legged knee immobilization in healthy, young men. Data were analyzed using with a two-way ANOVA (time $x$ treatment). In case of a significant main effect, Bonferroni posthoc tests were applied to locate the individual differences. A: Significant treatment $(P<0.01)$ effect. B: Significant time $(P<0.01)$ effect. ${ }^{*} P<0.05$ compared with corresponding pre immobilization values. 
At baseline, muscle fibre type composition showed $43 \pm 3$ and $57 \pm 3 \%$ type I and II muscle fibre, respectively, in the 5 day group, and $35 \pm 3$ and $65 \pm 3 \%$, respectively, in the 14 day group. No differences were observed between groups. There was a significant time effect $(P<0.01)$ for a greater type I muscle fibre proportion following immobilization with $10 \pm 7(P=0.10)$ and $26 \pm 12 \%(P<0.05)$ more type I muscle fibres following 5 and 14 days of immobilization, respectively.

\section{Skeletal muscle mRNA expression and protein content}
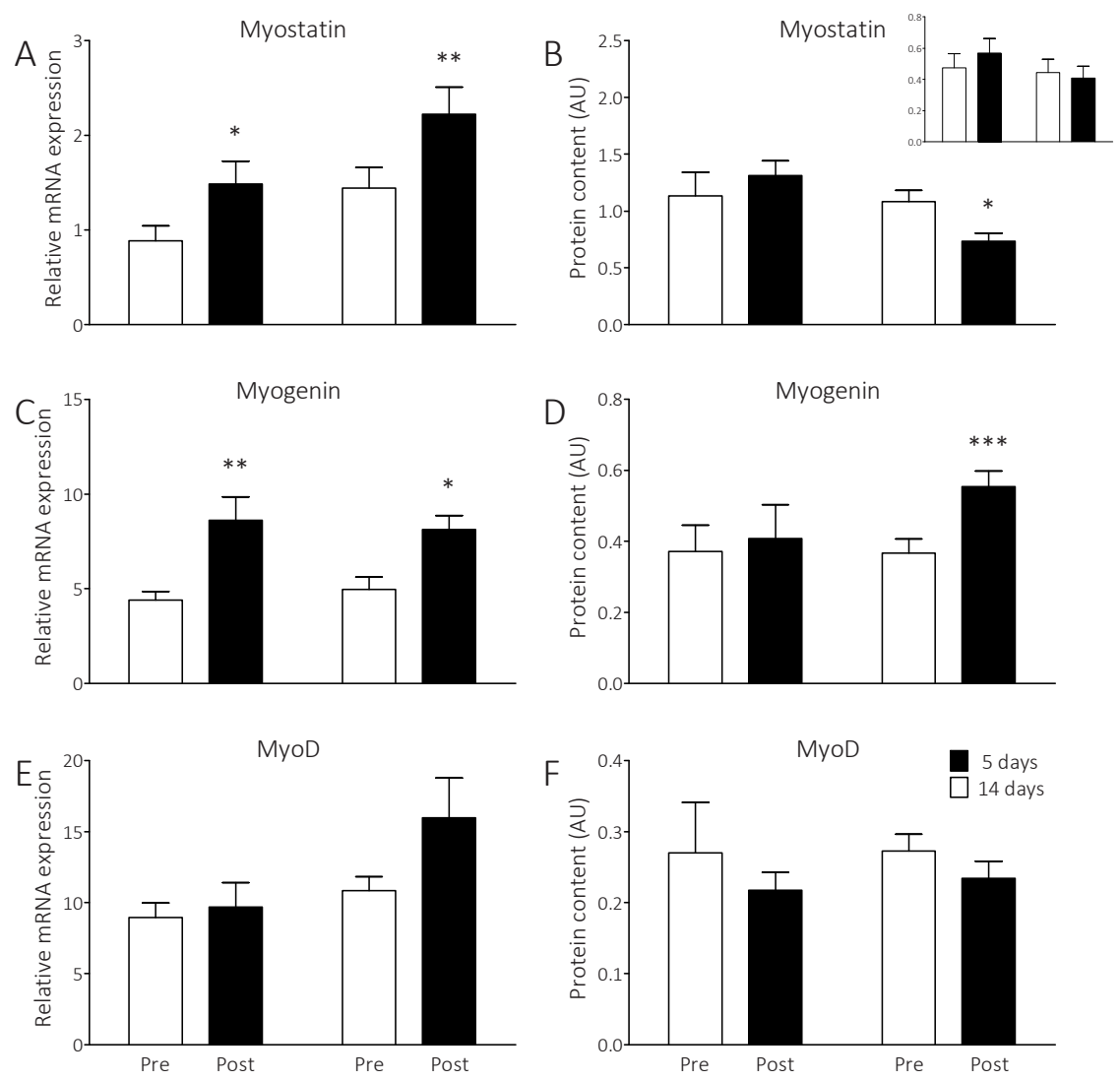

Figure 3 | Skeletal muscle mRNA expression and protein content of myostatin ( $\mathrm{A}$ and $\mathrm{B}$, respectively), myogenin ( $C$ and $D$, respectively) and myoD ( $E$ and $F$, respectively) before (Pre) and after (Post) either 5 ( $n=12$ ) or 14 days ( $n=12$ ) of one-legged knee immobilization in healthy, young men. Figure B represents the 52 (main) and 26 kDa (inset) isoforms of myostatin. Data were analyzed with a two-way ANOVA (time $x$ treatment). In case of a significant main effect, Bonferroni post-hoc tests were applied to locate the individual differences. A: Significant time $(P<0.001)$ and treatment $(P<0.05)$ effects. B: Significant interaction $(P<0.05)$ effect. C: Significant time $(P<0.001)$ effect. D: Significant time $(P<0.001)$ and interaction $(P<0.01)$ effects. E: Significant treatment $(P<0.05)$ effect. $\mathrm{F}$ : No significant effects were detected. ${ }^{*} P<0.05, * * P<0.01$ and $* * * P<0.001$ compared with corresponding Pre value. 
Figure 3 depicts the skeletal muscle mRNA expression and protein content of myostatin, myogenin, and MyoD. Muscle myostatin mRNA expression (A) increased significantly by 68 and $54 \%$ after $5(P<0.05)$ or $14(P<0.01)$ days of immobilization, respectively. While myostatin ( $52 \mathrm{kDa}$ isoform) protein content (B) remained unaltered in the 5 day group, 14 days of limb immobilization led to a $32 \%$ reduction $(P<0.05)$. No significant differences were observed between groups or over time in muscle protein content of the 26 $\mathrm{kDa}$ isoform of myostatin ( $\mathrm{B}$; inset). Muscle myogenin mRNA expression (C) increased significantly by 96 and $64 \%$ following $5(P<0.01)$ or $14(P<0.05)$ days of immobilization, respectively. However, the muscle protein content of myogenin (D) increased only in the 14 day group by $51 \%(P<0.001)$ and did not change in the 5 day group. Muscle myoD mRNA expression (E) and protein content (F) did not differ between groups and seemed to remain unaffected by either duration of immobilization.

The skeletal muscle mRNA expression data for selected genes implicated in the regulation of muscle mass, amino acid metabolism or oxidative metabolism are displayed in Figure 4. Muscle MAFBx mRNA expression (A) increased by 48 and $40 \%$ following $5(P<0.01)$ or $14(P<0.05)$ days of immobilization, respectively, while muscle MuRF1 (B) mRNA expression increased (56\%) only in the 5 day group. Muscle mRNA expression of FOXO1 (C), mTOR (D), LAT1/SLC (F), PAT1 (G) and FAK (J) were not altered by either duration of immobilization. Muscle mRNA expression of P70S6K (E) increased modestly (18\%; $P<0.05)$ following 5 but not 14 days of immobilization. PGC1 $\alpha(H)$ and citrate synthase (I) muscle mRNA expression declined by $73(P<0.01)$ and 33\% $(P<0.01)$ in the 5 day group, and $72(P<0.01)$ and 31\% $(P<0.01)$ in the 14 day group, both respectively. 

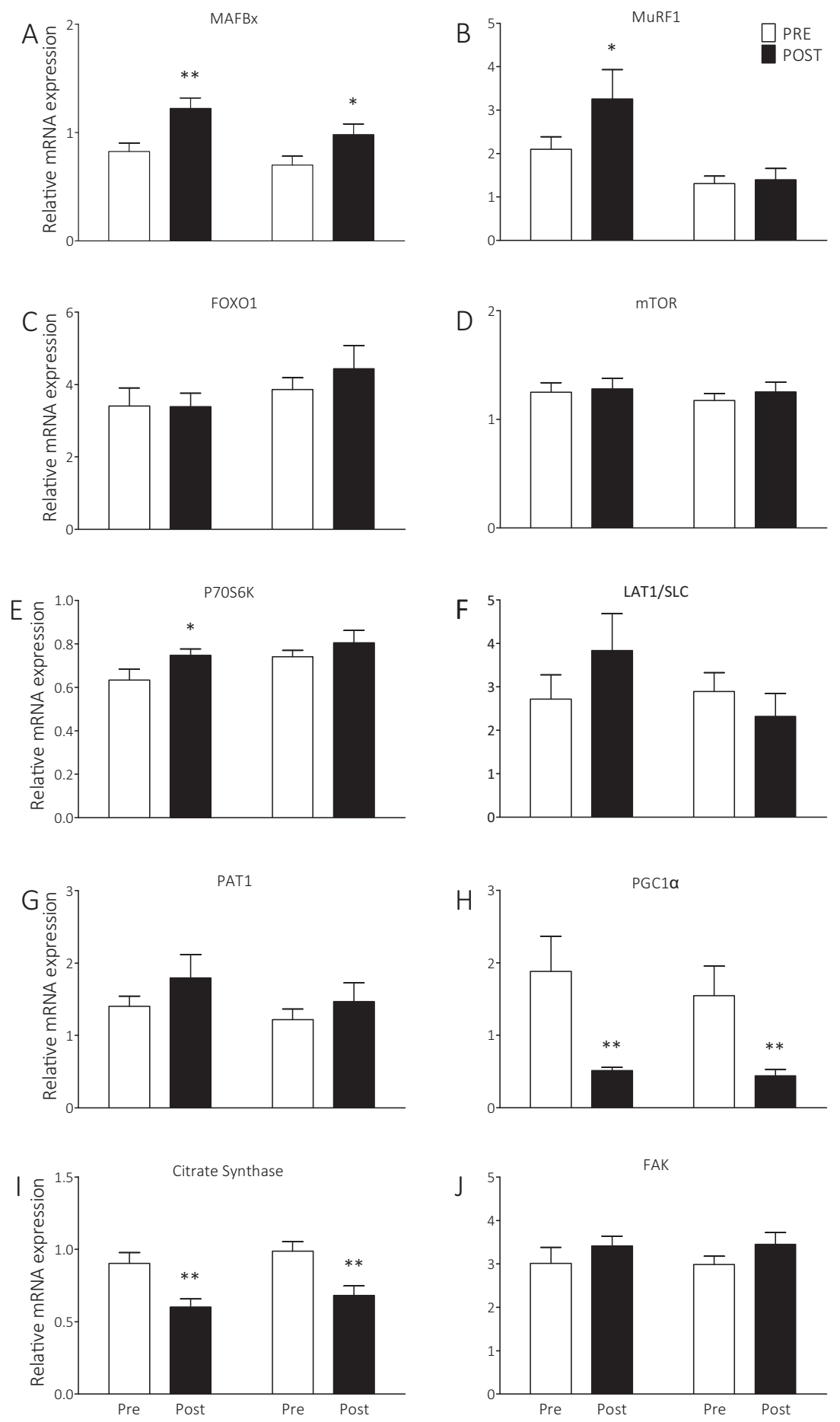
Figure 4 | Skeletal muscle mRNA expression of MAFBx (A), MuRF1 (B), FOXO1 (C), mTOR (D), P70S6K (E), LAT1/SLC (F), PAT1 (G), PGC1 $\alpha(\mathrm{H})$, citrate synthase (I) and FAK (J) before (Pre) and after (Post) either 5 $(n=12)$ or 14 days $(n=12)$ of one-legged knee immobilization in healthy, young men. Data were analyzed with a two-way ANOVA (time $x$ treatment). In case of a significant main effect, Bonferroni post-hoc tests were applied to locate the individual differences. A: Significant time $(P<0.001)$ effect. B: Significant treatment $(P<0.01)$ effect. C: No significant effects. D: No significant effects. E: Significant time $(P<0.05)$ effect. F: No significant effects. G: No significant effects. H: Significant time $(P<0.001)$ effect. I: Significant time $(P<0.001)$ effect. J: Significant time $(P<0.05)$ effect. $* P<0.05, * * P<0.01$ and $* * * P<0.001$ compared with corresponding Pre value. 


\section{DISCUSSION}

The principal novel finding of the present study is that skeletal muscle mass already declines substantially after only 5 days of limb immobilization. Moreover, we provide evidence for a divergent molecular signaling response within skeletal muscle tissue regarding the expression of myostatin and components of the ubiquitin-proteasome pathway during the early (5 days) compared with the later stages (14 days) of muscle disuse. These findings underline the clinical relevance of striving to prevent or minimize muscle loss even during short periods of bed rest or immobilization due to illness or injury.

The multitude of negative consequences that are brought about by the loss of skeletal muscle tissue following a period of bed rest or limb immobilization [17] make muscle disuse atrophy an important area for scientific research and clinical evaluation. Previous studies have demonstrated that muscle loss during limb immobilization generally occurs at a rate of $0.5-0.6 \%$ per day [17]. In keeping with this, in the present study, we report that 14 days of limb immobilization led to an $\sim 8.5 \%$ loss of quadriceps cross-sectional area (CSA; Figure 1A), representing $\sim 350 \mathrm{~g}$ lean tissue lost (Figure 1B). This considerable muscle atrophy was accompanied by a $25 \%$ loss of muscle strength (Figure $1 \mathrm{C}$ ) following 14 days of immobilization, underlining the detrimental impact of prolonged disuse on functional capacity. On a muscle fibre level, we report that 14 days of immobilization led to a clear atrophy of both type I and type II muscle fibres (Figure 2), with the latter being quantitatively most affected. These findings are in line with previous studies demonstrating disuse atrophy in both fibre types [39-42], with type II muscle fibres being the most susceptible [42].

Innovations within healthcare systems have reduced the duration that patients' typically remain hospitalized, such that 5-6 days is now the average stay for an elderly person admitted with acute illness [14]. However, to what extent such a short period of disuse may impact upon skeletal muscle mass has not yet been evaluated. This is the first study to show that only 5 days of limb immobilization already induces a $3.5 \%$ decline in quadriceps muscle CSA in vivo in humans (Figure 1A). We assessed muscle mass of the quadriceps as an entire unit due its important role in numerous daily functions. However, it is also true that the $3.5 \%$ observable atrophy may represent an underestimate of the muscles more specific to knee extension, since the $m$. rectus femoris did not atrophy in either group. The lack of atrophy in this specific muscle likely relates to its contribution to hip extension which may have still occurred to a certain extent during the immobilization period. Regardless, the decline in muscle CSA was accompanied by our observation of as much as $150 \mathrm{~g}$ lean mass lost from a single leg after merely 5 days of disuse (Figure 1B). Our data are in line with a previous short-term disuse study which reported a $3 \%$ loss of thigh muscle mass assessed by MRI after 7 days of bed rest in young men [43]. We were unable to detect any significant changes in muscle fibre size from the collected muscle biopsies following 5 days of immobilization. This is not 
surprising given the considerable inter- and intra- subject variation in muscle fibre size [44]. In fact, decreases in muscle mass likely need to exceed $\sim 6 \%$ before specific muscle fibre atrophy can be detected using immunohistochemistry in combination with fluorescence microscopy [44]. It should be noted that a recent study was able to show an $\sim 8$ and $12 \%$ decline in type I and II muscle fibre CSA, respectively, following only 4 days of immobilization [15]. The reason(s) for the apparent discrepancy between this and the present study is unclear, but could relate to a differing immobilization protocol (knee brace vs. casting) or less variability in the data. Importantly, the loss of muscle mass following 5 days of immobilization led to an even greater (relative) decline in leg muscle strength of $\sim 9 \%$ (Figure 1C), suggesting that already compromised patients could easily fall below a critical threshold with respect to carrying out normal daily activities following only a few days of illness or injury. A striking observation of the present study was that a substantial amount of the atrophy and loss of strength observed after 14 days was already present after only 5 days of limb immobilization. Since losses of strength over a more prolonged period seem mostly attributable to continued loss of muscle mass [45], this underlines the importance of intervening as early as possible when a period of disuse is unavoidable.

While muscle atrophy during prolonged disuse is generally attributed to declines in muscle protein synthesis rates $[2,10,17,46]$, evidence also suggests that a rapid increase in muscle protein breakdown during the first few days of disuse may also contribute to overall muscle loss [17, 18, 37, 38, 47, 48]. Myostatin, a proposed 'master' negative regulator of skeletal muscle mass in animals [24, 25] and humans [26], acts via the inhibition of mammalian target of rapamycin (mTOR) muscle protein synthesis signaling [27] and by initiating muscle protein breakdown through the ubiquitin proteasome system [30]. Consistent with previous animal [49] and human [12, 37, 50] disuse studies, we report a clear up-regulation of myostatin gene expression in response to 5 and 14 days of limb immobilization (Figure 3). In parallel, we observed a profound increase in gene expression of the two primary ubiquitin ligases, MAFBx and MuRF1 [51], after 5 days of immobilization, which became less striking after 14 days (Figure 4). These data extend on previous findings [20, 37, 38, 47, 52] by suggesting that an early and transient increase in ubiquitin-proteasome mediated muscle protein breakdown, possibly mediated via increased myostatin transcription, contributes to muscle disuse atrophy during the first few days of disuse. In support, it has previously been shown that anti-body directed inhibition of endogenous myostatin is capable of attenuating muscle atrophy in the short- but not long-term phase of hind-limb unloading in rodents [49]. However, it should be noted that we were unable to demonstrate that increased myostatin transcription led to the successful translation of either the (52 $\mathrm{kD})$ precursor or purported biologically active $(26 \mathrm{kD})$ isoforms of the protein. Clearly more research is warranted to directly assess muscle protein breakdown rates in combination with myostatin signaling during (short-term) disuse. Such information will allow us to better define their contribution to muscle disuse atrophy, and assess whether 
myostatin [53] and/or proteasome inhibition [54] are viable future targets for attenuating muscle disuse atrophy in humans.

Myostatin also plays a key role in myogenesis through the inhibition of the myogenic regulatory factors, most notably myogenin and $\operatorname{MyoD}[28,29,55]$. Here, we provide novel evidence that the up-regulation of myostatin transcription does not translate into increased protein levels during limb immobilization. In fact, after 14 days of limb immobilization, myostatin protein content ( $52 \mathrm{kD}$ isoform) had significantly declined by $32 \%$ (Figure 3 ). We extend on previous work with the observation that myogenin mRNA expression, considered mandatory for satellite cell differentiation and therefore myogenesis, increased after 5 and 14 days of immobilization, which translated into greater protein content after 14 days of immobilization (Figure 3). The present findings imply that prolonged disuse down-regulates myostatin at the functional level (i.e. protein) and thereby facilitates the successful translation of myogenin, possibly in anticipation of a forthcoming anabolic stimulus (i.e. re-ambulation). Therefore, it could be speculated that the regulation of myogenesis/satellite cells via myostatin and the myogenic regulatory factors may be of more physiological relevance in the regeneration of muscle mass (rather than in muscle atrophy) following more prolonged periods of disuse. As such, a disuse induced 'priming' of the myogenic machinery for an anabolic stimulus may explain the rapid rate of muscle growth generally observed in healthy individuals recovering from disuse atrophy $[12,56]$. This has important clinical relevance when considering the design of rehabilitative programs to improve muscle mass and function following disuse in various populations.

Concerning our measures of gene expression, an interesting consideration is the impact of disuse on total ribosome content and RNA abundance. It has been shown that limb disuse [57] and denervation [58] can result in a reduction in both de novo ribosomal RNA synthesis and global (ribosomal) RNA content. Though we did not make these measurements in the present study, we did not observe any differences in total RNA quality obtained from samples pre and post immobilization, nor did we see any changes over time in our housekeeping gene (18S). Moreover, any such changes in global RNA abundance would only make the elevated expression of the muscle atrogenes, myostatin, and myogenic regulatory factors all the more striking.

The present study illustrates the impact of only a few days of disuse on skeletal muscle mass and strength. Extending on this, we also report a decline in citrate synthase and PGC1 $\alpha$ gene expression after only 5 days of immobilization (Figure 4), suggesting that a decline in oxidative capacity and/or insulin sensitivity may also occur after only a few days of disuse. Thus, in clinically compromised populations (e.g. hospitalized elderly, critically ill, hip-fracture or acute illness patients), just a few days of bed rest or immobilization can likely already compromise functional capacity and metabolic health. Such patient groups are particularly vulnerable to atrophy during disuse since the presence of illness related stress responses are known to accelerate muscle loss during disuse $[59,60]$. Furthermore, short successive periods of muscle disuse atrophy 
throughout the lifespan will contribute significantly to the age-related loss of muscle mass and strength, and muscle quality [17-19,61]. Together with the observation that we seem to lose our ability to regain lost muscle tissue as we age [42], these data support the concept that successive short periods of bed rest or immobilization throughout the lifespan can contribute substantially to the development of sarcopenia and decline in metabolic health. As a whole, our data underline the importance of understanding the response to muscle disuse, both in terms of molecular signaling as well as physiological/functional changes, if we are to develop effective nutritional, exercise and/or pharmacological interventions aimed at attenuating muscle disuse atrophy during short periods of bed rest or immobilization.

In conclusion, limb immobilization leads to a rapid loss of skeletal muscle mass and strength, with substantial atrophy already occurring during the first 5 days of muscle disuse. These data imply that successive short periods of muscle disuse, due to illness or injury, can be of important clinical relevance and, therefore, effective interventional strategies should be designed to prevent muscle atrophy during such short periods of muscle disuse. 


\section{REFERENCES}

1. Deitrick, J.E., The effect of immobilization on metabolic and physiological functions of normal men. Bull $N$ Y Acad Med, 1948. 24(6): p. 364-75.

2. Gibson, J.N., D. Halliday, W.L. Morrison, P.J. Stoward, G.A. Hornsby, P.W. Watt, G. Murdoch, and M.J. Rennie, Decrease in human quadriceps muscle protein turnover consequent upon leg immobilization. Clin Sci (Lond), 1987. 72(4): p. 503-9.

3. Ingemann-Hansen, T. and J. Halkjaer-Kristensen, Computerized tomographic determination of human thigh components. The effects of immobilization in plaster and subsequent physical training. Scand J Rehabil Med, 1980. 12(1): p. 27-31.

4. White, M.J., C.T. Davies, and P. Brooksby, The effects of short-term voluntary immobilization on the contractile properties of the human triceps surae. Q J Exp Physiol, 1984. 69(4): p. 685-91.

5. LeBlanc, A.D., V.S. Schneider, H.J. Evans, C. Pientok, R. Rowe, and E. Spector, Regional changes in muscle mass following 17 weeks of bed rest. J Appl Physiol (1985), 1992. 73(5): p. 2172-8.

6. Haruna, Y., Y. Suzuki, K. Kawakubo, R. Yanagibori, and A. Gunji, Decremental reset in basal metabolism during 20-days bed rest. Acta Physiol Scand Suppl, 1994. 616: p. 43-9.

7. Tzankoff, S.P. and A.H. Norris, Effect of muscle mass decrease on age-related BMR changes. J Appl Physiol Respir Environ Exerc Physiol, 1977. 43(6): p. 1001-6.

8. Stuart, C.A., R.E. Shangraw, M.J. Prince, E.J. Peters, and R.R. Wolfe, Bed-rest-induced insulin resistance occurs primarily in muscle. Metabolism, 1988. 37(8): p. 802-6.

9. Ferrando, A.A., K.D. Tipton, M.M. Bamman, and R.R. Wolfe, Resistance exercise maintains skeletal muscle protein synthesis during bed rest. J Appl Physiol (1985), 1997. 82(3): p. 807-10.

10. Ferrando, A.A., H.W. Lane, C.A. Stuart, J. Davis-Street, and R.R. Wolfe, Prolonged bed rest decreases skeletal muscle and whole body protein synthesis. Am J Physiol, 1996. 270(4 Pt 1): p. E627-33.

11. Brooks, N., G.J. Cloutier, S.M. Cadena, J.E. Layne, C.A. Nelsen, A.M. Freed, R. Roubenoff, and C. Castaneda-Sceppa, Resistance training and timed essential amino acids protect against the loss of muscle mass and strength during 28 days of bed rest and energy deficit. J Appl Physiol, 2008. 105(1): p. 241-8.

12. Jones, S.W., R.J. Hill, P.A. Krasney, B. O'Conner, N. Peirce, and P.L. Greenhaff, Disuse atrophy and exercise rehabilitation in humans profoundly affects the expression of genes associated with the regulation of skeletal muscle mass. FASEB J, 2004. 18(9): p. 1025-7.

13. Paddon-Jones, D., M. Sheffield-Moore, R.J. Urban, A.P. Sanford, A. Aarsland, R.R. Wolfe, and A.A. Ferrando, Essential amino acid and carbohydrate supplementation ameliorates muscle protein loss in humans during 28 days bedrest. J Clin Endocrinol Metab, 2004. 89(9): p. 4351-8.

14. Fisher, S.R., Y.F. Kuo, J.E. Graham, K.J. Ottenbacher, and G.V. Ostir, Early ambulation and length of stay in older adults hospitalized for acute illness. Arch Intern Med, 2010. 170(21): p. 1942-3.

15. Suetta, C., U. Frandsen, L. Jensen, M.M. Jensen, J.G. Jespersen, L.G. Hvid, M. Bayer, S.J. Petersson, H.D. Schroder, J.L. Andersen, K.M. Heinemeier, P. Aagaard, P. Schjerling, and M. Kjaer, Aging affects the transcriptional regulation of human skeletal muscle disuse atrophy. PLoS One, 2012. 7(12): p. e51238.

16. Hvid, L.G., C. Suetta, P. Aagaard, M. Kjaer, U. Frandsen, and N. Ortenblad, Four days of muscle disuse impairs single fiber contractile function in young and old healthy men. Exp Gerontol, 2013. 48(2): p. 154-61.

17. Wall, B.T. and L.J. van Loon, Nutritional strategies to attenuate muscle disuse atrophy. Nutr Rev, 2013. 71(4): p. 195-208.

18. Wall, B.T., M.L. Dirks, and L.J. van Loon, Skeletal muscle atrophy during short-term disuse: implications for age-related sarcopenia. Ageing Res Rev, 2013. 12(4): p. 898-906.

19. English, K.L. and D. Paddon-Jones, Protecting muscle mass and function in older adults during bed rest. Curr Opin Clin Nutr Metab Care, 2010. 13(1): p. 34-9.

20. Glover, E.I., N. Yasuda, M.A. Tarnopolsky, A. Abadi, and S.M. Phillips, Little change in markers of protein breakdown and oxidative stress in humans in immobilization-induced skeletal muscle atrophy. Appl Physiol Nutr Metab, 2010. 35(2): p. 125-33. 
21. Marimuthu, K., A.J. Murton, and P.L. Greenhaff, Mechanisms regulating muscle mass during disuse atrophy and rehabilitation in humans. J Appl Physiol (1985), 2011. 110(2): p. 555-60.

22. Murton, A.J. and P.L. Greenhaff, Physiological control of muscle mass in humans during resistance exercise, disuse and rehabilitation. Curr Opin Clin Nutr Metab Care, 2010. 13(3): p. 249-54.

23. Phillips, S.M., E.I. Glover, and M.J. Rennie, Alterations of protein turnover underlying disuse atrophy in human skeletal muscle. J Appl Physiol (1985), 2009. 107(3): p. 645-54.

24. Mosher, D.S., P. Quignon, C.D. Bustamante, N.B. Sutter, C.S. Mellersh, H.G. Parker, and E.A. Ostrander, A mutation in the myostatin gene increases muscle mass and enhances racing performance in heterozygote dogs. PLoS Genet, 2007. 3(5): p. e79.

25. McPherron, A.C. and S.J. Lee, Double muscling in cattle due to mutations in the myostatin gene. Proc Natl Acad Sci U S A, 1997. 94(23): p. 12457-61.

26. Schuelke, M., K.R. Wagner, L.E. Stolz, C. Hubner, T. Riebel, W. Komen, T. Braun, J.F. Tobin, and S.J. Lee, Myostatin mutation associated with gross muscle hypertrophy in a child. N Engl J Med, 2004. 350(26): p. 2682-8.

27. Rodriguez, J., B. Vernus, M. Toubiana, E. Jublanc, L. Tintignac, S. Leibovitch, and A. Bonnieu, Myostatin inactivation increases myotube size through regulation of translational initiation machinery. J Cell Biochem, 2011. 112(12): p. 3531-42.

28. Amthor, H., R. Huang, I. McKinnell, B. Christ, R. Kambadur, M. Sharma, and K. Patel, The regulation and action of myostatin as a negative regulator of muscle development during avian embryogenesis. Dev Biol, 2002. 251(2): p. 241-57.

29. Muroya, S., K. Watanabe, S. Hayashi, M. Miyake, S. Konashi, Y. Sato, M. Takahashi, S. Kawahata, Y. Yoshikawa, H. Aso, K. Chikuni, and T. Yamaguchi, Muscle type-specific effect of myostatin deficiency on myogenic regulatory factor expression in adult double-muscled Japanese Shorthorn cattle. Anim Sci J, 2009. 80(6): p. 678-85.

30. McFarlane, C., E. Plummer, M. Thomas, A. Hennebry, M. Ashby, N. Ling, H. Smith, M. Sharma, and R. Kambadur, Myostatin induces cachexia by activating the ubiquitin proteolytic system through an NFkappaB-independent, FoxO1-dependent mechanism. J Cell Physiol, 2006. 209(2): p. 501-14.

31. Mayhew, J.L., J.L. Prinster, J.S. Ware, D.L. Zimmer, J.R. Arabas, and M.G. Bemben, Muscular endurance repetitions to predict bench press strength in men of different training levels. J Sports Med Phys Fitness, 1995. 35(2): p. 108-13.

32. Goodpaster, B.H., D.E. Kelley, F.L. Thaete, J. He, and R. Ross, Skeletal muscle attenuation determined by computed tomography is associated with skeletal muscle lipid content. J Appl Physiol, 2000. 89(1): p. 104-10.

33. Strandberg, S., M.L. Wretling, T. Wredmark, and A. Shalabi, Reliability of computed tomography measurements in assessment of thigh muscle cross-sectional area and attenuation. BMC Med Imaging, 2010. 10: p. 18.

34. Bergstrom, J., Percutaneous needle biopsy of skeletal muscle in physiological and clinical research. Scand J Clin Lab Invest, 1975. 35(7): p. 609-16.

35. Kraemer, W., and A. Fry. . Strength testing: Development and evaluation of methodology. In:. Physiological assessment of physical fitness, 1995. P. Maud and C. Foster (Eds.) Leeds: Human Kinetics: p. 115-133.

36. Edelstein, A., N. Amodaj, K. Hoover, R. Vale, and N. Stuurman, Computer control of microscopes using microManager. Curr Protoc Mol Biol, 2010. Chapter 14: p. Unit14 20.

37. Gustafsson, T., T. Osterlund, J.N. Flanagan, F. von Walden, T.A. Trappe, R.M. Linnehan, and P.A. Tesch, Effects of 3 days unloading on molecular regulators of muscle size in humans. J Appl Physiol, 2010. 109(3): p. 721-7.

38. Chen, Y.W., C.M. Gregory, M.T. Scarborough, R. Shi, G.A. Walter, and K. Vandenborne, Transcriptional pathways associated with skeletal muscle disuse atrophy in humans. Physiol Genomics, 2007. 31(3): p. 510-20.

39. Bamman, M.M., M.S. Clarke, D.L. Feeback, R.J. Talmadge, B.R. Stevens, S.A. Lieberman, and M.C. Greenisen, Impact of resistance exercise during bed rest on skeletal muscle sarcopenia and myosin isoform distribution. J Appl Physiol (1985), 1998. 84(1): p. 157-63. 
40. Yasuda, N., E.I. Glover, S.M. Phillips, R.J. Isfort, and M.A. Tarnopolsky, Sex-based differences in skeletal muscle function and morphology with short-term limb immobilization. J Appl Physiol, 2005. 99(3): p. 1085-92.

41. Veldhuizen, J.W., F.T. Verstappen, J.P. Vroemen, H. Kuipers, and J.M. Greep, Functional and morphological adaptations following four weeks of knee immobilization. Int J Sports Med, 1993. 14(5): p. 283-7.

42. Hvid, L., P. Aagaard, L. Justesen, M.L. Bayer, J.L. Andersen, N. Ortenblad, M. Kjaer, and C. Suetta, Effects of aging on muscle mechanical function and muscle fiber morphology during short-term immobilization and subsequent retraining. J Appl Physiol (1985), 2010. 109(6): p. 1628-34.

43. Ferrando, A.A., C.A. Stuart, D.G. Brunder, and G.R. Hillman, Magnetic resonance imaging quantitation of changes in muscle volume during 7 days of strict bed rest. Aviat Space Environ Med, 1995. 66(10): p. 976-81.

44. Nilwik, R., T. Snijders, M. Leenders, B.B. Groen, J. van Kranenburg, L.B. Verdijk, and L.J. van Loon, The decline in skeletal muscle mass with aging is mainly attributed to a reduction in type II muscle fiber size. Exp Gerontol, 2013. 48(5): p. 492-8.

45. Campbell, E.L., O.R. Seynnes, R. Bottinelli, J.S. McPhee, P.J. Atherton, D.A. Jones, G. Butler-Browne, and M.V. Narici, Skeletal muscle adaptations to physical inactivity and subsequent retraining in young men. Biogerontology, 2013. 14(3): p. 247-59.

46. Glover, E.I., S.M. Phillips, B.R. Oates, J.E. Tang, M.A. Tarnopolsky, A. Selby, K. Smith, and M.J. Rennie, Immobilization induces anabolic resistance in human myofibrillar protein synthesis with low and high dose amino acid infusion. J Physiol, 2008. 586(Pt 24): p. 6049-61.

47. Urso, M.L., A.G. Scrimgeour, Y.W. Chen, P.D. Thompson, and P.M. Clarkson, Analysis of human skeletal muscle after $48 \mathrm{~h}$ immobilization reveals alterations in mRNA and protein for extracellular matrix components. J Appl Physiol (1985), 2006. 101(4): p. 1136-48.

48. Tesch, P.A., F. von Walden, T. Gustafsson, R.M. Linnehan, and T.A. Trappe, Skeletal muscle proteolysis in response to short-term unloading in humans. J Appl Physiol (1985), 2008. 105(3): p. 902-6.

49. Murphy, K.T., V. Cobani, J.G. Ryall, C. Ibebunjo, and G.S. Lynch, Acute antibody-directed myostatin inhibition attenuates disuse muscle atrophy and weakness in mice. J Appl Physiol (1985), 2011. 110(4): p. 1065-72.

50. Bunn, J.A., T.W. Buford, M.C. Serra, R.B. Kreider, and D.S. Willoughby, Protein and Amino Acid Supplementation Does Not Alter Proteolytic Gene Expression following Immobilization. J Nutr Metab, 2011. 2011: p. 539690.

51. Murton, A.J., D. Constantin, and P.L. Greenhaff, The involvement of the ubiquitin proteasome system in human skeletal muscle remodelling and atrophy. Biochim Biophys Acta, 2008. 1782(12): p. 730-43.

52. Abadi, A., E.I. Glover, R.J. Isfort, S. Raha, A. Safdar, N. Yasuda, J.J. Kaczor, S. Melov, A. Hubbard, X. Qu, S.M. Phillips, and M. Tarnopolsky, Limb immobilization induces a coordinate down-regulation of mitochondrial and other metabolic pathways in men and women. PLoS One, 2009. 4(8): p. e6518.

53. Elliott, B., D. Renshaw, S. Getting, and R. Mackenzie, The central role of myostatin in skeletal muscle and whole body homeostasis. Acta Physiol (Oxf), 2012. 205(3): p. 324-40.

54. Hollinger, K. and J.T. Selsby, The physiological response of protease inhibition in dystrophic muscle. Acta Physiol (Oxf), 2013. 208(3): p. 234-44.

55. Krause, M.P., J. Moradi, S.K. Coleman, D.M. D'Souza, C. Liu, M.S. Kronenberg, D.W. Rowe, T.J. Hawke, and M. Hadjiargyrou, A novel GFP reporter mouse reveals Mustn1 expression in adult regenerating skeletal muscle, activated satellite cells and differentiating myoblasts. Acta Physiol (Oxf), 2013. 208(2): p. 180-90.

56. Hespel, P., B. Op't Eijnde, M. Van Leemputte, B. Urso, P.L. Greenhaff, V. Labarque, S. Dymarkowski, P. Van Hecke, and E.A. Richter, Oral creatine supplementation facilitates the rehabilitation of disuse atrophy and alters the expression of muscle myogenic factors in humans. J Physiol, 2001. 536(Pt 2): p. 625-33.

57. Gamrin, L., H.E. Berg, P. Essen, P.A. Tesch, E. Hultman, P.J. Garlick, M.A. McNurlan, and J. Wernerman, The effect of unloading on protein synthesis in human skeletal muscle. Acta Physiol Scand, 1998. 163(4): p. 369-77.

58. Machida, M., K. Takeda, H. Yokono, S. Ikemune, Y. Taniguchi, H. Kiyosawa, and T. Takemasa, Reduction of ribosome biogenesis with activation of the mTOR pathway in denervated atrophic muscle. J Cell Physiol, 2012. 227(4): p. 1569-76. 
59. Ferrando, A.A., C.A. Stuart, M. Sheffield-Moore, and R.R. Wolfe, Inactivity amplifies the catabolic response of skeletal muscle to cortisol. J Clin Endocrinol Metab, 1999. 84(10): p. 3515-21.

60. Fitts, R.H., J.G. Romatowski, J.R. Peters, D. Paddon-Jones, R.R. Wolfe, and A.A. Ferrando, The deleterious effects of bed rest on human skeletal muscle fibers are exacerbated by hypercortisolemia and ameliorated by dietary supplementation. Am J Physiol Cell Physiol, 2007. 293(1): p. C313-20.

61. Mithal, A., J.P. Bonjour, S. Boonen, P. Burckhardt, H. Degens, G. El Hajj Fuleihan, R. Josse, P. Lips, J. Morales Torres, R. Rizzoli, N. Yoshimura, D.A. Wahl, C. Cooper, B. Dawson-Hughes, and I.C.N.W. Group, Impact of nutrition on muscle mass, strength, and performance in older adults. Osteoporos Int, 2013. 24(5): p. 1555-66. 



\title{
CHAPTER
}

Short-term muscle disuse lowers myofibrillar protein synthesis rates and induces anabolic resistance to protein ingestion

\author{
Benjamin T Wall * \\ Marlou L Dirks * \\ Tim Snijders \\ Jan-Willem van Dijk \\ Mario Fritsch \\ Lex B Verdijk \\ Luc JC van Loon \\ *Joint first authors
}


ABSTRACT

Background Disuse leads to rapid loss of skeletal muscle mass and function. It has been hypothesized that short, successive periods of muscle disuse throughout the lifespan play an important role in the development of sarcopenia. The physiological mechanisms underlying short-term muscle disuse atrophy remain to be elucidated. We assessed the impact of five days of muscle disuse on postabsorptive and postprandial myofibrillar protein synthesis rates in humans.

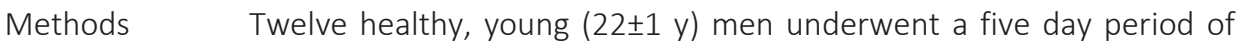
one-legged knee immobilization (full leg cast). Quadriceps crosssectional area (CSA) of both legs was assessed before and after immobilization. Continuous infusions of $\mathrm{L}-\left[\mathrm{ring}^{-}{ }^{2} \mathrm{H}_{5}\right]$-phenylalanine and L-[1${ }^{13} \mathrm{C}$ ]-leucine were combined with the ingestion of a $25 \mathrm{~g}$ bolus of intrinsically $\mathrm{L}-\left[1-{ }^{13} \mathrm{C}\right]$-phenylalanine and $\mathrm{L}-\left[1-{ }^{13} \mathrm{C}\right]$-leucine labelled dietary protein to assess myofibrillar muscle protein fractional synthetic rates (FSRs) in the immobilized and non-immobilized control leg.

Results Immobilization led to a $3.9 \pm 0.6 \%$ decrease in quadriceps muscle CSA of the immobilized leg. Based on the L-[ring $\left.-{ }^{2} \mathrm{H}_{5}\right]$-phenylalanine tracer, immobilization reduced postabsorptive myofibrillar protein synthesis rates by $41 \pm 13 \%\left(0.015 \pm 0.002\right.$ vs $\left.0.032 \pm 0.005 \% \mathrm{~h}^{-1} ; P<0.01\right)$ and postprandial myofibrillar protein synthesis rates by $53 \pm 4 \%(0.020 \pm 0.002$ vs $\left.0.044 \pm 0.003 \% \mathrm{~h}^{-1} ; P<0.01\right)$. Comparable results were found using the $\mathrm{L}-\left[1-{ }^{13} \mathrm{C}\right]$-leucine tracer. Following protein ingestion, myofibrillar protein-bound $\mathrm{L}-\left[1-{ }^{13} \mathrm{C}\right]$-phenylalanine enrichments were $53 \pm 18 \%$ lower in the immobilized compared with the control leg $(0.007 \pm 0.002$ and $0.015 \pm 0.002$ MPE, respectively; $P<0.05)$.

Conclusion We conclude that five days of muscle disuse substantially lowers postabsorptive myofibrillar protein synthesis rates and induces anabolic resistance to protein ingestion. 


\section{INTRODUCTION}

In otherwise healthy humans, the recovery from injury or illness often mandates a period of local (e.g. limb immobilization) or whole-body (e.g. bed rest) muscle disuse. It has long been recognized that a prolonged period of disuse (i.e. $>10$ days) leads to rapid skeletal muscle atrophy [1-3]. The resulting negative health consequences that accompany prolonged muscle disuse atrophy, such as a reduction in functional capacity [1, 2], insulin sensitivity [4] and basal metabolic rate [5], have been well documented. As such, muscle disuse atrophy is of great clinical relevance and therefore represents an important area for scientific investigation.

Although a single bout of prolonged disuse provides an acute metabolic and functional challenge to any individual, perhaps of more long-term relevance is the prevalence of (multiple) shorter periods of disuse that occur throughout the lifespan. For example, the average length of hospitalization for elderly patients admitted with acute illness is 5-6 days [6] and most periods of illness and injury that require home-based recovery/reduced physical activity generally last less than one week. We $[7,8]$, and others $[9,10]$ have hypothesized that the accumulation of such short periods of disuse over the lifespan represents an important factor in the development of age-related sarcopenia. In support, we recently reported that even a period of muscle disuse lasting only five days already leads to substantial losses of skeletal muscle mass and functional strength in young [7] and older [11] subjects.

Mechanistically, any sizeable loss of skeletal muscle mass must be underpinned by a persistent imbalance between muscle protein synthesis and breakdown rates. A body of early muscle atrophy studies performed in animal models comprehensively demonstrated that a decline in muscle protein synthesis rates was a key factor responsible for disuse atrophy [e.g. 12, 13, 14]. Critically, however, no data are currently available assessing in vivo muscle protein turnover rates during a period of short-term disuse (i.e. $<10$ days) in humans. Accordingly, the physiological mechanisms underlying skeletal muscle atrophy during short-term disuse in humans remain to be elucidated. Previous human studies, applying more prolonged periods of disuse, suggests that declines in the fasting muscle protein synthesis rates $[2,15,16]$ and/or a reduced responsiveness to the anabolic properties of amino acids/food intake [16-18] are important factors responsible for disuse atrophy. In the present study, we applied specifically produced intrinsically $\mathrm{L}-\left[1-{ }^{13} \mathrm{C}\right]$-phenylalanine and $\mathrm{L}-\left[1-{ }^{13} \mathrm{C}\right]$-leucine labelled dietary protein combined with continuous intravenous $\mathrm{L}-\left[\mathrm{ring}_{-}{ }^{2} \mathrm{H}_{5}\right]$-phenylalanine and $\mathrm{L}-\left[1-{ }^{13} \mathrm{C}\right]$-leucine infusions [19] to assess both postabsorptive and postprandial muscle protein synthesis rates as well as the metabolic fate of ingested protein following 5 days of leg immobilization in 12 healthy young men. 


\section{METHODS}

\section{Subjects}

Twelve healthy, young ( $22 \pm 1$ y) men volunteered to participate in the present study. Subjects' characteristics are presented in Table 1.

Table 1 | Subjects' characteristics $(n=12)$

\begin{tabular}{lc}
\hline Age $(\mathrm{y})$ & $22 \pm 1$ \\
Body mass $(\mathrm{kg})$ & $82.5 \pm 2.7$ \\
$\mathrm{BMI}\left(\mathrm{kg} \mathrm{m}^{-2}\right)$ & $24.6 \pm 0.7$ \\
Body fat (\% of body mass) & $19.4 \pm 1.2$ \\
Lean body mass (kg) & $63.5 \pm 1.6$ \\
Average leg lean mass (kg) & $10.9 \pm 0.4$ \\
HbA1c $(\%)$ & $5.0 \pm 0.1$ \\
\hline
\end{tabular}

Values represent means \pm SEM. HbA1c: glycosylated haemoglobin.

All subjects were fully informed of the nature and possible risks of the experimental procedures, before providing written informed consent. Subjects were screened to exclude any person with lower limb and/or back injuries sustained within a year prior to the study, a (family) history of thrombosis/cardiovascular disease, use of anticoagulants, musculoskeletal/orthopaedic/haemostatic disorders, or participation in any regular resistance training program within 6 months prior to the study. During screening, body composition (fat, fat-free mass and bone mineral content) were determined by dual-energy x-ray absorptiometry (DXA) scan (Hologic Inc., Discovery A, QDR series, Bedford, USA). Whole-body and regional lean mass and percent body fat were determined using the software package Apex version 2.3 (Hologic, Bedford, USA). The study was approved by the Medical Ethics Committee of the Maastricht University Medical Centre, Maastricht, the Netherlands in accordance with the guidelines set out in the Declaration of Helsinki.

\section{Experimental design}

Subjects participated in a single stable isotope tracer infusion experimental visit immediately following a five day period of one-legged knee immobilization by means of a full leg cast. During the experimental visit, continuous intravenous infusions of L-[ring- $\left.{ }^{2} \mathrm{H}_{5}\right]$ phenylalanine and $\mathrm{L}-\left[1-{ }^{13} \mathrm{C}\right]$-leucine were combined with the ingestion of $25 \mathrm{~g}$ intrinsically $\mathrm{L}-\left[1-{ }^{13} \mathrm{C}\right]$-phenylalanine and $\mathrm{L}-\left[1-{ }^{13} \mathrm{C}\right]$-leucine labelled dietary protein. Throughout the experimental visit, multiple blood samples were drawn and muscle samples were obtained from both legs in a previously validated, comprehensive approach to determine muscle protein metabolism [19]. This design allowed us to simultaneously assess 
postabsorptive and postprandial muscle protein synthesis rates, and the metabolic fate of the ingested protein, in the immobilized and non-immobilized control leg.

\section{Pre-testing}

Two days prior to the immobilization period, subjects participated in a single pre-testing session to assess skeletal muscle mass of the legs. Subjects arrived at the laboratory at $08.00 \mathrm{~h}$ and body weight was measured with a digital balance with an accuracy of $0.1 \mathrm{~kg}$ (SECA GmbH, Hamburg, Germany). Thereafter, a single slice CT-scan (Philips Brilliance 64, Philips Medical Systems, Best, the Netherlands) was performed to assess upper leg muscle cross-sectional area (CSA). The scanning characteristics were as follows: $120 \mathrm{kV}, 300$ $\mathrm{mA}$, rotation time of $0.75 \mathrm{~s}$, and a field of view of $500 \mathrm{~mm}$. With subjects lying supine with their legs extended and feet secured, a $3 \mathrm{~mm}$ thick axial image was taken $15 \mathrm{~cm}$ proximal to the top of the patella. The precise scan position was marked with semipermanent ink for the duration of the experimental protocol to ensure accurate repeat measurements. Muscle area of the legs was selected between 0 and 100 Hounsfield units [20], after which the quadriceps muscle was selected by manual tracing using ImageJ software (version 1.45d, National Institute of Health, Maryland, USA) [21-23]. Next, the leg to be immobilized was selected at random (counterbalanced for left and right legs) and subjects were instructed on, and familiarized with, the use of crutches (casting described below). On the morning of the experimental test day (i.e. the end of the immobilization period) the cast removal was immediately followed by a second CT scan.

\section{Limb immobilization}

Two days following pre-testing, subjects attended the Casting Room at Maastricht University Medical Centre at $08.00 \mathrm{~h}$ to have a full leg cast fitted to induce one-legged knee immobilization. First, a high moisture zinc paste bandage (Varicex ${ }^{\circledR} T$, Lohmann \& Rauscher, Germany) was placed around the calf to prevent the cast dropping, and a foam ankle band was applied (Collar ' $n$ ' Cuff ${ }^{\mathrm{TM}}$, Mölnlycke Health Care, Gothenburg, Sweden) to avoid pressure from the cast on the ankle joint. Thereafter, cotton padding was applied to the leg for skin protection (Cellona ${ }^{\circledR}$, Lohmann \& Rauscher,Neuwied, Germany) and a fibreglass and polyurethane resin lightweight cast (Delta-Lite ${ }^{\circledR}, \mathrm{BSN}$ Medical, Germany) was fitted to the leg. The circular leg cast extended from $10 \mathrm{~cm}$ above the ankle to approximately $25 \mathrm{~cm}$ above the patella. The knee was casted at a $30^{\circ}$ angle of flexion to prevent subjects performing any weight bearing on the casted limb. Subjects were provided with crutches for proper ambulation. Throughout the immobilization period, subjects were instructed to perform a series of daily simple ankle exercises (i.e. plantar and dorsal flexion, and circular movements of the entire foot) to keep the calf muscle pump activated in the immobilized leg, thereby minimizing the risk of developing a deep vein thrombosis. Prior to the start of the stable isotope infusion visit, subjects visited the Casting Room to have the cast removed and had a second CT scan 
performed as described above. Following cast removal, subjects were exclusively transported by wheelchair to prevent any weight bearing on the immobilized leg.

\section{Diet and physical activity}

All subjects received the same standardized meal the evening prior to the stable isotope infusion experimental visits ( $33 \pm 2 \mathrm{~kJ}^{\prime} \mathrm{kg}^{-1}$ body weight, providing 44 energy\% (En\%) carbohydrate, $22 \mathrm{En} \%$ protein, and $34 \mathrm{En} \%$ fat). All volunteers were instructed to refrain from alcohol intake and to keep their diet as constant as possible for the duration of the immobilization period.

\section{Experimental visits}

On the morning following the 5 day immobilization period (i.e. day 6 ) subjects arrived at the laboratory by taxi at 08:00 $\mathrm{h}$ for a stable isotope infusion experiment. Following cast removal and CT-scanning (described above), a polytetrafluoroethylene catheter was inserted into an antecubital vein for stable-isotope infusion. A second catheter was inserted into a heated dorsal hand vein of the contralateral arm after which the hand was placed in a hot box $\left(60^{\circ} \mathrm{C}\right)$ for arterialized blood sampling [24]. After a basal blood sample was collected $(t=-240 \mathrm{~min}$ ), the plasma phenylalanine and leucine pools were primed with a single intravenous dose $\left(2.0 \mu \mathrm{mol} \mathrm{kg}^{-1} \mathrm{~L}\right.$ - $\left[\mathrm{ring}_{-}{ }^{2} \mathrm{H}_{5}\right]$-phenylalanine; 4.0 $\mu \mathrm{mol} \mathrm{kg}^{-1} \mathrm{~L}-\left[1-{ }^{13} \mathrm{C}\right]$-leucine), after which continuous $\mathrm{L}-\left[\right.$ ring $\left.-{ }^{2} \mathrm{H}_{5}\right]$-phenylalanine $(0.06$ $\left.\mu \mathrm{mol} \mathrm{kg}^{-1} \mathrm{~min}^{-1}\right)$ and $\mathrm{L}-\left[1{ }^{13} \mathrm{C}\right]$-leucine $\left(0.10 \mu \mathrm{mol}^{1} \mathrm{~kg}^{-1} \mathrm{~min}^{-1}\right)$ infusions were started. After the subjects rested in a semi-supine position for $120 \mathrm{~min}$, a second blood sample was drawn and muscle biopsies were collected from the vastus lateralis muscle of both legs $(t=-120 \mathrm{~min}$ ). This signified the beginning of a $2 \mathrm{~h}$ period for the determination of postabsorptive muscle protein synthesis rates during which blood samples were collected every $30 \mathrm{~min}$ (i.e. $t=-90,-60,-30$ and $0 \mathrm{~min}$ ). At $t=0 \mathrm{~min}$, muscle biopsies were again collected from the vastus lateralis muscle of both legs. Immediately after these biopsies, subjects ingested a single bolus of $25 \mathrm{~g}$ intrinsically $\mathrm{L}-\left[1-{ }^{13} \mathrm{C}\right]$-phenylalanine and $\mathrm{L}$ $\left[1-{ }^{13} \mathrm{C}\right]$-leucine-labelled whey protein dissolved in $350 \mathrm{~mL}$ vanilla flavored water signifying the beginning of a $4 \mathrm{~h}$ period for the determination of postprandial muscle protein synthesis rates. We have previously shown [19] that the above described use of doubly labelled milk protein allows the measurement of postprandial muscle protein synthesis rates without the disturbance of steady state tracer conditions, with the concomitant determination of de novo muscle protein accretion from the ingested protein source (for full details of the approach please refer to [19]).

Following protein ingestion, subjects rested in a semi-supine position for another $240 \mathrm{~min}$, during which arterialized blood samples were collected at regular intervals $(t=$ $30,60,90,120,180$ and $240 \mathrm{~min}$ ) after which final muscle biopsies were collected from both legs $(t=240 \mathrm{~min}$ ) marking the end of the infusion period. 
Blood samples were collected into EDTA-containing tubes and centrifuged at $3500 \mathrm{~g}$ for $10 \mathrm{~min}$ at $4^{\circ} \mathrm{C}$. Aliquots of plasma were frozen in liquid nitrogen and stored at $-80^{\circ} \mathrm{C}$. The muscle biopsy samples were all taken from separate incisions and in different directions, at least $2 \mathrm{~cm}$ apart. Muscle biopsy samples were obtained from the middle region of the vastus lateralis, $\sim 15 \mathrm{~cm}$ above the patella and $\sim 3 \mathrm{~cm}$ below entry through the fascia, using the percutaneous needle biopsy technique [25]. Muscle samples were dissected carefully and freed from any visible non-muscle material and were immediately frozen in liquid nitrogen and stored at $-80^{\circ} \mathrm{C}$ until further analysis.

\section{Production of intrinsically labelled protein}

Intrinsically $\mathrm{L}-\left[1-{ }^{13} \mathrm{C}\right]$-phenylalanine and $\mathrm{L}-\left[1-{ }^{13} \mathrm{C}\right]$-leucine milk protein was obtained by a constant infusion of $\mathrm{L}-\left[1-{ }^{13} \mathrm{C}\right]$-phenylalanine $\left(455 \mu \mathrm{mol} \cdot \mathrm{min}^{-1}\right)$ and $\mathrm{L}-\left[1-{ }^{13} \mathrm{C}\right]$-leucine $(200$ $\mu \mathrm{mol} \cdot \mathrm{min}^{-1}$ ) maintained for $96 \mathrm{~h}$ in a lactating dairy cow [26-28]. The milk was collected, processed, and fractionated into the whey protein concentrate as previously described [27]. The $\mathrm{L}-\left[1-{ }^{13} \mathrm{C}\right]$-phenylalanine and $\mathrm{L}-\left[1-{ }^{13} \mathrm{C}\right]$-leucine enrichments in whey protein were measured by gas chromatography-combustion-isotope ratio mass spectrometry (GC-C-IRMS; MAT 252, Finnigan, Breman, Germany) and averaged 38.7 MPE and 9.3 MPE, respectively. The proteins met all chemical and bacteriologic specifications for human consumption.

\section{Plasma analyses}

Plasma glucose and insulin concentrations were analyzed using commercially available kits (Glucose HK CP, ABX Diagnostics, ref. A11A01667, Montpellier, France and Millipore, ref. HI-14K, Billerica, USA, respectively). Plasma amino acid concentrations and enrichments were determined by GCMS analysis (Agilent 7890A GC/5975C; MSD, Little Falls, DE, USA). Specifically, internal standards of $\left[\mathrm{U}-{ }^{13} \mathrm{C}_{6}\right]$-leucine and $\left[\mathrm{U}-{ }^{13} \mathrm{C}_{9}{ }^{15} \mathrm{~N}\right]$ phenylalanine, and $\left[\mathrm{U}_{-}{ }^{13} \mathrm{C}_{9}{ }^{15} \mathrm{~N}\right]$-tyrosine were added to the samples. The plasma was deproteinised on ice with $10 \mathrm{mg}$ of dry 5-sulfosalicylic acid. Free amino acids were purified using cation exchange chromatography (AG 50W-X8 resin; Bio-Rad Laboratories, Hercules, CA, USA). The free amino acids were converted to their $t$-butyldimethylsilyl (TBDMS) derivative before analysis by GCMS. The amino acid concentrations were determined using electron impact ionization by monitoring ions at mass/charge $(\mathrm{m} / \mathrm{z}) 302$ and 308 for unlabelled and $\left[\mathrm{U}-^{13} \mathrm{C}_{6}\right.$ ]-leucine respectively, 336 and 346 for unlabelled and $\left[\mathrm{U}-{ }^{13} \mathrm{C}_{9}{ }^{15} \mathrm{~N}\right]$-phenylalanine respectively, and 466 and 476 for unlabelled and $\left[\mathrm{U}-{ }^{13} \mathrm{C}_{9}{ }^{15} \mathrm{~N}\right]-$ tyrosine respectively. The plasma free $\mathrm{L}$-[ring- $\left.{ }^{2} \mathrm{H}_{5}\right]$-phenylalanine enrichments were measured from the $m / z$ ratios at $336(m+0)$ and $341(m+5)$, with $m+0$ representing the lowest molecular weight of the ion. For $L-\left[1-{ }^{13} \mathrm{C}\right]$-phenylalanine, $m / z 336(m+0)$ and 337 $(m+1)$ were monitored. For L- $\left[1-{ }^{13} \mathrm{C}\right]$-leucine, $\mathrm{m} / \mathrm{z} 302(\mathrm{~m}+0)$ and $303(m+1)$ were monitored. Standard regression curves were applied in all isotopic enrichment analyses to assess linearity of the mass spectrometer and to control for the stable isotope effects 
that occur during analysis. Phenylalanine and leucine enrichments were corrected for the presence of both the ${ }^{13} \mathrm{C}$ and ${ }^{2} \mathrm{H}$ isotopes.

\section{Muscle tracer analyses}

Myofibrillar protein enriched fractions were extracted from $50 \mathrm{mg}$ of wet muscle tissue by hand-homogenizing on ice using a Teflon pestle in a standard extraction buffer (7.5 $\mu \mathrm{l} \cdot \mathrm{mg}^{-1}$ ) [29]. The samples were spun at $1500 \mathrm{~g}$ for $10 \mathrm{~min}$ at $4^{\circ} \mathrm{C}$. The resultant supernatants that contained the sarcoplasmic proteins were removed. The remaining myofibrillar and collagen pellet were washed with $500 \mu \mathrm{l}$ of extraction buffer and spun at $700 \times \mathrm{g}$ for $10 \mathrm{~min}$ at $4^{\circ} \mathrm{C}$. The myofibrillar proteins were solubilized by adding $1.5 \mathrm{~mL}$ of $0.3 \mathrm{M}$ $\mathrm{NaOH}$ and heating at $50^{\circ} \mathrm{C}$ for $30 \mathrm{~min}$ with vortex mixing every $10 \mathrm{~min}$. Samples were centrifuged at $10000 \mathrm{~g}$ for $5 \mathrm{~min}$ at $4^{\circ} \mathrm{C}$, the supernatant containing the myofibrillarenriched fraction was collected and the collagen pellet was discarded. Myofibrillar proteins were precipitated by the addition of $1 \mathrm{~mL}$ of $1 \mathrm{M}$ PCA and spinning at $700 \mathrm{~g}$ for 10 $\min$ at $4^{\circ} \mathrm{C}$. The myofibrillar enriched proteins were washed twice with $70 \%$ ethanol. Amino acids were liberated by the addition of $2 \mathrm{~mL}$ of $6 \mathrm{M} \mathrm{HCL}$ and heating at $110^{\circ} \mathrm{C}$ for $24 \mathrm{~h}$. The hydrolysed myofibrillar protein fraction was dried under a nitrogen stream while being heated to $120^{\circ} \mathrm{C}$ and, subsequently, dissolved in a $25 \%$ acetic acid solution and passed over a Dowex exchange resin (AG 50W-X8, 100 - 200 mesh hydrogen form; Biorad, Hercules, CA, USA). The free amino acids were eluted with $2 \mathrm{M} \mathrm{NH}_{4} \mathrm{OH}$, dried, and the purified amino acids were derivatised into MTBSTFA-phenylethylamines [30, 31] to measure the $\mathrm{L}-\left[\right.$ ring $\left.-{ }^{2} \mathrm{H}_{5}\right]$-phenylalanine labelling using GCMS analysis as described previously [32]. Separate aliquots of the purified amino acids were converted to their $\mathrm{N}(\mathrm{O}, \mathrm{S})$-ethoxycarbonyl ethyl esters derivatives to determine the $\mathrm{L}-\left[1-{ }^{13} \mathrm{C}\right]$ phenylalanine and $\mathrm{L}-\left[1-{ }^{13} \mathrm{C}\right]$-leucine labelling of the myofibrillar proteins by gas chromatography combustion-isotope ratio mass spectrometry analysis (GC-C-IRMS; Trace GC Ultra, IRMS model MAT 253, Thermo Scientific, Breman, Germany). The derivatised amino acids were separated on a $30 \mathrm{~m} \times 0.25 \mathrm{~mm} \times 0.25 \mu \mathrm{m}$ DB-5 column (temperature program: $120^{\circ} \mathrm{C}$ for $10 \mathrm{~min} ; 3^{\circ} \mathrm{C} \cdot \mathrm{min}^{-1}$ ramp to $200^{\circ} \mathrm{C}$; $30^{\circ} \mathrm{C} \cdot \mathrm{min}^{-1}$ ramp to $300^{\circ} \mathrm{C}$; hold for $5 \mathrm{~min}$ ) prior to combustion. Standard regression curves were applied from a series of known standard enrichment values against the measured values to assess the linearity of the mass spectrometer and to account for any isotope fractionation that may have occurred during the analysis. In addition, western blot homogenates (see below) were used to determine the muscle intracellular free amino acid pool tracer enrichments using methods described previously [19].

\section{mRNA analyses}

Total RNA was isolated from 10-20 mg of frozen muscle tissue using TRIzol Reagent (Life Technologies, Invitrogen), according to the manufacturer's protocol. Total RNA quantification was carried out spectrophotometrically at $260 \mathrm{~nm}$ (NanoDrop ND-1000 
Spectrophotometer, Thermo Fisher Scientific, USA), and RNA purity was determined as the ratio of readings at $260 / 280 \mathrm{~nm}$. Thereafter, first strand cDNA was synthesized from

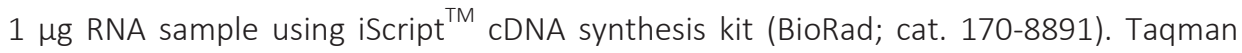
PCR was carried out using a 7300 Real Time PCR System (AppliedBiosystems, USA), with

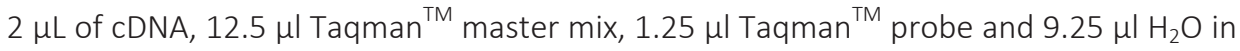
a $25 \mu \mathrm{L}$ final well volume. Each sample was run in duplicate, together with a serial dilution standard curve. The housekeeping gene $18 \mathrm{~S}$ was used as an internal control as this gene was unaffected by immobilization (mean $C t$ values were unaffected over time; data not shown) and has been used previously in similar studies [7, 33, 34]. Taqman primer/probe sets were obtained from Applied Biosystems (Foster City, USA): LAT1, PAT1, SNAT2, CD98, MAFBX, MURF1, FOXO1, IL-6, TNF $\alpha$ and 18S. The thermal cycling conditions used were: $2 \mathrm{~min}$ at $50^{\circ} \mathrm{C}, 10 \mathrm{~min}$ at $95^{\circ} \mathrm{C}$, followed by 40 cycles at $95^{\circ} \mathrm{C}$ for $15 \mathrm{~s}$ and $60^{\circ} \mathrm{C}$ for $1 \mathrm{~min}$. Ct values of the target genes were normalized to $\mathrm{C} t$ values of the internal control and final results were calculated as relative expression against the standard curve. To avoid redundancy, mRNA data in the fasted state are only presented in the second fasting biopsy.

\section{Western blotting}

A portion of each muscle sample frozen for biochemical analyses was homogenized in 7 volumes Tris buffer (20 mM Tris-HCL, 5 mM EDTA. $10 \mathrm{mM}$ Na-pyrosphospate, $100 \mathrm{mM}$ $\mathrm{NaF}, 2 \mathrm{mM}$ Na3VO4, 1\% Nonident P-40; $\mathrm{pH}$ 7.4) supplemented with the following protease and phosphatase inhibitors: Aprotinin $10 \mu \mathrm{g} \cdot \mathrm{mL}^{-1}$, Leupeptin $10 \mu \mathrm{g} \cdot \mathrm{mL}^{-1}$, Benzamidin $3 \mathrm{mM}$ and PMSF $1 \mathrm{mM}$. After homogenization, each muscle extract was centrifuged for 10 min at $10,000 \mathrm{~g}\left(4^{\circ} \mathrm{C}\right)$ and sample buffer was added to the supernatant to final concentrations of $60 \mathrm{mM}$ Tris, $10 \%$ glycerol, $20 \mathrm{mg} \cdot \mathrm{mL}^{-1} \mathrm{SDS}, 0.1 \mathrm{mM} \mathrm{DTT}, 20 \mu \mathrm{g} \cdot \mathrm{mL}^{-1}$ bromophenol blue. The supernatant was then heated for $5 \mathrm{~min}$ at $100^{\circ} \mathrm{C}$ and immediately placed on ice. Immediately before analyses, the muscle extraction sample was warmed to $50^{\circ} \mathrm{C}$ and centrifuged for $1 \mathrm{~min}$ at $13,000 \mathrm{~g}$ (RT). Total amount of sample loaded on the gel was based on weight (1.0 mg per lane). With the exception of mTOR, protein samples were run on a Criterion Precast TGX 4-20\% gel (Biorad Order No. 567-1094) for 10 min at $50 \mathrm{~V}$ (constant voltage) and $\pm 90 \mathrm{~min}$ at $150 \mathrm{~V}$ (constant voltage) and transferred onto a Trans-blot Turbo $0.2 \mu \mathrm{m}$ nitrocellulose membrane (Biorad Order No. 170-4159) in $7 \mathrm{~min}$ at $2.5 \mathrm{~A}$ and $25 \mathrm{~V}$. mTOR proteins were run and blotted under the same conditions but on a Criterion Precast XT 3-8\% Tris-acetate gel (Biorad order No. 345-0130). Specific proteins were detected by overnight incubation at $4^{\circ} \mathrm{C}$ on a shaker with specific antibodies in 50\% in PBS Odyssey blocking buffer (Li-Cor Biosciences Part No. 927-40000) after blocking for 60 min at RT in 50\% in PBS Odyssey blocking buffer. Polyclonal primary phospho-specific antibodies [ $\alpha$-tubulin (52 kDa; dilution 1:10 000, rabbit monoclonal IgG; Cell Signaling Tecnhologies, Beverly, MA, USA; Order No. 2125), anti-phospho-mTOR $\left(\mathrm{Ser}^{2448}\right)$, anti-phospho-S6K1 $\left(\mathrm{Thr}^{389}\right)$, anti-phospho-S6 $\left(\mathrm{Ser}^{235} / \mathrm{Ser}^{236}\right)$, anti-phospho-4E- 
BP1 $\left(\mathrm{Thr}^{37 / 46}\right)$, anti-mTOR, anti-S6K1, anti-RS6 and anti-4E-BP1 were purchased from Cell Signaling Technologies. Following incubation, membranes were washed 3 times 10 min in $0.1 \%$ PBS-Tween 20 and once for 10 min in PBS. Next, samples were incubated on a shaker ( $1 \mathrm{~h}$ at RT) with Infrared secondary antibodies, donkey anti-rabbit IRDYE 800 (Rockland, Cat. No. 611-732-127, dilution 1:10000) and donkey anti-mouse IRDYE 800CW (Li-Cor, Cat. No. 626-32212, dilution 1:10000) dissolved in 50\% PBS Odyssey blocking buffer. After a final wash step (3 x $10 \mathrm{~min}$ ) in 0.1\% Tween20-PBS and once $10 \mathrm{~min}$ in PBS, protein quantification was performed by scanning on an Odyssey Infrared Imaging System (LI-COR Biotechnology, Lincoln, NE) with local background subtraction and intensity of the bands expressed as arbitrary units. $\alpha$-Tubulin was used to standardize for the amount of protein loaded. Phosphorylation status as a proxy of activation of the signaling proteins was expressed relative to the total amount of each protein.

\section{Calculations}

The fractional synthetic rates (FSRs) of the myofibrillar proteins were calculated using the standard precursor-product equation:

$$
\operatorname{FSR}\left(\% \cdot h^{-1}\right)=\Delta \operatorname{Ep} /\left[E_{\text {precursor }} \times t\right] \times 100
$$

where $\Delta \mathrm{Ep}$ is the change in phenylalanine or leucine labelling between two muscle biopsies, $E_{\text {precursor }}$ is the average labelling over time curve (AUC) of the plasma precursor pool; and $t$ indicates the tracer incorporation time (hours) between two muscle biopsies.

\section{Statistics}

All data are expressed as means_SEM. A one-way ANOVA was used to assess changes over time in plasma glucose, insulin and amino acid concentrations. A two-way repeated measures ANOVA with time (pre and post) and leg (immobilized and nonimmobilized) as within-subjects factors was used to compare differences in quadriceps CSA. A two-way repeated measures ANOVA with time (fasting and fed) and leg (immobilized and non-immobilized) as within-subjects factors was used to compare differences in $\mathrm{L}-\left[1-{ }^{13} \mathrm{C}\right]$-leucine and $\mathrm{L}-\left[\right.$ ring- $\left.{ }^{2} \mathrm{H}_{5}\right]$-phenylalanine myofibrillar protein-bound enrichments and FSR and all mRNA and protein expression data. Myofibrillar protein-bound L$\left[1-{ }^{13} \mathrm{C}\right]$-phenylalanine enrichments and FSR were compared using paired $t$-tests. For all ANOVA analyses, when a significant time*leg interaction was detected, a Bonferoni post-hoc test was applied to locate the individual differences. Statistical significance was set at $P<0.05$. All calculations were performed by using GraphPad Prism version 5.0 (GraphPad Software, San Diego, CA, USA). 


\section{RESULTS}

\section{Muscle mass}

Muscle quadriceps cross-sectional area (CSA) determined by CT-scan of the mid-thigh is displayed in Figure 1.

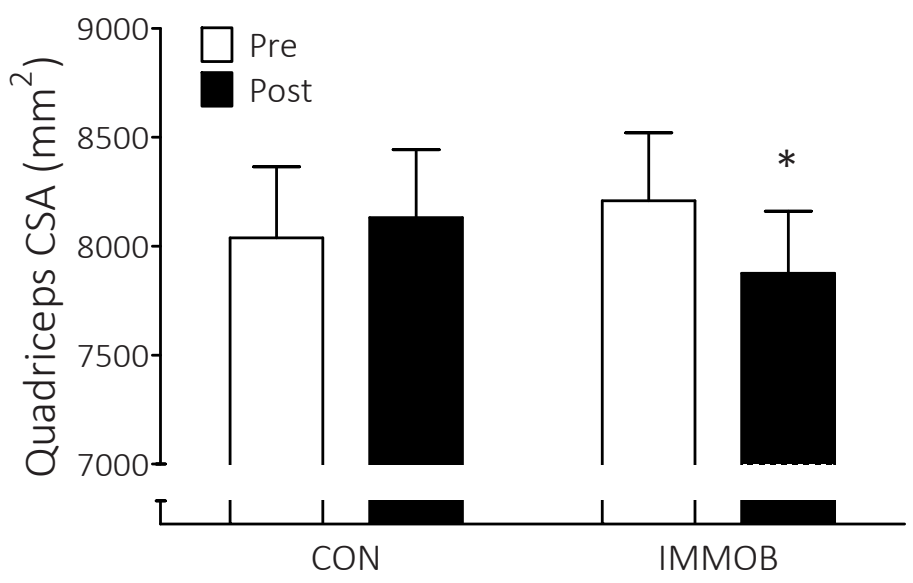

Figure 1 | Mean ( \pm SEM) quadriceps cross-sectional area (CSA) before (Pre) and after (Post) 5 days of onelegged knee immobilization in the immobilized (IMMOB) and non-immobilized control (CON) legs of healthy, young men $(n=12)$. Data were analyzed with a two-way repeated measures ANOVA (time $x$ leg) with Bonferroni post-hoc tests to locate differences: Significant time $(P<0.05)$ and interaction $(P<0.01)$ effects, ${ }^{*} P<0.05$ compared with corresponding Pre value.

Quadriceps CSA did not differ between legs at baseline and remained unchanged throughout the experiment in the non-immobilized leg (from 8039 \pm 326 to $8181 \pm 313$ $\left.\mathrm{mm}^{2} ; P=0.24\right)$. However, five days of immobilization resulted in a $3.9 \pm 0.6 \%$ decrease in quadriceps muscle CSA of the immobilized leg (from $8208 \pm 312$ to $7876 \pm 285 \mathrm{~mm}^{2}$; $P<0.0001)$.

\section{Plasma analyses}

Plasma glucose concentrations declined over time $(P<0.001)$ during the experimental visit (not shown). Plasma insulin concentrations (Figure $2 \mathrm{~A}$ ) showed a rapid and brief increase following protein ingestion up to $\sim 23 \mathrm{mU}^{-1}$ after $30 \mathrm{~min}$, before returning to baseline levels after 90 min (time effect; $P<0.001$ ). Plasma phenylalanine (Figure 2B), leucine (Figure 2C) and tyrosine (Figure 2D) concentrations increased (all $P<0.0001$ ) following protein ingestion and remained above basal levels for 90-180 min. The time course of plasma $\mathrm{L}-\left[\mathrm{ring}^{-}{ }^{2} \mathrm{H}_{5}\right]$-phenylalanine $(\mathrm{E}), \mathrm{L}-\left[1-{ }^{13} \mathrm{C}\right]$-leucine $(\mathrm{F})$ and $\mathrm{L}-\left[1-{ }^{13} \mathrm{C}\right]-$ phenylalanine $(\mathrm{G})$ enrichments are also illustrated in Figure 2. 

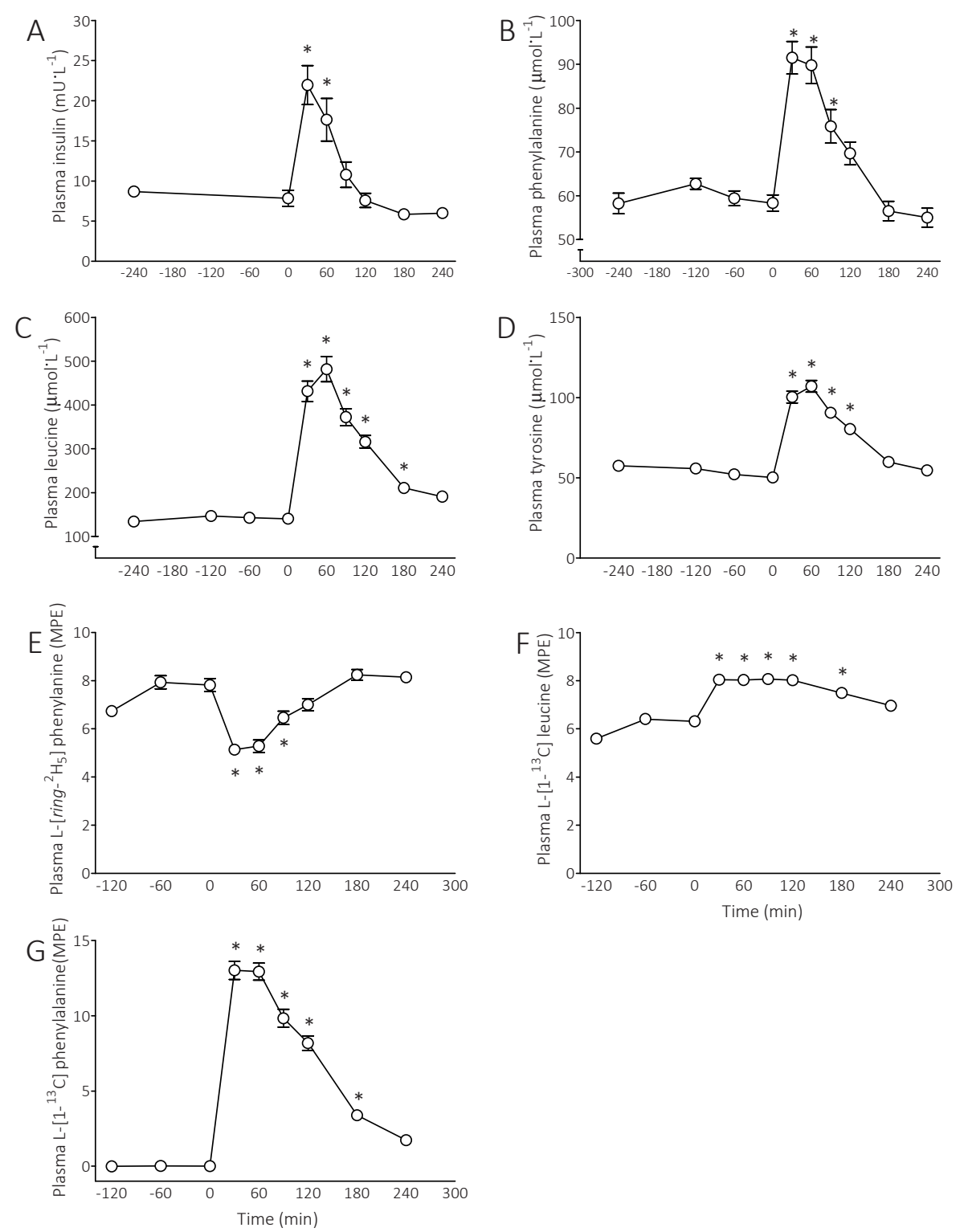

Figure 2 | Mean ( \pm SEM) plasma insulin (A), phenylalanine (B), leucine (C) and tyrosine (D) concentrations, and mean $\left( \pm\right.$ SEM) plasma $\mathrm{L}-\left[\right.$ ring- $\left.{ }^{2} \mathrm{H}_{5}\right]$-phenylalanine $(\mathrm{E}), \mathrm{L}-\left[1-{ }^{13} \mathrm{C}\right]$-leucine $(\mathrm{F})$ and $\mathrm{L}-\left[1-{ }^{13} \mathrm{C}\right]$-phenylalanine $(\mathrm{G})$ enrichments across time ( $x$ axis; minutes) before and following ingestion of $25 \mathrm{~g}$ protein (at $t=0$ min) after 5 days of one-legged knee immobilization in healthy, young men $(n=12)$. Data were analyzed with a one-way repeated measures ANOVA with Bonferonni post hoc tests applied to locate differences: $A, B, C, D, E, F$ and $G$ all showed significant time effects $(P<0.001)$ and * indicates values significantly different compared to ' $t=0$ '. 
During the postabsorptive period, plasma L-[ring- $\left.{ }^{2} \mathrm{H}_{5}\right]$-phenylalanine and L-[1- $\left.{ }^{13} \mathrm{C}\right]-$ leucine remained in steady-state at $\sim 7$ and $\sim 6 \mathrm{MPE}$, respectively. Following protein ingestion (at $t=0 \mathrm{~min}$ ), plasma L- $\left[\mathrm{ring}_{-}{ }^{2} \mathrm{H}_{5}\right]$-phenylalanine enrichments decreased for $90 \mathrm{~min}$ before returning to fasting, steady-state levels $(P<0.0001)$, while plasma $\mathrm{L}-\left[1{ }^{-13} \mathrm{C}\right]$ leucine enrichments increased and remained at a steady state of 8 MPE throughout the entire postprandial period $(P<0.0001)$. Following protein ingestion, plasma $\mathrm{L}-\left[1-{ }^{13} \mathrm{C}\right]-$ phenylalanine enrichments increased rapidly to 14 MPE after 30 min and remained elevated for $180 \mathrm{~min}$ into the postprandial period $(P<0.0001)$. These higher plasma enrichments of $\mathrm{L}-\left[1-{ }^{13} \mathrm{C}\right]$-phenylalanine compared with $\mathrm{L}-\left[1-{ }^{13} \mathrm{C}\right]$-leucine following protein ingestion reflects the greater enrichment of the $\mathrm{L}-\left[1-{ }^{13} \mathrm{C}\right]$-phenylalanine tracer in the intrinsically labelled protein. The greater rise in plasma leucine concentrations compared with phenylalanine is due to the higher leucine content (compared with phenylalanine) in whey protein.

\section{Skeletal muscle tracer analyses}

Based on the $\mathrm{L}-\left[\right.$ ring- $\left.^{2} \mathrm{H}_{5}\right]$-phenylalanine tracer and the plasma enrichment as the precursor pool, postabsorptive and postprandial myofibrillar FSRs were $41 \pm 13 \%$ $\left(0.015 \pm 0.002\right.$ vs $\left.0.032 \pm 0.005 \% \cdot h^{-1} ; P<0.01\right)$ and $53 \pm 4 \%\left(0.020 \pm 0.002\right.$ vs $0.044 \pm 0.003 \% \cdot h^{-}$ $\left.{ }^{1} ; P<0.01\right)$ lower, respectively, in the immobilized compared with the control leg. Protein ingestion increased myofibrillar FSR in both legs $(P<0.05)$, and when comparing delta change a trend was observed for a lower increase in the immobilized leg $(P=0.12)$. Individual postabsorptive and postprandial myofibrillar FSRs based on the L-[ring- $\left.{ }^{2} \mathrm{H}_{5}\right]$ phenylalanine tracer and plasma precursor are illustrated in Figure 3.
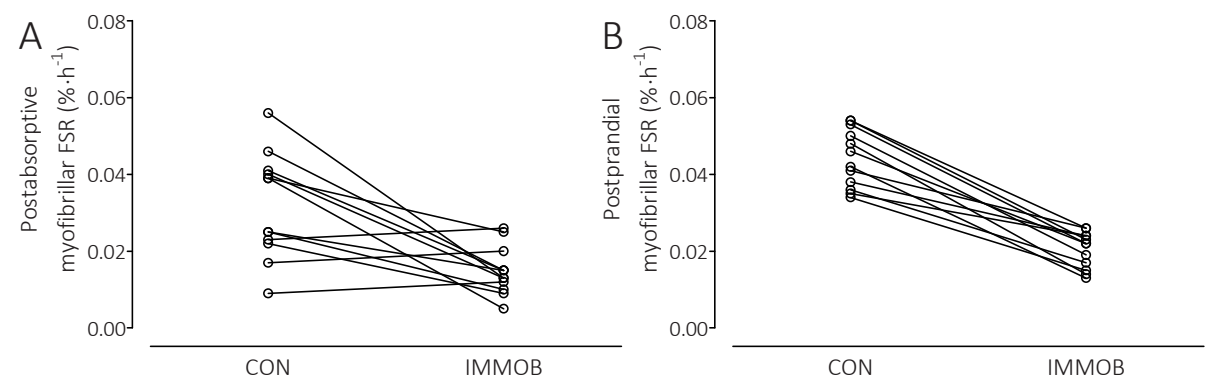

Figure 3 | Individual subjects' postabsorptive (A) and postprandial (B) fractional myofibrillar protein synthesis rates (FSR) calculated from L-[ring- $\left.{ }^{2} \mathrm{H}_{5}\right]$-phenylalanine following 5 days of one-legged knee immobilization in the immobilized (IMMOB) and non-immobilized control (CON) legs of healthy, young men ( $n=12)$. Data were analyzed with a two-way repeated measures ANOVA and significant main effects of protein ingestion $(P<0.05)$ and immobilization $(P<0.001)$ were detected (for interaction $P=0.16)$. 
Similar results were observed when the intracellular L-[ring- $\left.{ }^{2} \mathrm{H}_{5}\right]$-phenylalanine enrichments were used as the precursor pool, with postabsorptive and postprandial myofibrillar FSRs being $30 \pm 19 \%\left(0.026 \pm 0.004\right.$ vs $\left.0.051 \pm 0.008 \% \mathrm{~h}^{-1} ; \quad P<0.01\right)$ and $48 \pm 4 \%$ $\left(0.032 \pm 0.003\right.$ vs $\left.0.062 \pm 0.005 \% \mathrm{~h}^{-1} ; P<0.01\right)$ lower, respectively, in the immobilized compared with the control leg. Postprandial FSR calculations using L-[ring- $\left.{ }^{2} \mathrm{H}_{5}\right]-$ phenylalanine required the use of a modestly disturbed precursor pool (Figure 2E). However, comparable postabsorptive and postprandial myofibrillar FSRs were observed when using the $\mathrm{L}-\left[1-{ }^{13} \mathrm{C}\right]$-leucine tracer which, following a readjustment in enrichments levels during the transition from fasted to fed, presented a steady state plasma precursor pool in both conditions (Figure 2F). Specifically, postabsorptive $(0.011 \pm 0.012$ vs $\left.0.036 \pm 0.011 \% \mathrm{~h}^{-1} ; P<0.01\right)$ and postprandial $\left(0.023 \pm 0.003\right.$ vs $\left.0.055 \pm 0.007 \% \mathrm{~h}^{-1} ; P<0.01\right)$ myofibrillar FSRs were lower in the immobilized compared to control leg, respectively. Moreover, similar results were observed when the intracellular $\mathrm{L}-\left[1-{ }^{13} \mathrm{C}\right]$-leucine enrichments were used as the precursor pool, with postabsorptive $(0.018 \pm 0.004$ vs $\left.0.053 \pm 0.015 \% \mathrm{~h}^{-1} ; P<0.01\right)$ and postprandial $\left(0.044 \pm 0.007\right.$ vs $\left.0.102 \pm 0.014 \% \mathrm{~h}^{-1} ; P<0.01\right)$ myofibrillar FSRs being lower in the immobilized compared with control leg, respectively. Following protein ingestion, the increase in myofibrillar protein-bound $\mathrm{L}-\left[1-{ }^{13} \mathrm{C}\right]-$ phenylalanine enrichments were $53 \pm 18 \%$ lower in the immobilized compared with the control leg (0.007 \pm 0.002 and $0.015 \pm 0.002 \mathrm{MPE}$, respectively; $P<0.05$; Figure 4).

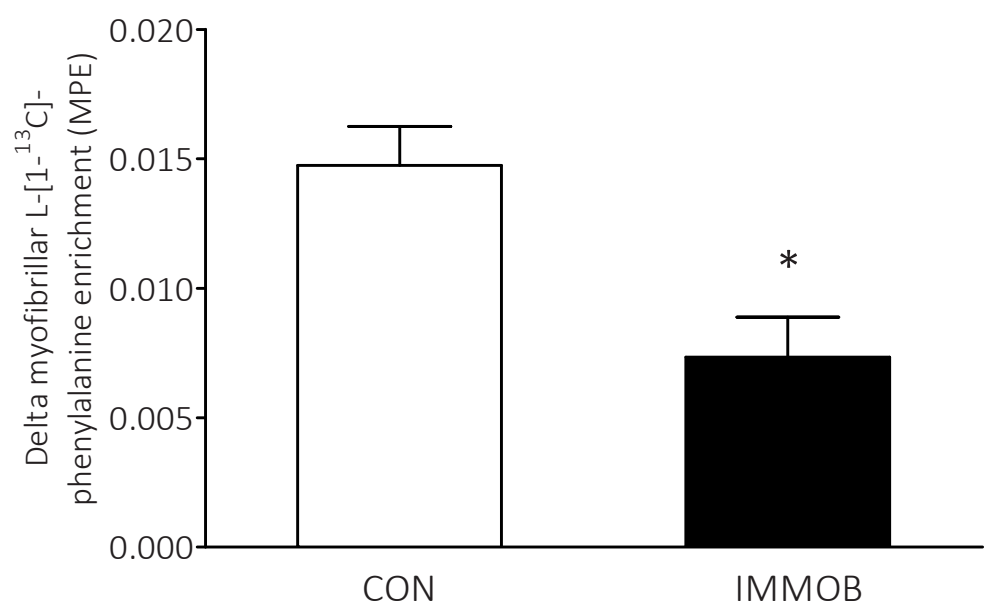

Figure 4 | Delta myofibrillar protein enrichment of $\mathrm{L}-\left[1-{ }^{13} \mathrm{C}\right]$-phenylalanine $4 \mathrm{~h}$ after the ingestion of $25 \mathrm{~g}$ intrinsically L-[1- $\left.{ }^{13} \mathrm{C}\right]$-phenylalanine labelled protein following 5 days of one-legged knee immobilization in the immobilized (IMMOB) and non-immobilized control (CON) legs of healthy, young men $(n=12)$. Data were analyzed with a paired t-test. $* P<0.05$ compared with $\mathrm{CON}$. 


\section{mRNA and cell signaling analyses}

The skeletal muscle mRNA expression of genes implicated in the regulation of intracellular amino acid transport are presented in Figure 5.
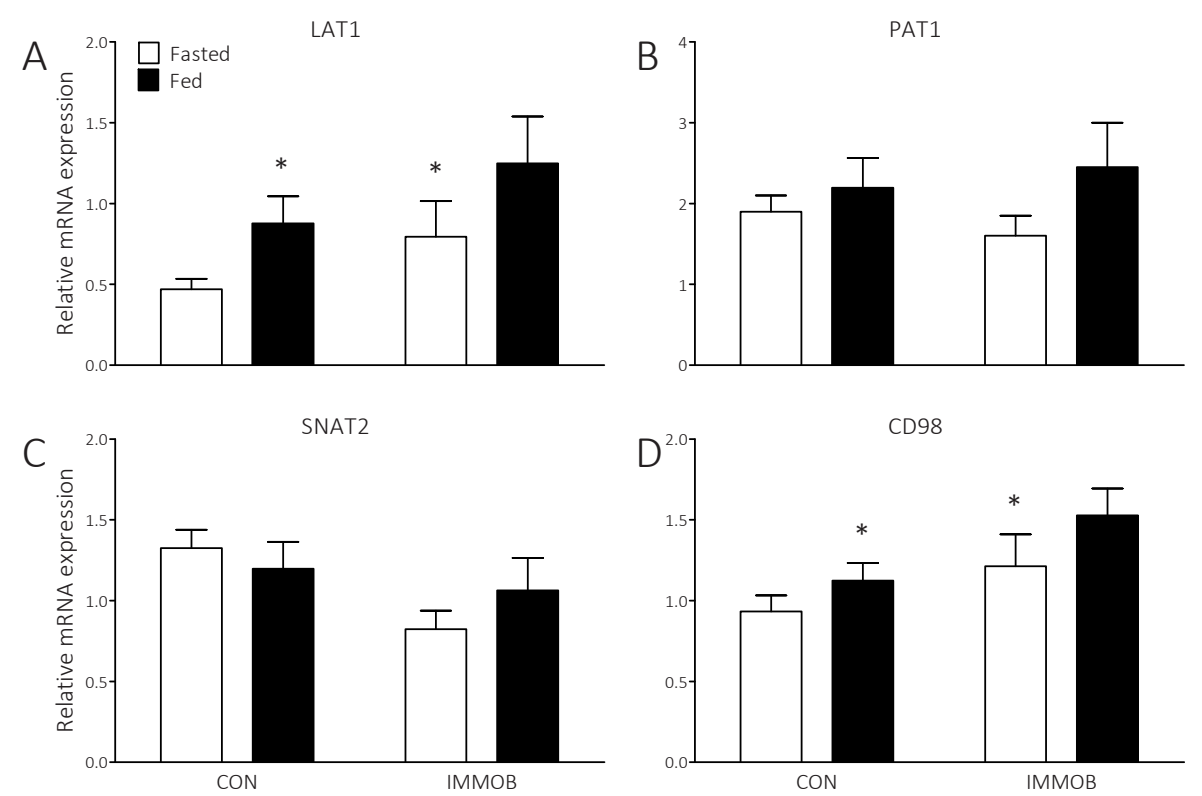

Figure 5 | Skeletal muscle mRNA expression of LAT1 (A), PAT1 (B), SNAT2 (C) and CD98 (D) in the fasted and fed ( $4 \mathrm{~h}$ following ingestion of $25 \mathrm{~g}$ protein) state following 5 days of one-legged knee immobilization in the immobilized (IMMOB) and non-immobilized control (CON) legs of healthy, young men $(n=12)$. Data were analyzed with two-way repeated measures ANOVAs. A: Significant main effect of protein ingestion $(P<0.05)$. B: No significant effects. C: No significant effects. D: Significant main effect of protein ingestion $(P<0.05) .{ }^{*} P<0.05$ compared with corresponding fasted value.

Muscle LAT1 mRNA expression $(A)$ increased with protein ingestion $(P<0.05)$ in both legs (by 87 and 57\% for control and immobilized leg, respectively) with no effect of immobilization. PAT1 (B) and SNAT2 (C) were not affected by protein ingestion or immobilization. Muscle CD98 mRNA expression (D) increased with protein ingestion $(P<0.05)$ in both legs (by 20 and $26 \%$ for control and immobilized leg, respectively) with no effect of immobilization. Muscle mRNA expression of genes involved in muscle protein breakdown and inflammation are shown in Figure 6. 

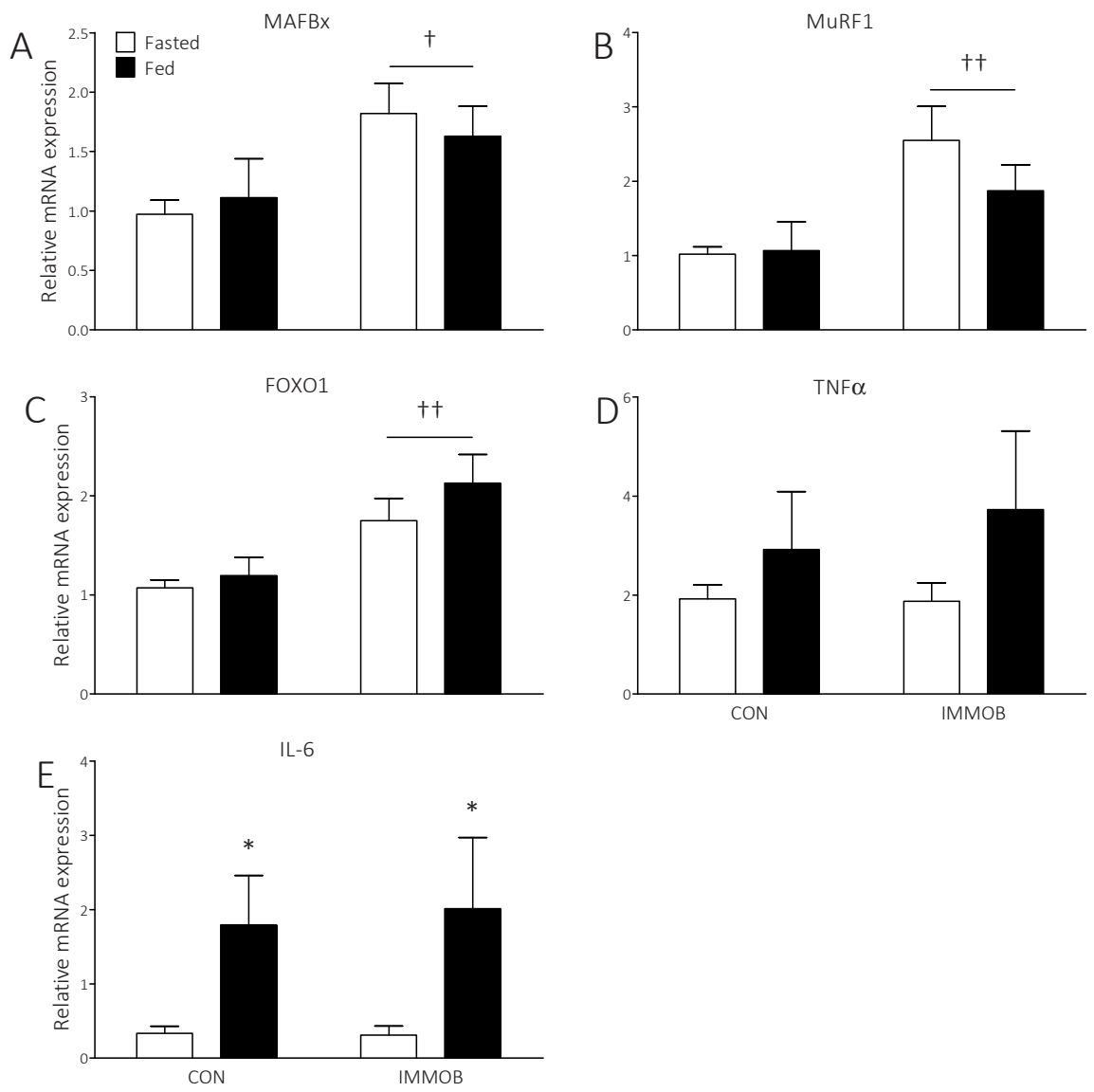

Figure 6 | Skeletal muscle mRNA expression of MAFBx (A), MuRF1 (B), FOXO1 (C), TNFa (D) and IL-6 (E) in the fasted and fed ( $4 \mathrm{~h}$ following ingestion of $25 \mathrm{~g}$ protein) state following 5 days of one-legged knee immobilization in the immobilized (IMMOB) and non-immobilized control (CON) legs of healthy, young men $(n=12)$. Data were analyzed with two-way repeated measures ANOVAs. A: Significant main effect of immobilization $(P<0.05)$. B: Significant main effect of immobilization $(P<0.01)$. C: Significant main effect of immobilization $(P<0.01)$. D: No significant effects. E: Significant main effect of protein ingestion $(P<0.05) .+P<0.05$ compared with corresponding CON values. ${ }^{*} P<0.05$ compared with corresponding fasted value.

Muscle MAFBx (A), MuRF1 (B) and FOXO1 (C) mRNA expression were all greater in the immobilized compared with control leg (all $P<0.05$ ) but were not affected by protein ingestion. Muscle TNFa mRNA expression was not affected by protein ingestion or immobilization and muscle IL-6 mRNA expression increased with protein ingestion $(P<0.05)$ but was not changed with immobilization. Figure 7 depicts the muscle phosphorylation status (presented as a ratio of phosphorylated to total protein) of key proteins involved in the initiation of muscle protein synthesis. 

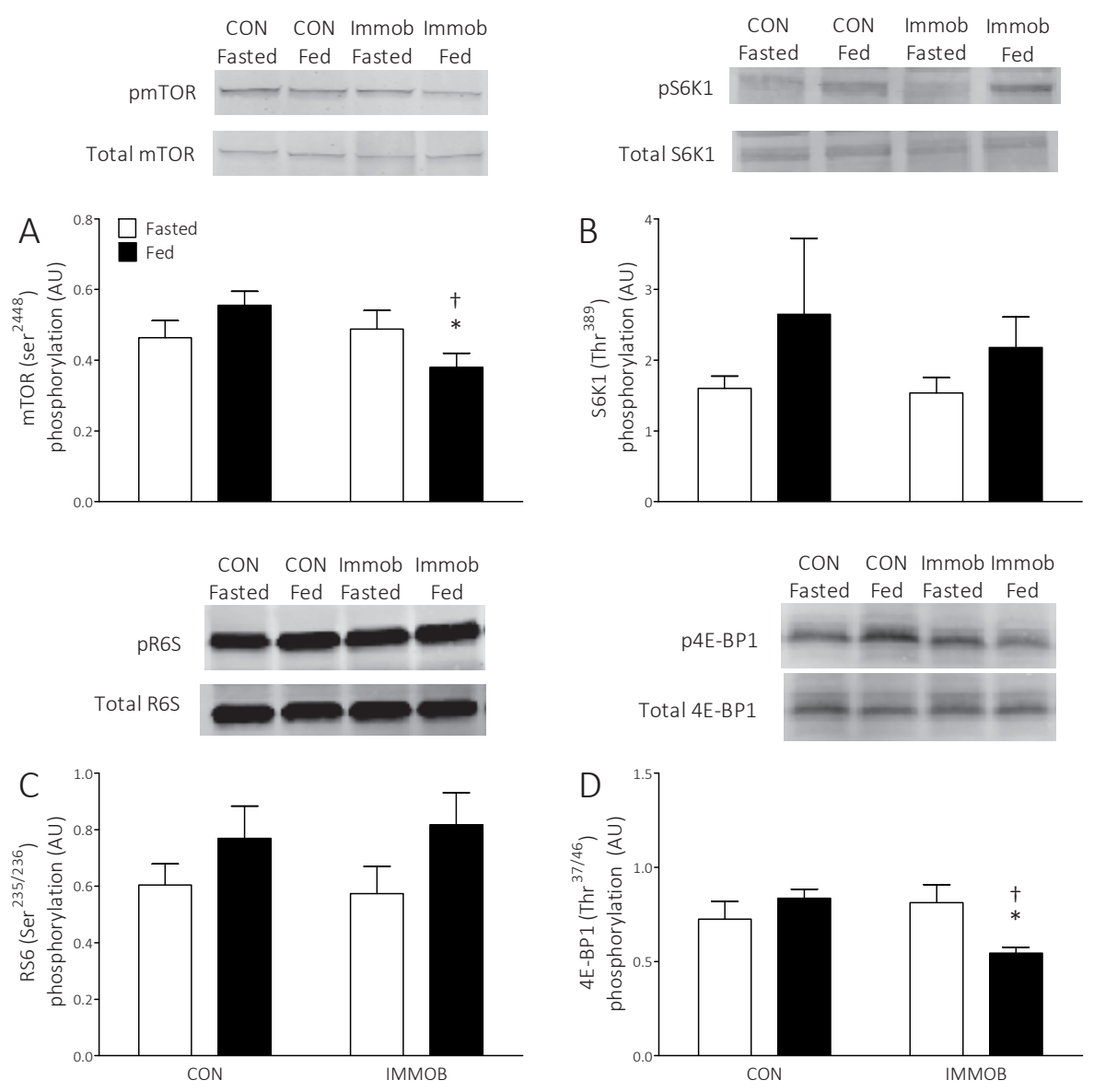

Figure 7 | Muscle phosphorylation status (presented as a ratio of phosphorylated to total protein) of mTOR (A) S6K (B), RS6 (C) and 4-EBP1 (D) in the fasted and fed (4 h following ingestion of $25 \mathrm{~g}$ protein) state following 5 days of one-legged knee immobilization in the immobilized (IMMOB) and non-immobilized control (CON) legs of healthy, young men $(n=12)$. Data were analyzed with two-way repeated measures ANOVAs. A: Significant interaction effect $(P<0.01)$. B: No significant effects. C: Significant main effect of feeding $(P<0.01)$. D: Significant interaction effect $(P<0.01)$. $+P<0.05$ compared with corresponding CON values. ${ }^{*} P<0.05$ compared with corresponding fasted value.

Protein ingestion increased the phosphorylation status of RS6 (C) in both legs (time effect; $P<0.01)$ and a similar trend $(P=0.14)$ was also observed for S6K1 (B). There was an interaction effect for mTOR (A) and 4-EBP1 (D) (both $P<0.05)$ such that the immobilized leg in the fed state showed lower phosphorylation status when compared with the corresponding control leg or fasted value. 


\section{DISCUSSION}

The present study demonstrates that the loss of quadriceps muscle mass observed during merely five days of muscle disuse can be attributed to a decline in basal myofibrillar protein synthesis rates and a reduced capacity of skeletal muscle tissue to utilize dietary protein derived amino acids for de novo myofibrillar protein synthesis in healthy males.

The present study demonstrates that the ( $\sim \%)$ loss of quadriceps cross-sectional area induced by 5 days of muscle disuse (Figure 1) is accompanied by an approximate halving of postabsorptive myofibrillar protein synthesis rates when compared with the non-immobilized, control leg (Figure 3). This striking decrease was comparable irrespective of whether the incorporation of L- $\left[\right.$ ring $\left._{-}{ }^{2} \mathrm{H}_{5}\right]$-phenylalanine or $\mathrm{L}-\left[1-{ }^{13} \mathrm{C}\right]$-leucine was used to calculate myofibrillar protein synthesis rates. Our data demonstrate that the decline in basal muscle protein synthetic rate that occurs during more prolonged immobilization and bed rest studies $[2,15,16]$ occurs much more rapidly than previously thought. Extending on this, we report that the reduced capacity to synthesize muscle proteins in the postabsorptive state is pronounced in the contractile myofibrillar proteins. Therefore, reduced basal myofibrillar protein synthesis rates can, at least in part, explain the considerable loss of muscle mass and function that rapidly occurs during only a few days of muscle disuse. As such, increasing basal myofibrillar protein synthesis rates should form a key therapeutic target for effective interventional strategies aiming to preserve muscle mass.

Skeletal muscle is maintained by the successive daily stimulation of muscle protein synthesis rates following food intake [35]. Previous studies, employing more prolonged disuse protocols, have suggested that a reduced responsiveness to the anabolic properties of amino acids may develop with prolonged disuse [16-18]. However, determining muscle protein synthesis rates in response to the ingestion of a bolus of dietary protein has its technical difficulties. The measurement of fractional muscle protein synthesis rate requires precursor pool enrichments to remain in a relative steady state between serial muscle biopsy collections. However, the ingestion of a meal-like bolus of dietary protein disturbs tracer steady state by diluting the precursor pool. Previous workers have attempted to obviate this problem by co-ingesting free labelled amino acids [36], ingesting small repeated boluses of protein [37], or simply by making non-steady state calculations [18]. All these approaches have their specific methodological limitations when assessing postprandial muscle protein synthesis rates following ingestion of a single bolus of dietary protein [19]. Recently, we produced milk proteins intrinsically labelled with high L- $\left[1-{ }^{13} \mathrm{C}\right]$-phenylalanine enrichment levels (>30 MPE) and lower levels of $\mathrm{L}-\left[1-{ }^{13} \mathrm{C}\right]$-leucine ( 8-10 MPE). The high enrichment $\mathrm{L}-\left[1-{ }^{13} \mathrm{C}\right]$-phenylalanine levels allow us to directly assess the use of dietary protein derived phenylalanine for de novo muscle protein synthesis, whereas the lower $\mathrm{L}-\left[1-{ }^{13} \mathrm{C}\right]$-leucine enrichment allows us to match the plasma L-[1- $\left.{ }^{13} \mathrm{C}\right]$-leucine precursor pool enrichment during a primed constant 
intravenous $\mathrm{L}-\left[1-{ }^{13} \mathrm{C}\right]$-leucine infusion. Using this approach, we have previously measured postprandial muscle protein synthesis rates under both steady and non-steadystate precursor pool conditions as well as assessing the metabolic fate of the ingested protein derived amino acids [19]. Here, we show that protein ingestion increased muscle protein synthesis rates above basal, postabsorptive values in the control leg $(0.032 \pm 0.005$ vs $0.044 \pm 0.003$; Figure 3$)$. Despite a rise in circulating plasma insulin and leucine concentrations known to be sufficient to support maximal protein anabolism (Figure 2) [38-40], postprandial myofibrillar protein synthesis rates were $>50 \%$ lower in the previously immobilized leg when compared with the control leg (Figure 3). The lower postprandial muscle protein synthetic rates in the immobilized leg were observed independent of which tracer was used to calculate FSR. It should be noted, however, that protein ingestion did still increase FSR in both legs and the delta increase in FSR only tended to be lower in the immobilized leg. This suggests that the absolute rate of FSR was more affected than the relative FSR response to food ingestion. However, the postprandial incorporation of dietary protein derived $\mathrm{L}-\left[1-{ }^{13} \mathrm{C}\right]$-phenylalanine in myofibrillar protein was more than 50\% lower in the immobilized versus control leg (Figure 5), supporting the concept that the anabolic response to feeding per se is diminished. Consequently, a few days of disuse is followed by rapid onset of anabolic resistance of muscle tissue to dietary protein ingestion, resulting in reduced postprandial myofibrillar protein accretion. It is therefore of important clinical relevance to evaluate strategies that can overcome disuse induced anabolic resistance and preserve muscle mass during short periods of muscle disuse. Effective strategies could include changing the amount [41, 42], type [43-46] or timing [47] of dietary protein administration, or applying mimetics for physical activity such as neuromuscular electrical stimulation [48, 49] to attenuate muscle disuse atrophy [50]. The fact that disused muscle is still responsive to protein ingestion implies that interventional strategies aiming to preserve muscle mass during a period of disuse should minimize time spent in the postabsorptive state.

Although anabolic resistance has now been reported in a variety of situations, the underlying mechanism(s) are poorly understood. Impairments could conceivably reside systemically (e.g. protein digestion and/or absorption $[15,43]$, or the postprandial hormonal response and subsequent microvascular perfusion $[51,52])$ or locally at the muscle tissue level (e.g. muscle amino acid uptake [53] and/or intramuscular signaling [54, 55]). We have previously provided evidence to suggest that the responsible mechanisms for disuse induced anabolic resistance are intracellular [18]. Intracellular muscle amino acid transport requires the presence of specific amino acid transporter proteins. Several of these amino acid transporters, including L-type amino acid transporter 1 (LAT1), sodium coupled neutral amino acid transporter 2 (SNAT2) and CD98/SLC3A2 (CD98), reside on the cell membrane, and are thought to work in concert to regulate intracellular amino acid flux. Proton assisted amino acid transporter (PAT1) is found in the lysosomal membrane and is thought to be instrumental in exporting amino acids from the lysosomal lumen into the cytosol. LAT1, SNAT2, CD98 and PAT1 mRNA and/or 
protein expression have all been shown to increase and coincide with a rise in muscle protein synthesis following essential amino acid ingestion and/or a single bout of resistance-type exercise $[53,56,57]$. Interestingly, the increased expression of LAT1 and SNAT2 following essential amino acid ingestion was shown to be blunted following 7 days of bed rest [17]. Taken together, these data have led to the hypothesis that the amino acid transporter expression response to increased amino acid availability may regulate postprandial muscle protein synthesis $[53,58]$. In the present study, we observed no differences in amino acid transporter expression levels between the immobilized and control leg (Figure 5). Our data confirm the responsiveness of amino acid transporter expression following protein ingestion but do not support the hypothesis that changes in amino acid transporter expression may be responsible for the local muscle disuse induced anabolic resistance. However, it should be acknowledged that we only present gene expression of these transporters, and this may not necessarily reflect their protein content and/or cellular location and involvement in amino acid transport.

Postprandial stimulation of muscle protein synthesis rates is initiated by a phosphorylation cascade where mammalian target of rapamycin (mTOR) and its downstream targets P70S6 protein kinase (P70S6K/S6K1), ribosomal protein S6 (RS6) and eukaryotic translation initiation factor $4 \mathrm{E}$ binding protein 1 (4E-BP1) are of central importance [59, 60]. Consistent with previous studies [e.g. 17, 61] we report that the ingestion of protein led to a modest general increase in the phosphorylation of these anabolic signaling proteins (Figure 7). Interestingly, immobilization significantly impaired postprandial activation of mTOR and its downstream target 4E-BP1 such that they actually decreased following protein ingestion; whereas P70S6K and its subsequent activation of S6K1 did not seem to be affected. Both the lack of robust feeding induced increases in phosphorylation of these proteins in the control leg, as well as the decrease in phosphorylation in the immobilized leg may be explained by our study design which allowed the determination of anabolic signaling at a single postprandial time-point, $4 \mathrm{~h}$ following protein ingestion. Due to its role in initiating the translation process, peak stimulation of this signaling cascade generally occurs 1-2 h following protein ingestion [57, 61-63] and begins to subside thereafter [57, 61-63]. It may be speculated, therefore, that peak stimulation was missed and we are observing the residual activation of these anabolic proteins in the control leg, and the reduced phosphorylation in the disused leg represents a reduced transduction of the anabolic signal through mTOR/4E-BP1, impairing the assembly of the active elF4F complex and/or via the suppression of translation initiation [59]. Consistent with these findings, we have recently observed that lipid induced anabolic resistance is also characterized by an impairment of postprandial 4E-BP1 activation [64]. Collectively, our data point to an intracellular signaling defect that likely underpins anabolic resistance under diverse circumstances. Future work aiming to elucidate the intracellular impairments underlying disuse induced anabolic resistance would likely require earlier and more frequent biopsy points to capture rapid, transient 
changes and determine a detailed time-course of intracellular responses to protein ingestion following disuse. Moreover, employing dynamic measures of intracellular amino acid flux are required to discriminate between anabolic signaling defects per se and simply a reduced amino acid supply to the intracellular signaling pathways following disuse. A final interesting consideration in light of our cell signaling data is the inclusion of younger subjects. Using a retrospective, database analysis, it has recently been shown that ageing is associated with a hyperphosphorylation of mTOR in the fasted state [65]. Moreover, it has also been reported that mTOR/P70S6K responsiveness to protein ingestion may be enhanced in older muscle [66], despite a reduced muscle protein synthetic response. These findings suggest a reduced efficiency of older muscle in terms of signal transduction or, at least, an altered dynamic relationship between cell signaling and muscle protein synthesis. In the same way, it could also be speculated that disuse may also alter the efficiency and/or kinetics or feeding induced mTOR activation, underlining the importance of conducting future time course based studies. Consequently, understanding the relationship between the relevant signaling pathways and muscle protein synthesis in disused young and older muscle remains an important future research direction.

Aside from changes in muscle protein synthesis, we assessed the gene expression of two primary ubiquitin ligases, Muscle Atrophy F-Box/atrogin-1 (MAFbx) and MuscleSpecific RING-finger protein 1 (MuRF1), and their key transcription factor, fork head box protein 01 (FOXO1) $[67,68]$ to obtain insight into possible changes in muscle protein breakdown. In keeping with our previous work [7, 11], the expression of these genes were markedly elevated in the immobilized compared with the control leg (Figure 6). These data are consistent with previous assertions that a rapid and transient rise in muscle protein breakdown may contribute to muscle disuse atrophy [3, 69-71], though it should be noted that these static molecular data cannot be considered as conclusive evidence for alterations in muscle protein breakdown. Indeed, recent reports have suggested that MuRF1 may also have a pivotal role in inhibiting muscle protein synthetic pathways [72] and thus could have contributed to the observed anabolic resistance. Although it has been suggested that a rapid rise in muscle protein breakdown at the onset of disuse may be provoked by a pro-inflammatory state [73, 74], we failed to detect any evidence of increased inflammation in the immobilized muscle tissue as indicated by IL-6 and TNF $\alpha$ gene expression (Figure 6). Irrespective of leg, protein ingestion resulted in a robust increase in IL-6 gene expression which likely reflects the shift in energy and substrate metabolism in the transition to the postprandial state, processes within which IL-6 plays a metabolic role $[75,76]$.

In conclusion, the present study demonstrates that a decline in postabsorptive myofibrillar protein synthesis rates and an impaired capacity to utilize dietary protein derived amino acids for de novo myofibrillar protein synthesis are key factors underlying short-term skeletal muscle disuse atrophy. Given the proposed role of muscle disuse in the development of age-related sarcopenia, it is critical that strategies aimed at stimu- 
lating muscle protein synthesis rates and/or overcoming anabolic resistance should be developed and applied as early as possible in an effort to preserve skeletal muscle mass and function.

\section{ACKNOWLEDGEMENTS}

The authors would also like to acknowledge Dr Henrike Hamer for her efforts in the initial set-up of this project, Antoine Zorenc and Joy Goessens for their technical assistance, the enthusiastic support of the staff at Maastricht University Hospital Casting Room and the volunteers for their participation. 


\section{REFERENCES}

1. Deitrick, J., The effect of immobilization on metabolic and physiological functions of normal men. Bull N Y Acad Med, 1948. 24(6): p. 364-75.

2. Gibson, J., D. Halliday, W. Morrison, P. Stoward, G. Hornsby, P. Watt, G. Murdoch, and M. Rennie, Decrease in human quadriceps muscle protein turnover consequent upon leg immobilization. Clin Sci (Lond), 1987. 72(4): p. 503-9.

3. Wall, B. and L. van Loon, Nutritional strategies to attenuate muscle disuse atrophy. Nutr Rev, 2012. 71(4): p. $195-208$.

4. Stuart, C., R. Shangraw, M. Prince, E. Peters, and R. Wolfe, Bed-rest-induced insulin resistance occurs primarily in muscle. Metabolism, 1988. 37(8): p. 802-6.

5. Haruna, Y., Y. Suzuki, K. Kawakubo, R. Yanagibori, and A. Gunji, Decremental reset in basal metabolism during 20-days bed rest. Acta Physiol Scand Suppl, 1994. 616: p. 43-9.

6. Fisher, S., Y. Kuo, J. Graham, K. Ottenbacher, and G. Ostir, Early ambulation and length of stay in older adults hospitalized for acute illness. Arch Intern Med, 2010. 170(21): p. 1942-3.

7. Wall, B., M. Dirks, T. Snijders, J. Senden, J. Dolmans, and L. van Loon, Substantial skeletal muscle loss occurs during only 5 days of disuse. Acta Physiol Scand, 2013. 210(3): p. 600-11.

8. Wall, B., M. Dirks, and L. van Loon, Skeletal muscle atrophy during short-term disuse: implications for agerelated sarcopenia. Ageing Res Rev, 2013. 12(4): p. 898-906.

9. English, K. and D. Paddon-Jones, Protecting muscle mass and function in older adults during bed rest. Curr Opin Clin Nutr Metab Care, 2010. 13(1): p. 34-9.

10. Booth, F. and K. Zwetsloot, Basic concepts about genes, inactivity and aging. Scand J Med Sci Sports., 2010. 20(1): p. 1-4.

11. Dirks, M., B. Wall, R. Nilwik, D. Weerts, L. Verdijk, and L. van Loon, Skeletal muscle disuse atrophy is not attenuated by dietary protein supplementation in healthy, older men. J Nutr, 2014. 144(8): p. 1196-203.

12. Goldspink, D., The effects of denervation on protein turnover of rat skeletal muscle. Biochem J, 1976. 156(1): p. 71-80.

13. Goldspink, D., The influence of immobilization and stretch on protein turnover of rat skeletal muscle. J Physiol, 1977. 264(1): p. 267-82.

14. Loughna, P., G. Goldspink, and D. Goldspink, Effect of inactivity and passive stretch on protein turnover in phasic and postural rat muscles. J Appl Physiol, 1986. 61(1): p. 173-9.

15. Ferrando, A., H. Lane, C. Stuart, J. Davis-Street, and R. Wolfe, Prolonged bed rest decreases skeletal muscle and whole body protein synthesis. Am J Physiol Endocrinol Metab, 1996. 270(4): p. 627-33.

16. Glover, E., S. Phillips, B. Oates, J. Tang, M. Tarnopolsky, A. Selby, K. Smith, and M. Rennie, Immobilization induces anabolic resistance in human myofibrillar protein synthesis with low and high dose amino acid infusion. J Physiol, 2008. 586: p. 6049-61.

17. Drummond, M., J. Dickinson, C. Fry, D. Walker, D. Gundermann, P. Reidy, K. Timmerman, M. Markofski, D. Paddon-Jones, B. Rasmussen, and E. Volpi, Bed rest impairs skeletal muscle amino acid transporter expression, mTORC1 signaling, and protein synthesis in response to essential amino acids in older adults. Am J Physiol Endocrinol Metab, 2012. 302(9): p. 1113-22.

18. Wall, B., T. Snijders, J. Senden, C. Ottenbros, A. Gijsen, L. Verdijk, and L. van Loon, Disuse impairs the muscle protein synthetic response to protein ingestion in healthy men. J Clin Endocrinol Metab, 2013. 98(12): p. 4872-8.

19. Burd, N.A., N.M. Cermak, I.W. Kouw, S.H. Gorissen, A.P. Gijsen, and L.J. van Loon, The use of doubly labeled milk protein to measure postprandial muscle protein synthesis rates in vivo in humans. J Appl Physiol (1985), 2014. 117(11): p. 1363-70.

20. Frayn, K. and P. Maycock, Skeletal muscle triacylglycerol in the rat: methods for sampling and measurement, and studies of biological variability. J Lipid Res, 1980. 21(1): p. 139-44.

21. Folch, J., M. Lees, and G. Sloane Stanley, A simple method for the isolation and purification of total lipides from animal tissues. J Biol Chem, 1957. 226(1): p. 497-509.

22. Goodpaster, B.H., D.E. Kelley, F.L. Thaete, J. He, and R. Ross, Skeletal muscle attenuation determined by computed tomography is associated with skeletal muscle lipid content. J Appl Physiol, 2000. 89(1): p. 10410. 
23. Strandberg, S., M.L. Wretling, T. Wredmark, and A. Shalabi, Reliability of computed tomography measurements in assessment of thigh muscle cross-sectional area and attenuation. BMC Med Imaging, 2010. 10: p. 18.

24. Abumrad, N.N., D. Rabin, M.P. Diamond, and W.W. Lacy, Use of a heated superficial hand vein as an alternative site for the measurement of amino acid concentrations and for the study of glucose and alanine kinetics in man. Metabolism, 1981. 30(9): p. 936-40.

25. Bergstrom, J., Percutaneous needle biopsy of skeletal muscle in physiological and clinical research. Scand J Clin Lab Invest, 1975. 35(7): p. 609-16.

26. van Loon, L.J., Y. Boirie, A.P. Gijsen, J. Fauquant, A.L. de Roos, A.K. Kies, S. Lemosquet, W.H. Saris, and R. Koopman, The production of intrinsically labeled milk protein provides a functional tool for human nutrition research. J Dairy Sci, 2009. 92(10): p. 4812-22.

27. Pennings, B., W.F. Pellikaan, J.M. Senden, A.M. van Vuuren, J. Sikkema, and L.J. van Loon, The production of intrinsically labeled milk and meat protein is feasible and provides functional tools for human nutrition research. J Dairy Sci, 2011. 94(9): p. 4366-73.

28. Burd, N.A., H.M. Hamer, B. Pennings, W.F. Pellikaan, J.M.G. Senden, A.P. Gijsen, and L.J.C. van Loon, Substantial Differences between Organ and Muscle Specific Tracer Incorporation Rates in a Lactating Dairy Cow. PLoS One, 2013. 8(6): p. e68109.

29. Koopman, R., A.H. Zorenc, R.J. Gransier, D. Cameron-Smith, and L.J. van Loon, Increase in S6K1 phosphorylation in human skeletal muscle following resistance exercise occurs mainly in type II muscle fibers. Am J Physiol Endocrinol Metab, 2006. 290(6): p. E1245-52.

30. Schwenk, W.F., P.J. Berg, B. Beaufrere, J.M. Miles, and M.W. Haymond, Use of t-butyldimethylsilylation in the gas chromatographic/mass spectrometric analysis of physiologic compounds found in plasma using electron-impact ionization. Anal Biochem, 1984. 141(1): p. 101-9.

31. Calder, A.G., S.E. Anderson, I. Grant, M.A. McNurlan, and P.J. Garlick, The determination of low d5phenylalanine enrichment (0.002-0.09 atom percent excess), after conversion to phenylethylamine, in relation to protein turnover studies by gas chromatography/electron ionization mass spectrometry. Rapid Commun Mass Spectrom, 1992. 6(7): p. 421-4.

32. Burd, N.A., B. Pennings, B.B. Groen, A.P. Gijsen, J.M. Senden, and L.J. van Loon, The single biopsy approach is reliable for the measurement of muscle protein synthesis rates in vivo in older men. J Appl Physiol, 2012. 113(6): p. 896-902.

33. Jones, S., R. Hill, P. Krasney, B. O'Conner, N. Peirce, and P. Greenhaff, Disuse atrophy and exercise rehabilitation in humans profoundly affects the expression of genes associated with the regulation of skeletal muscle mass. FASEB J, 2004. 18(9): p. 1025-7.

34. Chen, Y., C. Gregory, M. Scarborough, R. Shi, G. Walter, and K. Vandenborne, Transcriptional pathways associated with skeletal muscle disuse atrophy in humans. Physiol Genomics, 2007. 31(3): p. 510-20.

35. Rennie, M., R. Edwards, D. Halliday, D. Matthews, S. Wolman, and D. Millward, Muscle protein synthesis measured by stable isotope techniques in man: the effects of feeding and fasting. Clin Sci (Lond), 1982. 63(6): p. 519-23.

36. Burd, N., D. West, T. Rerecich, T. Prior, S. Baker, and S. Phillips, Validation of a single biopsy approach and bolus protein feeding to determine myofibrillar protein synthesis in stable isotope tracer studies in humans. Nutr Metab (Lond), 2011. 8: p. 15.

37. Koopman, R., L. Verdijk, R. Manders, A. Gijsen, M. Gorselink, E. Pijpers, A. Wagenmakers, and L. van Loon, Co-ingestion of protein and leucine stimulates muscle protein synthesis rates to the same extent in young and elderly lean men. Am J Clin Nutr., 2006. 84(3): p. 623-32.

38. Greenhaff, P., L. Karagounis, N. Peirce, E. Simpson, M. Hazell, R. Layfield, H. Wackerhage, K. Smith, P. Atherton, A. Selby, and M. Rennie, Disassociation between the effects of amino acids and insulin on signaling, ubiquitin ligases, and protein turnover in human muscle. Am J Physiol Endocrinol Metab, 2008. 295(3): p. 595-604.

39. Moore, D., M. Robinson, J. Fry, J. Tang, E. Glover, S. Wilkinson, T. Prior, M. Tarnopolsky, and S. Phillips, Ingested protein dose response of muscle and albumin protein synthesis after resistance exercise in young men. Am J Clin Nutr, 2009. 89(1): p. 161-8. 
40. Witard, O., S. Jackman, L. Breen, K. Smith, A. Selby, and K. Tipton, Myofibrillar muscle protein synthesis rates subsequent to a meal in response to increasing doses of whey protein at rest and after resistance exercise. Am J Clin Nutr, 2014. 99(1): p. 86-95.

41. Pennings, B., B. Groen, A. de Lange, A. Gijsen, A. Zorenc, J. Senden, and L. van Loon, Amino acid absorption and subsequent muscle protein accretion following graded intakes of whey protein in elderly men Am J Physiol Endocrinol Metab, 2012. 302(8): p. E992-9.

42. Yang, Y., L. Breen, N. Burd, A. Hector, T. Churchward-Venne, A. Josse, M. Tarnopolsky, and S. Phillips, Resistance exercise enhances myofibrillar protein synthesis with graded intakes of whey protein in older men. Br J Nutr, 2012. 7: p. 1-9.

43. Pennings, B., Y. Boirie, J. Senden, A. Gijsen, H. Kuipers, and L. van Loon, Whey protein stimulates postprandial muscle protein accretion more effectively than do casein and casein hydrolysate in older men. Am J Clin Nutr., 2011. 93(5): p. 997-1005.

44. Boirie, Y., M. Dangin, P. Gachon, M. Vasson, J. Maubois, and B. Beaufrère, Slow and fast dietary proteins differently modulate postprandial protein accretion. Proc Natl Acad Sci U S A, 1997. 94(26): p. 14930-5.

45. Koopman, R., N. Crombach, A. Gijsen, S. Walrand, J. Fauquant, A. Kies, S. Lemosquet, W. Saris, Y. Boirie, and L. van Loon, Ingestion of a protein hydrolysate is accompanied by an accelerated in vivo digestion and absorption rate when compared with its intact protein. Am J Clin Nutr, 2009. 90(1): p. 106-15.

46. Wall, B., H. Hamer, A. de Lange, A. Kiskini, B. Groen, J. Senden, A. Gijsen, L. Verdijk, and L. van Loon, Leucine co-ingestion improves post-prandial muscle protein accretion in elderly men. Clin Nutr, 2013. 32(3): p. 412-9.

47. Groen, B., P. Res, B. Pennings, E. Hertle, J. Senden, W. Saris, and L. van Loon, Intragastric protein administration stimulates overnight muscle protein synthesis in elderly men. Am J Physiol Endocrinol Metab, 2012. 302(1): p. 52-60.

48. Wall, B., M. Dirks, L. Verdijk, T. Snijders, D. Hansen, P. Vranckx, N. Burd, P. Dendale, and L. van Loon, Neuromuscular electrical stimulation increases muscle protein synthesis in elderly type 2 diabetic men. Am J Physiol Endocrinol Metab, 2012. 303(5): p. 614-23.

49. Dirks, M., B. Wall, T. Snijders, C. Ottenbros, L. Verdijk, and L. van Loon, Neuromuscular electrical stimulation prevents muscle disuse atrophy during leg immobilisation in humans. Acta Physiol, 2013. 210(3): p. 628-41.

50. Wall, B., J. Morton, and L. van Loon, Strategies to maintain skeletal muscle mass in the injured athlete: Nutritional considerations and exercise mimetics. Eur J Sport Sci, 2014. 15(1): p. 53-62.

51. Rasmussen, B., S. Fujita, R. Wolfe, B. Mittendorfer, M. Roy, V. Rowe, and E. Volpi, Insulin resistance of muscle protein metabolism in aging. FASEB J, 2006. 20(6): p. 768-9.

52. Timmerman, K., J. Lee, H. Dreyer, S. Dhanani, E. Glynn, C. Fry, M. Drummond, M. Sheffield-Moore, B. Rasmussen, and E. Volpi, Insulin stimulates human skeletal muscle protein synthesis via an indirect mechanism involving endothelial-dependent vasodilation and mammalian target of rapamycin complex 1 signaling. J Clin Endocrinol Metab., 2010. 95(8): p. 3848-57.

53. Drummond, M., E. Glynn, C. Fry, K. Timmerman, E. Volpi, and B. Rasmussen, An increase in essential amino acid availability upregulates amino acid transporter expression in human skeletal muscle. Am J Physiol Endocrinol Metab, 2010. 298(5): p. 1011-8.

54. Fry, C., M. Drummond, E. Glynn, J. Dickinson, D. Gundermann, K. Timmerman, D. Walker, S. Dhanani, E. Volpi, and B. Rasmussen, Aging impairs contraction-induced human skeletal muscle mTORC1 signaling and protein synthesis. Skelet Muscle, 2011. 1(1): p. 11.

55. Cuthbertson, D., K. Smith, J. Babraj, G. Leese, T. Waddell, P. Atherton, H. Wackerhage, P. Taylor, and M. Rennie, Anabolic signaling deficits underlie amino acid resistance of wasting, aging muscle. FASEB J, 2005. 19(3): p. 422-4.

56. Drummond, M., C. Fry, E. Glynn, K. Timmerman, J. Dickinson, D. Walker, D. Gundermann, E. Volpi, and B. Rasmussen, Skeletal muscle amino acid transporter expression is increased in young and older adults following resistance exercise. J Appl Physiol, 2011. 111(1): p. 135-42.

57. Churchward-Venne, T., N. Burd, C. Mitchell, D. West, A. Philp, G. Marcotte, S. Baker, K. Baar, and S. Phillips, Supplementation of a suboptimal protein dose with leucine or essential amino acids: effects on myofibrillar protein synthesis at rest and following resistance exercise in men. J Physiol, 2012. 590: p. 2751-65.

58. Dickinson, J. and B. Rasmussen, Amino acid transporters in the regulation of human skeletal muscle protein metabolism. Curr Opin Clin Nutr Metab Care, 2013. 16(6): p. 638-44. 
59. Kimball, S., P. Farrell, and L. Jefferson, Invited Review: Role of insulin in translational control of protein synthesis in skeletal muscle by amino acids or exercise. J Appl Physiol, 2002. 93(3): p. 1168-80.

60. Drummond, M., C. Fry, E. Glynn, H. Dreyer, S. Dhanani, K. Timmerman, E. Volpi, and B. Rasmussen, Rapamycin administration in humans blocks the contraction-induced increase in skeletal muscle protein synthesis. J Physiol, 2009. 587: p. 1535-46.

61. Glover, E., N. Yasuda, M. Tarnopolsky, A. Abadi, and S. Phillips, Little change in markers of protein breakdown and oxidative stress in humans in immobilization-induced skeletal muscle atrophy. Appl Physiol Nutr Metab, 2010. 35(2): p. 125-33.

62. West, D., N. Burd, V. Coffey, S. Baker, L. Burke, J. Hawley, D. Moore, T. Stellingwerff, and S. Phillips, Rapid aminoacidemia enhances myofibrillar protein synthesis and anabolic intramuscular signaling responses after resistance exercise. Am J Clin Nutr., 2011. 94(3): p. 795-803.

63. Drummond, M.J., J.M. Dickinson, C.S. Fry, D.K. Walker, D.M. Gundermann, P.T. Reidy, K.L. Timmerman, M.M. Markofski, D. Paddon-Jones, B.B. Rasmussen, and E. Volpi, Bed rest impairs skeletal muscle amino acid transporter expression, mTORC1 signaling, and protein synthesis in response to essential amino acids in older adults. Am J Physiol Endocrinol Metabo, 2012. 302(9): p. E1113-22.

64. Stephens, F.B., C. Chee, B.T. Wall, A.J. Murton, C.E. Shannon, L.J. van Loon, and K. Tsintzas, Lipid-induced insulin resistance is associated with an impaired skeletal muscle protein synthetic response to amino acid ingestion in healthy young men. Diabetes, 2015. 64(5): p. 1615-20.

65. Markofski, M., J. Dickinson, M. Drummond, C. Fry, S. Fujita, D. Gundermann, E. Glynn, K. Jennings, D. Paddon-Jones, P. Reidy, M. Sheffield-Moore, K. Timmerman, B. Rasmussen, and E. Volpi, Effect of age on basal muscle protein synthesis and mTORC1 signaling in a large cohort of young and older men and women. Exp Gerontol, 2015. 65: p. 1-7.

66. Farnfield, M., L. Breen, K. Carey, A. Garnham, and D. Cameron-Smith, Activation of mTOR signalling in young and old human skeletal muscle in response to combined resistance exercise and whey protein ingestion. Appl Physiol Nutr Metab, 2012. 37(1): p. 21-30.

67. Bodine, S., E. Latres, S. Baumhueter, V. Lai, L. Nunez, B. Clarke, W. Poueymirou, F. Panaro, E. Na, K. Dharmarajan, Z. Pan, D. Valenzuela, T. DeChiara, T. Stitt, G. Yancopoulos, and D. Glass, Identification of ubiquitin ligases required for skeletal muscle atrophy. Science, 2001. 23: p. 1704-8.

68. Gomes, M., S. Lecker, R. Jagoe, A. Navon, and A. Goldberg, Atrogin-1, a muscle-specific F-box protein highly expressed during muscle atrophy. Proc Natl Acad Sci U S A, 2001. 98(25): p. 14440-5.

69. Wall, B., M. Dirks, T. Snijders, J. Senden, F. Stephens, V. ML, and L. van Loon, Short-term muscle disuse atrophy is not associated with increased intramuscular lipid deposition or a decline in skeletal muscle oxidative capacity in young and older males. Exp Gerontol, 2014. 61: p. 76-83.

70. Urso, M., A. Scrimgeour, Y. Chen, P. Thompson, and P. Clarkson, Analysis of human skeletal muscle after $48 \mathrm{~h}$ immobilization reveals alterations in mRNA and protein for extracellular matrix components. J Appl Physiol, 2006. 101(4): p. 1136-48.

71. Marimuthu, K., A. Murton, and P. Greenhaff, Mechanisms regulating muscle mass during disuse atrophy and rehabilitation in humans. J Appl Physiol, 2011. 110(2): p. 555-60.

72. Baehr, L., J. Furlow, and S. Bodine, Muscle sparing in muscle RING finger 1 null mice: response to synthetic glucocorticoids. J Physiol, 2011. 589: p. 4759-76.

73. Drummond, M., K. Timmerman, M. Markofski, D. Walker, J. Dickinson, M. Jamaluddin, A. Brasier, B. Rasmussen, and E. Volpi, Short-term bed rest increases TLR4 and IL-6 expression in skeletal muscle of older adults. Am J Physiol Regul Integr Comp Physiol, 2013. 305(3): p. 216-23.

74. Li, H., S. Malhotra, and A. Kumar, Nuclear factor-kappa B signaling in skeletal muscle atrophy. J Mol Med (Berl), 2008. 86(10): p. 1113-26.

75. Stefanyk, L. and D. Dyck, The interaction between adipokines, diet and exercise on muscle insulin sensitivity. Curr Opin Clin Nutr Metab Care, 2010. 13(3): p. 255-9.

76. Febbraio, M., N. Hiscock, M. Sacchetti, C. Fischer, and B. Pedersen, Interleukin-6 is a novel factor mediating glucose homeostasis during skeletal muscle contraction. Diabetes, 2004. 53(7): p. 1643-8. 


\section{CHAPTER}

\section{Neuromuscular electrical stimulation prevents muscle disuse atrophy during leg immobilization in humans}

Marlou L Dirks Benjamin T Wall

Tim Snijders Chris LP Ottenbros

Lex B Verdijk Luc JC van Loon

Acta Physiol (Oxf) 2014 March;210(3):628-41 
ABSTRACT

Background Short periods of muscle disuse, due to illness or injury, result in substantial skeletal muscle atrophy. Recently we have shown that a single session of neuromuscular electrical stimulation (NMES) increases muscle protein synthesis rates.

Aim To investigate the capacity for daily NMES to attenuate muscle atrophy during short-term muscle disuse.

Methods Twenty-four healthy, young ( $23 \pm 1 \mathrm{y}$ ) males participated in the present study. Volunteers were subjected to 5 days of one-legged knee immobilization with (NMES; $n=12$ ) or without (CON; $n=12$ ) supervised NMES sessions (40 min sessions, twice daily). Two days prior to and immediately after the immobilization period, CT-scans and single leg oneRepetition Maximum (1RM) strength tests were performed to assess quadriceps muscle cross-sectional area (CSA) and leg muscle strength, respectively. Furthermore, muscle biopsies were taken to assess muscle fibre CSA, satellite cell content and mRNA and protein expression of selected genes.

Results In CON, immobilization reduced quadriceps CSA by $3.5 \pm 0.5 \%$ $(P<0.0001)$ and muscle strength by $9 \pm 2 \%(P<0.05)$. In contrast, no significant muscle loss was detected following immobilization in NMES although strength declined by $7 \pm 3 \%(P<0.05)$. Muscle MAFbx and MuRF1 mRNA expression increased following immobilization in CON $(P<0.001$ and $P=0.07$, respectively) whereas levels either declined $(P<0.01)$ or did not change in NMES, respectively. Immobilization led to an increase in muscle myostatin mRNA expression in CON $(P<0.05)$ but remained unchanged in NMES.

Conclusion During short-term disuse, NMES represents an effective interventional strategy to prevent the loss of muscle mass, but it does not allow preservation of muscle strength. NMES during disuse may be of important clinical relevance in both health and disease. 


\section{INTRODUCTION}

Situations such as the recovery from illness or injury require otherwise healthy individuals to undergo short periods of bed rest or limb immobilization. Under these circumstances there is a rapid loss of skeletal muscle mass [1-3] that leads to reduced functional capacity [1-4], loss of muscle strength [5], impaired insulin sensitivity [6], a decline in basal metabolic rate [7, 8], and a concomitant increase in body fat mass [9-11]. As a consequence, the extent of disuse atrophy that occurs due to illness or injury has previously been identified as an important predictor of the duration of hospitalization and subsequent rehabilitation [12].

During periods of disuse, muscle atrophy occurs as a consequence of an imbalance between muscle protein synthesis and breakdown rates. Previous studies, employing either 10-14 days of bed rest $[10,13]$ or 2-6 weeks of limb immobilization $[2,14-16]$ as models of disuse, have demonstrated impairments in both fasting and postprandial muscle protein synthesis rates without any discernible changes in muscle protein breakdown $[13,17]$. Maintaining a certain minimal level of physical activity during periods of muscle disuse can offset such impairments in postabsorptive or postprandial muscle protein synthesis rates $[11,18]$ and, as such, attenuate muscle tissue loss [19, 20]. Unfortunately, in many clinical situations physical activity is temporarily not feasible or simply impossible and, thus, surrogates should be sought to alleviate muscle disuse atrophy.

Neuromuscular electrical stimulation (NMES) offers an attractive alternative way to allow muscle contraction, thereby acting as a surrogate for habitual physical activity during periods of muscle disuse due to illness or injury. Recently, we applied contemporary stable isotope methodology with repeated muscle biopsy sampling to demonstrate that a single session of NMES increases muscle protein synthesis rates for several hours in vivo in men [21]. Moreover, self-administered NMES has previously been shown to maintain muscle protein synthesis rates during long-term recovery from tibia fracture [14], and clinically applied NMES has shown beneficial effects on skeletal muscle function in patients recovering from surgery $[22,23]$ or suffering from severe cardiac complications $[24,25]$. However, to date, the capacity of supervised NMES as an interventional strategy to counteract the loss of muscle mass and strength during a short period of disuse remains to be established. This may be of important clinical relevance as the loss of muscle mass and strength during short periods of bed rest or immobilization following illness or injury are believed to delay subsequent recovery and likely contribute substantially to the loss of muscle mass with ageing [26, 27].

In the present study we investigate the efficacy of NMES as a means to attenuate skeletal muscle disuse atrophy. We hypothesized that a twice daily supervised NMES program could preserve skeletal muscle mass and attenuate the loss of muscle strength during a 5 day period of leg immobilization. We assessed changes in muscle mass following 5 days of one-legged knee immobilization using a full leg cast in 24 healthy 
young men with or without twice daily supervised NMES sessions. Muscle mass was assessed at a limb level using CT and DXA scans, whereas muscle biopsies were obtained prior to and immediately after immobilization to assess changes in muscle fibre type characteristics and relevant myocellular signalling. 


\section{METHODS}

\section{Subjects}

A total of 24 healthy young males (age: $23 \pm 1 \mathrm{y}$; body mass: $76 \pm 2 \mathrm{~kg}$; body mass index [BMI] $22 \pm 1 \mathrm{~kg} \cdot \mathrm{m}^{-2}$ ) were included in the present study which was approved by the Medical Ethical Committee of the Maastricht University Medical Centre in accordance with the Declaration of Helsinki. Prior to the study, subjects completed a routine medical screening and general health questionnaire to ensure their suitability to take part. Exclusion criteria were: BMI below 18.5 or above $30 \mathrm{~kg} \cdot \mathrm{m}^{-2}$; any back, knee or shoulder complaints which may interfere with the use of crutches; type 2 diabetes mellitus (determined by HbA1c-values $>7.0 \%$ ); any family history of thrombosis; and/or severe cardiac problems. Furthermore, subjects who had performed structured and prolonged resistance type exercise training during the 6 months prior to the study were also excluded. All subjects were informed of the nature and possible risks of the experimental procedures, before their written informed consent was obtained. During screening, an estimation of one-Repetition Maximum (1RM) single leg knee extension strength (Technogym, Rotterdam, the Netherlands) was made using the multiple repetitions testing procedure [28].

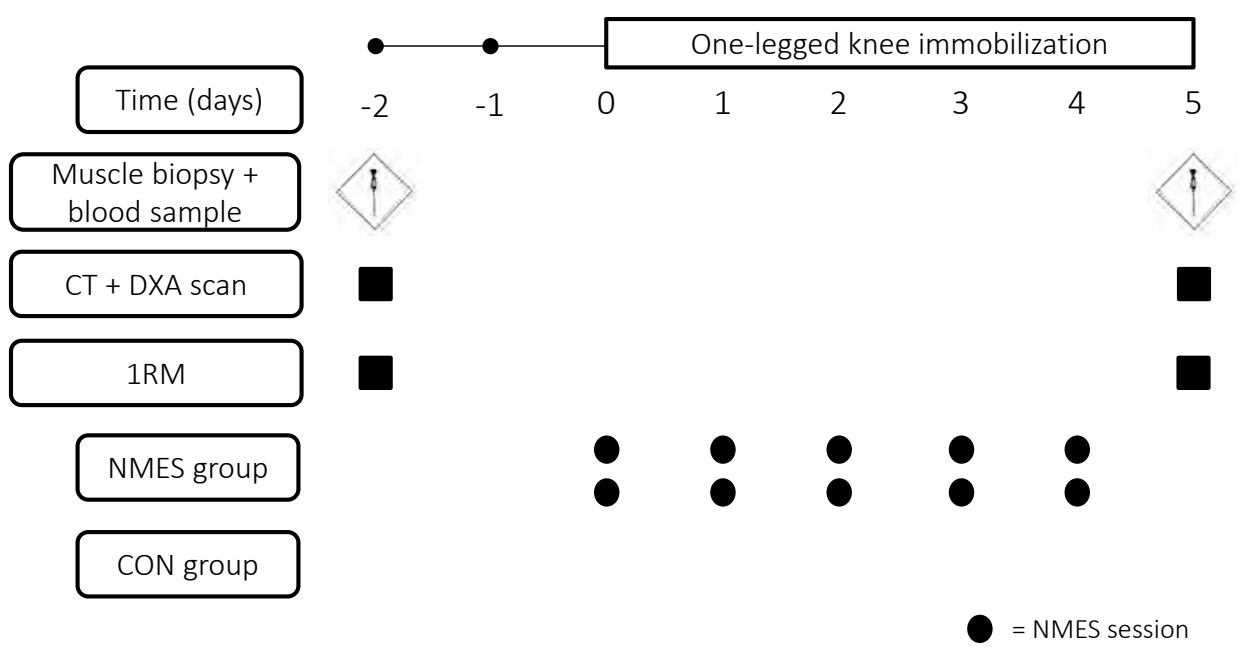

Figure 1 | Schematic representation of the experimental protocol. NMES = Neuromuscular electrical stimulation 


\section{Study design}

After inclusion, subjects were randomly allocated into either the control (CON; $n=12$ ) or the neuromuscular electrical stimulation (NMES; $n=12$ ) group. The experimental protocol is depicted in Figure 1. Both groups underwent a 5 day period of muscle disuse induced via one-legged knee immobilization by way of a full leg cast, either with (NMES group) or without (CON group) NMES performed twice daily under supervision at home. The leg to be immobilized was randomized and counter-balanced between left and right. On two separate test days, $48 \mathrm{~h}$ before and immediately after the immobilization period, single slice computed tomography (CT) scans were performed at the mid-thigh of both legs, whole-body dual energy x-ray absorptiometry (DXA) scans were taken, leg volume was measured by anthropometry [29], a single muscle biopsy and venous blood sample were collected, and one-legged knee extension strength (1RM) was assessed.

\section{Muscle mass and function}

Forty eight $\mathrm{h}$ prior to, and immediately after the immobilization period, subjects visited the laboratory in the fasted state for 2 identical test days (i.e. test days 1 and 2). During the test days, several measurements of muscle mass and function were performed. First, anatomical cross-sectional area (CSA) of the quadriceps muscle and whole thigh were assessed via a single slice CT scan (Philips Brilliance 64, Philips Medical Systems, Best, the Netherlands). The scanning characteristics were as follows: $120 \mathrm{kV}, 300 \mathrm{~mA}$, rotation time of $0.75 \mathrm{~s}$, and a field of view of $500 \mathrm{~mm}$. While the subjects were lying supine, legs extended and their feet secured, a $3 \mathrm{~mm}$ thick axial image was taken $15 \mathrm{~cm}$ proximal to the top of the patella. On test day 1 , the precise scanning position was marked with semi-permanent ink for replication on test day 2. CT-scans were analysed for the CSA of the whole thigh muscle as well as the quadriceps by manual tracing using ImageJ software (version 1.46d, National Institute of Health, Maryland, USA)[30]. Thereafter, body composition and bone mineral content were measured via DXA-scan (Hologic, Discovery A, QDR Series, Bedford, MA, USA). Whole-body and regional lean mass were determined using the system's software package Apex version 2.3. Leg volume of both legs was also assessed by anthropometry as described previously [29]. Maximal calf circumference of both legs was measured as part of the measurements to determine leg volume. Maximum strength was evaluated for each leg separately by one-Repetition Maximum (1RM) strength tests on a leg extension machine (Technogym, Rotterdam, the Netherlands). The estimations obtained during the screening visit were used to determine 1 RM as described previously [31].

\section{Blood and muscle sampling}

During test day 1 , fasting venous blood samples were collected to determine basal plasma glucose and insulin concentrations. Blood $(10 \mathrm{~mL})$ was collected into EDTA- 
containing tubes and directly centrifuged at $1,000 \mathrm{~g}$ for $10 \mathrm{~min}$ at $4^{\circ} \mathrm{C}$. Aliquots of plasma were immediately frozen in liquid nitrogen and stored at $-80^{\circ} \mathrm{C}$ until further analysis. Plasma glucose concentrations (Glucose HK CP, ABX Diagnostics, ref. A11A01667, Montpellier, France) were analysed with a COBAS FARA semi-automatic analyser (Roche, Basel, Switzerland). Plasma insulin concentrations were determined by radioimmunoassay (Millipore, ref. HI-14K, Billerica, MA, USA). Additionally, during test day 1 and 2, a single muscle biopsy sample was collected from the leg previously selected for immobilization. After local anaesthesia was induced, percutaneous needle biopsy samples were collected from the vastus lateralis muscle, approximately $15 \mathrm{~cm}$ above the patella [32]. Any visible non-muscle tissue was removed immediately, and part of the biopsy sample was embedded in Tissue-Tec (Sakura Finetek, Zoeterwoude, the Netherlands) before being frozen in liquid nitrogen-cooled isopentane, while another part was immediately frozen in liquid nitrogen. Muscle samples were subsequently stored at $80^{\circ} \mathrm{C}$ until further analyses.

\section{Leg immobilization}

Forty eight $\mathrm{h}$ following test day 1 , a full leg cast (randomized and counterbalanced for left and right legs) was applied in the plaster room of the Academic Hospital in Maastricht at 8:00 on the first day of the immobilization period. The leg cast extended from $\sim 5 \mathrm{~cm}$ above the ankle until $\sim 25 \mathrm{~cm}$ above the patella (i.e. approximately halfway up the upper leg). The cast was set so the knee joint was placed at a 30 degree angle of flexion to prevent subjects from performing weight-bearing activities with the casted leg. The immobilization period always comprised 3 week days and 2 weekend days. Additionally, for subjects assigned to the NMES group, placement of the electrodes for NMES was determined prior to fitting the cast (described below) and a small 'window' (a rectangle of approximately $12 \times 6 \mathrm{~cm}$ ) was cut in the cast $\sim 5 \mathrm{~cm}$ above the knee. Following the removal of this window, the section of cast was placed back from where it was removed and bandaged firmly in place. Subjects were given crutches and instructed on their correct usage before being provided with transportation home. Application of the cast signified the beginning of the immobilization period which continued for $5 d$, after which the cast was removed at 8.00 at the plaster room immediately prior to performing test day 2 .

\section{Neuromuscular electrical stimulation}

For subjects allocated to the NMES group, two NMES sessions were performed each day at the subjects' home for the duration of the 5 day immobilization period (i.e. 10 sessions in total). Neuromuscular electrical stimulation sessions were performed in the morning (7.00-12.00) and afternoon (13.00-18.00), with a minimum of $4 \mathrm{~h}$ between sessions. During each session, with the subject lying supine with a pillow placed under the knee to obviate the flexion angle, the window was removed from the cast and elec- 
trodes were placed on the distal part at the muscle belly of the $\mathrm{m}$. rectus femoris and the $m$. vastus lateralis, and at the inguinal area of both muscles. The position of the electrodes was re-marked each day with semi-permanent ink to ensure that location of the electrodes was not altered between sessions.

Stimulation was provided by an Enraf Nonius TensMed S84 stimulation device (Enraf Nonius, Rotterdam, the Netherlands) and 4, $2 \mathrm{~mm}$-thick, self-adhesive electrodes (50 $\mathrm{x}$ $50 \mathrm{~mm}$; Enraf Nonius), discharging biphasic symmetric rectangular-wave pulses. The NMES protocol consisted of a warm-up phase ( $5 \mathrm{~min}, 5 \mathrm{~Hz}, 250 \mu \mathrm{s})$, a stimulation period ( $30 \mathrm{~min}, 100 \mathrm{~Hz}, 400 \mu \mathrm{s}, 5 \mathrm{~s}$ on ( $0.75 \mathrm{~s}$ rise, $3.5 \mathrm{~s}$ contraction, $0.75 \mathrm{~s}$ fall) and $10 \mathrm{~s}$ off), and a cooling-down phase $(5 \mathrm{~min}, 5 \mathrm{~Hz}, 250 \mu \mathrm{s})$. Subjects set the intensity of the stimulation to a level at which full contractions of $m$. quadriceps femoris were visible and palpable, and the heel began to slightly lift. This protocol was based on our previous work [21] demonstrating an acute increase in muscle protein synthesis following a single bout of NMES and selected due to previous work using high-frequency (>60 Hz), high pulse duration (>250 $\mu$ s) NMES $[33,34]$. Researchers encouraged subjects to increase the intensity of the stimulation during each subsequent session to provide a 'progressive' stimulus.

\section{Dietary intake}

On the evening prior to both test days subjects received a standardized meal containing 2900 kJ providing 51 Energy\% (En\%) as carbohydrate, 32 En\% as fat, and 17 En\% as protein. Subjects completed weighted dietary intake records for the 5 day duration of the immobilization period as well as on a separate consecutive 5 day occasion before the immobilization period. The same 5 days of the week were selected for both recording periods. Dietary intake records were analysed with Dieetlnzicht software, based on NEVO table 2011.

\section{Muscle analyses}

The portion of the muscle biopsies frozen and mounted in Tissue-Tek was cut into $5 \mu \mathrm{m}$ thick cryosections using a cryostat at $-20^{\circ} \mathrm{C}$. Pre and post samples from one control and one NMES subject were mounted together on uncoated, pre-cleaned glass slides. Care was taken to correctly align the samples for cross-sectional fibre analyses. Muscle biopsies were stained for muscle fibre typing (FT) and satellite cell (SC) content as described in detail previously [35]. In short, slides were incubated with primary antibodies directed against myosin heavy chain (MHC)-I (A4.840, dilution 1:25; Developmental Studies Hybridoma Bank, lowa City, IA), laminin (polyclonal rabbit anti-laminin, dilution 1:50; Sigma, Zwijndrecht, the Netherlands) and CD56 (dilution 1:40; BD Biosciences, San Jose, CA). The following appropriate secondary antibodies were applied: goat anti-mouse IgM AlexaFluor555, goat anti-rabbit IgG AlexaFluor647, and Streptavidin Alexa 488 (dilution 1:500, 1:400, and 1:200, respectively; Molecular Probes, Invitrogen, Breda, the Nether- 
lands). Nuclei were stained with 4,6-diamidino-2-phenylindole (DAPI, $0.238 \mu \mathrm{M}$; Molecular Probes). Images were captured at 10x magnification with a fluorescent microscope equipped with an automatic stage, and analysed using ImageJ software (version 1.46r, National Institute of Health [30]). Mean numbers of $184 \pm 17$ and $220 \pm 22$ muscle fibres were analysed in the biopsy samples collected pre and post immobilization, respectively.

The portion of the muscle biopsy sample immediately frozen in liquid nitrogen was used to determine mRNA and protein expression of several target genes as described previously [36]. In short, total RNA was isolated from 10-20 mg of frozen muscle tissue, which was then quantified spectrophotometrically. Thereafter, RNA purity was determined and cDNA synthesis was performed, and Taqman PCR was carried out as reported previously using $18 \mathrm{~S}$ as a housekeeping gene [37]. Taqman primer/probe sets were obtained from Applied Biosystems (Foster City, USA) for the following genes of interest: mammalian target of rapamycin (mTOR), P70S6 kinase (P70S6K), myogenic factor 4 (myogenin), MyoD, myostatin, Atrogen-1/Muscle Atrophy F-box (MAFbx), Muscle RINGfinger protein-1 (MuRF1), Forkhead box protein O1 (FOXO1), Focal Adhesion Kinase (FAK), large neutral amino acid transporter 1 (LAT1) and Proton-coupled amino acid transporter 1 (PAT1). Ct values of the target genes were normalized to $\mathrm{Ct}$ values of the internal control $18 \mathrm{~S}$, and final results were calculated as relative expression against the standard curve.

Muscle samples ( 40 mg) for Western blotting analyses were analysed as described previously [37]. The antibodies used in this study were anti Myostatin (52 kDa; dilution 1:500; rabbit polyclonal IgG; Santa Cruz sc-6885-R), anti MyoD (37 kDa; dilution 1:1000; rabbit polyclonal IgG; Santa Cruz sc-760), anti Myogenin (34 kDa; dilution 1:500; rabbit polyclonal IgG; Santa Cruz sc-576) and anti $\alpha$-actin (42 kDa; dilution 1:160.000, mouse monoclonal IgM; Sigma A2172).

\section{Statistics}

All data are expressed as means \pm standard error of the mean (SEM). Baseline characteristics between groups were compared by means of an independent samples t-test. Preversus post-intervention data were analysed using repeated-measures ANOVA with time (pre vs post) as within-subjects factor and treatment (CON vs NMES) as betweensubjects factor. Pearson's Correlation Coefficient was used to test for significant correlations. For the muscle fibre analysis, fibre type (type I vs type II) was added to the repeated-measures ANOVA as a within-subjects factor. In case of a significant interaction, paired $t$ tests were performed to determine time effects within groups or within type I or $\mathrm{II}$ fibres and independent $\mathrm{t}$ tests for group differences in the pre- and postintervention values. Statistical significance was set at $P<0.05$. All calculations were performed using SPSS version 20.0 (Chicago, IL, USA). 


\section{RESULTS}

\section{Subjects}

Subjects' characteristics are provided in Table 1. No differences between the control (CON) and neuromuscular electrical stimulation (NMES) group were observed for any of the parameters.

Table 1 | Subjects' characteristics

\begin{tabular}{lcc}
\hline & CON $(n=12)$ & NMES $(n=12)$ \\
\hline Age $(\mathrm{y})$ & $22 \pm 1$ & $23 \pm 1$ \\
Body mass $(\mathrm{kg})$ & $74.4 \pm 3.5$ & $77.7 \pm 2.2$ \\
Height $(\mathrm{m})$ & $1.84 \pm 0.03$ & $1.84 \pm 0.02$ \\
BMI $\left(\mathrm{kg} \cdot \mathrm{m}^{-2}\right)$ & $21.9 \pm 1.0$ & $23.1 \pm 0.7$ \\
Leg volume (L) & $8.23 \pm 0.50$ & $8.15 \pm 0.30$ \\
Glucose $\left(\mathrm{mmol} \cdot \mathrm{L}^{-1}\right)$ & $5.01 \pm 0.11$ & $5.08 \pm 0.07$ \\
Insulin $\left(\mathrm{mU} \cdot \mathrm{L}^{-1}\right)$ & $8.77 \pm 0.70$ & $8.93 \pm 1.01$ \\
HOMA-index & $1.97 \pm 0.18$ & $2.03 \pm 0.26$ \\
Glycated haemoglobin (\%) & $5.1 \pm 0.1$ & $5.4 \pm 0.1$ \\
\hline
\end{tabular}

Values are means \pm SEM. Abbreviations: BMI, Body Mass Index; HOMA-index, Homeostatic Model Assessment Index [38].

\section{Dietary intake}

During the 5 days of immobilization the daily energy intake averaged $8.5 \pm 0.7$ and 8.7 $\pm 0.6 \mathrm{MJ} \cdot \mathrm{day}^{-1}$ in the CON and NMES group, respectively, with average daily protein intakes of $1.01 \pm 0.04$ and $1.00 \pm 0.08 \mathrm{~g} \cdot \mathrm{kg}$ body weight ${ }^{-1} \cdot$ day $^{-1}$. For both energy intake and protein intake, no significant interaction effects were found.

\section{Neuromuscular electrical stimulation}

The intensity of the NMES intervention for subjects in the NMES group averaged $20.8 \pm 1.6 \mathrm{~mA}$ during the first session and was progressively increased to $42.2 \pm 3.7 \mathrm{~mA}$ in the final session. The average NMES intensity across all sessions and all subjects was $30.6 \pm 2.2 \mathrm{~mA}$.

\section{Muscle mass}

For quadriceps muscle CSA, a significant time*treatment interaction was observed in the immobilized leg (Figure 2; $P<0.001$ ). Quadriceps CSA in the CON group had decreased by $3.5 \pm 0.5 \%$ (from $7504 \pm 342$ to $7238 \pm 324 \mathrm{~mm}^{2} ; P<0.001$ ), whereas in the NMES group no significant decrease in quadriceps CSA was detected (from 7740 259 to 
$\left.7675 \pm 254 \mathrm{~mm}^{2}: P=0.07\right)$. In agreement, a significant time*treatment interaction $(P<0.001)$ was also observed for changes in CSA of the whole-thigh muscle, which showed a $3.7 \pm 0.6 \%$ decrease in the CON group $(P<0.001)$, with no changes in the NMES group (-0.5 $\pm 0.4 \%$ : $P=0.192)$. In the non-immobilized leg, quadriceps and thigh muscle CSA did not show any changes following 5 days of immobilization in both the CON and NMES group.

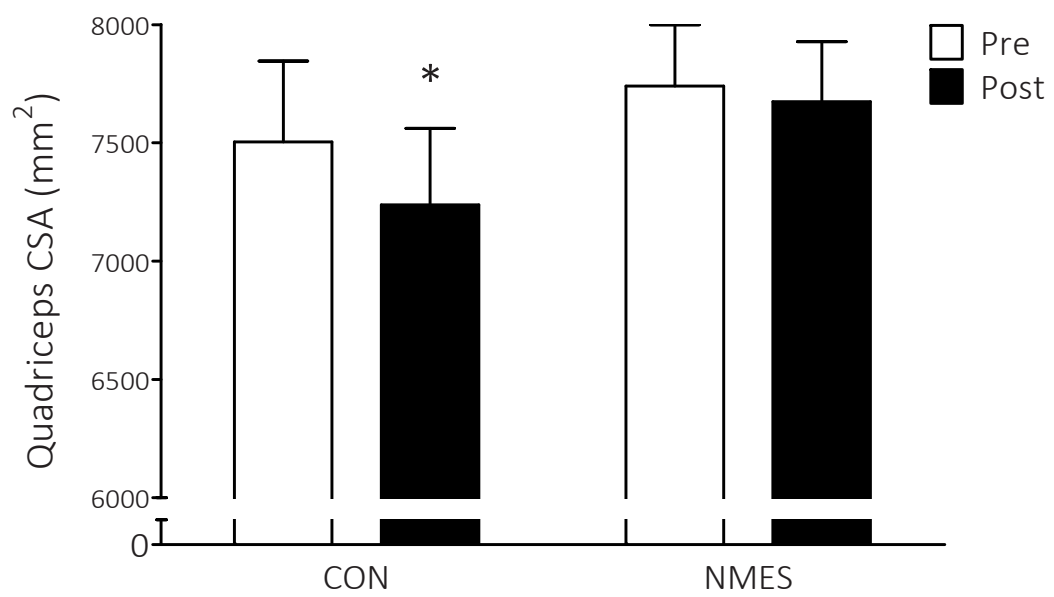

Figure 2 | Cross-sectional area (CSA) of $\mathrm{m}$. quadriceps femoris in the CON and NMES group, before and after 5 days of one-legged knee immobilization, as measured by single-slice CT scan. Data were analysed with a Repeated Measures ANOVA, and demonstrated a significant time*treatment interaction $(P=0.001)$. Data are expressed as means \pm SEM. $* P<0.05$; significantly different when compared with pre-immobilization values.

In line with the data on muscle CSA, a significant time*treatment interaction was observed for leg lean mass $(P<0.05)$. Subjects in the CON group lost on average $147 \pm 72 \mathrm{~g}$ of muscle tissue in the immobilized leg, representing $1.4 \pm 0.7 \%$ loss of leg muscle tissue $(P=0.066)$. In contrast, the NMES group showed an increase of $209 \pm 82 \mathrm{~g}(1.9 \pm 0.7 \%)$ in the immobilized leg after 5 days of immobilization $(P<0.05)$. No changes over time in leg lean mass were detected in the non-immobilized leg of subjects in the CON and NMES group $(P>0.05)$.

For leg volume and calf circumference, no changes over time (time effect, $P>0.05$ ) or between groups (interaction effect, $P>0.05$ ) were observed.

Scatter plots for correlations between NMES intensity and key outcome measures are presented as supplemental information in Figure 5. No significant correlations were found between the NMES intensity and delta quadriceps CSA (Figure 5A), delta muscle strength (Figure 5B), and delta leg lean mass (Figure 5C), respectively. 


\section{Muscle strength}

For muscle strength, a significant main effect of time ( $P=0.001)$ was detected in the immobilized leg such that one-legged 1 RM declined by $9.0 \pm 2.2 \%$ (from $77.9 \pm 3.9$ to $71.1 \pm 4.1 \mathrm{~kg}$ ) and $6.5 \pm 3.2 \%$ (from $78.3 \pm 4.5$ to $72.9 \pm 4.4 \mathrm{~kg}$ ) in the CON and NMES groups, respectively, with no differences between groups. Muscle strength in the nonimmobilized leg increased in both groups (time effect, $P<0.05$ ); from $78.8 \pm 4.4$ to $81.5 \pm 4.9 \mathrm{~kg}$ in the CON group and from $76.9 \pm 3.1$ to $81.9 \pm 3.4 \mathrm{~kg}$ in the NMES group.

Table 2 | Muscle fibre characteristics

\begin{tabular}{|c|c|c|c|c|c|}
\hline & & \multicolumn{2}{|c|}{ CON } & \multicolumn{2}{|c|}{ NMES } \\
\hline & $\begin{array}{l}\text { Fibre } \\
\text { type }\end{array}$ & Pre & Post & Pre & Post \\
\hline \multirow[t]{2}{*}{ Muscle fibre CSA $\left(\mu \mathrm{m}^{2}\right)$} & । & $5259 \pm 328$ & $5378 \pm 392$ & $5676 \pm 424$ & $5493 \pm 430$ \\
\hline & II & $6680 \pm 328 *$ & $6316 \pm 441$ & $5885 \pm 426$ & $6412 \pm 586+$ \\
\hline \multirow[t]{2}{*}{$\%$ Fibre (number) } & I & $43 \pm 3$ & $45 \pm 4$ & $52 \pm 3$ & $46 \pm 3$ \\
\hline & II & $57 \pm 3 *$ & $55 \pm 4$ & $48 \pm 3$ & $54 \pm 3$ \\
\hline \multirow[t]{2}{*}{ Nuclei per fibre } & I & $2.8 \pm 0.2$ & $2.9 \pm 0.2$ & $2.9 \pm 0.2$ & $2.7 \pm 0.2$ \\
\hline & II & $3.3 \pm 0.1$ & $3.3 \pm 0.2$ & $2.9 \pm 0.2$ & $2.9 \pm 0.2$ \\
\hline \multirow[t]{2}{*}{ Myonuclear domain $\left(\mu \mathrm{m}^{2}\right)$} & । & $1910 \pm 57$ & $1848 \pm 68$ & $1944 \pm 87$ & $1997 \pm 63$ \\
\hline & ॥ & $2057 \pm 103 *$ & $1935 \pm 79$ & $2004 \pm 89$ & $2233 \pm 83 \#$ \\
\hline \multirow[t]{2}{*}{ Number of SCs per fibre } & । & $0.090 \pm 0.007$ & $0.109 \pm 0.009$ & $0.115 \pm 0.011$ & $0.106 \pm 0.010$ \\
\hline & II & $0.072 \pm 0.006$ & $0.075 \pm 0.007$ & $0.075 \pm 0.011 *$ & $0.060 \pm 0.007$ \\
\hline \multirow[t]{2}{*}{ Number of SCs per $\mathrm{mm}^{2}$} & 1 & $17.3 \pm 0.3$ & $20.8 \pm 1.7$ & $20.8 \pm 2.0$ & $19.2 \pm 1.4$ \\
\hline & ॥ & $11.5 \pm 1.4^{*}$ & $12.5 \pm 1.6$ & $12.5 \pm 1.4^{*}$ & $9.6 \pm 1.0$ \\
\hline \multirow[t]{2}{*}{ SCs per myonuclei (\%) } & । & $3.3 \pm 0.2$ & $3.8 \pm 0.3$ & $4.0 \pm 0.4$ & $3.8 \pm 0.3$ \\
\hline & ॥ & $2.2 \pm 0.2 *$ & $2.4 \pm 0.3$ & $2.5 \pm 0.3 *$ & $2.1 \pm 0.2$ \\
\hline
\end{tabular}

Data represent means \pm SEM. Abbreviations: CSA, cross-sectional area; SC, satellite cell; SCs per myonuclei (\%), the number of SCS as a percentage of the total number of myonuclei (i.e. number of myonuclei + number of SCS). * Significantly different from type I fibre value $(P<0.05)$. + Significantly different from pre value in NMES group. \# Significantly different from CON post-immobilization value $(P<0.05)$

\section{Muscle fibre characteristics}

Muscle fibre characteristics are displayed in Table 2. Before the intervention, no significant difference was observed in type I and type II muscle fibre CSA between groups. A significant time*treatment*fibre type interaction was observed for muscle fibre CSA $(P<0.001)$. Separate analyses showed no significant change in both type I and type II muscle fibre CSA in the CON group after immobilization. In contrast, we observed a significant increase in type II muscle fibre CSA in the NMES group over time (from 5885 \pm 426 to $\left.6412 \pm 586 \mu^{2} ; P<0.05\right)$, whereas in type I fibres no time effect was observed 
$(P>0.05)$. Fibre distribution showed no differences at baseline between groups, and did not change over time in both groups $(P>0.05)$.

For myonuclear domain size, a significant time*treatment*fibre type interaction was observed $(P<0.05)$ and an overall effect of fibre type $(P<0.001)$, with larger myonuclear domain sizes in type II vs. type I fibres in both the CON and NMES group. No changes in type I myonuclear domain size were found $(P>0.05)$, while a significant time*treatment interaction was observed in type II fibres $(P<0.05)$ caused by a greater myonuclear domain in the NMES vs. CON group after immobilization. At baseline, no differences in SC content were observed between groups $(P>0.05)$. In addition, no changes over time were found for type I and type II SC content expressed per muscle fibre, per millimetre squared, or as a percentage of the total number of myonuclei $(P>0.05$ for all three parameters).

\section{mRNA and protein expression}

Figure 3 and 4 display the relative expression in skeletal muscle mRNA of selected genes of interest in the CON and NMES group, two days prior to and immediately following 5 days of one-legged knee-immobilization. No differences in mRNA expression of selected genes were observed between CON and NMES at baseline. For muscle myostatin mRNA expression, a significant time*treatment interaction was observed (Figure $3 A ; P<0.05$ ). Separate analysis showed a $68 \%$ increase following immobilization in the CON group $(P<0.05)$, whereas a trend for a decline was observed in the NMES group $(P=0.075)$. For muscle mRNA expression of MyoD (Figure $3 \mathrm{C}$ ) and myogenin (Figure 3E) a significant increase was observed over time $(P<0.05$ and $P<0.01$, respectively), with no differences between groups. 

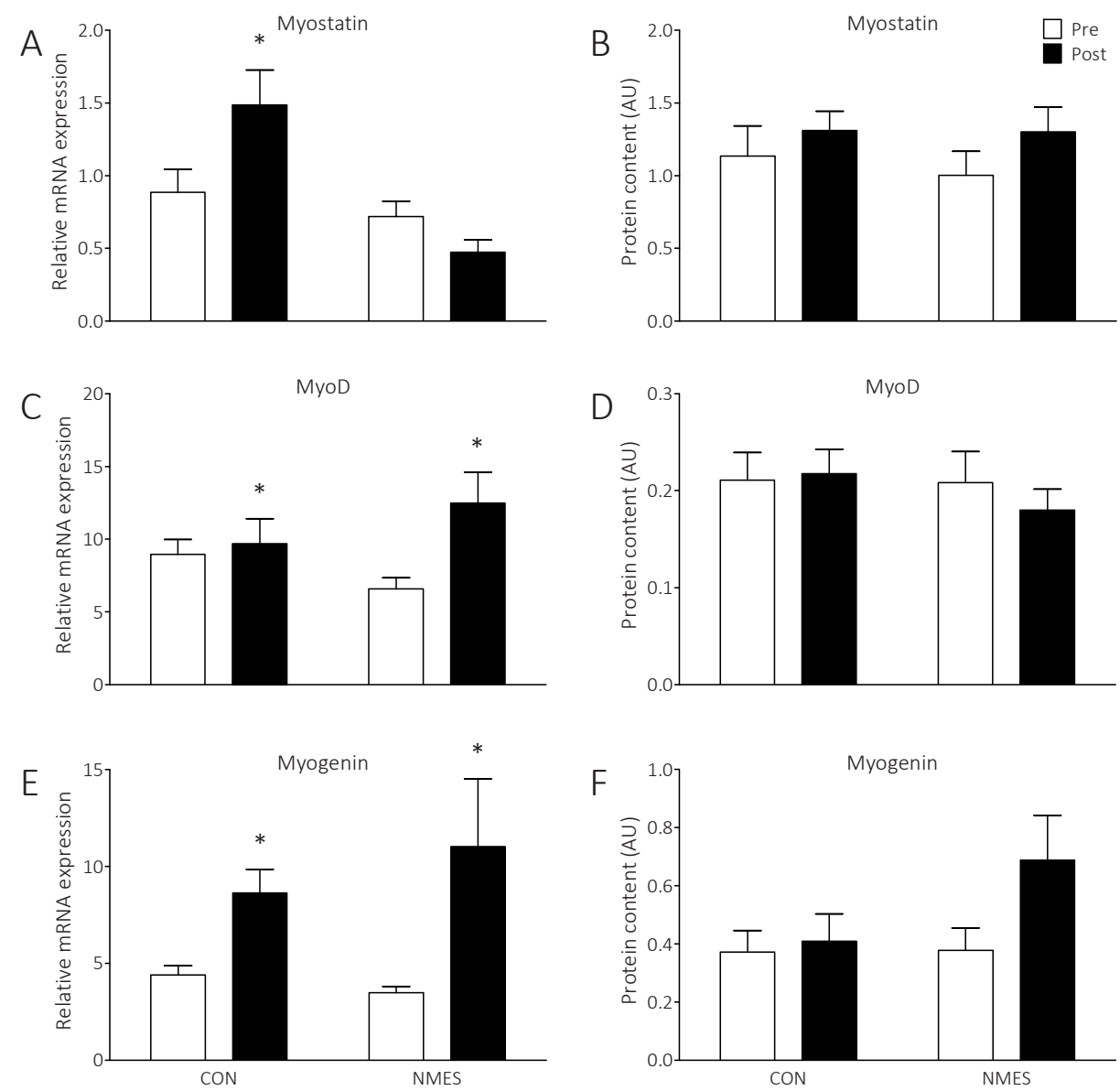

Figure 3 | Skeletal muscle mRNA expression of myostatin, MyoD and myogenin in the CON and NMES group before and after 5 days of one-legged knee immobilization. Data were analysed with a Repeated Measures ANOVA, and expressed as means \pm SEM. * $P<0.05$; significantly different when compared with preimmobilization values. 

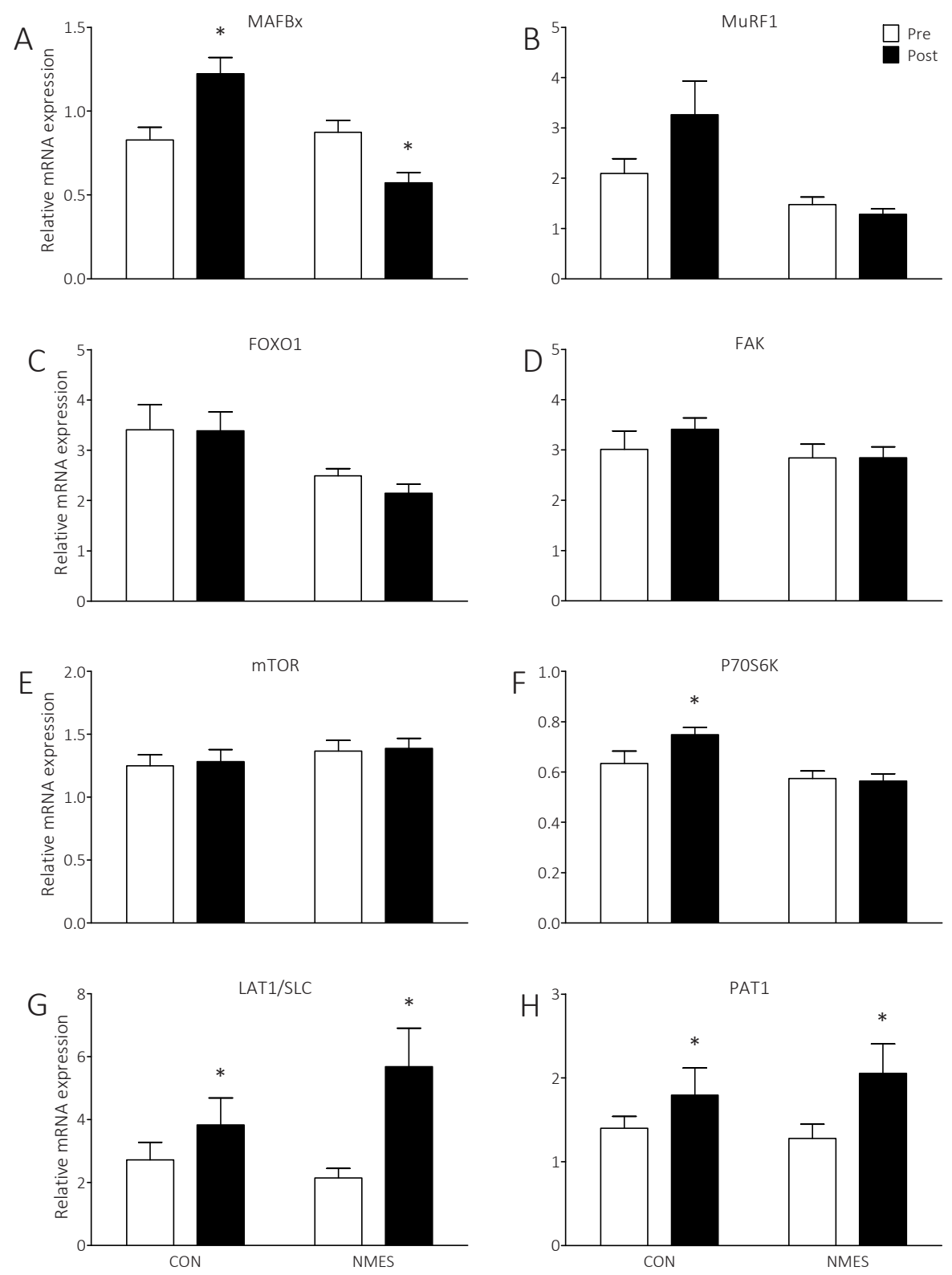

Figure 4 | Skeletal muscle mRNA expression of selected genes of interest in the CON and NMES group before and after 5 days of one-legged knee immobilization. Data were analysed with a Repeated Measures ANOVA. * $P<0.05$; significantly different when compared with pre-immobilization values. Data are expressed as means \pm SEM. Abbreviations: MAFbx, Muscle Atrophy F-box; MuRF1, Muscle RING-finger protein-1; FOXO1, Forkhead box protein 01; FAK, Focal Adhesion Kinase; LAT1, large neutral amino acid transporter 1; PAT1, Proton-coupled amino acid transporter 1. 
A significant time*treatment interaction was observed for the mRNA expression of muscle MAFbx (Figure 4A; $P<0.001$ ) and MuRF1 (Figure 4B; $P<0.05$ ). MAFBx mRNA expression was upregulated in the CON group (48\%; $P<0.001)$, whereas in the NMES group a decline was observed $(35 \%, P<0.05)$. MuRF1 mRNA expression tended to increase in the CON group (56\%, $P=0.066)$, while no change over time was observed in the NMES group $(P>0.05)$. No significant changes occurred over time or between groups in the muscle mRNA expression of FOXO1 (Figure 4C), mTOR (Figure 4E) or FAK (Figure 4D). A significant time*treatment interaction was observed for the muscle mRNA expression of P70S6K (Figure 4F; $P<0.05$ ), with an $18 \%$ upregulation following immobilization in the CON group $(P<0.01)$, whereas no change was observed in the NMES group $(P>0.05)$. Muscle mRNA expression of the amino acid transporters LAT1/SLC (Figure 4G) and PAT1 (Figure $4 \mathrm{H}$ ) had significantly increased following immobilization (both $P<0.05$ ), with no differences between groups.

Protein expression of myostatin, myoD and myogenin are presented in Figure 3. For both myostatin and MyoD, no changes in protein expression were observed (both $P>0.05)$. Myogenin protein expression tended to increase following immobilization $(P=0.054)$ with no differences between groups ( $P=0.122$ for time*treatment interaction). 


\section{DISCUSSION}

In the present study, we demonstrated that neuromuscular electrical stimulation (NMES) prevented skeletal muscle atrophy to occur during 5 days of one-legged knee immobilization. However, NMES could not rescue the loss of muscle strength during this short period of disuse. Moreover, we report that the molecular changes associated with muscle disuse atrophy can largely be prevented by the daily application of NMES.

Skeletal muscle disuse leads to a loss of muscle mass and strength and is accompanied by numerous negative health consequences [1-4, 6-11]. Based on previous studies, the rate of muscle loss during experimental lower limb immobilization is approximately $0.5 \%$ per day $[27,39]$. However, this loss does not appear to be linear with higher rates of muscle loss occurring during the first few days of disuse [40]. In the present study we report that merely 5 days of one-legged knee immobilization significantly decreased quadriceps muscle cross-sectional area by $3.5 \%$ in a group of healthy young males (Figure 2; CON group), representing $150 \mathrm{~g}$ of muscle tissue lost from the immobilized leg. When translating our observations of muscle loss in a single limb to a whole-body level, assuming that $60 \%$ of whole-body muscle loss occurs in the lower limbs, patients could lose as much as $1 \mathrm{~kg}$ of muscle tissue during 5 days of bed rest $[5,41]$. This is consistent with previous studies investigating the impact of 10 days of bed rest $[13,41]$. Furthermore, the 5 days of leg immobilization also resulted in a substantial $9.0 \pm 2.2 \%$ decline in leg strength. Clearly, these data demonstrate the impact of short periods of muscle disuse on muscle mass and strength and underline the clinical relevance to develop effective interventional strategies to attenuate muscle disuse atrophy and associated negative health consequences.

The use of NMES has been proposed as an interventional strategy to alleviate muscle loss in a variety of clinical conditions [14, 22-25]. Recently, we showed that a single NMES session stimulates muscle protein synthesis in vivo in men [21]. In the current study, we investigated whether the application of daily NMES could attenuate the loss of muscle mass during a short period of muscle disuse. Strikingly, the application of supervised NMES performed twice daily on the immobilized leg entirely prevented any disuse atrophy (Figure 2), with no measurable loss of muscle observed in the NMES group $(-0.8 \pm 0.4 \% ; P>0.05)$. Given the inherent variability of the measurement of muscle fibre size [42], we were unable to detect specific muscle fibre atrophy following only 5 days of disuse in the control group (Table 2). However, we did detect a small but significant increase in type II muscle fibre size following immobilization in the group receiving NMES. These data suggest that high-frequency NMES may exert its protective effect on skeletal muscle disuse atrophy predominantly through the recruitment of type II muscle fibres. This is of significant relevance as muscle loss due to more prolonged disuse [43, $44]$ and/or ageing $[45,46]$ has been attributed to specific type II muscle fibre atrophy [42]. It is important to view the present data in the context of the potential clinical benefits of applying NMES to preserve muscle mass during relatively short periods of mus- 
cle disuse. Previously, NMES has generally been applied during rehabilitation [47, 48], when muscle mass has already been lost and has to be regained. However, in the present study we clearly demonstrate the relevance of applying NMES during a period of disuse or bed rest to prevent muscle tissue loss.

Muscle disuse atrophy is generally accompanied by a substantial decline in muscle strength and impairments in functional capacity [1-3]. Previous studies performing onelegged knee immobilization have reported a decline in muscle strength ranging from 0.4 [49] to $4.2 \%$ per day [50] with an average muscle strength loss of $1.3 \%$ per day [27]. In the present study, we report that 5 days of limb immobilization resulted in a $9.0 \%$ loss of leg muscle strength (representing an average daily loss of $1.8 \%$ per day). Consistent with earlier reports $[4,50,51]$, we show a greater relative decline in muscle strength when compared to the loss of muscle mass. This is in agreement with previous suggestions that neuromuscular deconditioning during the early stages of training or disuse is mainly responsible for the rapid changes in muscle strength $[52,53]$. This also explains why the decline in muscle strength in the control group was only partially rescued with NMES (-6.5 $\pm 3.2 \%)$. We speculate that the application of NMES will likely further attenuate muscle strength loss during more prolonged periods of muscle disuse, when muscle mass loss becomes the key determinant of the decline in muscle strength. In agreement, previous work assessing the impact of prolonged NMES training has been shown to effectively increase muscle strength in healthy young subjects [33, 34], in CHD patients [54] and in patients suffering from septic shock [55].

Aside from assessing the impact of NMES on muscle mass and strength during a period of disuse, we also investigated some of the myocellular mechanisms that may be responsible for the NMES mediated prevention of muscle mass loss during immobilization. Skeletal muscle satellite cells (SCS) are essential for repair, maintenance and growth of myofibres [56-58]. Moreover, we have previously reported that type II fibre specific atrophy associated with ageing [59] and spinal cord injury [60] is also accompanied by a decline in SC content in these fibres. In the present study, we hypothesized that a better maintenance of muscle SC content in the NMES group contributes to the preservation of muscle mass. However, short-term immobilization did not alter SC content in either type I or II fibres in either the control or NMES group (Table 2). As such, the present data suggest that changes in SC content are not instrumental in the early development of disuse atrophy, nor the NMES mediated prevention of muscle loss. However, it cannot be ruled out that the rate of SC proliferation may be of more relevance during muscle atrophy (or NMES mediated prevention of muscle loss) observed over a more prolonged period of disuse [61]. Furthermore, we determined the mRNA and protein expression of key signalling proteins thought to be important in the regulation of muscle maintenance. Myostatin is regarded as a negative regulator of muscle mass in vivo $[62,63]$, primarily by inhibiting myogenesis $[64,65]$ via its inhibitory action on the myogenic regulatory factors [66], notably MyoD and myogenin [67, 68]. Consistent with the proposed role of myostatin, we report an increased mRNA expression in 
the CON group that was prevented in the NMES group (Figure 3). Moreover, the significant increase in the mRNA expression of MyoD and myogenin seemed to be larger in the NMES group but was observed in both groups, while this did not result in an increased muscle protein expression (Figure 3). Collectively these data are consistent with a role for myostatin in the NMES mediated maintenance of muscle mass during disuse.

Increased rates of muscle protein breakdown have been suggested to play a role in short-term (<10 days) muscle disuse atrophy [27, 69]. Muscle protein breakdown in humans is thought to be regulated primarily by the ubiquitin-proteasome pathway, with key roles for the ubiquitin ligases MAFbx and MuRF1 [70, 71], and their upstream transcription factor FOXO1 [72]. In accordance, in the present study we report that both MAFbx and MuRF1 mRNA expression increase with immobilization (Figure 4). Strikingly, these effects were prevented in the NMES group, suggesting that NMES may also help to preserve muscle mass during disuse by preventing an increase in muscle protein breakdown.

In the present study we applied NMES to the quadriceps only. This muscle group is particularly susceptible to muscle loss during whole-body disuse [5] and is functionally important to allow proper performance of daily living activities. From a clinical perspective, it could be speculated that multiple muscle groups should be targeted with NMES to ensure muscle mass maintenance during whole-body disuse. Although extending the use of NMES to multiple muscle groups could introduce practical constraints (e.g. skin irritation, antagonistic contractions, time constraints), optimizing such protocols will allow (more) effective clinical use of NMES. Given the role of skeletal muscle mass in metabolic homeostasis, muscle preservation during disuse would likely have a positive impact on preserving both metabolic health [73] and functional capacity.

The present study clearly demonstrates that merely a few days of disuse will lead to substantial loss of muscle mass and strength. Furthermore, NMES is identified as an effective interventional strategy to preserve muscle mass during such short periods of disuse. These data are of important clinical relevance as hospitalization following acute illness or injury is generally accompanied by a hospital stay of 6 days [74]. The loss of muscle mass and strength during such short (successive) periods of muscle disuse impairs functional capacity and hinders the subsequent rehabilitation upon discharge. In fact, it is now much speculated that the development of sarcopenia in the older population is, at least partly, attributed to the muscle loss that is experienced during short, successive periods of muscle disuse due to illness or injury occurring over the latter 2-3 decades of our lifespan [26, 40]. The use of NMES could also be of particular relevance to other patient groups and populations suffering from muscle atrophy, such as athletes recovering from injury [75], mechanically ventilated patients [55], spinal cord injured subjects [60], and post-surgery patients [23]. Preventing or attenuating the loss of muscle mass and strength during limb immobilization or bed rest likely minimizes the burden of muscle disuse, shortens hospital stay, and facilitates subsequent rehabilitation in both health and disease. 
In conclusion, NMES represents an effective interventional strategy to prevent the loss of muscle mass during short periods of muscle disuse. This is likely attributed to a stimulation of muscle protein synthesis and suppression of muscle protein breakdown. NMES forms a feasible strategy to prevent muscle loss and support subsequent rehabilitation during short periods of muscle disuse due to illness or injury.

\section{ACKNOWLEDGEMENTS}

The authors greatly acknowledge the cooperation and enthusiasm of the staff in the casting room of the Academic Hospital Maastricht. Furthermore, the assistance of Mellanie Geijen during the data collection, and the technical assistance by Joan MG Senden and Antoine Zorenc are very much appreciated. 


\section{REFERENCES}

1. Deitrick, J.E., The effect of immobilization on metabolic and physiological functions of normal men. Bull $N$ Y Acad Med, 1948. 24(6): p. 364-75.

2. Gibson, J.N., D. Halliday, W.L. Morrison, P.J. Stoward, G.A. Hornsby, P.W. Watt, G. Murdoch, and M.J. Rennie, Decrease in human quadriceps muscle protein turnover consequent upon leg immobilization. Clin Sci (Lond), 1987. 72(4): p. 503-9.

3. Ingemann-Hansen, T. and J. Halkjaer-Kristensen, Computerized tomographic determination of human thigh components. The effects of immobilization in plaster and subsequent physical training. Scand J Rehabil Med, 1980. 12(1): p. 27-31.

4. White, M.J., C.T. Davies, and P. Brooksby, The effects of short-term voluntary immobilization on the contractile properties of the human triceps surae. Q J Exp Physiol, 1984. 69(4): p. 685-91.

5. LeBlanc, A.D., V.S. Schneider, H.J. Evans, C. Pientok, R. Rowe, and E. Spector, Regional changes in muscle mass following 17 weeks of bed rest. J Appl Physiol, 1992. 73(5): p. 2172-8.

6. Stuart, C.A., R.E. Shangraw, M.J. Prince, E.J. Peters, and R.R. Wolfe, Bed-rest-induced insulin resistance occurs primarily in muscle. Metab Clin Exp, 1988. 37(8): p. 802-6.

7. Haruna, Y., Y. Suzuki, K. Kawakubo, R. Yanagibori, and A. Gunji, Decremental reset in basal metabolism during 20-days bed rest. Acta Physiol (Scand). Supplementum, 1994. 616: p. 43-9.

8. Tzankoff, S.P. and A.H. Norris, Effect of muscle mass decrease on age-related BMR changes. J Appl Physiol Respir Environ Exerc Physiol, 1977. 43(6): p. 1001-6.

9. Brooks, N., G.J. Cloutier, S.M. Cadena, J.E. Layne, C.A. Nelsen, A.M. Freed, R. Roubenoff, and C. Castaneda-Sceppa, Resistance training and timed essential amino acids protect against the loss of muscle mass and strength during 28 days of bed rest and energy deficit. J Appl Physiol, 2008. 105(1): p. 241-8.

10. Ferrando, A.A., H.W. Lane, C.A. Stuart, J. Davis-Street, and R.R. Wolfe, Prolonged bed rest decreases skeletal muscle and whole body protein synthesis. Am J Physiol, 1996. 270(4 Pt 1): p. E627-33.

11. Ferrando, A.A., K.D. Tipton, M.M. Bamman, and R.R. Wolfe, Resistance exercise maintains skeletal muscle protein synthesis during bed rest. J Appl Physiol, 1997. 82(3): p. 807-10.

12. Christensen, T., T. Bendix, and H. Kehlet, Fatigue and cardiorespiratory function following abdominal surgery. The British journal of surgery, 1982. 69(7): p. 417-9.

13. Kortebein, P., A. Ferrando, J. Lombeida, R. Wolfe, and W.J. Evans, Effect of 10 days of bed rest on skeletal muscle in healthy older adults. J Am Med Assoc, 2007. 297(16): p. 1772-4.

14. Gibson, J.N., K. Smith, and M.J. Rennie, Prevention of disuse muscle atrophy by means of electrical stimulation: maintenance of protein synthesis. Lancet, 1988. 2(8614): p. 767-70.

15. Glover, E.I., S.M. Phillips, B.R. Oates, J.E. Tang, M.A. Tarnopolsky, A. Selby, K. Smith, and M.J. Rennie, Immobilization induces anabolic resistance in human myofibrillar protein synthesis with low and high dose amino acid infusion. J Physiol, 2008. 586(Pt 24): p. 6049-61.

16. de Boer, M.D., A. Selby, P. Atherton, K. Smith, O.R. Seynnes, C.N. Maganaris, N. Maffulli, T. Movin, M.V. Narici, and M.J. Rennie, The temporal responses of protein synthesis, gene expression and cell signalling in human quadriceps muscle and patellar tendon to disuse. J Physiol, 2007. 585(Pt 1): p. 241-51.

17. Symons, T.B., M. Sheffield-Moore, D.L. Chinkes, A.A. Ferrando, and D. Paddon-Jones, Artificial gravity maintains skeletal muscle protein synthesis during 21 days of simulated microgravity. J Appl Physiol, 2009. 107(1): p. 34-8.

18. Burd, N.A., S.H. Gorissen, and L.J. van Loon, Anabolic resistance of muscle protein synthesis with aging. Exerc Sport Sci Rev, 2013. 41(3): p. 169-73.

19. Oates, B.R., E.I. Glover, D.W. West, J.L. Fry, M.A. Tarnopolsky, and S.M. Phillips, Low-volume resistance exercise attenuates the decline in strength and muscle mass associated with immobilization. Muscle Nerve, 2010. 42(4): p. 539-46.

20. Kawakami, Y., H. Akima, K. Kubo, Y. Muraoka, H. Hasegawa, M. Kouzaki, M. Imai, Y. Suzuki, A. Gunji, H. Kanehisa, and T. Fukunaga, Changes in muscle size, architecture, and neural activation after 20 days of bed rest with and without resistance exercise. European J Appl Physiol, 2001. 84(1-2): p. 7-12. 
21. Wall, B.T., M.L. Dirks, L.B. Verdijk, T. Snijders, D. Hansen, P. Vranckx, N.A. Burd, P. Dendale, and L.J. van Loon, Neuromuscular electrical stimulation increases muscle protein synthesis in elderly type 2 diabetic men. Am J Physiol Endocrinol Metab, 2012. 303(5): p. E614-23.

22. Snyder-Mackler, L., A. Delitto, S.W. Stralka, and S.L. Bailey, Use of electrical stimulation to enhance recovery of quadriceps femoris muscle force production in patients following anterior cruciate ligament reconstruction. Physical therapy, 1994. 74(10): p. 901-7.

23. Vinge, O., L. Edvardsen, F. Jensen, F.G. Jensen, J. Wernerman, and H. Kehlet, Effect of transcutaneous electrical muscle stimulation on postoperative muscle mass and protein synthesis. The British journal of surgery, 1996. 83(3): p. 360-3.

24. Banerjee, P., B. Caulfield, L. Crowe, and A.L. Clark, Prolonged electrical muscle stimulation exercise improves strength, peak VO2, and exercise capacity in patients with stable chronic heart failure. Journal of cardiac failure, 2009. 15(4): p. 319-26.

25. Vivodtzev, I., J.L. Pepin, G. Vottero, V. Mayer, B. Porsin, P. Levy, and B. Wuyam, Improvement in quadriceps strength and dyspnea in daily tasks after 1 month of electrical stimulation in severely deconditioned and malnourished COPD. Chest, 2006. 129(6): p. 1540-8.

26. English, K.L. and D. Paddon-Jones, Protecting muscle mass and function in older adults during bed rest. Curr Opin Clin Nutr Metab Care, 2010. 13(1): p. 34-9.

27. Wall, B.T. and L.J. van Loon, Nutritional strategies to attenuate muscle disuse atrophy. Nutr Rev, 2013. 71(4): p. 195-208.

28. Mayhew, J.L., J.L. Prinster, J.S. Ware, D.L. Zimmer, J.R. Arabas, and M.G. Bemben, Muscular endurance repetitions to predict bench press strength in men of different training levels. J Sports Med Phys Fitness, 1995. 35(2): p. 108-13.

29. Jones, P.R. and J. Pearson, Anthropometric determination of leg fat and muscle plus bone volumes in young male and female adults. J Physiol, 1969. 204(2): p. 63P-66P.

30. Strandberg, S., M.L. Wretling, T. Wredmark, and A. Shalabi, Reliability of computed tomography measurements in assessment of thigh muscle cross-sectional area and attenuation. BMC Med Imaging, 2010. 10: p. 18.

31. Verdijk, L.B., L. van Loon, K. Meijer, and H.H. Savelberg, One-repetition maximum strength test represents a valid means to assess leg strength in vivo in humans. J Sports Sci, 2009. 27(1): p. 59-68.

32. Bergstrom, J., Percutaneous needle biopsy of skeletal muscle in physiological and clinical research. Scand J Clin Lab Invest, 1975. 35(7): p. 609-16.

33. Gondin, J., L. Brocca, E. Bellinzona, G. D'Antona, N.A. Maffiuletti, D. Miotti, M.A. Pellegrino, and R. Bottinelli, Neuromuscular electrical stimulation training induces atypical adaptations of the human skeletal muscle phenotype: a functional and proteomic analysis. J Appl Physiol, 2011. 110(2): p. 433-50.

34. Maffiuletti, N.A., R. Zory, D. Miotti, M.A. Pellegrino, M. Jubeau, and R. Bottinelli, Neuromuscular adaptations to electrostimulation resistance training. Am J Phys Med Rehab, 2006. 85(2): p. 167-75.

35. Leenders, M., L.B. Verdijk, L. van der Hoeven, J. van Kranenburg, R. Nilwik, and L.J. van Loon, Elderly men and women benefit equally from prolonged resistance-type exercise training. J Gerontol A Biol Sci Med Sci, 2013. 68(7): p. 769-79.

36. Wall, B.T., M.L. Dirks, T. Snijders, J.M. Senden, J. Dolmans, and L.J. van Loon, Substantial skeletal muscle loss occurs during only 5 days of disuse. Acta Physiol (Oxf), 2013.

37. Wall, B.T., T. Snijders, J.M. Senden, C.L. Ottenbros, A.P. Gijsen, L.B. Verdijk, and L.J. van Loon, Disuse impairs the muscle protein synthetic response to protein ingestion in healthy men. J Clin Endocrinol Metab, 2013.

38. Phillips, S.M., E.I. Glover, and M.J. Rennie, Alterations of protein turnover underlying disuse atrophy in human skeletal muscle. J Appl Physiol, 2009. 107(3): p. 645-54.

39. Wall, B.T., M.L. Dirks, and L.J. van Loon, Skeletal muscle atrophy during short-term disuse: Implications for age-related sarcopenia. Ageing Res Rev, 2013.

40. Ferrando, A.A., D. Paddon-Jones, N.P. Hays, P. Kortebein, O. Ronsen, R.H. Williams, A. McComb, T.B. Symons, R.R. Wolfe, and W. Evans, EAA supplementation to increase nitrogen intake improves muscle function during bed rest in the elderly. Clin Nutr, 2010. 29(1): p. 18-23. 
41. Nilwik, R., T. Snijders, M. Leenders, B.B. Groen, J. van Kranenburg, L.B. Verdijk, and L.J. van Loon, The decline in skeletal muscle mass with aging is mainly attributed to a reduction in type II muscle fiber size. Exp Gerontol, 2013.

42. Bamman, M.M., M.S. Clarke, D.L. Feeback, R.J. Talmadge, B.R. Stevens, S.A. Lieberman, and M.C. Greenisen, Impact of resistance exercise during bed rest on skeletal muscle sarcopenia and myosin isoform distribution. J Appl Physiol, 1998. 84(1): p. 157-63.

43. Yasuda, N., E.I. Glover, S.M. Phillips, R.J. Isfort, and M.A. Tarnopolsky, Sex-based differences in skeletal muscle function and morphology with short-term limb immobilization. J Appl Physiol, 2005. 99(3): p. 1085-92.

44. Verdijk, L.B., R.A. Jonkers, B.G. Gleeson, M. Beelen, K. Meijer, H.H. Savelberg, W.K. Wodzig, P. Dendale, and L.J. van Loon, Protein supplementation before and after exercise does not further augment skeletal muscle hypertrophy after resistance training in elderly men. Am J Clin Nutr, 2009. 89(2): p. 608-16.

45. Tieland, M., O. van de Rest, M.L. Dirks, N. van der Zwaluw, M. Mensink, L.J. van Loon, and L.C. de Groot, Protein supplementation improves physical performance in frail elderly people: a randomized, doubleblind, placebo-controlled trial. J Am Med Dir Assoc, 2012. 13(8): p. 720-6.

46. Harris, S., J.P. LeMaitre, G. Mackenzie, K.A. Fox, and M.A. Denvir, A randomised study of home-based electrical stimulation of the legs and conventional bicycle exercise training for patients with chronic heart failure. Eur Heart J, 2003. 24(9): p. 871-8.

47. Neder, J.A., D. Sword, S.A. Ward, E. Mackay, L.M. Cochrane, and C.J. Clark, Home based neuromuscular stimulation as a new rehabilitative strategy for severely disabled patients with chronic obstructive pulmonary disease (COPD). Thorax, 2002. 57((4)): p. 333-7.

48. Seynnes, O.R., C.N. Maganaris, M.D. de Boer, P.E. di Prampero, and M.V. Narici, Early structural adaptations to unloading in the human calf muscles. Acta Physiol, 2008. 193(3): p. 265-74.

49. Thom, J.M., M.W. Thompson, P.A. Ruell, G.J. Bryant, J.S. Fonda, A.R. Harmer, X.A. Janse de Jonge, and S.K. Hunter, Effect of 10-day cast immobilization on sarcoplasmic reticulum calcium regulation in humans. Acta Physiol (Scand), 2001. 172(2): p. 141-7.

50. Jones, S.W., R.J. Hill, P.A. Krasney, B. O'Conner, N. Peirce, and P.L. Greenhaff, Disuse atrophy and exercise rehabilitation in humans profoundly affects the expression of genes associated with the regulation of skeletal muscle mass. FASEB J, 2004. 18(9): p. 1025-7.

51. Clark, B.C. and T.M. Manini, Sarcopenia =/= dynapenia. J Gerontol A Biol Sci Med Sci, 2008. 63(8): p. 829-34.

52. Enoka, R.M., Neuromechanics of Human Movement. Vol. 4. 2008: Human Kinetics.

53. Quittan, M., A. Sochor, G.F. Wiesinger, J. Kollmitzer, B. Sturm, R. Pacher, and W. Mayr, Strength improvement of knee extensor muscles in patients with chronic heart failure by neuromuscular electrical stimulation. Artif Organs, 1999. 23(5): p. 432-5.

54. Rodriguez, P.O., M. Setten, L.P. Maskin, I. Bonelli, S.R. Vidomlansky, S. Attie, S.L. Frosiani, S. Kozima, and R. Valentini, Muscle weakness in septic patients requiring mechanical ventilation: protective effect of transcutaneous neuromuscular electrical stimulation. J Crit Care, 2012. 27(3): p. 319 e1-8.

55. Mauro, A., Satellite cell of skeletal muscle fibers. J Biophys Biochem Cytol, 1961. 9: p. 493-5.

56. Moss, F.P. and C.P. Leblond, Nature of dividing nuclei in skeletal muscle of growing rats. J Cell Biol, 1970. 44(2): p. 459-62.

57. Moss, F.P. and C.P. Leblond, Satellite cells as the source of nuclei in muscles of growing rats. Anat Rec, 1971. 170(4): p. 421-35.

58. Verdijk, L.B., R. Koopman, G. Schaart, K. Meijer, H.H. Savelberg, and L.J. van Loon, Satellite cell content is specifically reduced in type II skeletal muscle fibers in the elderly. Am J Physiol Endocrinol Metab, 2007. 292(1): p. E151-7.

59. Verdijk, L.B., M.L. Dirks, T. Snijders, J.J. Prompers, M. Beelen, R.A. Jonkers, D.H. Thijssen, M.T. Hopman, and L.J. Van Loon, Reduced satellite cell numbers with spinal cord injury and aging in humans. Med Sci Sports Exerc, 2012. 44(12): p. 2322-30.

60. Guo, B.S., K.K. Cheung, S.S. Yeung, B.T. Zhang, and E.W. Yeung, Electrical stimulation influences satellite cell proliferation and apoptosis in unloading-induced muscle atrophy in mice. PloS One, 2012. 7(1): p. e30348. 
61. McPherron, A.C., A.M. Lawler, and S.J. Lee, Regulation of skeletal muscle mass in mice by a new TGF-beta superfamily member. Nature, 1997. 387(6628): p. 83-90.

62. Elliott, B., D. Renshaw, S. Getting, and R. Mackenzie, The central role of myostatin in skeletal muscle and whole body homeostasis. Acta Physiol (Oxf), 2012. 205(3): p. 324-40.

63. Lee, S.J. and A.C. McPherron, Regulation of myostatin activity and muscle growth. P Natl Acad Sci USA, 2001. 98(16): p. 9306-11.

64. Thomas, M., B. Langley, C. Berry, M. Sharma, S. Kirk, J. Bass, and R. Kambadur, Myostatin, a negative regulator of muscle growth, functions by inhibiting myoblast proliferation. J Biol Chem, 2000. 275(51): p. 40235-43.

65. Amthor, H., R. Huang, I. McKinnell, B. Christ, R. Kambadur, M. Sharma, and K. Patel, The regulation and action of myostatin as a negative regulator of muscle development during avian embryogenesis. Dev Biol, 2002. 251(2): p. 241-57.

66. Sabourin, L.A. and M.A. Rudnicki, The molecular regulation of myogenesis. Clin Genet, 2000. 57(1): p. 16-25.

67. Snijders, T., L.B. Verdijk, and L.J. van Loon, The impact of sarcopenia and exercise training on skeletal muscle satellite cells. Ageing Res Rev, 2009. 8(4): p. 328-38.

68. Tesch, P.A., F. von Walden, T. Gustafsson, R.M. Linnehan, and T.A. Trappe, Skeletal muscle proteolysis in response to short-term unloading in humans. J Appl Physiol, 2008. 105(3): p. 902-6.

69. Bodine, S.C., E. Latres, S. Baumhueter, V.K. Lai, L. Nunez, B.A. Clarke, W.T. Poueymirou, F.J. Panaro, E. Na, K. Dharmarajan, Z.Q. Pan, D.M. Valenzuela, T.M. DeChiara, T.N. Stitt, G.D. Yancopoulos, and D.J. Glass, Identification of ubiquitin ligases required for skeletal muscle atrophy. Science, 2001. 294(5547): p. 1704-8.

70. Gomes, M.D., S.H. Lecker, R.T. Jagoe, A. Navon, and A.L. Goldberg, Atrogin-1, a muscle-specific F-box protein highly expressed during muscle atrophy. P Natl Acad Sci USA, 2001. 98(25): p. 14440-5.

71. Murton, A.J., D. Constantin, and P.L. Greenhaff, The involvement of the ubiquitin proteasome system in human skeletal muscle remodelling and atrophy. Biochim Biophys Acta, 2008. 1782(12): p. 730-43.

72. Nair, K.S., Aging muscle. Am J Clin Nutr, 2005. 81(5): p. 953-63.

73. Fisher, S.R., Y.F. Kuo, J.E. Graham, K.J. Ottenbacher, and G.V. Ostir, Early ambulation and length of stay in older adults hospitalized for acute illness. Arch Intern Med, 2010. 170(21): p. 1942-3.

74. Khalid, M., A. Brannigan, and T. Burke, Calf muscle wasting after tibial shaft fracture. Br J Sports Med, 2006. 40(6): p. 552-3.

75. Matthews, D.R., J.P. Hosker, A.S. Rudenski, B.A. Naylor, D.F. Treacher, and R.C. Turner, Homeostasis model assessment: insulin resistance and beta-cell function from fasting plasma glucose and insulin concentrations in man. Diabetologia, 1985. 28(7): p. 412-9. 


\section{CHAPTER}

\section{Neuromuscular electrical stimulation prevents muscle wasting in critically ill, comatose patients}

Marlou L Dirks

Dominique Hansen

Aimé van Assche

Paul Dendale

Luc JC van Loon

Clin Sci (Lond) 2015 March;128(6):357-65 
ABSTRACT

Background Fully-sedated patients, being treated in the ICU, experience substantial skeletal muscle loss. Consequently, survival rate is reduced and full recovery after awakening is compromised. Neuromuscular electrical stimulation (NMES) represents an effective method to stimulate muscle protein synthesis and alleviate muscle disuse atrophy in healthy subjects.

Methods We investigated the efficacy of twice-daily NMES to alleviate muscle loss in six fully-sedated ICU patients admitted for acute critical illness ( $n=3$ males, $n=3$ females; age $63 \pm 6$ y; APACHE ॥ disease severityscore: $29 \pm 2$ ). One leg was subjected to twice-daily NMES of the quadriceps muscle for a period of $7 \pm 1 d$ while the other leg acted as nonstimulated control (CON). Directly before the first and on the morning after the final NMES session, quadriceps muscle biopsies were collected from both legs to assess muscle fibre-type specific cross-sectional area (CSA). Furthermore, phosphorylation status of key proteins involved in the regulation of muscle protein synthesis was assessed, and mRNA expression of selected genes was measured.

Results In the CON leg, type I and type II muscle fibre CSA decreased by $16 \pm 9$ and $24 \pm 7 \%$, respectively $(P<0.05)$. No muscle atrophy was observed in the stimulated leg. NMES increased mTOR phosphorylation by $19 \%$ when compared to baseline $(P<0.05)$, with no changes in the CON leg. Furthermore, mRNA expression of key genes involved in muscle protein breakdown either declined (FOXO1; $P<0.05)$ or remained unchanged (MAFBx and MuRF1), with no differences between legs.

Conclusion In conclusion, NMES represents an effective and feasible interventional strategy to prevent skeletal muscle atrophy in critically ill, comatose patients. 


\section{INTRODUCTION}

Critically ill patients suffer from extensive muscle wasting, which occurs rapidly at the onset of an ICU stay [1-3]. Aside from an increased risk of mortality [4, 5], consequences to this muscle loss include muscle weakness, prolonged mechanical ventilation, fatigue, decreases in muscle strength, impaired glucose homeostasis and delayed recovery and rehabilitation [6-9]. Muscle atrophy in ICU patients exceeds that seen in normal hospitalized or bedridden persons $[10,11]$. Moreover, ICU patients who are mechanically ventilated and deeply sedated are thought to be even more susceptible to muscle wasting and subsequent negative health consequences due to a complete lack of muscle contraction. Despite this, no data are currently available concerning muscle fibre atrophy in this specific ICU patient subpopulation.

Early ambulation has been proven a successful rehabilitation strategy in nonsedated ICU patients in terms of improving functional outcomes and overall prognosis [12]. However, in fully-sedated patients, early ambulation is not feasible and, as such, alternative strategies should be defined to alleviate muscle wasting. Neuromuscular electrical stimulation (NMES) is an effective means to invoke involuntary muscle contractions. Previously, NMES has been shown to attenuate the loss of muscle mass and strength experienced by non-sedated ICU patients [13] and healthy individuals subjected to limb immobilization [14]. However, the potential for NMES to rescue muscle mass in fully-sedated, comatose ICU patients has not been investigated. In the present study, we investigated our hypothesis that daily NMES attenuates skeletal muscle fibre atrophy in fully-sedated, comatose ICU patients. Fully-sedated ICU patients, expected to be sedated for a minimum of three days, were included in the present study. NMES was performed twice-daily on the quadriceps of one leg, whereas the other leg served as a sham-treated control. Prior to and immediately after the intervention, plasma samples were taken to assess any systemic changes in amino acid availability during the experiment, and muscle biopsies were taken from both legs to assess muscle fibre atrophy and myocellular characteristics. Additionally, RT-PCR and Western blotting were performed on collected muscle tissue samples to assess the potential impact of NMES on basal mRNA and protein expression levels of key genes involved in the regulation of muscle mass maintenance. 


\section{METHODS}

\section{Patients}

All patients admitted to the Intensive Care Unit (ICU) of Jessa Hospital, Hasselt, Belgium between March 2012 and July 2013 were assessed for eligibility for the present study (see Supplemental Figure 1). Patients admitted to the ICU were screened by the nursing staff, and were excluded if one or more of the following exclusion criteria were met: $<18$ or $>80$ y old, not expected to undergo complete sedation, suffering from spinal cord injury, recent arterial surgery on the legs, local wounds that prohibit the application of neuromuscular electrical stimulation (NMES), chronic use of corticosteroids, intake of certain antithrombotic drugs, or the presence of an implantable cardioverterdefibrillator (ICD) and/or pacemaker. Secondly, the expected sedation time was estimated by the responsible physician and patients were excluded if this was $<3$ days. All patients who were excluded based on an expected short sedation time were reevaluated after $24 \mathrm{~h}$, and included if the revised expected sedation time was $>3$ days. Participants were accepted into the study after written informed consent was obtained from their legal representatives. The study was approved by the Medical Ethical Committee of the Jessa Hospital in accordance with the Declaration of Helsinki.

\section{Study design}

An overview of the experimental protocol is depicted in Supplemental Figure 2. Patients were included in the study directly after informed consent was obtained from their legal representatives, which was generally given within 2.5 days after admission to the ICU (depicted in column 'Time to inclusion' in Table 1). After this, patient's legs were randomly assigned as either the control (CON) or stimulated (NMES) leg, counterbalanced for left and right legs. Randomization was performed by an independent investigator, and treatment allocation was performed by using sequentially labelled envelopes which were opened after inclusion of subjects. Baseline measurements were then taken, which consisted of assessment of leg circumference (measured at different locations on the upper leg), obtaining an arterial blood sample, and obtaining a muscle biopsy from both legs. After the pre-measurements, NMES was performed twice-daily on one leg (NMES) whereas the other leg served as a control (CON). Post-measurements were performed on the final day of sedation, with a minimum study duration of 3 days and a maximum of 10 days. The study duration for each patient is depicted in Table 1. Postmeasurements were performed prior to subjects being awake. Standard medical care was not altered, and passive mobilization was performed on both legs according to standard care procedures. 


\section{Data collection}

At baseline, data on demographic and clinical characteristics of the patients were obtained, including information necessary to determine the severity of illness. These data were scored according to the Acute Physiology and Chronic Health Evaluation II (APACHE II) system with higher values indicating more severe illness and more therapeutic interventions, respectively [15]. Arterial blood samples were collected from the catheter already placed in the arteria radialis. Blood $(10 \mathrm{~mL})$ was collected into EDTAcontaining tubes and immediately centrifuged at $1,000 \mathrm{~g}$ for $10 \mathrm{~min}$ at $4^{\circ} \mathrm{C}$. Aliquots of plasma were directly snap-frozen in liquid nitrogen and stored at $-80^{\circ} \mathrm{C}$ until further analysis. Processing and storage of the samples was done by UBiLim (Universitaire Biobank Limburg, Hasselt, Belgium). Plasma amino acid concentrations were measured using ultra-performance liquid chromatography tandem mass spectrometry as described previously [16], and results are displayed in Supplemental Table 2.

In addition, during the pre- and post-measurements, a muscle biopsy sample was collected from each leg. After injection of local anesthesia, percutaneous needle biopsy samples were collected from $m$. vastus lateralis, approximately $15 \mathrm{~cm}$ above the patella using the Bergström technique [17]. 


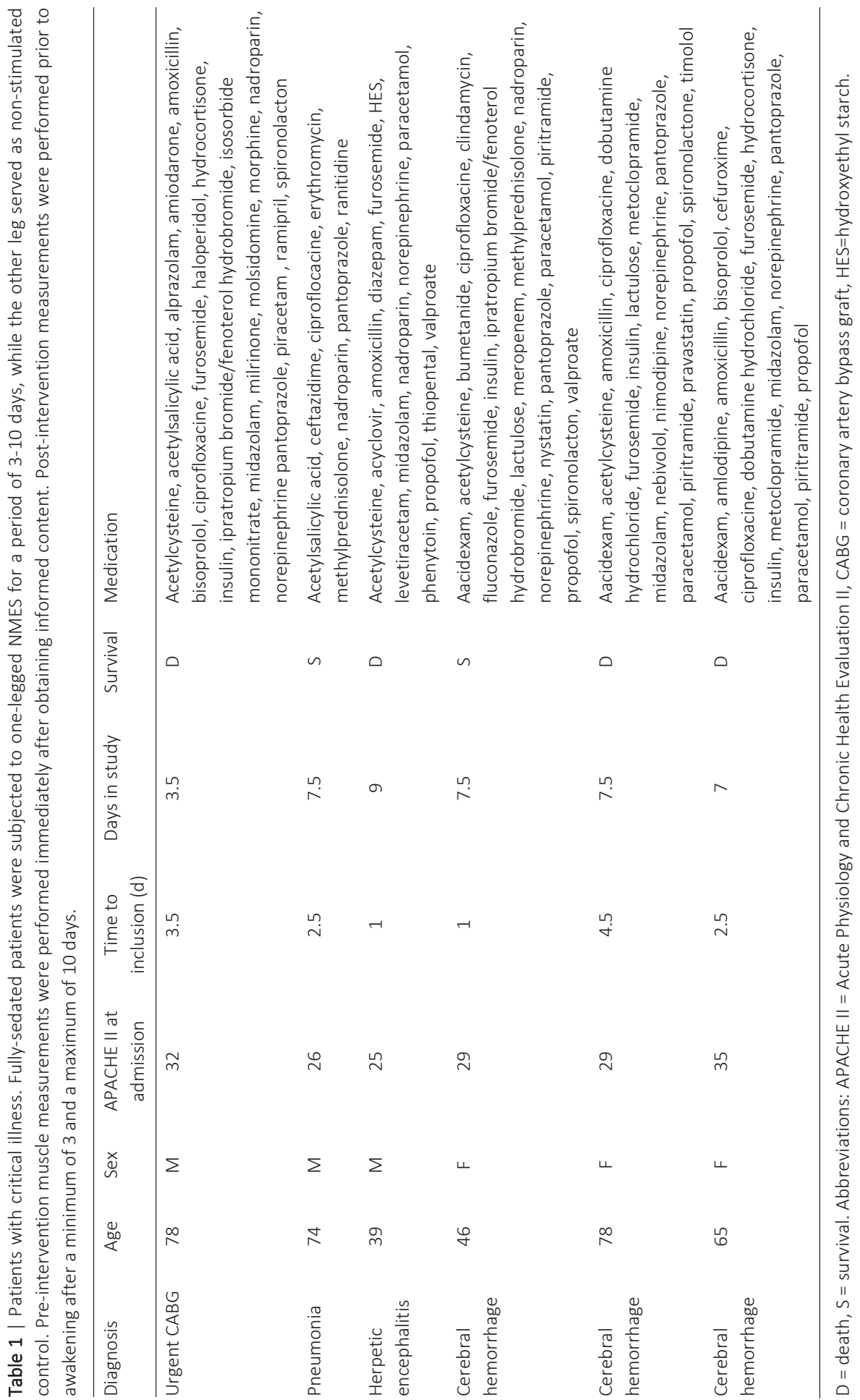




\section{Neuromuscular electrical stimulation}

Neuromuscular electrical stimulation sessions were performed both in the morning (11:00 AM) and afternoon (4.30 PM). Four self-adhesive electrodes ( $2 \mathrm{~mm}$ thick, $50 \times 50$ $\mathrm{mm}$ ) were placed on the distal part at the muscle belly of the $\mathrm{m}$. rectus femoris and the $m$. vastus lateralis, and at the inguinal area of both muscles. The electrodes were connected to an Enraf-Nonius TensMed S84 stimulation device (Enraf-Nonius, Rotterdam, the Netherlands), discharging biphasic symmetric rectangular-wave pulses. The position of the electrodes was re-marked daily with a semi-permanent marker to maintain the same location of stimulation for each session. The NMES protocol was composed of a warm-up phase ( $5 \mathrm{~min}, 5 \mathrm{~Hz}, 250 \mu \mathrm{s}$ ), a stimulation period ( $30 \mathrm{~min}, 100 \mathrm{~Hz}, 400 \mu \mathrm{s}, 5 \mathrm{~s}$ on ( $0.75 \mathrm{~s}$ rise, $3.5 \mathrm{~s}$ contraction, $0.75 \mathrm{~s}$ fall) and $10 \mathrm{~s}$ off), and a cooling-down phase ( 5 $\min , 5 \mathrm{~Hz}, 250 \mu \mathrm{s})$. The intensity of the stimulation was set to a level at which full contractions of $\mathrm{m}$. quadriceps femoris were both visible and palpable. The intensity was raised approximately every $3 \mathrm{~min}$ when a full muscle contraction was no longer achieved with the current intensity. This protocol was based on our previous work showing increased rates of muscle protein synthesis after a single bout of NMES [18], and applied on the immobilized leg of healthy young adults [14]. During the NMES sessions, four electrodes and compatible cables were also applied to the control leg to standardize all procedures (representing a sham treatment).

\section{Dietary intake}

When patients were hemodynamically stable, enteral feeding was started according to routine guidelines of the ICU at Jessa Hospital as early as possible. Patients were fed Nutrison Multi Fibre (containing 420 kJ, 16 En\% protein, 49 En\% carbohydrates, and 35 En\% fat per $100 \mathrm{~mL}$ ). Generally, patients were fed maximally $80 \mathrm{~mL}$ per hour with short intervals during which nutritional supply was paused. Gastric emptying was determined by the nursing staff, and food administration was altered accordingly. Nutritional support was not modulated and was applied according to the standard medical care in this ICU.

\section{Muscle analyses}

Muscle samples were freed from any visible non-muscle tissue and separated into different sections; the first part ( $\sim 30 \mathrm{mg}$ ) was imbedded in Tissue-Tek (Sakura Finetek, Zoeterwoude, the Netherlands), frozen on liquid nitrogen cooled isopentane and used to determine muscle fibre-type specific cross-sectional area (CSA) and satellite cell content as done previously [19]. The second part ( 15 mg) was snap frozen in liquid nitrogen and used for real time-PCR analysis to determine mRNA expression of selected genes as described before $[14,20]$, and compared with mRNA expression of $n=6$ healthy, age- and gender-matched controls. The third part ( $\sim 40 \mathrm{mg}$ ) was snap frozen in liquid nitrogen for Western Blot analysis to determine the total content and phosphory- 
lation status of several key proteins of interest as described previously [18]. All muscle analyses were performed by an investigator blinded to treatment.

\section{Statistics}

Based on data from previous studies in healthy subjects in our laboratory $[14,21]$, we calculated that 8 patients would be required to detect a $8 \%$ difference in muscle fibre CSA between CON and NMES over 7 days (using an $\alpha$ level of 0.05 and a $\beta$ level of 0.10). All data presented are expressed as means \pm SEM. Baseline differences between legs were compared with a paired samples t-test. Pre- and post-intervention data were analyzed using repeated measures analysis of variance (ANOVA) with time (pre vs post) and treatment (CON vs NMES) as factors. Fibre type (type I vs type II) was added as a third within-subjects factor when analyzing all muscle fibre characteristics. In case of significant interaction (time $x$ treatment), paired-samples t-tests were performed to determine time effects within the CON and NMES leg separately. Alternatively, when a time $x$ treatment effect was observed for muscle fibre characteristics, a 2-way ANOVA was performed for the CON and NMES leg separately, with time and treatment as factors. For the mRNA analyses, differences between patients and healthy controls were tested by means of an independent samples t-test between the mean value of the CON and NMES leg in patients and the values observed in healthy controls. Statistical analyses were performed using the SPSS version 20.0 software package (SPSS Inc., Chicago, IL, USA), with $P<0.05$ as the value for statistical significance. 


\section{RESULTS}

\section{Patients}

Between March 2012 and July 2013, 9 patients were included in the present study. Two patients awoke after $<3$ study days and one patient died. Therefore, the presented results represent data collected from 6 patients. Clinical characteristics of the included patients are listed in Table 1. Energy intake per day averaged 5.31 $\pm 0.56 \mathrm{MJ}$, with a mean protein intake of $0.56 \pm 0.06 \mathrm{~g} \cdot \mathrm{kg}$ body weight ${ }^{-1} \cdot$ day $^{-1}$.

\section{Neuromuscular electrical stimulation}

Within 5 min of the start of the actual 30 min stimulation period, a full muscle contraction was achieved. The intensity of the NMES intervention for subjects averaged $29.9 \mathrm{~mA}$ during the first session and was progressively increased to $32.3 \mathrm{~mA}$ in the final session.

\section{Muscle fibre characteristics}

Figure 1 illustrates the delta change in muscle fibre cross-sectional area (CSA) in both the NMES and CON legs throughout the study.

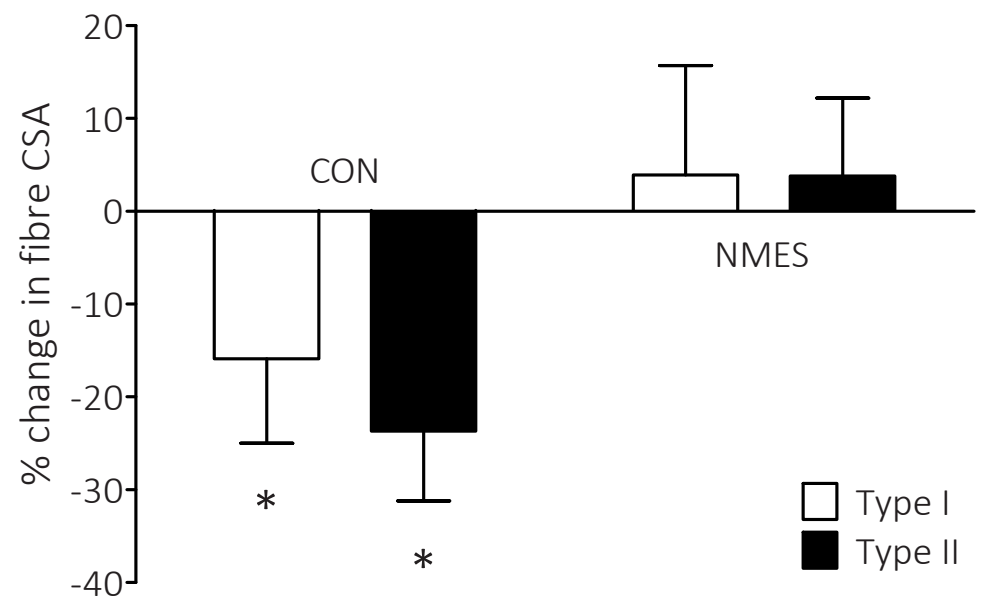

Figure 1 | Changes in muscle fibre cross-sectional area (CSA) in the control (CON) and stimulated (NMES) leg of sedated patients, after $7 \pm 1$ days of twice-daily NMES. A significant interaction effect $(P<0.05)$ was observed, and a time effect in the CON leg $(P<0.05)$. * Significantly different from zero $(P<0.05)$.

Table 2 details skeletal muscle fibre type specific characteristics at baseline and following $7 \pm 1 \mathrm{~d}$ of full sedation in both legs. In the CON leg, a significant decline of $16 \pm 9 \%$ and $24 \pm 7 \%$ was observed in type I and II muscle fibre CSA, respectively (time effect; $P<0.05$ ). In contrast, the NMES leg showed no atrophy in either type I or II muscle fibres (time $x$ 
treatment interaction effect; $P<0.05)$. Muscle fibre type distribution showed an overall significant time $\mathrm{x}$ treatment interaction effect (see Table 2; $P<0.05$ ), with a shift from type I towards type II fibres in the CON leg, and a shift towards more type I fibres in the NMES leg. At baseline, satellite cell content was greater in type I vs type II muscle fibres (expressed per muscle fibre, per millimeter squared, and as a percentage of total myonuclei). No differences in muscle fibre type specific myonuclear content, myonuclear domain size or satellite cell content were observed between legs or over time.

Table 2 | Muscle fibre characteristics

\begin{tabular}{|c|c|c|c|c|c|}
\hline & & \multicolumn{2}{|c|}{ CON } & \multicolumn{2}{|c|}{ NMES } \\
\hline & $\begin{array}{l}\text { Fibre } \\
\text { type }\end{array}$ & Pre & Post & Pre & Post \\
\hline \multirow[t]{2}{*}{ Muscle fibre CSA $\left(\mu \mathrm{m}^{2}\right)$} & I & $4560 \pm 261$ & $3879 \pm 484^{*}$ & $4414 \pm 441$ & $4512 \pm 550$ \\
\hline & II & $3412 \pm 530$ & $2647 \pm 512 *$ & $3168 \pm 607$ & $3246 \pm 590$ \\
\hline \multirow[t]{2}{*}{$\%$ Fibre } & 1 & $53 \pm 8+$ & $45 \pm 5+$ & $42 \pm 6+$ & $46 \pm 6+$ \\
\hline & $\|$ & $47 \pm 8+$ & $55 \pm 5+$ & $58 \pm 6+$ & $54 \pm 6+$ \\
\hline \multirow[t]{2}{*}{$\%$ Fibre area } & 1 & $59 \pm 9$ & $56 \pm 8$ & $51 \pm 8$ & $55 \pm 7$ \\
\hline & ॥ & $41 \pm 9$ & $44 \pm 8$ & $49 \pm 8$ & $45 \pm 7$ \\
\hline \multirow[t]{2}{*}{ Nuclei per fibre } & I & $2.4 \pm 0.1$ & $2.5 \pm 0.2$ & $2.3 \pm 0.2$ & $2.6 \pm 0.2$ \\
\hline & $\|$ & $2.1 \pm 0.2 \#$ & $2.1 \pm 0.2 \#$ & $1.9 \pm 0.2 \#$ & $2.3 \pm 0.3 \#$ \\
\hline \multirow[t]{2}{*}{ Myonuclear domain $\left(\mu \mathrm{m}^{2}\right)$} & । & $1853 \pm 69$ & $1574 \pm 183$ & $1931 \pm 110$ & $1760 \pm 142$ \\
\hline & ॥ & $1573 \pm 162 \#$ & $1255 \pm 203 \#$ & $1618 \pm 153 \#$ & $1452 \pm 220 \#$ \\
\hline \multirow[t]{2}{*}{ Number of SCs per fibre } & । & $0.083 \pm 0.014$ & $0.085 \pm 0.012$ & $0.075 \pm 0.006$ & $0.092 \pm 0.006$ \\
\hline & $\|$ & $0.061 \pm 0.015 \#$ & $0.049 \pm 0.013 \#$ & $0.048 \pm 0.008 \#$ & $0.055 \pm 0.009 \#$ \\
\hline \multirow[t]{2}{*}{ Number of SCs per $\mathrm{mm}^{2}$} & 1 & $18.8 \pm 2.9$ & $22.7 \pm 2.7$ & $17.6 \pm 1.7$ & $22.1 \pm 4.1$ \\
\hline & ॥ & $18.6 \pm 2.9 \#$ & $17.8 \pm 2.0 \#$ & $17.0 \pm 3.9 \#$ & $19.8 \pm 4.6 \#$ \\
\hline \multirow[t]{2}{*}{ SCs/myonuclei (\%) } & । & $3.4 \pm 0.5$ & $3.5 \pm 0.4$ & $3.4 \pm 0.4$ & $3.7 \pm 0.4$ \\
\hline & II & $2.7 \pm 0.4 \#$ & $2.3 \pm 0.5 \#$ & $2.5 \pm 0.3 \#$ & $2.5 \pm 0.3 \#$ \\
\hline
\end{tabular}

Data represent means \pm SEM. Abbreviations: SC, satellite cell; SCs/myonuclei (\%), the number of SCS as a percentage of the total number of myonuclei (i.e. number of myonuclei + number of SCs). * Significantly different from pre-intervention value $(P<0.05)$. \# Significantly different from type I fibre value $(P<0.05)$. $\dagger$ Significant treatment*time*fibre type interaction effect $(P<0.05)$

\section{mRNA expression}

Figure 2 displays the relative muscle mRNA expression of key genes involved in the regulation of muscle protein synthesis and breakdown in the CON and NMES leg before and after the intervention, as well as for a group of healthy, age- and gender-matched controls. 

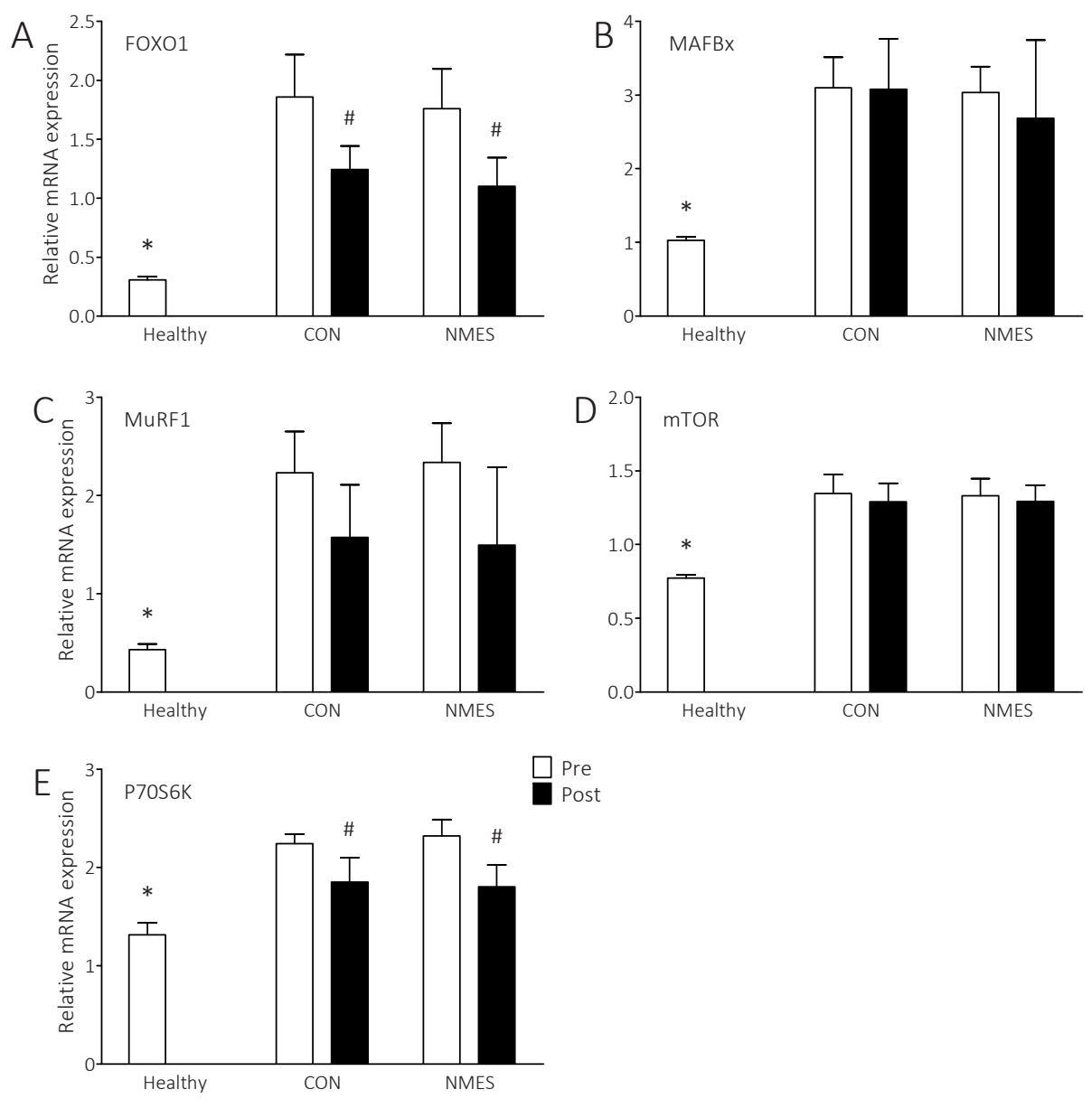

Figure 2 | Skeletal muscle mRNA expression of genes of interest. Abbreviations: FOXO1, Forkhead box protein O1; MAFbx, Muscle Atrophy F-box; MuRF1, Muscle RING-finger protein-1; mTOR, mammalian target of rapamycin; P70S6K, P70S6 kinase. * Significantly different from patients at baseline $(P<0.05)$. \# Significantly different from pre-value $(P<0.05)$.

At baseline, mRNA expression did not differ between NMES and CON legs. However, MAFBx, MURF1, FOXO1, mTOR and P70S6K were all more highly expressed in the patients compared with healthy controls $(P<0.01)$. There was a significant time effect $(P<0.05)$ such that FOXO1 and P70S6K expression decreased during the period of sedation, with no differences between legs. Expression levels for all other genes did not reveal any interaction or time effects. The mRNA expression of additional genes involved in the regulation of myogenesis, oxidative metabolism, mechano-sensing and cellular amino acid transport are presented in Supplemental Figure 3. 


\section{Signaling proteins}

The skeletal muscle content and phosphorylation status of key proteins involved in the regulation of muscle protein synthesis are displayed in Figure 3.
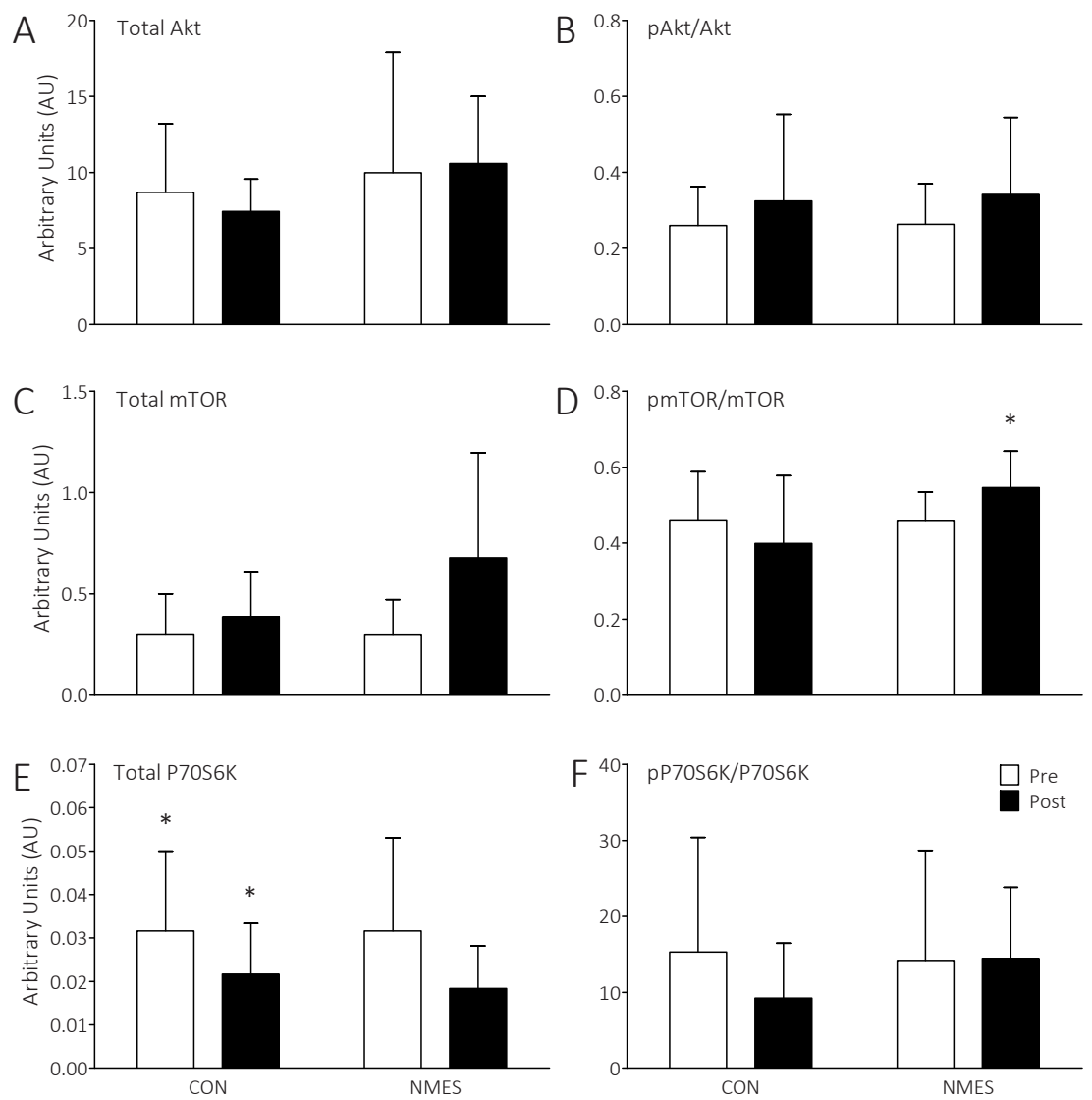

Figure 3 | Skeletal muscle protein expression of Akt, mTOR and P70S6K in the control (CON) and stimulated (NMES) leg, before (white bars) and after (black bars) $7 \pm 1$ days of twice-daily NMES. Left graphs: total protein expression, right graphs: phosphorylated/total expression. Abbreviations: mTOR, mammalian target of rapamycin; P70S6K, P70S6 kinase. * Significantly different from pre-intervention values $(P<0.05)$.

Neither total protein content, nor phosphorylation status of Akt was affected by time or the intervention (both $P>0.05$ ). Whereas muscle mTOR content was unaffected by time or treatment, a significant time $x$ treatment interaction effect $(P<0.05)$ was found for the phosphorylation status of mTOR. mTOR phosphorylation increased by as much as $19 \pm 5 \%$ in the NMES leg $(P<0.05)$, with no changes in the CON leg $(P>0.05)$. Muscle P70S6K total protein content decreased following the intervention in both legs (time effect, $P<0.05)$, without changes in phosphorylation status $(P>0.05)$. 


\section{DISCUSSION}

In the present study, we demonstrate for the first time that fully-sedated patients experience substantial type I and type II muscle fibre atrophy during a $\sim 7$ stay in the ICU. Daily application of neuromuscular electrical stimulation (NMES) effectively prevents skeletal muscle fibre atrophy, offering an effective and feasible interventional strategy to alleviate muscle wasting in comatose ICU patients.

General admission to the ICU has been shown to cause substantial muscle wasting [22] with a decline in type I and type II muscle fibre cross-sectional area of 3 and $4 \%$ per day, respectively [2]. In keeping with this, we show a 2.8 and $4.4 \%$ decline in muscle fibre size in type I and II muscle fibres, respectively, in fully-sedated patients (i.e. no possibility of voluntary muscle contraction) during on average 7 days in the ICU (Figure 1). By way of comparison, muscle atrophy brought about by disuse only in healthy humans (i.e. limb immobilization) leads to a 0.5 and $0.9 \%$ per day decline in type I and II muscle fibre cross-sectional area (CSA), respectively [21]. This implies that the mechanisms responsible for muscle wasting in the ICU are not simply attributed to disuse. One possible contributing factor could be inadequate nutritional status. Sufficient dietary protein is considered a key factor in the maintenance of muscle mass [23-25], and previous research has shown that sufficient protein intake is associated with reduced mortality rates in critically ill patients $[26,27]$. In the current study, patients received $0.56 \pm 0.06 \mathrm{~g}$ protein $\cdot \mathrm{kg}$ body weight ${ }^{-1} \cdot \mathrm{day}^{-1}$, which is below the current guidelines of 1.3 $2.0 \mathrm{~g}$ protein $\cdot \mathrm{kg}$ body weight ${ }^{-1} \cdot \mathrm{day}^{-1}$ recommended during critical illness $[28,29]$, and has likely contributed to the extensive level of muscle wasting. In support, plasma amino acid concentrations in our patients declined throughout the sedated state (Supplemental Table 2). In agreement, previous work has reported declines in circulating amino acid concentrations during critical illness [30]. Such a decline in circulating amino acid concentrations likely reduces amino acid uptake in muscle [31] and, as such, could modulate the efficacy of NMES as a means to stimulate muscle protein synthesis rates.

From a mechanistic viewpoint, disuse atrophy has been primarily attributed to declines in muscle protein synthesis rates [20,32-34]. However, it has been suggested that in various conditions associated with rapid muscle wasting a multitude of other factors (e.g. increased inflammation, higher metabolic stress responses etc.) may stimulate muscle proteolysis, driving much of the muscle loss [35]. In line with this, we see evidence of the severely metabolically compromised condition of our patients as demonstrated by numerous clinical chemistry indictors obtained throughout the study (e.g. high white blood cell counts and C-reactive protein (CRP) concentrations; Supplemental Table 1). In keeping with this, molecular markers that have been used as a proxy for changes in muscle protein breakdown rate were elevated upon admission to the ICU, when compared with a group of healthy subjects (i.e. MAFBx, MuRF1 and FOXO1; Figure 2). The subsequent decline in the expression levels of these genes suggest a decline in muscle protein turnover during hospital stay but expression levels remained elevated 
when compared to healthy controls. This is not unexpected given the metabolic stress response upon ICU admission [36]. In contrast to previous work investigating the impact of NMES on an immobilized leg [14], we observed no significant differences in the expression levels of various genes between the stimulated and unstimulated leg in this comatose ICU setting. The absence of such differences may be attributed to various factors, but underline our understanding that changes in the expression and phosphorylation levels of various genes being used as a proxy for changes in muscle protein breakdown and synthesis do not necessarily represent changes in muscle protein breakdown and synthesis rates and do not necessarily translate to a net increase or decrease in muscle mass [37]. Taken together, the present data highlight the need for immediate and effective intervention at the onset of ICU admission to stimulate muscle protein synthesis and inhibit proteolysis, thereby preventing or attenuating extensive muscle wasting. An interesting observation in the stimulated leg was that NMES reversed the decline in phosphorylation status of mTOR (Figure 3D), which seems to be in line with previous work showing that NMES increases muscle protein synthesis rates [18].

Daily application of NMES has been shown to prevent muscle atrophy in healthy subjects during a week of leg immobilization [14]. Moreover, clinical trials have demonstrated beneficial effects of NMES on muscle function in various bed-rested populations, including patients suffering from $\operatorname{COPD}[38,39]$ and sepsis $[40,41]$. The current study demonstrates, for the first time, that NMES is capable of preventing muscle wasting in fully-sedated patients during 7 days in the ICU (with a $+7 \pm 12 \%$ change in mixed muscle fibre CSA in the stimulated leg compared with a $-21 \pm 8 \%$ decline in mixed muscle fibre CSA in the control leg; Figure 1). The prevention of muscle atrophy in these individuals can have profound clinical implications. For instance, maintaining muscle mass during critical illness has been shown to reduce mortality rates [4, 5]. Additionally, since muscle mass is vital for functional capacity [42], metabolic homeostasis [9], and immune function [43], maintaining muscle mass during an ICU stay is essential to allow proper recovery during rehabilitation. As such, preventing muscle wasting is imperative for promoting quality of life after hospital discharge and reducing the likelihood of rehospitalization. NMES in fully-sedated patients can be easily applied by nursing staff, is relatively cheap and does not seem to cause any adverse effects on vital parameters during or after the sessions [44]. Some difficulties applying NMES in ICU patients have been reported previously and are likely due to increased skin/soft tissue impedance and/or edema [13]. Despite experiencing similar problems in the present study, all NMES sessions could be successfully performed without any adverse effects. Taken together, our data demonstrate that NMES is practical and feasible as a countermeasure for muscle wasting in clinically compromised ICU patients. Future studies should address whether these findings would translate into longer-term benefits such as increased survival rates, reduced hospitalization length of stay and/or improved rehabilitation outcomes. 


\section{CONCLUSION}

NMES represents an effective and feasible interventional strategy to prevent skeletal muscle wasting in critically ill, comatose patients.

\section{ACKNOWLEDGEMENTS}

We gratefully acknowledge the enthusiasm and assistance of the physicians and nursing staff of the ICU in Jessa Hospital. We would also like to thank Marika Leenders and Lex B Verdijk for their practical support and Benjamin T Wall for his assistance in drafting the manuscript. Furthermore, we are thankful for the assistance of dr. E. Bijnens, Department of Radiology at Jessa Hospital, and for the support and assistance provided by Biobank UbiLim at Jessa Hospital for processing and storage of the samples. 


\section{REFERENCES}

1. Monk, D.N., L.D. Plank, G. Franch-Arcas, P.J. Finn, S.J. Streat, and G.L. Hill, Sequential changes in the metabolic response in critically injured patients during the first 25 days after blunt trauma. Ann Surg, 1996. 223(4): p. 395-405.

2. Helliwell, T.R., A. Wilkinson, R.D. Griffiths, P. McClelland, T.E. Palmer, and J.M. Bone, Muscle fibre atrophy in critically ill patients is associated with the loss of myosin filaments and the presence of lysosomal enzymes and ubiquitin. Neuropath Appl Neuro, 1998. 24(6): p. 507-17.

3. Latronico, N. and C.F. Bolton, Critical illness polyneuropathy and myopathy: a major cause of muscle weakness and paralysis. Lancet Neurol, 2011. 10(10): p. 931-41.

4. Weijs, P.J., W.G. Looijaard, I.M. Dekker, S.N. Stapel, A.R. Girbes, H.M. Oudemans-van Straaten, and A. Beishuizen, Low skeletal muscle area is a risk factor for mortality in mechanically ventilated critically ill patients. Crit Care, 2014. 18(1): p. R12.

5. Moisey, L.L., M. Mourtzakis, B.A. Cotton, T. Premji, D.K. Heyland, C.E. Wade, E. Bulger, R.A. Kozar, N. for the, and C. Rehabilitation Investigators, Skeletal muscle predicts ventilator-free days, ICU-free days, and mortality in elderly ICU patients. Crit Care, 2013. 17(5): p. R206.

6. Levine, S., T. Nguyen, N. Taylor, M.E. Friscia, M.T. Budak, P. Rothenberg, J. Zhu, R. Sachdeva, S. Sonnad, L.R. Kaiser, N.A. Rubinstein, S.K. Powers, and J.B. Shrager, Rapid disuse atrophy of diaphragm fibers in mechanically ventilated humans. N Engl J Med, 2008. 358(13): p. 1327-35.

7. Herridge, M.S., Legacy of intensive care unit-acquired weakness. Crit Care Med, 2009. 37(10 Suppl): p. S457-61.

8. Truong, A.D., E. Fan, R.G. Brower, and D.M. Needham, Bench-to-bedside review: mobilizing patients in the intensive care unit--from pathophysiology to clinical trials. Crit Care, 2009. 13(4): p. 216.

9. Nair, K.S., Aging muscle. Am J Clin Nutr, 2005. 81(5): p. 953-63.

10. Puthucheary, Z.A., J. Rawal, M. McPhail, B. Connolly, G. Ratnayake, P. Chan, N.S. Hopkinson, R. Padhke, T. Dew, P.S. Sidhu, C. Velloso, J. Seymour, C.C. Agley, A. Selby, M. Limb, L.M. Edwards, K. Smith, A. Rowlerson, M.J. Rennie, J. Moxham, S.D. Harridge, N. Hart, and H.E. Montgomery, Acute skeletal muscle wasting in critical illness. J Am Med Assoc, 2013. 310(15): p. 1591-600.

11. Wall, B.T. and L.J. van Loon, Nutritional strategies to attenuate muscle disuse atrophy. Nutr Rev, 2013. 71(4): p. 195-208.

12. Schweickert, W.D., M.C. Pohlman, A.S. Pohlman, C. Nigos, A.J. Pawlik, C.L. Esbrook, L. Spears, M. Miller, M. Franczyk, D. Deprizio, G.A. Schmidt, A. Bowman, R. Barr, K.E. McCallister, J.B. Hall, and J.P. Kress, Early physical and occupational therapy in mechanically ventilated, critically ill patients: a randomised controlled trial. Lancet, 2009. 373(9678): p. 1874-82.

13. Rodriguez, P.O., M. Setten, L.P. Maskin, I. Bonelli, S.R. Vidomlansky, S. Attie, S.L. Frosiani, S. Kozima, and R. Valentini, Muscle weakness in septic patients requiring mechanical ventilation: protective effect of transcutaneous neuromuscular electrical stimulation. J Crit Care, 2012. 27(3): p. 319 e1-8.

14. Dirks, M.L., B.T. Wall, T. Snijders, C.L. Ottenbros, L.B. Verdijk, and L.J. van Loon, Neuromuscular electrical stimulation prevents muscle disuse atrophy during leg immobilization in humans. Acta Physiol (Oxf), 2014. 210(3): p. 628-41.

15. Knaus, W.A., E.A. Draper, D.P. Wagner, and J.E. Zimmerman, APACHE II: a severity of disease classification system. Crit Care Med, 1985. 13(10): p. 818-29.

16. Waterval, W.A., J.L. Scheijen, M.M. Ortmans-Ploemen, C.D. Habets-van der Poel, and J. Bierau, Quantitative UPLC-MS/MS analysis of underivatised amino acids in body fluids is a reliable tool for the diagnosis and follow-up of patients with inborn errors of metabolism. Clin Chim Acta, 2009. 407(1-2): p. 36-42.

17. Bergstrom, J., Percutaneous needle biopsy of skeletal muscle in physiological and clinical research. Scand J Clin Lab Invest, 1975. 35(7): p. 609-16.

18. Wall, B.T., M.L. Dirks, L.B. Verdijk, T. Snijders, D. Hansen, P. Vranckx, N.A. Burd, P. Dendale, and L.J. van Loon, Neuromuscular electrical stimulation increases muscle protein synthesis in elderly type 2 diabetic men. Am J Physiol Endocrinol Metab, 2012. 303(5): p. E614-23. 
19. Leenders, M., L.B. Verdijk, L. van der Hoeven, J. van Kranenburg, R. Nilwik, and L.J. van Loon, Elderly men and women benefit equally from prolonged resistance-type exercise training. J Gerontol A Biol Sci Med Sci, 2013. 68(7): p. 769-79.

20. Wall, B.T., T. Snijders, J.M. Senden, C.L. Ottenbros, A.P. Gijsen, L.B. Verdijk, and L.J. van Loon, Disuse impairs the muscle protein synthetic response to protein ingestion in healthy men. J Clin Endocrinol Metab, 2013. 98(12): p. 4872-81.

21. Snijders, T., B.T. Wall, M.L. Dirks, J.M. Senden, F. Hartgens, J. Dolmans, M. Losen, L.B. Verdijk, and L.J. van Loon, Muscle disuse atrophy is not accompanied by changes in skeletal muscle satellite cell content. Clin Sci (Lond), 2014. 126(8): p. 557-66.

22. Reid, C.L., I.T. Campbell, and R.A. Little, Muscle wasting and energy balance in critical illness. Clin Nutr, 2004. 23(2): p. 273-80.

23. Paddon-Jones, D., M. Sheffield-Moore, X.J. Zhang, E. Volpi, S.E. Wolf, A. Aarsland, A.A. Ferrando, and R.R. Wolfe, Amino acid ingestion improves muscle protein synthesis in the young and elderly. Am J Physiol Endocrinol Metab, 2004. 286(3): p. E321-8.

24. Rennie, M.J., R.H. Edwards, D. Halliday, D.E. Matthews, S.L. Wolman, and D.J. Millward, Muscle protein synthesis measured by stable isotope techniques in man: the effects of feeding and fasting. Clin Sci (Lond), 1982. 63(6): p. 519-23.

25. Volpi, E., B. Mittendorfer, S.E. Wolf, and R.R. Wolfe, Oral amino acids stimulate muscle protein anabolism in the elderly despite higher first-pass splanchnic extraction. Am J Physiol, 1999. 277(3 Pt 1): p. E513-20.

26. Strack van Schijndel, R.J., P.J. Weijs, R.H. Koopmans, H.P. Sauerwein, A. Beishuizen, and A.R. Girbes, Optimal nutrition during the period of mechanical ventilation decreases mortality in critically ill, longterm acute female patients: a prospective observational cohort study. Crit Care, 2009. 13(4): p. R132.

27. Weijs, P.J., S.N. Stapel, S.D. de Groot, R.H. Driessen, E. de Jong, A.R. Girbes, R.J. Strack van Schijndel, and A. Beishuizen, Optimal protein and energy nutrition decreases mortality in mechanically ventilated, critically ill patients: a prospective observational cohort study. JPEN J Parenter Enteral Nutr, 2012. 36(1): p. 60-8.

28. Martindale, R.G., S.A. McClave, V.W. Vanek, M. McCarthy, P. Roberts, B. Taylor, J.B. Ochoa, L. Napolitano, G. Cresci, M. American College of Critical Care, and A.S.P.E.N.B.o. Directors, Guidelines for the provision and assessment of nutrition support therapy in the adult critically ill patient: Society of Critical Care Medicine and American Society for Parenteral and Enteral Nutrition: Executive Summary. Crit Care Med, 2009. 37(5): p. 1757-61.

29. Singer, P., M.M. Berger, G. Van den Berghe, G. Biolo, P. Calder, A. Forbes, R. Griffiths, G. Kreyman, X. Leverve, C. Pichard, and Espen, ESPEN Guidelines on Parenteral Nutrition: intensive care. Clin Nutr, 2009. 28(4): p. 387-400.

30. Jespersen, J.G., A. Nedergaard, S. Reitelseder, U.R. Mikkelsen, K.J. Dideriksen, J. Agergaard, F. Kreiner, F.C. Pott, P. Schjerling, and M. Kjaer, Activated protein synthesis and suppressed protein breakdown signaling in skeletal muscle of critically ill patients. PLoS One, 2011. 6(3): p. e18090.

31. Pennings, B., B. Groen, A. de Lange, A.P. Gijsen, A.H. Zorenc, J.M. Senden, and L.J. van Loon, Amino acid absorption and subsequent muscle protein accretion following graded intakes of whey protein in elderly men. Am J Physiol Endocrinol Metab, 2012. 302(8): p. E992-9.

32. Biolo, G., B. Ciocchi, M. Lebenstedt, R. Barazzoni, M. Zanetti, P. Platen, M. Heer, and G. Guarnieri, Shortterm bed rest impairs amino acid-induced protein anabolism in humans. J Physiol, 2004. 558(Pt 2): p. 381-8.

33. Biolo, G., B. Ciocchi, M. Lebenstedt, M. Heer, and G. Guarnieri, Sensitivity of whole body protein synthesis to amino acid administration during short-term bed rest. J Gravit Physiol, 2002. 9(1): p. P197-8.

34. Glover, E.I., S.M. Phillips, B.R. Oates, J.E. Tang, M.A. Tarnopolsky, A. Selby, K. Smith, and M.J. Rennie, Immobilization induces anabolic resistance in human myofibrillar protein synthesis with low and high dose amino acid infusion. J Physiol, 2008. 586(Pt 24): p. 6049-61.

35. Egerman, M.A. and D.J. Glass, Signaling pathways controlling skeletal muscle mass. Crit Rev Biochem Mol Biol, 2014. 49(1): p. 59-68.

36. Constantin, D., J. McCullough, R.P. Mahajan, and P.L. Greenhaff, Novel events in the molecular regulation of muscle mass in critically ill patients. J Physiol, 2011. 589(Pt 15): p. 3883-95. 
37. Greenhaff, P.L., L.G. Karagounis, N. Peirce, E.J. Simpson, M. Hazell, R. Layfield, H. Wackerhage, K. Smith, P. Atherton, A. Selby, and M.J. Rennie, Disassociation between the effects of amino acids and insulin on signaling, ubiquitin ligases, and protein turnover in human muscle. Am J Physiol Endocrinol Metab, 2008. 295(3): p. E595-604.

38. Abdellaoui, A., C. Prefaut, F. Gouzi, A. Couillard, M. Coisy-Quivy, G. Hugon, N. Molinari, T. Lafontaine, O. Jonquet, D. Laoudj-Chenivesse, and M. Hayot, Skeletal muscle effects of electrostimulation after COPD exacerbation: a pilot study. Eur Respir J, 2011. 38(4): p. 781-8.

39. Zanotti, E., G. Felicetti, M. Maini, and C. Fracchia, Peripheral muscle strength training in bed-bound patients with COPD receiving mechanical ventilation: effect of electrical stimulation. Chest, 2003. 124(1): p. 292-6.

40. Poulsen, J.B., K. Moller, C.V. Jensen, S. Weisdorf, H. Kehlet, and A. Perner, Effect of transcutaneous electrical muscle stimulation on muscle volume in patients with septic shock. Crit Care Med, 2011. 39(3): p. 456-61.

41. Rodriguez, P.O., M. Setten, L.P. Maskin, I. Bonelli, S.R. Vidomlansky, S. Attie, S.L. Frosiani, S. Kozima, and R. Valentini, Muscle weakness in septic patients requiring mechanical ventilation: Protective effect of transcutaneous neuromuscular electrical stimulation. J Crit Care, 2011.

42. Maughan, R.J., J.S. Watson, and J. Weir, Strength and cross-sectional area of human skeletal muscle. J Physiol, 1983. 338: p. 37-49.

43. Nielsen, S. and B.K. Pedersen, Skeletal muscle as an immunogenic organ. Curr Opin Pharmacol, 2008. 8(3): p. 346-51.

44. Meesen, R.L.J., P. Dendale, K. Cuypers, J. Berger, A. Hermans, H. Thijs, and O. Levin, Neuromuscular Electrical Stimulation as a Possible Means to Prevent Muscle Tissue Wasting in Artificially Ventilated and Sedated Patients in the Intensive Care Unit: A Pilot Study. Neuromodulation, 2010. 13: p. 315-321. 
SUPPLEMENTAL MATERIAL

SUPPLEMENTAL METHODS

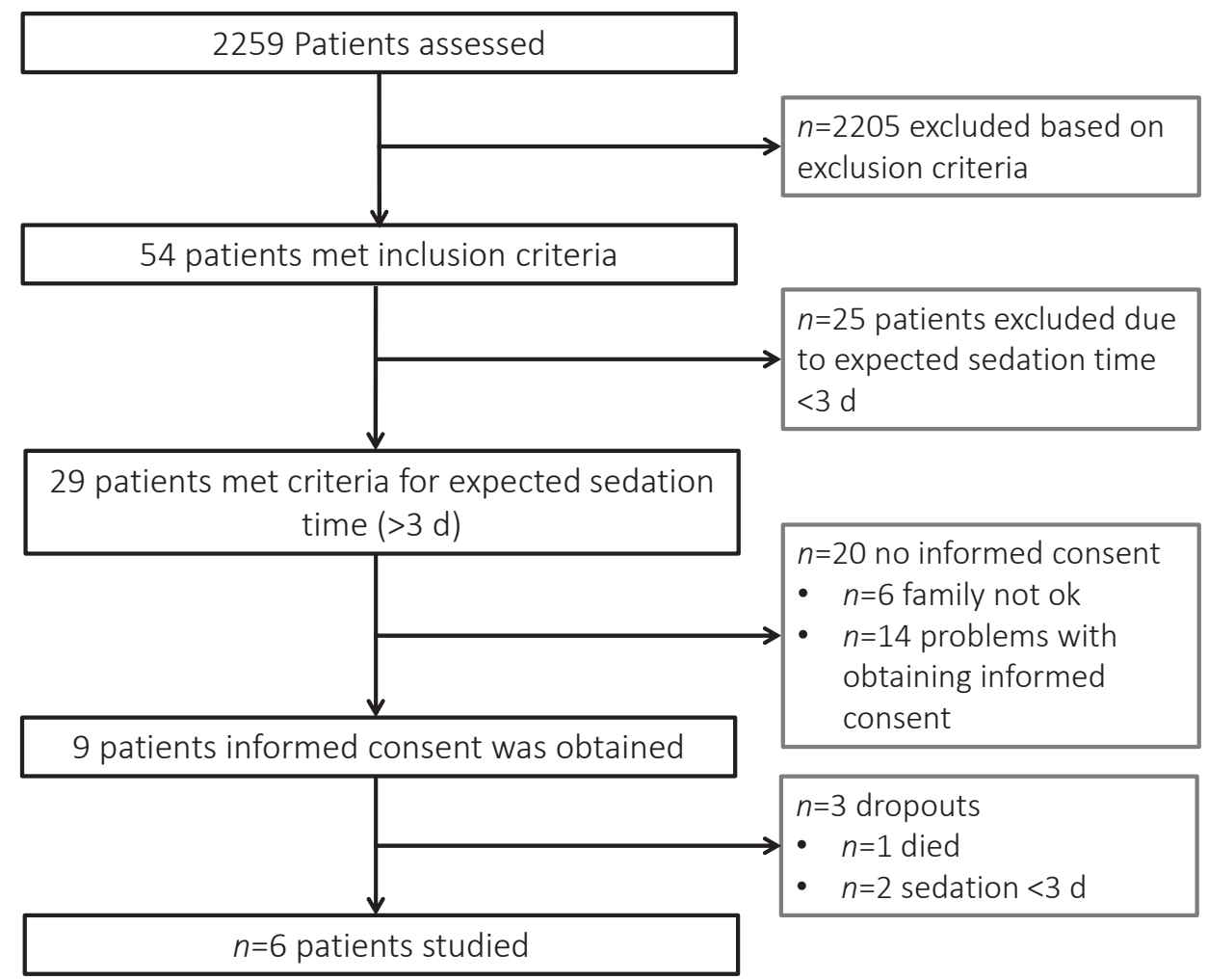

Supplemental Figure 1 | Patient flow 


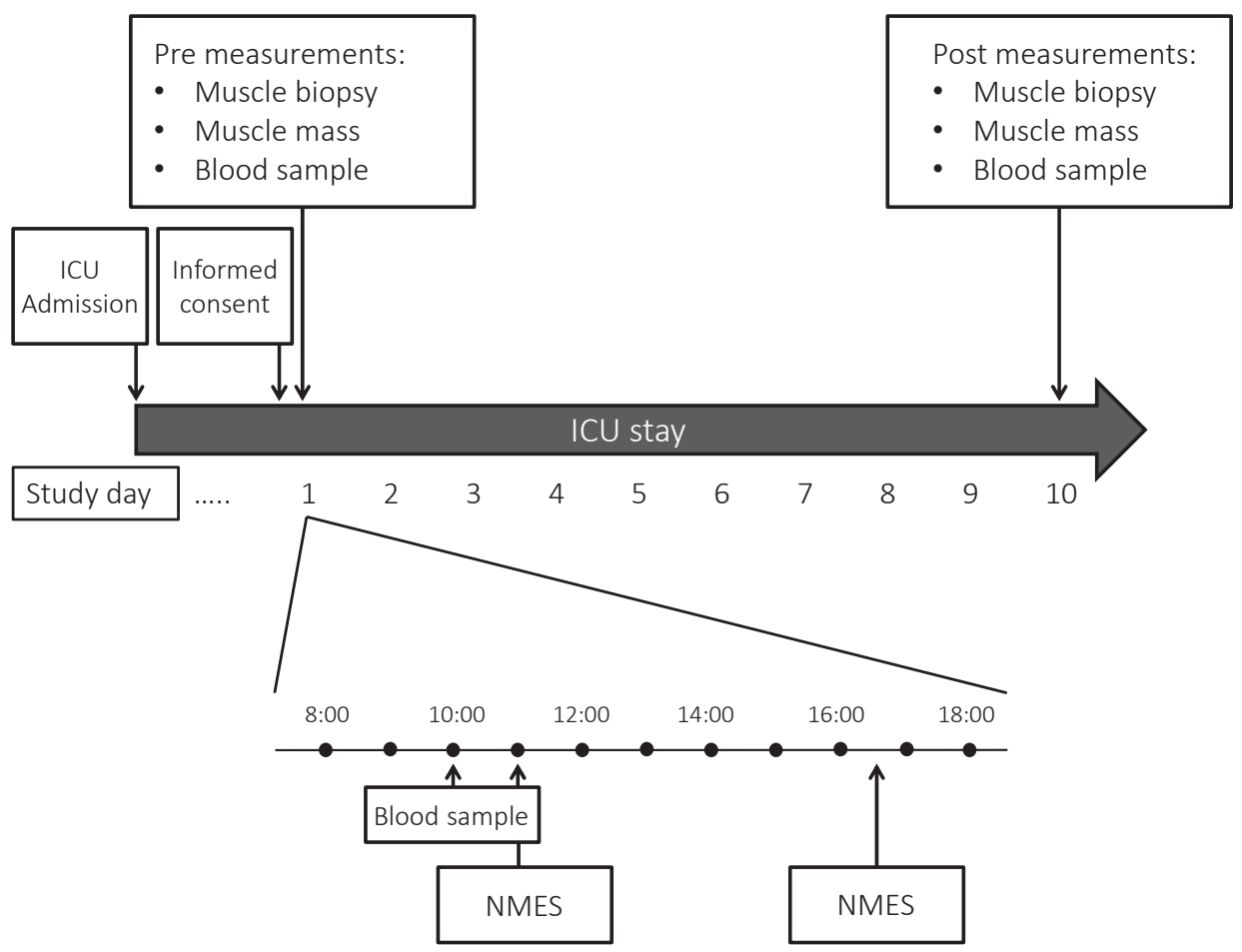

Supplemental Figure 2 | Overview of the experimental protocol. Upper part: Overview of the study, in which fully-sedated patients were subjected to minimally 3 days and maximally 10 days of NMES. Post-intervention measurements were performed prior to patient awakening. Lower part: detailed overview of each study day. '...' represents the duration between ICU admission and obtaining informed consent from the patient's legal representatives (i.e. on average 2.5 days).

\section{Leg circumference measurements}

In the morning of day 1 , circumference of the legs was measured using a tape measure at 5, 10 and $20 \mathrm{~cm}$ proximal to the top of the patella. The positions of leg circumference measurements were marked with semi-permanent ink to ensure the exact same measuring position during the post-measurements.

\section{Muscle analysis}

The part of the muscle sample that was mounted and frozen in Tissue-Tek (Sakura Finetek, Zoeterwoude, the Netherlands) was cut into $5 \mu \mathrm{m}$ thick cryosections using a cryostat at $-20^{\circ} \mathrm{C}$. Pre- and post-intervention samples of the NMES and CON leg of each subject were mounted together on uncoated, pre-cleaned glass slides, while care was taken to properly align the samples for cross-sectional fibre analyses. Stainings were performed to measure muscle fibre typing (FT) and satellite cell (SC) content. To do this, slides were incubated with primary antibodies directed against myosin heavy chain 
(MHC)-I (A4.840, dilution 1:25; Developmental Studies Hybridoma Bank, lowa City, IA), laminin (polyclonal rabbit anti-laminin, dilution 1:50; Sigma, Zwijndrecht, the Netherlands) and Pax7 (neat; cell supernatant from cells obtained from the Developmental Studies Hybridoma Bank, lowa City, IA, USA). Pax7 has been extensively used in the determination of SC content in human skeletal muscle by us [1] and others [2]. After washing, the appropriate secondary antibodies were applied: goat anti-rabbit IgG AlexaFluor647, goat anti-mouse IgM AlexaFluor555, and Streptavidin Alexa 488 (dilution 1:400, 1:500, and 1:200, respectively; Molecular Probes, Invitrogen, Breda, the Netherlands). Myonuclei were stained with 4,6-diamidino-2-phenylindole (DAPI, $0.238 \mu \mathrm{M}$; Molecular Probes). Both primary and secondary antibodies were diluted in $0.1 \%$ Bovine Serum Albumin (BSA) in $0.1 \%$ Tween- phosphate-buffered saline (PBS). All incubation steps were performed at room temperature. Staining of the muscle tissue was done as follows. After fixation in acetone for $5 \mathrm{~min}$, slides were air dried and incubated with 3\% BSA in $0.1 \%$ Tween-PBS for $30 \mathrm{~min}$. Slides were washed in PBS for $5 \mathrm{~min}$, and incubated with Pax7 for $2 \mathrm{~h}$. Thereafter, slides were washed (standard washing protocol: 5 min $0.1 \%$ Tween-PBS, $2 \times 5$ min PBS) and incubated with goat anti-mouse Biotin (dilution 1:133, Vector Laboratories, Inc., Burlingame, CA, USA) for $60 \mathrm{~min}$, washed again, and incubated with Streptavidin for $60 \mathrm{~min}$. After washing, primary antibodies against laminin and MHC-I were applied for $45 \mathrm{~min}$. Slides were then washed and incubated with the appropriate secondary antibodies, diluted together with DAPI. After a last washing step, slides were mounted with cover glasses Mowiol (Calbiochem, Amsterdam, the Netherlands). Staining procedures resulted in images with nuclei stained in blue, Pax7 in green, MHC-I in red, and laminin in far-red. Images were visualized and automatically captured at 10x magnification with a fluorescent microscope equipped with an automatic stage (IX81 motorized inverted microscope, Olympus, Hamburg, Germany) and EXi Aqua CCD camera (QImaging, Surrey, BC, Canada). Image acquisition was done by Micromanager 1.4 software [3], and images were analyzed with Image J software package (version 1.46r, National Institute of Health [4]). Recording and analyzing of the images was done by an investigator blinded to subject coding. As a measure of fibre circularity, form factors were calculated by using the following formula: $(4 \pi \cdot C S A) \cdot(\text { perimeter })^{-2}$. No differences in fibre circularity were observed between legs or between pre- and post-intervention samples. Mean numbers of $257 \pm 33$ and $238 \pm 23$ muscle fibres were analyzed in the pre- and post-intervention samples, respectively.

The part of the muscle sample that was frozen in liquid nitrogen was used to determine mRNA and protein expression of several genes of interest. Frozen muscle (10-20 mg) was used for mRNA isolation using Tri Reagent (Sigma-Aldrich, St. Louis, MO, USA), according to the manufacturer's protocol. Quantification of total RNA was carried out spectrophotometrically at $260 \mathrm{~nm}$ using the NanoDrop ND-1000 Spectrophotometer (Thermo Fisher Scientific, Madison, WI, USA), and RNA purity was determined as the ratio of readings at $260 / 280 \mathrm{~nm}$. Thereafter, first strand cDNA was synthesized from 1 $\mu \mathrm{g}$ RNA sample using random primers (Promega, Madison, WI, USA) and PowerScript 
Reverse Transcriptase (Applied Biosystems, Foster City, CA, USA). Taqman PCR was carried out using an ABI Prism 7000 sequence detector (Applied Biosystems, USA), with $2 \mu \mathrm{L}$ of $\mathrm{cDNA}, 18 \mu \mathrm{l} \cdot \mathrm{L}^{-1}$ of each primer, $5 \mu \mathrm{l} \cdot \mathrm{L}^{-1}$ probe, and Universal Taqman $2 \times \mathrm{PCR}$ mastermix (Eurogentec S.A., Seraing, Belgium) in a $25 \mu \mathrm{L}$ final volume. Each sample was run in duplicate, in duplex reactions. A separate standard curve was included for each gene, with serial dilutions of cDNA synthesized in parallel with the study samples. 18S was selected as a housekeeping gene to be used as an internal control, as we have used it previously and have shown that it does not change with muscle disuse $[5,6]$, i.e. mean $\mathrm{Ct}$ values did not change over time in either leg (data not shown). Taqman primer/probe sets (Applied Biosystems) were obtained for the following genes of interest: mammalian target of rapamycin (mTOR), P70S6 kinase (P70S6K), myogenic factor 4 (myogenin), MyoD, myostatin, Atrogin-1/Muscle Atrophy F-box (MAFbx), Muscle RINGfinger protein-1 (MuRF1), Forkhead box protein O1 (FOXO1), Focal Adhesion Kinase (FAK), large neutral amino acid transporter (LAT1) and Proton-coupled amino acid transporter 1 (PAT1). All genes of interest were labelled with the fluorescent reporter FAM. Thermal cycling was performed using the following conditions: 2 min at $50^{\circ} \mathrm{C}, 10$ min at $950 \mathrm{C}$, followed by 40 cycles at $95^{\circ} \mathrm{C}$ for $15 \mathrm{~s}$ and $60^{\circ} \mathrm{C}$ for $1 \mathrm{~min}$. Ct values of the genes of interest were normalized to $\mathrm{C} t$ values of $18 \mathrm{~S}$, and these values were calculated as relative expression against the standard curve. Muscle samples from age- and sexmatched healthy controls were analyzed to compare pre-intervention values of the patients in the study to healthy volunteers.

Muscle homogenates for Western blotting analysis were made using $40 \mathrm{mg}$ of muscle tissue in 7x volumes Tris buffer $(20 \mathrm{mM}$ Tris-HCL, $5 \mathrm{mM}$ EDTA. $10 \mathrm{mM}$ Napyrosphospate, $100 \mathrm{mM} \mathrm{NaF}, 2 \mathrm{mM} \mathrm{Na3VO4,} \mathrm{1 \%} \mathrm{Nonident} \mathrm{P-40;} \mathrm{pH} \mathrm{7.4)} \mathrm{supplemented}$ with the following protease and phosphatase inhibitors: Aprotinin $10 \mu \mathrm{g} \cdot \mathrm{mL}^{-1}$, Leupeptin $10 \mu \mathrm{g} \cdot \mathrm{mL}^{-1}$, Benzamidin $3 \mathrm{mM}$ and PMSF $1 \mathrm{mM}$. Muscle samples were centrifuged for 10 min at $10,000 \mathrm{~g}\left(4^{\circ} \mathrm{C}\right.$ ), where after sample buffer (final concentration: $60 \mathrm{mM}$ Tris, 5\% glycerol, $20 \mathrm{mg} \cdot \mathrm{mL}^{-1} \mathrm{SDS}, 0.1 \mathrm{mM} \mathrm{DTT}, 20 \mu \mathrm{g} \cdot \mathrm{mL}^{-1}$ bromophenolblue) was added to the supernatant. The solution was then boiled for $5 \mathrm{~min}$ at $100^{\circ} \mathrm{C}$, after which the samples were put on ice. Directly before the commencement of the analyses, the extraction sample was warmed to $50^{\circ} \mathrm{C}$ and centrifuged for $1 \mathrm{~min}$ at $1,000 \mathrm{~g}$ at room temperature. Each lane on the gel was loaded with a total of $50 \mu \mathrm{g}$ muscle sample. Protein samples (for (p)Akt, (p)P70S6K and $\alpha$-actin quantification) were run on a Criterion 'any kDa' gel (Biorad Order No. 567-1124) for $10 \mathrm{~min}$ at $50 \mathrm{~V}$ (constant voltage) and $\pm 90 \mathrm{~min}$ at $150 \mathrm{~V}$ (constant voltage) and transferred onto a Trans-blot Turbo $0.2 \mu \mathrm{m}$ nitrocellulose membrane (Biorad Order No. 170-4159) in $7 \mathrm{~min}$ at 2.5A and 25V. For (p)mTOR protein a Tris-acetate gel was used (Biorad Order nr. 345-0129), and the gel was transferred onto a Trans-blot Turbo $0.2 \mu \mathrm{m}$ nitrocellulose membrane (Biorad Order No. 170-4159) in 10 $\min$ at $1.3 \mathrm{~A}$ and $25 \mathrm{~V}$. Specific proteins were detected by overnight incubation at $4^{\circ} \mathrm{C}$ on a shaker with specific antibodies in 50\% PBS/Odyssey blocking buffer (Li-Cor Biosciences Part No. 927-40000) after blocking for $60 \mathrm{~min}$ at RT in 50\% PBS/Odyssey blocking buffer. 
Antibodies that were used in this study were anti-Akt (60 kDa; dilution 1:1000, \#9272 Cell Signaling) and anti-phospho-Akt (Ser ${ }^{473} ; 60$ kDa, dilution 1:1000, \#9271 Cell Signalling), anti-mTOR (289 kDa; dilution 1:1000, \#2972 Cell Signalling) and anti-phosphomTOR (Ser ${ }^{2448}$; 289 kDa, dilution 1:1000, \#2971 Cell Signalling), anti-P70S6K (70 kDa; dilution 1:1000, \#9202 Cell Signalling), anti-phospho P70S6K ( $\mathrm{Thr}^{389}$; 70kDa, dilution 1:1000, \#9206 Cell Signalling) and anti $\alpha$-actin (42 kDa; dilution 1:100.000, mouse monoclonal IgM, Sigma A2172). After incubation, membranes were washed $3 \times 10 \mathrm{~min}$ in $0.1 \%$ PBS-Tween and $1 \times 10$ min with PBS. Samples were incubated for $1 \mathrm{~h}$ at room temperature with the following secondary antibodies: donkey anti-rabbit IRDYE 680 (LiCor, Cat. No. 926-32223, dilution 1:10000) and donkey anti-mouse IRDYE 800CW (LiCor, Cat. No. 926-32212, dilution 1:10000) dissolved in 50\% PBS Odyssey blocking buffer. After the last washing step ( $3 \times 5$ min in $0.1 \%$ Tween20-PBS and $1 \times 10$ min with PBS), protein quantification was performed by scanning on an Odyssey Infrared Imaging System (LI-COR Biotechnology, Lincoln, NE, USA). 


\section{SUPPLEMENTAL RESULTS}

\section{Laboratory results}

Supplemental Table1 | Average laboratory values during the study period

\begin{tabular}{lcc}
\hline & Value & Reference value \\
\hline White blood cell count $\left(* 10^{9} \cdot \mathrm{L}^{-1}\right)$ & $15.9 \pm 2.5$ & $4.5-11.0$ \\
Lymphocytes $\left(* 10^{9} \cdot \mathrm{L}^{-1}\right)$ & $0.8 \pm 0.1$ & $1.01-3.38$ \\
Monocytes $\left(* 10^{9} \cdot \mathrm{L}^{-1}\right)$ & $0.90 \pm 0.30$ & $<0.82$ \\
CRP $\left(\mathrm{mg} \cdot \mathrm{dL}^{-1}\right)$ & $9.1 \pm 2.3$ & $<0.5$ \\
Ureum $\left(\mathrm{mg} \cdot \mathrm{dL}^{-1}\right)$ & $74.6 \pm 10.9$ & $17-43$ \\
Creatinine $\left(\mathrm{mg}^{\mathrm{d}} \mathrm{dL}\right)$ & $0.9 \pm 0.1$ & $0.51-0.95$ \\
Total plasma protein $\left(\mathrm{g} \cdot \mathrm{dL}^{-1}\right)$ & $5.7 \pm 0.2$ & $6.6-8.3$ \\
Albumin $\left(\mathrm{g} \cdot \mathrm{dL}^{-1}\right)$ & $2.8 \pm 0.1$ & $3.5-5.2$ \\
\hline
\end{tabular}

$\mathrm{CRP}=\mathrm{C}$-reactive protein

\section{Plasma amino acid concentrations}

Plasma concentrations of the measured amino acids are displayed in Supplemental Table 2. Following the intervention, significant decreases in the concentrations of alanine, histidine and phenylalanine were observed (all $P<0.05$ ), whereas for leucine a trend for a decline was seen $(P=0.065)$. 
Supplemental Table 2 | Plasma AA concentrations

\begin{tabular}{|c|c|c|}
\hline & Pre & Post \\
\hline$\alpha$-aminobutyric acid & $47 \pm 10$ & $29 \pm 4$ \\
\hline Alanine & $369 \pm 50$ & $241 \pm 31 *$ \\
\hline Arginine & $66 \pm 16$ & $47 \pm 8$ \\
\hline Asparagine & $63 \pm 10$ & $44 \pm 5$ \\
\hline Aspartic acid & $4 \pm 1$ & $6 \pm 2$ \\
\hline Citrulline & $18 \pm 3$ & $23 \pm 4$ \\
\hline Cysteine & $40 \pm 11$ & $48 \pm 9$ \\
\hline Glutamic acid & $46 \pm 15$ & $73 \pm 20$ \\
\hline Glutamine & $470 \pm 59$ & $430 \pm 70$ \\
\hline Glycine & $263 \pm 67$ & $189 \pm 24$ \\
\hline Histidine & $81 \pm 4$ & $55 \pm 6 *$ \\
\hline Isoleucine & $77 \pm 6$ & $60 \pm 7$ \\
\hline Leucine & $158 \pm 9$ & $111 \pm 14$ \\
\hline Lysine & $218 \pm 38$ & $145 \pm 19$ \\
\hline Methionine & $40 \pm 6$ & $28 \pm 5$ \\
\hline Ornithine & $90 \pm 18$ & $78 \pm 11$ \\
\hline Phenylalanine & $99 \pm 10$ & $71 \pm 8^{*}$ \\
\hline Proline & $161 \pm 21$ & 15833 \\
\hline Serine & $85 \pm 14$ & $69 \pm 9$ \\
\hline Taurine & $42 \pm 12$ & $45 \pm 8$ \\
\hline Threonine & $139 \pm 28$ & $91 \pm 16$ \\
\hline Tryptophan & $34 \pm 7$ & $31 \pm 6$ \\
\hline Tyrosine & $77 \pm 13$ & $61 \pm 8$ \\
\hline Valine & $283 \pm 22$ & $222 \pm 24$ \\
\hline
\end{tabular}

Data are presented as means \pm SEM.* Significantly different from pre-value $(P<0.05)$. All values are presented as $\mu \mathrm{mol} \cdot \mathrm{L}^{-1}$.

\section{Leg circumference}

No baseline differences in leg circumference between NMES and CON legs were observed, and no effect of time or treatment occurred (both interaction and time effect $P>0.05)$.

mRNA analysis

For myostatin (Supplemental Figure 3A), myoD (Supplemental Figure 3B), and myogenin (Supplemental Figure $3 C$ ), no differences were observed when compared with healthy controls and no interaction effect was found for the CON and NMES leg (all $P>0.05$ ). FAK (Supplemental Figure 3D) was higher in patients than in healthy controls $(P<0.05)$, but 
was not changed over time or between legs $(P>0.05)$. For both PGC-1 $\alpha$ (Supplemental Figure $3 \mathrm{E}$ ) and citrate synthase (Supplemental Figure 3F) no differences between healthy controls and patients were observed (both $P<0.05)$. Although not significantly $(P>0.05)$, PGC-1 $\alpha$ tended to decrease over time in both legs. Citrate synthase showed a significant time*treatment interaction $(P<0.05)$; when both legs were tested separately with a paired-samples t-test a trend for a decline in the CON leg $(P=0.059)$ was observed, while in the NMES leg no decrease was observed $(P>0.05)$. The amino acid transporters LAT1 (Supplemental Figure 3G) and PAT1 (Supplemental Figure 3H) were not different (LAT1) or significantly higher (PAT1; $P<0.01$ ) in patients compared with healthy controls, whereas over time no changes were observed in either gene (both $P>0.05$ ). 

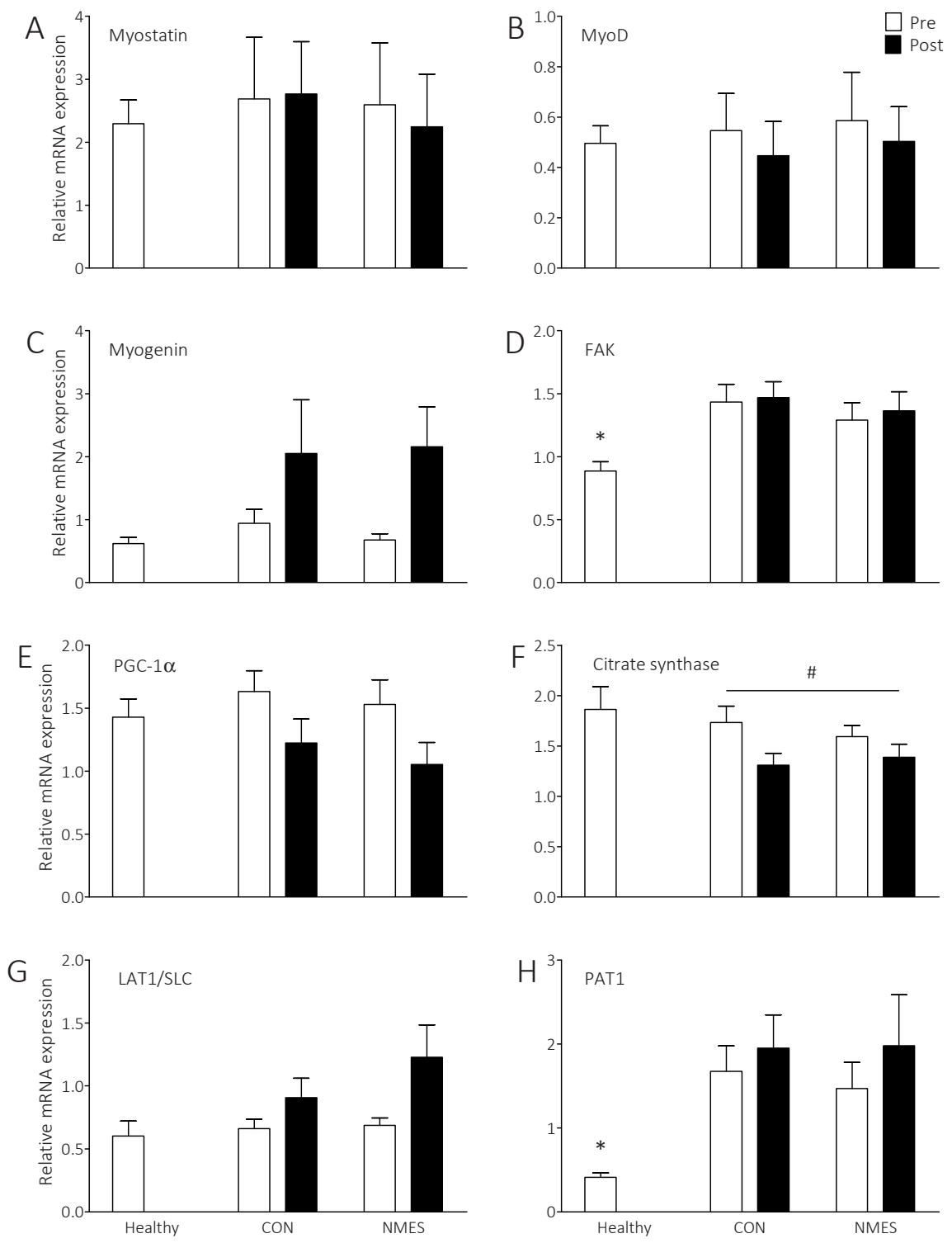

Supplemental Figure 3 | Skeletal muscle mRNA expression of genes of interest in the CON and NMES leg of fully-sedated ICU patients (right part of the figure) versus healthy controls (left column), before (white bars) and after (black bars) $7 \pm 1$ days of twice-daily NMES. Abbreviations: FAK, Focal Adhesion Kinase; LAT1, Large Neutral Amino Acid Transporter 1; PAT1, Proton-coupled amino acid transporter 1; PGC-1 $\alpha$, Peroxisome proliferator-activated receptor gamma coactivator 1-alpha. * Significantly different from the mean preintervention value of the CON and NMES legs. \# Significant interaction effect $(P<0.05)$. 


\section{SUPPLEMENTAL REFERENCES}

1 Verdijk LB, Koopman R, Schaart G, Meijer K, Savelberg HH, van Loon LJ. Satellite cell content is specifically reduced in type II skeletal muscle fibers in the elderly. Am J Physiol Endocrinol Metab 2007; 292: E151-7.

2 Mackey AL, Andersen LL, Frandsen U, Suetta C, Sjogaard G. Distribution of myogenic progenitor cells and myonuclei is altered in women with vs. those without chronically painful trapezius muscle. J Appl Physiol 2010; 109: 1920-9.

3 Edelstein A, Amodaj N, Hoover K, Vale R, Stuurman N. Computer control of microscopes using $\mu$ Manager. Curr Protoc Mol Biol 2010; Chapter 14.

4 Strandberg S, Wretling ML, Wredmark T, Shalabi A. Reliability of computed tomography measurements in assessment of thigh muscle cross-sectional area and attenuation. BMC Med Imaging 2010; 10: 18.

5 Wall BT, Snijders T, Senden JM, Ottenbros CL, Gijsen AP, Verdijk LB, van Loon LJ. Disuse impairs the muscle protein synthetic response to protein ingestion in healthy men. J Clin Endocrinol Metab 2013; 98 : 4872-81.

6 Dirks ML, Wall BT, Snijders T, Ottenbros CL, Verdijk LB, van Loon LJ. Neuromuscular electrical stimulation prevents muscle disuse atrophy during leg immobilization in humans. Acta Physiol (Oxf) 2014; 210: 628-41. 


\section{CHAPTER}

\section{Skeletal muscle disuse atrophy is not attenuated by dietary protein supplementation in healthy, older men}

Marlou L Dirks

Benjamin T Wall

Rachel Nilwik

Daniëlle HJM Weerts

Lex B Verdijk

Luc JC van Loon 
ABSTRACT

Background Short successive periods of muscle disuse, due to injury or illness, can contribute significantly to the loss of muscle mass with ageing (sarcopenia). It has been suggested that increasing the protein content of the diet may be an effective dietary strategy to attenuate muscle disuse atrophy. We hypothesized that protein supplementation twicedaily would preserve muscle mass during a short period of limb immobilization.

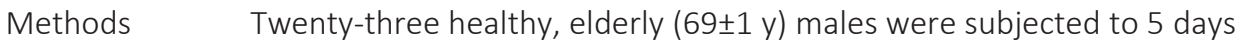
of one-legged knee immobilization by means of a full leg cast with (PRO group; $n=11$ ) or without (CON group; $n=12$ ) administration of a dietary protein supplement (20.7 g protein, $9.3 \mathrm{~g}$ carbohydrate, and $3.0 \mathrm{~g}$ fat) twice daily. Two days prior to and immediately after the immobilization period, single slice CT-scans of the quadriceps and single leg 1-Repetition Maximum (1RM) strength tests were performed to assess muscle cross-sectional area (CSA) and leg muscle strength, respectively. Additionally, muscle biopsies were collected to assess muscle fibre characteristics, and mRNA and protein expression of selected genes.

Results Immobilization decreased quadriceps CSA by $1.5 \pm 0.7 \%(P<0.05)$ and $2.0 \pm 0.6 \%(P<0.05)$, and muscle strength by $8.3 \pm 3.3 \%(P<0.05)$ and $9.3 \pm 1.6 \%(P<0.05)$ in the CON and PRO groups, respectively; without differences between groups. Skeletal muscle myostatin, myogenin, and MuRF1 mRNA expression increased following immobilization in both groups $(P<0.05)$, while muscle MAFbx mRNA expression increased in the PRO group only $(P<0.05)$.

Conclusion In conclusion, dietary protein supplementation ( $20 \mathrm{~g}$ twice daily) does not attenuate muscle loss during short-term muscle disuse in healthy older men. Clinical trial registration: NCT01588808 


\section{INTRODUCTION}

A period of prolonged (i.e. several weeks) muscle disuse, due to illness or injury, can lead to substantial loss of skeletal muscle mass and strength in otherwise healthy individuals. The resulting negative health consequences, such as impaired functional capacity [1-3], decreased muscle strength [4], the onset of insulin resistance [5], and a decline in basal metabolic rate $[6,7]$, are of particular concern to elderly individuals, who are already functionally and/or metabolically compromised. Recently, we [8, 9] as well as others [10] have shown that even a few days of disuse can already lead to significant losses of muscle mass and strength in young and old men. These findings are of particular clinical relevance as hospitalization of elderly due to acute illness generally results in an average 5-7 day hospital stay [11]. It has been hypothesized that such short successive periods of muscle disuse occurring throughout the lifespan may be instrumental in the progressive loss of muscle mass that occurs with ageing $[12,13]$.

Any substantial loss of skeletal muscle mass due to muscle disuse must be attributed to a chronic imbalance between muscle protein synthesis and breakdown rates. A decline in basal (postabsorptive) muscle protein synthesis rates has been reported following both bed rest [14-16] as well as limb immobilization [17-19]. Furthermore, recent work from our laboratory [20] as well as others [17, 21, 22] has shown that the muscle protein synthetic response to protein or amino acid administration becomes blunted following a period of disuse. Additionally, there is some indirect evidence that increases in muscle protein breakdown rates occur during the initial first few days of muscle disuse only [23-25]. As such, it is now widely thought that declines in both postabsorptive and postprandial muscle protein synthesis rates play the major causal role in the loss of muscle mass during a period of disuse $[26,27]$. Dietary protein intake stimulates muscle protein synthesis rates and inhibits muscle protein breakdown, and thereby allows net muscle protein accretion [28]. Accordingly, it has been speculated that maintaining or even increasing dietary protein intake can attenuate muscle loss during a period of disuse $[12,27]$. In support, intervention studies have shown high-dose, essential amino acid supplementation to attenuate muscle loss during prolonged bed rest in young [2931] and elderly individuals [32]. However, the potential for a practical dietary protein feeding strategy to alleviate muscle loss during short-term disuse in the elderly population remains to be investigated.

In the present study, we investigated our hypothesis that dietary protein supplementation attenuates muscle loss during a short period of muscle disuse in older men. To test this hypothesis, 23 healthy elderly men were selected to participate in a study during which they were subjected to 5 days of one-legged knee immobilization with or without dietary protein supplementation ( $20 \mathrm{~g}$ protein twice daily). Muscle mass and strength were assessed prior to and immediately after immobilization, and muscle biopsy samples were collected to assess muscle fibre characteristics and associated myocellular signaling. 


\section{METHODS}

\section{Subjects}

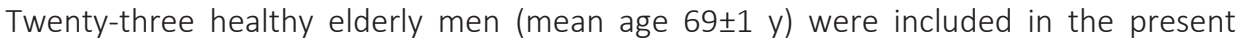
study. Prior to inclusion, a general health questionnaire was filled in by the subjects and a routine medical screening was completed to exclude individuals with BMI below 18.5 or above $30 \mathrm{~kg} \cdot \mathrm{m}^{-2}$; any back, knee or shoulder complaints that could interfere with the use of crutches; a (family) history of thrombosis; type 2 diabetes mellitus (determined by HbA1c values $>7.0 \%$ ); severe cardiac problems; or a history of performing prolonged resistance-type exercise in the six months preceding the start of the study. All subjects were informed on the nature and risks of the experiment before written informed consent was obtained. The present study was approved by the Medical Ethical Committee of Maastricht University Medical Centre in accordance with the Declaration of Helsinki.

\section{Experimental outline}

An overview of the experimental protocol is depicted in Figure 1.

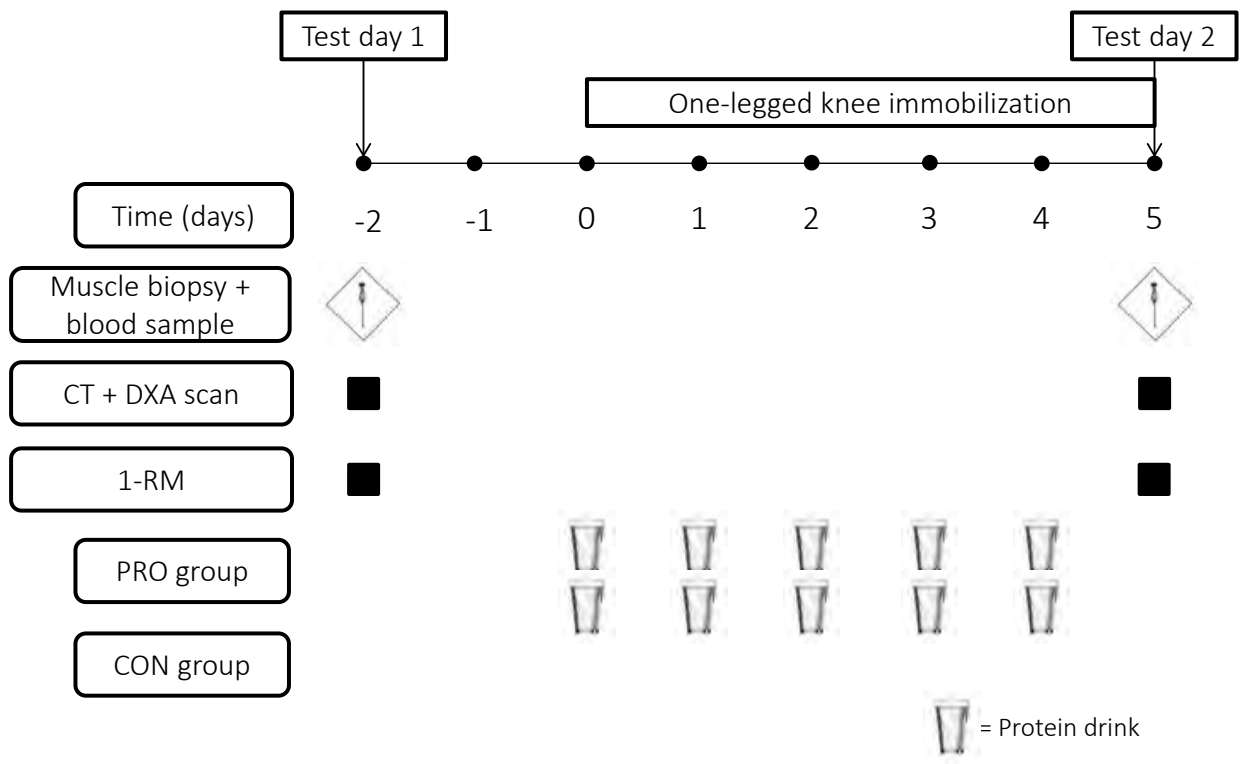

Figure 1 | Outline of the experimental protocol. Two groups of healthy elderly males were included to undergo 5 days of one-legged knee-immobilization, with (PRO; $n=11)$ or without (CON; $n=12$ ) protein supplementation ( 20 g protein twice daily).

After inclusion into the study, subjects were randomly allocated to either the control (CON, $n=12$ ) or the protein (PRO, $n=11$ ) group. Both groups were subjected to five days 
of muscle disuse induced by way of a full leg cast. The immobilized leg was randomly allocated and counter-balanced between left and right. Two days prior to casting and directly after cast removal, a series of measurements was performed. Single slice computed tomography (CT) scans were performed at the mid-thigh of both legs, wholebody dual energy $\mathrm{x}$-ray absorptiometry (DXA) scans were taken, a single muscle biopsy from the immobilized leg and venous blood sample were collected, and one-legged knee extension strength (1RM, one-Repetition Maximum) was assessed for both legs.

\section{Muscle mass and function tests}

Forty eight hours prior to, and directly after the casting period, subjects visited the laboratory for two identical test days (i.e. test days 1 and 2). During these test days, multiple measurements of muscle mass and function were performed. Firstly, the anatomical cross-sectional area (CSA) of $m$. quadriceps femoris and whole thigh were assessed via a single slice computed tomography (CT) scan (Philips Brilliance 64, Philips Medical Systems, Best, The Netherlands) as done before [8]. With subjects placed in a supine position, their legs extended and their feet secured, a $3 \mathrm{~mm}$ thick axial image was taken $15 \mathrm{~cm}$ proximal to the top of the patella. On test day 1 the exact scanning position was marked with semi-permanent ink for replication on test day 2. ImageJ software (version 1.46r, National Institute of Health, Bethesda, MD, USA) was used to analyze CT scan images for the cross-sectional area of all thigh muscles as well as the quadriceps muscle separately. Secondly, a DXA scan (Hologic, Discovery A, QDR Series, Bedford, MA, USA) was used to determine body composition and bone mineral content. Leg lean mass was determined using the system's software package Apex version 2.3. Maximal muscle strength was determined for each leg individually by 1 RM strength tests on a leg extension machine (Technogym, Rotterdam, the Netherlands) as done before $[8,33]$.

\section{Blood and muscle sampling}

Fasting venous blood samples were collected for determination of basal plasma glucose and insulin concentrations on test day 1 and 2 . Blood $(10 \mathrm{~mL})$ was collected in EDTAcontaining tubes and immediately centrifuged at 1,000g for $10 \mathrm{~min}$ at $4^{\circ} \mathrm{C}$. Aliquots of plasma were snap frozen in liquid nitrogen and stored at $-80^{\circ} \mathrm{C}$ until further analysis. Plasma glucose, free fatty acids, and triglyceride concentrations were analyzed with a ABX Pentra 400 analyzer (Horiba Diagnostics, Montpellier, France) with test kits from ABX Diagnostics (Montpellier, France), whereas plasma insulin concentrations were determined by radioimmunoassay (Millipore, ref. HI-14K, Billerica, MA, USA). Plasma amino acid concentrations were measured using ultra-performance liquid chromatography tandem mass spectrometry as described previously [34].

Muscle biopsies were taken from $m$. vastus lateralis of the immobilized leg prior to casting and immediately after cast removal, prior to performing any weight bearing 
activities. Biopsies were taken at the same time (08.30 AM) in the morning after an overnight fast and the same standardized meal was provided the evening prior to muscle biopsy collection. Percutaneous muscle biopsies were taken from $m$. vastus lateralis with the Bergstrom technique, approximately $15 \mathrm{~cm}$ above the patella. The collected muscle was freed from any visible non-muscle tissue, processed immediately, and stored at $-80^{\circ} \mathrm{C}$ until further analysis.

\section{Leg immobilization}

Two days after performing test day 1, at 8:00 in the morning, a full leg cast (randomized and counterbalanced for left and right leg) was applied in the casting room of the Academic Hospital in Maastricht. This marked the start of the 5 day immobilization period that always contained 3 week days and 2 weekend days. The cast extended from $\sim 5 \mathrm{~cm}$ above the ankle until $\sim 25 \mathrm{~cm}$ above the patella. A $\sim 30$ degree angle of flexion of the knee joint was established in order to prevent subjects from performing weight-bearing activities with the immobilized leg. Subjects received crutches and were instructed on the correct usage before being provided with transportation home. The cast was removed at 8:00 on the morning of test day 2 , after exactly 5 days of immobilization.

\section{Protein supplementation}

Subjects were randomly allocated to the group receiving a high whey protein leucineenriched oral nutritional supplement (PRO) or the control group receiving no supplement (CON). Subjects allocated to the PRO group consumed the first drink in the laboratory on the morning of casting and were instructed to consume one drink directly after breakfast and one drink immediately prior to sleep on each day during immobilization (i.e. twice-daily, 10 drinks in total). Each drink provided 635 kJ, 21 g protein, 9 g carbohydrates, $3 \mathrm{~g}$ fat, and a mixture of vitamins, minerals and fibres. Supplemental Table 1 depicts the composition of the study product.

\section{Dietary intake}

Standardized meals, containing $2.9 \mathrm{MJ}$ and providing 51 Energy\% (En\%) as carbohydrate, $32 \mathrm{En} \%$ as fat, and $17 \mathrm{En} \%$ as protein, were consumed on the evening prior to both test days. Weighted dietary intake records were completed by the subjects for the 5 day duration of the immobilization period as well as on a separate consecutive 5 day occasion either before or after (randomly allocated to avoid recording bias) the immobilization period. The same 5 days of the week were selected for both recording periods. DieetInzicht software [35], based on the NEVO table 2011, was used to analyze dietary intake records. 


\section{Muscle analysis}

Muscle samples were freed from any visible non-muscle tissue and separated into two sections. The first part ( $30 \mathrm{mg}$ ) was imbedded in Tissue-Tek (Sakura Finetek, Zoeterwoude, the Netherlands), frozen on liquid nitrogen cooled isopentane and used to determine muscle fibre-type specific cross-sectional area (CSA) and satellite cell content as done previously [8]. The second part ( $15 \mathrm{mg}$ ) was snap frozen in liquid nitrogen and used for real time-PCR analysis to determine mRNA expression of selected genes as described before $[8,20]$. A detailed overview of the muscle analyses is presented in the supplemental material.

\section{Statistics}

All data are expressed as means \pm SEM. Baseline values between groups were compared by means of an independent samples t-test. Pre- versus post-immobilization data were analyzed using Repeated Measures ANOVA with treatment (CON vs PRO) as betweensubjects factor and time (pre- vs post-immobilization) as within-subjects factor. Fibre type (type I vs type II) was added to the test as a within-subjects factor when performing the statistical analyses for the muscle data. In case of a significant main effect, paired-samples $t$ tests were executed to determine time effects within treatment groups or within fibre types, and independent-samples t tests were performed to determine group differences in pre- and post-immobilization values. When a significant main effect was detected, Bonferroni's post hoc test was applied to locate the differences. A P-value of $<0.05$ was used to determine statistical significance. All data were analyzed using SPSS version 20.0 (SPSS Inc., Chicago, IL, USA). 


\section{RESULTS}

\section{Subjects}

Subjects' characteristics are provided in Table 1. No baseline differences between the control (CON) and protein (PRO) groups were observed for age, height, weight, BMI, glucose, insulin, HOMA, or HbA1c levels at baseline. Glucose, insulin, and HOMA were measured pre- and post-intervention, and did not change over time in either group.

Table 1 | Subjects' characteristics of healthy older men in the control (CON) and protein supplemented (PRO) groups

\begin{tabular}{lcc}
\hline & CON $(\mathrm{n}=12)$ & PRO $(\mathrm{n}=11)$ \\
\hline Age $(\mathrm{y})$ & $70 \pm 1$ & $68 \pm 1$ \\
Body mass $(\mathrm{kg})$ & $82.9 \pm 3.0$ & $79.6 \pm 2.4$ \\
Height $(\mathrm{m})$ & $1.74 \pm 0.02$ & $1.74 \pm 0.02$ \\
BMI $\left(\mathrm{kg} \cdot \mathrm{m}^{-2}\right)$ & $27.3 \pm 0.6$ & $26.4 \pm 0.8$ \\
Leg volume $(\mathrm{L})$ & $7.96 \pm 0.28$ & $7.90 \pm 0.35$ \\
Plasma glucose $\left(\mathrm{mmol} \cdot \mathrm{L}^{-1}\right)$ & $5.6 \pm 0.1$ & $5.7 \pm 0.1$ \\
Plasma insulin $\left(\mathrm{uU} \cdot \mathrm{mL}^{-1}\right)$ & $11.7 \pm 1.4$ & $9.9 \pm 1.0$ \\
Glycated hemoglobin $(\%)$ & $5.4 \pm 0.1$ & $5.7 \pm 0.1$ \\
HOMA-index & $3.0 \pm 0.4$ & $2.6 \pm 0.3$ \\
Glycated hemoglobin $\left(\mathrm{mmol} \cdot \mathrm{mol}^{-1}\right)$ & $35.9 \pm 1.2$ & $38.4 \pm 1.3$ \\
\hline
\end{tabular}

Values are means \pm SEM. Abbreviations: HOMA-index, Homeostatic Model Assessment Index. No differences were observed between groups ( $P>0.05$ for all variables).

\section{Muscle mass and strength}

Quadriceps muscle cross-sectional area (CSA) is displayed in Figure 2A. At baseline, no differences were observed in quadriceps or whole leg muscle CSA between groups $(P>0.05$ for both parameters). Five days of immobilization caused significant muscle atrophy of the quadriceps (time effect, $P<0.001$; see Figure $2 \mathrm{~A}$ ) and the whole leg (time effect, $P<0.05$; from $13.3 \pm 5.4$ to $13.2 \pm 5.3 \mathrm{~cm}^{2}(-0.7 \pm 0.6 \%)$ in $\mathrm{CON}$ and from $12.6 \pm 4.2$ to $12.4 \pm 4.6 \mathrm{~cm}^{2}(-1.6 \pm 0.6 \%)$ in $\left.\mathrm{PRO}\right)$ with no differences between groups ( $\mathrm{P}$ interaction $>0.05$ for both parameters). Immobilization did not affect whole-body or leg lean mass in either group (data not shown; both $P>0.05$ ). Leg muscle strength data are presented in Figure 2B. Maximal leg muscle strength had decreased following immobilization in the CON and PRO group (time effect, $P<0.001$ ), with no differences between groups ( $P$-interaction $>0.05)$. 

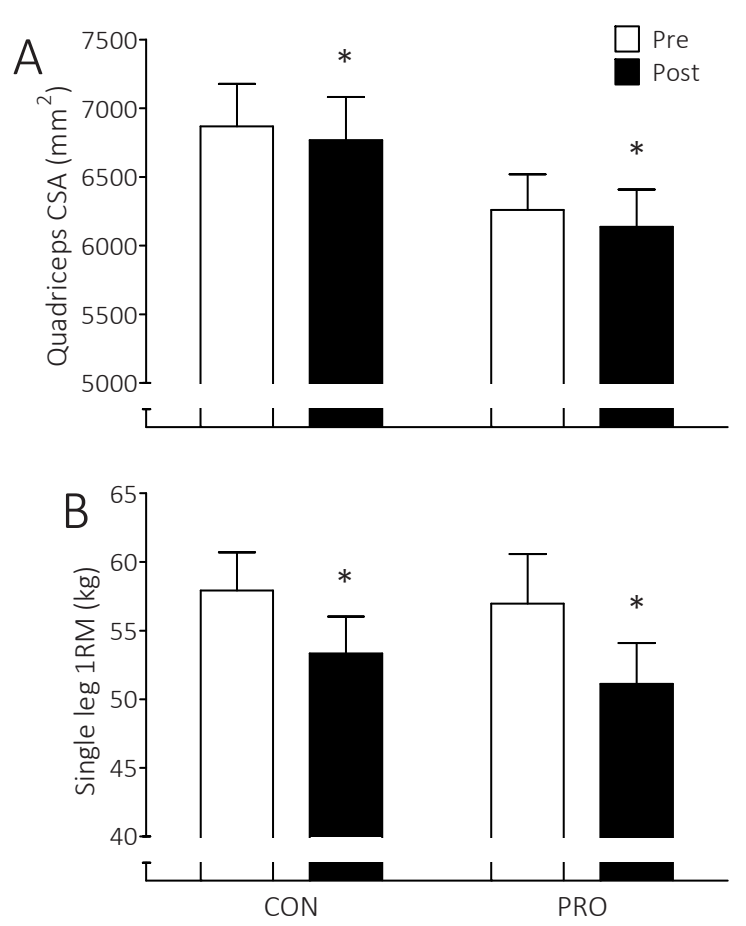

Figure 2 | A Cross-sectional area (CSA) of $m$. quadriceps femoris in healthy elderly participants in the CON $(n=12)$ and PRO $(n=11)$ groups, measured by single-slice CT scan 48 h prior to and immediately following 5 days of leg immobilization. B Leg muscle strength as measured by 1 RM, in both the CON and PRO group. Data are expressed as means \pm SEM. ${ }^{*} P<0.05$; significantly different when compared with pre-immobilization values.

\section{Dietary intake}

Table 2 shows data for subjects' habitual diet for 5 days under free living conditions and during the 5 day immobilization period. No differences in habitual diet were observed between groups (all measured parameters $P>0.05$ ). Habitual diet did not change due to immobilization in the CON group ( $P>0.05)$, whereas in the PRO group, twice-daily ingestion of the protein drink significantly increased protein intake (expressed as $\mathrm{g} \cdot \mathrm{day}^{-1}, \mathrm{~g} \cdot \mathrm{kg}$ body weight ${ }^{-1} \cdot$ day $^{-1}$, and En\%) compared with baseline $(P<0.05)$ and the CON group $(P<0.05)$. Habitual protein intake averaged $1.1 \mathrm{~g} \cdot \mathrm{kg}$ body weight ${ }^{-1} \cdot \mathrm{day}^{-1}$ and was increased to $1.6 \mathrm{~g} \cdot \mathrm{kg}$ body weight ${ }^{-1} \cdot \mathrm{day}^{-1}$ during the immobilization period in the PRO group. Energy intake in the PRO group was maintained during immobilization; a relatively higher amount of energy was received from protein, at the expense of energy from fat $(P<0.05)$. 
Table 2 | Dietary intake of healthy elderly subjects under free-living conditions and during a 5-day period of leg immobilization, with (PRO) or without (CON) supplementation.

\begin{tabular}{lcccc}
\hline & \multicolumn{2}{c}{ CON $(n=12)$} & \multicolumn{2}{c}{ PRO $(n=11)$} \\
\hline & Free living & Immobilization & Free living & Immobilization \\
\hline Energy intake $\left(\mathrm{MJ} \cdot\right.$ day $^{-1}$ ) & $8.82 \pm 0.62$ & $9.03 \pm 0.46$ & $8.73 \pm 0.54$ & $9.50 \pm 0.49$ \\
Protein intake $\left(\mathrm{g} \cdot\right.$ day $\left.^{-1}\right)$ & $85 \pm 9$ & $86 \pm 4$ & $90 \pm 4$ & $125 \pm 6 *$ \\
Protein $\left(\mathrm{g} \cdot \mathrm{kg}\right.$ body weight $\left.{ }^{-1} \cdot \mathrm{day}^{-1}\right)$ & $1.04 \pm 0.12$ & $1.05 \pm 0.06$ & $1.14 \pm 0.07$ & $1.60 \pm 0.11 *$ \\
Protein (En\%) & $16.7 \pm 1.2$ & $16.4 \pm 0.7$ & $18.0 \pm 0.9$ & $22.9 \pm 1.0 *$ \\
Fat (En\%) & $31.6 \pm 1.3$ & $32.9 \pm 2.3$ & $29.5 \pm 1.8$ & $25.8 \pm 1.2 *$ \\
Carbohydrate (En\%) & $51.7 \pm 2.0$ & $50.6 \pm 2.1$ & $52.5 \pm 2.1$ & $51.3 \pm 1.5$ \\
\hline
\end{tabular}

Data represent means \pm SEM. Data in the PRO group are expressed including twice-daily intake of the protein supplement. * Significantly different from free living value $(P<0.05)$

\section{Plasma analyses}

Plasma amino acid concentrations (Supplemental Table 2) were increased in both groups for alanine, cysteine, phenylalanine, threonine, and tryptophan (all $P<0.05$ ). For valine $(P$-interaction $<0.05)$, an increase following immobilization was observed in the PRO group only $(P<0.05)$. All other measured amino acids were not changed following immobilization (all $P>0.05$ ). Immobilization, with or without protein supplementation, did not influence plasma free fatty acid (CON: from $384 \pm 33$ to $354 \pm 33 \mu \mathrm{mol} \cdot \mathrm{L}^{-1}$; PRO: from $446 \pm 48$ to $404 \pm 46 \mu \mathrm{mol} \cdot \mathrm{L}^{-1}$ ) or triglyceride (CON: from $1190 \pm 210$ to $1270 \pm 92$ $\mu \mathrm{mol} \cdot \mathrm{L}^{-1}$; PRO: from $968 \pm 88$ to $1110 \pm 118 \mu \mathrm{mol} \cdot \mathrm{L}^{-1}$ ) concentrations (both $P>0.05$ ).

\section{Muscle fibre characteristics}

Muscle fibre characteristics are displayed in Table 3. At baseline, no differences between groups were observed for any of the variables. No measurable decline in muscle fibre CSA was observed following immobilization in either group $(P>0.05)$. Although no changes in myonuclear content were observed following immobilization ( $P>0.05)$, myonuclear domain size decreased in both fibre types in both CON and PRO (time effect, $P<0.05)$. At baseline, satellite cell (SC) content expressed per muscle fibre, per millimetre squared, and as a percentage of the total number of myonuclei was higher in type I compared with type II fibres ( $P<0.05$ for all three parameters). No changes over time or differences between groups were observed $(P>0.05)$. 
Table 3 | Muscle fibre characteristics of healthy elderly individuals before (pre) and after (post) 5 days of leg immobilization, with (PRO) or without (CON) supplementation.

\begin{tabular}{|c|c|c|c|c|c|}
\hline & \multirow[b]{2}{*}{$\begin{array}{l}\text { Fibre } \\
\text { type }\end{array}$} & \multicolumn{2}{|c|}{$\operatorname{CON}(n=12)$} & \multicolumn{2}{|c|}{$\operatorname{PRO}(n=11)$} \\
\hline & & Pre & Post & Pre & Post \\
\hline \multirow[t]{2}{*}{ Muscle fibre CSA $\left(\mu \mathrm{m}^{2}\right)$} & I & $5654 \pm 391$ & $5037 \pm 487$ & $5646 \pm 469$ & $5370 \pm 379$ \\
\hline & ॥ & $5592 \pm 564$ & $5000 \pm 525$ & $5131 \pm 390$ & $5027 \pm 356$ \\
\hline \multirow[t]{2}{*}{ Fibre (\%) } & I & $49 \pm 3$ & $44 \pm 3$ & $48 \pm 5$ & $48 \pm 5$ \\
\hline & ॥ & $51 \pm 3$ & $56 \pm 3$ & $52 \pm 5$ & $52 \pm 5$ \\
\hline \multirow[t]{2}{*}{ Fibre (area\%) } & । & $50 \pm 4$ & $45 \pm 3$ & $51 \pm 5$ & $49 \pm 6$ \\
\hline & ॥ & $50 \pm 4$ & $55 \pm 3$ & $49 \pm 5$ & $51 \pm 6$ \\
\hline \multirow[t]{2}{*}{ Nuclei (n/fibre) } & । & $2.8 \pm 0.1$ & $2.9 \pm 0.3$ & $2.8 \pm 0.2$ & $2.9 \pm 0.2$ \\
\hline & ॥ & $2.8 \pm 0.1$ & $2.8 \pm 0.2$ & $2.8 \pm 0.2$ & $2.8 \pm 0.2$ \\
\hline \multirow[t]{2}{*}{ Myonuclear domain $\left(\mu \mathrm{m}^{2}\right)$} & । & $2026 \pm 86$ & $1716 \pm 106 *$ & $2035 \pm 89$ & $1914 \pm 97 *$ \\
\hline & ॥ & $2072 \pm 112$ & $1770 \pm 126^{*}$ & $1843 \pm 111$ & $1791 \pm 112 *$ \\
\hline \multirow[t]{2}{*}{ SC (n/fibre) } & । & $0.101 \pm 0.014$ & $0.091 \pm 0.013$ & $0.099 \pm 0.010$ & $0.099 \pm 0.007$ \\
\hline & $\|$ & $0.056 \pm 0.008 \#$ & $0.055 \pm 0.009 \#$ & $0.062 \pm 0.006 \#$ & $0.060 \pm 0.006 \#$ \\
\hline \multirow[t]{2}{*}{$\mathrm{SC}\left(\mathrm{n} / \mathrm{mm}^{2}\right)$} & । & $18.1 \pm 2.5$ & $18.0 \pm 2.3$ & $17.7 \pm 2.1$ & $19.1 \pm 1.6$ \\
\hline & ॥ & $10.1 \pm 1.5 \#$ & $10.3 \pm 1.4 \#$ & $11.8 \pm 1.1 \#$ & $12.7 \pm 1.6 \#$ \\
\hline \multirow[t]{2}{*}{ SC (n/myonuclei, \%) } & । & $3.6 \pm 0.4$ & $3.3 \pm 0.5$ & $3.7 \pm 0.5$ & $3.6 \pm 0.3$ \\
\hline & ॥ & $1.9 \pm 0.2 \#$ & $2.0 \pm 0.3 \#$ & $2.2 \pm 0.2 \#$ & $2.2 \pm 0.2 \#$ \\
\hline
\end{tabular}

Data represent means \pm SEM. Abbreviations: CSA, cross-sectional area; SC, satellite cell; SC, $\mathrm{n} /$ myonuclei (\%), the number of SCS as a percentage of the total number of myonuclei (i.e. number of myonuclei + number of $\mathrm{SCS})$. \# Significantly different from values in type I fibre $(P<0.05)$. * Significantly different from preimmobilization values $(P<0.05)$

\section{mRNA expression}

Figure 3 and Supplemental Figure 1 display the skeletal muscle mRNA expression of the selected genes of interest. Muscle mRNA expression of myostatin (Figure $3 \mathrm{~A}$ ) and myogenin (Figure $3 C$ ) increased following immobilization in both groups $(P<0.05)$, whereas myoD (Figure $3 \mathrm{~B}$ ) tended towards an increase in both groups $(P=0.07)$. MAFBx mRNA expression (Figure 3D) showed a significant time*treatment interaction $(P<0.05)$ with a significant increase only detected in the PRO group $(P<0.05)$ following immobilization. Muscle MuRF1 mRNA (Figure 3E) significantly increased in both groups (time effect, $P<0.01)$. There was a trend for an interaction effect $(P=0.07)$ such that MuRF1 mRNA expression was increased to a greater extent in PRO $(P<0.05)$ compared with CON $(P>0.05)$. For the mRNA expression of both amino acid transporters LAT1 (Supplemental Figure 1D) and PAT1 (Supplemental Figure 1E), a significant time effect was found $(P<0.01$ for both genes) such that expression was upregulated following immobilization in both groups. All other genes showed no significant changes between or within groups. 

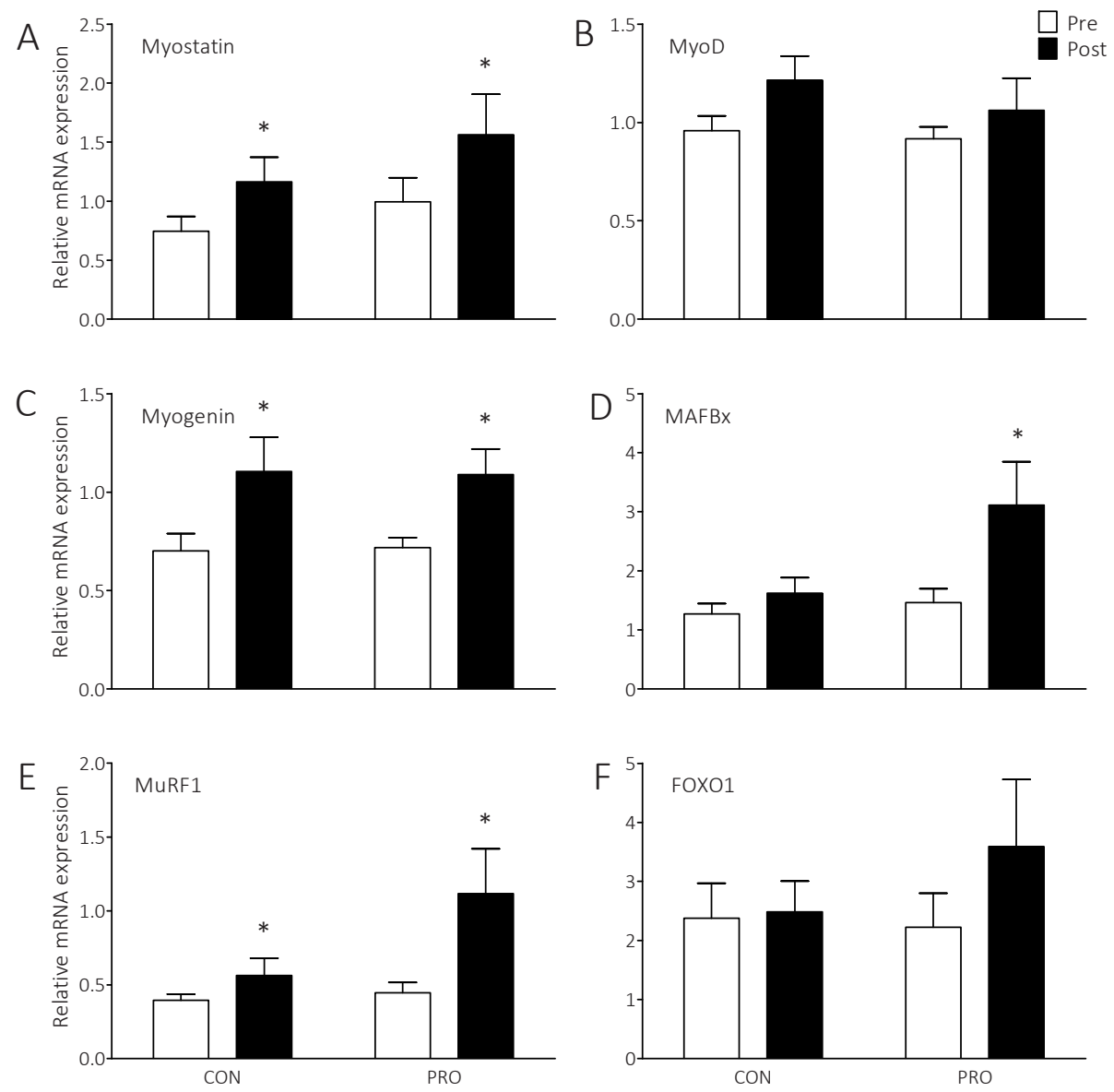

Figure 3 | Skeletal muscle mRNA expression of selected genes involved in myogenesis (A-B-C) and muscle proteolysis (D-E-F), measured $48 \mathrm{~h}$ prior to and immediately following 5 days of one-legged knee immobilization in healthy elderly men in the $\operatorname{CON}(n=12)$ and PRO $(n=11)$ group. ${ }^{*} P<0.05$; significantly different when compared with pre-immobilization values. Data are expressed as means $\pm S E M$. Abbreviations: MAFBx, Muscle Atrophy F-box; MuRF1, Muscle RING-finger protein-1; FOX01, Forkhead box protein O1. 


\section{DISCUSSION}

In the present study, we demonstrate that merely 5 days of one-legged knee immobilization leads to substantial skeletal muscle mass and strength loss in healthy, elderly men. Increasing dietary protein intake by supplementing $\sim 20 \mathrm{~g}$ protein twice-daily did not attenuate the loss of muscle mass or strength during 5 days of muscle disuse in older males.

A period of prolonged muscle disuse and the associated muscle atrophy causes numerous negative health consequences $[1,2,4,6]$, and the occurrence of successive periods of muscle disuse likely represents a key factor responsible for the loss of muscle mass during the later decades of our lifespan [13]. In the present study, we report that only 5 days of muscle disuse already leads to substantial loss of muscle mass ($1.5 \pm 0.7 \%$; Figure $2 \mathrm{~A})$ and strength $(-8.3 \pm 3.3 \%$; Figure $2 \mathrm{~B})$ in older individuals. These data are in line with recent data from our group in which we observed similar muscle mass and strength losses in younger individuals [8]. Furthermore, Suetta et al. reported significant muscle fibre atrophy after 4 days of immobilization in both young and older individuals [10]. The rapid muscle atrophy observed in our older subjects after merely 5 days of leg immobilization is of important clinical significance, as successive short periods of muscle disuse due to illness or injury are highly prevalent during the later stages of our lifespan [36]. In line, the average length of hospitalization for elderly patients admitted with acute illness is $5-7$ days [11]. The observed muscle loss is of particular relevance as the older population has difficulty to regain skeletal muscle mass and strength following a period of disuse [37]. Even when applying rehabilitative resistancetype exercise training after a period of disuse, muscle mass does not seem to be restored after 4 weeks of intense supervised training [37]. For these reasons, it is presently believed that the impact of short successive episodes of muscle disuse may be of key relevance in the development of sarcopenia [13].

Practical and effective interventional strategies are needed to prevent or attenuate muscle mass and strength loss during short periods of muscle disuse in healthy elderly as well as more clinically compromised subpopulations. It has been proposed that simply increasing the protein content of the diet may alleviate the loss of muscle tissue during a period of disuse $[12,27]$. Indeed, studies focusing on mimicking prolonged hospitalization (i.e. >2-3 weeks bed rest under tightly controlled dietary conditions) have shown that supplementation with high doses of crystalline essential amino acids ( $\sim 50 \mathrm{~g}$, equivalent to $\sim 100-150 \mathrm{~g}$ intact protein) attenuates the loss of muscle mass [29-31]. Given the clinical relevance of short, successive periods of muscle disuse, we assessed the efficacy of a more practical and feasible dietary strategy to attenuate muscle loss during a short period of limb immobilization under free living conditions. Increasing dietary protein intake from 1.1 to $1.6 \mathrm{~g} \cdot \mathrm{kg}$ body weight ${ }^{-1} \cdot \mathrm{day}^{-1}$ did not rescue the loss of muscle mass or strength observed during a 5 day period of leg immobilization (Figure 2). The apparent discrepancy between the outcome of the present study and previous 
work in prolonged bed rest studies may be attributed to differences in protein intake in the control group. In the present study the control group retained normal habitual energy and protein intake $\left(1.1 \mathrm{~g} \cdot \mathrm{kg}\right.$ body weight $\left.{ }^{-1} \cdot \mathrm{day}^{-1}\right)$ whereas the protein group received additional supplementation $\left(1.6 \mathrm{~g} \cdot \mathrm{kg}\right.$ body weight $\left.{ }^{-1} \cdot \mathrm{day}^{-1}\right)$. In contrast, in previous bed rest studies that show benefits of amino acid supplementation on muscle mass maintenance, the control groups generally consumed dietary protein at a level no higher than $0.8 \mathrm{~g} \cdot \mathrm{kg}$ body weight ${ }^{-1} \cdot$ day $^{-1}$ [29-31]. Consequently, we speculate that maintaining dietary protein intake is required to prevent muscle loss during disuse, but that increasing dietary protein intake above habitual levels does not further alleviate muscle loss during disuse $[38,39]$. This would be of particular relevance in institutionalized or hospitalized elderly who are unable to maintain habitual dietary protein consumption during more prolonged periods of muscle disuse due to illness or injury. Additional considerations of the present nutritional intervention include the type and timing of protein administered. We selected whey protein in the present study as we have previously shown it leads to greater postprandial muscle protein accretion compared with casein protein in healthy elderly men [40]. We chose to supplement volunteers at breakfast time since we have previously shown that community dwelling elderly individuals generally consume inadequate amounts of protein at breakfast [41]. Specifically, the supplement was consumed directly after breakfast to avoid volunteers compensating for the supplement by consuming less breakfast and therefore ensuring adequate protein was consumed. This was achieved given that the PRO group consumed $(36 \pm 2 \mathrm{~g}$ at this meal compared to the CON group who only consumed $13 \pm 1 \mathrm{~g}$, the latter being an amount insufficient to properly stimulate muscle protein synthesis rates [42]. We opted to deliver the second supplement immediately prior to sleep, since we have recently shown that such a strategy effectively stimulates overnight muscle protein synthesis rates [43]. However, it is also true that these beneficial effects on nocturnal muscle protein synthesis were obtained with the ingestion (or intragastric administration) of large amounts of casein protein, to ensure a more sustained hyperaminoacidemia throughout the night [44]. Accordingly, it could be speculated that future nutritional strategies aimed at attenuating muscle disuse atrophy may wish to consider incorporating large boluses of casein as a pre-bedtime meal. In contrast, it could also be hypothesized that ingestion of a large bolus of dietary protein prior to sleep increases both muscle protein synthesis and breakdown rates, without net muscle protein accretion [45]. Though previous work has shown improvements in overnight whole-body protein balance following protein administration in healthy older men [43] and in young adults during overnight recovery from exercise [46], we cannot exclude that such improvements in overnight protein balance may not occur in a setting of muscle disuse.

Besides assessing the impact of protein supplementation on muscle mass and strength during short-term disuse, we wished to gain insight into the underlying myocellular mechanisms involved in muscle disuse atrophy and/or muscle mass maintenance. Muscle loss during short-term muscle disuse is thought to be, at least partly, 
mediated by accelerated rates of muscle protein breakdown [13]. Myostatin is known as a negative regulator of muscle growth in vivo [47], and acts through multiple pathways including the stimulation of muscle protein breakdown [48]. Consistent with this role, we observed increases in myostatin mRNA expression (Figure 3) and in markers of muscle protein breakdown (i.e. increased gene expression of MAFBx and MuRF1; Figure $3)$. This is in line with previous findings [10] and our own work in young men [8,9], and supportive of a role for muscle protein breakdown in short-term muscle atrophy, possibly mediated through increased myostatin transcription. Given the lack of effect of protein supplementation on muscle mass in the present study, it is not surprising that we observed no attenuation of the rise in myostatin and markers of proteolysis. In fact, we actually observed that MAFBx and MuRF1 gene expression increased to a greater extent in the PRO group (Figure 3), supporting the idea that increasing dietary protein intake beyond the habitual dietary protein intake level may strongly stimulate overall protein turnover rates.

Myostatin is also reported to regulate muscle size by acting via the inhibition of myogenesis through its inhibitory action on the myogenic regulatory factors [49]. However, in line with our previous work [8,50], we report that the disuse-induced increase in myostatin expression does not coincide with impaired expression of the myogenic regulatory factors (i.e. MyoD and myogenin, Figure 3). Moreover, no alterations in muscle satellite cell content were observed, suggesting that the mechanisms underlying shortterm disuse atrophy do not require alterations in myogenesis or satellite cell content. Recent data have suggested that the expression of specific amino acid transporters within skeletal muscle provide a site of regulation for muscle protein synthesis [51]. As such, we analyzed the gene expression of Large Neutral Amino Acid Transporter 1 (LAT1/SLC) and Proton-coupled amino acid transporter 1 (PAT1) which are thought to be the key transporters facilitating intramuscular transport of BCAAs particularly in response to nutrition [52]. Interestingly, LAT1 and PAT1 mRNA expression (Supplemental Figure 1) increased following immobilization in both groups, possibly indicating a compensatory mechanism by which atrophying muscle attempts to 'scavenge' circulating amino acids as a substrate for muscle protein synthesis.

In the present study we show that protein supplementation on top of a diet containing ample protein (1.1 g.kg body weight ${ }^{-1} \cdot$ day $^{-1}$ ) does not alleviate muscle loss during short-term single leg disuse. This shows that besides maintaining dietary protein intake, other strategies are warranted to help maintain muscle mass. Where possible, performing some degree of exercise should be considered during disuse [39]. In conditions where exercise is not feasible due to injury or illness, low-volume physical activity [53] or even exercise surrogates [8] could be suggested. Furthermore, other nutritional compounds, such as creatine or omega-3 fatty acids, may support muscle maintenance during disuse [27]. An often under-appreciated consideration is how dietary strategies could support rehabilitation following a period of disuse. This area has been comparatively under studied [54-57] but, given the opportunity to combine nutrition with re- 
ambulation and/or physical exercise, future research should address how dietary protein and/or other nutritional strategies could best be used to facilitate the rapid and complete restoration of muscle mass following a period of disuse.

In short, we conclude that short-term muscle disuse results in a substantial decline in both muscle mass and strength in older individuals. Increasing dietary protein intake during short-term muscle disuse on top of a diet providing $>1.0 \mathrm{~g} \cdot \mathrm{kg}$ body weight ${ }^{-1} \cdot$ day $^{-1}$ does not alleviate muscle disuse atrophy in healthy, elderly men.

\section{ACKNOWLEDGEMENTS}

We greatly appreciate the expertise of the staff in the casting room of the Academic Hospital Maastricht. Additionally, the technical assistance of Antoine Zorenc has been of great help while performing the muscle analyses. We would also like to thank Danone Nutricia Research for the provision of the protein beverages used in this study. 


\section{REFERENCES}

1. Deitrick, J.E., The effect of immobilization on metabolic and physiological functions of normal men. Bull $N$ Y Acad Med, 1948. 24(6): p. 364-75.

2. Ingemann-Hansen, T. and J. Halkjaer-Kristensen, Computerized tomographic determination of human thigh components. The effects of immobilization in plaster and subsequent physical training. Scand J Rehabil Med, 1980. 12(1): p. 27-31.

3. White, M.J., C.T. Davies, and P. Brooksby, The effects of short-term voluntary immobilization on the contractile properties of the human triceps surae. Q J Exp Physiol, 1984. 69(4): p. 685-91.

4. LeBlanc, A.D., V.S. Schneider, H.J. Evans, C. Pientok, R. Rowe, and E. Spector, Regional changes in muscle mass following 17 weeks of bed rest. J Appl Physiol, 1992. 73(5): p. 2172-8.

5. Stuart, C.A., R.E. Shangraw, M.J. Prince, E.J. Peters, and R.R. Wolfe, Bed-rest-induced insulin resistance occurs primarily in muscle. Metab Clin Exp, 1988. 37(8): p. 802-6.

6. Haruna, Y., Y. Suzuki, K. Kawakubo, R. Yanagibori, and A. Gunji, Decremental reset in basal metabolism during 20-days bed rest. Acta Physiol (Scand). Supplementum, 1994. 616: p. 43-9.

7. Tzankoff, S.P. and A.H. Norris, Effect of muscle mass decrease on age-related BMR changes. J Appl Physiol Respir Environ Exerc Physiol, 1977. 43(6): p. 1001-6.

8. Dirks, M.L., B.T. Wall, T. Snijders, C.L. Ottenbros, L.B. Verdijk, and L.J. van Loon, Neuromuscular electrical stimulation prevents muscle disuse atrophy during leg immobilization in humans. Acta Physiol (Oxf), 2014. 210(3): p. 628-41.

9. Wall, B.T., M.L. Dirks, T. Snijders, J.M. Senden, J. Dolmans, and L.J. van Loon, Substantial skeletal muscle loss occurs during only 5 days of disuse. Acta Physiol (Oxf), 2014. 210(3): p. 600-11.

10. Suetta, C., U. Frandsen, L. Jensen, M.M. Jensen, J.G. Jespersen, L.G. Hvid, M. Bayer, S.J. Petersson, H.D. Schroder, J.L. Andersen, K.M. Heinemeier, P. Aagaard, P. Schjerling, and M. Kjaer, Aging affects the transcriptional regulation of human skeletal muscle disuse atrophy. PloS One, 2012. 7(12): p. e51238.

11. Fisher, S.R., Y.F. Kuo, J.E. Graham, K.J. Ottenbacher, and G.V. Ostir, Early ambulation and length of stay in older adults hospitalized for acute illness. Arch Intern Med, 2010. 170(21): p. 1942-3.

12. English, K.L. and D. Paddon-Jones, Protecting muscle mass and function in older adults during bed rest. Curr Opin Clin Nutr Metab Care, 2010. 13(1): p. 34-9.

13. Wall, B.T., M.L. Dirks, and L.J. van Loon, Skeletal muscle atrophy during short-term disuse: implications for age-related sarcopenia. Ageing Res Rev, 2013. 12(4): p. 898-906.

14. Ferrando, A.A., H.W. Lane, C.A. Stuart, J. Davis-Street, and R.R. Wolfe, Prolonged bed rest decreases skeletal muscle and whole body protein synthesis. Am J Physiol, 1996. 270(4 Pt 1): p. E627-33.

15. Ferrando, A.A., K.D. Tipton, M.M. Bamman, and R.R. Wolfe, Resistance exercise maintains skeletal muscle protein synthesis during bed rest. J Appl Physiol, 1997. 82(3): p. 807-10.

16. Kortebein, P., A. Ferrando, J. Lombeida, R. Wolfe, and W.J. Evans, Effect of 10 days of bed rest on skeletal muscle in healthy older adults. J Am Med Assoc, 2007. 297(16): p. 1772-4.

17. Glover, E.I., S.M. Phillips, B.R. Oates, J.E. Tang, M.A. Tarnopolsky, A. Selby, K. Smith, and M.J. Rennie, Immobilization induces anabolic resistance in human myofibrillar protein synthesis with low and high dose amino acid infusion. J Physiol, 2008. 586(Pt 24): p. 6049-61.

18. Gibson, J.N., D. Halliday, W.L. Morrison, P.J. Stoward, G.A. Hornsby, P.W. Watt, G. Murdoch, and M.J. Rennie, Decrease in human quadriceps muscle protein turnover consequent upon leg immobilization. Clin Sci (Lond), 1987. 72(4): p. 503-9.

19. de Boer, M.D., A. Selby, P. Atherton, K. Smith, O.R. Seynnes, C.N. Maganaris, N. Maffulli, T. Movin, M.V. Narici, and M.J. Rennie, The temporal responses of protein synthesis, gene expression and cell signalling in human quadriceps muscle and patellar tendon to disuse. J Physiol, 2007. 585(Pt 1): p. 241-51.

20. Wall, B.T., T. Snijders, J.M. Senden, C.L. Ottenbros, A.P. Gijsen, L.B. Verdijk, and L.J. van Loon, Disuse impairs the muscle protein synthetic response to protein ingestion in healthy men. J Clin Endocrinol Metab, 2013. 98(12): p. 4872-81. 
21. Biolo, G., B. Ciocchi, M. Lebenstedt, R. Barazzoni, M. Zanetti, P. Platen, M. Heer, and G. Guarnieri, Shortterm bed rest impairs amino acid-induced protein anabolism in humans. J Physiol, 2004. 558(Pt 2): p. 381-8.

22. Biolo, G., B. Ciocchi, M. Lebenstedt, M. Heer, and G. Guarnieri, Sensitivity of whole body protein synthesis to amino acid administration during short-term bed rest. J Gravit Physiol, 2002. 9(1): p. P197-8.

23. Abadi, A., E.I. Glover, R.J. Isfort, S. Raha, A. Safdar, N. Yasuda, J.J. Kaczor, S. Melov, A. Hubbard, X. Qu, S.M. Phillips, and M. Tarnopolsky, Limb immobilization induces a coordinate down-regulation of mitochondrial and other metabolic pathways in men and women. PLoS One, 2009. 4(8): p. e6518.

24. Glover, E.I., N. Yasuda, M.A. Tarnopolsky, A. Abadi, and S.M. Phillips, Little change in markers of protein breakdown and oxidative stress in humans in immobilization-induced skeletal muscle atrophy. Appl Physiol Nutr Metab, 2010. 35(2): p. 125-33.

25. Gustafsson, T., T. Osterlund, J.N. Flanagan, F. von Walden, T.A. Trappe, R.M. Linnehan, and P.A. Tesch, Effects of 3 days unloading on molecular regulators of muscle size in humans. J Appl Physiol, 2010. 109(3): p. 721-7.

26. Phillips, S.M., E.I. Glover, and M.J. Rennie, Alterations of protein turnover underlying disuse atrophy in human skeletal muscle. J Appl Physiol, 2009. 107(3): p. 645-54.

27. Wall, B.T. and L.J. van Loon, Nutritional strategies to attenuate muscle disuse atrophy. Nutr Rev, 2013. 71(4): p. 195-208.

28. Rennie, M.J., R.H. Edwards, D. Halliday, D.E. Matthews, S.L. Wolman, and D.J. Millward, Muscle protein synthesis measured by stable isotope techniques in man: the effects of feeding and fasting. Clin Sci (Lond), 1982. 63(6): p. 519-23.

29. Paddon-Jones, D., M. Sheffield-Moore, R.J. Urban, A.P. Sanford, A. Aarsland, R.R. Wolfe, and A.A. Ferrando, Essential amino acid and carbohydrate supplementation ameliorates muscle protein loss in humans during 28 days bedrest. J Clin Endocrinol Metab, 2004. 89(9): p. 4351-8.

30. Stein, T.P., M.R. Donaldson, M.J. Leskiw, M.D. Schluter, D.W. Baggett, and G. Boden, Branched-chain amino acid supplementation during bed rest: effect on recovery. J Appl Physiol, 2003. 94(4): p. 1345-52.

31. Stuart, C.A., R.E. Shangraw, E.J. Peters, and R.R. Wolfe, Effect of dietary protein on bed-rest-related changes in whole-body-protein synthesis. Am J Clin Nutr, 1990. 52(3): p. 509-14.

32. Ferrando, A.A., D. Paddon-Jones, N.P. Hays, P. Kortebein, O. Ronsen, R.H. Williams, A. McComb, T.B. Symons, R.R. Wolfe, and W. Evans, EAA supplementation to increase nitrogen intake improves muscle function during bed rest in the elderly. Clin Nutr, 2010. 29(1): p. 18-23.

33. Verdijk, L.B., R. Koopman, G. Schaart, K. Meijer, H.H. Savelberg, and L.J. van Loon, Satellite cell content is specifically reduced in type II skeletal muscle fibers in the elderly. Am J Physiol Endocrinol Metab, 2007. 292(1): p. E151-7.

34. Waterval, W.A., J.L. Scheijen, M.M. Ortmans-Ploemen, C.D. Habets-van der Poel, and J. Bierau, Quantitative UPLC-MS/MS analysis of underivatised amino acids in body fluids is a reliable tool for the diagnosis and follow-up of patients with inborn errors of metabolism. Clin Chim Acta, 2009. 407(1-2): p. 36-42.

35. Bonstato. Dieetinzicht. 2012 [cited 2013 July 18]; Available from: www.dieetinzicht.nl.

36. Hallal, P.C., L.B. Andersen, F.C. Bull, R. Guthold, W. Haskell, U. Ekelund, and G. Lancet Physical Activity Series Working, Global physical activity levels: surveillance progress, pitfalls, and prospects. Lancet, 2012. 380(9838): p. 247-57.

37. Suetta, C., U. Frandsen, A.L. Mackey, L. Jensen, L.G. Hvid, M.L. Bayer, S.J. Petersson, H.D. Schroder, J.L. Andersen, P. Aagaard, P. Schjerling, and M. Kjaer, Ageing is associated with diminished muscle re-growth and myogenic precursor cell expansion early after immobility-induced atrophy in human skeletal muscle. J Physiol, 2013. 591(Pt 15): p. 3789-804.

38. Stein, T.P. and S. Blanc, Does protein supplementation prevent muscle disuse atrophy and loss of strength? Crit Rev Food Sci, 2011. 51(9): p. 828-34.

39. Trappe, T.A., N.A. Burd, E.S. Louis, G.A. Lee, and S.W. Trappe, Influence of concurrent exercise or nutrition countermeasures on thigh and calf muscle size and function during 60 days of bed rest in women. Acta Physiol (Oxf), 2007. 191(2): p. 147-59. 
40. Pennings, B., Y. Boirie, J.M.G. Senden, A.P. Gijsen, H. Kuipers, and L.J.C. van Loon, Whey protein stimulates postprandial muscle protein accretion more effectively than do casein and casein hydrolysate in older men. Am J Clin Nutr, 2011. 93(5): p. 997-1005.

41. Tieland, M., K.J. Borgonjen-Van den Berg, L.J. van Loon, and L.C. de Groot, Dietary protein intake in community-dwelling, frail, and institutionalized elderly people: scope for improvement. Eur J Nutr, 2012. 51(2): p. 173-9.

42. Pennings, B., B. Groen, A. de Lange, A.P. Gijsen, A.H. Zorenc, J.M. Senden, and L.J. van Loon, Amino acid absorption and subsequent muscle protein accretion following graded intakes of whey protein in elderly men. Am J Physiol Endocrinol Metab, 2012. 302(8): p. E992-9.

43. Groen, B.B., P.T. Res, B. Pennings, E. Hertle, J.M. Senden, W.H. Saris, and L.J. van Loon, Intragastric protein administration stimulates overnight muscle protein synthesis in elderly men. Am J Physiol Endocrinol Metab, 2012. 302(1): p. E52-60.

44. Boirie, Y., M. Dangin, P. Gachon, M.P. Vasson, J.L. Maubois, and B. Beaufrere, Slow and fast dietary proteins differently modulate postprandial protein accretion. Proc Natl Acad Sci U S A, 1997. 94(26): p. 14930-5.

45. Garlick, P.J., M.A. McNurlan, and P.E. Ballmer, Influence of dietary protein intake on whole-body protein turnover in humans. Diabetes Care, 1991. 14(12): p. 1189-98.

46. Res, P.T., B. Groen, B. Pennings, M. Beelen, G.A. Wallis, A.P. Gijsen, J.M. Senden, and V.A.N.L. LJ, Protein ingestion before sleep improves postexercise overnight recovery. Med Sci Sports Exerc, 2012. 44(8): p. 1560-9.

47. McPherron, A.C., A.M. Lawler, and S.J. Lee, Regulation of skeletal muscle mass in mice by a new TGF-beta superfamily member. Nature, 1997. 387(6628): p. 83-90.

48. McFarlane, C., E. Plummer, M. Thomas, A. Hennebry, M. Ashby, N. Ling, H. Smith, M. Sharma, and R. Kambadur, Myostatin induces cachexia by activating the ubiquitin proteolytic system through an NFkappaB-independent, FoxO1-dependent mechanism. J Cell Physiol, 2006. 209(2): p. 501-14.

49. Amthor, H., R. Huang, I. McKinnell, B. Christ, R. Kambadur, M. Sharma, and K. Patel, The regulation and action of myostatin as a negative regulator of muscle development during avian embryogenesis. Dev Biol, 2002. 251(2): p. 241-57.

50. Snijders, T., B.T. Wall, M.L. Dirks, J.M. Senden, F. Hartgens, J. Dolmans, M. Losen, L.B. Verdijk, and L.J. van Loon, Muscle disuse atrophy is not accompanied by changes in skeletal muscle satellite cell content. Clin Sci (Lond), 2014. 126(8): p. 557-66.

51. Dickinson, J.M. and B.B. Rasmussen, Essential amino acid sensing, signaling, and transport in the regulation of human muscle protein metabolism. Curr Opin Clin Nutr Metab Care, 2011. 14(1): p. 83-8.

52. Dickinson, J.M. and B.B. Rasmussen, Amino acid transporters in the regulation of human skeletal muscle protein metabolism. Curr Opin Clin Nutr Metab Care, 2013. 16(6): p. 638-44.

53. Oates, B.R., E.I. Glover, D.W. West, J.L. Fry, M.A. Tarnopolsky, and S.M. Phillips, Low-volume resistance exercise attenuates the decline in strength and muscle mass associated with immobilization. Muscle Nerve, 2010. 42(4): p. 539-46.

54. Hespel, P., B. Op't Eijnde, M. Van Leemputte, B. Urso, P.L. Greenhaff, V. Labarque, S. Dymarkowski, P. Van Hecke, and E.A. Richter, Oral creatine supplementation facilitates the rehabilitation of disuse atrophy and alters the expression of muscle myogenic factors in humans. J Physiol, 2001. 536(Pt 2): p. 625-33.

55. Magne, H., I. Savary-Auzeloux, C. Migne, M.A. Peyron, L. Combaret, D. Remond, and D. Dardevet, Unilateral hindlimb casting induced a delayed generalized muscle atrophy during rehabilitation that is prevented by a whey or a high protein diet but not a free leucine-enriched diet. PLoS One, 2013. 8(8): p. e70130.

56. Savary-Auzeloux, I., H. Magne, C. Migne, M. Oberli, D. Breuille, M. Faure, K. Vidal, M. Perrot, D. Remond, L. Combaret, and D. Dardevet, A dietary supplementation with leucine and antioxidants is capable to accelerate muscle mass recovery after immobilization in adult rats. PLoS One, 2013. 8(11): p. e81495.

57. Alway, S.E., S.L. Pereira, N.K. Edens, Y. Hao, and B.T. Bennett, beta-Hydroxy-beta-methylbutyrate (HMB) enhances the proliferation of satellite cells in fast muscles of aged rats during recovery from disuse atrophy. Exp Gerontol, 2013. 48(9): p. 973-84. 


\section{SUPPLEMENTAL MATERIAL}

Supplemental Table 1 | Composition of the study product

\begin{tabular}{|c|c|c|}
\hline Component & Unit & PRO \\
\hline Energy & $\mathrm{kcal} / \mathrm{kJ}$ & $150 / 635$ \\
\hline Protein & $\%$ & 55 \\
\hline Carbohydrates & $\%$ & 25 \\
\hline Fat & $\%$ & 18 \\
\hline Fibre & $\%$ & 2 \\
\hline \multicolumn{3}{|l|}{ Protein } \\
\hline Total & g & 20.7 \\
\hline Total EAA & g & 10.6 \\
\hline Total leucine & $\mathrm{g}$ & 2.8 \\
\hline Total phenylalanine & g & 0.6 \\
\hline \multicolumn{3}{|l|}{ Carbohydrates } \\
\hline Total & g & 9.4 \\
\hline Sugars & g & 4.2 \\
\hline \multicolumn{3}{|l|}{ Fat } \\
\hline Total & g & 3.0 \\
\hline Saturated & g & 0.8 \\
\hline \multicolumn{3}{|l|}{ Fibre } \\
\hline Total & g & 1.3 \\
\hline Soluble & g & 1.3 \\
\hline \multicolumn{3}{|l|}{ Minerals } \\
\hline Sodium & mg & 150 \\
\hline Potassium & mg & 279 \\
\hline Chloride & mg & 70 \\
\hline Calcium & mg & 500 \\
\hline Phosphorus & mg & 250 \\
\hline Magnesium & mg & 37 \\
\hline \multicolumn{3}{|l|}{ Trace elements } \\
\hline Iron & mg & 2.4 \\
\hline Zinc & mg & 2.2 \\
\hline Copper & $\mu g$ & 270 \\
\hline Manganese & mg & 0.50 \\
\hline Fluoride & mg & 0.15 \\
\hline Molybdenum & $\mu g$ & 15 \\
\hline Selenium & $\mu g$ & 15 \\
\hline Chromium & $\mu g$ & 7.5 \\
\hline lodine & $\mu \mathrm{g}$ & 20 \\
\hline
\end{tabular}




\begin{tabular}{lcc}
\hline Component & Unit & PRO \\
\hline Vitamins & $\mu \mathrm{g}-\mathrm{RE}$ & 152 \\
Vitamin A & $\mu \mathrm{g}$ & 20 \\
Cholecalciferol & $\mathrm{mg} \alpha$-TE & 7.5 \\
Vitamin E & $\mu \mathrm{g}$ & 12 \\
Phylloquinone & $\mathrm{mg}$ & 0.23 \\
Thiamin & $\mathrm{mg}$ & 0.25 \\
Riboflavin & $\mathrm{mg} \mathrm{NE}$ & 8.8 \\
Niacin & $\mathrm{mg}$ & 0.81 \\
Pantothenic acid & $\mathrm{mg}$ & 0.76 \\
Vitamin B6 & $\mu \mathrm{g}$ & 203 \\
Folic acid & $\mu \mathrm{g}$ & 3.0 \\
Vitamin B12 & $\mu \mathrm{g}$ & 6.1 \\
Biotin & $\mu \mathrm{g}$ & 32 \\
Vitamin C & & \\
Extra additions & $\mathrm{mg}$ & 0.30 \\
Carotenoids & $\mathrm{mg}$ & 56 \\
Choline &
\end{tabular}

Data are presented as mean values, $n=23$. Abbreviations used: $E A A$, essential amino acid; $N E$, niacin equivalents; RE, retinol equivalents; $\alpha$-TE, $\alpha$-tocopherol equivalents.

\section{Muscle analyses}

Muscle samples that were mounted and frozen in Tissue-Tek were cut into $5 \mu \mathrm{m}$ thick cryosections using a cryostat at $-20^{\circ} \mathrm{C}$. Samples were carefully aligned for cross-sectional fibre analyses. Pre and post immobilization samples from one PRO and one CON subject were mounted together on uncoated, pre-cleaned glass slides. All biopsies were stained for muscle fibre type (FT) and satellite cell (SC) content. At the start of the staining procedure, glass slides were incubated with primary antibodies against myosin heavy chain (MHC)-I (A4.840, dilution 1:25, Developmental Studies Hybridoma Bank, lowa City, IA), laminin (polyclonal rabbit anti-laminin, dilution 1:50; Sigma, Zwijndrecht, the Netherlands) and CD56 (dilution 1:40; BD Biosciences, San Jose, CA). CD56 has been used in previous research by ourselves [1-3] and others [4] for determination of SC content in human skeletal muscle. After washing, slides were incubated with the appropriate secondary antibodies: goat anti-rabbit IgG AlexaFluor647, goat anti-mouse IgM AlexaFluor555, and Streptavidin Alexa 488 (dilution 1:400, 1:500, and 1:200, respectively; Molecular Probes, Invitrogen, Breda, the Netherlands). Nuclei were stained with 4,6diamidino-2-phenylindole (DAPI, $0.238 \mu \mathrm{M}$; Molecular Probes). All incubations steps were done at room temperature. Both primary and secondary antibodies were diluted in $0.1 \%$ Bovine Serum Albumin (BSA) in 0.1\% Tween- phosphate-buffered saline (PBS). The staining procedure was done as follows. After slides were fixated in acetone for 5 
min, slides were air dried and incubated for 30 min with 3\% BSA in 0.1\% Tween-PBS. After a 5 min washing step with PBS, slides were incubated with CD56 in 0.1\% BSA in $0.1 \%$ Tween-PBS for $2 \mathrm{~h}$. Afterwards slides were washed (standard washing protocol: 5 min $0.1 \%$ Tween-PBS, $2 \times 5$ min PBS) and incubated with goat anti-mouse Biotin (dilution 1:133, Vector Laboratories, Inc., Burlingame, CA) for 60 min. After washing, slides were incubated with Steptavidin for $30 \mathrm{~min}$. Thereafter, slides were washed and incubated with primary antibodies against MHC-1 and laminin for $30 \mathrm{~min}$. Slides were washed and the appropriate secondary antibodies were applied, diluted together with DAPI. After a final washing step, all slides were mounted with cover glasses using Mowiol (Calbiochem, Amsterdam, the Netherlands). Staining procedures resulted in nuclei stained in blue, CD56 in green, MHC-I in red, and laminin in far-red. Images were visualized and automatically captured at 10x magnification with a fluorescent microscope equipped with an automatic stage (IX81 motorised inverted microscope, Olympus, Hamburg, Germany) and EXi Aqua CCD camera (Qlmaging, Surrey, BC, Canada). Image acquisition was performed by Micro-Manager 1.4 software as done before [5]. Analysis of the recorded images was performed by an investigator blinded to subject coding. To assess fibre circularity, form factors were calculated by using the following formula: $(4 \pi \cdot \mathrm{CSA}) \cdot(\text { perimeter })^{-2}$. Fibre circularity did not change over time or between groups. Mean numbers of $148 \pm 12$ and $151 \pm 12$ fibres were analyzed in pre- and postimmobilization samples, respectively.

The part of the muscle that was directly frozen in liquid nitrogen was used to determine mRNA expression of several genes of interest. Total RNA was isolated by using Tri Reagent (Sigma-Aldrich) on 10-20 mg of frozen muscle, according to the manufacturer's protocol. Quantification of total RNA was carried out spectrophotometrically at $260 \mathrm{~nm}$ (NanoDrop ND-1000 Spectrophotometer, Thermo Fisher Scientific, USA), and RNA purity was determined as the ratio of readings at 260/280 nm. Subsequently, first strand cDNA was synthesized from $1 \mu \mathrm{g}$ RNA sample using random primers (Promega) and PowerScript Reverse Transcriptase (AppliedBiosystems, USA). Taqman PCR was carried out using an $\mathrm{ABI}$ Prism 7000 sequence detector (AppliedBiosystems, USA), with $2 \mu \mathrm{L}$ of CDNA, $18 \mu \mathrm{l} \cdot \mathrm{L}^{-1}$ of each primer, $5 \mu \mathrm{l} \cdot \mathrm{L}^{-1}$ probe, and Universal Taqman $2 \times \mathrm{PCR}$ mastermix (Eurogentec) in a final volume of $25 \mu \mathrm{L}$. Each sample was run in duplicate, in duplex reactions, with a separate standard curve included for each gene (serial dilutions of cDNA synthesized in parallel with the study sample). 18S was used as a housekeeping gene as an internal control, and similarly to previous human immobilization studies [6, 7] it seemed unaffected by treatment (i.e. mean $\mathrm{C} t$ values did not change over time in each of the intervention groups; data not shown). Taqman primer/probe sets (Applied Biosystems, Foster City, USA) were obtained for the following genes of interest: mammalian target of rapamycin (mTOR), P70S6 kinase (P70S6K), myogenic factor 4 (myogenin), MyoD, myostatin, Atrogin-1/Muscle Atrophy F-box (MAFbx), Muscle RING-finger protein-1 (MuRF1), Forkhead box protein O1 (FOXO1), Focal Adhesion Kinase (FAK), large neutral amino acid transporter 1 (LAT1) and Proton-coupled amino acid trans- 
porter 1 (PAT1). All genes of interest were labelled with the fluorescent reporter FAM. The thermal cycling conditions used were: $2 \mathrm{~min}$ at $50^{\circ} \mathrm{C}, 10 \mathrm{~min}$ at $95^{\circ} \mathrm{C}$, followed by 40 cycles at $95^{\circ} \mathrm{C}$ for $15 \mathrm{~s}$ and $60^{\circ} \mathrm{C}$ for $1 \mathrm{~min}$. Ct values of the genes of interest were normalized to $\mathrm{Ct}$ values of the housekeeping gene, and final results were calculated as relative expression against the standard curve.

Supplemental Table 2 | Plasma amino acid concentrations measured $48 \mathrm{~h}$ prior to and immediately following 5 days of one-legged knee immobilization in healthy elderly men with (PRO; $n=11$ ) or without (CON; $n=12$ ) twice-daily protein supplementation.

\begin{tabular}{|c|c|c|c|c|}
\hline & \multicolumn{2}{|c|}{$\operatorname{CON}(n=12)$} & \multicolumn{2}{|c|}{$\operatorname{PRO}(n=11)$} \\
\hline & Pre & Post & Pre & Post \\
\hline & $\mu \mathrm{mol} \cdot \mathrm{L}^{-1}$ & $\mu \mathrm{mol} \cdot \mathrm{L}^{-1}$ & $\mu \mathrm{mol} \cdot \mathrm{L}^{-1}$ & $\mu \mathrm{mol} \cdot \mathrm{L}^{-1}$ \\
\hline$\alpha$-aminobutyric acid & $30 \pm 4$ & $25 \pm 2$ & $27 \pm 2$ & $30 \pm 4$ \\
\hline Alanine & $393 \pm 36$ & $485 \pm 36 *$ & $379 \pm 25$ & $423 \pm 22 *$ \\
\hline Arginine & $88 \pm 5$ & $90 \pm 4$ & $84 \pm 3$ & $81 \pm 4$ \\
\hline Asparagine & $47 \pm 2$ & $47 \pm 2$ & $45 \pm 2$ & $45 \pm 2$ \\
\hline Aspartic acid & $5 \pm 1$ & $5 \pm 1$ & $4 \pm 1$ & $4 \pm 1$ \\
\hline Citrulline & $39 \pm 3$ & $36 \pm 2$ & $35 \pm 1$ & $38 \pm 3$ \\
\hline Cysteine & $42 \pm 3$ & $46 \pm 2 *$ & $44 \pm 2$ & $50 \pm 2 *$ \\
\hline Glutamic acid & $69 \pm 10$ & $68 \pm 7$ & $58 \pm 9$ & $59 \pm 9$ \\
\hline Glutamine & $605 \pm 29$ & $622 \pm 27$ & $575 \pm 39$ & $540 \pm 31$ \\
\hline Glycine & $217 \pm 11$ & $237 \pm 12$ & $204 \pm 10$ & $194 \pm 13$ \\
\hline Histidine & $86 \pm 5$ & $86 \pm 2$ & $83 \pm 4$ & $84 \pm 3$ \\
\hline Isoleucine & $70 \pm 5$ & $73 \pm 4$ & $66 \pm 4$ & $76 \pm 8$ \\
\hline Leucine & $138 \pm 8$ & $137 \pm 5$ & $128 \pm 7$ & $154 \pm 16$ \\
\hline Lysine & $190 \pm 10$ & $191 \pm 7$ & $177 \pm 9$ & $212 \pm 14$ \\
\hline Methionine & $28 \pm 2$ & $29 \pm 1$ & $27 \pm 1$ & $30 \pm 2$ \\
\hline Ornithine & $60 \pm 4$ & $62 \pm 3$ & $58 \pm 4$ & $60 \pm 4$ \\
\hline Phenylalanine & $59 \pm 3$ & $60 \pm 2 *$ & $56 \pm 3$ & $64 \pm 2 *$ \\
\hline Proline & $218 \pm 27$ & $223 \pm 22$ & $169 \pm 14$ & $192 \pm 12$ \\
\hline Serine & $90 \pm 6$ & $93 \pm 5$ & $85 \pm 5$ & $89 \pm 7$ \\
\hline Taurine & $81 \pm 8$ & $89 \pm 6$ & $96 \pm 10$ & $82 \pm 7$ \\
\hline Threonine & $129 \pm 7$ & $135 \pm 8 *$ & $120 \pm 7$ & $154 \pm 15 *$ \\
\hline Tryptophan & $56 \pm 4$ & $59 \pm 3 *$ & $53 \pm 3$ & $62 \pm 4^{*}$ \\
\hline Tyrosine & $67 \pm 4$ & $69 \pm 3$ & $65 \pm 4$ & $71 \pm 3$ \\
\hline Valine & $261 \pm 17$ & $259 \pm 9$ & $240 \pm 12$ & $288 \pm 19 *$ \\
\hline
\end{tabular}

Data are presented as means \pm SEM, $n=23$. * Significantly different from pre-immobilization value $(P<0.05)$. 
No significant time*treatment nor time effects were found for mTOR (Supplemental Figure 1A), P706SK (Supplemental Figure 1B), and FAK (Supplemental Figure 1C: all $P>0.05)$.
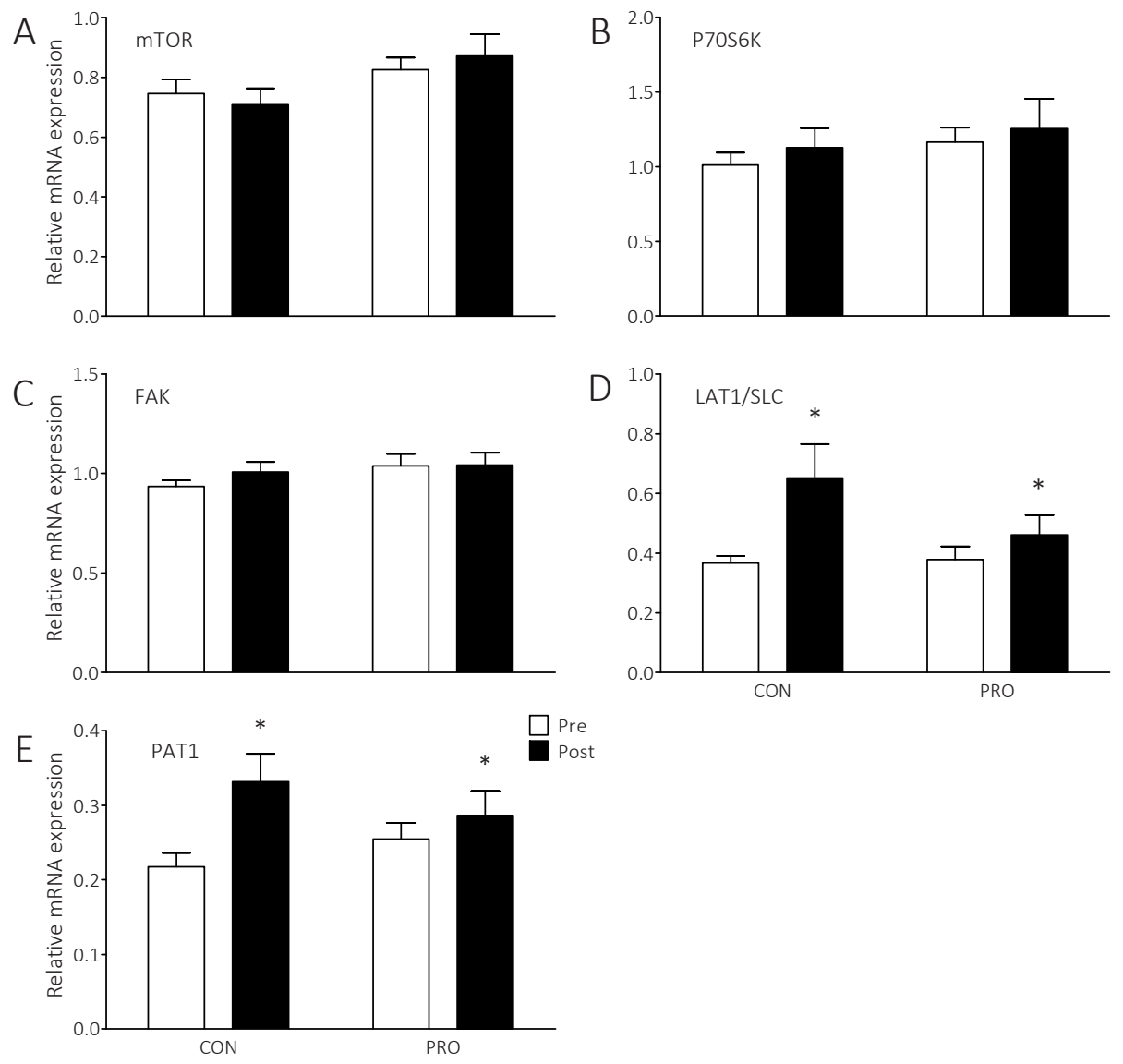

Supplemental Figure 1 | mRNA expression of anabolic genes of interest in the CON $(n=12)$ and PRO $(n=11)$ group $48 \mathrm{~h}$ prior to and immediately following 5 days of leg immobilization. ${ }^{*} P<0.05$; significantly different when compared with pre-immobilization values. Data are expressed as means \pm SEM, $n=23$. Abbreviations: FAK, Focal Adhesion Kinase; LAT1, large neutral amino acid transporter; mTOR, mammalian target of rapamycin; PAT1, proton-coupled amino acid transporter 1; P70S6K, P70S6 kinase. 


\section{SUPPLEMENTAL REFERENCES}

1. Snijders, T., L.B. Verdijk, M. Beelen, B.R. McKay, G. Parise, F. Kadi, and L.J. van Loon, A single bout of exercise activates skeletal muscle satellite cells during subsequent overnight recovery. Exp Physiol, 2012. 97(6): p. 762-73.

2. Verdijk, L.B., R. Koopman, G. Schaart, K. Meijer, H.H. Savelberg, and L.J. van Loon, Satellite cell content is specifically reduced in type II skeletal muscle fibers in the elderly. Am J Physiol Endocrinol Metab, 2007. 292(1): p. E151-7.

3. Verdijk, L.B., M.L. Dirks, T. Snijders, J.J. Prompers, M. Beelen, R.A. Jonkers, D.H. Thijssen, M.T. Hopman, and L.J. Van Loon, Reduced satellite cell numbers with spinal cord injury and aging in humans. Med Sci Sports Exerc, 2012. 44(12): p. 2322-30.

4. Kadi, F., N. Charifi, C. Denis, and J. Lexell, Satellite cells and myonuclei in young and elderly women and men. Muscle Nerve, 2004. 29(1): p. 120-7.

5. Snijders, T., B.T. Wall, M.L. Dirks, J.M. Senden, F. Hartgens, J. Dolmans, M. Losen, L.B. Verdijk, and L.J. van Loon, Muscle disuse atrophy is not accompanied by changes in skeletal muscle satellite cell content. Clin Sci (Lond), 2014. 126(8): p. 557-66.

6. Dirks, M.L., B.T. Wall, T. Snijders, C.L. Ottenbros, L.B. Verdijk, and L.J. van Loon, Neuromuscular electrical stimulation prevents muscle disuse atrophy during leg immobilization in humans. Acta Physiol (Oxf), 2014. 210(3): p. 628-41.

7. Wall, B.T., T. Snijders, J.M. Senden, C.L. Ottenbros, A.P. Gijsen, L.B. Verdijk, and L.J. van Loon, Disuse impairs the muscle protein synthetic response to protein ingestion in healthy men. J Clin Endocrinol Metab, 2013. 98(12): p. 4872-81. 



\title{
CHAPTER
}

\section{A single session of neuromuscular electrical stimulation does not augment postprandial muscle protein accretion}

\author{
Marlou L Dirks \\ Benjamin T Wall \\ Irene Fleur Kramer \\ Antoine $\mathrm{H}$ Zorenc \\ Joy PB Goessens \\ Annemie P Gijsen \\ Luc JC van Loon
}


ABSTRACT

Background The loss of muscle mass and strength that occurs with ageing, termed sarcopenia, has been (at least partly) attributed to an impaired muscle protein synthetic response to food intake. We previously showed that neuromuscular electrical stimulation (NMES) can stimulate fasting muscle protein synthesis rates and prevent muscle atrophy during disuse. We hypothesized that NMES prior to protein ingestion would increase postprandial muscle protein accretion.

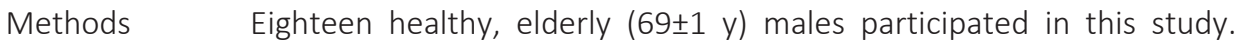
After performing a 70 min unilateral NMES protocol, subjects ingested $20 \mathrm{~g}$ intrinsically L-[1- $\left.{ }^{13} \mathrm{C}\right]$-phenylalanine-labelled casein. Plasma samples and muscle biopsies were collected to assess postprandial mixed muscle and myofibrillar protein accretion, as well as associated myocellular signaling, during a 4 hour postprandial period in both the control (CON) and stimulated (NMES) leg.

Results Protein ingestion resulted in rapid increases in both plasma phenylalanine concentrations and $\mathrm{L}-\left[1-{ }^{13} \mathrm{C}\right]$-phenylalanine enrichments, which remained elevated during the entire $4 \mathrm{~h}$ postprandial period $(P<0.05)$. Mixed muscle protein-bound $\mathrm{L}-\left[1-{ }^{13} \mathrm{C}\right]$-phenylalanine enrichments significantly increased over time following protein ingestion, with no differences between the CON $(0.0164 \pm 0.0019 \mathrm{MPE})$ and NMES (0.0164 $\pm 0.0019 \mathrm{MPE})$ leg $(P>0.05)$. In agreement, no differences were observed in the postprandial rise in myofibrillar protein-bound L-[1${ }^{13} \mathrm{C}$ ]-phenylalanine enrichments between the CON and NMES legs $(0.0115 \pm 0.0014$ vs $0.0133 \pm 0.0013 \mathrm{MPE}$, respectively; $P>0.05)$. Significant increases in mTOR and P70S6K phosphorylation status were observed in the NMES stimulated leg only $(P<0.05)$.

Conclusion A single session of NMES prior to food intake does not augment postprandial muscle protein accretion in healthy, older men. 


\section{INTRODUCTION}

Ageing is accompanied by declines in skeletal muscle mass and strength, termed sarcopenia [1]. A less than optimal diet and sedentary lifestyle are factors contributing to sarcopenia [1, 2]. However, the underlying mechanisms remain to be elucidated. From a physiological perspective, any loss of muscle mass must be attributed to an imbalance between muscle protein synthesis and breakdown rates. Research has generally demonstrated that basal (i.e. postabsorptive) muscle protein synthesis [3-6] and breakdown $[7,8]$ rates do not change with advancing age. As such, research has since focused on the impact of ageing on the anabolic response to food intake. Recent work has shown that the skeletal muscle protein synthetic response to dietary protein ingestion is impaired in older individuals $[4,6,9]$. This 'anabolic resistance' to food intake is now regarded as a key factor in the etiology of sarcopenia $[6,10]$. Accordingly, we $[3,11,12]$ and many others $[4,13-15]$ have begun to investigate ways to overcome anabolic resistance in older individuals in an effort to develop more effective strategies to attenuate age-related muscle loss and support healthy ageing.

One strategy that has been shown to be effective to increase the postprandial muscle protein synthetic response to feeding is physical activity performed prior to food ingestion [16-20]. By combining the ingestion of a meal-like bolus (i.e. 20 g) of intrinsically labelled milk protein with the continuous infusion of stable isotope-labelled amino acids, we were able to show that a single bout of physical activity performed prior to protein ingestion augments the postprandial use of dietary protein derived amino acid for de novo muscle protein synthesis [16]. However, some conditions do not allow an increase in physical activity level. For example, acute periods of illness or injury necessitate short periods of bed rest or limb immobilization. Such successive short periods of local or whole-body muscle disuse increase anabolic resistance to feeding and contribute to the development of sarcopenia during the lifespan [21, 22]. Therefore, alternative strategies to maximize the postprandial muscle protein synthetic response to food ingestion are warranted in both health and disease.

In situations where physical activity levels are reduced, neuromuscular electrical stimulation (NMES) may be used as an alternative means to elicit muscle contraction. We have previously shown that NMES increases (fasting) muscle protein synthesis rates [23], and can be applied effectively to prevent muscle atrophy during short periods of muscle disuse in young men [24] as well as critically ill patients [25]. In the present study, we hypothesized that a single bout of NMES improves postprandial protein accretion by increasing the postprandial use of dietary protein derived amino acids for de novo muscle protein synthesis in older adults. To test this hypothesis, we selected 18 healthy older males who were subjected to 70 min of unilateral NMES followed by the ingestion of 20 g intrinsically L-[1-13C]-phenylalanine-labelled casein protein. This was combined with regular blood and muscle tissue sampling to assess postprandial protein accretion and underlying myocellular signaling in both the stimulated and non-stimulated leg. 


\section{METHODS}

\section{Subjects}

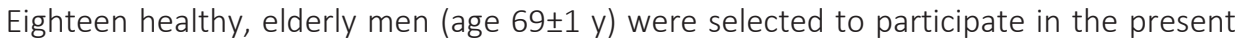
study. Subjects were excluded if one of the following criteria were met: BMI below 18.5 or above $30 \mathrm{~kg} \cdot \mathrm{m}^{-2}$, type 2 diabetes mellitus, use of non-steroidal anti-inflammatory drugs, presence of a pacemaker or implantable cardioverter defibrillator, or having participated in any regular resistance-type exercise program within 6 months prior to the study. Subjects' characteristics are displayed in Table 1. All subjects were informed on the nature and risks of the study before written informed consent was obtained. The study was approved by the Medical Ethical Committee of the Maastricht University Medical Centre in accordance with the Declaration of Helsinki.

Table 1 | Subjects' characteristics

\begin{tabular}{lc}
\hline Age $(\mathrm{y})$ & $69 \pm 1$ \\
Weight $(\mathrm{kg})$ & $83.4 \pm 2.2$ \\
BMI $\left(\mathrm{kg} \cdot \mathrm{m}^{-2}\right)$ & $26.5 \pm 0.6$ \\
Body fat (\% body weight) & $22.0 \pm 1.0$ \\
Lean body mass $(\mathrm{kg})$ & $62.5 \pm 1.5$ \\
Leg lean mass $(\mathrm{kg})$ & $10.0 \pm 0.3$ \\
Quadriceps CSA (mm ${ }^{2} *$ & $7151 \pm 266$ \\
Leg volume (L) & $8.4 \pm 0.2$ \\
Basal plasma glucose $\left(\mathrm{mmol}^{-1}\right)$ & $5.7 \pm 0.1$ \\
Basal plasma insulin $\left(\mathrm{mU}^{-1} \mathrm{~L}^{-1}\right.$ & $10.2 \pm 1.0$ \\
HbA1c $(\%)$ & $5.4 \pm 0.1$ \\
OGIS (mL·min $\left.{ }^{-1} \cdot \mathrm{m}^{-2}\right)$ & $433 \pm 9$ \\
\hline
\end{tabular}

Values represent means \pm SEM. BMI, body mass index; CSA, cross-sectional area; HbA1c, glycosylated hemoglobin; OGIS, oral glucose insulin sensitivity. * Data from $n=10$ participants

\section{Pretesting}

All subjects filled out a health questionnaire and completed a routine medical screening before inclusion into the study. During this visit, an Oral Glucose Tolerance Test (OGTT) was performed in a fasted state [26] to test for type 2 diabetes mellitus, and height and weight were measured. A second visit was performed to assess body composition via whole-body dual energy $\mathrm{x}$-ray absorptiometry (DXA) and single-slice computed tomography (CT) of $\mathrm{m}$. quadriceps femoris, at $15 \mathrm{~cm}$ above the patella. Also during this visit, subjects were familiarized with the NMES protocol to be used in the experimental visit (see below for details). 


\section{Diet and physical activity prior to testing}

The evening prior to the test day, subjects consumed a standardized meal containing $2900 \mathrm{~kJ}$ providing 51 energy\% (En\%) as carbohydrate, 32 En\% as fat, and 17 En\% as protein. All subjects received instructions to refrain from any sort of heavy physical activity and to keep their diet as constant as possible during the $48 \mathrm{~h}$ prior to the test day.

\section{Experimental protocol}

An overview of the experimental protocol is depicted in Figure 1.

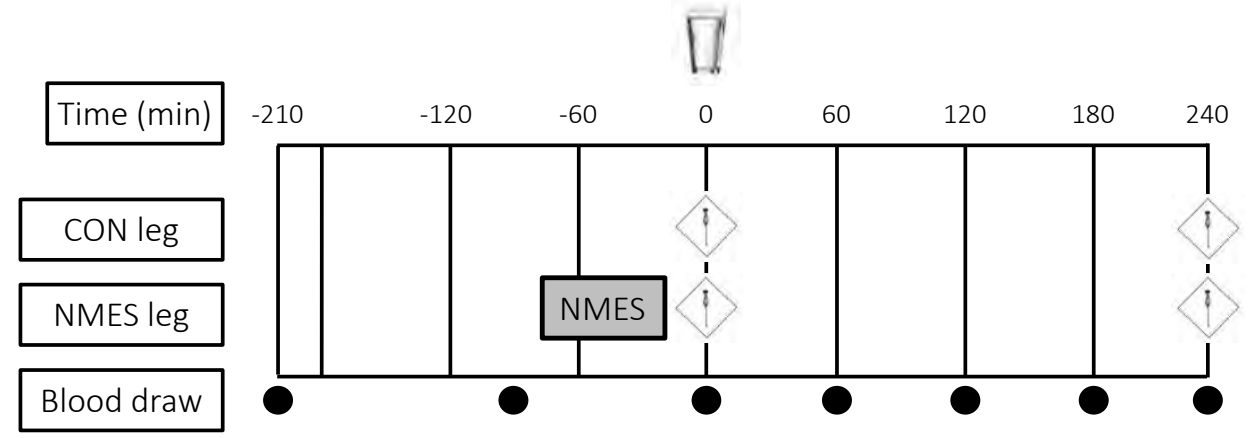

Figure 1 | Outline of the experimental protocol. Eighteen healthy, older men ingested a protein drink containing $20 \mathrm{~g}$ casein following unilateral NMES (neuromuscular electrical stimulation).

After an overnight fast, subjects arrived at the laboratory at 8:00 AM for a single test day. While resting in a supine position on a bed, a catheter was placed in a heated dorsal hand vein and placed in a hot box at $60^{\circ} \mathrm{C}$ for arterialized venous blood sampling [94]. After collection of a basal arterialized blood sample at $t=-210 \mathrm{~min}$, a blood sample was collected $120 \mathrm{~min}(t=-90 \mathrm{~min}$ ) after the baseline sample. After this, an NMES protocol (see below for details) was started at $t=-70 \mathrm{~min}$. After terminating the NMES session at $t=0 \mathrm{~min}$, a blood sample was taken, and muscle biopsies were collected from both the stimulated (NMES) and the non-stimulated (CON) leg within approximately 5 min after the end of the NMES protocol. Subjects then received a test drink containing $20 \mathrm{~g}$ intrinsically L-[1- $\left.{ }^{13} \mathrm{C}\right]$-phenylalanine-labelled protein. The consumption of this drink signified the beginning of a $4 \mathrm{~h}$ postprandial period. Arterialized blood samples were subsequently collected every $60 \mathrm{~min}$ with the final sample being taken at $t=240 \mathrm{~min}$. At the same time (at $t=240 \mathrm{~min}$ ), muscle biopsy samples were taken from both the NMES and CON leg.

Arterialized venous blood samples were collected into pre-cooled EDTA-containing tubes and centrifuged at $1000 \mathrm{~g}$ for $10 \mathrm{~min}$ at $4^{\circ} \mathrm{C}$. Aliquots of plasma were snap frozen in liquid nitrogen and stored at $-80^{\circ} \mathrm{C}$ until further analysis. Muscle biopsy samples were collected from the middle region of $m$. vastus lateralis, $\sim 15 \mathrm{~cm}$ above the patella [28]. 
Any visible non-muscle tissue was removed, and the muscle sample was frozen in liquid nitrogen. Subsequently, samples were stored at $-80^{\circ} \mathrm{C}$ until further analysis.

\section{Neuromuscular electrical stimulation}

After inclusion, one of the subjects' legs was randomly allocated to receive 70 min of NMES during the experimental visit. Prior to the NMES session, subjects were placed in a supine position with a pillow underneath both knees to instigate light knee flexion. Four self-adhesive electrodes (50 × 50 mm; Enraf-Nonius, Rotterdam, the Netherlands) were placed on the distal part at the muscle belly of the $\mathrm{m}$. rectus femoris and the $\mathrm{m}$. vastus lateralis, and at the inguinal area of both muscles of both legs. The electrodes were connected to an Enraf-Nonius TensMed S84 stimulation device, discharging biphasic symmetric rectangular-wave pulses. However, NMES was only applied to one leg (NMES) while the other leg served as a sham-treated control (CON). The 70-min protocol consisted of a warm-up phase ( $5 \mathrm{~min}, 5 \mathrm{~Hz}, 250 \mu \mathrm{s}$ ), a stimulation period (60 min, $100 \mathrm{~Hz}, 400 \mu \mathrm{s}, 5 \mathrm{~s}$ on ( $0.75 \mathrm{~s}$ rise, $3.5 \mathrm{~s}$ contraction, $0.75 \mathrm{~s}$ fall) and $10 \mathrm{~s}$ off), and a cooling-down phase $(5 \mathrm{~min}, 5 \mathrm{~Hz}, 250 \mu \mathrm{s})$. This protocol was selected as we previously demonstrated it is effective in preventing muscle atrophy during short-term disuse in young men [24] and critically ill patients [25]. Subjects were encouraged to continuously adjust the intensity of the stimulation to the level where a full contraction of m. quadriceps femoris was both visible and palpable, with the heel slightly being lifted from the bed. The NMES protocol was completed by all subjects. The maximal intensity of the 70 min NMES session averaged 35.9 $\pm 2.7 \mathrm{~mA}$, whereas the average intensity across all subjects and sessions averaged $26.1 \pm 1.5 \mathrm{~mA}$.

\section{Preparation of intrinsically labelled protein}

Intrinsically L-[1- $\left.{ }^{13} \mathrm{C}\right]$-phenylalanine-labelled micellar casein protein was obtained by infusing a Holstein cow with large quantities of $\mathrm{L}-\left[1{ }^{-13} \mathrm{C}\right]$-phenylalanine, collecting milk, and purifying the casein fraction as described previously [29]. The average $L-\left[1-{ }^{13} \mathrm{C}\right]-$ phenylalanine enrichment was 38.7 mole percent excess (MPE). All subjects received a drink with $20 \mathrm{~g}$ casein in a total volume of $350 \mathrm{~mL}$, flavored with vanilla flavor.

\section{Plasma analyses}

Plasma glucose and insulin concentrations were analyzed by Dr. Stein und Kollegen Laboratories (Mönchengladbach, Germany) using commercially available kits (GLUC3, Roche, Ref: 05168791 190, and Immunologic, Roche, Ref: 12017547 122, respectively). Plasma amino acid concentrations and enrichments were determined by GC-MS (Agilent 7890A GC/5975C; MSD, Little Falls, DE, USA). Plasma phenylalanine was converted to its tert-butyl dimethylsilyl (TBDMS) derivative before analysis by GC-MS by using electron impact ionization by monitoring ions at mass/charge $(\mathrm{m} / \mathrm{z}) 336$ and 337 for 
unlabelled and L-[1- $\left.{ }^{13} \mathrm{C}\right]$-labelled phenylalanine, respectively [12]. Standard regression curves were applied from a series of known standard enrichment values against the measured values to assess the linearity of the mass spectrometer and to account for any isotope fractionation which may have occurred during the analysis. Phenylalanine enrichments were corrected for the presence of the ${ }^{13} \mathrm{C}$ isotopes.

\section{Muscle tissue analyses}

Mixed muscle protein-bound enrichments were determined in a piece of wet muscle ( 45 mg) as described previously [3]. Briefly, muscle was freeze-dried, and collagen, blood and other visible non-muscle material was removed under a dissecting microscope. After homogenizing and incubating samples in ice-cold $2 \%$ perchloric acid (PCA), samples were centrifuged. The supernatant was collected for determination of L-[1$\left.{ }^{13} \mathrm{C}\right]$-phenylalanine enrichments in the muscle free amino acid pool using GC-MS analysis [12]. The mixed muscle protein pellet washed, hydrolyzed overnight, and dried under a nitrogen stream. Next, free amino acids were dissolved in $50 \%$ acetic acid solution and passed over cation exchange AG 50W0X8 resin columns. To determine the L-[1- $\left.{ }^{13} \mathrm{C}\right]$ phenylalanine enrichment, the purified amino acids were derivatized into their $N(O, S)$ ethoxycarbonyl ethyl ester derivatives with ethyl chloroformate (ECF), and then measured by GC-C-IRMS (MAT 253; Thermo Scientific, Bremen, Germany) using a DB5-MScolumn (no. 122122-5532; Agilent J+W, USA), GC Isolink, and monitoring ion masses 44, 45 , and 46 . By establishing the relation between the enrichment of a series of $L-\left[1-{ }^{13} \mathrm{C}\right]-$ phenylalanine standards of variable enrichments and the enrichments of the $N(O, S)$ ethoxycarbonyl ethyl esters of these standards, the mixed muscle protein-bound enrichment of phenylalanine was determined.

Myofibrillar protein enriched fractions were extracted from wet muscle tissue as described elsewhere [30]. In short, 50 mg wet muscle tissue was manually homogenized on ice using a Teflon pestle in a standard extraction buffer, after which the samples were centrifuged and the supernatants containing sarcoplasmic proteins were removed. In an additional step, the myofibrillar fraction-containing supernatant was collected and the collagen pellet was removed. The remaining myofibrillar fraction was purified and hydrolyzed, such that the free amino acids remained and could be dried under a nitrogen stream. The enrichment of the derivative was measured by GC-C-IRMS by using a DB5-MS-column (no. 122-5532; Agilent J+W, USA), GC Isolink, and monitoring of ion masses 44,45 , and 46 . By establishing the relationship between the enrichment of a series of $\mathrm{L}-\left[1-{ }^{13} \mathrm{C}\right]$-phenylalanine standards of variable enrichment, the myofibrillar protein-bound enrichment of phenylalanine was determined. Standard regression curves were applied to assess the linearity of the mass spectrometer and to control for the loss of tracer. Muscle protein deposition from the ingested casein over the $4 \mathrm{~h}$ postprandial period was expressed as the relative increase of $\mathrm{L}-\left[1-{ }^{13} \mathrm{C}\right]$-phenylalanine enrichment in muscle tissue. 
Western blot analyses were performed as described previously [25]. In short, 30 mg muscle tissue was homogenized and protein quantification was performed. After protein quantification, the gels were transferred onto a nitrocellulose membrane. Specific proteins were detected by overnight incubation with the following antibodies: anti-mTOR (289 kDa; dilution 1:1000, \#2972 Cell Signaling, Danvers, MA, USA) and anti-phosphomTOR (Ser ${ }^{2448}$; 289 kDa, dilution 1:1000, \#2971 Cell Signaling), anti-P70S6K (70 kDa; dilution 1:1000, \#9202 Cell Signaling) and anti-phospho P70S6K (Thr ${ }^{389} ; 70 \mathrm{kDa}$, dilution 1:1000, \#9206 Cell Signaling), anti-RS6 (32 kDa; dilution 1:1000; \#2217 Cell Signaling) and anti-phospho-RS6 (Ser ${ }^{235} / \mathrm{Ser}^{236}, 32$ kDa; dilution 1:1000; \#4856 Cell Signaling) and anti $\alpha$ tubulin (52 kDa; dilution 1:1000; \#2125 Cell Signaling). The complementary secondary antibodies applied were IRDye 680 donkey anti-rabbit (Cat. No. 926-32223, dilution 1:10000, Li-Cor, Lincoln, NE, USA) and IRDye 800CW donkey anti-mouse (Cat. No. 92632212, dilution 1:10000, Li-Cor). Protein quantification was performed by scanning on an Odyssey Infrared Imaging System (LI-COR Biotechnology, Lincoln, NE, USA).

\section{Statistics}

All data are expressed as means \pm SEM. Differences in baseline leg values (i.e. left vs right) were determined using a paired samples t-test. A one-way repeated measures analysis of variance (ANOVA) with time as within-subjects factor was used to analyze effects in plasma concentrations and enrichments. Differences in protein-bound L-[1${ }^{13} \mathrm{C}$ ]-phenylalanine enrichments between legs after $4 \mathrm{~h}$ incorporation were analyzed using a paired-samples t-test. When a significant main effect was detected, Bonferroni's post hoc test was applied to locate the differences. Statistical analyses were performed using the SPSS version 22.0 software package (SPSS Inc., Chicago, IL, USA), with statistical significance set at $P<0.05$. 


\section{RESULTS}

\section{Plasma analyses}

For plasma glucose and insulin concentrations, depicted in Figure 2, a significant time effect was observed (both $P<0.001$ ).
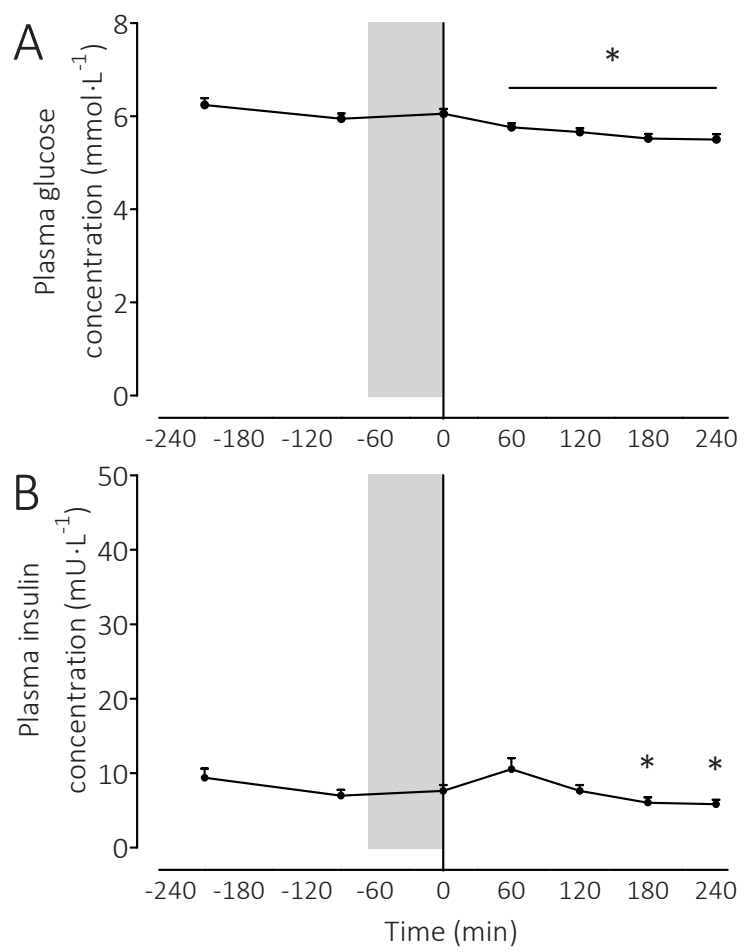

Figure 2 | Means \pm SEM plasma glucose (A) and insulin (B) concentrations prior to and following ingestion of 20 g casein. The gray bar represents a 70 min NMES protocol. Data were analyzed with one-way repeated measures ANOVA with time as within-subjects factor. A significant time effect $(P<0.001)$ was found for both glucose and insulin. * Significantly different from $t=0 \mathrm{~min}(P<0.05)$.

During the postprandial period, plasma glucose and insulin concentrations averaged $5.6 \pm 0.1 \mathrm{mmol} \cdot \mathrm{L}^{-1}$ and $7.5 \pm 0.8 \mathrm{mU} \cdot \mathrm{L}^{-1}$, respectively. Figure 3 displays plasma concentrations of phenylalanine (A), tyrosine (B), and leucine (C). At the start of the experiment, fasting plasma phenylalanine, tyrosine, and leucine concentrations averaged $54 \pm 1,62 \pm 2$ and $128 \pm 5 \mu \mathrm{M}$, respectively. Following the ingestion of $20 \mathrm{~g}$ casein, at $t=0 \mathrm{~min}$, concentrations of these three amino acids increased rapidly (time effect; $P<0.001$ ) and remained elevated until the end of the experiment. 

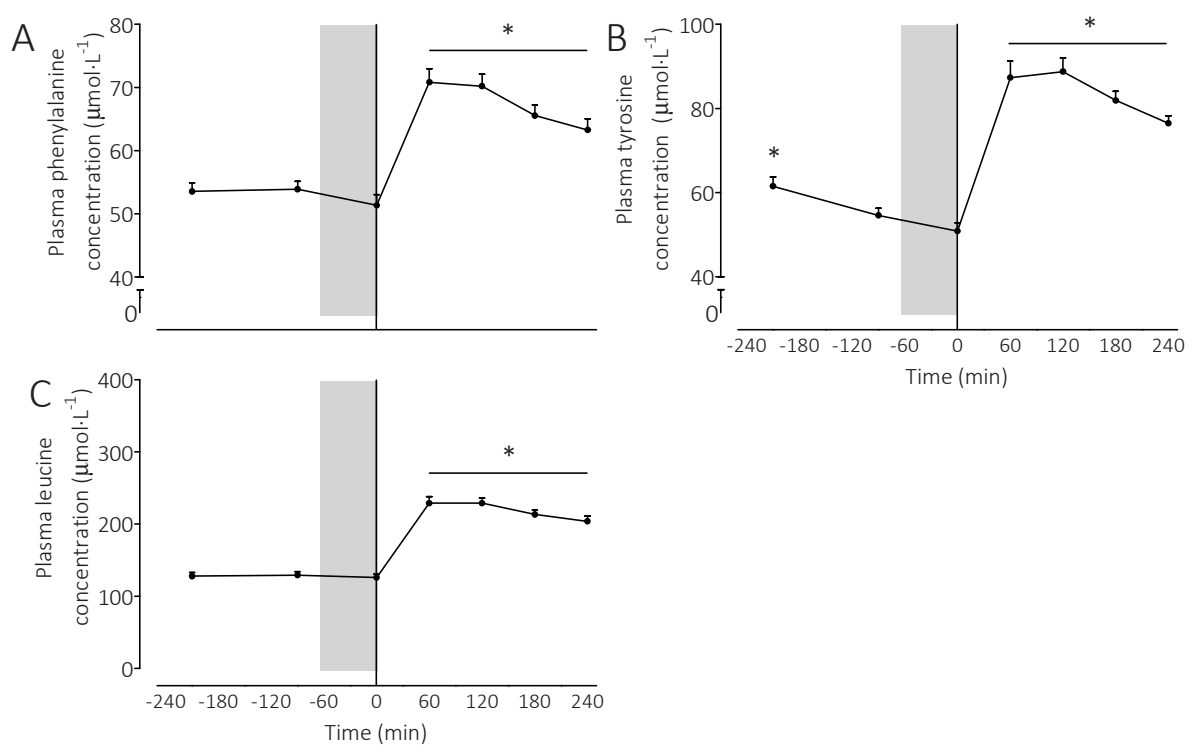

Figure 3 | Means \pm SEM plasma phenylalanine (A), tyrosine (B), and leucine $(C)$ concentrations during the fasting period ( $t=-210 \mathrm{until} 0 \mathrm{~min}$ ) and following the ingestion of $20 \mathrm{~g}$ casein. The gray bar represents the 70 min NMES protocol. Data were analyzed with one-way repeated measures ANOVA with time as withinsubjects factor. For all amino acids, significant time effects were observed (all $P<0.001)$. * Significantly different from $t=0 \mathrm{~min}(P<0.05)$. 
Figure 4 depicts plasma enrichments of $\mathrm{L}-\left[1-{ }^{13} \mathrm{C}\right]$-phenylalanine. After ingestion of the protein beverage, plasma L- $\left[1-{ }^{13} \mathrm{C}\right]$-phenylalanine enrichments increased $(P<0.001)$, and remained elevated throughout the $4 \mathrm{~h}$ postprandial period.

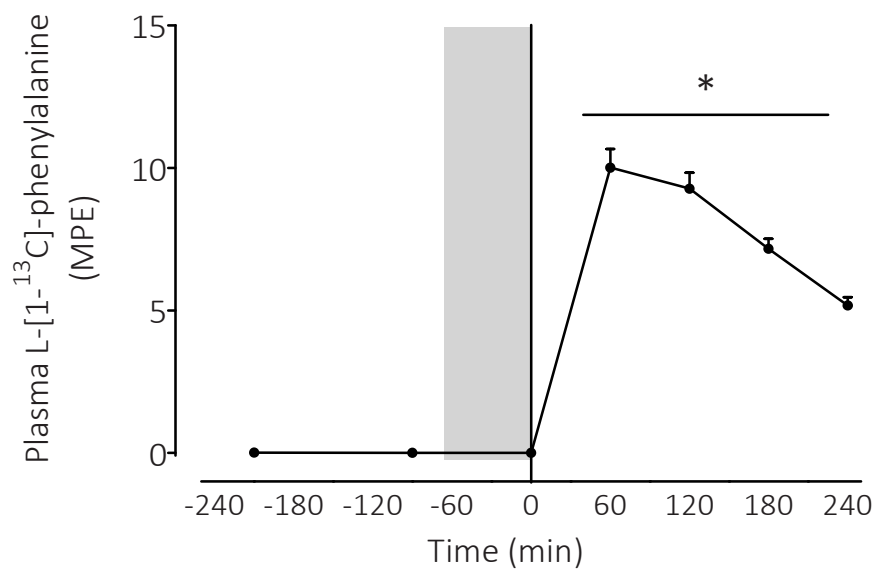

Figure 4 | Plasma L-[1- $\left.{ }^{13} \mathrm{C}\right]$-phenylalanine enrichments. The 70 min NMES protocol is visualized by the grey bar. Values are expressed as means \pm SEM. Data were analyzed with one-way repeated measures ANOVA with time as within-subjects factor. A significant time effect was found $(P<0.001)$ * Significantly different from $t=$ $0 \min (P<0.05)$.

\section{Muscle tracer analyses}

Muscle free $\mathrm{L}-\left[1-{ }^{13} \mathrm{C}\right]$-phenylalanine enrichments averaged 4.1 \pm 0.2 and $4.1 \pm 0.2 \mathrm{MPE}$ at $4 \mathrm{~h}$ after protein ingestion in the CON and NMES leg, respectively, which did not differ $(P>0.05)$. Figure 5A depicts mixed muscle $\mathrm{L}-\left[1-{ }^{13} \mathrm{C}\right]$-phenylalanine enrichments following ingestion of $20 \mathrm{~g}$ casein in the CON and NMES leg. Four hours after the ingestion of $20 \mathrm{~g}$ casein protein, mixed muscle L-[1- $\left.{ }^{13} \mathrm{C}\right]$-phenylalanine enrichments did not differ between legs: $0.0164 \pm 0.0019$ and $0.0164 \pm 0.0019$ MPE in the CON and NMES leg, respectively $(P>0.05) . \mathrm{L}-\left[1-{ }^{13} \mathrm{C}\right]$-phenylalanine enrichments of the intracellular free amino acid pool were 4.074 \pm 0.183 and $4.115 \pm 0.163 \mathrm{MPE}$ in the CON and NMES leg, respectively $(P>0.05)$. 

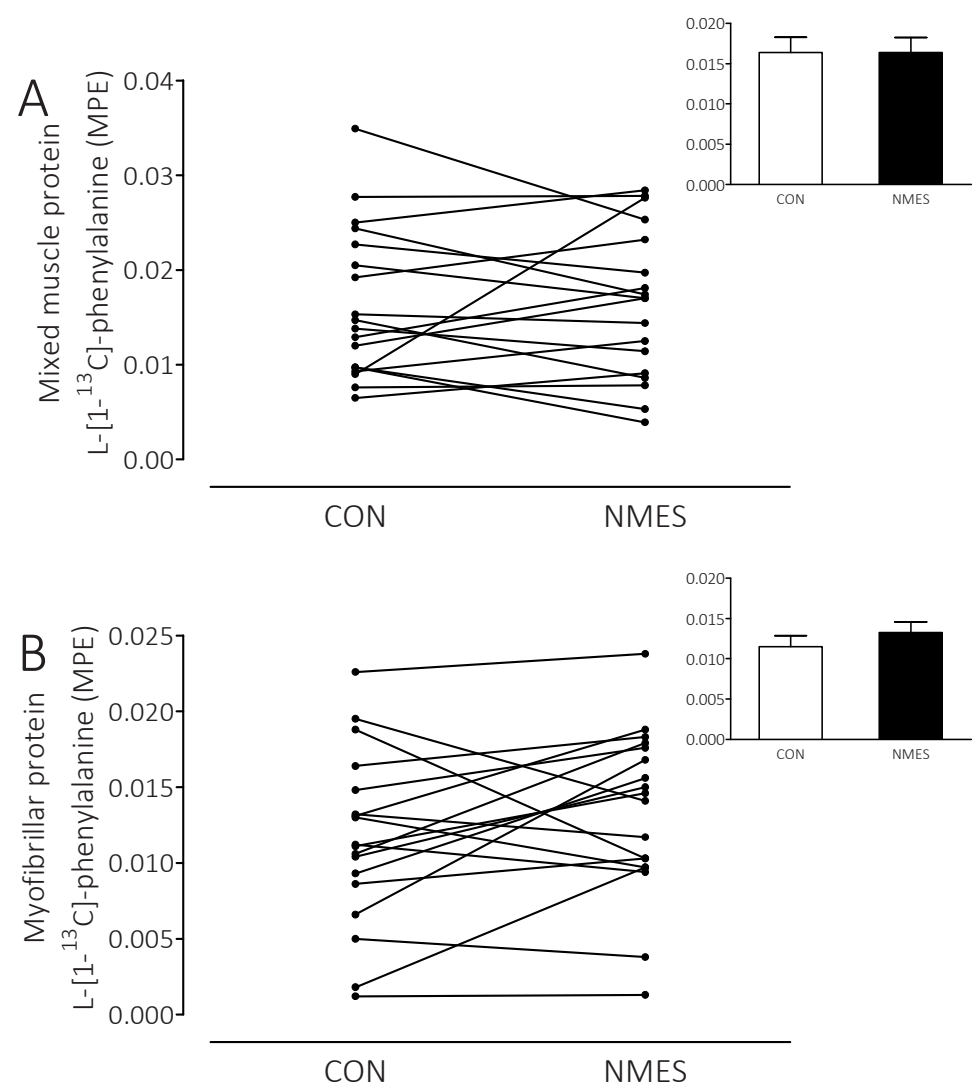

CON

NMES

Figure 5 | Individual subjects' mixed muscle (A) and myofibrillar protein-bound (B) L-[1- $\left.{ }^{13} \mathrm{C}\right]$-phenylalanine enrichments (MPE) over a $4 \mathrm{~h}$ period following ingestion of $20 \mathrm{~g}$ casein, in the CON and NMES leg. Data are presented as means \pm SEM.

Myofibrillar protein-bound $\mathrm{L}-\left[1-{ }^{13} \mathrm{C}\right]$-phenylalanine enrichments are presented in Figure 5B. Ingestion of $20 \mathrm{~g}$ casein resulted in an increase in $\mathrm{L}-\left[1{ }^{-13} \mathrm{C}\right]$-phenylalanine enrichments up to $0.0115 \pm 0.0014$ and $0.0133 \pm 0.0013 \mathrm{MPE}$ in the CON and NMES leg, respectively $(P>0.05)$.

\section{Signaling proteins}

The muscle phosphorylation status of selected proteins involved in the regulation of muscle protein synthesis is displayed in Figure 6. 

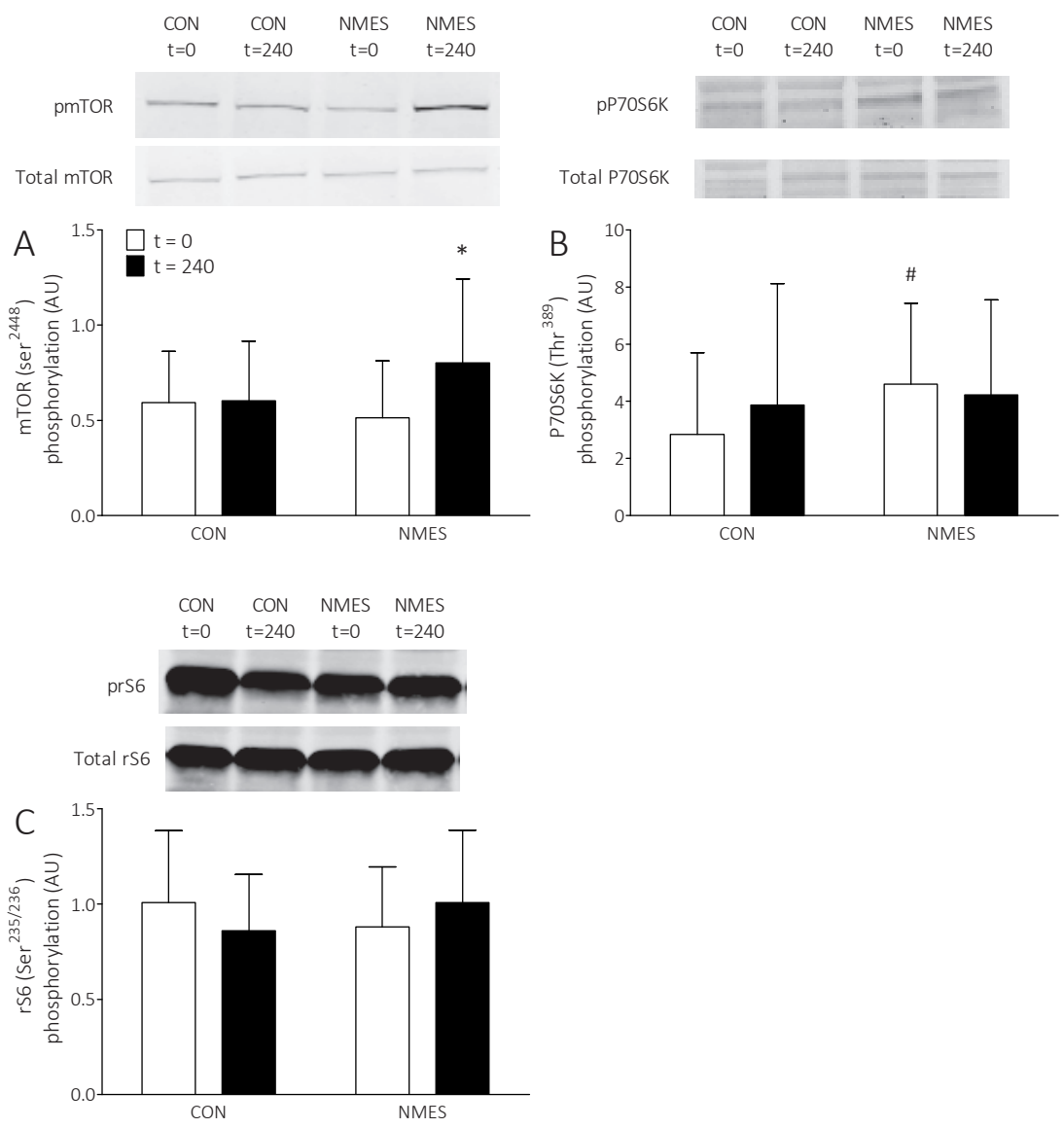

Figure 6 | Skeletal muscle phosphorylation status (expressed as means \pm SEM) of selected proteins in the control (CON) and stimulated (NMES) leg. Muscle samples were taken directly after ( $t=0 \mathrm{~min})$ ingestion of 20 g casein protein, and $4 \mathrm{~h}$ thereafter $(t=240 \mathrm{~min}) .{ }^{*}$ Significantly different from $t=0 \mathrm{~min}$. \# Significantly different from CON. Abbreviations: mTOR, mammalian target of rapamycin; P70S6K, P70S6 kinase; RS6, ribosomal protein S6.

Data are expressed as the ratios between the phosphorylated protein and the total protein content. Directly after cessation of the NMES, for P70S6K, a higher phosphorylation status was observed in the NMES leg when compared to the CON leg $(P<0.05$, Figure 6B). Following protein ingestion, the phosphorylation status of mTOR (Figure 6A) significantly increased over time in the NMES leg only (interaction effect; $P<0.05$ ). No changes in the phosphorylation status of P70S6K were observed after protein ingestion between legs or over time. Despite a significant interaction effect for RS6 (Figure 6C; $P<0.01)$, no changes over time were found in the CON and NMES legs. 


\section{DISCUSSION}

In the present study we show that neuromuscular electrical stimulation (NMES) prior to protein ingestion does not augment the use of dietary protein derived amino acids for de novo muscle protein accretion in healthy, older males. Nevertheless, we observed significant increases in mTOR and P70S6K phosphorylation in muscle following the bout of NMES.

Ageing is accompanied by declines in skeletal muscle mass and strength, called sarcopenia [1]. Previous research has shown that the older population possesses a blunted skeletal muscle protein synthetic response to food intake, termed 'anabolic resistance' $[4,6,9]$. This anabolic resistance is now believed to represent a key factor in the etiology of sarcopenia $[6,10]$. In the current study, intake of a meal-like amount of $20 \mathrm{~g}$ intrinsically labelled casein led to a rapid increase in plasma amino acid concentrations (Figure 3), which was accompanied by an increase in plasma L-[1- $\left.{ }^{13} \mathrm{C}\right]$-phenylalanine enrichment that remained elevated for the entire $4 \mathrm{~h}$ postprandial period (Figure 4). This demonstrates a clear amino acid signal to stimulate anabolism as well as a tracer precursor allowing the determination of postprandial muscle protein accretion. Indeed, these dietary protein derived amino acids were rapidly used for de novo muscle protein synthesis, as evidenced by the $\sim 0.016 \mathrm{MPE}$ increase in muscle protein-bound L-[1- $\left.{ }^{13} \mathrm{C}\right]$ phenylalanine in mixed muscle tissue and $0.012 \mathrm{MPE}$ in the myofibrillar fraction of the muscle tissue obtained in the control leg $4 \mathrm{~h}$ after protein ingestion (Figure 5 ). The use of intrinsically L-[1- $\left.{ }^{13} \mathrm{C}\right]$-phenylalanine labelled protein allows us to assess the percentage of the ingested protein that was released into the circulation and used for de novo muscle protein synthesis [31]. Based on the assumption that $\mathrm{L}-\left[1-{ }^{13} \mathrm{C}\right]$-phenylalanine enrichments in $m$. vastus lateralis would be representative of most other muscle groups, we calculated that a total of $0.037 \pm 0.004 \mathrm{~g} \mathrm{~L}-\left[1-{ }^{13} \mathrm{C}\right]$-phenylalanine had been incorporated in all appendicular lean tissue during the entire $4 \mathrm{~h}$ postprandial period. This translates to $2.0 \pm 0.2 \mathrm{~g}$ muscle protein, and equals $9.9 \pm 1.2 \%$ of the ingested dietary protein derived amino acids that were incorporated in de novo muscle protein. These data are in line with our recent calculations [31] and demonstrate the possibilities of using intrinsically labelled protein to demonstrate the metabolic fate of dietary protein derived amino acid in vivo in humans [29].

Physical activity performed prior to food intake has been shown to further increase postprandial muscle protein synthesis compared with food intake alone [16-20], and to augment the use of protein derived amino acids for de novo muscle protein synthesis [16]. Currently, it remains unknown to what extent the stimulating properties of physical activity are attributed to its impact on skeletal muscle perfusion or whether the effects are predominantly intramuscular. As maintaining or increasing physical activity can be compromised in various clinical and non-clinical settings, exercise mimetics such as NMES may be used to evoke involuntary contractions to reintroduce some level of physical activity. Indeed, previous work from our group has shown that local NMES can 
increase postabsorptive muscle protein synthesis rates by as much as $27 \%$ when compared to the non-stimulated, control leg [23]. To date, no studies have assessed the impact of NMES on the postprandial muscle protein synthetic response to feeding. In the current study, we assessed postprandial protein accretion following ingestion of a single bolus of intrinsically labelled protein in an electrically stimulated (NMES) and a non-stimulated, control leg. Despite the 70 min of neuromuscular electrical stimulation prior to protein ingestion we observed no differences in the muscle free $\mathrm{L}-\left[1-{ }^{13} \mathrm{C}\right]$ phenylalanine enrichments or the deposition of dietary protein derived amino acids into de novo muscle protein between both legs $(0.0164 \pm 0.0019$ vs $0.0164 \pm 0.0019$ MPE and $0.0115 \pm 0.0014$ vs $0.0133 \pm 0.0013 \mathrm{MPE}$ for the increase in $\mathrm{L}-\left[1-{ }^{13} \mathrm{C}\right]$-phenylalanine enrichment in mixed muscle protein and myofibrillar protein, respectively; Figure 5). Clearly, a single session of NMES prior to protein ingestion was not sufficient to modulate the metabolic fate of the dietary protein derived amino acids and did not augment postprandial protein deposition in the stimulated leg of these healthy, older males.

The postprandial stimulation of muscle protein synthesis is initiated by a phosphorylation cascade in which mammalian target of rapamycin (mTOR) and its downstream effectors P70S6 kinase (P70S6K) and ribosomal protein S6 (RS6) are key players [32, 33]. This pathway is not only activated by protein intake, but also by physical activity (as reviewed in [34]). Here we show that protein ingestion did not lead to changes in activation of mTOR, P70S6K, and RS6 in the control leg (Figure 6). This is not surprising considering our low dose of protein administered [35] as well as previous work showing the peak of this translation initiation process to generally occur $1-2 \mathrm{~h}$ following protein ingestion, and to subside thereafter [36-38]. Of course, the timing of our muscle biopsy collection that was chosen to optimally measure muscle protein-bound enrichments was likely not optimal for the detection of changes in anabolic signaling, which had probably subsided by then. However, we observed an early increase in the phosphorylation of P70S6K immediately following NMES (Figure 6). This is in agreement with our previous work demonstrating that an acute bout of NMES stimulates muscle protein synthesis in the postabsorptive state, possibly via a similar rise in P70S6K signaling [23], but is rather contradictory to previous studies showing an increase in P70S6K to occur only several hours after the cessation of exercise [36, 38]. Furthermore, we observed a significant increase in mTOR activation $4 \mathrm{~h}$ after NMES (Figure 6). This is in line with previous data showing greater mTOR phosphorylation at $4 \mathrm{~h}$ after NMES [23] and $3 \mathrm{~h}$ postexercise [39] in older individuals. Clearly, the NMES did induce an anabolic stimulus, but this did not seem strong enough to augment the postprandial muscle protein synthetic response to feeding.

Previously, we have shown that muscle loss during disuse can be prevented by the application of NMES in both young males during short-term immobilization [24] as well as in critically ill patients in a comatose state [25]. This has been, at least partly, attributed to the increase in basal muscle protein synthesis rate that can be observed after performing a single session of NMES [23]. In the present study, we assessed 
whether NMES augmenting the muscle protein synthetic response to feeding may go some way to explaining the beneficial effect on muscle retention during disuse. In contrast to our hypothesis, we failed to detect a stimulatory effect of NMES on postprandial muscle protein accretion. This implies that NMES may particularly impact upon basal protein synthesis rates, as opposed to postprandial protein handling, in healthy older men. Although muscle disuse is associated with anabolic resistance to food intake [38, $40,41]$, based on the present data it could be suggested that the observed efficacy of NMES to prevent disuse atrophy is primarily attained in the basal state $[40,42,43]$. Though our results demonstrate that NMES does not affect postprandial protein handling in healthy, active individuals, we cannot exclude that NMES may modulate postprandial protein handling in a more clinically compromised state, where anabolic sensitivity to food intake is further reduced $[37,38,40,41]$ or in fact under situations where larger amounts, or more anabolic dietary proteins are provided to older subjects. Obviously, the efficacy of NMES combined with nutritional support may be of particular relevance for older hospitalized patients, who are losing muscle partially due to low dietary protein intake [44-46]. Furthermore, it should be noted that we assessed the effect of a single bout of NMES only, and we cannot rule out any synergistic effects of multiple, repetitive NMES sessions performed over time.

In conclusion, a single session of NMES prior to protein ingestion does not augment postprandial muscle protein accretion in healthy, older males.

\section{ACKNOWLEDGEMENTS}

We gratefully acknowledge the enthusiastic support of Rinske Franssen (NUTRIM School of Nutrition and Translational Research in Metabolism) in this study. 


\section{REFERENCES}

1. Koopman, R. and L.J. van Loon, Aging, exercise, and muscle protein metabolism. J Appl Physiol, 2009. 106(6): p. 2040-8.

2. Rolland, Y., S. Czerwinski, G. Abellan Van Kan, J.E. Morley, M. Cesari, G. Onder, J. Woo, R. Baumgartner, F. Pillard, Y. Boirie, W.M. Chumlea, and B. Vellas, Sarcopenia: its assessment, etiology, pathogenesis, consequences and future perspectives. J Nutr Health Aging, 2008. 12(7): p. 433-50.

3. Gorissen, S.H., N.A. Burd, H.M. Hamer, A.P. Gijsen, B.B. Groen, and L.J. van Loon, Carbohydrate coingestion delays dietary protein digestion and absorption but does not modulate postprandial muscle protein accretion. J Clin Endocrinol Metab, 2014. 99(6): p. 2250-8.

4. Cuthbertson, D., K. Smith, J. Babraj, G. Leese, T. Waddell, P. Atherton, H. Wackerhage, P.M. Taylor, and M.J. Rennie, Anabolic signaling deficits underlie amino acid resistance of wasting, aging muscle. FASEB J, 2005. 19(3): p. 422-4.

5. Volpi, E., B. Mittendorfer, B.B. Rasmussen, and R.R. Wolfe, The response of muscle protein anabolism to combined hyperaminoacidemia and glucose-induced hyperinsulinemia is impaired in the elderly. J Clin Endocrinol Metab, 2000. 85(12): p. 4481-90.

6. Wall, B.T., S.H. Gorissen, B. Pennings, R. Koopman, B.B. Groen, L.B. Verdijk, and L.J. van Loon, Aging Is Accompanied by a Blunted Muscle Protein Synthetic Response to Protein Ingestion. PLoS One, 2015. 10(11): p. e0140903.

7. Volpi, E., B. Mittendorfer, S.E. Wolf, and R.R. Wolfe, Oral amino acids stimulate muscle protein anabolism in the elderly despite higher first-pass splanchnic extraction. Am J Physiol, 1999. 277(3 Pt 1): p. E513-20.

8. Wilkes, E.A., A.L. Selby, P.J. Atherton, R. Patel, D. Rankin, K. Smith, and M.J. Rennie, Blunting of insulin inhibition of proteolysis in legs of older subjects may contribute to age-related sarcopenia. Am J Clin Nutr, 2009. 90(5): p. 1343-50.

9. Guillet, C., M. Prod'homme, M. Balage, P. Gachon, C. Giraudet, L. Morin, J. Grizard, and Y. Boirie, Impaired anabolic response of muscle protein synthesis is associated with S6K1 dysregulation in elderly humans. FASEB J, 2004. 18(13): p. 1586-7.

10. Moore, D.R., Keeping older muscle "young" through dietary protein and physical activity. Adv Nutr, 2014. 5(5): p. 599S-607S.

11. Koopman, R., N. Crombach, A.P. Gijsen, S. Walrand, J. Fauquant, A.K. Kies, S. Lemosquet, W.H.M. Saris, Y. Boirie, and L.J.C. van Loon, Ingestion of a protein hydrolysate is accompanied by an accelerated in vivo digestion and absorption rate when compared with its intact protein. Am J Clin Nutr, 2009. 90(1): p. 106-115.

12. Wall, B.T., H.M. Hamer, A. de Lange, A. Kiskini, B.B. Groen, J.M. Senden, A.P. Gijsen, L.B. Verdijk, and L.J. van Loon, Leucine co-ingestion improves post-prandial muscle protein accretion in elderly men. Clin Nutr, 2013. 32(3): p. 412-9.

13. Rieu, I., M. Balage, C. Sornet, C. Giraudet, E. Pujos, J. Grizard, L. Mosoni, and D. Dardevet, Leucine supplementation improves muscle protein synthesis in elderly men independently of hyperaminoacidaemia. J Physiol, 2006. 575(Pt 1): p. 305-15.

14. Smith, G.I., P. Atherton, D.N. Reeds, B.S. Mohammed, D. Rankin, M.J. Rennie, and B. Mittendorfer, Dietary omega-3 fatty acid supplementation increases the rate of muscle protein synthesis in older adults: a randomized controlled trial. Am J Clin Nutr, 2011. 93(2): p. 402-12.

15. Kumar, V., A. Selby, D. Rankin, R. Patel, P. Atherton, W. Hildebrandt, J. Williams, K. Smith, O. Seynnes, N. Hiscock, and M.J. Rennie, Age-related differences in the dose-response relationship of muscle protein synthesis to resistance exercise in young and old men. J Physiol, 2009. 587(Pt 1): p. 211-7.

16. Pennings, B., R. Koopman, M. Beelen, J.M.G. Senden, W.H.M. Saris, and L.J.C. van Loon, Exercising before protein intake allows for greater use of dietary protein-derived amino acids for de novo muscle protein synthesis in both young and elderly men. Am J Clin Nutr, 2011. 93(2): p. 322-331.

17. Tang, J.E., D.R. Moore, G.W. Kujbida, M.A. Tarnopolsky, and S.M. Phillips, Ingestion of whey hydrolysate, casein, or soy protein isolate: effects on mixed muscle protein synthesis at rest and following resistance exercise in young men. J Appl Physiol, 2009. 107(3): p. 987-92. 
18. Burd, N.A., Y. Yang, D.R. Moore, J.E. Tang, M.A. Tarnopolsky, and S.M. Phillips, Greater stimulation of myofibrillar protein synthesis with ingestion of whey protein isolate $\mathrm{v}$. micellar casein at rest and after resistance exercise in elderly men. Br J Nutr, 2012. 108(6): p. 958-62.

19. Yang, Y., L. Breen, N.A. Burd, A.J. Hector, T.A. Churchward-Venne, A.R. Josse, M.A. Tarnopolsky, and S.M. Phillips, Resistance exercise enhances myofibrillar protein synthesis with graded intakes of whey protein in older men. Brit J Nutr, 2012: p. 1-9.

20. Witard, O.C., M. Tieland, M. Beelen, K.D. Tipton, L.J. van Loon, and R. Koopman, Resistance exercise increases postprandial muscle protein synthesis in humans. Med Sci Sports Exer, 2009. 41(1): p. 144-54.

21. Wall, B.T., M.L. Dirks, and L.J. van Loon, Skeletal muscle atrophy during short-term disuse: implications for age-related sarcopenia. Ageing Res Rev, 2013. 12(4): p. 898-906.

22. Hallal, P.C., L.B. Andersen, F.C. Bull, R. Guthold, W. Haskell, U. Ekelund, and G. Lancet Physical Activity Series Working, Global physical activity levels: surveillance progress, pitfalls, and prospects. Lancet, 2012. 380(9838): p. 247-57.

23. Wall, B.T., M.L. Dirks, L.B. Verdijk, T. Snijders, D. Hansen, P. Vranckx, N.A. Burd, P. Dendale, and L.J. van Loon, Neuromuscular electrical stimulation increases muscle protein synthesis in elderly type 2 diabetic men. Am J Physiol Endocrinol Metab, 2012. 303(5): p. E614-23.

24. Dirks, M.L., B.T. Wall, T. Snijders, C.L. Ottenbros, L.B. Verdijk, and L.J. van Loon, Neuromuscular electrical stimulation prevents muscle disuse atrophy during leg immobilization in humans. Acta Physiol (Oxf), 2014. 210(3): p. 628-41.

25. Dirks, M.L., D. Hansen, A. Van Assche, P. Dendale, and L.J. Van Loon, Neuromuscular electrical stimulation prevents muscle wasting in critically ill comatose patients. Clin Sci (Lond), 2015. 128(6): p. 357-65.

26. Alberti, K.G. and P.Z. Zimmet, Definition, diagnosis and classification of diabetes mellitus and its complications. Part 1: diagnosis and classification of diabetes mellitus provisional report of a WHO consultation. Diabet Med, 1998. 15(7): p. 539-53.

27. Abumrad, N.N., D. Rabin, M.P. Diamond, and W.W. Lacy, Use of a heated superficial hand vein as an alternative site for the measurement of amino acid concentrations and for the study of glucose and alanine kinetics in man. Metabolism, 1981. 30(9): p. 936-40.

28. Bergstrom, J., Percutaneous needle biopsy of skeletal muscle in physiological and clinical research. Scand J Clin Lab Invest, 1975. 35(7): p. 609-16.

29. van Loon, L.J., Y. Boirie, A.P. Gijsen, J. Fauquant, A.L. de Roos, A.K. Kies, S. Lemosquet, W.H. Saris, and R. Koopman, The production of intrinsically labeled milk protein provides a functional tool for human nutrition research. J Dairy Sci, 2009. 92(10): p. 4812-22.

30. Burd, N.A., N.M. Cermak, I.W. Kouw, S.H. Gorissen, A.P. Gijsen, and L.J. van Loon, The use of doubly labeled milk protein to measure postprandial muscle protein synthesis rates in vivo in humans. J Appl Physiol, 2014. 117(11): p. 1363-70.

31. Groen, B.B., A.M. Horstman, H.M. Hamer, M. de Haan, J. van Kranenburg, J. Bierau, M. Poeze, W.K. Wodzig, B.B. Rasmussen, and L.J. van Loon, Post-Prandial Protein Handling: You Are What You Just Ate. PLoS One, 2015. 10(11): p. e0141582.

32. Ronnestad, B.R., H. Nygaard, and T. Raastad, Physiological elevation of endogenous hormones results in superior strength training adaptation. Eur J Appl Physiol, 2011. 111(9): p. 2249-59.

33. Zebrowska, A., Z. Waskiewicz, A. Zajac, Z. Gasior, H. Galbo, and J. Langfort, IGF-1 response to arm exercise with eccentric and concentric muscle contractions in resistance-trained athletes with left ventricular hypertrophy. Int J Sports Med, 2013. 34(2): p. 116-22.

34. West, D.W., N.A. Burd, J.E. Tang, D.R. Moore, A.W. Staples, A.M. Holwerda, S.K. Baker, and S.M. Phillips, Elevations in ostensibly anabolic hormones with resistance exercise enhance neither training-induced muscle hypertrophy nor strength of the elbow flexors. J Appl Physiol, 2010. 108(1): p. 60-7.

35. West, D.W., G.W. Kujbida, D.R. Moore, P. Atherton, N.A. Burd, J.P. Padzik, M. De Lisio, J.E. Tang, G. Parise, M.J. Rennie, S.K. Baker, and S.M. Phillips, Resistance exercise-induced increases in putative anabolic hormones do not enhance muscle protein synthesis or intracellular signalling in young men. J Physiol, 2009. 587(Pt 21): p. 5239-47. 
36. Drummond, M.J., C.S. Fry, E.L. Glynn, H.C. Dreyer, S. Dhanani, K.L. Timmerman, E. Volpi, and B.B. Rasmussen, Rapamycin administration in humans blocks the contraction-induced increase in skeletal muscle protein synthesis. J Physiol, 2009. 587(Pt 7): p. 1535-46.

37. Kimball, S.R., P.A. Farrell, and L.S. Jefferson, Invited Review: Role of insulin in translational control of protein synthesis in skeletal muscle by amino acids or exercise. J Appl Physiol, 2002. 93(3): p. 1168-80.

38. Watson, K. and K. Baar, mTOR and the health benefits of exercise. Semin Cell Dev Biol, 2014. 36: p. 130-9.

39. Pennings, B., B. Groen, A. de Lange, A.P. Gijsen, A.H. Zorenc, J.M. Senden, and L.J. van Loon, Amino acid absorption and subsequent muscle protein accretion following graded intakes of whey protein in elderly men. Am J Physiol Endocrinol Metab, 2012. 302(8): p. E992-9.

40. Churchward-Venne, T.A., N.A. Burd, C.J. Mitchell, D.W. West, A. Philp, G.R. Marcotte, S.K. Baker, K. Baar, and S.M. Phillips, Supplementation of a suboptimal protein dose with leucine or essential amino acids: effects on myofibrillar protein synthesis at rest and following resistance exercise in men. J Physiol, 2012. 590(Pt 11): p. 2751-65.

41. Drummond, M.J., J.M. Dickinson, C.S. Fry, D.K. Walker, D.M. Gundermann, P.T. Reidy, K.L. Timmerman, M.M. Markofski, D. Paddon-Jones, B.B. Rasmussen, and E. Volpi, Bed rest impairs skeletal muscle amino acid transporter expression, mTORC1 signaling, and protein synthesis in response to essential amino acids in older adults. Am j Physiol Endocrinol Metab, 2012. 302(9): p. E1113-22.

42. Glover, E.I., S.M. Phillips, B.R. Oates, J.E. Tang, M.A. Tarnopolsky, A. Selby, K. Smith, and M.J. Rennie, Immobilization induces anabolic resistance in human myofibrillar protein synthesis with low and high dose amino acid infusion. J Physiol, 2008. 586(Pt 24): p. 6049-61.

43. Beelen, M., A. Zorenc, B. Pennings, J.M. Senden, H. Kuipers, and L.J. van Loon, Impact of protein coingestion on muscle protein synthesis during continuous endurance type exercise. Am J Physiol Endocrinol Metab, 2011. 300(6): p. E945-54.

44. Drummond, M.J., H.C. Dreyer, B. Pennings, C.S. Fry, S. Dhanani, E.L. Dillon, M. Sheffield-Moore, E. Volpi, and B.B. Rasmussen, Skeletal muscle protein anabolic response to resistance exercise and essential amino acids is delayed with aging. J Appl Physiol, 2008. 104(5): p. 1452-61.

45. Wall, B.T., M.L. Dirks, T. Snijders, J.W. van Dijk, M. Fritsch, L.B. Verdijk, and L.J. van Loon, Short-term muscle disuse lowers myofibrillar protein synthesis rates and induces anabolic resistance to protein ingestion. Am J Physiol Endocrinol Metab, 2016. 310(2): p. E137-47.

46. Wall, B.T., T. Snijders, J.M. Senden, C.L. Ottenbros, A.P. Gijsen, L.B. Verdijk, and L.J. van Loon, Disuse impairs the muscle protein synthetic response to protein ingestion in healthy men. J Clin Endocrinol Metab, 2013. 98(12): p. 4872-81.

47. Ferrando, A.A., H.W. Lane, C.A. Stuart, J. Davis-Street, and R.R. Wolfe, Prolonged bed rest decreases skeletal muscle and whole body protein synthesis. Am J Physiol, 1996. 270(4 Pt 1): p. E627-33.

48. Gibson, J.N., D. Halliday, W.L. Morrison, P.J. Stoward, G.A. Hornsby, P.W. Watt, G. Murdoch, and M.J. Rennie, Decrease in human quadriceps muscle protein turnover consequent upon leg immobilization. Clin Sci (Lond), 1987. 72(4): p. 503-9.

49. Wall, B.T. and L.J. van Loon, Nutritional strategies to attenuate muscle disuse atrophy. Nutr Rev, 2013. 71(4): p. 195-208.

50. Stein, T.P. and S. Blanc, Does protein supplementation prevent muscle disuse atrophy and loss of strength? Crit Rev Food Sci, 2011. 51(9): p. 828-34.

51. Dirks, M.L., B.T. Wall, R. Nilwik, D.H. Weerts, L.B. Verdijk, and L.J. van Loon, Skeletal muscle disuse atrophy is not attenuated by dietary protein supplementation in healthy older men. J Nutr, 2014. 144(8): p. 1196203. 



\section{CHAPTER}

\section{Neuromuscular electrical stimulation increases postprandial muscle protein accretion during overnight sleep}

Marlou L Dirks

Bart BL Groen

Rinske Franssen

Janneau van Kranenburg

Luc JC van Loon

* Joint first authors

Submitted 
ABSTRACT

Background Short periods of skeletal muscle disuse, due to illness or injury, result in substantial skeletal muscle atrophy. Recently, we showed that both neuromuscular electrical stimulation (NMES) as well as dietary protein ingestion prior to sleep represent effective strategies to stimulate muscle protein synthesis rates. In this study we test our hypothesis that NMES can augment the impact of protein ingestion prior to sleep to stimulate overnight muscle protein accretion.

Methods Twenty healthy, elderly ( $69 \pm 1$ y) males were subjected to $24 \mathrm{~h}$ of bed rest, starting at 8:00 AM. In the evening, subjects were subjected to a 70 min one-legged NMES protocol, while the other leg served as nonstimulated control (CON). Immediately following NMES, a drink containing $40 \mathrm{~g}$ intrinsically L-[1- $\left.{ }^{13} \mathrm{C}\right]$-phenylalanine labelled protein was ingested prior to sleep. Repeated blood samples were taken throughout the night, and muscle biopsies were obtained from both legs in the evening and on the following morning ( $8 \mathrm{~h}$ after protein ingestion) to assess dietary protein derived L-[1- $\left.{ }^{13} \mathrm{C}\right]$-phenylalanine enrichment in myofibrillar protein.

Results Plasma phenylalanine concentrations and $\mathrm{L}-\left[1-{ }^{13} \mathrm{C}\right]$-phenylalanine enrichments increased significantly following protein ingestion and remained elevated for up to 6 hours $(P<0.05)$. During overnight sleep, myofibrillar protein-bound $\mathrm{L}-\left[1-{ }^{13} \mathrm{C}\right]$-phenylalanine enrichments increased to a greater extent in the stimulated compared with the control leg $(0.0344 \pm 0.0019$ vs $0.0297 \pm 0.0016 \mathrm{MPE}$, respectively; $P<0.01)$, representing $18 \pm \%$ greater incorporation in the NMES leg.

Conclusion The combined application of NMES with protein feeding prior to sleep increases overnight muscle protein accretion in elderly men. 


\section{INTRODUCTION}

Ageing is associated with a progressive loss of skeletal muscle mass, often referred to as sarcopenia [1]. The loss of muscle mass reduces strength, impairs functional capacity, and increases the risk of developing chronic metabolic diseases [2]. Sarcopenia is facilitated by a combination of factors, which include a reduced sensitivity to anabolic stimuli $[3,4]$, a less than optimal diet [5], and a more sedentary lifestyle [5]. It has recently been suggested that short successive periods of reduced physical activity, due to illness or injury, may form a key factor responsible for the progressive loss of muscle tissue in the older population [6]. To attenuate muscle loss during such short periods of bed rest in the elderly population, various interventional strategies have been suggested [7].

The ingestion of dietary protein directly stimulates muscle protein synthesis [8]. Aeging is associated with an impaired muscle protein synthetic response to feeding, previously coined 'anabolic resistance' [9]. Numerous research groups are now looking for effective dietary strategies to compensate for this anabolic resistance by increasing the amount of dietary protein ingested [10], changing the protein source applied [11, $12]$, co-ingesting additional free leucine $[13,14]$ and altering the timing of protein ingestion [15]. Rather than improving the muscle protein synthetic response to the ingestion of a single meal, we investigated the possibility of increasing overnight muscle protein accretion by dietary protein administration prior to and/or during sleep $[16,17]$. Our work shows that protein ingestion prior to sleep may represent an effective strategy to stimulate overnight muscle protein synthesis, thereby providing a practical strategy to attenuate muscle loss during periods characterized by anabolic resistance.

Physical activity is severely restricted during a period of bed rest due to illness or injury. The loss of physical activity reduces the muscle protein synthetic response to protein feeding $[18,19]$. Neuromuscular electrical stimulation (NMES) represents an exercise mimetic to allow muscle contractions to occur and may be used to attenuate disuse related anabolic resistance. In agreement, a single session of NMES has been shown to increase muscle protein synthesis rates [20]. Furthermore, NMES has been applied successfully to prevent muscle atrophy during 5 days of limb immobilization [21]. We hypothesize that NMES can further stimulate the muscle protein synthetic response to protein feeding, resulting in greater postprandial muscle protein accretion.

We hypothesized that combining NMES with dietary protein feeding prior to sleep could further stimulate overnight muscle protein accretion. If supported, the combined application of NMES and protein feeding prior to sleep may be used to attenuate muscle loss during short periods of hospitalization in a compromised elderly population. To test our hypothesis, we performed unilateral NMES in the evening after a day of bed rest in 20 healthy elderly males. Subjects ingested specifically produced intrinsically L$\left[1-{ }^{13} \mathrm{C}\right]$-phenylalanine labelled protein [8] to assess overnight muscle protein accretion. By taking blood samples throughout the night and muscle biopsies before and after sleep from both legs we assessed overnight muscle protein accretion in the stimulated 
and non-stimulated leg. This is the first study to show that the combined application of NMES and protein feeding prior to sleep stimulates overnight muscle protein accretion, thereby providing a novel interventional strategy to prevent or attenuate muscle loss during short periods of hospitalization in older subjects. 


\section{METHODS}

\section{Subjects}

Twenty healthy, elderly males (age: $69 \pm 1$ y) were included in the present study that was approved by the Medical Ethical Committee of the Maastricht University Medical Centre in accordance with the Declaration of Helsinki. Prior to the study, subjects completed a routine medical screening and general health questionnaire to ensure their suitability to participate. Exclusion criteria were: BMI below 18.5 or above $30 \mathrm{~kg} \cdot \mathrm{m}^{2}$, type 2 diabetes mellitus (determined by oral glucose tolerance testing), severe cardiac problems, and implantation of a pacemaker and/or implantable cardioverter-defibrillator (ICD). Furthermore, subjects who had performed structured and prolonged resistance type exercise training during the 6 months prior to the study were excluded. All subjects were informed of the nature and possible risks of the experimental procedures, before their written informed consent was obtained.

\section{Pretesting}

Following an overnight fast, the subjects arrived at the laboratory at $8.00 \mathrm{AM}$ in the morning of the day of pretesting by car or public transportation. A catheter (Baxter BV, Utrecht, the Netherlands) was inserted into an antecubital vein for venous blood sampling. All participants performed an oral glucose tolerance test before inclusion in the study. Plasma glucose concentrations were measured to determine oral glucose tolerance and/or the presence of type 2 diabetes according to 2006 American Diabetes Association guidelines [22]. Leg volume was measured by anthropometry as described by Jones and Pearson [23]. Directly after the oral glucose tolerance test, body weight, body composition, and bone mineral content were measured with Dual Energy X-Ray Absorptiometry (DXA; Lunar Prodigy Advance; GE Health Care, Madison, WI, USA). The system's software package (en-CORE 2005, version 9.15.00) was used to determine wholebody and regional lean and fat mass. Finally, all subjects performed a short session of neuromuscular electrical stimulation (NMES) to familiarize them with the intervention and to determine the required stimulation intensity for full muscle contractions during testing.

\section{Diet and physical activity prior and during testing}

All volunteers were instructed to refrain from any vigorous physical activity and to keep their diet as constant as possible 3 days prior to the trial. On the day of the experiment, subjects consumed a standardized breakfast, mid-morning snack, lunch, mid-afternoon snack, and dinner while being in the hospital. The standardized diet ( 3 meals and 2 snacks) provided $7.7 \mathrm{MJ}$, with 60\% energy (En\%) provided by carbohydrate, 22 En\% provided by fat, and $18 \mathrm{En} \%$ provided by protein. Total protein intake averaged $1.35 \pm 0.02 \mathrm{~g} \cdot \mathrm{kg}$ body weight ${ }^{-1}$ during the test day. 


\section{Study protocol}

After inclusion, one of the subjects' legs was randomly allocated to receive neuromuscular electrical stimulation (NMES), while the other leg served as a (non-stimulated) control (CON) leg. The experimental protocol is depicted in Figure 1.

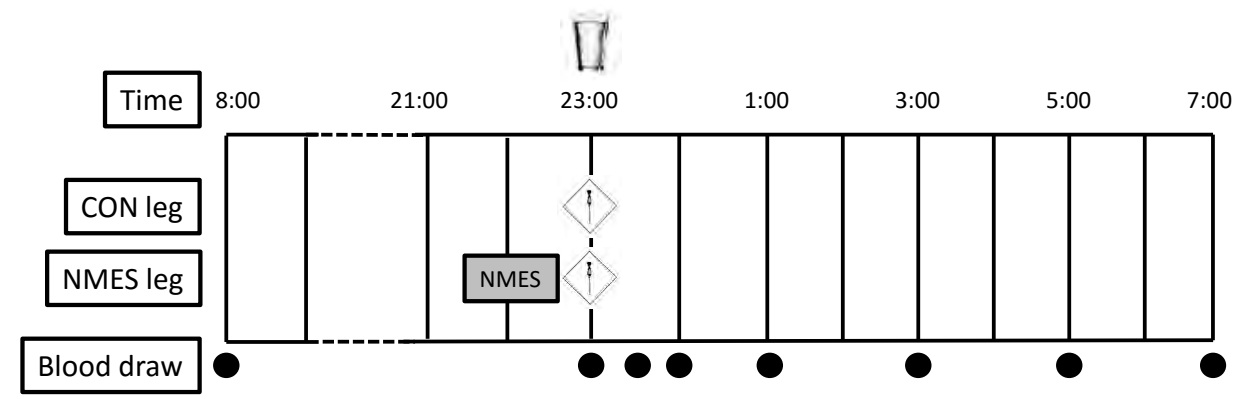

Figure 1 | Outline of the experimental protocol. NMES, neuromuscular electrical stimulation.

At $8.00 \mathrm{AM}$, following an overnight fast, subjects arrived at the laboratory where a first blood sample was obtained by venipuncture to determine fasting plasma glucose and insulin levels. During the rest of the day, subjects remained in a supine position in their hospital bed. At 7:30 PM, A Teflon catheter was inserted in a dorsal hand vein for blood sampling and a first blood sample $(8 \mathrm{~mL})$ was drawn at 8:00 PM. At night, a $30 \mathrm{~cm}$ extension line was connected to the cannula to allow overnight blood sampling without waking the subjects. A 70 min NMES protocol was performed at 9:30 PM. Immediately following the NMES, a muscle biopsy was collected from the vastus lateralis muscle $(t=$ 0 h) from both legs (NMES and CON). Directly after the biopsy procedure, subjects received the test drink, consisting of $40 \mathrm{~g}$ intrinsically $\mathrm{L}-\left[1-{ }^{13} \mathrm{C}\right]$-phenylalanine labelled casein protein dissolved in a total volume of $450 \mathrm{~mL}$. After consuming the test drink, subjects prepared to go to sleep. Blood samples ( $8 \mathrm{~mL})$ were collected at $t=0,1 / 2,1,2$, 4, 6 and $8 \mathrm{~h}$ after protein feeding. The next morning, directly after waking up, muscle biopsies from both legs were taken from a new incision, proximal from the first incision, at $t=8 \mathrm{~h}$ after protein feeding.

Venous blood samples were collected into EDTA-containing tubes and centrifuged at $1000 \mathrm{~g}$ for $10 \mathrm{~min}$ at $4^{\circ} \mathrm{C}$. Aliquots of plasma were frozen in liquid nitrogen and stored at $-80^{\circ} \mathrm{C}$ until further analysis. Muscle biopsy samples were obtained from the middle region of the vastus lateralis muscle, $15 \mathrm{~cm}$ above the patella and $\sim 3 \mathrm{~cm}$ below entry through the fascia using the percutaneous needle biopsy technique [24]. Muscle biopsy samples were carefully dissected and freed from any visible non-muscle material and then immediately frozen in liquid nitrogen and stored at $-80^{\circ} \mathrm{C}$ until further analysis. 


\section{Neuromuscular electrical stimulation}

All subjects performed a single session of neuromuscular electrical stimulation (NMES) and a simultaneous sham session (CON) at the opposite leg at 9:30 PM. During this session, subjects were lying supine and electrodes were placed on the distal part at the muscle belly of the $\mathrm{m}$. rectus femoris and the $m$. vastus lateralis, and at the inguinal area of both muscles. Stimulation was provided by an Enraf-Nonius TensMed S84 stimulation device (Enraf-Nonius, Rotterdam, the Netherlands) and four, $2 \mathrm{~mm}$-thick, selfadhesive electrodes $(50 \times 50 \mathrm{~mm})$, discharging biphasic symmetric rectangular-wave pulses. The NMES protocol comprised of a warm-up phase ( $5 \mathrm{~min}, 5 \mathrm{~Hz}, 250 \mu \mathrm{s}$ ), a stimulation phase ( $60 \mathrm{~min}, 100 \mathrm{~Hz}, 400 \mu \mathrm{s}, 5 \mathrm{~s}$ on ( $0.75 \mathrm{~s}$ rise, $3.5 \mathrm{~s}$ contraction, $0.75 \mathrm{~s}$ fall) and $10 \mathrm{~s}$ off), and a cooling-down phase ( $5 \mathrm{~min}, 5 \mathrm{~Hz}, 250 \mu \mathrm{s})$. Subjects set the intensity of the stimulation to a level at which full contractions of $m$. quadriceps femoris were both visible and palpable, and the heel began to slightly lift. This protocol was based on our previous work [25] demonstrating an increase in muscle protein synthesis during a 6 $\mathrm{h}$ period following a single bout of NMES. The same protocol was also used in a previous study showing that high-frequency $(>60 \mathrm{~Hz}$ ), high pulse duration (>250 $\mu \mathrm{s})$ NMES is effective in preventing muscle atrophy during short-term muscle disuse [21]. Subjects were encouraged to increase the intensity of the stimulation during the entire session to provide a 'progressive' stimulus. The maximal intensity of the 70 min NMES session averaged $36.2 \pm 1.8 \mathrm{~mA}$, whereas the average intensity across all sessions and all subjects was $27.3 \pm 1.3 \mathrm{~mA}$. All subjects completed the NMES protocol.

\section{Intrinsically labelled protein}

Intrinsically L-[1- $\left.{ }^{13} \mathrm{C}\right]$-phenylalanine-labelled casein protein was obtained by infusing a Holstein cow with large quantities of $\mathrm{L}-\left[1-{ }^{13} \mathrm{C}\right]$-phenylalanine, collecting milk, and purifying the casein fraction as described previously [26]. The $\mathrm{L}-\left[1-{ }^{13} \mathrm{C}\right]$-phenylalanine enrichment in the casein fraction averaged 35.8 mole percent excess (MPE). The casein protein met all chemical and bacteriological specifications for human consumption. Subjects received a total beverage volume of $450 \mathrm{~mL}$, which provided 40 g casein protein. Drinks were flavored by adding $2 \mathrm{~mL}$ vanilla flavor (Givaudan, Naarden, the Netherlands) per L beverage.

\section{Plasma analyses}

Plasma glucose (Uni Kit III, 07367204, Roche, Basel, Switzerland) concentrations were analyzed with the COBAS-FARA semi-automatic analyzer (Roche). Insulin was analyzed by radio-immunoassay (Insulin RIA kit, LINCO Research Inc., St. Charles, MO, USA). Plasma amino acid concentrations and enrichments were determined by GC-MS (Agilent 7890A GC/5975C; MSD, Little Falls, DE, USA). Specifically, internal standards of [U$\left.{ }^{13} \mathrm{C}_{6}\right]$-leucine, $\left[\mathrm{U}_{-}{ }^{13} \mathrm{C}_{9}{ }^{15} \mathrm{~N}\right]$-phenylalanine, and $\left[\mathrm{U}^{13} \mathrm{C}_{9}{ }^{15} \mathrm{~N}\right]$-tyrosine were added to the samples. The plasma was deproteinized on ice with dry 5-sulfosalicylic acid. Free amino 
acids were purified using cation exchange AG 50W-X8 resin (mesh size: 100-200, ionic form: hydrogen (Bio-Rad Laboratories, Hercules, CA, USA)) columns. The free amino acids were converted to their tert-butyl dimethylsilyl (TBDMS) derivative before analysis by GC-MS. The amino acid concentrations were determined using electron impact ionization by monitoring ions at mass/charge $(\mathrm{m} / \mathrm{z}) 302$ and 308 for unlabelled and $\left[\mathrm{U}_{-}{ }^{13} \mathrm{C}_{6}\right]$ leucine respectively, 336 and 346 for unlabelled and $\left[\mathrm{U}_{-}^{13} \mathrm{C}_{9}{ }^{15} \mathrm{~N}\right]$-phenylalanine, respectively. For L-[1- $\left.{ }^{13} \mathrm{C}\right]$-phenylalanine, $\mathrm{m} / \mathrm{z} 336(m+0)$ and $337(m+1)$ were monitored. Standard regression curves were applied from a series of known standard enrichment values against the measured values to assess the linearity of the mass spectrometer and to account for any isotope fractionation that may have occurred during the analysis. Phenylalanine enrichments were corrected for the presence of the ${ }^{13} \mathrm{C}$ isotopes. Standard regression curves were applied in all isotopic enrichment analyses to assess the linearity of the mass spectrometer and to control for the loss of tracer. Enrichments (MPE) were corrected for the natural abundance of ${ }^{13} \mathrm{C}$ phenylalanine [27].

\section{Muscle analyses}

Myofibrillar protein enriched fractions were extracted from wet muscle tissue as described previously [28]. In short, $50 \mathrm{mg}$ wet muscle tissue was manually homogenized on ice using a Teflon pestle in a standard extraction buffer, after which the samples were centrifuged and the supernatants containing sarcoplasmic proteins were removed. In an additional step, the collagen-containing supernatant was removed. The remaining myofibrillar fraction was purified and hydrolyzed, such that the free amino acids remained and could be dried. The enrichment of the derivative was measured by GC-C-IRMS (MAT 253; Thermo Scientific, Bremen, Germany) by using a DB5DB5-MScolumn (no. 122122-5532; Agilent J+W, USA), GC Isolink, and monitoring of ion masses 44,45 and 46 . By establishing the relationship between the enrichment of a series of L$\left[1-{ }^{13} \mathrm{C}\right]$-phenylalanine standards of variable enrichment, the myofibrillar protein-bound enrichment of phenylalanine was determined. Standard regression curves were applied to assess the linearity of the mass spectrometer and to control for the loss of tracer. Muscle protein deposition from the ingested casein over the $8 \mathrm{~h}$ postprandial period was expressed in the relative increase of $\mathrm{L}-\left[1-{ }^{13} \mathrm{C}\right]$-phenylalanine enrichment in muscle tissue.

\section{Statistics}

All data are expressed as means \pm SEM. Differences in baseline leg values (i.e. NMES vs CON) were determined using a paired, two-tailed Student's t-Test. Differences in protein-bound $\mathrm{L}-\left[1-{ }^{13} \mathrm{C}\right]$-phenylalanine enrichments and FSR between the NMES and CON legs after $8 \mathrm{~h}$ incorporation were analyzed using a paired-samples t-test. In case of a significant effect, a post hoc test with Bonferroni correction was used to locate significant differences. Statistical significance was set at $P<0.05$. All calculations were performed by using the SPSS statistics 22 software package (SPSS Inc., Chicago, IL, USA). 


\section{RESULTS}

\section{Plasma analyses}

Plasma insulin concentrations are presented in Figure 2. Provision of intrinsically labelled casein resulted in a small but significant increase in circulating plasma insulin concentrations (time effect; $P<0.001$ ). Plasma insulin concentrations averaged 20.6 \pm 2.4 $\mathrm{mU} \cdot \mathrm{L}^{-1}$ during the overnight period, and reached peak values of $34.7 \pm 5.9 \mathrm{mU} \cdot \mathrm{L}^{-1}(P<0.01$ versus $t=0)$.

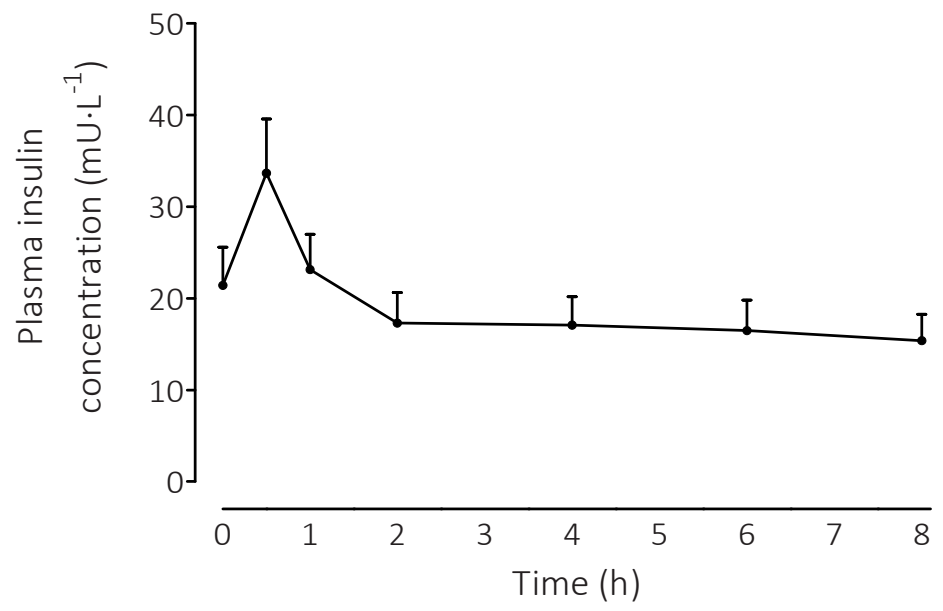

Figure 2 | Plasma insulin concentrations, expressed as means \pm SEM. Data were analyzed with repeated measures ANOVA. Plasma insulin: time effect, $P<0.001$.

Plasma glucose concentrations declined following protein ingestion (time effect; $P<0.001)$, and became significantly different from baseline values from $t=2 \mathrm{~h}$ onwards $(P<0.05)$. Overnight plasma glucose concentrations averaged $5.6 \pm 0.1 \mathrm{mmol} \cdot \mathrm{L}^{-1}$. Figure 3 depicts plasma leucine and phenylalanine concentrations during the overnight period. At the start of the experiment, fasting plasma leucine and phenylalanine concentrations averaged $133 \pm 4$ and $57 \pm 1 \mu \mathrm{M}$, respectively. In the evening, prior to ingestion of the protein drink, concentrations averaged $138 \pm 7$ and $64 \pm 1 \mu \mathrm{M}$, respectively. Directly after protein ingestion ( 10:30 PM), plasma leucine and phenylalanine concentrations increased rapidly (time effects; $P<0.001$ ), and remained elevated throughout most of the night. Peak plasma leucine $(284 \pm 11 \mu \mathrm{M})$ and phenylalanine $(89 \pm 3 \mu \mathrm{M})$ concentrations were reached at $182 \pm 19$ and $155 \pm 25$ min after protein ingestion, respectively. 

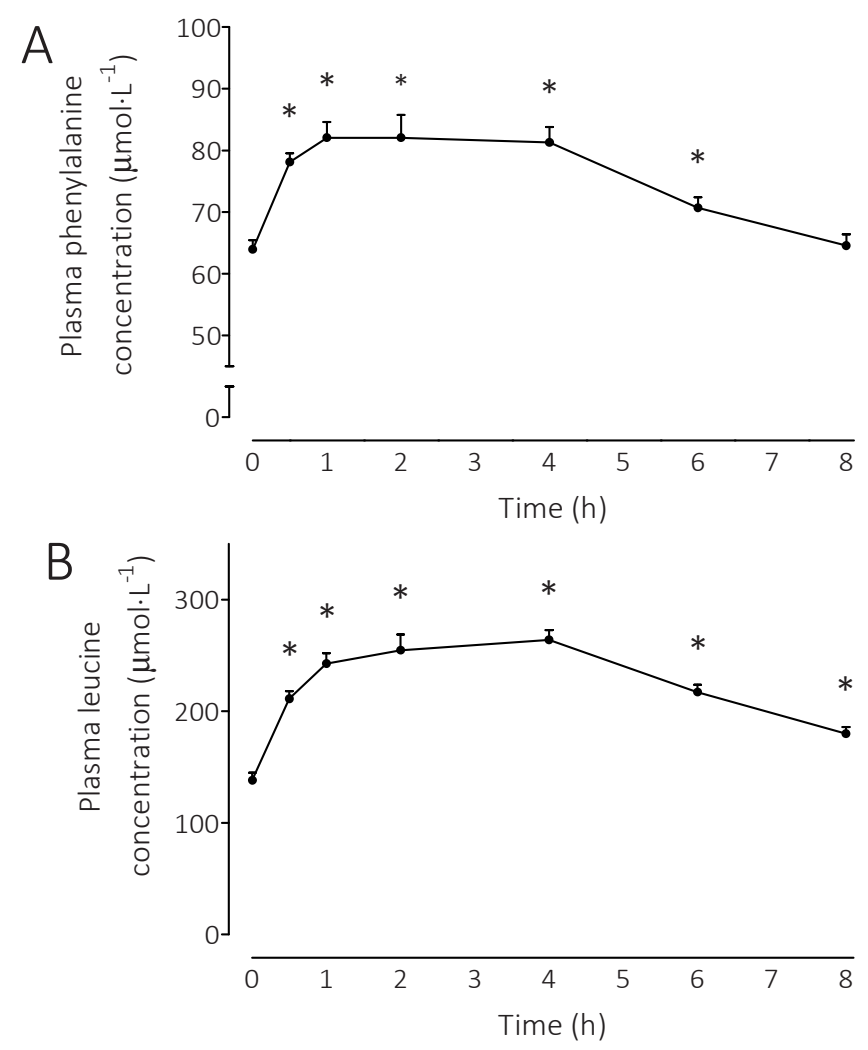

Figure 3 | Plasma leucine (A) and phenylalanine (B) concentrations. Values are expressed as means \pm SEM. Data were analyzed with repeated measures ANOVA. Plasma leucine: time effect, $P<0.001$; plasma phenylalanine: time effect, $P<0.001 ;$ *Significantly different from baseline value.

\section{Stable isotope tracer analyses}

Plasma L- $\left[1-{ }^{13} \mathrm{C}\right]$-phenylalanine enrichments over time are presented in Figure 4. Plasma $\mathrm{L}-\left[1-{ }^{13} \mathrm{C}\right]$-phenylalanine enrichments increased rapidly after ingestion of the intrinsically $\mathrm{L}-\left[1-{ }^{13} \mathrm{C}\right]$-phenylalanine labelled casein protein, with plasma $\mathrm{L}-\left[1-{ }^{13} \mathrm{C}\right]$-phenylalanine enrichment levels reaching peak values of $12.2 \pm 0.5 \mathrm{MPE}$ at $174 \pm 16$ min after protein consumption. Plasma L-[1 $\left[{ }^{13} \mathrm{C}\right]$-phenylalanine enrichments remained significantly elevated for the remaining night (time effect; $P<0.001$ ). 


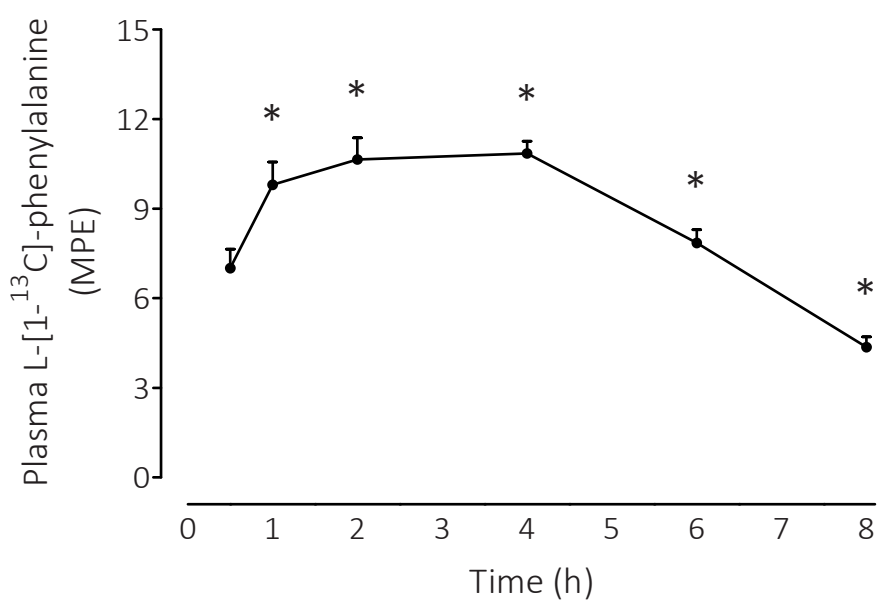

Figure 4 | Plasma L-[1- $\left.{ }^{13} \mathrm{C}\right]$-phenylalanine enrichments. Values are expressed as means \pm SEM. Data were analyzed with repeated measures ANOVA. Plasma phenylalanine: time effect, $P<0.001$; *Significantly different from baseline values.

Myofibrillar muscle protein-bound $\mathrm{L}-\left[1-{ }^{13} \mathrm{C}\right]$-phenylalanine enrichments are presented in Figure 5. Muscle tissue analyses were performed on muscle biopsy samples collected before, and $8 \mathrm{~h}$ after ingestion of the intrinsically $\mathrm{L}-\left[1-{ }^{13} \mathrm{C}\right]$-phenylalanine labelled casein. After the $8 \mathrm{~h}$ overnight period, $\mathrm{L}-\left[1-{ }^{13} \mathrm{C}\right]$-phenylalanine enrichments had increased up to $0.0297 \pm 0.0016$ MPE in the CON leg and 0.0344 \pm 0.0019 MPE in the NMES leg, $(P<0.01)$, representing $18 \pm 6 \%$ greater enrichments in the NMES leg compared with the CON leg.

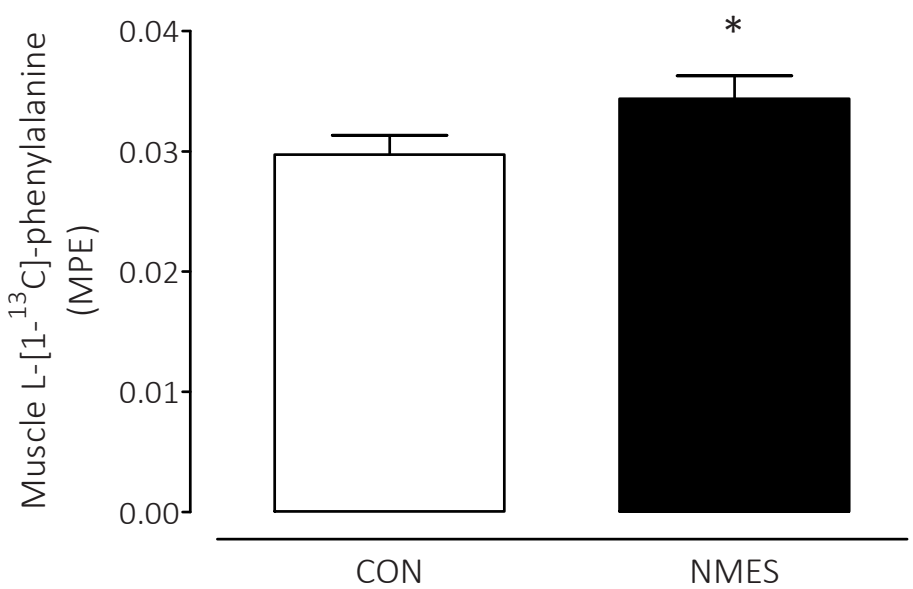

Figure 5 | Muscle tissue L-[1- $\left.{ }^{13} \mathrm{C}\right]$-phenylalanine enrichments. Values are expressed as means \pm SEM. Data were analyzed with a paired student's t-test. *Significantly different from the control leg $(P<0.01)$. 


\section{DISCUSSION}

The present study demonstrates for the first time that ingestion of $40 \mathrm{~g}$ casein protein prior to sleep leads to overnight de novo myofibrillar protein accretion in healthy, elderly men after a day of bed rest. The overnight accretion of newly formed muscle protein was augmented when neuromuscular electrical stimulation (NMES) was applied prior to protein ingestion.

Short periods of bed rest or immobilization due to injury or illness lead to substantial loss of skeletal muscle mass and strength [29-32]. The decline in muscle tissue has been, at least partly, attributed to a blunted muscle protein synthetic response to food ingestion [33]. This so-called anabolic resistance to protein ingestion can be compensated for by changing the dietary protein source [34,35], the amount of ingested protein [36-38] and/or the distribution of protein intake throughout the day [39-41]. Recently, we introduced the concept of stimulating overnight muscle protein synthesis by providing dietary protein during sleep in older males [16]. Intragastric protein administration via a nasogastric tube during overnight sleep was followed by proper dietary protein digestion and absorption, which increased plasma amino acid availability and stimulated de novo muscle protein synthesis in healthy elderly males [16]. In the current study, $40 \mathrm{~g}$ casein protein was ingested prior to sleep to test the concept of nighttime protein feeding in a more practically applicable manner. We show that pre-sleep ingestion of intrinsically $\mathrm{L}-\left[1-{ }^{13} \mathrm{C}\right]$-phenylalanine labelled casein leads to a substantial postprandial rise in circulating plasma phenylalanine concentrations (Figure 3 ) and L-[1- $\left.{ }^{13} \mathrm{C}\right]$ phenylalanine enrichments (Figure 4). The increase in plasma L-[1- $\left.{ }^{13} \mathrm{C}\right]$-labelled phenylalanine clearly demonstrates that protein ingested prior to sleep is followed by proper digestion and absorption throughout the remainder of the night. Dietary protein derived amino acids were subsequently used for de novo myofibrillar protein synthesis as evidenced by the increase in myofibrillar protein-bound L-[1- $\left.{ }^{13} \mathrm{C}\right]$-phenylalanine enrichment in both legs in the $8 \mathrm{~h}$ following protein ingestion (Figure 5). The results of our study are in line with our previous findings and show that protein ingestion prior to sleep leads to proper protein digestion, amino acid absorption and net myofibrillar protein accretion throughout overnight sleep [16], and demonstrate that protein ingestion prior to sleep forms a practical and effective nutritional strategy to stimulate overnight muscle protein accretion.

Besides dietary protein ingestion, physical activity is a key anabolic stimulus that is required to preserve skeletal muscle mass. In a clinical setting, lack of sufficient physical activity causes anabolic resistance [33], resulting in rapid muscle loss [3, 42, 43]. Recently we introduced NMES as a surrogate for physical activity to increase the sensitivity of skeletal muscle tissue to the anabolic properties of protein administration [44] and, as such, to attenuate muscle mass loss during a short period of immobilization [21]. We hypothesized that NMES performed in the evening sensitizes skeletal muscle tissue to the anabolic properties of overnight protein feeding. In the present study, we electrically stimulated one leg, whereas the other leg was sham stimulated. This means that 
electrodes and wiring were applied to both legs but only one leg was actually electrically stimulated. After protein feeding, the stimulated leg showed an $18 \pm 6 \%$ greater increase in muscle protein-bound $\mathrm{L}-\left[1-{ }^{13} \mathrm{C}\right]$-phenylalanine enrichment when compared with the control leg $(0.0344 \pm 0.0019$ vs $0.0297 \pm 0.0016$, respectively) assessed over 8 hours of subsequent sleep $(P<0.01$; Figure 5$)$. NMES effectively increased the sensitivity of the skeletal muscle tissue to protein feeding with more of the ingested protein being directed towards de novo muscle protein accretion during overnight sleep.

NMES has been proposed as an interventional strategy that can be used to evoke involuntary muscle contraction during muscle disuse, when individuals experience difficulties performing voluntary muscle contraction. The combined application of NMES and protein feeding prior to sleep may be of particular relevance in more clinically compromised individuals. An interesting population that could benefit greatly from such an intervention are clinically compromised elderly subjects that are hospitalized for a short period following a fall and/or a fracture. Most elderly individuals have difficulty regaining skeletal muscle mass after a short period of disuse [45], which impairs rehabilitation and contributes greatly to the development of frailty. Therefore, interventional strategies that can preserve skeletal muscle mass during a short period of muscle disuse are of paramount importance. From a practical point of view, the use of NMES has been shown to be both applicable and feasible during muscle disuse. In all studies performed in our laboratory $[20,21]$, the treatment was well tolerated and no contra-indications or side effects were observed. Furthermore, the costs associated with NMES are relatively low, and hospital staff can easily apply the technique. Long-term effects of this treatment on muscle mass remain to be investigated, but we expect our results to be applicable for bedridden patients as we have even observed muscle mass preservation following the application of NMES in a setting of accelerated muscle loss in comatose patients [46].

In conclusion, this is the first study to show that protein ingestion prior to sleep represents an effective interventional strategy to stimulate overnight skeletal muscle protein accretion in vivo in healthy, older men. The application of NMES in the evening can further augment postprandial muscle protein accretion following protein ingestion prior to sleep. The application of NMES combined with protein ingestion prior to sleep represents a feasible strategy to optimize overnight muscle protein accretion, and may be of great clinical benefit to attenuate muscle loss in clinically compromised individuals.

\section{ACKNOWLEDGEMENTS}

We gratefully acknowledge the enthusiastic support of Benjamin T Wall and the subjects who volunteered to participate in this experiment, and the assistance of Anne Gingrich. 


\section{REFERENCES}

1. Koopman, R. and L. van Loon, Aging, exercise, and muscle protein metabolism. J Appl Physiol (Bethesda, Md. : 1985), 2009. 106(6): p. 2040-2048.

2. Evans, W., What is sarcopenia? J Gerontol A Biol Sci Med Sci, 1995. 50 Spec No: p. 5-8.

3. Cuthbertson, D., K. Smith, J. Babraj, G. Leese, T. Waddell, P. Atherton, H. Wackerhage, P.M. Taylor, and M.J. Rennie, Anabolic signaling deficits underlie amino acid resistance of wasting, aging muscle. FASEB J, 2005. 19(3): p. 422-4.

4. Kumar, D., C. Idzikowski, D.L. Wingate, E.E. Soffer, P. Thompson, and C. Siderfin, Relationship between enteric migrating motor complex and the sleep cycle. Am J Physiol, 1990. 259(6 Pt 1): p. G983-90.

5. Evans, W.J., Protein nutrition, exercise and aging. J Am Coll Nutr, 2004. 23(6 Suppl): p. 601S-609S.

6. Wall, B.T., M.L. Dirks, and L.J. van Loon, Skeletal muscle atrophy during short-term disuse: implications for age-related sarcopenia. Ageing Res Rev, 2013. 12(4): p. 898-906.

7. Breen, L. and S. Phillips, Skeletal muscle protein metabolism in the elderly: Interventions to counteract the 'anabolic resistance' of ageing. Nutr Metab, 2011. 8: p. 68.

8. van Loon, L., Y. Boirie, A. Gijsen, J. Fauquant, A. de Roos, A. Kies, S. Lemosquet, W. Saris, and R. Koopman, The production of intrinsically labeled milk protein provides a functional tool for human nutrition research. Journal of dairy science, 2009. 92(10): p. 4812-4822.

9. Burd, N.A., S.H. Gorissen, and L.J. van Loon, Anabolic resistance of muscle protein synthesis with aging. Exerc Sport Sci Rev, 2013. 41(3): p. 169-73.

10. Volpi, E., A.A. Ferrando, C.W. Yeckel, K.D. Tipton, and R.R. Wolfe, Exogenous amino acids stimulate net muscle protein synthesis in the elderly. J Clin Invest, 1998. 101(9): p. 2000-7.

11. Boirie, Y., M. Dangin, P. Gachon, M.P. Vasson, J.L. Maubois, and B. Beaufrere, Slow and fast dietary proteins differently modulate postprandial protein accretion. Proc Natl Acad Sci U S A, 1997. 94(26): p. 14930-5.

12. Pennings, B., Y. Boirie, J.M. Senden, A.P. Gijsen, H. Kuipers, and L.J. van Loon, Whey protein stimulates postprandial muscle protein accretion more effectively than do casein and casein hydrolysate in older men. Am J Clin Nutr, 2011. 93(2): p. 322-31.

13. Koopman, R., L.B. Verdijk, M. Beelen, M. Gorselink, A.N. Kruseman, A.J. Wagenmakers, H. Kuipers, and L.J. van Loon, Co-ingestion of leucine with protein does not further augment post-exercise muscle protein synthesis rates in elderly men. Br J Nutr, 2008. 99(3): p. 571-80.

14. Rieu, I., M. Balage, C. Sornet, C. Giraudet, E. Pujos, J. Grizard, L. Mosoni, and D. Dardevet, Leucine supplementation improves muscle protein synthesis in elderly men independently of hyperaminoacidaemia. J Physiol, 2006. 575(Pt 1): p. 305-15.

15. Esmarck, B., J. Andersen, S. Olsen, E. Richter, M. Mizuno, and M. Kjaer, Timing of postexercise protein intake is important for muscle hypertrophy with resistance training in elderly humans. J Physiol, 2001. 535(Pt 1): p. 301-311.

16. Groen, B.B., P.T. Res, B. Pennings, E. Hertle, J.M. Senden, W.H. Saris, and L.J. van Loon, Intragastric protein administration stimulates overnight muscle protein synthesis in elderly men. Am J Physiol Endocrinol Metab, 2012. 302(1): p. E52-60.

17. Res, P.T., B. Groen, B. Pennings, M. Beelen, G.A. Wallis, A.P. Gijsen, J.M. Senden, and L.J. van Loon, Protein ingestion before sleep improves postexercise overnight recovery. Med Sci Sports Exerc, 2012. 44(8): p. 1560-9.

18. Glover, E.I., S.M. Phillips, B.R. Oates, J.E. Tang, M.A. Tarnopolsky, A. Selby, K. Smith, and M.J. Rennie, Immobilization induces anabolic resistance in human myofibrillar protein synthesis with low and high dose amino acid infusion. J Physiol, 2008. 586(Pt 24): p. 6049-61.

19. Wall, B.T., T. Snijders, J.M. Senden, C.L. Ottenbros, A.P. Gijsen, L.B. Verdijk, and L.J. van Loon, Disuse impairs the muscle protein synthetic response to protein ingestion in healthy men. J Clin Endocrinol Metab, 2013. 98(12): p. 4872-81. 
20. Wall, B.T., M.L. Dirks, L.B. Verdijk, T. Snijders, D. Hansen, P. Vranckx, N.A. Burd, P. Dendale, and L.J. van Loon, Neuromuscular electrical stimulation increases muscle protein synthesis in elderly type 2 diabetic men. Am J Physiol Endocrinol Metab, 2012. 303(5): p. E614-23.

21. Dirks, M.L., B.T. Wall, T. Snijders, C.L. Ottenbros, L.B. Verdijk, and L.J. van Loon, Neuromuscular electrical stimulation prevents muscle disuse atrophy during leg immobilization in humans. Acta Physiol (Oxf), 2014. 210(3): p. 628-41.

22. Expert Committee on the, D. and M. Classification of Diabetes, Report of the expert committee on the diagnosis and classification of diabetes mellitus. Diabetes care, 2003. 26 Suppl 1: p. 20.

23. Jones, P.R. and J. Pearson, Anthropometric determination of leg fat and muscle plus bone volumes in young male and female adults. J Physiol, 1969. 204(2): p. 63P-66P.

24. Bergstrom, J., Percutaneous needle biopsy of skeletal muscle in physiological and clinical research. Scand J Clin Lab Invest, 1975. 35(7): p. 609-16.

25. Wall, B.T., H.M. Hamer, A. de Lange, A. Kiskini, B.B. Groen, J.M. Senden, A.P. Gijsen, L.B. Verdijk, and L.J. van Loon, Leucine co-ingestion improves post-prandial muscle protein accretion in elderly men. Clin Nutr, 2013. 32(3): p. 412-9.

26. van Loon, L.J., Y. Boirie, A.P. Gijsen, J. Fauquant, A.L. de Roos, A.K. Kies, S. Lemosquet, W.H. Saris, and R. Koopman, The production of intrinsically labeled milk protein provides a functional tool for human nutrition research. J Dairy Sci, 2009. 92(10): p. 4812-22.

27. Biolo, G., P. Tessari, S. Inchiostro, D. Bruttomesso, C. Fongher, L. Sabadin, M. Fratton, A. Valerio, and A. Tiengo, Leucine and phenylalanine kinetics during mixed meal ingestion: a multiple tracer approach. AM j Physiol, 1992. 262(4 Pt 1): p. 63.

28. Burd, N.A., N.M. Cermak, I.W. Kouw, S.H. Gorissen, A.P. Gijsen, and L.J. van Loon, The use of doubly labeled milk protein to measure postprandial muscle protein synthesis rates in vivo in humans. J Appl Physiol, 2014. 117(11): p. 1363-70.

29. Deitrick, J.E., The effect of immobilization on metabolic and physiological functions of normal men. Bull $N$ Y Acad Med, 1948. 24(6): p. 364-75.

30. Haruna, Y., Y. Suzuki, K. Kawakubo, R. Yanagibori, and A. Gunji, Decremental reset in basal metabolism during 20-days bed rest. Acta Physiol (Scand). Supplementum, 1994. 616: p. 43-9.

31. Ingemann-Hansen, T. and J. Halkjaer-Kristensen, Computerized tomographic determination of human thigh components. The effects of immobilization in plaster and subsequent physical training. Scand J Rehabil Med, 1980. 12(1): p. 27-31.

32. LeBlanc, A.D., V.S. Schneider, H.J. Evans, C. Pientok, R. Rowe, and E. Spector, Regional changes in muscle mass following 17 weeks of bed rest. J Appl Physiol, 1992. 73(5): p. 2172-8.

33. Wall, B.T., T. Snijders, J.M. Senden, C.L. Ottenbros, A.P. Gijsen, L.B. Verdijk, and L.J. van Loon, Disuse impairs the muscle protein synthetic response to protein ingestion in healthy men. J Clin Endocrinol Metab, 2013. 98(12): p. 4872-4881.

34. Luiking, Y.C., M.P. Engelen, P.B. Soeters, Y. Boirie, and N.E. Deutz, Differential metabolic effects of casein and soy protein meals on skeletal muscle in healthy volunteers. Clin Nutr, 2011. 30(1): p. 65-72.

35. Tipton, K.D., T.A. Elliott, M.G. Cree, S.E. Wolf, A.P. Sanford, and R.R. Wolfe, Ingestion of casein and whey proteins result in muscle anabolism after resistance exercise. Med Sci Sports Exer, 2004. 36(12): p. 20732081.

36. Pennings, B., B. Groen, A. de Lange, A.P. Gijsen, A.H. Zorenc, J.M. Senden, and L.J. van Loon, Amino acid absorption and subsequent muscle protein accretion following graded intakes of whey protein in elderly men. Am J Physiol Endocrinol Metab, 2012. 302(8): p. E992-9.

37. Volpi, E., A.A. Ferrando, C.W. Yeckel, K.D. Tipton, and R.R. Wolfe, Exogenous amino acids stimulate net muscle protein synthesis in the elderly. J Clin Invest, 1998. 101(9): p. 2000-7.

38. Moore, D.R., M.J. Robinson, J.L. Fry, J.E. Tang, E.I. Glover, S.B. Wilkinson, T. Prior, M.A. Tarnopolsky, and S.M. Phillips, Ingested protein dose response of muscle and albumin protein synthesis after resistance exercise in young men. Am J Clin Nutr, 2009. 89(1): p. 161-168.

39. Wall, B.T. and L.J. van Loon, Nutritional strategies to attenuate muscle disuse atrophy. Nutr Rev, 2013. 71(4): p. 195-208. 
40. Areta, J.L., L.M. Burke, M.L. Ross, D.M. Camera, D.W. West, E.M. Broad, N.A. Jeacocke, D.R. Moore, T. Stellingwerff, S.M. Phillips, J.A. Hawley, and V.G. Coffey, Timing and distribution of protein ingestion during prolonged recovery from resistance exercise alters myofibrillar protein synthesis. J Physiol, 2013. 591(Pt 9): p. 2319-2331.

41. Walrand, S. and Y. Boirie, Optimizing protein intake in aging. Curr Opin Clin Nutr Metab Care, 2005. 8(1): p. 89-94.

42. Guillet, C., M. Prod'homme, M. Balage, P. Gachon, C. Giraudet, L. Morin, J. Grizard, and Y. Boirie, Impaired anabolic response of muscle protein synthesis is associated with S6K1 dysregulation in elderly humans. FASEB J, 2004. 18(13): p. 1586-1587.

43. Rasmussen, B.B., S. Fujita, R.R. Wolfe, B. Mittendorfer, M. Roy, V.L. Rowe, and E. Volpi, Insulin resistance of muscle protein metabolism in aging. FASEB J, 2006. 20(6): p. 768-9.

44. Wall, B.T., M.L. Dirks, L.B. Verdijk, T. Snijders, D. Hansen, P. Vranckx, N.A. Burd, P. Dendale, and L.J. van Loon, Neuromuscular electrical stimulation increases muscle protein synthesis in elderly type 2 diabetic men. Am J Physiol Endocrinol Metab, 2012. 303(5): p. 23.

45. Suetta, C., U. Frandsen, A.L. Mackey, L. Jensen, L.G. Hvid, M.L. Bayer, S.J. Petersson, H.D. Schroder, J.L. Andersen, P. Aagaard, P. Schjerling, and M. Kjaer, Ageing is associated with diminished muscle re-growth and myogenic precursor cell expansion early after immobility-induced atrophy in human skeletal muscle. J Physiol, 2013. 591(Pt 15): p. 3789-804.

46. Dirks, M.L., D. Hansen, A. Van Assche, P. Dendale, and L.J. Van Loon, Neuromuscular electrical stimulation prevents muscle wasting in critically ill comatose patients. Clin Sci (Lond), 2015. 128(6): p. 357-65. 


\section{CHAPTER}

\section{The impact of bed rest versus leg immobilization on muscle disuse atrophy}

Marlou L Dirks

Evelien MP Backx

Benjamin T Wall

Lex B Verdijk

LuC JC van Loon

Acta Physiol (Oxf). 2016 Sept;218(1):10-2 
ABSTRACT

Background Short periods of muscle disuse, caused by injury or illness, result in a loss of muscle mass and strength. Muscle disuse can be localized to a limb (i.e. leg immobilization due to bone fracture), or may occur on a whole-body level (i.e. bed rest during critical illness or following surgery). A direct comparison of the rate of muscle atrophy and loss of muscle strength between both conditions is lacking. We tested our hypothesis that one week of bed rest leads to greater muscle loss when compared with limb immobilization.

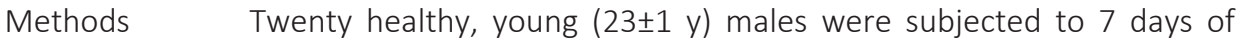
either leg immobilization via a full leg cast $(n=10)$, or strict bed rest $(n=10)$. Before and immediately after the disuse period, single-slice CT-scans and one-Repetition Maximum (1RM) strength tests were performed to assess quadriceps cross-sectional area (CSA) and maximal leg muscle strength, respectively. Additionally, muscle biopsies were taken from $m$. vastus lateralis to assess muscle fibre-type specific CSA.

Results Seven days of muscle disuse resulted in a substantial decline in quadriceps CSA in both the limb immobilization $(-5.4 \pm 1.0 \%)$ and bed-rested $(-3.2 \pm 0.9 \% ; P<0.01)$ group $(P<0.001)$, with no significant differences between groups $(P>0.05)$. Maximal leg muscle strength declined by $6 \pm 3 \%$ and $8 \pm 2 \%$, respectively $(P<0.01)$, with no differences between groups $(P>0.05)$. No significant changes in type I and II muscle fibre CSA were observed within one week of disuse ( $P>0.05)$.

Conclusion Seven days of muscle disuse following leg immobilization and bed rest leads to substantial declines in skeletal muscle mass and strength. Although the rate of muscle atrophy does not differ between both models of disuse, more total lean mass is lost following bed rest compared with single leg immobilization. 


\section{INTRODUCTION}

An injury or illness often requires a short period of muscle disuse in an otherwise healthy person. Such short periods are generally mandated to facilitate recovery, but concurrently cause substantial loss of muscle mass and strength [1-3]. Such skeletal muscle disuse has been shown to lead to various negative health consequences such as an increase in fat mass $[4,5]$, a decline in basal metabolic rate $[6,7]$, and the development of glucose intolerance and insulin resistance $[8,9]$. Preservation of muscle mass during short periods of disuse is of important clinical relevance and deserves more focus in present clinical research.

Muscle disuse can be localized to a limb, such as seen following arm or leg immobilization after ligament damage or bone fracture. Alternatively, bed rest may be required following trauma, surgery, or during (critical) illness. Both limb immobilization [1-3, 1013] and bed rest [4, 9, 14-17] have been shown to be accompanied by substantial declines in both muscle mass and strength (as reviewed in [18]). The extent of muscle mass and strength loss is likely dependent upon the amount of muscle tissue that is subjected to disuse. Whereas muscle loss during limb immobilization can be regarded as a direct result of local muscle disuse, bed rest may result in greater muscle loss due to the amount of muscle tissue that experiences disuse as well as various systemic factors (e.g. hormonal changes [9, 19-21], or systemic inflammation [22]) that may increase the rate of muscle atrophy. In the current scientific literature, both models are typically applied to investigate the impact of disuse on the loss of muscle mass and function. However, whether both models of disuse actually lead to comparable local muscle loss remains to be assessed.

In the present study, we compare the rate of muscle atrophy and loss of muscle strength following one week of one-legged knee immobilization versus one week of strict bed rest. We hypothesized that the rate of muscle atrophy would be greater during bed rest when compared with leg immobilization. To test our hypothesis, we subjected 20 healthy, young males to 7 days of either single leg immobilization or strict bed rest. Measures of muscle mass, muscle strength, and muscle fibre type-specific crosssectional area were performed to investigate the impact of both models of muscle disuse. 


\section{METHODS}

\section{Participants}

Between September 2013 and April 2015, 20 healthy, young males (age: $23 \pm 1$ y, BMI $23.2 \pm 0.7 \mathrm{~kg} \cdot \mathrm{m}^{-2}$ ) were included in the present study. These volunteers were part of two previous studies (NCT02109380/NCT01894737) which represented part of a larger project investigating the role of muscle disuse on muscle mass and function. The current article describes a comparison between age- and BMI-matched male participants who underwent 7 days of either single leg immobilization or bed rest. Prior to inclusion, participants completed a general health questionnaire and routine medical screening to ensure their eligibility to participate. Male individuals, 18-35 years old, were excluded if they matched one or more of the following exclusion criteria: BMI below 18.5 or above $30 \mathrm{~kg} \cdot \mathrm{m}^{-2}$, a (family) history of thrombosis, diabetes mellitus, and any back, knee or shoulder complaints. Furthermore, individuals who underwent surgery or participated in progressive resistance training in the past 6 months were excluded from participation. The present study was approved by the Medical Ethical Committee of Maastricht University Medical Centre in accordance with the Declaration of Helsinki. All participants were informed on the nature and possible risks of the experimental procedures, after which written informed consent was obtained. As part of the screening visit, an estimation of one-Repetition Maximum (1RM) knee extension strength (Technogym, Rotterdam, the Netherlands) was made by using the multiple repetitions testing procedure [23].

\section{Experimental outline}

Twenty-four to $48 \mathrm{~h}$ prior to, and immediately after the period of disuse, participants visited the laboratory in a fasted state for 2 identical test days (i.e. test days 1 and 2) during which measures of muscle mass and function were performed. On test day 1 , firstly, a single-slice CT scan of the upper leg was performed. Next, a muscle biopsy was taken from the vastus lateralis muscle. Lastly, a 1RM leg strength test was performed. One or two days later, participants returned to the laboratory to start their period of muscle disuse (described below). A second test day was performed following 7 days of muscle disuse. On that day, the CT scan and muscle biopsy sampling were completed before any weight bearing was performed.

\section{One week of muscle disuse}

Participants were subjected to 7 days of muscle disuse via a leg cast, or by means of strict bed rest. Forty-eight $\mathrm{h}$ following test day 1 , participants in the immobilization group reported to the plaster room of the Maastricht University Medical Centre at 8:00 AM on the first day of the immobilization period for the application of a full leg cast. The cast 
extended from halfway up the upper leg ( $25 \mathrm{~cm}$ above the patella) until $\sim 5 \mathrm{~cm}$ above the ankle. The cast was applied with the knee joint in an approximate 30 degree angle of flexion to prevent participants from performing weight-bearing activities with the immobilized leg. Participants received crutches and were given instructions on their correct usage. Application of the cast signified the beginning of the immobilization period, which ended following exactly 7 days by cast removal, immediately prior to test day 2 .

Participants who were included in the bed rest group reported to the laboratory on the morning after test day 1, to start a 7-day period of strict bed rest at 8:00 AM. From that moment on, participants remained in a (semi-) supine position. They were allowed to use a pillow and slightly elevate the bed-back to perform their daily activities. All hygiene and sanitary activities were done while lying down. The bed rest period ended after exactly 7 days, immediately prior to test day 2 .

\section{Muscle mass}

During test days 1 and 2, anatomical cross-section of the quadriceps, hamstring, and whole-thigh muscles, as well as leg circumference, were assessed via a single-slice Computed Tomography (CT) scan (Philips Brilliance 64, Philips Medical Systems, Best, the Netherlands). Images were obtained by using the following scanning characteristics: $120 \mathrm{kV}, 300 \mathrm{~mA}$, rotation time of $0.75 \mathrm{~s}$, and a field of view of $500 \mathrm{~mm}$. While the participants were lying supine, legs extended and their feet secured, a $3 \mathrm{~mm}$ thick axial image was taken $15 \mathrm{~cm}$ proximal to the top of the patella. To ensure proper replication of the scanning position on test day 2 , the precise scanning location was marked with semipermanent ink on test day 1. CT-scans were analysed for the CSA of quadriceps, hamstring, and whole-thigh muscles, as well as leg circumference, by manual tracing using ImageJ software version 1.49s (National Institute of Health, Maryland, USA, [24]) using 0-100 Hounsfield Units for muscle tissue [25].

\section{Muscle strength}

Maximum leg strength was evaluated by a 1RM strength test on a leg extension machine (Technogym). The estimated $1 R M$ values during the screening visit were used to determine 1 RM as described previously [26]. For the participants in the immobilization group, leg strength was measured during a one-legged test with the immobilized leg, whereas two-legged 1RM was assessed in the bed rested group.

\section{Muscle tissue sampling}

During test day 1 and 2, a muscle biopsy was collected from $m$. vastus lateralis via the Bergström technique [27], at approximately $15 \mathrm{~cm}$ above the patella. Any visible nonmuscle tissue was removed, and the biopsy was carefully embedded in Tissue Tek (Sakura Finetek, Zoeterwoude, the Netherlands) and frozen in liquid nitrogen-cooled iso- 
pentane. Muscle samples were subsequently stored at $-80^{\circ} \mathrm{C}$ until the analysis were performed.

\section{Dietary intake}

On the evening prior to both test days, participants consumed a controlled meal to standardize food intake. During the period of muscle disuse, participants in the immobilization group were instructed to maintain their habitual food intake. Prior to and during the immobilization period, participants filled in 3-day dietary records, which were subsequently analysed by Dieetinzicht software (www.dieetinzicht.nl, based on NEVO table 2011). During the immobilization period, participants reported average energy intakes of $8.0 \mathrm{MJ} \cdot \mathrm{d}^{-1}$ and protein intakes of $1.1 \mathrm{~g} \cdot \mathrm{kg}$ body weight ${ }^{-1} \cdot \mathrm{d}^{-1}$, which was not different from their habitual diet $(P>0.05)$. Participants in the bed rest group were fed standardized nutrition for 7 days prior to and during the 7 day bed rest period, based on indirect calorimetry measurements for basal metabolic rate and an activity factor of 1.55 and 1.35 , respectively. This resulted in an energy intake of $9.2 \mathrm{MJ} \cdot \mathrm{d}^{-1}$ and protein intake of $0.98 \mathrm{~g} \cdot \mathrm{kg}$ body weight ${ }^{-1} \cdot \mathrm{d}^{-1}$ during bed rest.

\section{Muscle tissue analyses}

In preparation for muscle fibre analyses, a section of muscle mounted and frozen in Tissue-Tek was cut into $5 \mu \mathrm{m}$ thick cryosections using a cryostat at $-20^{\circ} \mathrm{C}$. Pre- and postdisuse samples of each subject were mounted together on uncoated, pre-cleaned glass slides, thereby carefully aligning the samples for cross-sectional fibre analyses. An immunohistochemical staining was performed to assess muscle fibre-type specific crosssectional area (CSA) as described below. Tissue was fixated in acetone for 5 min, after which the slides were air dried and incubated with 3\% BSA in 0.1\% Tween-PBS for 30 min. After rinsing with PBS for 5 min, slides were incubated with primary antibodies directed against myosin heavy chain (MHC)-I (A4.840, dilution 1:25; DSHB) and laminin (polyclonal rabbit anti-laminin, dilution 1:50; Sigma, Zwijndrecht, the Netherlands) for $30 \mathrm{~min}$. Next, slides were washed and the appropriate secondary antibodies were applied: goat anti-rabbit IgG AlexaFluor647 and goat anti-mouse IgM AlexaFluor555 (dilution 1:400 and 1:500, respectively; Molecular Probes). Both primary and secondary antibodies were diluted in $0.1 \%$ Bovine Serum Albumin (BSA) in $0.1 \%$ Tween- phosphate-buffered saline (PBS). All incubation steps were performed at room temperature. After a last washing step, cover glasses were mounted by Mowiol (Calbiochem, Amsterdam, the Netherlands). As a result of the staining procedure, MHC-I was stained in red and laminin in far-red. On the next day, images were visualized and automatically captured at 10x magnification with a Olympus BX51 fluorescence microscope with customized spinning disk unit (DSU; Olympus, Zoeterwoude, the Netherlands) with a ultra-high sensitivity monochrome electron multiplier CCD camera (1000 x 1000 pixels, C9100-02, Hamamatsu Photonics, Hamamatsu City, Japan). Image acquisition was done by Mi- 
cromanager 1.4 software [28], and images were analysed with ImageJ. Recording and analysing of the images was performed by an investigator blinded to subject coding. As a measure of fibre circularity, form factors were calculated by using the following formula: $(4 \pi \cdot C S A) \cdot(\text { perimeter })^{-2}$. No difference in fibre circularity was observed between pre- and post-disuse samples. On average, $203 \pm 24$ and $234 \pm 33$ muscle fibres were analysed in the pre- and post-disuse samples, respectively.

\section{Statistics}

All data presented are expressed as meanststandard error of the mean (SEM). An independent samples t-test was used at baseline to test for differences between groups. Pre- versus post-intervention data were analysed using Repeated Measures ANOVA with time (pre vs post) as within-subjects factor and treatment (immobilization vs bed rest) as between-subjects factor. For muscle fibre-specific analysis, fibre type (type I vs type II) was added to the Repeated Measures ANOVA as a within-subjects factor. In case of a significant interaction, paired t tests were performed to determine time effects within groups or within type I or II fibres, and independent t tests for group differences in the pre- and post-intervention values. All statistical analyses were performed using SPSS version 22.0 (SPSS Inc., Chicago, IL, USA), and $P<0.05$ was used to determine statistical significance. 


\section{RESULTS}

\section{Participants}

Participants' characteristics are displayed in Table 1. No difference between the immobilization and bed rest groups were observed for any of the parameters.

Table 1 | Participants' characteristics

\begin{tabular}{lccc}
\hline & Immobilization $(n=10)$ & Bed rest $(n=10)$ & $P$-value \\
\hline Age $(\mathrm{y})$ & $23 \pm 1$ & $23 \pm 1$ & 0.942 \\
Body weight $(\mathrm{kg})$ & $72.3 \pm 4.1$ & $74.9 \pm 2.3$ & 0.601 \\
Height $(\mathrm{m})$ & $1.76 \pm 0.03$ & $1.81 \pm 0.02$ & 0.208 \\
BMI $\left(\mathrm{kg} \cdot \mathrm{m}^{-2}\right)$ & $23.5 \pm 1.1$ & $23.0 \pm 0.9$ & 0.756 \\
\hline
\end{tabular}

Values represent means \pm SEM. BMI, body mass index

\section{Muscle mass}

Quadriceps cross-sectional area (CSA) declined following 7 days of disuse in both groups though no significant interaction based on treatment was observed (Figure 1; time effect $P<0.001$, time $x$ treatment interaction $P=0.097$ ), such that in the immobilization group a $5.4 \pm 1.0 \%$ decline (from $7646 \pm 399$ to $7229 \pm 373 \mathrm{~mm}^{2}$ ) was observed and participants in the bed rest group lost $3.2 \pm 0.9 \%$ (from $7900 \pm 315$ to $7664 \pm 354 \mathrm{~mm}^{2}$ ). Wholethigh muscle CSA decreased similarly in both groups (mean decline $-2.8 \pm 0.6 \%$; $P<0.001$ ), whereas no change in hamstring CSA was observed $(P>0.05)$. Leg circumference declined following the intervention, without differences between groups (mean decrease $1.1 \pm 0.2 \%, P<0.001$ ). Hounsfield units (HUs) from the whole-thigh muscle increased in the bed rest group only (time $x$ treatment interaction; $P<0.05$ ), whereas HUs from the quadriceps increased similarly in both groups $(P<0.01)$. On a muscle fibre level, no changes in muscle fibre CSA of type I $(6132 \pm 539$ to $6290 \pm 654$ and $6650 \pm 725$ to $\left.6218 \pm 662 \mu \mathrm{m}^{2}\right)$ or II fibres $\left(7561 \pm 728\right.$ to $7166 \pm 714$ and $6542 \pm 746$ to $5982 \pm 525 \mu^{2}$ ) were observed following 7 days of either leg immobilization or bed rest, respectively. 


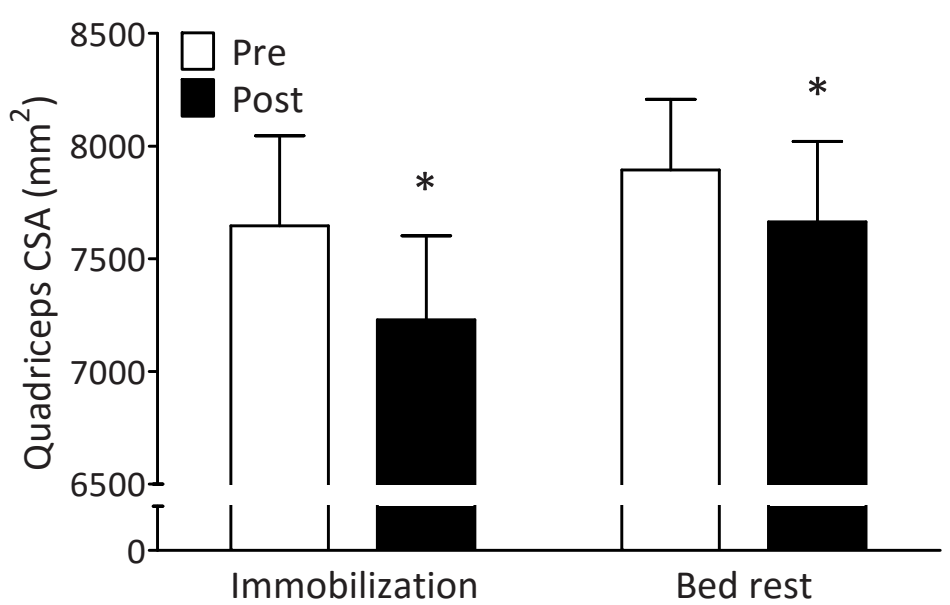

Figure 1 | Cross-sectional area of $m$. quadriceps femoris in the immobilization and bed rest group, before and after 7 days of muscle disuse, as measured by single-slice CT scan. Values are presented as means \pm SEM. Data were analysed with a Repeated Measures ANOVA with treatment and time as factors, and demonstrated a significant time effect $(P<0.001)$ with no differences between groups $(P>0.05)$. Significantly different from pre

\section{Muscle strength}

In the immobilization group, one-legged maximal strength declined from $56 \pm 5$ to $53 \pm 5$ $\mathrm{kg}$, representing a $6 \pm 3 \%$ decline. In the bed rest group, two-legged maximal strength decreased from $128 \pm 7$ to $117 \pm 7 \mathrm{~kg}$, which represented an $8 \pm 2 \%$ decline in leg strength. The decline in muscle strength was significant in both groups (time effect, $P<0.01$ ), with no differences between groups (time $\mathrm{x}$ treatment interaction; $P=0.522$ ). 


\section{DISCUSSION}

The current study is the first to directly compare the rate of muscle atrophy and the loss of muscle strength following a short period of single leg immobilization versus bed rest. We demonstrate that 7 days of leg immobilization and bed rest lead to a $4.3 \pm 0.7 \%$ decline in muscle mass and a concomitant $7 \pm 1 \%$ decline in muscle strength. Whereas local leg muscle loss did not differ between models, more lean mass was lost following bed rest compared with leg immobilization.

A short period of muscle disuse, which is often necessary for the recovery from injury or illness, leads to negative health consequences such as the loss of muscle mass and strength [1-3], a decline in basal metabolic rate [6, 7], and the development of insulin resistance [9]. The two most widely used models to study muscle disuse are bed rest and limb immobilization. While both models have been shown to result in substantial muscle loss, there are fundamental differences between these experimental settings such as the total amount of muscle tissue subjected to disuse, the extent to which free movements are possible, and the amount of weight bearing that can be performed. Moreover, it is important to acknowledge that in a bed rest setting, systemic factors may contribute to greater rates of muscle atrophy $[29,30]$. Consequently, we hypothesised that bed rest would elicit a greater local rate of muscle atrophy when compared with single leg immobilization. Contrary to our hypothesis, we report an equivalent decline in muscle mass following short-term muscle disuse in both models. Irrespective of the model, we show that seven days of disuse leads to $\sim 0.6 \%$ muscle loss per day when assessed at the level of the leg (Figure 1). These data are in line with previous work showing an approximate $0.5-0.7 \%$ muscle loss per day during muscle disuse (as reviewed in [18]). By way of comparison, the $\sim 140 \mathrm{~g}$ loss of leg muscle tissue (representing 3.2\% of quadriceps cross-sectional area loss) measured via DXA following bed rest translates to $220 \mathrm{~g}$ leg muscle loss (representing $5.4 \%$ of quadriceps cross-sectional area loss) following leg immobilization (Figure 2). However, due to the obvious involvement of the entire body during bed rest, there is a difference in the absolute amount of muscle that is lost after a week of disuse. Therefore, while the relative rate of muscle atrophy did not differ between models, the $\sim 1.4 \mathrm{~kg}$ muscle loss that was observed following whole-body bed rest evidently exceeds the $\sim 220 \mathrm{~g}$ leg muscle loss following limb immobilization. 
Leg immobilization

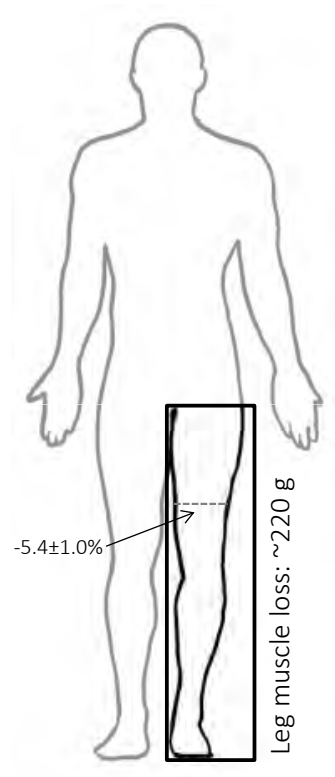

Total loss: $220 \mathrm{~g}$
Bed rest

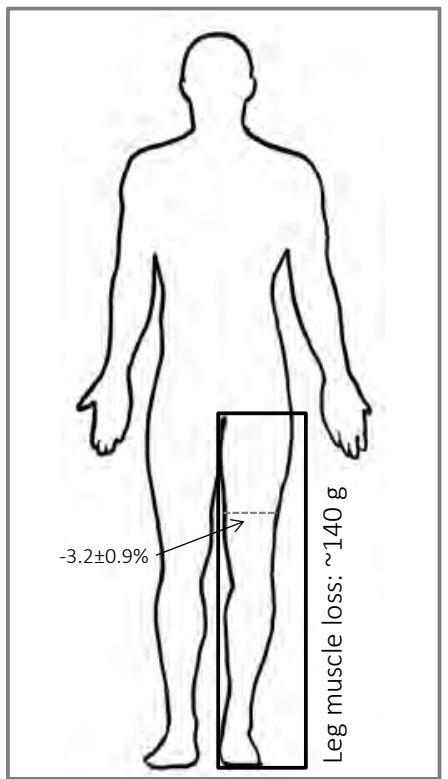

Total loss: $1400 \mathrm{~g}$

Figure 2 | Loss of leg lean mass and total lean mass following 7 days of muscle disuse in a model of leg immobilization versus bed rest. Data on leg lean mass following leg immobilization are extrapolated from muscle loss measured via quadriceps cross-sectional area (CSA; $5.4 \pm 1.0$ and $3.2 \pm 0.9 \%$ decline in quadriceps CSA following 7 days of leg immobilization and bed rest, respectively) and DXA scans made following 7 days of bed rest.

Theoretically, systemic changes associated with whole-body disuse may result in a greater degree of muscle loss compared with limb immobilization. Such factors could include increased inflammation [22], whole-body insulin resistance [8, 9, 19, 31], or increased levels of stress hormones [20,21]. However, given there is no difference in the rate of muscle loss between local leg immobilization and whole-body bed rest, there is no reason to suggest that such systemic changes affect muscle loss in a short-term bed rest setting, at least in healthy young volunteers. Indeed, previous work has shown the absence of systemic alterations, including inflammation [32,33] and circulating stress hormones [34], following short-term bed rest in healthy individuals. Despite the potential absence of such changes, it is true that bed rest will have altered several other physiological factors such as cardiac output [35], tissue perfusion [36], and whole-body and peripheral insulin sensitivity $[9,19,31]$. Since these factors apparently did not modulate muscle loss in the present study, our data support the concept that uncomplicated disuse is largely a local intracellular process. Of course, more complicated disuse experienced during bed rest following major surgery or acute illness may be compli- 
cated by systemic factors and the presence of pharmaceutical treatment. More accelerated muscle atrophy has been observed following bed rest due to critical illness [37, 38]. Moreover, extreme muscle wasting (i.e. five-fold greater than in uncomplicated disuse) has been observed in conditions where a lack of any voluntary motor control is evident, such as in fully sedated, comatose patients [39]. Indeed, in such situations large increases in C-reactive protein and white blood cells $[39,40]$ and other inflammatory parameters such as IL-6 and TNF- $\alpha$ [41] have been reported. Furthermore, bedridden patients are often hyperglycaemic [41] and are treated with a wide range of drugs that likely influence metabolism (reviewed in [42]). It is of additional importance to keep in mind that in situations of clinical muscle disuse, an individual's nutritional intake will be altered due to decreased energy requirements [43, 44], reduced appetite [45, 46], and/or other routes of food administration (e.g. nasogastric or intravenous feeding). In such situations, the decrease in energy intake and concomitant reduction in protein intake will further accelerate muscle atrophy. These and other factors that potentially influence muscle atrophy during both clinical and experimental bed rest are displayed in Figure 3.

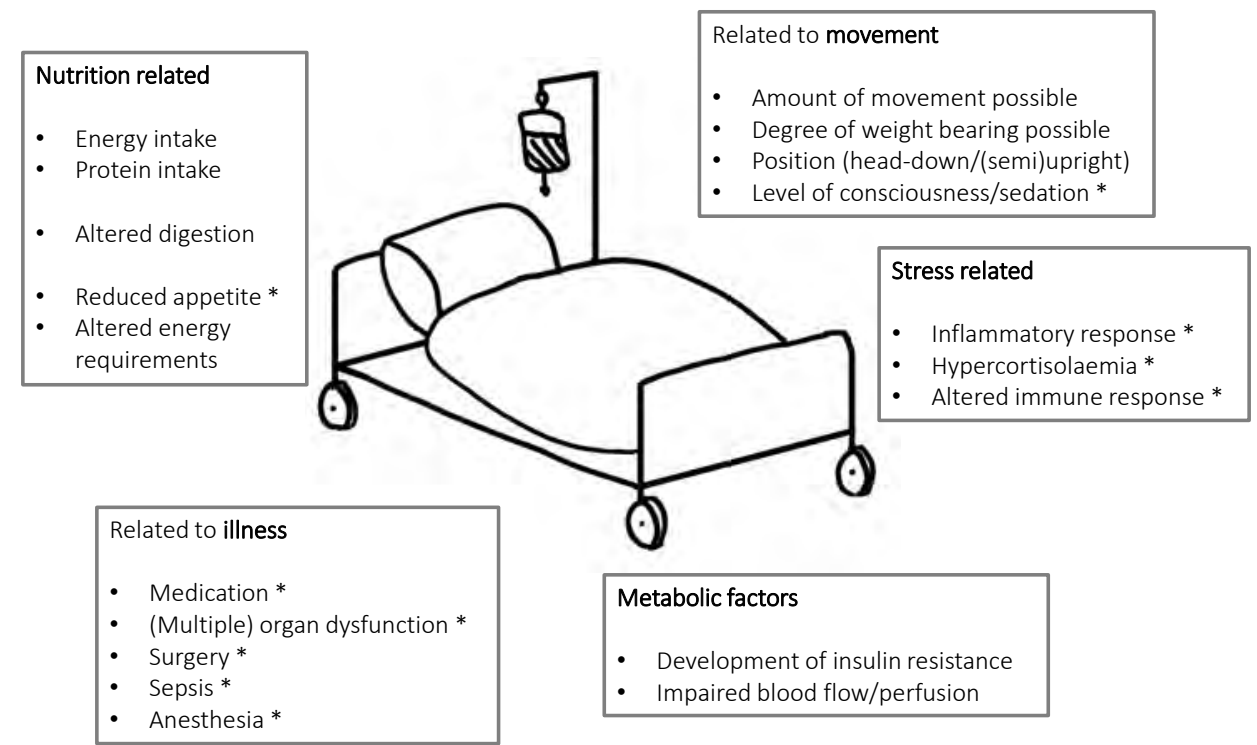

Figure 3 | Overview of systemic factors that potentially influence muscle atrophy during clinical and experimental bed rest. Factors marked with * are predominantly important in clinical situations of bed rest, whereas the other factors are relevant in both a clinical and experimental bed rest setting

Although systemic factors clearly play a role in clinically relevant situations of complicated muscle wasting, our data demonstrate that removing the mechanical stimulus forms the key factor bringing about muscle atrophy in uncomplicated, 'healthy' disuse, and that the model applied to induce this is of less relevance. For the proper assess- 
ment of muscle atrophy in short-term disuse studies, irrespective of the disuse model used, sensitive measures of muscle mass are clearly warranted. This is evident when considering the whole limb muscle atrophy we have observed was not evident on the muscle fibre level. While this is likely partially due to the intra- and inter-subject variation in muscle fibre cross-sectional area [47], the effect of oedema-induced fibre swelling, which is observed with changes in muscle fibre size, should not be disregarded [48]. Therefore, for the sensitive measurement of changes in muscle mass, techniques such as Computed Tomography (CT) or Magnetic Resonance Imaging (MRI) are preferred.

Muscle disuse has been shown to induce a substantial decline in muscle strength [1, $2,12,49]$. Due to the differences in the amount of movement and degree of weight bearing possible, it could be suggested that the loss of muscle strength differs between bed rest and immobilization. Specifically, based on separate studies, the loss of muscle strength during bed rest appears to be $\sim 0.8 \%$ per day, whereas muscle strength decreases by $\sim 1-3 \%$ per day during lower limb immobilization (reviewed in reference [18]). A review by Clark showed corroborative data from separate studies that suggest a more pronounced loss of muscle strength with immobilization [50]. In contrast, when we directly compared muscle strength loss in the present study, with age-, sex-, and BMImatched participants and with the same leg extension machine, we observed no differences in leg strength loss between the immobilization and bed rest model. Both models induced an approximate $7 \%$ decline in leg strength following 7 days of disuse (i.e. 1\% decrease per day), which is in line with previous work from us [10-12] as well as others $[18,51]$. Our data demonstrate that the neuromuscular component of muscle strength loss, generally attributed to a change in motor unit recruitment [52], is not so much influenced by the amount of movement possible in both models. This could imply that the amount of gravity or weight bearing is a more important factor mediating muscle strength loss, rather than the degree of movement possible.

The current study is the first to directly compare the rate of muscle atrophy and loss of muscle strength during short-term leg immobilization versus bed rest. Given the magnitude of muscle mass and strength losses, our data underline the clinical relevance of preserving muscle mass even during short periods of disuse. From an experimental point of view, it could be argued that mechanistic studies aimed at understanding disuse atrophy may be better suited to an immobilization model in order to isolate the effect of muscle disuse per se, without necessary changes in habitual dietary intake and/or systemic adaptations. In contrast, the bed rest model offers a more directly relevant model from a clinical perspective as it represents a period of hospitalization and its practical implications. An experimental consideration of applying this model is the obvious necessity to control an individual's dietary intake away from their habitual diet and with altered energy requirements $[53,54]$. Although the rate of muscle atrophy at the level of the leg is comparable, care is warranted when extrapolating results on muscle loss from leg immobilization to a whole-body level, as different muscle groups show different rates of muscle atrophy [49]. Therefore, researchers should carefully 
consider all influencing factors when choosing the experimental model to address their research question. We conclude that short-term muscle disuse leads to substantial declines in muscle mass and strength. Though both models result in similar relative rates of muscle loss, greater whole-body muscle loss is evident during bed rest.

\section{ACKNOWLEDGEMENTS}

The authors gratefully acknowledge the commitment and time investment of all participants in the study. The assistance of Bas van de Valk in the execution of the study was greatly appreciated. 


\section{REFERENCES}

1. Deitrick, J.E., The effect of immobilization on metabolic and physiological functions of normal men. Bull $N$ Y Acad Med, 1948. 24(6): p. 364-75.

2. Ingemann-Hansen, T. and J. Halkjaer-Kristensen, Computerized tomographic determination of human thigh components. The effects of immobilization in plaster and subsequent physical training. Scand J Rehabil Med, 1980. 12(1): p. 27-31.

3. Wall, B.T., M.L. Dirks, T. Snijders, J.M. Senden, J. Dolmans, and L.J. van Loon, Substantial skeletal muscle loss occurs during only 5 days of disuse. Acta Physiol (Oxf), 2014. 210(3): p. 600-11.

4. Ferrando, A.A., H.W. Lane, C.A. Stuart, J. Davis-Street, and R.R. Wolfe, Prolonged bed rest decreases skeletal muscle and whole body protein synthesis. Am J Physiol, 1996. 270(4 Pt 1): p. E627-33.

5. Brooks, N., G.J. Cloutier, S.M. Cadena, J.E. Layne, C.A. Nelsen, A.M. Freed, R. Roubenoff, and C. Castaneda-Sceppa, Resistance training and timed essential amino acids protect against the loss of muscle mass and strength during 28 days of bed rest and energy deficit. J Appl Physiol, 2008. 105(1): p. 241-8.

6. Haruna, Y., Y. Suzuki, K. Kawakubo, R. Yanagibori, and A. Gunji, Decremental reset in basal metabolism during 20-days bed rest. Acta Physiol (Scand). Supplementum, 1994. 616: p. 43-9.

7. Tzankoff, S.P. and A.H. Norris, Effect of muscle mass decrease on age-related BMR changes. J Appl Physiol Respir Environ Exerc Physiol, 1977. 43(6): p. 1001-6.

8. Bergouignan, A., F. Rudwill, C. Simon, and S. Blanc, Physical inactivity as the culprit of metabolic inflexibility: evidence from bed-rest studies. J Appl Physiol, 2011. 111(4): p. 1201-10.

9. Stuart, C.A., R.E. Shangraw, M.J. Prince, E.J. Peters, and R.R. Wolfe, Bed-rest-induced insulin resistance occurs primarily in muscle. Metab Clin Exp, 1988. 37(8): p. 802-6.

10. Dirks, M.L., B.T. Wall, R. Nilwik, D.H. Weerts, L.B. Verdijk, and L.J. van Loon, Skeletal muscle disuse atrophy is not attenuated by dietary protein supplementation in healthy older men. J Nutr, 2014. 144(8): p. 1196-203.

11. Dirks, M.L., B.T. Wall, T. Snijders, C.L. Ottenbros, L.B. Verdijk, and L.J. van Loon, Neuromuscular electrical stimulation prevents muscle disuse atrophy during leg immobilization in humans. Acta Physiol (Oxf), 2014. 210(3): p. 628-41.

12. Snijders, T., B.T. Wall, M.L. Dirks, J.M. Senden, F. Hartgens, J. Dolmans, M. Losen, L.B. Verdijk, and L.J. van Loon, Muscle disuse atrophy is not accompanied by changes in skeletal muscle satellite cell content. Clin Sci (Lond), 2014. 126(8): p. 557-66.

13. Wall, B.T., M.L. Dirks, T. Snijders, J.W. van Dijk, M. Fritsch, L.B. Verdijk, and L.J. van Loon, Short-term muscle disuse lowers myofibrillar protein synthesis rates and induces anabolic resistance to protein ingestion. Am J Physiol Endocrinol Metab, 2016. 310(2): p. E137-47.

14. Paddon-Jones, D., M. Sheffield-Moore, R.J. Urban, A.P. Sanford, A. Aarsland, R.R. Wolfe, and A.A. Ferrando, Essential amino acid and carbohydrate supplementation ameliorates muscle protein loss in humans during 28 days bedrest. J Clin Endocrinol Metab, 2004. 89(9): p. 4351-8.

15. Shangraw, R.E., C.A. Stuart, M.J. Prince, E.J. Peters, and R.R. Wolfe, Insulin responsiveness of protein metabolism in vivo following bedrest in humans. Am J Physiol, 1988. 255(4 Pt 1): p. E548-58.

16. Ferrando, A.A., D. Paddon-Jones, N.P. Hays, P. Kortebein, O. Ronsen, R.H. Williams, A. McComb, T.B. Symons, R.R. Wolfe, and W. Evans, EAA supplementation to increase nitrogen intake improves muscle function during bed rest in the elderly. Clin Nutr, 2010. 29(1): p. 18-23.

17. Kortebein, P., A. Ferrando, J. Lombeida, R. Wolfe, and W.J. Evans, Effect of 10 days of bed rest on skeletal muscle in healthy older adults. J Am Med Assoc, 2007. 297(16): p. 1772-4.

18. Wall, B.T. and L.J. van Loon, Nutritional strategies to attenuate muscle disuse atrophy. Nutr Rev, 2013. 71(4): p. 195-208.

19. Alibegovic, A.C., L. Hojbjerre, M.P. Sonne, G. van Hall, B. Stallknecht, F. Dela, and A. Vaag, Impact of 9 days of bed rest on hepatic and peripheral insulin action, insulin secretion, and whole-body lipolysis in healthy young male offspring of patients with type 2 diabetes. Diabetes, 2009. 58(12): p. 2749-56. 
20. Liang, X., L. Zhang, Y. Wan, X. Yu, Y. Guo, X. Chen, C. Tan, T. Huang, H. Shen, X. Chen, H. Li, K. Lv, F. Sun, S. Chen, and J. Guo, Changes in the diurnal rhythms during a 45-day head-down bed rest. PLoS One, 2012. 7(10): p. e47984.

21. Ferrando, A.A., C.A. Stuart, M. Sheffield-Moore, and R.R. Wolfe, Inactivity amplifies the catabolic response of skeletal muscle to cortisol. J Clin Endocrinol Metab, 1999. 84(10): p. 3515-21.

22. Jurdana, M., Z. Jenko-Praznikar, N. Mohorko, A. Petelin, T. Jakus, B. Simunic, and R. Pisot, Impact of 14day bed rest on serum adipokines and low-grade inflammation in younger and older adults. Age (Dordr), 2015. 37(6): p. 116.

23. Mayhew, J.L., J.L. Prinster, J.S. Ware, D.L. Zimmer, J.R. Arabas, and M.G. Bemben, Muscular endurance repetitions to predict bench press strength in men of different training levels. J Sports Med Phys Fitness, 1995. 35(2): p. 108-13.

24. Strandberg, S., M.L. Wretling, T. Wredmark, and A. Shalabi, Reliability of computed tomography measurements in assessment of thigh muscle cross-sectional area and attenuation. BMC Med Imaging, 2010. 10: p. 18.

25. Goodpaster, B.H., D.E. Kelley, F.L. Thaete, J. He, and R. Ross, Skeletal muscle attenuation determined by computed tomography is associated with skeletal muscle lipid content. J Appl Physiol, 2000. 89(1): p. 104-10.

26. Verdijk, L.B., L. van Loon, K. Meijer, and H.H. Savelberg, One-repetition maximum strength test represents a valid means to assess leg strength in vivo in humans. J Sports Sci, 2009. 27(1): p. 59-68.

27. Bergstrom, J., Percutaneous needle biopsy of skeletal muscle in physiological and clinical research. Scand J Clin Lab Invest, 1975. 35(7): p. 609-16.

28. Edelstein, A., N. Amodaj, K. Hoover, R. Vale, and N. Stuurman, Computer control of microscopes using uManager. Curr Protoc Mol Biol, 2010. Chapter 14(Unit14.20).

29. Paddon-Jones, D., M. Sheffield-Moore, R.J. Urban, A. Aarsland, R.R. Wolfe, and A.A. Ferrando, The catabolic effects of prolonged inactivity and acute hypercortisolemia are offset by dietary supplementation. J Clin Endocrinol Metab, 2005. 90(3): p. 1453-9.

30. Paddon-Jones, D., M. Sheffield-Moore, M.G. Cree, S.J. Hewlings, A. Aarsland, R.R. Wolfe, and A.A. Ferrando, Atrophy and Impaired Muscle Protein Synthesis during Prolonged Inactivity and Stress. J Clin Endocrinol Metab, 2006. 91(12): p. 4836-4841.

31. Mikines, K.J., E.A. Richter, F. Dela, and H. Galbo, Seven days of bed rest decrease insulin action on glucose uptake in leg and whole body. J Appl Physiol, 1991. 70(3): p. 1245-54.

32. Drummond, M.J., K.L. Timmerman, M.M. Markofski, D.K. Walker, J.M. Dickinson, M. J Am Med Assocluddin, A.R. Brasier, B.B. Rasmussen, and E. Volpi, Short-term bed rest increases TLR4 and IL-6 expression in skeletal muscle of older adults. Am J Physiol Regul Integr Comp Physiol, 2013. 305(3): p. R216-23.

33. Hamburg, N.M., C.J. McMackin, A.L. Huang, S.M. Shenouda, M.E. Widlansky, E. Schulz, N. Gokce, N.B. Ruderman, J.F. Keaney, Jr., and J.A. Vita, Physical inactivity rapidly induces insulin resistance and microvascular dysfunction in healthy volunteers. Arterioscler Thromb Vasc Biol, 2007. 27(12): p. 2650-6.

34. Chouker, A., B. Feuerecker, S. Matzel, I. Kaufmann, C. Strewe, M. Hoerl, G. Schelling, and M. Feuerecker, Psychoneuroendocrine alterations during 5 days of head-down tilt bed rest and artificial gravity interventions. Eur J Appl Physiol, 2013. 113(8): p. 2057-65.

35. Saltin, B., G. Blomqvist, J.H. Mitchell, R.L. Johnson, Jr., K. Wildenthal, and C.B. Chapman, Response to exercise after bed rest and after training. Circulation, 1968. 38(5 Suppl): p. VII1-78.

36. Broderick, B.J., D.E. O'Briain, P.P. Breen, S.R. Kearns, and G. Olaighin, A hemodynamic study of popliteal vein blood flow: the effect of bed rest and electrically elicited calf muscle contractions. Conf Proc IEEE Eng Med Biol Soc, 2009. 2009: p. 2149-52.

37. Reid, C.L., I.T. Campbell, and R.A. Little, Muscle wasting and energy balance in critical illness. Clin Nutr, 2004. 23(2): p. 273-80.

38. Puthucheary, Z.A., J. Rawal, M. McPhail, B. Connolly, G. Ratnayake, P. Chan, N.S. Hopkinson, R. Padhke, T. Dew, P.S. Sidhu, C. Velloso, J. Seymour, C.C. Agley, A. Selby, M. Limb, L.M. Edwards, K. Smith, A. Rowlerson, M.J. Rennie, J. Moxham, S.D. Harridge, N. Hart, and H.E. Montgomery, Acute skeletal muscle wasting in critical illness. J Am Med Assoc, 2013. 310(15): p. 1591-600. 
39. Dirks, M.L., D. Hansen, A. Van Assche, P. Dendale, and L.J. Van Loon, Neuromuscular electrical stimulation prevents muscle wasting in critically ill comatose patients. Clin Sci (Lond), 2015. 128(6): p. 357-65.

40. Constantin, D., J. McCullough, R.P. Mahajan, and P.L. Greenhaff, Novel events in the molecular regulation of muscle mass in critically ill patients. J Physiol, 2011. 589(Pt 15): p. 3883-95.

41. Jespersen, J.G., A. Nedergaard, S. Reitelseder, U.R. Mikkelsen, K.J. Dideriksen, J. Agergaard, F. Kreiner, F.C. Pott, P. Schjerling, and M. Kjaer, Activated protein synthesis and suppressed protein breakdown signaling in skeletal muscle of critically ill patients. PLoS One, 2011. 6(3): p. e18090.

42. Jones, J.D., H.L. Kirsch, R.L. Wortmann, and M.H. Pillinger, The causes of drug-induced muscle toxicity. Curr Opin Rheumatol, 2014. 26(6): p. 697-703.

43. Blanc, S., S. Normand, P. Ritz, C. Pachiaudi, L. Vico, C. Gharib, and G. Gauquelin-Koch, Energy and water metabolism, body composition, and hormonal changes induced by 42 days of enforced inactivity and simulated weightlessness. J Clin Endocrinol Metab, 1998. 83(12): p. 4289-97.

44. Ritz, P., A. Maillet, S. Blanc, and R.J. Stubbs, Observations in energy and macronutrient intake during prolonged bed-rest in a head-down tilt position. Clin Nutr, 1999. 18(4): p. 203-7.

45. Harris, C.L. and C. Fraser, Malnutrition in the institutionalized elderly: the effects on wound healing. Ostomy Wound Manage, 2004. 50(10): p. 54-63.

46. Nematy, M., J.E. O'Flynn, L. Wandrag, A.E. Brynes, S.J. Brett, M. Patterson, M.A. Ghatei, S.R. Bloom, and G.S. Frost, Changes in appetite related gut hormones in intensive care unit patients: a pilot cohort study. Crit Care, 2006. 10(1): p. R10.

47. Nilwik, R., T. Snijders, M. Leenders, B.B. Groen, J. van Kranenburg, L.B. Verdijk, and L.J. van Loon, The decline in skeletal muscle mass with aging is mainly attributed to a reduction in type II muscle fiber size. Exp Gerontol, 2013. 48(5): p. 492-8.

48. Damas, F., S.M. Phillips, M.E. Lixandrao, F.C. Vechin, C.A. Libardi, H. Roschel, V. Tricoli, and C. Ugrinowitsch, Early resistance training-induced increases in muscle cross-sectional area are concomitant with edema-induced muscle swelling. Eur J Appl Physiol, 2016. 116(1): p. 49-56.

49. LeBlanc, A.D., V.S. Schneider, H.J. Evans, C. Pientok, R. Rowe, and E. Spector, Regional changes in muscle mass following 17 weeks of bed rest. J Appl Physiol, 1992. 73(5): p. 2172-8.

50. Clark, B.C., In vivo alterations in skeletal muscle form and function after disuse atrophy. Med Sci Sports Exerc, 2009. 41(10): p. 1869-75.

51. Hvid, L., P. Aagaard, L. Justesen, M.L. Bayer, J.L. Andersen, N. Ortenblad, M. Kjaer, and C. Suetta, Effects of aging on muscle mechanical function and muscle fiber morphology during short-term immobilization and subsequent retraining. J Appl Physiol, 2010. 109(6): p. 1628-34.

52. Clark, B.C. and T.M. Manini, Sarcopenia =/= dynapenia. J Gerontol A Biol Sci Med Sci, 2008. 63(8): p. 829-34.

53. Biolo, G., B. Ciocchi, M. Stulle, A. Bosutti, R. Barazzoni, M. Zanetti, R. Antonione, M. Lebenstedt, P. Platen, M. Heer, and G. Guarnieri, Calorie restriction accelerates the catabolism of lean body mass during 2 wk of bed rest. Am J Clin Nutr, 2007. 86(2): p. 366-72.

54. Weiss, E.P., S.B. Racette, D.T. Villareal, L. Fontana, K. Steger-May, K.B. Schechtman, S. Klein, A.A. Ehsani, J.O. Holloszy, and C.G. Washington University School of Medicine, Lower extremity muscle size and strength and aerobic capacity decrease with caloric restriction but not with exercise-induced weight loss. J Appl Physiol, 2007. 102(2): p. 634-40. 



\title{
CHAPTER
}

\section{One week of bed rest leads to substantial muscle atrophy and induces whole-body insulin resistance in the absence of skeletal muscle lipid accumulation}

\author{
Marlou L Dirks \\ Benjamin T Wall \\ Bas van de Valk \\ Tanya M Holloway \\ Graham P Holloway \\ Adrian Chabowski \\ Gijs H Goossens \\ Luc JC van Loon
}




\section{ABSTRACT}

Background Short ( $<10$ days) periods of muscle disuse, often necessary for recovery from illness or injury, lead to various negative health consequences. The present study investigated mechanisms underlying disuseinduced insulin resistance, taking into account muscle atrophy.

Methods Ten healthy, young males (age: $23 \pm 1 \mathrm{y}, \mathrm{BMI}: 23.0 \pm 0.9 \mathrm{~kg} \cdot \mathrm{m}^{-2}$ ) were subjected to one week of strict bed rest. Prior to and after bed rest, lean body mass (DXA) and quadriceps cross-sectional area (CSA; CT) were assessed, and $\mathrm{VO}_{2}$ peak and leg strength were determined. Whole-body insulin sensitivity was measured using a hyperinsulinemic-euglycemic clamp. Additionally, muscle biopsies were collected to assess muscle lipid (fraction) content and various markers of mitochondrial and vascular content.

Results Bed rest resulted in $1.4 \pm 0.2 \mathrm{~kg}$ lean tissue loss and a $3.2 \pm 0.9 \%$ decline in quadriceps CSA (both $P<0.01$ ). $V_{2}$ peak and $1 \mathrm{RM}$ declined by $6.4 \pm 2.3(P<0.05)$ and $6.9 \pm 1.4 \%(P<0.01)$, respectively. Bed rest induced a $29 \pm 5 \%$ decrease in whole-body insulin sensitivity $(P<0.01)$. This was accompanied by a decline in muscle oxidative capacity, without alterations in skeletal muscle lipid content or saturation level, markers of oxidative stress, or capillary density.

Conclusion In conclusion, one week of bed rest substantially reduces skeletal muscle mass and lowers whole-body insulin sensitivity, without affecting mechanisms implicated in high-fat diet-induced insulin resistance. 


\section{INTRODUCTION}

Recovery from injury or illness generally necessitates a period of bed rest, often as a consequence of hospitalization. Prolonged ( $>10$ days) periods of bed rest have been shown to induce substantial changes in body composition and are accompanied by overall metabolic decline $[1,2]$. Though this model of prolonged physical inactivity has taught us much about muscle disuse atrophy, it may be of limited clinical relevance to most patients who are, on average, hospitalized for less than 7 days [3]. Recent data from our laboratory as well as others show that merely a few days of disuse substantially reduces skeletal muscle mass and strength $[2,4-6]$. As a consequence, it has been suggested that the accumulation of such short ( $<10$ days), successive periods of bed rest or immobilization may largely be responsible for the loss of muscle mass and metabolic decline observed throughout the lifespan [7, 8].

Impairments in metabolic health following prolonged disuse have been well described and include a decline in glucose tolerance and insulin sensitivity [1, 9], a decrease in resting fat oxidation [10], an increase in mitochondrial ROS production [11], and a decline in basal metabolic rate [12]. As the decline in metabolic health predisposes to greater morbidity and mortality of patients [13], it is of major clinical relevance to understand the mechanisms responsible for this decline in metabolic health. Prolonged disuse has been associated with substantial loss of muscle mass and/or gain in fat mass [2]. Such changes in body composition lower the body's capacity for blood glucose disposal, and may contribute to the decline in metabolic health. However, changes in body composition can only partly explain the observed metabolic decline, as reduced insulin sensitivity has been observed during bed rest before measurable changes in body composition became apparent $[1,2]$. We hypothesize that the substantial muscle atrophy caused by short-term bed rest will contribute to, but not fully explain, the vast decline in metabolic health.

One of the key hallmarks of metabolic health is insulin sensitivity. Earlier studies have demonstrated that short periods of bed rest impair glucose tolerance and lower whole-body and/or peripheral insulin sensitivity [14-18]. The development of insulin resistance under conditions of lipid oversupply (e.g. type 2 diabetes mellitus, (high-fat) overfeeding) has been associated with lipid deposition in skeletal muscle [19], and, more specifically, with an increase in intramuscular lipid intermediates such as diacylglycerols (DAGs), ceramides, and long-chain fatty acyl-CoA, which impair insulin signaling (as reviewed in [20]). Furthermore, both muscle disuse atrophy [21, 22] and the development of insulin resistance [23] have also been attributed to a decline in mitochondrial content and/or impairments in skeletal muscle mitochondrial function. Additionally, impairments in micro- and macrovascular function have been linked to peripheral insulin resistance [24]. So far, it is unclear which mechanism(s) contribute to the proposed development of insulin resistance during short-term bed rest. 
The objective of the present study was to assess mechanisms that may contribute to the development of insulin resistance during short-term muscle disuse, taken into account the expected muscle atrophy. To achieve this we subjected healthy, young males to one week of strict bed rest and used comprehensive measures of muscle mass and muscle function in combination with detailed metabolic phenotyping (e.g. whole-body insulin sensitivity, substrate metabolism, skeletal muscle lipid content and composition, muscle oxidative capacity, and capillary density) to determine their possible contribution to the development of disuse-induced whole-body insulin resistance. Importantly, this was conducted under energy-balanced conditions to eliminate the contribution of overfeeding to our results. We hypothesized that bed rest-induced insulin resistance is attributed to mechanisms known to induce insulin resistance in chronic metabolic disease, i.e. ectopic lipid deposition, intramuscular accumulation of lipid intermediates, a decline in mitochondrial content, and/or impairment in skeletal muscle capillarization. Here we demonstrate that short-term bed rest leads to skeletal muscle atrophy, pronounced whole-body insulin resistance, and a decline in skeletal muscle oxidative capacity. Strikingly, these effects do not seem to be mediated via mechanisms involved in obesity-related insulin resistance such as skeletal muscle lipid accumulation, oxidative stress and micro- and/or macrovascular dysfunction. 


\section{METHODS}

\section{Subjects}

Ten healthy young men (age $23 \pm 1$ y) were included in the present study. Subjects' characteristics are presented in Table 1. Prior to inclusion in the study, subjects filled in a general health questionnaire and completed a routine medical screening to ensure their eligibility to take part in the study. Exclusion criteria were a BMI below 18.5 or above 30 $\mathrm{kg} \cdot \mathrm{m}^{-2}$, a (family) history of thrombosis, type 2 diabetes mellitus (determined by $\mathrm{HbA} 1 \mathrm{c}$ values $\left.>7.0 \%\left(53 \mathrm{mmol} \cdot \mathrm{mol}^{-1}\right)\right)$, and any back, knee or shoulder complaints. Furthermore, subjects who had been involved in structured and prolonged resistance-type exercise training during the 6 months prior to the study were also excluded. All subjects were informed on the nature and risks of the experiment before written informed consent was obtained. During the screening visit, a fasting blood sample was taken to assess $\mathrm{HbA1c}$, resting energy expenditure was measured with the use of a ventilated hood, and a one-Repetition Maximum (1RM) estimation test was performed. The present study was approved by the Medical Ethical Committee of Maastricht University Medical Centre (registration number 14-3-013) in accordance with the Declaration of Helsinki.

Table 1 | Subjects' characteristics

\begin{tabular}{lc}
\hline Age $(\mathrm{y})$ & $23 \pm 1$ \\
Body mass $(\mathrm{kg})$ & $74.9 \pm 2.3$ \\
Height $(\mathrm{m})$ & $1.81 \pm 0.02$ \\
BMI $\left(\mathrm{kg} \cdot \mathrm{m}^{-2}\right)$ & $23.0 \pm 0.9$ \\
Fasting glucose $\left(\mathrm{mmol} \cdot \mathrm{L}^{-1}\right)$ & $5.7 \pm 0.2$ \\
Fasting insulin $\left(\mathrm{mU} \cdot \mathrm{L}^{-1}\right)$ & $7.2 \pm 1.8$ \\
HbA1c $(\%)$ & $5.1 \pm 0.1$ \\
RMR $\left(\mathrm{MJ} \cdot \mathrm{d}^{-1}\right)$ & $7.2 \pm 0.2$ \\
\hline
\end{tabular}

BMI, body mass index; HbA1c, glycated haemoglobin; RMR, resting metabolic rate

\section{Experimental outline}

The experimental protocol is depicted in Supplemental Figure 1. After inclusion into the study, subjects visited the university for a pre-testing visit during which the 1 RM and VO2peak tests were performed. Following this visit, a 7-day period of standardized nutrition was started. On day 6 of the controlled diet, a mixed meal tolerance test was performed. The day after, on day 7 of the standardized diet and the day prior to bed rest, test day 1 was scheduled. During this day a muscle biopsy was taken from the $m$. vastus lateralis, and a hyperinsulinemic-euglycemic clamp, CT- and DXA scans were performed. The next morning, subjects arrived at the laboratory to start the bed rest 
period. The meal tolerance test was repeated on day 6 of bed rest. After exactly seven days of bed rest, test day 1 was repeated and subjects were allowed to go home. On the next day, subjects returned to the laboratory to repeat the 1RM and VO2peak tests.

\section{One week of bed rest}

To mimic the effects of a standard hospitalization procedure, subjects underwent a 7day period of strict bed rest. On the morning of day 1 , subjects reported to the laboratory in the fasted state at 8:00. From that moment on, subjects remained in bed. During the day, subjects were permitted to use a pillow and elevation of the bed-back to perform their daily activities. All hygiene and sanitary activities were performed on the bed. Every morning subjects were woken at 8:00 and lights were turned off at 23.00. Participants were monitored continuously by the research team.

\section{Dietary intake}

During the screening visit, resting energy expenditure was measured by indirect calorimetry, using an open-circuit ventilated hood system (Omnical, Maastricht University, Maastricht, the Netherlands; [25]). For seven days prior to bed rest subjects were given standardized food to prepare and consume at home. During the bed rest period itself, dietary intake was entirely controlled. During the pre-bed rest period, subjects received all food products and prepared the meals at home. In that week, subjects reported to the laboratory once or twice to allow adjustments of the diet in response to body weight changes (when necessary), to keep body weight stable. During bed rest, energy intake was increased when subjects reported being hungry. Energy requirements were estimated based on indirect calorimetry data, multiplied by an activity factor (AF) of 1.55 (prior to bed rest) and 1.35 (during bed rest). Macronutrient composition of the diet was identical before and during the bed rest period (Supplemental Table 1).

\section{Body composition}

During test days 1 and 2 (prior to and immediately after bed rest, respectively), anatomical cross-sectional area (CSA) of the quadriceps muscle, hamstrings and whole thigh were assessed via a single slice CT scan (Philips Brilliance 64, Philips Medical Systems, Best, the Netherlands). Whilst subjects were lying supine, with their legs extended and their feet secured, a $3 \mathrm{~mm}$ thick axial image was taken $15 \mathrm{~cm}$ proximal to the top of the patella. On test day 1 , the precise scanning position was marked with semi-permanent ink for replication on test day 2. Next, a single slice CT scan at the level of the upper border of the L3 vertebra was taken to assess total muscle CSA (i.e. all paraspinal and abdominal muscle). For this scan, subjects were lying in a prone position, with their chin resting on both hands. The following scanning characteristics were used: $120 \mathrm{kV}, 300$ $\mathrm{mA}$, rotation time of $0.75 \mathrm{~s}$, and a field of view of $500 \times 500 \mathrm{~mm}$. CT-scans were analyzed 
for the CSA of the whole thigh muscle as well as the quadriceps and hamstring muscles, and for total muscle CSA at the level of the L3 vertebra by manual tracing using ImageJ software (version 1.48t, National Institute of Health, Maryland, USA, [26]). The L3 Skeletal Muscle Index (L3 SMI) was calculated by dividing the paraspinal muscle area by height squared. Tissue with Hounsfield units (HU) between -29 and $+150 \mathrm{HU}$ was selected as muscle tissue. The L3 CT scans were also used to determine intramuscular adipose tissue (IMAT), visceral adipose tissue (VAT) and subcutaneous adipose tissue (SAT), using SliceOmatic software (version 5.0, Tomovision, Montreal, QC, Canada) as described previously [27]. Body composition was measured via DXA (Dual Energy X-Ray Absorptiometry; Hologic, Discovery A, QDR Series, Bedford, MA, USA). The system's software package Apex version 2.3 was used to determine whole-body and regional lean mass, fat mass and bone mineral content.

\section{Insulin sensitivity}

On the day prior to bed rest, and directly after one week of bed rest, a hyperinsulinemic-euglycemic clamp was performed to assess whole-body insulin sensitivity. At the applied level of hyperinsulinemia, hepatic glucose output will be minimal $[28,29]$. Therefore, the presented whole-body insulin sensitivity data presented here mainly reflect peripheral insulin sensitivity. Due to the choices for the setup of this experiment, this protocol does not allow assessment of the impact of bed rest on maximal insulin responsiveness. Before the start of the experiment, a Teflon cannula was inserted anterogradely in an antecubital vein of the forearm for the infusion of $20 \%$ glucose (Baxter B.V., Utrecht, the Netherlands) and insulin $\left(40 \mathrm{mU} \cdot \mathrm{m}^{-2} \cdot \mathrm{min}^{-1}\right.$; Novorapid, Novo Nordisk Farma, Alphen aan den Rijn, the Netherlands). On the contralateral hand, a second cannula was inserted into a superficial dorsal hand vein. From this catheter, arterialized venous blood was obtained by heating the hand in a hot-box $\left(60^{\circ} \mathrm{C}\right)$. A small amount of blood was drawn every 5 min throughout the entire $2.5 \mathrm{~h}$ clamp to determine glucose concentration (ABL800 Flex, Radiometer Medical, Denmark). The amount of glucose infused was altered to maintain euglycaemia at $5.0 \mathrm{mmol} \cdot \mathrm{L}^{-1}$. The last $30 \mathrm{~min}$ of the clamp were used to calculate the mean glucose infusion rate.

At baseline and during the last 30 min of the clamp, fasting and insulin-stimulated energy expenditure and substrate oxidation were assessed by indirect calorimetry using an open-circuit ventilated hood system (Omnical, Maastricht University, Maastricht, the Netherlands, [25]). From these data, total fat and carbohydrate oxidation rates and metabolic flexibility were calculated as described before [30]. To test glucose tolerance in a practical manner, a meal tolerance test was performed two days prior to bed rest and on day 6 of bed rest at 08:30 as part of the standardized diet. Before and after bed rest, subjects received identical test meals which provided $7.6 \pm 0.2 \mathrm{kcal} \cdot \mathrm{kg}$ body weight$1,72 \pm 1 \mathrm{~g}$ carbohydrate $(52 \pm 0.4 \mathrm{En} \%), 19 \pm 0.3 \mathrm{~g}$ fat $(31 \pm 0.4 \mathrm{En} \%)$ and $24 \pm 0.1 \mathrm{~g}$ protein $(17 \pm 0.2 \mathrm{En} \%)$. While subjects were in an overnight fasted state, an antecubital vein was 
cannulated to allow repeated blood sampling. Prior to breakfast, and at $t=30,60,90$ and 120 min following meal ingestion, a blood sample was collected in a supine position to assess plasma glucose and insulin concentrations. The disposition index (DI), as a measure of beta cell function, was calculated using the following formula: $\mathrm{DI}=(\mid 120-$ IO/G120-G0) x OGIS.

\section{Muscle function tests}

Eight or nine days before, and on the day after the 7 day bed rest, an incremental cycle ergometer test was performed with $40 \mathrm{~W}$ increments every 3 min to determine peak oxygen uptake (VO2peak). Next, 1RM strength tests on a leg press and leg extension device (Technogym, Rotterdam, the Netherlands) were performed to determine maximal leg strength. The estimations obtained during the screening visit, obtained via the multiple repetitions testing procedure [31], were used to determine 1RM as described previously [32]. In short, after warming up, the load was set at 90-95\% of the estimated maximum strength and increased after each successful lift until failure. A 2 min resting period was allowed between subsequent attempts. A repetition was deemed valid if the participant was able to complete the entire lift in a controlled manner without assistance. Finally, maximal grip strength was determined using a Jamar handheld dynamometer (model BK-7498; Fred Sammons, Inc., Burr Ridge, IL, USA). Three consecutive measures were recorded for both hands, and maximal grip strength of both hands was averaged to calculate mean maximal grip strength [33].

\section{Blood and muscle sampling}

During the meal tolerance tests and on each day of bed rest, blood samples were collected in EDTA-containing tubes and directly centrifuged at $1,000 \mathrm{~g}$ for $10 \mathrm{~min}$ at $4^{\circ} \mathrm{C}$. Aliquots of plasma were snap frozen in liquid nitrogen and stored at $-80^{\circ} \mathrm{C}$ until further analysis. Additionally, before and after bed rest a single muscle biopsy was collected from the vastus lateralis muscle. After local anesthesia was induced, a percutaneous needle biopsy was taken approximately $15 \mathrm{~cm}$ above the patella [34]. Any visible nonmuscle tissue was directly removed, and part of the biopsy sample was embedded in Tissue-Tek (4583, Sakura Finetek, Zoeterwoude, the Netherlands) before being frozen in liquid nitrogen-cooled isopentane. All remaining muscle tissue was immediately frozen in liquid nitrogen. Muscle samples were subsequently stored at $-80^{\circ} \mathrm{C}$ until further analyses.

\section{Plasma biochemistry}

Plasma glucose and insulin concentrations were analyzed using commercially available kits (GLUC3, Roche, Ref: 05168791 190, and Immunologic, Roche, Ref: 12017547122 (inter-assay CV $4.9 \%$ and intra-assay CV 1.5\%), respectively). Plasma free fatty acid con- 
centrations were analyzed with an ABX Pentra 400 analyzer (Horiba Diagnostics, Montpellier, France) with test kits purchased from ABX Diagnostics (Montpellier, France).

\section{Skeletal muscle analyses}

\section{Fibre typing}

Muscle biopsies were stained for muscle fibre typing (FT) as described previously [35]. The section of the muscle that was mounted and frozen in Tissue-Tek was cut into $5 \mu \mathrm{m}$ thick cryosections using a cryostat at $-20^{\circ} \mathrm{C}$. Pre- and post- bed rest samples of each subject were mounted together on uncoated, pre-cleaned glass slides, thereby carefully aligning the samples for cross-sectional fibre analyses. Stainings were performed to analyze muscle fibre-type specific cross-sectional area (CSA) and intramyocellular triglyceride (IMTG) content. To measure fibre-type specific CSA, slides were incubated with primary antibodies directed against myosin heavy chain (MHC)-I (A4.840, dilution 1:25; DSHB) and laminin (polyclonal rabbit anti-laminin, L9393, dilution 1:50; SigmaAldrich, Zwijndrecht, the Netherlands). After washing, the appropriate secondary antibodies were applied: goat anti-rabbit IgG AlexaFluor647 and goat anti-mouse IgM AlexaFluor555 (A-21245 and A-21422; dilution 1:400 and 1:500, respectively; Molecular Probes, Invitrogen, Breda, the Netherlands). Myonuclei were stained with 4,6diamidino-2-phenylindole (DAPI, D1306, $0.238 \mu \mathrm{M}$, Molecular Probes). Both primary and secondary antibodies were diluted in 0.1\% Bovine Serum Albumin (BSA, A7906, Sigma-Aldrich) in $0.1 \%$ Tween 20 (P2287, Sigma-Aldrich) dissolved in phosphatebuffered saline (PBS). Incubation of antibodies was performed at room temperature. Skeletal muscle tissue was stained as follows. Tissue was fixated in acetone for 5 min, after which the slides were air dried for $15 \mathrm{~min}$ and incubated with 3\% BSA in $0.1 \%$ Tween-PBS for $30 \mathrm{~min}$. Slides were then washed (standard washing protocol: $5 \mathrm{~min}$ $0.1 \%$ Tween-PBS, 2x5 min PBS) and incubated with the first antibodies for $45 \mathrm{~min}$. After washing, slides were incubated with the secondary antibodies, diluted together with DAPI, for $45 \mathrm{~min}$. After a last washing step, cover glasses were mounted by Mowiol (475904-100GM, Calbiochem, Amsterdam, the Netherlands). As a result of the staining procedure, nuclei were stained in blue, MHC-I in red, and laminin in far-red. Images were visualized and automatically captured at 10x magnification with a Olympus BX51 fluorescence microscope with customized spinning disk unit (DSU; Olympus, Zoeterwoude, the Netherlands) with a ultra-high sensitivity monochrome electron multiplier CCD camera (1000 × 1000 pixels, C9100-02, Hamamatsu Photonics, Hamamatsu City, Japan). Image acquisition was done by Micromanager 1.4 software [36], and images were analyzed with ImageJ. The images were recorded and analyzed by an investigator blinded to subject coding. As a measure of fibre circularity, form factors were calculated 
by using the following formula: $(4 \pi \cdot \mathrm{CSA}) \cdot(\text { perimeter })^{-2}$. On average, $176 \pm 31$ and $212 \pm 60$ muscle fibres were analyzed in the pre- and post-bed rest samples, respectively.

\section{Capillary density}

An immunohistochemical staining for skeletal muscle capillarization (Figure 6D) was performed as described previously [37]. Slides with muscle cryosections of $5 \mu \mathrm{m}$ were taken from the $-80^{\circ} \mathrm{C}$ freezer and thawed for $30 \mathrm{~min}$ at room temperature. After fixation for $5 \mathrm{~min}$ with acetone, samples were air dried again for $15 \mathrm{~min}$. Slides were then incubated for 45 min with CD31 (dilution 1:50; M0823; DAKO, Glostrup, Denmark). Slides were then washed (standard washing protocol $3 \times 5$ min PBS). After that, a 45 min incubation step with goat anti-mouse biotin (BA-2000, dilution 1:200; Vector Laboratories, Burlingame, CA, USA) was started, and a standard wash was performed. Next, slides were incubated with Avidin Texas Red (A2006, dilution 1:400, Vector Laboratories), and antibodies against MHC-I (A4.840, dilution 1:25, DSHB), and laminin (polyclonal rabbit anti-laminin, dilution 1:50, L9393, Sigma-Aldrich) for $45 \mathrm{~min}$ and washed. In the final incubation step, goat anti-mouse IgM AlexaFluor488 and goat anti-rabbit IgG AlexaFluor350 (A-21042 and A-11046, dilution 1:200 and 1:133, respectively; Molecular Probes) were applied for $30 \mathrm{~min}$. After washing, slides were mounted with Mowiol. The staining procedure resulted in images with laminin in blue, MHC-I in green, and CD31 in red. Images were automatically captured at 10 x magnification with a Olympus BX51 fluorescence microscope with customized spinning disk unit (DSU; Olympus) with a ultra-high sensitivity monochrome electron multiplier CCD camera (1000 × 1000 pixels, C9100-02, Hamamatsu Photonics). Image acquisition was done by Micromanager 1.4 software [36], and images were analyzed with ImageJ. The images were recorded and analyzed by an investigator blinded to subject coding. In all images, a minimum of 30 fibres were counted per fibre type. The number of capillaries was counted, and expressed as capillary-to-fibre ratio and capillary-to-fibre perimeter exchange index (CFPE; number of capillaries per $1000 \mu \mathrm{m}$ perimeter).

\section{Fibre-type specific lipid content, lipid fractions, and saturation}

An Oil red O (ORO) staining was performed to analyze muscle fibre-type specific IMTG content, based on previous work [38]. Freshly cut samples were air dried for $30 \mathrm{~min}$, and fixated in 3.7\% formaldehyde (1040051000, Merck Millipore, Darmstadt, Germany) for $60 \mathrm{~min}$. After rinsing $3 \times 30 \mathrm{sec}$ with Milli-Q, slides were incubated for 5 min in $0.5 \%$ Triton X-100 (108643, Merck Millipore) in PBS. Slides were then washed for $3 \times 5$ min with PBS, and incubated for 45 min with primary antibodies against MHC-I (A4.951, dilution 1:25, DSHB) and laminin (polyclonal rabbit anti-laminin, dilution 1:50; L9393, Sigma-Aldrich) in 0.05\% Tween-PBS. After washing (1x5 min 0.05\% Tween-PBS, 2x5 min PBS) slides were incubated with the appropriate secondary antibodies: goat anti-mouse IgG1AlexaFluor488 and goat anti-rabbit IgG AlexaFluor350 (A-21121 and A-11046; dilu- 
tion 1:133 and 1:200, respectively; Molecular Probes, Invitrogen). Slides were then washed (1×5 min 0.05\% Tween-PBS, 2x5 min PBS) and rinsed for $30 \mathrm{~s}$ with Milli-Q, after which slides were placed in the ORO solution for $30 \mathrm{~min}$. This solution was made by dissolving 250 mg Oil Red O powder (O0625-25G, Sigma-Aldrich) in 50 mL 60\% triethylphosphate (538728, Sigma-Aldrich). Of this solution, $48 \mathrm{~mL}$ was added to $32 \mathrm{~mL}$ of Milli$\mathrm{Q}$, which was then filtered using a paper folding filter. After incubation with the ORO solution, slides were rinsed with Milli-Q for $3 \times 30 \mathrm{sec}$, and placed under slow running cold tap water, before being mounted with cover glasses and Mowiol. The staining procedure resulted in images with laminin in blue, MHC-I in green, and ORO in red. Images were semi-automatically captured at 40x magnification with using a Nikon E800 fluorescent microscope coupled with a Nikon DS-Fi1c camera (Nikon Instruments, Amsterdam, the Netherlands) using the NIS-Elements BR software package version 4.20.01. Analysis of the images was done using Image software by an investigator blinded to subject coding. No differences in fibre circularity were observed between pre- and post-bed rest samples. On average, $34 \pm 1$ muscle fibres were analyzed in both the pre- and post-bed rest samples. A representative image of the ORO staining is displayed in Figure $3 \mathrm{~A}$.

To determine intramuscular lipid content and the degree of saturation, 50 mg wet muscle was used as described elsewhere [39]. Total lipid was extracted using chloroform-methanol (1:1 vol/vol) and internal standards, and thereafter evaporated under nitrogen at $37^{\circ} \mathrm{C}$. The extracted lipids were separated into triacylglycerol (TAG), diacylglycerol (DAG), free fatty acids (FFA) and phospholipid (PL) by thin-layer chromatography and transferred into separate tubes. After incubation with methanol, pentane was added to the samples, which were then vortexed and centrifuged. The pentane extracts (upper phase) were isolated and the residues were evaporated under nitrogen at $37^{\circ} \mathrm{C}$. Finally, the residues were dissolved in iso-octane and FA concentrations in the fractions were determined using an analytical gas chromatograph (GC-2010 Plus, Shimadzu, Kyoto, Japan). Muscle ceramide content and ceramide fatty acid species were analyzed as described previously [40].

\section{Enzyme activities}

For mitochondrial enzyme activities, $10 \mathrm{mg}$ of the muscle was immediately homogenized in $100 \mathrm{vol} / \mathrm{wt}$ of a $100 \mathrm{mM}$ potassium phosphate buffer and used for the measurements of maximal $\beta$ - hydroxyacyl-CoA dehydrogenase ( $\beta$-HAD) and citrate synthase (CS) activities. Total muscle $\beta$-HAD activity was measured in Tris $\mathrm{HCl}$ buffer (50 mM TrisHCl, $2 \mathrm{mM}$ EDTA, and $250 \mu \mathrm{M} \mathrm{NADH}, \mathrm{pH} 7.0$ ) and 0.04\% Triton-X. The reaction was started by adding $100 \mu \mathrm{M}$ acetoacetyl-CoA, and absorbance was measured at $340 \mathrm{~nm}$ over a 2 min period $\left(37^{\circ} \mathrm{C}\right)[41]$. The CS activity was assayed spectrophotometrically at $37^{\circ} \mathrm{C}$ by measuring the disappearance of $\mathrm{NADH}$ at $412 \mathrm{~nm}$ [42]. 


\section{Western Blotting}

Muscle was homogenized as previously described [43], $10 \mu \mathrm{L}$ of protein was loaded and standard SDS-PAGE procedures were followed. Antibodies included total and phosphorylated Akt (Cell Signaling Inc, Danvers, MA, USA), OXPHOS (MitoSciences, Eugene, OR, USA), Vascular Endothelial Growth Factor (VEGF), hypoxia inducible factor-1 $\alpha$ (HIF-1 $\alpha$ ), endothelial nitric oxide synthase (eNOS), catalase, and SOD2 (all Abcam, Cambridge, UK), 4-HNE (Alpha Diagnostics, San Antonio, TX, USA), COXIV (Invitrogen), and $\alpha$-tubulin (Abcam) as a loading control. Protein carbonylation (Oxyblot, Millipore) was determined according to manufacturer's instructions. Ponceau staining was used to confirm equal loading for antibodies that required the entire membrane (e.g. 4HNE and protein carbonylation). All samples for a given protein were detected on the same membrane using chemiluminescence and the FluorChem HD imaging system (Alpha Innotech, Santa Clara, CA, USA).

\section{Statistics}

All data are expressed as means \pm SEM. Changes over time were analyzed using a paired samples Student's t-test (before vs after bed rest) or a One-Way ANOVA (for daily measurements) using a Bonferroni post-hoc test. Muscle characteristics were analyzed using a Repeated Measures ANOVA with time (before vs after bed rest) and fibre type (type I vs type II) as within-subjects factors. In case of a significant main effect, paired samples t tests were performed to assess time effects within fibre types. For the Oil Red $\mathrm{O}$ analyses, region (SS vs IMF) was added as a within-subjects factor. Statistical significance was set at $P<0.05$. All data were analyzed using SPSS version 22.0 (SPSS Inc., Chicago, IL, USA). 


\section{RESULTS}

\section{Body composition}

Figure 1 displays the effect of short-term bed rest on skeletal muscle mass as assessed by DXA (Figure 1A) and CT (Figure 1B). After one week of bed rest, participants lost $1.4 \pm 0.2 \mathrm{~kg}$ [range: 0.6 to $2.8 \mathrm{~kg}$ ] lean tissue mass (Figure $1 \mathrm{~A} ; P<0.01$ ), representing a $2.5 \pm 0.4 \%$ loss of lean tissue mass.
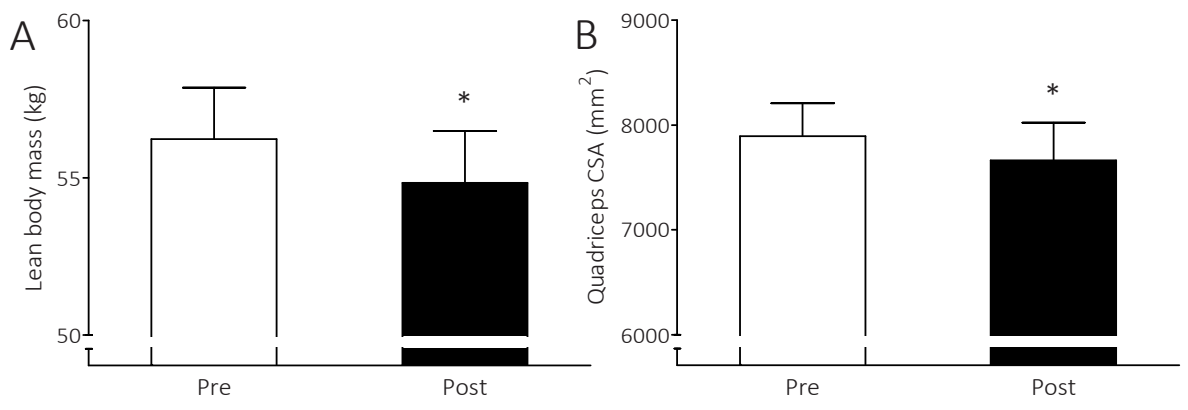

Figure 1 | One week of bed rest leads to a substantial decline in muscle mass. A: Whole-body lean mass declined by $1.4 \pm 0.2 \mathrm{~kg}$ following bed rest. B: Cross-sectional area (CSA) of $m$. quadriceps femoris declined by $3.2 \pm 0.9 \%$. Data represent mean \pm SEM. * Significantly different from pre-bed rest value $(P<0.05)$.

Lean tissue was mainly lost from the trunk $(1.0 \pm 0.2 \mathrm{~kg})$ and legs $(0.28 \pm 0.12 \mathrm{~kg})($ Table 2$)$. Fat mass did not change during one-week of bed rest as participants were fed in energy balance $(-0.2 \pm 0.1 \mathrm{~kg} ; P>0.05)$. A $3.2 \pm 0.9 \%$ decline in cross-sectional area (CSA) of $\mathrm{m}$. quadriceps femoris was observed following bed rest (from $7900 \pm 315$ to $7664 \pm 354 \mathrm{~mm}^{2}$; $P<0.01$, Figure 1B). CSA of the whole thigh muscle had declined by $2.2 \pm 1.0 \%(P<0.05)$. CT scans at the level of the L3 vertebra showed a $1.3 \pm 0.4 \%$ decline in total muscle CSA $(P<0.01)$. As a consequence, the L3 SMI had declined from $51.9 \pm 2.5$ to $51.1 \pm 2.4 \mathrm{~cm}^{2}$ $(P<0.01)$. Analyses performed with SliceOmatic revealed no changes in intermuscular adipose tissue $(P>0.05)$ and visceral adipose tissue $(P>0.05)$. Subcutaneous adipose tissue declined from $93 \pm 28$ to $89 \pm 27 \mathrm{~mm}^{2}(P<0.01)$. Following bed rest, non-significant declines in type I (from 6650 \pm 725 to $6218 \pm 662 \mu \mathrm{m}^{2}$ ) and type II muscle fibre CSA (from $6542 \pm 746$ to $5982 \pm 525 \mu^{2}$ ) were observed ( $P>0.05$; Supplemental Table 2$)$. No differences in fibre circularity were observed between pre- and post-bed rest samples. 
Table 2 | Body composition

\begin{tabular}{lccc}
\hline & Pre & Post & $P$-value \\
\hline Total mass $(\mathrm{kg})$ & $75.3 \pm 2.8$ & $73.7 \pm 2.9$ & $<0.001$ \\
Total lean mass $(\mathrm{kg})$ & $56.2 \pm 1.6$ & $54.8 \pm 1.7$ & $<0.001$ \\
Lean mass trunk $(\mathrm{kg})$ & $27.1 \pm 0.8$ & $26.1 \pm 0.8$ & 0.001 \\
Leg lean mass $(\mathrm{kg})$ & $9.6 \pm 0.3$ & $9.4 \pm 0.3$ & 0.042 \\
Arm lean mass $(\mathrm{kg})$ & $3.4 \pm 0.2$ & $3.4 \pm 0.2$ & 0.183 \\
ALM $(\mathrm{kg})$ & $25.9 \pm 0.9$ & $25.6 \pm 0.9$ & 0.034 \\
SMMI (kg·m ${ }^{-2}$ ) & $7.8 \pm 0.2$ & $7.7 \pm 0.2$ & 0.026 \\
Total fat mass $(\mathrm{kg})$ & $16.3 \pm 2.3$ & $16.1 \pm 2.3$ & 0.082 \\
Fat percentage $(\%)$ & $21.2 \pm 2.3$ & $21.3 \pm 2.3$ & 0.403 \\
BMC $(\mathrm{kg})$ & $2.8 \pm 0.1$ & $2.7 \pm 0.1$ & 0.616 \\
\hline
\end{tabular}

Values (means \pm SEM) represent parameters of body composition from $n=9$ healthy, male volunteers before (pre) and after (post) one week of strict bed rest, as measured by DXA. ALM, appendicular lean mass; BMC, bone mineral content; SMMI, skeletal muscle mass index

\section{Insulin sensitivity and glycemic control}

Glucose infusion rate (GIR) during the euglycemic-hyperinsulinemic clamp had declined by $29 \pm 5 \%$ (range: $9-53 \% ; P<0.01$ ) following one week of bed rest (Figure $2 \mathrm{~A}$ ). Adjustment of GIR for total body weight rather than lean body mass yielded similar results ($29 \pm 5 \% ; P<0.01)$. Postprandial plasma glucose and insulin concentrations observed during the meal tolerance tests are displayed in Figure $2 \mathrm{C}$ and $\mathrm{D}$. For plasma glucose, the area under the curve (AUC) and incremental area under the curve (iAUC) did not differ between both tests (both $P>0.05$ ). In contrast, plasma insulin concentrations showed a significant increase in AUC (from $4963 \pm 779$ to $6944 \pm 513 \mathrm{mU} \cdot \mathrm{L}^{-1} \cdot \mathrm{min}^{-1} ; P<0.05$ ) and iAUC (from $4213 \pm 773$ to $5736 \pm 430 \mathrm{mU} \cdot \mathrm{L}^{-1} \cdot \mathrm{min}^{-1} ; P<0.05$ ) following bed rest. Fasting plasma glucose concentrations (Figure 2B) averaged $5.7 \pm 0.2 \mathrm{mmol} \cdot \mathrm{L}^{-1}$ prior to bed rest, and did not change during the bed rest period $(P>0.05)$. For plasma insulin concentrations (Figure $2 \mathrm{~B})$, a significant time effect $(P<0.001)$ was observed such that fasting insulin concentrations had increased from $7.2 \pm 1.8 \mathrm{mU} \cdot \mathrm{L}^{-1}$ at baseline to $11.8 \pm 1.8 \mathrm{mU} \cdot \mathrm{L}^{-1}$ after one week of bed rest. Consequently, the Homeostasis Model Assessment of Insulin Resistance (HOMA-IR) index increased from $1.9 \pm 0.5$ to $3.1 \pm 0.5$ from day 1 to $8(P<0.01)$. The calculated disposition index (DI) was $-7043 \pm 11949$ and $16945 \pm 9972$ pre- and postbed rest, respectively $(P>0.05)$. 

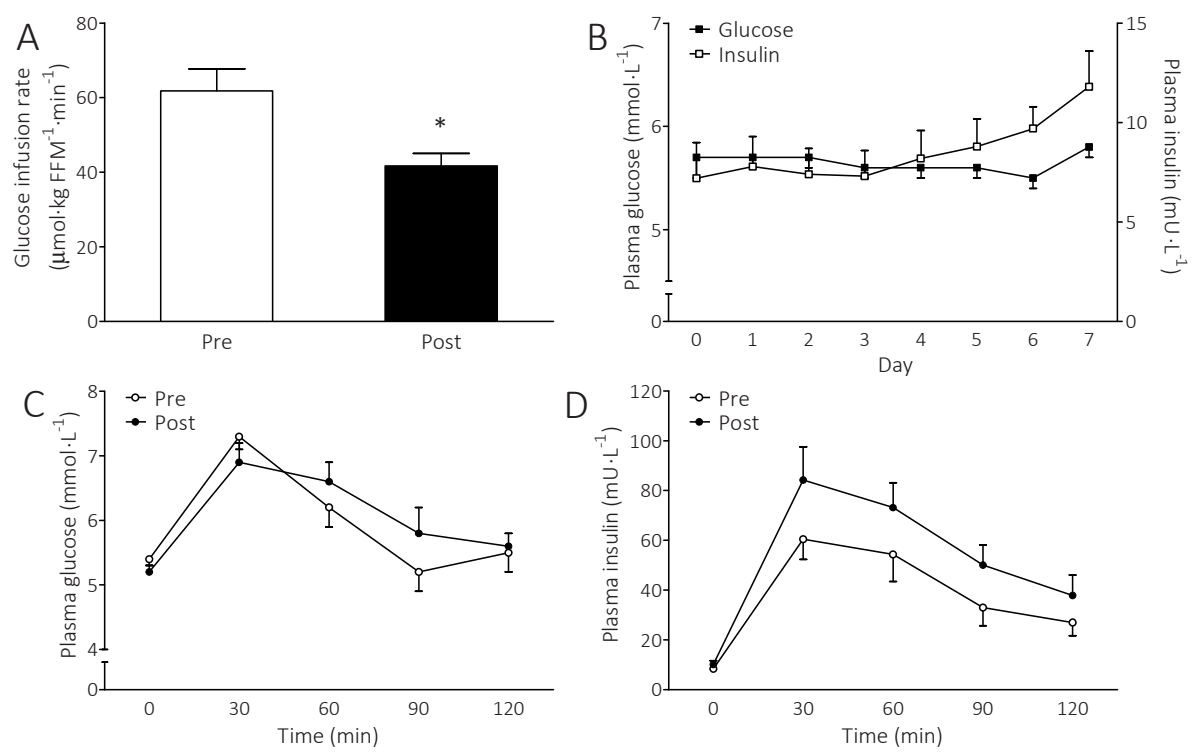

Figure 2 | Insulin sensitivity and postprandial glycemic control decline following one week of strict bed rest. Glucose infusion rates $(A)$ declined by $29 \pm 5 \%$ following bed rest $(P<0.01)$. B: Postabsorptive plasma glucose and insulin concentrations on day 1-7 during bed rest. Insulin concentrations increased over time during bed rest $(P<0.001)$. Postprandial plasma glucose and insulin concentrations in the meal tolerance tests pre- and post-bed rest are depicted in $C$ and $D$, respectively. For glucose, no changes in iAUC were observed $(P>0.05)$, whereas iAUC for insulin were increased following bed rest $(P<0.05)$. Data are shown as means \pm SEM. * Significantly different from pre-bed rest value $(P<0.05)$.

\section{Energy expenditure and whole-body substrate oxidation}

Resting metabolic rate, as measured by indirect calorimetry, tended to decline from $1694 \pm 47$ to $1624 \pm 34 \mathrm{kcal} \cdot \mathrm{d}^{-1}(-3.8 \pm 2.0 \%, P=0.070)$ following bed rest. When corrected for the total lean tissue mass, no such trend was observed $(P>0.05)$. During both the pre- and post-bed rest clamps, energy expenditure was increased during insulin infusion (time effect; $P<0.01$ ). Stimulation by insulin increased the respiratory quotient from $0.84 \pm 0.01$ during the baseline period to $0.93 \pm 0.01$ during exogenous insulin infusion $(P<0.001)$, without differences between pre- and post-bed rest values. Additionally, carbohydrate oxidation rates were increased during the pre- and post-bed rest clamp (baseline: $0.13 \pm 0.01$, hyperinsulinemia $\left.0.24 \pm 0.01 \mathrm{~g} \cdot \mathrm{min}^{-1} ; P<0.001\right)$. Fat oxidation rates decreased from $0.056 \pm 0.007$ (baseline) to $0.011 \pm 0.005 \mathrm{~g} \cdot \mathrm{min}^{-1}$ (insulin) during the prebed rest clamp, and from $0.047 \pm 0.004$ to $0.014 \pm 0.004 \mathrm{~g} \cdot \mathrm{min}^{-1}$ during the post-bed rest clamp (effect of insulin, $P<0.001$; trend for time $x$ treatment effect, $P=0.065$ ). Total protein content and phosphorylation status of both Akt $\left(\mathrm{Ser}^{473}\right)$ and Akt $\left(\mathrm{Thr}^{308}\right)$, measured in fasted biopsies, were not altered following bed rest $(P>0.05)$. 


\section{Functional outcomes}

A significant decline in 1 RM leg press strength, from $211 \pm 16$ to $196 \pm 45 \mathrm{~kg}(-7 \pm 1 \%$; $P<0.01)$, was observed following bed rest. Similarly, leg extension strength decreased from $128 \pm 7$ to $117 \pm 7 \mathrm{~kg}(-8 \pm 2 \% ; P<0.05)$. Following bed rest, no changes in hand grip strength were observed: grip strength averaged $45 \pm 2 \mathrm{~kg}$ prior to bed rest and $46 \pm 2 \mathrm{~kg}$ after the 7 day intervention $(P>0.05)$. Results from the cycle ergometer test showed a decline in peak oxygen uptake from $3332 \pm 200$ to $3100 \pm 162 \mathrm{~mL} \cdot \mathrm{min}^{-1}$, representing a $6.4 \pm 2.3 \%$ loss in VO2peak following bed rest $(P<0.05)$ at a maximal workload of $260 \pm 16$ vs $246 \pm 15 \mathrm{~W}$, respectively $(P<0.05)$.

\section{Lipid metabolism}

Plasma free fatty acid concentrations (FFAs, Supplemental Figure 2) showed a time effect $(P<0.001)$ during bed rest. Post-hoc analyses revealed that values on day 7 of bed rest were greater than on days 2 through $5(P<0.05)$. At baseline, results from the Oil Red $O$ staining showed a greater lipid area percentage in type I than type II muscle fibres $(P<0.05$, Figure $3 \mathrm{~B})$, with smaller droplets in type I vs type II fibres in the SS region $(P<0.05)$. Following bed rest, no changes in lipid area percentage were observed $(P>0.05)$. Droplet size (Figure $3 C)$ changed, such that a significant time $x$ fibre type interaction was found $(P<0.01)$. Based on this interaction, we showed greater lipid droplets in type I vs type II fibres following bed rest $(P<0.01)$. 

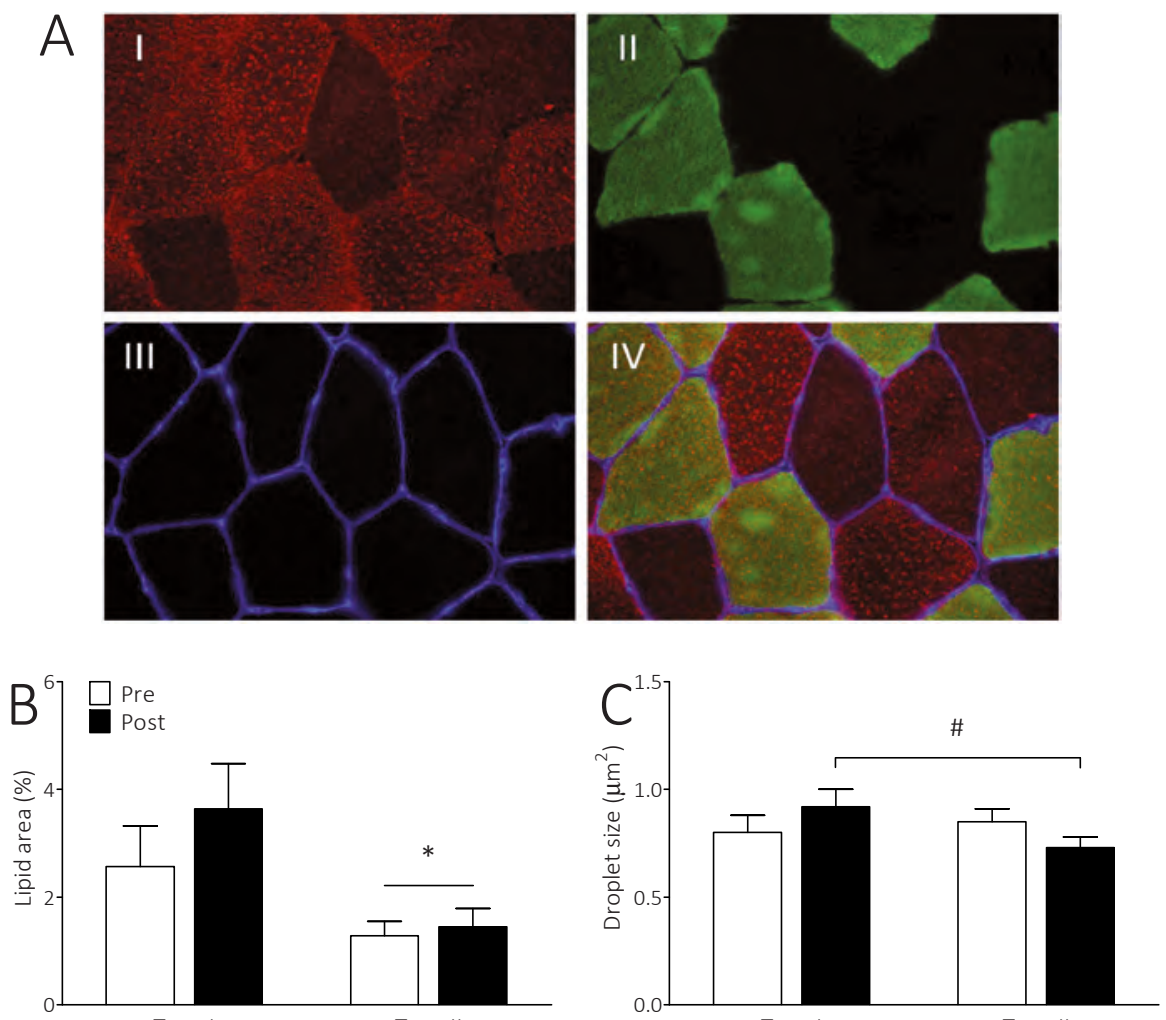

Type I

Type II

Type I

Type II

Figure 3 | Skeletal muscle lipid contents prior to and following one week of bed rest in healthy, young males. Values represent means \pm SEM. 3A represents an image of the Oil Red $O$ staining, made by immunofluorescence microscopy with a magnification of 40x. I: Oil Red O, II: MHC-I, III: laminin, IV, combined image. The lipid area percentage is depicted in $3 \mathrm{~B}$, and lipid droplet size in $3 \mathrm{C}$. * Significantly different from type I fibres $(P<0.05)$. \# Significant difference between type I and II post-bed rest values $(P<0.05)$.

Skeletal muscle lipid content of the measured lipid pools did not change with bed rest (all $P>0.05$, Figure 4). In the phospholipid pool, the percentage saturation increased $(P<0.05$; Supplemental Table 3$)$. For the three other pools, the proportion of polyunsaturated fatty acids (PUFAs) increased or tended to increase. Although contents of some specific fatty acid species was altered following bedrest, no changes in total contents of any of the measured lipid pools were observed (Supplemental Table 4). 

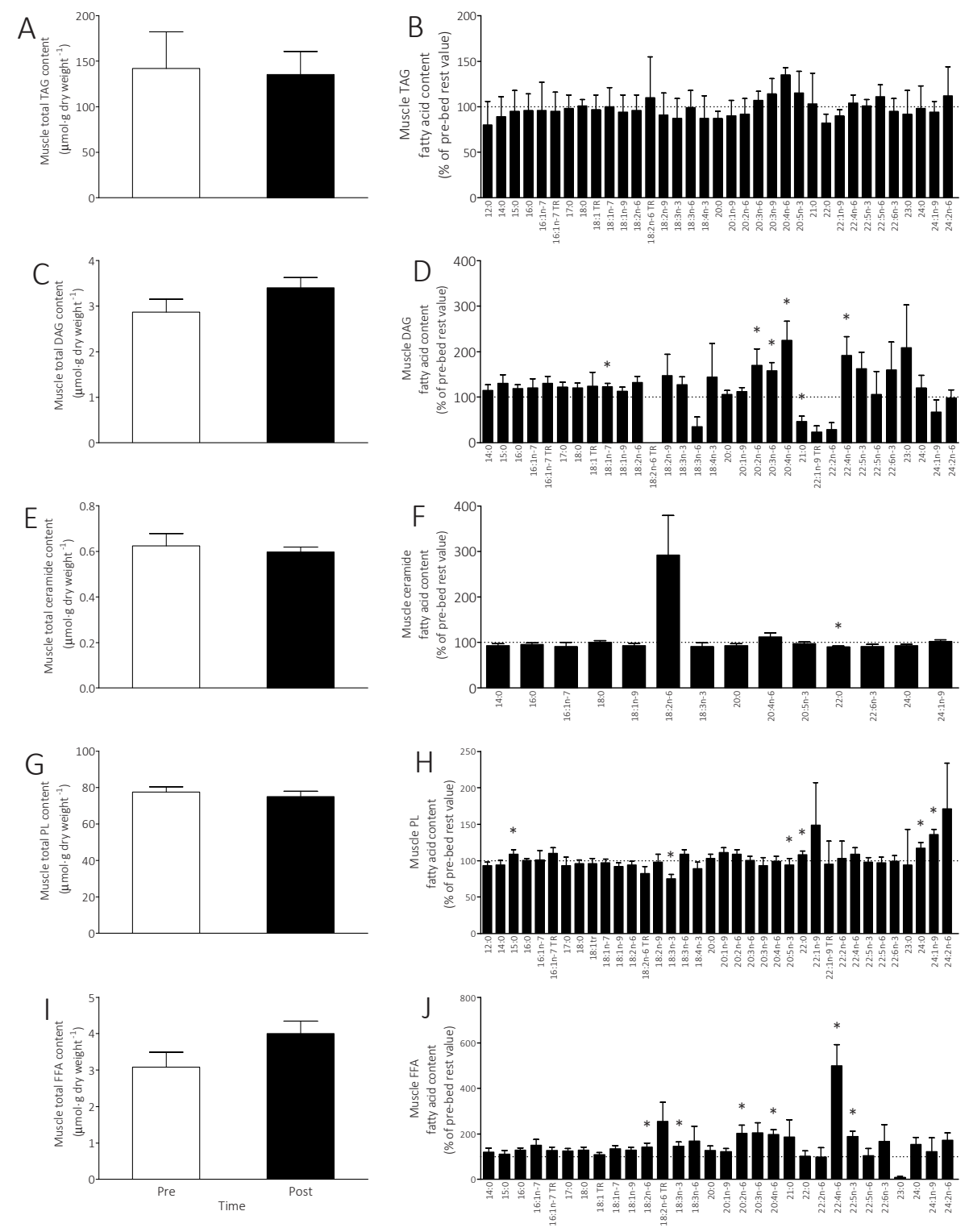

Fatty acid species

Figure 4 | Skeletal muscle total TAG (A), DAG (C), ceramide (E), phospholipid (G), and free fatty acid (I) content, as well as specific fatty acid species within the different lipid_pools. Total content is depicted in the panels on the left, and specific fatty acid species are depicted in panels on the right. Values in panel B, D, F, G, and $\mathrm{J}$ are expressed as relative change from pre-bed rest values (indicated by the dotted line). * Significantly different from pre-bed rest value $(P<0.05)$. 


\section{Oxidative capacity}

Figure 5 depicts results on various parameters of mitochondrial content. Citrate synthase $(C S, 5 A)$ activity decreased by $8 \pm 3 \%$ following bed rest $(P<0.05)$. Activity of $\beta-H A D$ $(5 B)$ tended to decrease by $9 \pm 6 \%(P=0.071)$. Protein content of the different complexes of the OXPHOS system all decreased or tended to decrease, as depicted in 5C. Lipid peroxidation, determined by 4-HNE content, did not change following bed rest (5D, $P>0.05)$. For protein carbonylation (5E) content, a trend for a decline was observed $(P=0.075)$. Both SOD2 (5F) and catalase (5G) protein expression did not change following one week of bed rest $(P>0.05)$.
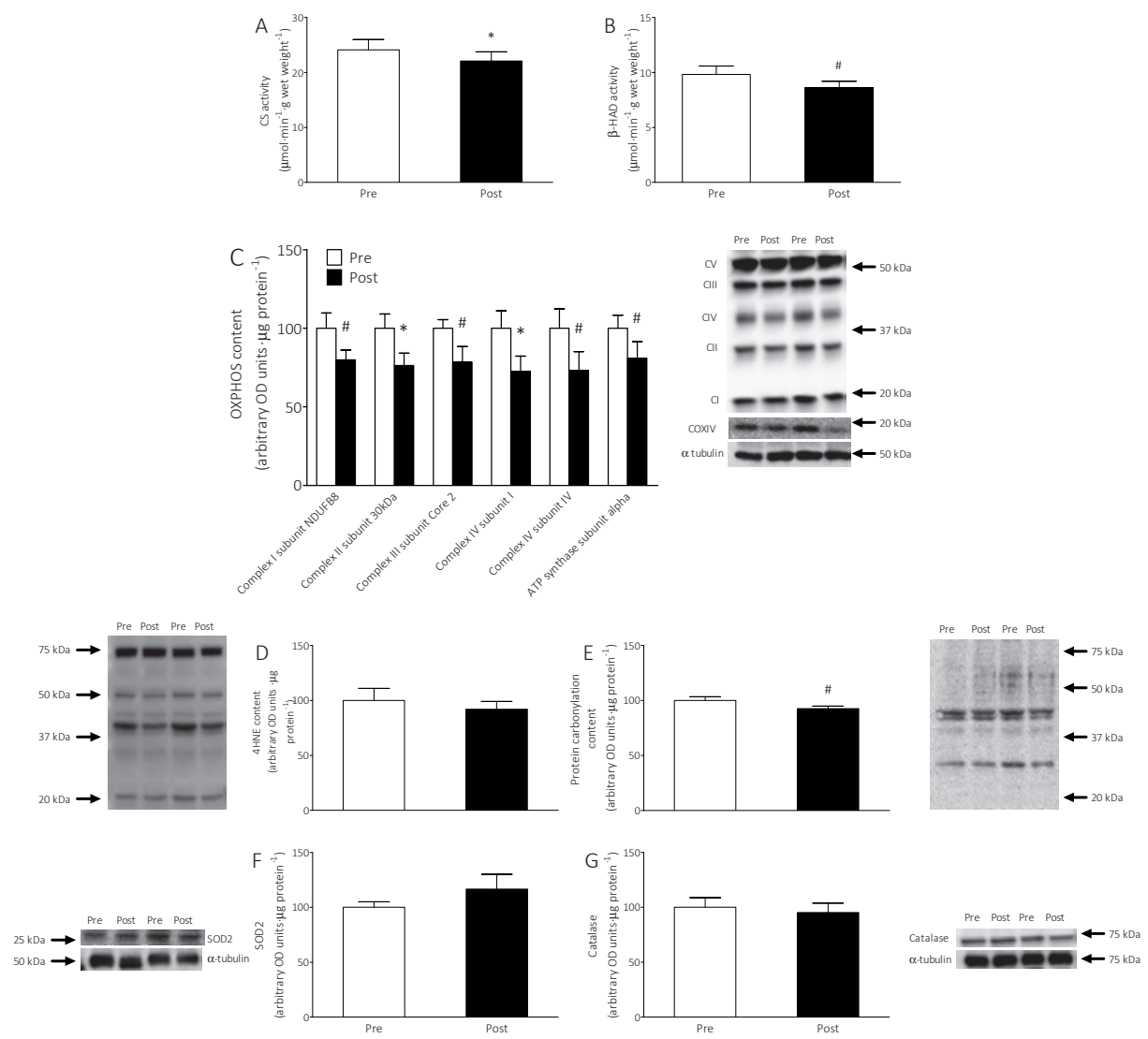

Figure 5 | Seven days of strict bed rest leads to a decline in mitochondrial function. Citrate synthase activity $(C S, A)$ decreased $(P<0.05)$, whereas $\beta-H A D$ activity tended $(P=0.071)$ to decrease $(B)$. The protein contents of the different complexes of the oxidative phosphorylation are displayed in 5C. Figure 5D-5G depict protein expression of $4 \mathrm{HNE}$, protein carbonylation, SOD2 (predicted MW of 27kDa), and catalase (60kDa), respectiveIy. Data represent means \pm SEM. * Significantly different from pre-bed rest $(P<0.05)$. \# Trend for a difference from pre-bed rest value $(P<0.10)$. 


\section{Vascularization}

Seven days of bed rest did not lead to significant changes in VEGF $(-13 \pm 10 \%, P>0.05)$ and eNOS $(-12 \pm 13 \%, P=0.086)$ protein expression (Figure $6 \mathrm{~A}+\mathrm{C}$ ). For HIF-1 $\alpha$ protein expression (6B), a $35 \pm 11 \%$ increase was observed following bed rest $(P<0.05)$. Bed rest did not lead to changes in capillary density, or oxidative exchange across the muscle bed, as shown by the capillary-to-fibre ratio $(6 \mathrm{E})$ and the capillary-to-fibre perimeter exchange index (CFPE index; 6F).
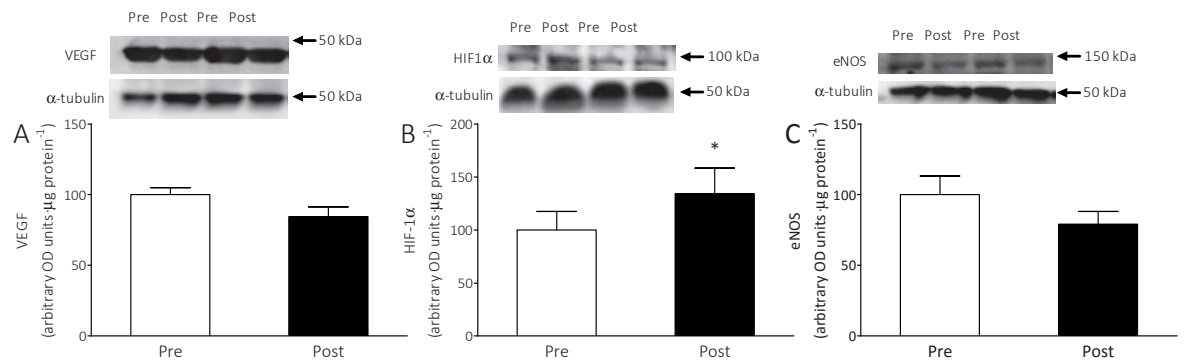

D
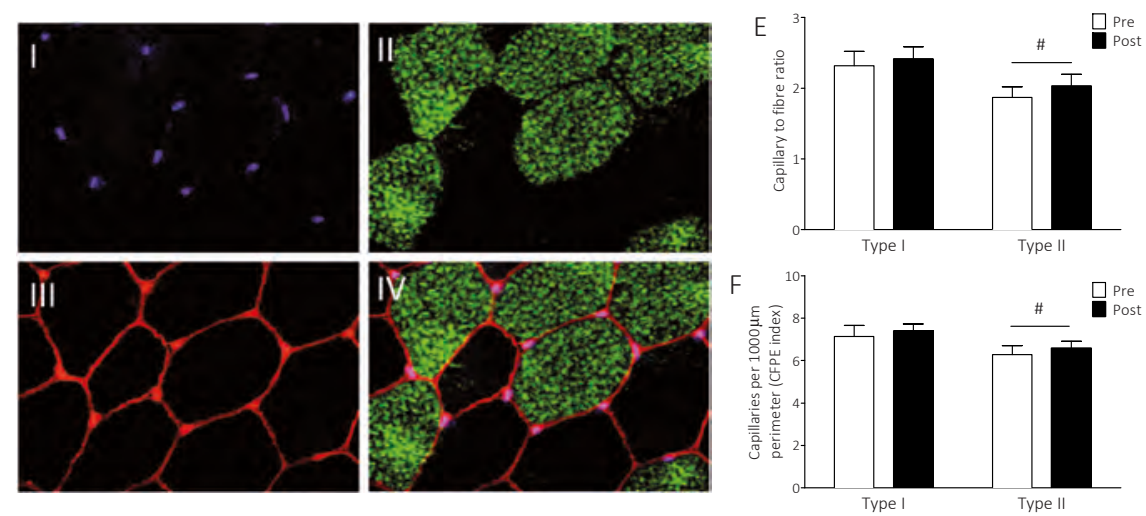

Figure 6 | Skeletal muscle capillary content is not altered following short-term bed rest. Values are presented as means \pm SEM. No changes in VEGF (Vascular Endothelial Growth Factor, 6A, predicted MW 43kDa) protein expression were observed. A significant increase in HIF-1 (Hypoxia-Inducible Factor-1 $\alpha, 6 \mathrm{~B}, 97 \mathrm{kDa}$ ) protein expression was observed following bed rest. Total eNOS (endothelial nitric oxide synthase, 6C, 133kDa) protein expression tended to decline following one week of bed rest $(P=0.086)$. $6 \mathrm{D}$ represents an immunohistochemical image of the CD31 staining, made by microscopy with a magnification of 20x. I: CD31, II: MHC-I, III: laminin, IV, combined image. No changes in capillary-to-fibre ratio (6E) and capillary-to-fibre perimeter exchange index (CFPE index; 6 F) were observed. * Significantly different from values prior to bed rest $(P<0.05)$. \# Significantly different from type I fibres $(P<0.05)$. 


\section{DISCUSSION}

In the present study, we observed that merely one week of bed rest strongly reduced muscle mass, strength, and physical performance. Bed rest resulted in the onset of severe whole-body insulin resistance and a strong decline in skeletal muscle oxidative capacity, both of which occurred in the absence of lipid accumulation or a decline in capillary density in skeletal muscle tissue.

The impact of prolonged bed rest upon skeletal muscle mass and metabolic health has been studied extensively $[1,2]$. Though the model of prolonged disuse is of substantial scientific importance, it may be of more clinical relevance to study short periods of disuse, as patients are typically hospitalized for up to 7 days [3]. Recently, we showed that even 5 days of disuse can lead to a $\sim 4 \%$ decline in muscle mass and a concomitant $\sim 9 \%$ decline in muscle strength [5]. In keeping with this, in the present study we report a $3.2 \%$ decline in quadriceps cross-sectional area (CSA) following one week of bed rest (Figure 1B)[2]. On a whole-body level, this translated to a $1.4 \pm 0.2 \mathrm{~kg}$ loss of lean tissue (Figure 1A), which is equivalent to $200 \mathrm{~g}$ lean tissue loss per day. In comparison, it took a group of healthy, young males 12 weeks of progressive resistance-type exercise training to gain the equivalent amount of lean tissue $(1.7 \mathrm{~kg})$ [44]. Thus, we can lose as much muscle in one week of bed rest as we can gain by 12 weeks of intense resistance type exercise training. Furthermore, the loss of muscle was accompanied by a substantial $\sim 8 \%$ decline in muscle strength and a $6 \%$ reduction in VO2peak. These findings clearly demonstrate that even a short period of disuse has severe consequences for muscle mass and physical performance, an effect that is unlikely compensated for during rehabilitation. As a consequence, it has been suggested that successive periods of bed rest or immobilization may be responsible for the progressive decline in muscle mass throughout our lifespan [7, 8].

The loss of skeletal muscle mass and/or strength during hospitalization has been shown to be predictive of morbidity and mortality [13]. This may be more related to the impact of disuse on metabolic health than to the decline in muscle mass per se. Therefore, in the present study we also aimed to assess the impact of short-term disuse on metabolic health. We performed hyperinsulinemic-euglycemic clamps prior to and after one week of bed rest to assess whole-body insulin sensitivity, and observed a substantial $\sim 30 \%$ decline in glucose disposal (Figure 2A). Under these conditions, hepatic glucose output is strongly diminished and skeletal muscle is responsible for $\sim 85 \%$ of glucose disposal [29]. This implies that merely one week of bed rest can lower insulin sensitivity by as much as $30 \%$. These findings are in line with previous studies, demonstrating similar declines in whole-body and/or peripheral insulin sensitivity following 7-9 days of bed rest $[14-16,18]$. This decline in whole-body insulin sensitivity manifested in a greater postprandial insulin response required to maintain normoglycaemia following bed rest (Figure $2 \mathrm{C}+\mathrm{D}$ ), illustrating the impact of physical inactivity on day to day metabolic control. Supporting the concept that profound insulin resistance manifested with bed 
rest, relatively insensitive population markers such as the HOMA-IR index also increased during the intervention. Interestingly, the increase in HOMA-IR over time did not occur until 4 days of bed rest and was entirely attributed to an increase in postabsorptive insulin concentrations (Figure 2B). Thus, it could be suggested that disuse-induced insulin resistance occurs even more rapidly than one week [9]. Previous work aiming to elucidate the impact of bed rest on insulin signaling has shown that bed rest induced insulin resistance is accompanied by reductions in the contents and/or activity of key proteins regulating glucose uptake and storage in muscle, such as GLUT4, hexokinase 2, and glycogen synthase [18]. However, the decline in insulin sensitivity following bed rest could not be explained by impaired insulin and AMPK signaling, as Akt and AS160 signaling seemed to remain intact following short-term bed rest [45]. Consequently, other mechanisms are likely to be responsible for the development of insulin resistance following short-term bed rest.

Despite substantial muscle atrophy, a 3\% decline in lean mass likely cannot explain the observed $\sim 30 \%$ decline in whole-body insulin sensitivity. As such, during short-term disuse, other mechanisms must contribute to the development of whole-body insulin resistance. Ectopic lipid deposition has often been suggested to lead to the development of insulin resistance in situations of lipid oversupply [19]. While previous studies have reported increases in intramuscular lipid deposition following prolonged bed rest [1], the impact of short-term disuse on skeletal muscle lipid accumulation has been comparatively under investigated [46]. In line with our previous findings [46], in the current study, we did not detect a measurable increase in type I or II muscle fibre lipid content (Figure 3B). We extend on these findings by reporting no increase in subsarcolemmal lipid depots, which have been suggested to more specifically contribute to the development of insulin resistance [47]. Of course, it could be speculated that an intracellular increase in specific fatty acid intermediates, such as diacylglycerols (DAGs), fatty acyl-CoA, ceramides, and/or free fatty acids may be responsible for impairments in insulin receptor function and glucose trafficking [20]. Therefore, we also measured muscle lipid content of various lipid fractions (Figure 4). In line with our fibre-type specific data, we did not observe changes in lipid content of the various lipid fractions, including DAGs, following one week bed rest. Whereas we did see changes in some specific DAG species (Supplemental Table 4), these were not the 18:2 species that have been specifically linked to insulin resistance [48]. Whereas previous work has been inconclusive about the role for ceramides in the development of insulin resistance [4951], we demonstrate no change in total content and only minor changes in specific fatty acid species within the ceramide pool following bed rest, thereby likely ruling out a mediating role for ceramides in the development of insulin resistance during bed rest. Furthermore, the degree of saturation of specifically the DAG pool has been reported to be increased in insulin resistant men when compared to control subjects [52]. However, we failed to observe any changes in the degree of saturation of the various lipid pools, but actually observed a relative increase in polyunsaturated fatty acids (PUFAs, Supplemental Table 3 ) in the different lipid pools. This can potentially be explained by a pref- 
erential oxidation of saturated fatty acids during disuse, which has been suggested previously [10]. Collectively, changes in lipid content and/or lipid composition in skeletal muscle tissue following bed rest are unlikely to explain the observed development of insulin resistance, and therefore other processes must be implicated.

Mitochondrial dysfunction, and specifically the release of mitochondrial reactive oxygen species (ROS), has been postulated as a key factor in the development of muscle disuse atrophy [23] and insulin resistance [53, 54]. Indeed, previous disuse studies have demonstrated a decrease in mitochondrial protein content and enzyme activities, the onset of mitochondrial respiratory dysfunction, and an increase in ROS emission in situations of muscle atrophy $[11,22,55,56]$. In keeping with this, we show a tendency for a decline in $\beta-\mathrm{HAD}$ (Figure 4B) and a significant $8 \%$ decline in citrate synthase activity (Figure 4A), indicative of a decline in mitochondrial content [57]. Similarly, protein content of all complexes of oxidative phosphorylation (OXPHOS, Figure 4C) decreased with bed rest. Given the lack of a fibre type shift away from oxidative fibres (Supplemental Table 2) that is normally observed following prolonged bed rest, these changes cannot be explained by differences in fibre type distribution. Additionally, it has been suggested that short-term bed rest could lead to oxidative stress, which in turn triggers the imbalance between muscle protein synthesis and breakdown [58]. However, we did not find increases in either 4-HNE or protein carbonylation suggesting the absence of overt oxidative damage. These findings are in contrast to a previous report analyzing markers of oxidative damage following a longer period of bed rest [59], suggesting that oxidative damage is a consequence of longer periods of bed rest. Given these data, it was not surprising that no changes in the antioxidants superoxide dismutase 2 (SOD2) and catalase (Figure $4 \mathrm{~F}+\mathrm{G}$ ) were found, as they would usually be increased in the presence of oxidative stress. Previous work by Abadi and colleagues [22] indicates that muscle oxidative capacity is impaired following short-term disuse. We extend on these findings by confirming actual declines in muscle oxidative capacity following bed rest, and suggest that, despite not having measured the GSH:GSSG ratio to assess short-term redox status, overt oxidative stress does not seem to play a role in the rapid development of insulin resistance during up to one week of bed rest. While time course studies are clearly warranted to look at instigating factors of muscle atrophy and the rapid development of insulin resistance, our data suggest that impairments in oxidative capacity may (partly) contribute to the observed decline in insulin sensitivity during short-term bed rest.

As in vivo peripheral insulin sensitivity can also be modulated by changes in macroand microvascular function [60], we also evaluated the effect of bed rest on various angiogenic markers by measuring the expression of vascular endothelial growth factor (VEGF) and endothelial nitric oxide synthase (eNOS), as well as hypoxia-inducible factor$1 \alpha$ (HIF-1 $\alpha$ ). These data suggest potential early adaptive responses following one week of bed rest, as the expression of eNOS tended to decrease while an increase in HIF-1 $\alpha$ was seen (Figure 5). However, this did not result in actual changes in skeletal muscle capillary density as measured by immunohistochemistry. This is in line with previous 
work showing no changes in capillary density following bed rest [16, 55]. Consequently, our data do not provide evidence that a decline in capillary networks contributes to the rapid decline in whole-body insulin sensitivity that was observed following one week of bed rest.

The magnitude of changes that we observed following merely one week of bed rest underlines the impact of short-term muscle disuse, as this study demonstrates that one week of bed rest can result in a similar amount of muscle mass and strength loss as can be regained within months of intense rehabilitation $[35,44]$. These changes in lean mass and muscle strength were observed despite our participants being in energy balance, suggesting that the impact of bed rest in undernourished individuals will be even greater. Next to the decline in muscle mass and function, the observed loss in metabolic health during disuse is of paramount importance. By means of comparison, the measured decline in insulin sensitivity (i.e. $~ 30 \%$ ) is similar to the difference between a normal glucose tolerant individual and a type 2 diabetes patient [52], and is equivalent to a decline that is observed following $30-40$ years of ageing $[23,61]$. As the decline in muscle mass, strength, and peripheral insulin sensitivity have been shown to be good proxy markers for patient outcomes following hospitalization [62], our results emphasize the importance of finding practical and effective interventional strategies that can be applied immediately following the onset of muscle disuse.

We conclude that short-term muscle disuse leads to substantial declines in muscle mass and function, and is associated with the development of peripheral insulin resistance and a decrease in skeletal muscle oxidative capacity. Whereas we are still unclear on the molecular mechanisms responsible, our findings clearly indicate that intramuscular lipid accumulation (implicated in high-fat diet induced insulin resistance), impairments in mitochondrial function and changes in capillary density in skeletal muscle tissue cannot be held responsible for the rapid onset of insulin resistance during a short period of bed rest. Clearly, early interventions are warranted to prevent or attenuate the negative functional and metabolic consequences of short-term bed rest.

\section{ACKNOWLEDGEMENTS}

The authors greatly acknowledge the enthusiasm and dedication of the participants in this study. We also thank Wendy Sluijsmans and Hasibe Aydeniz (both part of NUTRIM School of Nutrition and Translational Research in Metabolism) for technical expertise during the muscle analyses. The practical assistance of Imre Kouw, Irene Fleur Kramer, Kirsten van der Beek, Jorn Trommelen, Jean Nyakayiru, Philippe Pinckaers, Rinske Franssen, Armand Linkens, Kevin Paulussen, Evelien Backx, and Chantal Strijbos (all part of NUTRIM School of Nutrition and Translational Research in Metabolism) was greatly appreciated. 


\section{REFERENCES}

1. Bergouignan, A., F. Rudwill, C. Simon, and S. Blanc, Physical inactivity as the culprit of metabolic inflexibility: evidence from bed-rest studies. J Appl Physiol, 2011. 111(4): p. 1201-10.

2. Wall, B.T. and L.J. van Loon, Nutritional strategies to attenuate muscle disuse atrophy. Nutr Rev, 2013. 71(4): p. 195-208.

3. Union, E. Hospital discharges and length of stay statistics. 2015 October 2015 02/17/2016]; Available from: http://ec.europa.eu/eurostat/statistics-explained/index.php/Hospital_discharges_and_length_of_ stay_statistics.

4. Dirks, M.L., B.T. Wall, R. Nilwik, D.H. Weerts, L.B. Verdijk, and L.J. van Loon, Skeletal muscle disuse atrophy is not attenuated by dietary protein supplementation in healthy older men. J Nutr, 2014. 144(8): p. 1196203.

5. Dirks, M.L., B.T. Wall, T. Snijders, C.L. Ottenbros, L.B. Verdijk, and L.J. van Loon, Neuromuscular electrical stimulation prevents muscle disuse atrophy during leg immobilization in humans. Acta Physiol (Oxf), 2014. 210(3): p. 628-41.

6. Suetta, C., U. Frandsen, A.L. Mackey, L. Jensen, L.G. Hvid, M.L. Bayer, S.J. Petersson, H.D. Schroder, J.L. Andersen, P. Aagaard, P. Schjerling, and M. Kjaer, Ageing is associated with diminished muscle re-growth and myogenic precursor cell expansion early after immobility-induced atrophy in human skeletal muscle. J Physiol, 2013. 591(Pt 15): p. 3789-804.

7. English, K.L. and D. Paddon-Jones, Protecting muscle mass and function in older adults during bed rest. Curr Opin Clin Nutr Metab Care, 2010. 13(1): p. 34-9.

8. Wall, B.T., M.L. Dirks, and L.J. van Loon, Skeletal muscle atrophy during short-term disuse: implications for age-related sarcopenia. Ageing Res Rev, 2013. 12(4): p. 898-906.

9. Yanagibori, R., Y. Suzuki, K. Kawakubo, Y. Makita, and A. Gunji, Carbohydrate and lipid metabolism after 20 days of bed rest. Acta Physiol Scand Suppl, 1994. 616: p. 51-7.

10. Bergouignan, A., D.A. Schoeller, S. Normand, G. Gauquelin-Koch, M. Laville, T. Shriver, M. Desage, Y. Le Maho, H. Ohshima, C. Gharib, and S. Blanc, Effect of physical inactivity on the oxidation of saturated and monounsaturated dietary Fatty acids: results of a randomized trial. PLoS Clin Trials, 2006. 1(5): p. e27.

11. Gram, M., A. Vigelso, T. Yokota, J.W. Helge, F. Dela, and M. Hey-Mogensen, Skeletal muscle mitochondrial $\mathrm{HO}$ emission increases with immobilization and decreases after aerobic training in young and older men. J Physiol, 2015.

12. Haruna, Y., Y. Suzuki, K. Kawakubo, R. Yanagibori, and A. Gunji, Decremental reset in basal metabolism during 20-days bed rest. Acta Physiol (Scand). Supplementum, 1994. 616: p. 43-9.

13. Weijs, P.J., W.G. Looijaard, I.M. Dekker, S.N. Stapel, A.R. Girbes, H.M. Oudemans-van Straaten, and A. Beishuizen, Low skeletal muscle area is a risk factor for mortality in mechanically ventilated critically ill patients. Crit Care, 2014. 18(1): p. R12.

14. Alibegovic, A.C., L. Hojbjerre, M.P. Sonne, G. van Hall, B. Stallknecht, F. Dela, and A. Vaag, Impact of 9 days of bed rest on hepatic and peripheral insulin action, insulin secretion, and whole-body lipolysis in healthy young male offspring of patients with type 2 diabetes. Diabetes, 2009. 58(12): p. 2749-56.

15. Stuart, C.A., R.E. Shangraw, M.J. Prince, E.J. Peters, and R.R. Wolfe, Bed-rest-induced insulin resistance occurs primarily in muscle. Metab Clin Exp, 1988. 37(8): p. 802-6.

16. Mikines, K.J., E.A. Richter, F. Dela, and H. Galbo, Seven days of bed rest decrease insulin action on glucose uptake in leg and whole body. J Appl Physiol, 1991. 70(3): p. 1245-54.

17. Sonne, M.P., A.C. Alibegovic, L. Hojbjerre, A. Vaag, B. Stallknecht, and F. Dela, Effect of 10 days of bedrest on metabolic and vascular insulin action: a study in individuals at risk for type 2 diabetes. J Appl Physiol, 2010. 108(4): p. 830-7.

18. Bienso, R.S., S. Ringholm, K. Kiilerich, N.J. Aachmann-Andersen, R. Krogh-Madsen, B. Guerra, P. Plomgaard, G. van Hall, J.T. Treebak, B. Saltin, C. Lundby, J.A. Calbet, H. Pilegaard, and J.F. Wojtaszewski, GLUT4 and glycogen synthase are key players in bed rest-induced insulin resistance. Diabetes, 2012. 61(5): p. 1090-9. 
19. Krssak, M., K. Falk Petersen, A. Dresner, L. DiPietro, S.M. Vogel, D.L. Rothman, M. Roden, and G.I. Shulman, Intramyocellular lipid concentrations are correlated with insulin sensitivity in humans: a $1 \mathrm{H}$ NMR spectroscopy study. Diabetologia, 1999. 42(1): p. 113-6.

20. Bosma, M., S. Kersten, M.K. Hesselink, and P. Schrauwen, Re-evaluating lipotoxic triggers in skeletal muscle: relating intramyocellular lipid metabolism to insulin sensitivity. Prog Lipid Res, 2012. 51(1): p. 36-49.

21. Max, S.R., Disuse atrophy of skeletal muscle: loss of functional activity of mitochondria. Biochem Biophys Res Commun, 1972. 46(3): p. 1394-8.

22. Abadi, A., E.I. Glover, R.J. Isfort, S. Raha, A. Safdar, N. Yasuda, J.J. Kaczor, S. Melov, A. Hubbard, X. Qu, S.M. Phillips, and M. Tarnopolsky, Limb immobilization induces a coordinate down-regulation of mitochondrial and other metabolic pathways in men and women. PLoS One, 2009. 4(8): p. e6518.

23. Petersen, K.F., D. Befroy, S. Dufour, J. Dziura, C. Ariyan, D.L. Rothman, L. DiPietro, G.W. Cline, and G.I. Shulman, Mitochondrial dysfunction in the elderly: possible role in insulin resistance. Science, 2003. 300(5622): p. 1140-2.

24. Muris, D.M., A.J. Houben, M.T. Schram, and C.D. Stehouwer, Microvascular dysfunction is associated with a higher incidence of type 2 diabetes mellitus: a systematic review and meta-analysis. Arterioscler Thromb Vasc Biol, 2012. 32(12): p. 3082-94.

25. Schoffelen, P.F., K.R. Westerterp, W.H. Saris, and F. Ten Hoor, A dual-respiration chamber system with automated calibration. J Appl Physiol, 1997. 83(6): p. 2064-72.

26. Strandberg, S., M.L. Wretling, T. Wredmark, and A. Shalabi, Reliability of computed tomography measurements in assessment of thigh muscle cross-sectional area and attenuation. BMC Med Imaging, 2010. 10: p. 18.

27. Veasey Rodrigues, H., V.E. Baracos, J.J. Wheler, H.A. Parsons, D.S. Hong, A. Naing, S. Fu, G. Falchoock, A.M. Tsimberidou, S. Piha-Paul, G. Chisholm, and R. Kurzrock, Body composition and survival in the early clinical trials setting. Eur J Cancer, 2013. 49(15): p. 3068-75.

28. Conte, C., E. Fabbrini, M. Kars, B. Mittendorfer, B.W. Patterson, and S. Klein, Multiorgan insulin sensitivity in lean and obese subjects. Diabetes Care, 2012. 35(6): p. 1316-21.

29. DeFronzo, R.A., E. Jacot, E. Jequier, E. Maeder, J. Wahren, and J.P. Felber, The effect of insulin on the disposal of intravenous glucose. Results from indirect calorimetry and hepatic and femoral venous catheterization. Diabetes, 1981. 30(12): p. 1000-7.

30. Most, J., G.H. Goossens, J.W. Jocken, and E.E. Blaak, Short-term supplementation with a specific combination of dietary polyphenols increases energy expenditure and alters substrate metabolism in overweight subjects. Int J Obes (Lond), 2014. 38(5): p. 698-706.

31. Mayhew, J.L., J.L. Prinster, J.S. Ware, D.L. Zimmer, J.R. Arabas, and M.G. Bemben, Muscular endurance repetitions to predict bench press strength in men of different training levels. J Sports Med Phys Fitness, 1995. 35(2): p. 108-13.

32. Verdijk, L.B., R. Koopman, G. Schaart, K. Meijer, H.H. Savelberg, and L.J. van Loon, Satellite cell content is specifically reduced in type II skeletal muscle fibers in the elderly. Am J Physiol Endocrinol Metab, 2007. 292(1): p. E151-7.

33. Guralnik, J.M., L. Ferrucci, B.W. Penninx, J.D. Kasper, S.G. Leveille, K. Bandeen-Roche, and L.P. Fried, New and worsening conditions and change in physical and cognitive performance during weekly evaluations over 6 months: the Women's Health and Aging Study. J Gerontol A Biol Sci Med Sci, 1999. 54(8): p. M410-22.

34. Bergstrom, J., Percutaneous needle biopsy of skeletal muscle in physiological and clinical research. Scand J Clin Lab Invest, 1975. 35(7): p. 609-16.

35. Leenders, M., L.B. Verdijk, L. van der Hoeven, J. van Kranenburg, R. Nilwik, and L.J. van Loon, Elderly men and women benefit equally from prolonged resistance-type exercise training. J Gerontol A Biol Sci Med Sci, 2013. 68(7): p. 769-79.

36. Edelstein, A., N. Amodaj, K. Hoover, R. Vale, and N. Stuurman, Computer control of microscopes using microManager. Curr Protoc Mol Biol, 2010. Chapter 14: p. Unit14 20.

37. Groen, B.B., H.M. Hamer, T. Snijders, J. van Kranenburg, D. Frijns, H. Vink, and L.J. van Loon, Skeletal muscle capillary density and microvascular function are compromised with aging and type 2 diabetes. J Appl Physiol, 2014. 116(8): p. 998-1005. 
38. Koopman, R., G. Schaart, and M.K. Hesselink, Optimisation of oil red O staining permits combination with immunofluorescence and automated quantification of lipids. Histochem Cell Biol, 2001. 116(1): p. 63-8.

39. Moors, C.C., E.E. Blaak, N.J. van der Zijl, M. Diamant, and G.H. Goossens, The effects of long-term valsartan treatment on skeletal muscle fatty acid handling in humans with impaired glucose metabolism. J Clin Endocrinol Metab, 2013. 98(5): p. E891-6.

40. Beaudoin, M.S., C.G. Perry, A.M. Arkell, A. Chabowski, J.A. Simpson, D.C. Wright, and G.P. Holloway, Impairments in mitochondrial palmitoyl-CoA respiratory kinetics that precede development of diabetic cardiomyopathy are prevented by resveratrol in ZDF rats. J Physiol, 2014. 592(12): p. 2519-33.

41. Bergmeyer, H.U., Methods of enzymatic analysis. 2d English ed. 1974, Weinheim (NY): Verlag Chemie; Academic Press.

42. Srere, P.A., Citrate synthase. Methods Enzymol, 1969. 13: p. 3-11.

43. Herbst, E.A., S. Paglialunga, C. Gerling, J. Whitfield, K. Mukai, A. Chabowski, G.J. Heigenhauser, L.L. Spriet, and G.P. Holloway, Omega-3 supplementation alters mitochondrial membrane composition and respiration kinetics in human skeletal muscle. J Physiol, 2014. 592(Pt 6): p. 1341-52.

44. Snijders, T., P.T. Res, J.S. Smeets, S. van Vliet, J. van Kranenburg, K. Maase, A.K. Kies, L.B. Verdijk, and L.J. van Loon, Protein Ingestion before Sleep Increases Muscle Mass and Strength Gains during Prolonged Resistance-Type Exercise Training in Healthy Young Men. J Nutr, 2015. 145(6): p. 1178-84.

45. Mortensen, B., M. Friedrichsen, N.R. Andersen, A.C. Alibegovic, L. Hojbjerre, M.P. Sonne, B. Stallknecht, F. Dela, J.F. Wojtaszewski, and A. Vaag, Physical inactivity affects skeletal muscle insulin signaling in a birth weight-dependent manner. J Diabetes Complications, 2014. 28(1): p. 71-8.

46. Wall, B.T., M.L. Dirks, T. Snijders, F.B. Stephens, J.M. Senden, M.L. Verscheijden, and L.J. van Loon, Shortterm muscle disuse atrophy is not associated with increased intramuscular lipid deposition or a decline in the maximal activity of key mitochondrial enzymes in young and older males. Exp Gerontol, 2015. 61: p. 76-83.

47. Nielsen, J., M. Mogensen, B.F. Vind, K. Sahlin, K. Hojlund, H.D. Schroder, and N. Ortenblad, Increased subsarcolemmal lipids in type 2 diabetes: effect of training on localization of lipids, mitochondria, and glycogen in sedentary human skeletal muscle. Am J Physiol Endocrinol Metab, 2010. 298(3): p. E706-13.

48. Nowotny, B., L. Zahiragic, D. Krog, P.J. Nowotny, C. Herder, M. Carstensen, T. Yoshimura, J. Szendroedi, E. Phielix, P. Schadewaldt, N.C. Schloot, G.I. Shulman, and M. Roden, Mechanisms underlying the onset of oral lipid-induced skeletal muscle insulin resistance in humans. Diabetes, 2013. 62(7): p. 2240-8.

49. Adams, J.M., 2nd, T. Pratipanawatr, R. Berria, E. Wang, R.A. DeFronzo, M.C. Sullards, and L.J. Mandarino, Ceramide content is increased in skeletal muscle from obese insulin-resistant humans. Diabetes, 2004. 53(1): p. 25-31.

50. Bonen, A., S.S. Jain, L.A. Snook, X.X. Han, Y. Yoshida, K.H. Buddo, J.S. Lally, E.D. Pask, S. Paglialunga, M.S. Beaudoin, J.F. Glatz, J.J. Luiken, E. Harasim, D.C. Wright, A. Chabowski, and G.P. Holloway, Extremely rapid increase in fatty acid transport and intramyocellular lipid accumulation but markedly delayed insulin resistance after high fat feeding in rats. Diabetologia, 2015.

51. Sitnick, M.T., M.K. Basantani, L. Cai, G. Schoiswohl, C.F. Yazbeck, G. Distefano, V. Ritov, J.P. DeLany, R. Schreiber, D.B. Stolz, N.P. Gardner, P.C. Kienesberger, T. Pulinilkunnil, R. Zechner, B.H. Goodpaster, P. Coen, and E.E. Kershaw, Skeletal muscle triacylglycerol hydrolysis does not influence metabolic complications of obesity. Diabetes, 2013. 62(10): p. 3350-61.

52. Jocken, J.W., G.H. Goossens, H. Boon, R.R. Mason, Y. Essers, B. Havekes, M.J. Watt, L.J. van Loon, and E.E. Blaak, Insulin-mediated suppression of lipolysis in adipose tissue and skeletal muscle of obese type 2 diabetic men and men with normal glucose tolerance. Diabetologia, 2013. 56(10): p. 2255-65.

53. Anderson, E.J., M.E. Lustig, K.E. Boyle, T.L. Woodlief, D.A. Kane, C.T. Lin, J.W. Price, 3rd, L. Kang, P.S. Rabinovitch, H.H. Szeto, J.A. Houmard, R.N. Cortright, D.H. Wasserman, and P.D. Neufer, Mitochondrial $\mathrm{H} 2 \mathrm{O} 2$ emission and cellular redox state link excess fat intake to insulin resistance in both rodents and humans. J Clin Invest, 2009. 119(3): p. 573-81.

54. Lee, H.Y., C.S. Choi, A.L. Birkenfeld, T.C. Alves, F.R. Jornayvaz, M.J. Jurczak, D. Zhang, W.K. Woo, G.S. Shadel, W. Ladiges, P.S. Rabinovitch, J.H. Santos, K.F. Petersen, V.T. Samuel, and G.I. Shulman, Targeted 
expression of catalase to mitochondria prevents age-associated reductions in mitochondrial function and insulin resistance. Cell Metab, 2010. 12(6): p. 668-74.

55. Ringholm, S., R.S. Bienso, K. Kiilerich, A. Guadalupe-Grau, N.J. Aachmann-Andersen, B. Saltin, P. Plomgaard, C. Lundby, J.F. Wojtaszewski, J.A. Calbet, and H. Pilegaard, Bed rest reduces metabolic protein content and abolishes exercise-induced mRNA responses in human skeletal muscle. Am J Physiol Endocrinol Metab, 2011. 301(4): p. E649-58.

56. Levine, S., T. Nguyen, N. Taylor, M.E. Friscia, M.T. Budak, P. Rothenberg, J. Zhu, R. Sachdeva, S. Sonnad, L.R. Kaiser, N.A. Rubinstein, S.K. Powers, and J.B. Shrager, Rapid disuse atrophy of diaphragm fibers in mechanically ventilated humans. N Engl J Med, 2008. 358(13): p. 1327-35.

57. Larsen, S., J. Nielsen, C.N. Hansen, L.B. Nielsen, F. Wibrand, N. Stride, H.D. Schroder, R. Boushel, J.W. Helge, F. Dela, and M. Hey-Mogensen, Biomarkers of mitochondrial content in skeletal muscle of healthy young human subjects. J Physiol, 2012. 590(Pt 14): p. 3349-60.

58. Powers, S.K., A.N. Kavazis, and K.C. DeRuisseau, Mechanisms of disuse muscle atrophy: role of oxidative stress. Am J Physiol Regul Integr Comp Physiol, 2005. 288(2): p. R337-44.

59. Agostini, F., L. Dalla Libera, J. Rittweger, S. Mazzucco, M. Jurdana, I.B. Mekjavic, R. Pisot, L. Gorza, M. Narici, and G. Biolo, Effects of inactivity on human muscle glutathione synthesis by a double-tracer and single-biopsy approach. J Physiol, 2010. 588(Pt 24): p. 5089-104.

60. Lillioja, S., A.A. Young, C.L. Culter, J.L. Ivy, W.G. Abbott, J.K. Zawadzki, H. Yki-Jarvinen, L. Christin, T.W. Secomb, and C. Bogardus, Skeletal muscle capillary density and fiber type are possible determinants of in vivo insulin resistance in man. J Clin Invest, 1987. 80(2): p. 415-24.

61. Defronzo, R.A., Glucose intolerance and aging: evidence for tissue insensitivity to insulin. Diabetes, 1979. 28(12): p. 1095-101.

62. van den Berghe, G., P. Wouters, F. Weekers, C. Verwaest, F. Bruyninckx, M. Schetz, D. Vlasselaers, P. Ferdinande, P. Lauwers, and R. Bouillon, Intensive insulin therapy in critically ill patients. N Engl J Med, 2001. 345(19): p. 1359-67. 


\section{SUPPLEMENTAL MATERIAL}

\section{SUPPLEMENTAL METHODS}

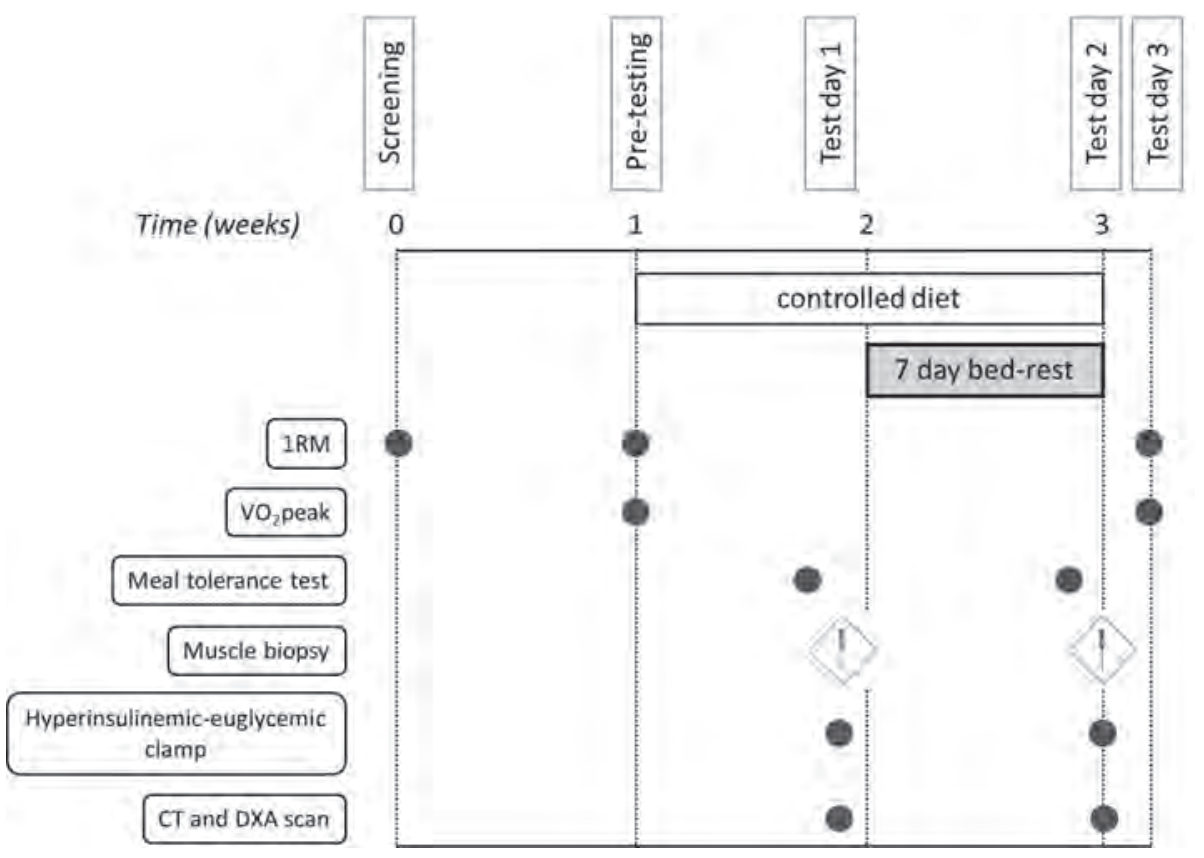

Supplemental Figure 1 | Schematic outline of the study protocol. CT, Computed Tomography; DXA, Dual Energy X-Ray Absorptiometry; 1RM, 1-Repetition Maximum

Supplemental Table 1 | Food intake before and during 7 days of strict bed rest

\begin{tabular}{lccc}
\hline & Pre-bed rest & Bed rest & P-value \\
\hline Energy $\left(\mathrm{kcal} \cdot \mathrm{d}^{-1}\right)$ & $2589 \pm 50$ & $2205 \pm 47$ & $<0.001$ \\
Protein $\left(\mathrm{g} \cdot \mathrm{kg} \mathrm{bw}{ }^{-1} \cdot \mathrm{d}^{-1}\right)$ & $1.16 \pm 0.02$ & $0.98 \pm 0.04$ & $<0.001$ \\
Protein $\left(\mathrm{g} \cdot \mathrm{d}^{-1}\right)$ & $88 \pm 2$ & $73 \pm 1$ & $<0.001$ \\
Fat $\left(\mathrm{g} \cdot \mathrm{d}^{-1}\right)$ & $88 \pm 2$ & $73 \pm 3$ & 0.004 \\
Carbohydrate $\left(\mathrm{g} \cdot \mathrm{d}^{-1}\right)$ & $343 \pm 9$ & $297 \pm 4$ & $<0.001$ \\
Protein $($ En\%) & $14.0 \pm 0.3$ & $13.7 \pm 0.2$ & 0.354 \\
Fat $($ En\%) & $31.6 \pm 0.6$ & $30.7 \pm 0.7$ & 0.428 \\
Carbohydrate $($ En\%) & $54.4 \pm 0.8$ & $55.6 \pm 0.7$ & 0.368 \\
\hline
\end{tabular}

Values represent means \pm SEM. Bw, body weight; En\%, Energy percentage 


\section{SUPPLEMENTAL RESULTS}

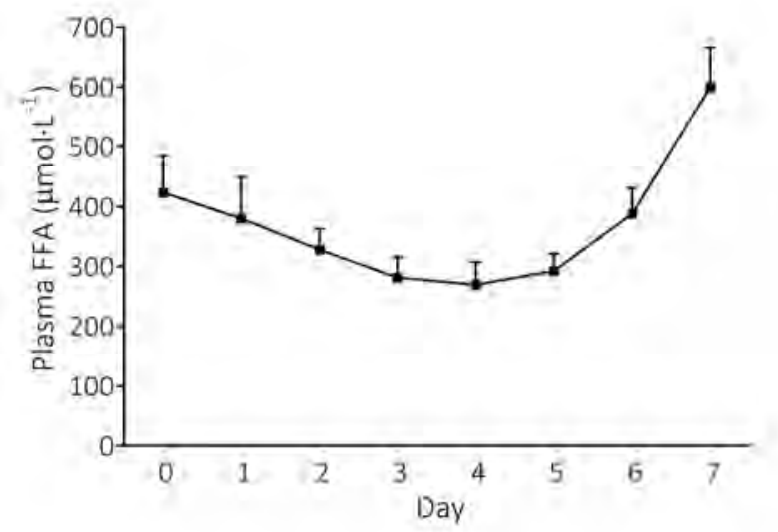

Supplemental Figure 2 | Fasting plasma free fatty acid (FFA) concentration on each day of the bed rest period.

Supplemental Table 2 | Muscle fibre cross-sectional area (CSA), fibre distribution and myonuclear content

\begin{tabular}{lcccc}
\hline & Fibre type & Pre & Post & $P$-value \\
\hline Muscle fibre CSA $\left(\mu \mathrm{m}^{2}\right)$ & I & $6650 \pm 725$ & $6218 \pm 662$ & 0.538 \\
\% Fibre (number) & II & $6542 \pm 746$ & $5982 \pm 525$ & 0.353 \\
\% Fibre (area) & I & $48 \pm 4$ & $40 \pm 3$ & 0.102 \\
Nuclei per fibre & I & $48 \pm 5$ & $41 \pm 4$ & 0.215 \\
& I & $3.8 \pm 0.3$ & $3.8 \pm 0.4$ & 0.914 \\
Myonuclear domain $\left(\mu \mathrm{m}^{2}\right)$ & $\mathrm{I}$ & $3.5 \pm 0.3$ & $3.8 \pm 0.2$ & 0.303 \\
& $\mathrm{I}$ & $1763 \pm 157$ & $1621 \pm 79$ & 0.450 \\
\hline
\end{tabular}

Data are presented as means \pm SEM. CSA, cross-sectional area. 
Supplemental Table 3 | Skeletal muscle lipid content and saturation

\begin{tabular}{|c|c|c|c|}
\hline & Pre & Post & $P$-value \\
\hline \multicolumn{4}{|l|}{ TAG } \\
\hline Total $\left(\mu \mathrm{mol} \cdot \mathrm{g}\right.$ dry weight $\left.{ }^{-1}\right)$ & $142 \pm 40$ & $135 \pm 25$ & 0.750 \\
\hline Saturation (\%) & $36 \pm 1$ & $36 \pm 1$ & 0.558 \\
\hline MUFA (\%) & $51 \pm 1$ & $49 \pm 1$ & 0.275 \\
\hline PUFA (\%) & $14 \pm 1$ & $14 \pm 1$ & 0.069 \\
\hline \multicolumn{4}{|l|}{ DAG } \\
\hline Total ( $\mu \mathrm{mol} \cdot \mathrm{g}$ dry weight $\left.{ }^{-1}\right)$ & $2.9 \pm 0.3$ & $3.4 \pm 0.2$ & 0.132 \\
\hline Saturation (\%) & $41 \pm 1$ & $42 \pm 1$ & 0.752 \\
\hline MUFA (\%) & $47 \pm 1$ & $45 \pm 1$ & 0.170 \\
\hline PUFA (\%) & $12 \pm 1$ & $13 \pm 1$ & 0.020 \\
\hline \multicolumn{4}{|l|}{ Ceramides } \\
\hline Total $\left(\mu \mathrm{mol} \cdot \mathrm{g}\right.$ dry weight $\left.{ }^{-1}\right)$ & $0.6 \pm 0.5$ & $0.6 \pm 0.2$ & 0.574 \\
\hline Saturation (\%) & $75 \pm 0$ & $74 \pm 0$ & 0.358 \\
\hline MUFA (\%) & $17 \pm 0$ & $17 \pm 0$ & 0.950 \\
\hline PUFA (\%) & $8 \pm 0$ & $8 \pm 0$ & 0.161 \\
\hline \multicolumn{4}{|l|}{$P L$} \\
\hline Total ( $\mu \mathrm{mol} \cdot \mathrm{g}$ dry weight ${ }^{-1}$ ) & $77.5 \pm 2.9$ & $75.0 \pm 3.0$ & 0.259 \\
\hline Saturation (\%) & $39 \pm 0$ & $40 \pm 1$ & 0.019 \\
\hline MUFA (\%) & $10 \pm 0$ & $9 \pm 0$ & 0.707 \\
\hline PUFA (\%) & $52 \pm 1$ & $51 \pm 1$ & 0.072 \\
\hline \multicolumn{4}{|l|}{ FFA } \\
\hline Total $\left(\mu \mathrm{mol} \cdot g\right.$ dry weight $\left.{ }^{-1}\right)$ & $3.1 \pm 0.4$ & $4.0 \pm 0.3$ & 0.085 \\
\hline Saturation (\%) & $51 \pm 1$ & $49 \pm 1$ & 0.231 \\
\hline MUFA (\%) & $39 \pm 1$ & $39 \pm 1$ & 0.771 \\
\hline PUFA (\%) & $10 \pm 1$ & $12 \pm 1$ & 0.045 \\
\hline
\end{tabular}

Values (means \pm SEM) represent skeletal muscle lipid content and degree of saturation of various lipid fractions, prior to and following 7 days of strict bed rest. DAG, diacylglycerol; FFA, free fatty acid; MUFA, monounsaturated fatty acid; PL, phospholipid; PUFA, polyunsaturated fatty acid; TAG, triacylglycerol. 


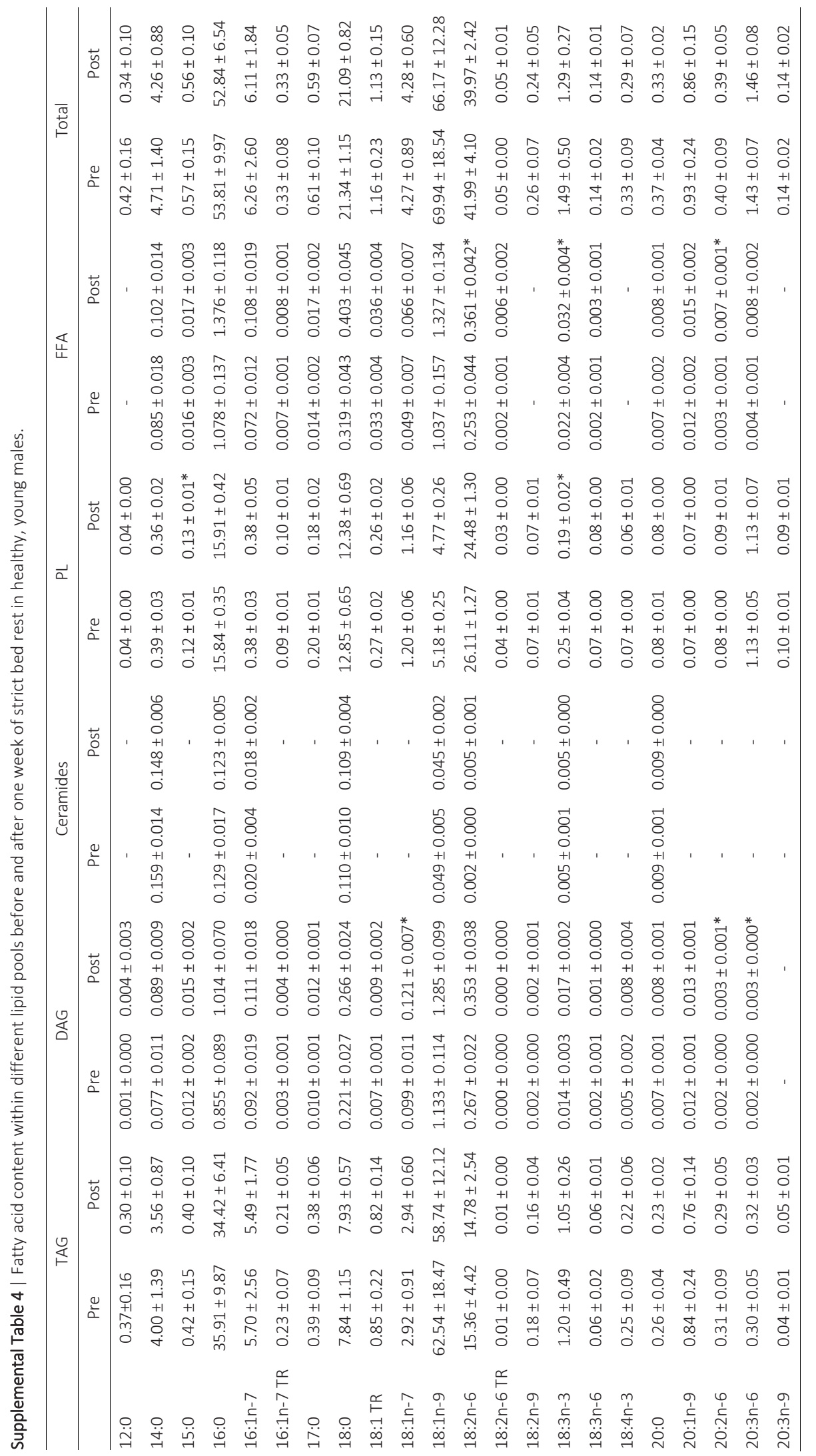




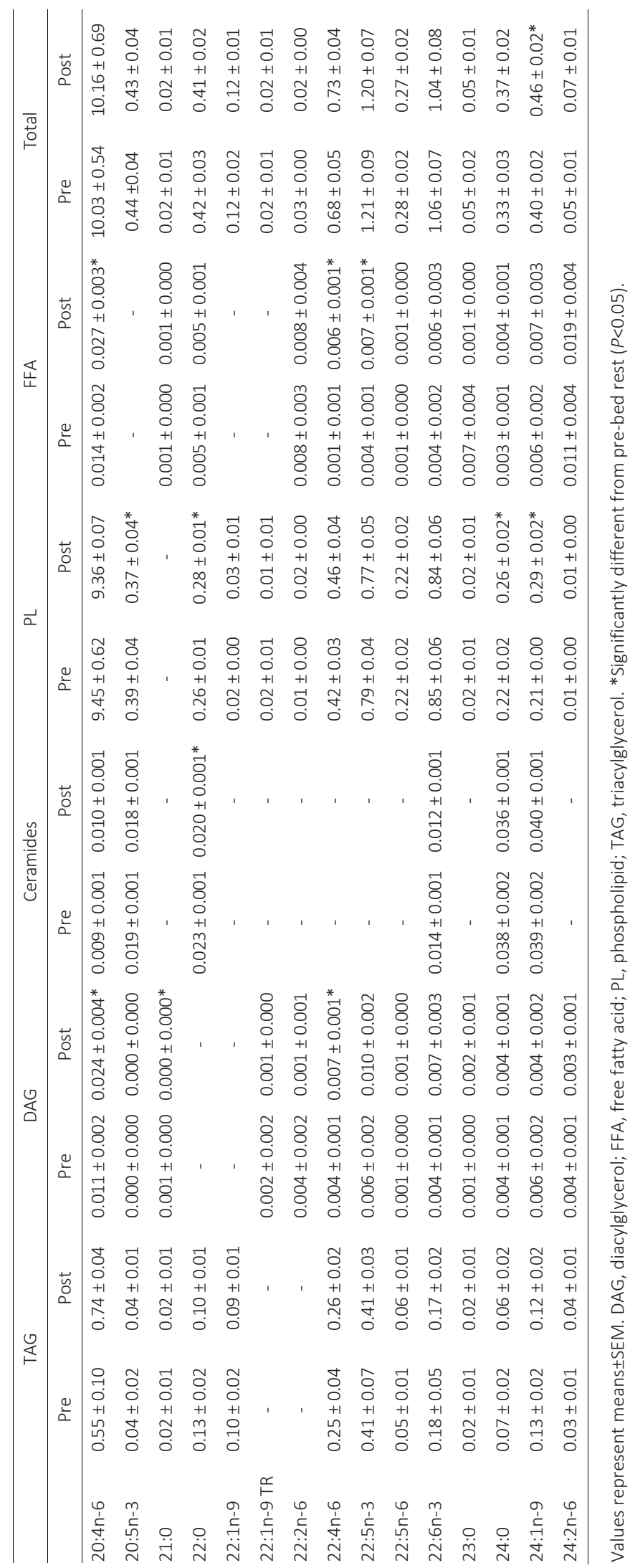



CHAPTER

General Discussion 
Various situations, such as the recovery from illness or rehabilitation after injury, often necessitate a period of physical inactivity in otherwise healthy individuals. This muscle disuse is initiated to enhance the recovery process, but is accompanied by the loss of muscle mass and muscle strength, as well as other negative health consequences. In this thesis, we studied the underlying physiological mechanisms responsible for muscle disuse atrophy, and assessed various nutritional and/or exercise interventional strategies to prevent muscle atrophy during a period of disuse. In this final chapter we will address the implications of the presented findings, and discuss them in a broader perspective. At the end of this chapter we have defined important goals for future research in this area.

\section{The impact of short-term disuse}

Most work addressing the impact of disuse on muscle atrophy in humans has applied experimental disuse models, such as limb immobilization and bed rest, for several weeks (e.g. [1-10]). Fewer studies have tried to address the impact of short(er) periods of bed rest or limb immobilization on muscle mass loss (e.g. [11-14]). This is of particular relevance as the average hospital length of stay for patients of all ages and all reasons of admission is 7 days [15]. Previous studies have shown a decline in functional strength after 4 days of leg immobilization [16, 17], but the effect of such a short period of immobilization on muscle mass had not been assessed before. Therefore, in Chapter 2, we assessed the loss of muscle mass and strength following 5 vs 14 days of leg immobilization in healthy, young men. We demonstrate that merely 5 days of leg immobilization already leads to a substantial decline in muscle mass. Next to the $3.5 \%$ muscle loss that was observed, a concomitant 9\% loss of muscle strength was seen (Chapter 2, [18]). Such short periods of disuse not only occur during hospitalization, but can also occur during recovery from illness or minor injury at home. These repeated 'catabolic crises' are now suggested to contribute to the persistent loss of muscle mass with ageing [19, 20]. This is due to the fact that older individuals generally do not regain all muscle tissue that was lost during a period of disuse [10, 21, 22]. More specifically, Hvid and coworkers have shown that elderly individuals experience more difficulties when trying to re-gain muscle lost after a period of disuse when compared to their younger counterparts [21]. The impact of multiple, successive short periods of disuse may largely, if not entirely, explain the 3-8\% muscle loss that is observed per decade after the age of 30 $[19,23]$. This 'accelerated ageing' further stresses the importance of focussing on both the prevention of muscle loss during disuse, as well as regaining muscle loss during subsequent rehabilitation.

Any net loss of muscle mass must be underpinned by an increase in muscle protein breakdown, a decrease in muscle protein synthesis, or a combination of both. Longterm muscle disuse has been shown to be accompanied by declines in both basal $[2,4$, $6,24-29]$ and postprandial muscle protein synthesis rates [26, 30, 31]. No studies have 
measured basal or postprandial muscle protein synthesis rates following short-term muscle disuse. Therefore, in Chapter 3, we subjected twelve healthy, young males to 5 days of one-legged knee immobilization. Following the immobilization period, we used stable isotope tracer infusions combined with the ingestion of intrinsically labelled protein to assess postabsorptive and postprandial muscle protein synthesis rates in the immobilized and control leg. This study showed that merely 5 days of immobilization reduces postabsorptive myofibrillar protein synthesis rates by $41 \pm 13 \%$ and postprandial myofibrillar protein synthesis rates by as much as $53 \pm 4 \%$ (Chapter 3, [32]). Additionally, indirect data from static markers suggested that the onset of disuse leads to an increase in muscle protein breakdown [33-36]. Therefore we suggested that muscle atrophy during short-term disuse is caused by a temporary increase in muscle protein breakdown and a large decrease in muscle protein synthesis (Figure 3).

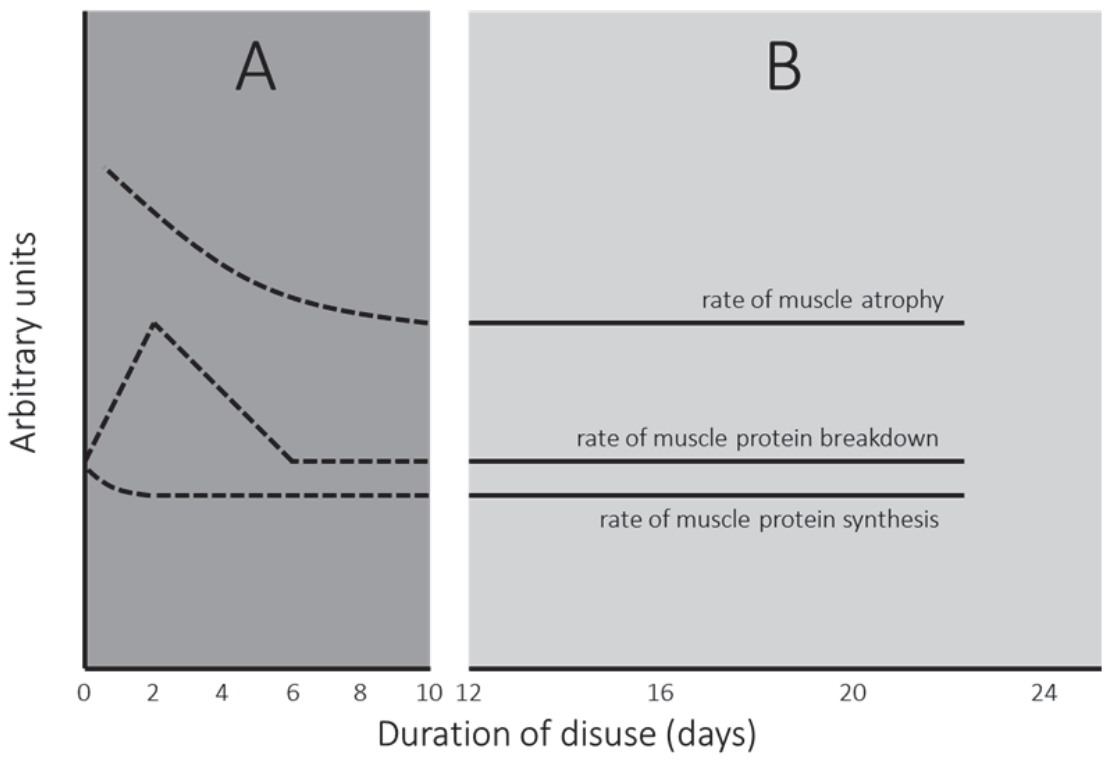

Figure 1 | Theoretical time course of the development of muscle disuse atrophy, combined with the associated changes in muscle protein synthesis and breakdown. Section A depicts the hypothesis obtained from indirect data that the onset of disuse is accompanied by an increase in muscle protein breakdown and a decrease in muscle protein synthesis, resulting in rapid muscle atrophy. Section B illustrates the data available that show that prolonged disuse (>10 days) results in a more modest rate of muscle atrophy when compared with short-term disuse, which is primarily caused by a decline in muscle protein synthesis. Adapted from [42].

However, the relative contribution of the decline in basal muscle protein synthesis, the impaired muscle protein synthetic response to feeding, and the increase in muscle protein breakdown to muscle atrophy remains to be elucidated. As it is a technical challenge to measure muscle protein breakdown rates in vivo in humans, there is a lack of 
studies investigating the role of muscle disuse on muscle protein breakdown. Consequently, many researchers emphasize the contribution of muscle protein synthesis to muscle atrophy. However, it is important to consider whether interventional strategies should target muscle protein synthesis or muscle protein breakdown to maximize their effect. Interventions that can stimulate basal and postprandial muscle protein synthesis, as well as inhibit muscle protein breakdown in the first few days of disuse may be the most effective in preventing muscle loss during a (short) period of disuse.

\section{INTERVENTIONAL STRATEGIES}

\section{Neuromuscular electrical stimulation}

Physical activity is one of the more potent stimuli that can increase muscle protein synthesis [37]. The introduction of an exercise regimen during a period of bed rest has been shown to effectively maintain muscle protein synthesis rates [27]. Furthermore, it has been shown that muscle atrophy can be attenuated or even abolished when a relatively low dose of exercise is applied [38-40]. While performing physical activity is undoubtedly the most effective intervention to preserve muscle mass, it is often impossible to maintain a certain level of physical activity during periods of muscle disuse. Immobilized individuals, such as injured athletes, will be severely limited in their training possibilities due to the presence of a cast or brace that rigorously restricts range of motion. Additionally, individuals who are bedridden due to general weakness or illness are likely unable to perform any activities at all. In such situations, an exercise mimetic such as neuromuscular electrical stimulation (NMES) could be applied to induce electrically evoked muscle contractions and, as such, stimulate muscle anabolism [41]. While the technique has been used in physical therapy practice over the past decades, it has recently gained a lot of attention from physiologists due to its influence on skeletal muscle mass during injury or rehabilitation. Previously, we performed a study in our laboratory in which subjects underwent 60 min of high-frequency, high-intensity NMES. We showed that NMES led to $27 \%$ greater muscle protein synthesis rates in the stimulated leg when compared to the non-exercised contralateral control leg [42]. This positive effect of NMES on muscle protein synthesis translates to beneficial effects on muscle mass: we showed a preservation of muscle mass following 5 days of leg immobilization in healthy, young individuals (Chapter 4, [43]). This is in line with others who have demonstrated the efficacy of NMES in preventing muscle loss in various clinical conditions [25, 44-49]. Moreover, we recently showed that NMES has the capacity to fully prevent the dramatic muscle wasting that is observed in critically ill, comatose, Intensive Care Unit (ICU) patients (Chapter 5, [50]). Data obtained in these studies suggest that, in line with mechanistic data [42], the benefits of NMES are, at least partly, attributed to an increase in postabsorptive muscle protein synthesis rates [25]. In support, 
we showed an increase in mTOR phosphorylation following 7 consecutive days of NMES, suggesting a beneficial effect of repeated bouts of NMES on basal muscle protein synthesis rates (Chapter 5, [50]). However, of interest, some data also pointed to an attenuation of muscle protein breakdown. This was indicated by a reversal of skeletal muscle mRNA expression of intermediates in the ubiquitin proteasome pathway, following multiple days with twice-daily NMES (Chapter 4, [43]). The increase in ubiquitin proteasome pathway activity during disuse without NMES is characteristic for the transient rise in muscle protein breakdown, which likely contributes to the observed muscle atrophy during short-term disuse. The parallel occurrence of an increase in muscle protein synthesis and a suppression of muscle protein breakdown in our studies is indicative of a more positive muscle protein balance when NMES is applied on a regular basis. These data underline the effectiveness of NMES in a disuse setting, and the need for it to be applied directly following the onset of muscle disuse.

While NMES is clearly an effective tool for attenuating muscle disuse atrophy, little information is available on how it can be (more) effectively applied. To specify its impact on skeletal muscle tissue, the long-term effect of high-frequency ( $>50 \mathrm{~Hz}$ ) NMES has been investigated in a fibre-type specific manner. Increases in both type I and II muscle fibre size have been observed in free-living, non-immobilized healthy individuals [51-53] and patients $[50,54]$ following multiple sessions of NMES training. More specifically, this effect has been shown to be located mainly in the type Ila fibres $[55,56]$. In situations of muscle disuse, we have shown that NMES leads to relatively greater changes in type II fibre size compared with type I fibre size [43, 50]. Specifically, NMES prevented the $16 \%$ decrease in type I and the $24 \%$ decrease in type II muscle fibre size in comatose patients [50]. Moreover, NMES applied during immobilization even led to specific type II muscle fibre hypertrophy [43]. The specific effect of NMES on type II fibres may be, at least partly, due to their more superficial location than type I fibres, as demonstrated by Sjöström and co-workers [57]. This makes NMES a highly relevant interventional strategy in various situations where individuals suffer from predominant type II muscle fibre atrophy, such as following injury [58], during immobilization [1,59] or critical illness [50], but also with ageing $[60,61]$. Obviously, in our studies we have examined the efficacy of NMES on one muscle group only. An important next step would be to target multiple muscle groups to maximize the relevance of applying NMES in clinical practice. Although the quadriceps femoris is one of the muscle groups most susceptible to atrophy [9], large muscle groups such as the hamstrings, calves, and (lower) back muscles, that also atrophy substantially [9], would likely benefit from treatment with NMES. Applying NMES to multiple muscle groups should be performed under guided supervision, given the importance of correct placement of electrodes and adjustment of the intensity to ensure full contractions throughout the session. Although newly developed products such as whole-body NMES suits have simplified the use of NMES in healthy individuals, further development of such products is essential to make their use feasible for more clinically compromised patients. Despite the demonstrated potential of NMES 
as a training modality during disuse, normal voluntary contractions remain the first choice in clinical practice to alleviate or prevent muscle atrophy. Physical activity such as passive mobilisation or performing exercises in bed or at the bedside need to be applied more frequently in various clinical settings. Strategies to stimulate physical activity are required to change the current mindset in clinical practice, to motivate patients to remain (more) active rather than turning passive and inactive. Since there are many opportunities to implement physical activity or NMES during a period of disuse, it is crucial to define approaches suitable to the various patient groups. It is important not to focus on the effects of exercise (mimetics) on muscle mass only, but also investigate the impact on metabolic health, functional capacity, quality of life, and long-term outcomes such as morbidity and mortality.

\section{Protein or amino acid supplementation}

Muscle protein synthesis can be strongly modulated by the intake of food, and specifically dietary protein [62-64]. Protein or essential amino acid ingestion acutely stimulates muscle protein synthesis rates in a dose-dependent manner $[65,66]$. In healthy, young individuals, a dose of 15-20 g of a high-quality protein (or 7.5-10 g crystalline essential amino acids) is sufficient to maximally stimulate muscle protein synthesis rates $[65,67$, 68]. However, as disuse is accompanied by anabolic resistance to protein intake [26, 3032] a larger bolus is necessary to stimulate postprandial muscle protein synthesis rates during muscle disuse. It has been shown that anabolic resistance in other situations (e.g. normal ageing) can be overcome by increasing the amount [69] or changing the type of protein [70]. Although it remains to be established if changing the amount or type of protein can augment the postprandial muscle protein synthetic response during muscle disuse, it certainly offers a promising strategy to attenuate disuse atrophy.

In healthy individuals, dietary protein intake provides $~ 15$ percent of total energy intake. For an $80 \mathrm{~kg}$ male, consuming $9 \mathrm{MJ}$ per day [71], this would result in a daily habitual protein intake of $\sim 80 \mathrm{~g}$. However, during situations of muscle disuse, total energy and protein intake are reduced due to the decline in energy requirement $[72,73]$ and/or a reduction in appetite $[74,75]$. Individuals will therefore consume less dietary protein, which will compromise muscle mass preservation. As such, it has been proposed that an overall increase in the protein or amino acid content of the diet could alleviate muscle loss during a period of disuse $[19,76,77]$. Despite a relative paucity of data, studies focusing on mimicking hospitalization have indeed shown that supplementation with high doses of crystalline amino acids attenuates the loss of muscle mass during disuse [7, 78, 79]. In contrast, we (Chapter 6, [71]) and others [80] have shown no beneficial effect of protein supplementation during a period of physical inactivity. This apparent discrepancy can potentially be explained by differences in the impact of free amino acids versus intact protein on muscle mass preservation [81]. However it is more likely that differences between studies are attributed to the level of amino acid or 
protein provision applied in those studies. Beneficial effects of protein or amino acid supplementation were only observed in those studies in which the control group consumed protein at a level no higher than the current Recommended Daily Allowance (RDA), i.e. $0.8 \mathrm{~g} \cdot \mathrm{kg}$ body weight ${ }^{-1} \cdot \mathrm{day}^{-1}$ [82]. Consequently, this would mean that populations at risk for undernutrition during muscle disuse, such as institutionalized or hospitalized elderly $[83,84]$, are more likely to benefit from protein supplementation to maintain ample daily protein intake. Dietary protein intake is prerequisite to maintain muscle mass during disuse. However, increasing protein intake above habitual intake levels does not further attenuate muscle mass loss during a period of disuse (Chapter 6, [71]). Additional considerations that should be regarded when optimising protein intake in various groups are, besides determining the optimal quantity of protein, the timing of ingestion of the supplement and the type of protein that is given [85]. Protein supplementation can be timed together with a relatively low-protein meal such as breakfast or lunch [86], to maximize the postprandial muscle protein synthetic response to meal ingestion. Although the threshold for the maximal postprandial stimulation of muscle protein synthesis during disuse remains to be elucidated, it is clear that proper meal distribution can play an important role in optimizing $24 \mathrm{~h}$ muscle protein balance. To date, it remains to be established how much protein is required to maximally stimulate muscle protein synthesis rates during periods of physical inactivity. Additionally, the type of protein that is consumed may also modulate the postprandial muscle protein synthetic response. Ingestion of more rapidly digestible whey protein has been shown to lead to greater increases in postprandial muscle protein synthesis rates when compared with casein ingestion [70]. The greater anabolic properties of whey protein can be, at least partly, attributed to the greater leucine content [70]. Previous work from our group [87] and others [88-90] has shown that increasing the leucine content of a meal can augment postprandial muscle protein synthesis rates. Furthermore, leucine has also been shown to have anti-proteolytic properties [91-93]. Therefore, leucine supplementation may represent an effective strategy to preserve muscle mass during a period of disuse. In support, recent work demonstrates that leucine supplementation can preserve muscle mass during 7 days, but not 14 days, of bed rest [94]. Similar suggestions have been made for supplementation with leucine's metabolite $\beta$-Hydroxy $\beta$ methylbutyric acid (HMB, [8]). Future studies will likely address the impact of free amino acid supplementation to preserve muscle mass loss during short periods of disuse.

Other nutritional compounds have been identified that may contribute to preserve muscle mass during a short period of disuse [76]. Such nutritional compounds that can be applied during muscle disuse or subsequent rehabilitation include creatine $[95,96]$, fish-oil-derived omega 3 fatty acids [97], green tea extract [98], and vitamin D [99, 100]. Another promising nutritional compound, ursolic acid, has been shown to reduce atrophy in mice via an inhibition of atrophy-associated skeletal muscle mRNA expression and a positive effect on insulin signalling $[101,102]$. Whereas it remains to be established if its effects are similar in humans, it could be suggested that ursolic acid can help 
to preserve muscle quality as well as metabolic health during a period of disuse. Similarly, the compound resveratrol has recently gained much attention for its proposed ability to inhibit muscle atrophy under cachectic and atrophic conditions. The effects of resveratrol during muscle disuse in animal models include the downregulation of proinflammatory cytokines and an upregulation of the level of Akt, AMPK and SIRT1 [103, 104]. However, the efficacy of resveratrol supplementation to exert these effects in an in vivo human model remains uninvestigated.

\section{Combined intervention of NMES and protein ingestion}

Neuromuscular electrical stimulation has been discussed previously as an effective method to stimulate muscle protein synthesis rates in an overnight fasted state [42]. However, people spend approximately half of their day under the influence of their last meal, i.e. in the postprandial state. Since disuse-induced anabolic resistance likely plays a key role in the process of muscle atrophy, we were looking for a strategy to optimize the postprandial response to the ingestion of a protein meal. A previous study from our laboratory showed that anabolic resistance to protein intake can be overcome by performing physical activity prior to food ingestion [105]. Therefore, at present, the optimal approach to attenuate muscle disuse atrophy likely lies in the parallel application of NMES and nutritional modulation. Up until recently, no data were available that assessed the combined effect of NMES and dietary protein intake. We recently performed two studies to assess the surplus effects of NMES on the postprandial muscle protein synthetic response to protein ingestion. In contrast to what we hypothesized, we showed that a single bout of NMES performed immediately prior to intake of a meal-like amount of protein did not augment the postprandial muscle protein synthetic response (Chapter 7). In that study, conducted in healthy, active, older participants, NMES did not lead to additional stimulation of de novo muscle protein accretion after ingestion of 20 g casein protein. In a second study, we used NMES to increase the postprandial muscle protein synthetic response prior to sleep. We created conditions simulating hospitalization by subjecting participants to one day of bed rest before the NMES session (Chapter 8). In contrast to the previous study, the use of NMES resulted in $\sim 18 \%$ greater muscle protein synthesis rates when compared to the non-stimulated leg. These results show that although the surplus effect of NMES is not present in active individuals (Chapter 7), NMES can sensitize the muscle of older, physically less active, individuals to the anabolic properties of protein feeding (Chapter 8). Up until now, these combined strategies of protein feeding and NMES have not been extrapolated to a multiple-day model of disuse. However, we speculate that proper timing of NMES, immediately before a meal, will overcome anabolic resistance and therefore attenuate skeletal muscle loss during a period of muscle disuse. Of course this also requires new insights and technological developments to allow translation of these findings into to relevant interventions to support muscle mass preservation during disuse. 


\section{Bed rest as a model for muscle disuse atrophy}

In the scientific literature, muscle disuse in humans is generally studied using a limb immobilization or bed rest model. Although both models are used to investigate underlying mechanisms of muscle loss and to test the efficacy of interventional strategies, this may not be justified due to potential differences between models (i.e. systemic factors or the amount of muscle action possible). Whereas some researchers have tried to extrapolate results of limb immobilization studies to a whole-body disuse setting, this may lead to incorrect results based on different rates of atrophy between muscle groups [9]. Conceptually, it is possible that interventions to preserve muscle mass would need to differ when disuse either occurs locally (via limb immobilization) or on a wholebody level (bed rest). Additionally, different rates of muscle atrophy could be accompanied by a different impact on metabolic health. Although previous work, based on combined results of separate studies, has suggested that immobilization leads to more pronounced muscle loss than bed rest [106], no direct comparison of the rate and amount of muscle loss between leg immobilization and bed rest existed. In Chapter 9, we compared twenty healthy males who participated in either a 7-day leg immobilization study or a 7-day bed rest study. We showed that both leg immobilization and bed rest led to considerable declines in muscle mass $(-5.4 \pm 1.0$ vs $-3.2 \pm 0.9 \%$, respectively) and strength $(-6 \pm 3$ vs $-8 \pm 2 \%$, respectively; Chapter 9$)$. Although the rate of muscle atrophy was comparable between both models, the total muscle loss during bed rest clearly exceeded that during immobilization due to the total amount of inactive tissue. Indeed, we demonstrated that merely one week of bed rest leads to a $1.4 \mathrm{~kg}$ loss of muscle tissue in healthy, young individuals (Chapter 10). This muscle atrophy was accompanied by a severe metabolic decline, such that 7 days of strict bed rest led to a $\sim 30 \%$ decline in insulin sensitivity (Chapter 10). This development of insulin resistance was accompanied by a decline in muscle oxidative capacity, without alterations in skeletal muscle lipid content or saturation level, markers of oxidative stress, or capillary density. Whereas the molecular mechanisms responsible remain to be elucidated, our findings clearly indicate that bed rest-induced insulin resistance is not associated with mechanisms implicated in high-fat diet-induced insulin resistance. This study demonstrates that interventional strategies should not only focus on preventing muscle loss during a period of disuse, but should also aim to attenuate the decline in metabolic health that occurs during disuse in a bed rest model.

In conclusion, both experimental models of disuse lead to comparable rates of muscle atrophy and can therefore both be used to induce muscle loss in an experimental setting. However, the choice for which experimental model to use depends on the research question. When specifically studying mechanisms underlying muscle disuse atrophy, the limb immobilization model is appropriate as it isolates inactivity without the need for altering habitual dietary intake. In contrast, in more clinically relevant situations the bed rest model is of utmost value to mimic a period of hospitalization. In a 
clinical setting, the functional impairment that occurs with limb immobilization can be relatively easily attenuated or even prevented by the strategies described in this thesis. In contrast, the metabolic decline that is associated with bed rest is more problematic given the fact that not only skeletal muscle tissue is affected, but due to inter-organ crosstalk other organs such as liver and adipose tissue are involved.

\section{FUTURE RESEARCH}

Over the past years, the impact of protein supplementation during muscle disuse has hardly been investigated. Not only is it challenging to subject healthy individuals to muscle disuse, the fact that nutritional intake should be thoroughly controlled brings about an extra difficulty. It is crucial that in the near future work will be undertaken to establish the dose-responsiveness of protein intake and the subsequent muscle protein synthetic response to feeding, in order to assess how much protein should be ingested per meal in patients experiencing disuse. When the optimal protein dose to maximally stimulate muscle protein synthesis during disuse has been established in both young and elderly individuals, we should seek for ways to implement this knowledge in clinical practice. This can be done by distributing protein intake such that the required protein intake is achieved in every main meal, as well as prior to sleep. Alternatively, it is possible to accomplish adequate protein consumption via supplementing with protein or amino acids on top of a habitual diet. In parallel, other nutritional compounds such as leucine or HMB should be tested for their suggested effect on muscle protein synthesis and breakdown, and consequently on muscle mass, during short-term disuse.

Neuromuscular electrical stimulation (NMES) is a feasible technique to re-introduce a certain level of physical activity. Whereas we demonstrated the proof of principle in an experimental setting, future studies should aim to apply NMES in a clinical setting by means of targeting multiple muscle groups. Not only its effect on muscle mass, but also the metabolic effects of NMES as well as long-term outcomes such as muscle function, morbidity, and mortality should be examined. Eventually, the combined strategy of NMES and protein feeding should be optimized and tested in a multiple-day model of disuse, in order to overcome anabolic resistance and limit muscle loss.

The focus of this thesis was on the effect of various interventions to prevent or attenuate muscle loss during muscle disuse. Although the results discussed are limited to the effect on muscle mass only, both NMES [51, 107-110] and the discussed nutritional interventions $[6,7,111,112]$ could have an important impact on muscle quality or metabolic health during disuse. Similarly, these interventions are very likely to have potential benefits during the recovery from muscle disuse $[10,96,113,114]$. Therefore, it is important to keep in mind that a strategy that does not alleviate muscle atrophy during disuse might still be able to affect metabolic health or recovery. Finally, as an improve- 
ment in metabolic health could possibly lead to an attenuation of muscle atrophy and vice versa, it may be relevant to test the efficacy of an intervention on both muscle quantity and muscle quality simultaneously.

\section{CONCLUSIONS}

Various situations, such as the recovery from illness or rehabilitation after injury, often necessitate a period of skeletal muscle disuse and lead to muscle loss in otherwise healthy individuals. Even a few days of bed rest or immobilization can lead to substantial declines in muscle mass and strength and are accompanied by a decline in metabolic health. Re-introduction of some level of physical activity by the application of neuromuscular electrical stimulation (NMES) can augment postabsorptive and/or postprandial muscle protein synthesis rates and can prevent muscle loss during short-term disuse in various clinical populations. Maintenance of habitual dietary protein consumption is prerequisite for muscle maintenance, but further increasing protein intake does not prevent muscle loss during disuse. Combining the anabolic properties of physical activity (surrogates) with nutritional support will have synergistic effects and both should, therefore, be applied in interventions aiming to prevent or alleviate muscle atrophy during short periods of disuse. 


\section{REFERENCES}

1. Snijders, T., B.T. Wall, M.L. Dirks, J.M. Senden, F. Hartgens, J. Dolmans, M. Losen, L.B. Verdijk, and L.J. van Loon, Muscle disuse atrophy is not accompanied by changes in skeletal muscle satellite cell content. Clin Sci (Lond), 2014. 126(8): p. 557-66.

2. Gibson, J.N., D. Halliday, W.L. Morrison, P.J. Stoward, G.A. Hornsby, P.W. Watt, G. Murdoch, and M.J. Rennie, Decrease in human quadriceps muscle protein turnover consequent upon leg immobilization. Clin Sci (Lond), 1987. 72(4): p. 503-9.

3. Deitrick, J.E., The effect of immobilization on metabolic and physiological functions of normal men. Bull $N$ Y Acad Med, 1948. 24(6): p. 364-75.

4. Ferrando, A.A., H.W. Lane, C.A. Stuart, J. Davis-Street, and R.R. Wolfe, Prolonged bed rest decreases skeletal muscle and whole body protein synthesis. Am J Physiol, 1996. 270(4 Pt 1): p. E627-33.

5. Biolo, G., B. Ciocchi, M. Lebenstedt, R. Barazzoni, M. Zanetti, P. Platen, M. Heer, and G. Guarnieri, Shortterm bed rest impairs amino acid-induced protein anabolism in humans. J Physiol, 2004. 558(Pt 2): p. 381-8.

6. Ferrando, A.A., D. Paddon-Jones, N.P. Hays, P. Kortebein, O. Ronsen, R.H. Williams, A. McComb, T.B. Symons, R.R. Wolfe, and W. Evans, EAA supplementation to increase nitrogen intake improves muscle function during bed rest in the elderly. Clin Nutr, 2010. 29(1): p. 18-23.

7. Paddon-Jones, D., M. Sheffield-Moore, R.J. Urban, A.P. Sanford, A. Aarsland, R.R. Wolfe, and A.A. Ferrando, Essential amino acid and carbohydrate supplementation ameliorates muscle protein loss in humans during 28 days bedrest. J Clin Endocrinol Metab, 2004. 89(9): p. 4351-8.

8. Deutz, N.E., S.L. Pereira, N.P. Hays, J.S. Oliver, N.K. Edens, C.M. Evans, and R.R. Wolfe, Effect of betahydroxy-beta-methylbutyrate (HMB) on lean body mass during 10 days of bed rest in older adults. Clin Nutr, 2013. 32(5): p. 704-12.

9. LeBlanc, A.D., V.S. Schneider, H.J. Evans, C. Pientok, R. Rowe, and E. Spector, Regional changes in muscle mass following 17 weeks of bed rest. J Appl Physiol, 1992. 73(5): p. 2172-8.

10. Suetta, C., U. Frandsen, A.L. Mackey, L. Jensen, L.G. Hvid, M.L. Bayer, S.J. Petersson, H.D. Schroder, J.L. Andersen, P. Aagaard, P. Schjerling, and M. Kjaer, Ageing is associated with diminished muscle re-growth and myogenic precursor cell expansion early after immobility-induced atrophy in human skeletal muscle. J Physiol, 2013. 591(Pt 15): p. 3789-804.

11. Ferrando, A.A., C.A. Stuart, D.G. Brunder, and G.R. Hillman, Magnetic resonance imaging quantitation of changes in muscle volume during 7 days of strict bed rest. Aviat Space Environ Med, 1995. 66(10): p. $976-81$.

12. Lindboe, C.F. and C.S. Platou, Effect of immobilization of short duration on the muscle fibre size. Clin Physiol, 1984. 4(2): p. 183-8.

13. Wall, B.T., M.L. Dirks, T. Snijders, F.B. Stephens, J.M. Senden, M.L. Verscheijden, and L.J. van Loon, Shortterm muscle disuse atrophy is not associated with increased intramuscular lipid deposition or a decline in the maximal activity of key mitochondrial enzymes in young and older males. Exp Gerontol, 2015. 61: p. 76-83.

14. Tanner, R.E., L.B. Brunker, J. Agergaard, K.M. Barrows, R.A. Briggs, O.S. Kwon, L.M. Young, P.N. Hopkins, E. Volpi, R.L. Marcus, P.C. LaStayo, and M.J. Drummond, Age-related differences in lean mass, protein synthesis and skeletal muscle markers of proteolysis after bed rest and exercise rehabilitation. J Physiol, 2015. 593(18): p. 4259-73.

15. Union, E. Hospital discharges and length of stay statistics. 2015 October 2015 02/17/2016]; Available from: http://ec.europa.eu/eurostat/statistics-explained/index.php/Hospital_discharges_and_length_of_ stay_statistics.

16. Suetta, C., U. Frandsen, L. Jensen, M.M. Jensen, J.G. Jespersen, L.G. Hvid, M. Bayer, S.J. Petersson, H.D. Schroder, J.L. Andersen, K.M. Heinemeier, P. Aagaard, P. Schjerling, and M. Kjaer, Aging affects the transcriptional regulation of human skeletal muscle disuse atrophy. PloS One, 2012. 7(12): p. e51238.

17. Hvid, L.G., C. Suetta, P. Aagaard, M. Kjaer, U. Frandsen, and N. Ortenblad, Four days of muscle disuse impairs single fiber contractile function in young and old healthy men. Exp Gerontol, 2013. 48(2): p. $154-61$. 
18. Wall, B.T., M.L. Dirks, T. Snijders, J.M. Senden, J. Dolmans, and L.J. van Loon, Substantial skeletal muscle loss occurs during only 5 days of disuse. Acta Physiol (Oxf), 2014. 210(3): p. 600-11.

19. English, K.L. and D. Paddon-Jones, Protecting muscle mass and function in older adults during bed rest. Curr Opin Clin Nutr Metab Care, 2010. 13(1): p. 34-9.

20. Wall, B.T., M.L. Dirks, and L.J. van Loon, Skeletal muscle atrophy during short-term disuse: implications for age-related sarcopenia. Ageing Res Rev, 2013. 12(4): p. 898-906.

21. Hvid, L., P. Aagaard, L. Justesen, M.L. Bayer, J.L. Andersen, N. Ortenblad, M. Kjaer, and C. Suetta, Effects of aging on muscle mechanical function and muscle fiber morphology during short-term immobilization and subsequent retraining. J Appl Physiol, 2010. 109(6): p. 1628-34.

22. Pisot, R., U. Marusic, G. Biolo, S. Mazzucco, S. Lazzer, B. Grassi, C. Reggiani, L. Toniolo, P.E. di Prampero, A. Passaro, M. Narici, S. Mohammed, J. Rittweger, M. Gasparini, M. Gabrijelcic Blenkus, and B. Simunic, Greater loss in muscle mass and function but smaller metabolic alterations in older compared with younger men following 2 wk of bed rest and recovery. J Appl Physiol, 2016. 120(8): p. 922-9.

23. Balagopal, P., O.E. Rooyackers, D.B. Adey, P.A. Ades, and K.S. Nair, Effects of aging on in vivo synthesis of skeletal muscle myosin heavy-chain and sarcoplasmic protein in humans. Am J Physiol, 1997. 273(4 Pt 1): p. E790-800.

24. de Boer, M.D., A. Selby, P. Atherton, K. Smith, O.R. Seynnes, C.N. Maganaris, N. Maffulli, T. Movin, M.V. Narici, and M.J. Rennie, The temporal responses of protein synthesis, gene expression and cell signalling in human quadriceps muscle and patellar tendon to disuse. J Physiol, 2007. 585(Pt 1): p. 241-51.

25. Gibson, J.N., K. Smith, and M.J. Rennie, Prevention of disuse muscle atrophy by means of electrical stimulation: maintenance of protein synthesis. Lancet, 1988. 2(8614): p. 767-70.

26. Glover, E.I., S.M. Phillips, B.R. Oates, J.E. Tang, M.A. Tarnopolsky, A. Selby, K. Smith, and M.J. Rennie, Immobilization induces anabolic resistance in human myofibrillar protein synthesis with low and high dose amino acid infusion. J Physiol, 2008. 586(Pt 24): p. 6049-61.

27. Ferrando, A.A., K.D. Tipton, M.M. Bamman, and R.R. Wolfe, Resistance exercise maintains skeletal muscle protein synthesis during bed rest. J Appl Physiol, 1997. 82(3): p. 807-10.

28. Kortebein, P., A. Ferrando, J. Lombeida, R. Wolfe, and W.J. Evans, Effect of 10 days of bed rest on skeletal muscle in healthy older adults. J Am Med Assoc, 2007. 297(16): p. 1772-4.

29. Symons, T.B., M. Sheffield-Moore, D.L. Chinkes, A.A. Ferrando, and D. Paddon-Jones, Artificial gravity maintains skeletal muscle protein synthesis during 21 days of simulated microgravity. J Appl Physiol, 2009. 107(1): p. 34-8.

30. Drummond, M.J., J.M. Dickinson, C.S. Fry, D.K. Walker, D.M. Gundermann, P.T. Reidy, K.L. Timmerman, M.M. Markofski, D. Paddon-Jones, B.B. Rasmussen, and E. Volpi, Bed rest impairs skeletal muscle amino acid transporter expression, mTORC1 signaling, and protein synthesis in response to essential amino acids in older adults. Am j Physiol Endocrinol Metab, 2012. 302(9): p. E1113-22.

31. Wall, B.T., T. Snijders, J.M. Senden, C.L. Ottenbros, A.P. Gijsen, L.B. Verdijk, and L.J. van Loon, Disuse impairs the muscle protein synthetic response to protein ingestion in healthy men. J Clin Endocrinol Metab, 2013. 98(12): p. 4872-81.

32. Wall, B.T., M.L. Dirks, T. Snijders, J.W. van Dijk, M. Fritsch, L.B. Verdijk, and L.J. van Loon, Short-term muscle disuse lowers myofibrillar protein synthesis rates and induces anabolic resistance to protein ingestion. Am J Physiol Endocrinol Metab, 2016. 310(2): p. E137-47.

33. Tesch, P.A., F. von Walden, T. Gustafsson, R.M. Linnehan, and T.A. Trappe, Skeletal muscle proteolysis in response to short-term unloading in humans. J Appl Physiol, 2008. 105(3): p. 902-6.

34. Abadi, A., E.I. Glover, R.J. Isfort, S. Raha, A. Safdar, N. Yasuda, J.J. Kaczor, S. Melov, A. Hubbard, X. Qu, S.M. Phillips, and M. Tarnopolsky, Limb immobilization induces a coordinate down-regulation of mitochondrial and other metabolic pathways in men and women. PLoS One, 2009. 4(8): p. e6518.

35. Urso, M.L., A.G. Scrimgeour, Y.W. Chen, P.D. Thompson, and P.M. Clarkson, Analysis of human skeletal muscle after $48 \mathrm{~h}$ immobilization reveals alterations in mRNA and protein for extracellular matrix components. J Appl Physiol, 2006. 101(4): p. 1136-48. 
36. Reich, K.A., Y.W. Chen, P.D. Thompson, E.P. Hoffman, and P.M. Clarkson, Forty-eight hours of unloading and $24 \mathrm{~h}$ of reloading lead to changes in global gene expression patterns related to ubiquitination and oxidative stress in humans. J Appl Physiol, 2010. 109(5): p. 1404-15.

37. Koopman, R. and L.J. van Loon, Aging, exercise, and muscle protein metabolism. J Appl Physiol, 2009. 106(6): p. 2040-8.

38. Oates, B.R., E.I. Glover, D.W. West, J.L. Fry, M.A. Tarnopolsky, and S.M. Phillips, Low-volume resistance exercise attenuates the decline in strength and muscle mass associated with immobilization. Muscle Nerve, 2010. 42(4): p. 539-46.

39. Brooks, N., G.J. Cloutier, S.M. Cadena, J.E. Layne, C.A. Nelsen, A.M. Freed, R. Roubenoff, and C. Castaneda-Sceppa, Resistance training and timed essential amino acids protect against the loss of muscle mass and strength during 28 days of bed rest and energy deficit. J Appl Physiol, 2008. 105(1): p. 241-8.

40. Alkner, B.A. and P.A. Tesch, Efficacy of a gravity-independent resistance exercise device as a countermeasure to muscle atrophy during 29-day bed rest. Acta Physiol Scand, 2004. 181(3): p. 345-57.

41. Hainaut, K. and J. Duchateau, Neuromuscular electrical stimulation and voluntary exercise. Sports Med, 1992. 14(2): p. 100-13.

42. Wall, B.T., M.L. Dirks, L.B. Verdijk, T. Snijders, D. Hansen, P. Vranckx, N.A. Burd, P. Dendale, and L.J. van Loon, Neuromuscular electrical stimulation increases muscle protein synthesis in elderly type 2 diabetic men. Am J Physiol Endocrinol Metab, 2012. 303(5): p. E614-23.

43. Dirks, M.L., B.T. Wall, T. Snijders, C.L. Ottenbros, L.B. Verdijk, and L.J. van Loon, Neuromuscular electrical stimulation prevents muscle disuse atrophy during leg immobilization in humans. Acta Physiol (Oxf), 2014. 210(3): p. 628-41.

44. Vinge, O., L. Edvardsen, F. Jensen, F.G. Jensen, J. Wernerman, and H. Kehlet, Effect of transcutaneous electrical muscle stimulation on postoperative muscle mass and protein synthesis. Br J Surg, 1996. 83(3): p. 360-3.

45. Vivodtzev, I., J.L. Pepin, G. Vottero, V. Mayer, B. Porsin, P. Levy, and B. Wuyam, Improvement in quadriceps strength and dyspnea in daily tasks after 1 month of electrical stimulation in severely deconditioned and malnourished COPD. Chest, 2006. 129(6): p. 1540-8.

46. Gibson, J.N., W.L. Morrison, C.M. Scrimgeour, K. Smith, P.J. Stoward, and M.J. Rennie, Effects of therapeutic percutaneous electrical stimulation of atrophic human quadriceps on muscle composition, protein synthesis and contractile properties. Eur J Clin Invest, 1989. 19(2): p. 206-12.

47. Gerovasili, V., K. Stefanidis, K. Vitzilaios, E. Karatzanos, P. Politis, A. Koroneos, A. Chatzimichail, C. Routsi, C. Roussos, and S. Nanas, Electrical muscle stimulation preserves the muscle mass of critically ill patients: a randomized study. Crit Care, 2009. 13(5): p. R161.

48. Meesen, R.L.J., P. Dendale, K. Cuypers, J. Berger, A. Hermans, H. Thijs, and O. Levin, Neuromuscular Electrical Stimulation as a Possible Means to Prevent Muscle Tissue Wasting in Artificially Ventilated and Sedated Patients in the Intensive Care Unit: A Pilot Study. Neuromodulation, 2010. 13: p. 315-321.

49. Quittan, M., A. Sochor, G.F. Wiesinger, J. Kollmitzer, B. Sturm, R. Pacher, and W. Mayr, Strength improvement of knee extensor muscles in patients with chronic heart failure by neuromuscular electrical stimulation. Artif Organs, 1999. 23(5): p. 432-5.

50. Dirks, M.L., D. Hansen, A. Van Assche, P. Dendale, and L.J. Van Loon, Neuromuscular electrical stimulation prevents muscle wasting in critically ill comatose patients. Clin Sci (Lond), 2015. 128(6): p. 357-65.

51. Gondin, J., L. Brocca, E. Bellinzona, G. D'Antona, N.A. Maffiuletti, D. Miotti, M.A. Pellegrino, and R. Bottinelli, Neuromuscular electrical stimulation training induces atypical adaptations of the human skeletal muscle phenotype: a functional and proteomic analysis. J Appl Physiol, 2011. 110(2): p. 433-50.

52. Sinacore, D.R., A. Delitto, D.S. King, and S.J. Rose, Type II fiber activation with electrical stimulation: a preliminary report. Phys Ther, 1990. 70(7): p. 416-22.

53. Maffiuletti, N.A., R. Zory, D. Miotti, M.A. Pellegrino, M. Jubeau, and R. Bottinelli, Neuromuscular adaptations to electrostimulation resistance training. Am J Phys Med Rehab, 2006. 85(2): p. 167-75.

54. Dal Corso, S., L. Napolis, C. Malaguti, A.C. Gimenes, A. Albuquerque, C.R. Nogueira, M.B. De Fuccio, R.D. Pereira, A. Bulle, N. McFarlane, L.E. Nery, and J.A. Neder, Skeletal muscle structure and function in re- 
sponse to electrical stimulation in moderately impaired COPD patients. Respir Med, 2007. 101(6): p. 1236-43.

55. Sillen, M.J., F.M. Franssen, H.R. Gosker, E.F. Wouters, and M.A. Spruit, Metabolic and structural changes in lower-limb skeletal muscle following neuromuscular electrical stimulation: a systematic review. PLoS One, 2013. 8(9): p. e69391.

56. Perez, M., A. Lucia, J.L. Rivero, A.L. Serrano, J.A. Calbet, M.A. Delgado, and J.L. Chicharro, Effects of transcutaneous short-term electrical stimulation on $\mathrm{M}$. vastus lateralis characteristics of healthy young men. Pflug Arch Eur J Phy, 2002. 443(5-6): p. 866-74.

57. Sjostrom, M., D.Y. Downham, and J. Lexell, Distribution of different fiber types in human skeletal muscles: why is there a difference within a fascicle? Muscle Nerve, 1986. 9(1): p. 30-6.

58. Wall, B.T., J.P. Morton, and L.J. van Loon, Strategies to maintain skeletal muscle mass in the injured athlete: nutritional considerations and exercise mimetics. Eur J Sport Sci, 2015. 15(1): p. 53-62.

59. Hvid, L.G., N. Ortenblad, P. Aagaard, M. Kjaer, and C. Suetta, Effects of ageing on single muscle fibre contractile function following short-term immobilisation. J Physiol, 2011. 589(Pt 19): p. 4745-57.

60. Lexell, J., C.C. Taylor, and M. Sjostrom, What is the cause of the ageing atrophy? Total number, size and proportion of different fiber types studied in whole vastus lateralis muscle from 15- to 83-year-old men. J Neurol Sci, 1988. 84(2-3): p. 275-94.

61. Larsson, L., B. Sjodin, and J. Karlsson, Histochemical and biochemical changes in human skeletal muscle with age in sedentary males, age 22--65 years. Acta Physiol Scand, 1978. 103(1): p. 31-9.

62. Volpi, E., M. Sheffield-Moore, B.B. Rasmussen, and R.R. Wolfe, Basal muscle amino acid kinetics and protein synthesis in healthy young and older men. J Am Med Assoc, 2001. 286(10): p. 1206-12.

63. Banos, G., P.M. Daniel, S.R. Moorhouse, and O.E. Pratt, The movement of amino acids between blood and skeletal muscle in the rat. J Physiol, 1973. 235(2): p. 459-75.

64. Silk, D.B., G.K. Grimble, and R.G. Rees, Protein digestion and amino acid and peptide absorption. Proc Nutr Soc, 1985. 44(1): p. 63-72.

65. Bohe, J., A. Low, R.R. Wolfe, and M.J. Rennie, Human muscle protein synthesis is modulated by extracellular, not intramuscular amino acid availability: a dose-response study. J Physiol, 2003. 552(Pt 1): p. 315-24.

66. Bohe, J., J.F. Low, R.R. Wolfe, and M.J. Rennie, Latency and duration of stimulation of human muscle protein synthesis during continuous infusion of amino acids. J Physiol, 2001. 532(Pt 2): p. 575-9.

67. Cuthbertson, D., K. Smith, J. Babraj, G. Leese, T. Waddell, P. Atherton, H. Wackerhage, P.M. Taylor, and M.J. Rennie, Anabolic signaling deficits underlie amino acid resistance of wasting, aging muscle. FASEB J, 2005. 19(3): p. 422-4.

68. Breen, L. and S.M. Phillips, Skeletal muscle protein metabolism in the elderly: Interventions to counteract the 'anabolic resistance' of ageing. Nutr Metab (Lond), 2011. 8: p. 68.

69. Pennings, B., B. Groen, A. de Lange, A.P. Gijsen, A.H. Zorenc, J.M. Senden, and L.J. van Loon, Amino acid absorption and subsequent muscle protein accretion following graded intakes of whey protein in elderly men. Am J Physiol Endocrinol Metab, 2012. 302(8): p. E992-9.

70. Pennings, B., Y. Boirie, J.M.G. Senden, A.P. Gijsen, H. Kuipers, and L.J.C. van Loon, Whey protein stimulates postprandial muscle protein accretion more effectively than do casein and casein hydrolysate in older men. Am J Clin Nutr, 2011. 93(5): p. 997-1005.

71. Dirks, M.L., B.T. Wall, R. Nilwik, D.H. Weerts, L.B. Verdijk, and L.J. van Loon, Skeletal muscle disuse atrophy is not attenuated by dietary protein supplementation in healthy older men. J Nutr, 2014. 144(8): p. 1196-203.

72. Blanc, S., S. Normand, P. Ritz, C. Pachiaudi, L. Vico, C. Gharib, and G. Gauquelin-Koch, Energy and water metabolism, body composition, and hormonal changes induced by 42 days of enforced inactivity and simulated weightlessness. J Clin Endocrinol Metab, 1998. 83(12): p. 4289-97.

73. Ritz, P., A. Maillet, S. Blanc, and R.J. Stubbs, Observations in energy and macronutrient intake during prolonged bed-rest in a head-down tilt position. Clin Nutr, 1999. 18(4): p. 203-7.

74. Harris, C.L. and C. Fraser, Malnutrition in the institutionalized elderly: the effects on wound healing. Ostomy Wound Manage, 2004. 50(10): p. 54-63. 
75. Nematy, M., J.E. O'Flynn, L. Wandrag, A.E. Brynes, S.J. Brett, M. Patterson, M.A. Ghatei, S.R. Bloom, and G.S. Frost, Changes in appetite related gut hormones in intensive care unit patients: a pilot cohort study. Crit Care, 2006. 10(1): p. R10.

76. Wall, B.T. and L.J. van Loon, Nutritional strategies to attenuate muscle disuse atrophy. Nutr Rev, 2013. 71(4): p. 195-208.

77. Paddon-Jones, D., R.R. Wolfe, and A.A. Ferrando, Amino acid supplementation for reversing bed rest and steroid myopathies. J Nutr, 2005. 135(7): p. 1809S-1812S.

78. Stein, T.P., M.R. Donaldson, M.J. Leskiw, M.D. Schluter, D.W. Baggett, and G. Boden, Branched-chain amino acid supplementation during bed rest: effect on recovery. J Appl Physiol, 2003. 94(4): p. 1345-52.

79. Stuart, C.A., R.E. Shangraw, E.J. Peters, and R.R. Wolfe, Effect of dietary protein on bed-rest-related changes in whole-body-protein synthesis. Am J Clin Nutr, 1990. 52(3): p. 509-14.

80. Trappe, T.A., N.A. Burd, E.S. Louis, G.A. Lee, and S.W. Trappe, Influence of concurrent exercise or nutrition countermeasures on thigh and calf muscle size and function during 60 days of bed rest in women. Acta Physiol (Oxf), 2007. 191(2): p. 147-59.

81. Dangin, M., Y. Boirie, C. Garcia-Rodenas, P. Gachon, J. Fauquant, P. Callier, O. Ballevre, and B. Beaufrere, The digestion rate of protein is an independent regulating factor of postprandial protein retention. Am J Physiol Endocrinol Metab, 2001. 280(2): p. E340-8.

82. Stein, T.P. and S. Blanc, Does protein supplementation prevent muscle disuse atrophy and loss of strength? Crit Rev Food Sci, 2011. 51(9): p. 828-34.

83. Sorensen, J., J. Kondrup, J. Prokopowicz, M. Schiesser, L. Krahenbuhl, R. Meier, M. Liberda, and O.s.g. Euro, EuroOOPS: an international, multicentre study to implement nutritional risk screening and evaluate clinical outcome. Clin Nutr, 2008. 27(3): p. 340-9.

84. Kruizenga, H.M., N.J. Wierdsma, M.A. van Bokhorst, S. de van der, H.J. Haollander, C.F. JonkersSchuitema, E. van der Heijden, G.C. Melis, and W.A. van Staveren, Screening of nutritional status in The Netherlands. Clin Nutr, 2003. 22(2): p. 147-52.

85. Wall, B.T., N.M. Cermak, and L.J. van Loon, Dietary protein considerations to support active aging. Sports Med, 2014. 44 Suppl 2: p. S185-94.

86. Tieland, M., K.J. Borgonjen-Van den Berg, L.J. van Loon, and L.C. de Groot, Dietary protein intake in community-dwelling, frail, and institutionalized elderly people: scope for improvement. Eur J Nutr, 2012. 51(2): p. 173-9.

87. Wall, B.T., H.M. Hamer, A. de Lange, A. Kiskini, B.B. Groen, J.M. Senden, A.P. Gijsen, L.B. Verdijk, and L.J. van Loon, Leucine co-ingestion improves post-prandial muscle protein accretion in elderly men. Clin Nutr, 2013. 32(3): p. 412-9.

88. Katsanos, C.S., H. Kobayashi, M. Sheffield-Moore, A. Aarsland, and R.R. Wolfe, A high proportion of leucine is required for optimal stimulation of the rate of muscle protein synthesis by essential amino acids in the elderly. Am J Physiol Endocrinol Metab, 2006. 291(2): p. E381-7.

89. Rieu, I., M. Balage, C. Sornet, C. Giraudet, E. Pujos, J. Grizard, L. Mosoni, and D. Dardevet, Leucine supplementation improves muscle protein synthesis in elderly men independently of hyperaminoacidaemia. J Physiol, 2006. 575(Pt 1): p. 305-15.

90. Casperson, S.L., M. Sheffield-Moore, S.J. Hewlings, and D. Paddon-Jones, Leucine supplementation chronically improves muscle protein synthesis in older adults consuming the RDA for protein. Clin Nutr, 2012. 31(4): p. 512-9.

91. Leenders, M. and L.J. van Loon, Leucine as a pharmaconutrient to prevent and treat sarcopenia and type 2 diabetes. Nutr Rev, 2011. 69(11): p. 675-89.

92. Nair, K.S., R.G. Schwartz, and S. Welle, Leucine as a regulator of whole body and skeletal muscle protein metabolism in humans. Am J Physiol, 1992. 263(5 Pt 1): p. E928-34.

93. Dodd, K.M. and A.R. Tee, Leucine and mTORC1: a complex relationship. Am J Physiol Endocrinol Metab, 2012. 302(11): p. E1329-42.

94. English, K.L., J.A. Mettler, J.B. Ellison, M.M. Mamerow, E. Arentson-Lantz, J.M. Pattarini, R. Ploutz-Snyder, M. Sheffield-Moore, and D. Paddon-Jones, Leucine partially protects muscle mass and function during bed rest in middle-aged adults. Am J Clin Nutr, 2016. 103(2): p. 465-73. 
95. Johnston, A.P., D.G. Burke, L.G. MacNeil, and D.G. Candow, Effect of creatine supplementation during castinduced immobilization on the preservation of muscle mass, strength, and endurance. J Strength Cond Res, 2009. 23(1): p. 116-20.

96. Hespel, P., B. Op't Eijnde, M. Van Leemputte, B. Urso, P.L. Greenhaff, V. Labarque, S. Dymarkowski, P. Van Hecke, and E.A. Richter, Oral creatine supplementation facilitates the rehabilitation of disuse atrophy and alters the expression of muscle myogenic factors in humans. J Physiol, 2001. 536(Pt 2): p. 625-33.

97. Smith, G.I., S. Julliand, D.N. Reeds, D.R. Sinacore, S. Klein, and B. Mittendorfer, Fish oil-derived n-3 PUFA therapy increases muscle mass and function in healthy older adults. Am J Clin Nutr, 2015.

98. Alway, S.E., B.T. Bennett, J.C. Wilson, J. Sperringer, J.S. Mohamed, N.K. Edens, and S.L. Pereira, Green tea extract attenuates muscle loss and improves muscle function during disuse, but fails to improve muscle recovery following unloading in aged rats. J Appl Physiol, 2015. 118(3): p. 319-30.

99. Ceglia, L., Vitamin D and skeletal muscle tissue and function. Mol Aspects Med, 2008. 29(6): p. 407-14.

100.Bischoff-Ferrari, H.A., T. Dietrich, E.J. Orav, F.B. Hu, Y. Zhang, E.W. Karlson, and B. Dawson-Hughes, Higher 25-hydroxyvitamin $D$ concentrations are associated with better lower-extremity function in both active and inactive persons aged $>$ or $=60$ y. Am J Clin Nutr, 2004. 80(3): p. 752-8.

101.Kunkel, S.D., M. Suneja, S.M. Ebert, K.S. Bongers, D.K. Fox, S.E. Malmberg, F. Alipour, R.K. Shields, and C.M. Adams, mRNA expression signatures of human skeletal muscle atrophy identify a natural compound that increases muscle mass. Cell Metab, 2011. 13(6): p. 627-38.

102.Adams, C.M., S.M. Ebert, and M.C. Dyle, Use of mRNA expression signatures to discover small molecule inhibitors of skeletal muscle atrophy. Curr Opin Clin Nutr Metab Care, 2015. 18(3): p. 263-8.

103. Momken, I., L. Stevens, A. Bergouignan, D. Desplanches, F. Rudwill, I. Chery, A. Zahariev, S. Zahn, T.P. Stein, J.L. Sebedio, E. Pujos-Guillot, M. Falempin, C. Simon, V. Coxam, T. Andrianjafiniony, G. GauquelinKoch, F. Picquet, and S. Blanc, Resveratrol prevents the wasting disorders of mechanical unloading by acting as a physical exercise mimetic in the rat. FASEB J, 2011. 25(10): p. 3646-60.

104.Alamdari, N., Z. Aversa, E. Castillero, A. Gurav, V. Petkova, S. Tizio, and P.O. Hasselgren, Resveratrol prevents dexamethasone-induced expression of the muscle atrophy-related ubiquitin ligases atrogin-1 and MuRF1 in cultured myotubes through a SIRT1-dependent mechanism. Biochem Biophys Res Commun, 2012. 417(1): p. 528-33.

105.Pennings, B., R. Koopman, M. Beelen, J.M.G. Senden, W.H.M. Saris, and L.J.C. van Loon, Exercising before protein intake allows for greater use of dietary protein-derived amino acids for de novo muscle protein synthesis in both young and elderly men. Am J Clin Nutr, 2011. 93(2): p. 322-331.

106.Clark, B.C., In vivo alterations in skeletal muscle form and function after disuse atrophy. Med Sci Sports Exerc, 2009. 41(10): p. 1869-75.

107. Bourjeily-Habr, G., C.L. Rochester, F. Palermo, P. Snyder, and V. Mohsenin, Randomised controlled trial of transcutaneous electrical muscle stimulation of the lower extremities in patients with chronic obstructive pulmonary disease. Thorax, 2002. 57(12): p. 1045-9.

108.Gerovasili, V., E. Tripodaki, E. Karatzanos, T. Pitsolis, V. Markaki, D. Zervakis, C. Routsi, C. Roussos, and S. Nanas, Short-term systemic effect of electrical muscle stimulation in critically ill patients. Chest, 2009. 136(5): p. 1249-56.

109.Rodriguez, P.O., M. Setten, L.P. Maskin, I. Bonelli, S.R. Vidomlansky, S. Attie, S.L. Frosiani, S. Kozima, and R. Valentini, Muscle weakness in septic patients requiring mechanical ventilation: protective effect of transcutaneous neuromuscular electrical stimulation. J Crit Care, 2012. 27(3): p. 319 e1-8.

110.Theriault, R., G. Theriault, and J.A. Simoneau, Human skeletal muscle adaptation in response to chronic low-frequency electrical stimulation. J Appl Physiol, 1994. 77(4): p. 1885-9.

111.Fitts, R.H., J.G. Romatowski, J.R. Peters, D. Paddon-Jones, R.R. Wolfe, and A.A. Ferrando, The deleterious effects of bed rest on human skeletal muscle fibers are exacerbated by hypercortisolemia and ameliorated by dietary supplementation. Am J Physiol Cell Physiol, 2007. 293(1): p. C313-20.

112.Op 't Eijnde, B., B. Urso, E.A. Richter, P.L. Greenhaff, and P. Hespel, Effect of oral creatine supplementation on human muscle GLUT4 protein content after immobilization. Diabetes, 2001. 50(1): p. 18-23. 
113.Savary-Auzeloux, I., H. Magne, C. Migne, M. Oberli, D. Breuille, M. Faure, K. Vidal, M. Perrot, D. Remond, L. Combaret, and D. Dardevet, A dietary supplementation with leucine and antioxidants is capable to accelerate muscle mass recovery after immobilization in adult rats. PLoS One, 2013. 8(11): p. e81495.

114. Milsom, J., P. Barreira, D.J. Burgess, Z. Iqbal, and J.P. Morton, Case study: Muscle atrophy and hypertrophy in a premier league soccer player during rehabilitation from ACL injury. Int J Sport Nutr Exerc Metab, 2014. 24(5): p. 543-52. 
Summary

Samenvatting 


\section{SUMMARY}

Various situations, such as the recovery from illness or rehabilitation after injury, may necessitate a period of physical inactivity in otherwise healthy individuals. This muscle disuse during a period of leg immobilization or bed rest leads to the loss of muscle mass and strength and can have numerous resultant negative health consequences. We need insight in the mechanism(s) responsible for disuse-related muscle atrophy to develop effective interventional strategies to preserve muscle mass and offset the negative health consequences of muscle disuse. In this thesis we examine the various aspects of skeletal muscle disuse atrophy and investigate the efficacy of various interventional strategies to combat disuse atrophy.

The effects of prolonged (two weeks or longer) muscle disuse have been extensively studied in the past. However, given that the average hospital length of stay is currently seven days, it is of importance to focus on the effects of short-term disuse. Therefore, we first assessed the impact of short (five days) versus long (fourteen days) term impact of leg immobilization on muscle mass and muscle strength in healthy, young volunteers (Chapter 2). As expected, we observed a substantial decline in muscle mass and strength following fourteen days of leg immobilization. However, even a mere five days of leg immobilization resulted in a 3.5\% decrease in muscle mass and a $9 \%$ decline in muscle strength. This underlines the impact of short periods of muscle disuse, and emphasizes the need to prevent or attenuate muscle loss during short periods of muscle disuse. The loss of muscle mass is caused by a decline in muscle protein synthesis, a decline in muscle protein breakdown, or a combination of both. Therefore, we studied the impact of 5 days of leg immobilization on muscle protein synthesis in a fasted state and following ingestion of a protein-rich drink in Chapter 3. In that chapter we showed that muscle protein synthesis rates in the immobilized leg were considerably lower in both the postabsorptive as well as the postprandial state when compared with the nonimmobilized control leg. This work shows that merely five days of disuse lowers fasting muscle protein synthesis and decreases the sensitivity of the muscle to dietary protein intake, a phenomenon called 'anabolic resistance'. Therefore, strategies to prevent or attenuate muscle loss during disuse should focus on stimulating muscle protein synthesis, as well as overcoming anabolic resistance.

Physical activity is one of the main anabolic stimuli required to maintain skeletal muscle mass. However, during muscle disuse it is often impossible to perform physical activity due to weakness or restriction of movement imposed by a cast. In Chapter 4 and 5 , we applied neuromuscular electrical stimulation (NMES) to maintain some level of physical activity during short-term muscle disuse. We demonstrate that in healthy, young adults, NMES is effective to prevent muscle loss during five days of one-legged immobilization (Chapter 4). In a follow-up study, we showed that NMES applied in critically ill, comatose patients in the Intensive Care Unit (ICU) fully prevented the large 
amount of muscle that is typically lost in these patients (Chapter 5). These two studies emphasize the importance of some level of muscle contraction to preserve muscle mass during a period of muscle disuse.

We started this thesis by describing the impact of short-term disuse on muscle mass and muscle protein synthesis. Such short periods are of particular relevance to the ageing society, as the accumulation of successive periods of disuse is thought to contribute to the muscle loss observed with ageing, termed sarcopenia. In Chapter 6, we assessed the impact of protein supplementation on muscle mass and strength during five days of immobilization in healthy, elderly volunteers. We show that twice-daily intake of a protein supplement does not prevent the loss of muscle mass and strength. Based on this we concluded that protein supplementation is not of additional value when an individual's habitual dietary protein intake is maintained at a supraoptimal level. To overcome the disuse-induced anabolic resistance we applied NMES as a strategy to increase the postprandial muscle protein synthetic response to protein ingestion in the older population. We applied the combined approach of using NMES together with protein ingestion in Chapter 7 and 8. By using intrinsically labelled protein we were able to analyse how much of the ingested protein was eventually built into contractile muscle proteins. In Chapter 7 we show that NMES does not augment muscle protein accretion in healthy, recreationally active men. In Chapter 8 , a comparable group of healthy elderly remained bed-rested for a full day at the University. In the evening we applied NMES after which we provided participants with a protein drink. From this study we concluded that, in contrast to what we showed in Chapter 7, NMES represents an effective strategy to stimulate muscle protein accretion following protein ingestion in a setting of reduced physical activity.

Muscle disuse can be localized to a limb (e.g. when an arm or leg is immobilized due to a bone fracture) or to the whole body (e.g. when a patient undergoes bed rest during illness or following surgery). Until now, both models are used interchangeably when studying muscle disuse, without knowing the extent of muscle atrophy that they cause. Therefore, we directly compared the loss of muscle mass and strength during seven days of leg immobilization versus seven days of bed rest in Chapter 9. We demonstrate that both models of muscle disuse lead to substantial declines in muscle mass and strength. Although no differences in leg muscle loss were observed, total muscle loss during bed rest exceeded that during leg immobilization only due to the total amount of muscle tissue involved in the disuse. The considerable amount of muscle loss following bed rest inevitably has an impact on muscle function and metabolic health. To investigate this, we subjected ten healthy, young males to seven days of strict bed rest (Chapter 10). We showed substantial declines in muscle mass, muscle function, and metabolic health. Effective strategies should therefore not only focus on maintaining muscle mass and function during short periods of bed rest, but should also aim to preserve metabolic health. 
The final chapter of this thesis (Chapter 11) encompasses a broad discussion on the implications of the work described. This thesis demonstrates that effective interventional strategies are warranted to prevent or attenuate the loss of muscle mass, strength, and metabolic health during short-term muscle disuse. Promising strategies include NMES plus pre-sleep protein feeding. Given the considerable muscle loss during merely five to seven days of muscle disuse, it is of paramount importance to apply effective intervention strategies before, during, and after a period of muscle disuse. 


\section{SAMENVATTING}

Korte perioden van fysieke inactiviteit zijn vaak nodig voor het herstel tijdens ziekte of na een blessure, en vinden bijvoorbeeld plaats wanneer een patiënt bedrust dient te houden of een been in het gips gezet wordt. Zulke perioden van inactiviteit leiden tot het verlies van spiermassa (ook wel spieratrofie genoemd), een afname in spierkracht en vele andere negatieve gevolgen. Gezien de negatieve gevolgen van fysieke inactiviteit, is het van belang om te begrijpen waarom het verlies van spiermassa en -kracht plaatsvindt. Op deze manier is het mogelijk om interventiestrategieën te kunnen ontwikkelen die effectief zijn in het afremmen of voorkomen van spieratrofie.

De effecten van langdurige fysieke inactiviteit (langer dan twee weken) zijn in het verleden uitgebreid in kaart gebracht. Het is echter ook van belang om te kijken naar kortere perioden van fysieke inactiviteit, omdat de gemiddelde ziekenhuisopname voor patiënten tegenwoordig slechts zeven dagen duurt. Daarom hebben we in Hoofdstuk 2 gekeken naar de impact van kortdurende versus langdurende fysieke inactiviteit op spiermassa en -kracht. Hierbij hebben we gezonde, jonge mannen met een been in het gips gezet gedurende vijf of veertien dagen. Zoals verwacht zagen we dat er na veertien dagen immobilisatie een sterke daling in spiermassa en -kracht te zien was. Echter, zelfs na vijf dagen immobilisatie was er al een afname van 3,5\% in spiermassa en van $9 \%$ in spierkracht zichtbaar. Dit illustreert de invloed van slechts enkele dagen fysieke inactiviteit, en toont bovendien het belang om te begrijpen waarom deze spieratrofie optreedt. Verlies van spiermassa wordt veroorzaakt door een vermindering van de spiereiwitopbouw, een verhoging van de spiereiwitafbraak, of een combinatie van beide factoren. Daarom hebben we in Hoofdstuk 3 onderzocht wat het effect van vijf dagen immobilisatie van één been op de spiereiwitopbouw is, in zowel nuchtere toestand als ook na inname van een eiwitrijke drank. In dat hoofdstuk hebben we laten zien dat in zowel de nuchtere als de gevoede toestand de snelheid van de spiereiwitopbouw sterk verlaagd was, wanneer we dit vergeleken met de waarden in het niet-geïmmobiliseerde controlebeen. Fysieke inactiviteit leidt dus niet alleen tot een verlaging van de spiereiwitopbouw in nuchtere toestand maar zorgt ook voor een verminderde gevoeligheid van de spier voor de anabole werking van eiwit ingenomen via de voeding. Dit fenomeen wordt 'anabole resistentie' genoemd. Het is daarom nodig om ons bij het ontwikkelen van interventiestrategieën ter vermindering van atrofie zowel te richten op het stimuleren van de spiereiwitopbouw, als het tegengaan van anabole resistentie.

Een van de belangrijkste stimuli voor de opbouw van spieren is fysieke activiteit. Het is echter tijdens een periode van fysieke inactiviteit vaak niet mogelijk om te trainen of oefeningen te doen, omdat patiënten zich zwak voelen of omdat hun bewegingen worden beperkt door een gipskoker. In Hoofdstuk 4 en 5 hebben we daarom neuromusculaire electrostimulatie (NMES) toegepast om een beperkte mate van spieractiviteit te behouden tijdens een periode van inactiviteit. We hebben laten zien dat NMES effectief 
is in het volledig voorkomen van spieratrofie tijdens vijf dagen immobilisatie in gezonde, jonge mannen (Hoofdstuk 4). Daarnaast hebben we NMES toegepast in ernstig zieke, comateuze patiënten op de Intensive Care. Niet alleen hebben we laten zien dat deze patiënten ongeveer vijf keer zo snel spiermassa verliezen als gezonde personen tijdens inactiviteit, maar ook dat NMES in staat is om deze atrofie volledig tegen te gaan (Hoofdstuk 5). Deze twee studies laten zien dat spiercontracties essentieel zijn om spierverlies tijdens kortdurende fysieke inactiviteit te verminderen.

We zijn dit proefschrift begonnen met het beschrijven van de impact van kortdurende fysieke inactiviteit op spiermassa, -kracht, en -eiwitopbouw. Zulke perioden zijn voornamelijk van belang voor ouderen omdat gedacht wordt dat het herhaaldelijk voorkomen van zulke perioden bijdraagt aan sarcopenie, het spierverlies dat we zien met veroudering. Naast fysieke activiteit is voeding, en vooral de hoeveelheid eiwit in de voeding, een belangrijke stimulus voor spieropbouw. Om die reden hebben we in Hoofdstuk 6 gekeken of we met eiwitsuppletie het verlies van spiermassa en spierkracht kunnen afremmen bij gezonde ouderen die gedurende vijf dagen geïmmobiliseerd werden. We tonen aan dat het tweemaal daags innemen van een eiwitsupplement de spieratrofie en het verlies van spierkracht niet kan voorkomen. Op basis hiervan nemen we aan dat eiwitsuppletie geen meerwaarde heeft wanneer de habituele eiwitinname voldoende is. Hierna zijn we gaan kijken hoe we de spiereiwitopbouw na eiwitinname verder kunnen stimuleren bij oudere mensen. We hebben hiervoor NMES toegepast, omdat een eerdere studie uit onze onderzoeksgroep heeft laten zien dat NMES de spiereiwitopbouw kan stimuleren. De gecombineerde aanpak van het uitvoeren van NMES en de inname van eiwit hebben we toegepast in Hoofdstuk 7 en 8 . Hierbij hebben we NMES toegepast op een van beide benen, terwijl het andere been als controlebeen gold. Door het gebruik van intrinsiek gelabeld eiwit waren we in staat om te analyseren hoeveel van het ingenomen eiwit uiteindelijk in contractiele spiereiwitten in de spier was ingebouwd. Uit Hoofdstuk 7 blijkt dat het toepassen van NMES voorafgaand aan inname van een eiwitdrank niet zorgt voor een meetbare toename in spiereiwitopbouw in actieve, gezonde ouderen. Parallel hieraan heeft in Hoofdstuk 8 een vergelijkbare groep gezonde ouderen een hele dag bedrust gehouden, waarna we 's avonds voor het slapen NMES hebben toegepast en een eiwitdrank hebben gegeven. De conclusie van dit onderzoek was dat, in tegenstelling tot de resultaten in Hoofdstuk 7, NMES wél effectief is in het stimuleren van de spiereiwitopbouw na inname van een eiwitdrank in een situatie van verminderde fysieke activiteit.

Fysieke inactiviteit kan lokaal geïnduceerd worden (bijvoorbeeld door een gipskoker om een arm of been na een botbreuk) of op heel-lichaamsniveau plaatsvinden (zoals wanneer een patiënt bedrust dient te houden tijdens ziekte of na een operatie). Tot op heden worden beide modellen naast elkaar gebruikt voor het bestuderen van fysieke inactiviteit, zonder daarbij te weten wat de snelheid van spieratrofie is in beide situaties. Om die reden hebben we in Hoofdstuk 9 een directe vergelijking gemaakt van de snelheid van zowel spierverlies als spierkrachtafname tussen zeven dagen beenimmobi- 
lisatie en zeven dagen bedrust. Uit deze vergelijking blijkt dat er geen verschil was in de snelheid van atrofie, maar dat bedrust wel leidt tot een groter totaal spiermassaverlies vanwege de grotere hoeveelheid spierweefsel die verminderd actief is. Het is onvermijdelijk dat spierverlies een substantiële invloed heeft op het functioneren van de spier en de hormoonhuishouding. Om dit te onderzoeken hebben in Hoofdstuk 10 tien gezonde, jonge mannen gedurende zeven dagen volledige bedrust gehouden. Dit resulteerde in een substantiële afname in spiermassa, spierfunctie, en metabole gezondheid. Deze resultaten laten zien dat interventiestrategieën tijdens fysieke inactiviteit zich niet alleen moeten richten op het voorkomen van spieratrofie, maar ook op het behouden van metabole gezondheid.

Dit proefschrift sluit af met een discussie van de onderzoeksresultaten (Hoofdstuk 11) en de implicaties hiervan. De bevindingen van dit proefschrift demonstreren dat we effectieve interventies nodig hebben om spieratrofie, het verlies van spierkracht en de metabole achteruitgang tijdens een periode van fysieke inactiviteit te verminderen of zelfs te voorkomen. Veelbelovende strategieën hiervoor zijn het toepassen van NMES en het geven van eiwitrijke voeding, waarbij rekening gehouden moet worden met het tijdstip van eiwitinname. Gezien het substantiële spierverlies dat al optreedt tijdens vijf tot zeven dagen fysieke inactiviteit, is het van groot belang om effectieve interventies zowel voor, tijdens als na een periode van inactiviteit toe te passen. 

Valorization 


\section{VALORIZATION}

\section{Relevance}

Hospitalization, often required during illness or following surgery, is associated with high health care costs. To illustrate this, the costs for one hospital bed per day in the Netherlands is 200 euro (based on primary, secondary and tertiary hospitals, [1]). At present, individuals who are admitted to the hospital have an average length of stay of less than 7 days [2]. During such periods of muscle disuse, patients suffer from muscle loss, which has been shown to be predictive of mortality [3]. Periods of hospitalization have been shown to be of particular relevance to the elderly population. Currently, the length of hospital stay has been demonstrated to increase with advancing age from the age of 40 [2]. Periods of muscle disuse not only occur during hospital admission, but also when an individual is struck with illness at home. While younger individuals demonstrate a full natural recovery after a period of disuse, recovery from disuse in elderly has been shown to be impaired [4]. More specifically, a 4-week progressive resistance-type exercise program was insufficient to fully regain lost muscle mass in the elderly [4]. The accumulation of such short periods of muscle disuse has been thought to contribute to the loss of muscle mass and strength with ageing, termed sarcopenia $[5,6]$. Due to the greater longevity, the subpopulation of elderly individuals aged 80 year and older is currently the fastest growing subpopulation in the developed world [7]. In the Netherlands, it has been estimated that in 2040 the number of people aged 65 and over will have increased to 4.8 million [8]. Together with the fact that life expectancy keeps on increasing [8], there will be more elderly people who are at risk for the negative consequences of muscle disuse in the next decades.

Nowadays, the Netherlands have more day care patients than in-patients [2], which demonstrates that we are able to minimize hospital stay. Moreover, in-patients are dismissed earlier, which is demonstrated by a reduction in average hospital length of stay from 7.5 days in 2003 to 5.2 days in 2013 [2]. However, a possible hazard could be that in the eyes of our physicians and health care workers, it is no longer worth to focus on preventing muscle loss during this decreased length of stay.

\section{Target groups}

In the present thesis, we have investigated strategies to combat muscle atrophy during short-term muscle disuse in a controlled laboratory setting. Even though within the scientific community there is consensus that merely a few days of muscle disuse already leads to various negative health consequences, our findings have not yet been translated to the general public. Firstly, amongst patients awareness should be created of the impact of even short-term muscle disuse. Obviously, it should be emphasized that such periods should be avoided or shortened as much as possible. It is the task of physicians and health care workers to educate patients in this. During hospital stay, patients are 
currently not encouraged to be active: their food is served at the bed side and an appointment with their physician takes places in the patients' room. As a result, elderly hospitalized patients spend $83 \%$ of their entire time in bed [9]. Of their time out of bed, they spend a mere 43 minutes per day standing or walking [9]. There is a clear role for policy makers here, as the mindset and logistics in hospitals need to be altered to change the current situation. Examples of minimizing physical inactivity in hospitals could entail serving meals in a shared dining room, assuring that patients visit their physician in her/his consulting room, and implementing digital (e.g. via television or internet) or real life physical activity or exercise (e.g. guided by a physical therapist). In order to achieve this, policy makers have to provide financial and (infra)structural support. Lastly, in the future insurance companies can benefit from the beneficial effects of NMES, applied with or without (more) protein rich nutrition. However, they will likely wait with implementing such a technique until the long-term effects are demonstrated in a large multicentre trial.

The results of this thesis are of great relevance for people who undergo a (short) period of disuse, and consequently lose a substantial amount of muscle mass. Muscle disuse is associated with specific type II muscle fibre atrophy [10]. Such fibre-type specific atrophy is not only seen in situations of muscle disuse, but also occurs in patients with cancer cachexia and chronic obstructive pulmonary disease. There is limited knowledge on the efficacy of NMES and protein ingestion in these patient groups. However, based on the results of this thesis, these interventional strategies are promising to have a beneficial effect in other situations of selective type II muscle fibre atrophy.

\section{Products and activities}

The current thesis demonstrates the efficacy of NMES to evoke muscle contractions and prevent muscle disuse atrophy. However, NMES is not a new technique: already in 1987 its value was demonstrated in a patient cohort by Gibson and co-workers [11]. The studies in the present thesis have advanced our knowledge on the applicability of NMES by confirming its efficacy during short-term disuse in healthy individuals (Chapter 4) and comatose patients (Chapter 5). Moreover, we have applied NMES in combination with nutritional intake (Chapter 7 and 8) to optimize the treatment of patients undergoing a period of disuse. Future large, multicentre trials should be conducted to assess the long-term impact of NMES. Obviously, it is important to focus on outcomes such as hospital length of stay, morbidity, and mortality rates in such large clinical trials. Additionally, it is relevant to also assess the long-term effect of NMES during hospitalization (e.g. 1-year following hospital discharge) on muscle function, metabolic health, and quality of life. In parallel, NMES can theoretically be safely used by individuals who undergo a period of muscle disuse. However, before NMES can be used in an unsupervised manner, technological developments are necessary to improve user friendliness. Devices should be able to adjust the stimulation intensity based on feedback on the extent of 
muscle contraction, so that throughout the session a full muscle contraction can be guaranteed without any input from the user. Currently, NMES devices are not yet able to perform in this way. This implies that if hospitals want to introduce the technique as it stands now, all sessions will have to be guided by a physical therapist or nurse. Given the time commitment of the procedure, this will decrease the cost-effectiveness. It is therefore necessary to re-design these devices.

As part of this thesis we tested the efficacy of protein supplementation to attenuate muscle atrophy during short-term immobilization in elderly volunteers (Chapter 6). There we showed that twice-daily protein supplementation did not affect muscle atrophy, suggesting protein supplementation is not of additional value when an individual's habitual protein intake is maintained at a sufficient level. This is line with previous work, which suggested that protein supplementation is of surplus value in individuals consuming no more than $0.8 \mathrm{~g}$ per $\mathrm{kg}$ bodyweight per day [12], which is the current Recommended Dietary Allowance (RDA) for protein intake. Nutrition companies should continue their work in optimizing food products that are suitable for stimulating muscle protein synthesis and thereby minimizing muscle loss in undernourished patients. It is crucial that food intake is monitored thoroughly at hospital admission and during hospital stay, in order to tailor dietary intake plans to individual patient's needs. In healthy individuals, it is possible that not total daily intake, but distribution of intake (i.e. feeding pattern) and type of protein are more important. More knowledge on the effect of dietary feeding pattern and type of protein on muscle atrophy is necessary to develop more specific clinical nutrition products.

\section{Personal perspective}

Throughout the years of my PhD trajectory, it became clear to me that scientists tend to focus on a small topic while sometimes losing overview of the other processes that are possibly implicated. In muscle disuse research, many advances in the field of muscle protein metabolism have been made over the past years. However, as demonstrated in Chapter 10, muscle disuse not only leads to muscle atrophy but also leads to a disturbance in metabolic health, demonstrated by a marked reduction in insulin sensitivity. To date, there are few data available on the interaction between muscle disuse atrophy and the disuse-induced decline in metabolic health. I look forward to continue working on this topic in the future, to unravel the impact of muscle disuse on both muscle quantity and quality. 


\section{REFERENCES}

1. Adam, T., D.B. Evans, and C.J. Murray, Econometric estimation of country-specific hospital costs. Cost Eff Resour Alloc, 2003. 1(1): p. 3.

2. Union, E. Hospital discharges and length of stay statistics. 2015 October 2015 02/17/2016]; Available from: http://ec.europa.eu/eurostat/statistics-explained/index.php/Hospital_discharges_and_length_of_ stay_statistics.

3. Moisey, L.L., M. Mourtzakis, B.A. Cotton, T. Premji, D.K. Heyland, C.E. Wade, E. Bulger, R.A. Kozar, N. for the, and C. Rehabilitation Investigators, Skeletal muscle predicts ventilator-free days, ICU-free days, and mortality in elderly ICU patients. Crit Care, 2013. 17(5): p. R206.

4. Suetta, C., U. Frandsen, A.L. Mackey, L. Jensen, L.G. Hvid, M.L. Bayer, S.J. Petersson, H.D. Schroder, J.L. Andersen, P. Aagaard, P. Schjerling, and M. Kjaer, Ageing is associated with diminished muscle re-growth and myogenic precursor cell expansion early after immobility-induced atrophy in human skeletal muscle. J Physiol, 2013. 591(Pt 15): p. 3789-804.

5. English, K.L. and D. Paddon-Jones, Protecting muscle mass and function in older adults during bed rest. Curr Opin Clin Nutr Metab Care, 2010. 13(1): p. 34-9.

6. Wall, B.T., M.L. Dirks, and L.J. van Loon, Skeletal muscle atrophy during short-term disuse: implications for age-related sarcopenia. Ageing Res Rev, 2013. 12(4): p. 898-906.

7. WHO. What are the public health implications of global ageing? 2011 06-06-2016]; Available from: http://www.who.int/features/qa/42/en/. 



\section{Dankwoord \\ Curriculum Vitae \\ List of publications}




\section{DANKWOORD}

Een totaal van 665 dagen inactiviteit, 81 paar krukken, 406 spierbiopten en 10.820 minuten electrostimulatie. Niet alleen dat, maar ook de hulp van anderen was hard nodig voor het afronden van dit proefschrift. Daarom is het nu de hoogste tijd om de mensen om me heen te bedanken!

Luc, als Luyksgestelse was het gelukkig niet moeilijk om een stageplek bij M3 te bemachtigen. Ik ben, na dik 9 jaar onderdeel van deze groep te zijn geweest, nog steeds blij dat ik destijds als stagiaire ben begonnen met de paraplegenstudie. Sindsdien zijn de mogelijkheden eindeloos geweest, heb je me altijd gesteund in wat ik wilde doen en ben ik je enorm dankbaar voor de hoeveelheid 'wat minder voor de hand liggende' studies die ik in de afgelopen jaren heb kunnen doen. Naast het feit dat je hebt gezorgd voor een stevige wetenschappelijke basis, ben ik je zeer erkentelijk dat je me basic life skills zoals het gebruik van koffiecreamer op een kampvuur hebt bijgebracht. Bedankt voor alles, ge komt de schoai mèr es terug hoale hè!

Lex, er is heel wat veranderd vanaf het moment dat je in je oranje hoodie achter je pc zat en ik je kwam storen met vragen over T1 en T2, tot het moment dat je nu als mijn co-promotor fungeert. Ik was je destijds al dankbaar voor je hulp bij mijn stage terwijl je zelf in de afrondende fase van je proefschrift zat, maar ben dat nu des temeer. Bedankt dat de deur altijd open stond wanneer dat nodig was!

Ben, aka BWBW, I don't really know where to start! Ever since the beginning of my PhD trajectory, we have been working together on a mutual topic of interest, i.e. muscle disuse atrophy. We formed a great team and were very productive over the past years, but more importantly, we also became good friends. Friday afternoon wasn't complete without Belgian 'office beers' on ice in a styrofoam box, which we combined with anything worthy of discussing in a social scientific event we called 'Beer \& Data'. I am surprised how we could find something interesting to chat about week after week. Thank you so much for everything, and thank you for being there as my co-promotor now. It's been epic so far, and I am looking forward to working together again in Exeter!

Graag zou ik deze mogelijkheid ook aangrijpen om de gipsmeesters (John, Chris, MarieLouise, Danielle, Mario, Anniek) te bedanken voor de fijne samenwerking en gezellige sfeer. Bedankt ook alle collega's van HB/BW sectie A, met name Anja, Dorien, Gijs, Hasibe, Jasper, Jos, Loek en Wendy, voor de hulp met studies en analyses alsook de goeie sfeer en leuke vrijdagmiddagborrels. A special thanks to the co-authors on the papers in this thesis: Adrian, Bart, Bas, Graham, Rinske, and Tanya. Aimé, Dominique, Pascal en Paul, hartelijk dank voor jullie betrokkenheid en vertrouwen tijdens de studie in het Virga Jessa. 
Cleo, Desiree B en Desiree $M$; hartelijk dank voor jullie administratieve hulp, mentale steun, en onontbeerlijke relativeringsvermogen.

M3'ers, wat vind ik het fijn dat jullie mijn collega's waren! Bedankt Andy, Annemie, Astrid, Bart P, Cas, Cindy, Evelien, Fleur, Gabriel, Henrike, Jean, Jorn, Joy, Jan-Willem, Kristin, Milou, Naomi, Nick, Letty, Sofie, Stefan, en Tim voor de fijne samenwerking in de afgelopen jaren. Jno, bedankt voor je inzet bij analyses en de onvergetelijke fontein/zee-momenten op congressen. Joan, je hulp als clamp-koning bij de tweede bedruststudie, compleet met boeiende verhalen, was super. Joey en Philippe, wat knap dat jullie het (bijna tot het einde) hebben uitgehouden met mij als roomie. Marika, superbedankt voor je praktische hulp en broodnodige mentale steun bij uitvoer van de comastudie. Rachel, M3 en ikzelf gaan je verfrissende nuchterheid en ImageJ skills nog missen. Twannie, bedankt voor al die Westerns, PCRs, en gepeuterde vezels van de afgelopen jaren. Tyler, thanks for your 'support' at Thembi when we deserved a Westie after acceptance of a paper.

Im, een speciaal woord van dank voor jou. Ondanks dat we in het begin nogal sceptisch waren over elkaar ('wie is in hemelsnaam toch die Imre waar iedereen het over heeft?!'), ben ik ontzettend blij dat we in de jaren erna zulke goede vriendinnen zijn geworden. Met jou is er altijd iets te beleven, is niks te gek, en ik vind je dan ook een van de gaafste mensen die ik ken. Jarenlang huisgenoten met jou zijn betekende veel wijn en kaas, rotondeschoenen passen, tandenpoetsen op de badrand, veel 'bomen' over het leven en kort-maar-krachtige teksten op de spiegel; ik had het voor geen goud willen missen! Homies 4 life!

Amb, wat was het fijn om de afgelopen jaren met jou te kunnen spuien over onze promotietrajecten. Hoewel er altijd wel iets tegen zat, was dat vaak na een avond wijnproeven tot 'behapbaar' teruggebracht zodat we er weer tegenaan konden. Ik vind het gaaf om te zien dat je je ambities met duizelingwekkende efficiëntie najaagt, zonder daarbij die relaxte houding te verliezen ('alles is relatief'). Fijn dat je erbij bent tijdens mijn promotie, al kon je er misschien niet meer onderuit nadat je mij tot zowel ceremoniemeester bij jullie bruiloft als paranimf bij je eigen promotie had gebombardeerd...

Dat jullie een goed team vormen hebben jullie al laten zien, al was dat in een iets andere setting in de kroeg in Gerlos. Bedankt dat jullie naast me staan!

Vrienden en familie zijn absoluut onmisbaar tijdens een promotietraject. Zonder de aanwezigheid van de mensen hieronder was het traject ongetwijfeld een stuk minder aangenaam en waarschijnlijk ook minder succesvol geweest!

Emma, Hanneke en Joke; wat een toffe tijd hebben we gehad op 4B in Wageningen! Samen eten, spelletjes doen, en niet te vergeten avonden lang strijden met Singstar voordat we naar de Bunker gingen. De skills die ik daarbij heb opgedaan zijn tijdens de bedruststudies flink van pas gekomen! Tegenwoordig is Singstar bij ons minder populair 
(tijd voor nieuwe DVD's?!), maar daarvoor in de plaats kwamen lekker eten en drinken. Geen vervelende verandering!

Britt, wij gaan al waaaaay back, vanaf de tijd dat de burritos van de Pijp nog de talk of the town waren, men in de kleine pauze petitfourtjes föhnde en je twee kauwgums op Freedent-achtige wijze in je mond stopte na een klein ongelukje. Sindsdien zijn we allebei onze eigen weg gegaan, maar wel wetende dat het 'toch wel goed zit'. Ik vind het vet om te zien dat je altijd vol ideeën zit, je eindeloze energie hebt, en je het beste voorbeeld bent van dat je tegelijkertijd moeder én gaaf kunt zijn!

Suus, Veertje, Carlala en Marije; de afgelopen jaren waren onze weekendjes (London baby, een of andere 'k*tstad', Keulen, enzovoort) een heerlijke afleiding van het normale leven. Met jullie is het altijd goed, ligt de veritas in de vino, en kun je ook gewoon eten bestellen als je geen honger hebt maar als het alweer twee uur geleden is sinds je voor het laatst wat hebt gegeten. Ons bezoek aan Oktoberfest in München zal ik niet snel vergeten, en ik hoop dat we dat in de komende jaren nog eens dunnetjes over gaan doen!

Loes, we zijn inmiddels al ongeveer 25 jaar vriendinnen, uit de tijd van Biek en Poertel. Ik vind het tof dat je altijd enthousiast en betrokken bent, en altijd in bent om iets leuks te gaan doen! Ook al mag je mij daarbij (iets met een oranje jurkje op vakantie) soms wel wat meer terug fluiten... ;) Fijn dat we elkaar de laatste tijd vaker zien!

Eef, Iris en Amb; tijdens de eerste maanden van onze opleiding kwamen we elkaar tegen, ergens in de collegebanken tussen de rijen met appeltjes en Spa Blauw flesjes. Daarna volgden veel etentjes, drankjes, en weekendjes (met etentjes en drankjes). Ondanks diezelfde basis zijn we allemaal toch compleet anders terecht gekomen, leuk om te zien!

Eef, in 2008 hebben we samen voor de eerste keer de Vierdaagse gelopen, en daarna heb je me nog twee keer weten over te halen. Ik heb het daarbij gelaten... Maar dat jij sinds die eerste keer ieder jaar bent geweest, laat zien wat voor doorzetter je bent. Ik heb ontzettend veel respect voor je!

Iris, het is ongelofelijk hoe alles bij jou altijd op z'n pootjes terecht komt. Voor een (internationale) verhuizing of het krijgen van een kind draai jij je hand niet om. Daarnaast ben je ook nog eens ontzettend goed in het onderhouden van je Nederlandse vrienden en het bijhouden van alles wat er in Nederland gebeurt (beter dan dat wij in Nederland dat doen!). Fijn dat jullie je weer in Nederland kunnen gaan settelen, maar mocht je de UK missen ben je altijd welkom in Exeter!

Amb, wat hebben we de afgelopen jaren veel kunnen genieten van onze gezamenlijke hobby's eten en wijn. Fijn dat we altijd tijd konden vrijmaken hiervoor! En ik zal het hier nog maar eens bevestigen voordat we het vergeten: die Vinologenopleiding komt er! 
Frans, Tonnie, Wilco, Marloes en Frank; bedankt voor jullie interesse in mijn proefschrift en toekomstplannen in de afgelopen tijd. Fijn dat de koffie (en een biertje) altijd klaar staat!

Jo, Miep, Willem en Marie; heel erg bedankt voor alle luisterende oren, avonden met bier en geouwehoer in Luyksgestel of Antwerpen, meedenken met de lay-out van dit proefschrift, 'geleende' stoelen en droge Dirks-humor (bedankt, Jo!) die alles gemakkelijk alsook hilarisch maakt. Jo, bedankt voor alle relativerende woorden van de afgelopen jaren. Miep, gaaf dat je zo betrokken bent geweest bij het hele proces. Willem, fijn om te merken dat we altijd zo op dezelfde lijn zitten, ook al zien we elkaar niet vaak. Marie, tof (op z'n Antwerps!) dat je m’n broertje zo gelukkig maakt!

Rinus, de laatste woorden van dit dankwoord zijn voor jou. Bedankt voor de rust en liefde die je me geeft, en voor je soms hilarische maar altijd onmisbare relativeringsvermogen. Dat we elkaar hebben leren kennen tijdens de laatste fase van mijn promotie heeft ervoor gezorgd dat je me op goede en minder goede momenten hebt meegemaakt en daardoor heb ik extra veel zin en vertrouwen in wat er komen gaat. Gaaf dat je vanaf het begin enthousiast bent geweest om mee naar het buitenland te gaan, dus: op naar de pints! 


\section{CURRICULUM VITAE}

Marlou Dirks was born on September 8, 1985 in Luyksgestel, the Netherlands. She completed secondary school at the Rythovius College in Eersel in 2004. In the same year, she started her Bachelor program of Nutrition and Health at Wageningen University. In 2007 she obtained her BSc diploma after writing her BSc thesis entitled 'Skeletal muscle atrophy'. Immediately after that, she started her Master's degree with a yearlong internship in the research group of Prof Luc van Loon at the department of Human Movement Sciences at Maastricht University. There she performed a human volunteer intervention study in paraplegic individuals, and gained analytical experience in performing immunohistochemical muscle analyses.

After obtaining her MSc degree in 2009, she started working as a research assistant on the ProMuscle project at the division of Human Nutrition at Wageningen University. There, she worked on a large randomized controlled trial to investigate the impact of protein supplementation with and without resistance-type exercise training on muscle mass and physical performance in frail elderly participants. In 2011, Marlou returned back to Maastricht and started her PhD project under the supervision of Prof Luc van Loon at Maastricht University. In the years that followed, she performed numerous human intervention studies to assess the impact of short-term muscle disuse and test interventional strategies that may alleviate muscle loss during a period of inactivity. Throughout the years, Marlou was awarded the Charles M. Tipton Student Research Award from the American College of Sports Medicine (ACSM) and the ECSS Young Investigators Award from the European College of Sport Sciences (ECSS) in 2014, as well as the International Early Career Physiologist Travel Award from the American Physiological Society (APS) in 2015. In January 2016, Marlou started working as a postdoctoral fellow on a large project investigating the effect of dietary feeding pattern on muscle mass and metabolic health during short-term bed rest under the supervision of Prof Luc van Loon. Currently, Marlou is working as a postdoctoral fellow at the University of Exeter under the supervision of Dr Francis Stephens. There she is continuing her research on the impact of (in-)activity and nutrition on skeletal muscle metabolism. 


\section{LIST OF PUBLICATIONS}

\section{Published}

Dirks ML, Wall BT, van de Valk B, Holloway TM, Holloway GP, Chabowski A, Goossens $\mathrm{GH}$, van Loon $\mathrm{L}$. One week of bed rest leads to substantial muscle atrophy and induces whole-body insulin resistance in the absence of skeletal muscle lipid accumulation. Diabetes 2016 Jun 29. pii: db151661

Dirks ML, Wall BT, Kramer IF, Zorenc AH, Goessens J, Gijsen AP, van Loon LJ. A single session of neuromuscular electrical stimulation does not augment postprandial muscle protein accretion. Am J Physiol Endocrinol Metab 2016 Jul 1;311(1):E278-85

Dirks ML, Backx EM, Wall BT, Verdijk LB, van Loon LJ. May bed rest cause greater muscle loss than limb immobilization? Acta Physiol (Oxf). 2016 Sep;218(1):10-2

Wall BW*, Dirks ML*, Snijders T, van Dijk JW, Fritsch M, Verdijk LB, van Loon JC. Shortterm muscle disuse lowers myofibrillar protein synthesis rates and induces anabolic resistance to protein ingestion. Am J Physiol Endocrinol Metab. 2016 Jan 15;310(2):E137-47

*Joint first authors

Ninivaggi $\mathrm{M}$, de Laat $\mathrm{M}$, Lancé MM, Cicken C, Pelkmans L, Bloemen S, Dirks ML, van Loon LJ, Govers-Riemslag JW, Lindhout T, Konings J, de Laat B. Hypoxia induces a prothrombotic state independently of the physical activity. PLoS One. 2015 Oct 30;10(10):e0141797

Churchward-Venne TA, Tieland M, Verdijk LB, Leenders M, Dirks ML, de Groot LC, van Loon LJ. There are no nonresponders to resistance-type exercise training in older men and women. J Am Med Dir Assoc. 2015 May 1;165):400-11

Wall BT, Dirks ML, Snijders T, Stephens FB, Senden JM, Verscheijden ML, van Loon LJ. Short-term muscle disuse atrophy is not associated with increased intramuscular lipid deposition or a decline in the maximal activity of key mitochondrial enzymes in young and older males. Exp Gerontol. 2014 Nov 29;61C:76-83

Dirks ML, Hansen D, Van Assche A, Dendale P, van Loon LJ. Neuromuscular electrical stimulation precents muscle wasting in critically ill, comatose patients. Clin Sci (Lond). 2015 Mar 1;128(6):357-65 
Dirks ML, Wall BT, Nilwik R, Weerts DH, Verdijk LB, van Loon LJ. Skeletal muscle disuse atrophy is not attenuated by dietary protein supplementation in healthy older men. J Nutr. 2014 Aug;144(8):1196-203

Dirks ML, Wall BT, Snijders T, Ottenbros CL, Verdijk LB, van Loon LJ. Neuromuscular electrical stimulation prevents muscle disuse atrophy during leg immobilization in humans. Acta Physiol (Oxf). Mar 2014;210(3):628-641

Snijders T, Wall BT, Dirks ML, Senden JM, Hartgens F, Dolmans J, Losen M, Verdijk LB, van Loon LJ. Muscle disuse atrophy is not accompanied by changes in skeletal muscle satellite cell content. Clin Sci (Lond). 2014 Apr 1;126(8):557-66

Wall BT, Dirks ML, Snijders T, Senden JM, Dolmans J, van Loon LJ. Substantial skeletal muscle loss occurs during only 5 days of disuse. Acta Physiol (Oxf). Mar 2014;210(3):600-611

Wall BT, Dirks ML, van Loon LJ. Skeletal muscle atrophy during short-term disuse: Implications for age-related sarcopenia. Ageing Res Rev. Sep 2013;12(4):898-906.

Tieland M, van de Rest O, Dirks ML, van der Zwaluw N, Mensink M, van Loon LJ, de Groot LC. Protein supplementation improves physical performance in frail elderly people: a randomized, double-blind, placebo-controlled trial. J Am Med Dir Assoc. Oct 2012;13(8):720-726.

Verdijk LB, Dirks ML, Snijders T, Prompers JJ, Beelen M, Jonkers RA, Thijssen DH, Hopman MT, van Loon LJ. Reduced satellite cell numbers with spinal cord injury and aging in humans. Med Sci Sports Exerc. Dec 2012;44(12):2322-2330.

Tieland M, Dirks ML, van der Zwaluw N, Verdijk LB, van de Rest O, de Groot LC, van Loon LJ. Protein supplementation increases muscle mass gain during prolonged resistancetype exercise training in frail elderly people: a randomized, double-blind, placebocontrolled trial. J Am Med Dir Assoc. Oct 2012;13(8):713-719.

Wall BT, Dirks ML, Verdijk LB, Snijders T, Hansen D, Vranckx P, Burd NA, Dendale P, van Loon LJ. Neuromuscular electrical stimulation increases muscle protein synthesis in elderly type 2 diabetic men. Am J Physiol Endocrinol Metab. Sep 1 2012;303(5):E614-623.

Jonkers RA, Dirks ML, Nabuurs Cl, de Feyter HM, Praet SF, Nicolay K, van Loon LJ, Prompers JJ. Myofibrillar distribution of succinate dehydrogenase activity and lipid stores differs in skeletal muscle tissue of paraplegic subjects. Am J Physiol Endocrinol Metab. Feb 2012;302(3):E365-373. 
Submitted for publication

Dirks ML*, Groen BB*, Franssen R, van Kranenburg J, van Loon LJ. Neuromuscular electrical stimulation increases postprandial muscle protein accretion during overnight sleep. Submitted

*Joint first authors

Prepared for submission

Dirks ML, Wall BT, van Loon LJ. Interventional strategies to combat muscle disuse atrophy. In preparation 
Financial support for the printing of this dissertation was kindly provided by

Danone Research - Centre for Specialised Nutrition

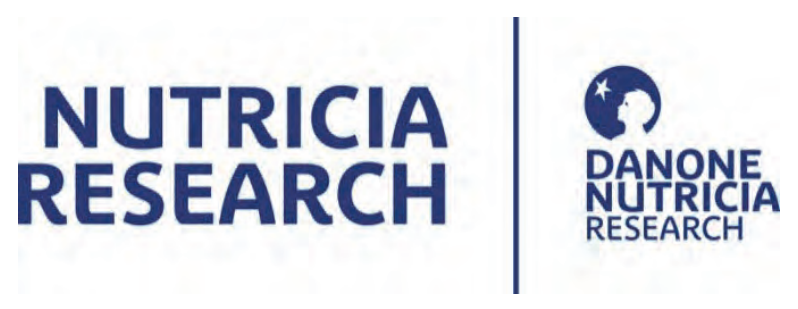

Enraf-Nonius

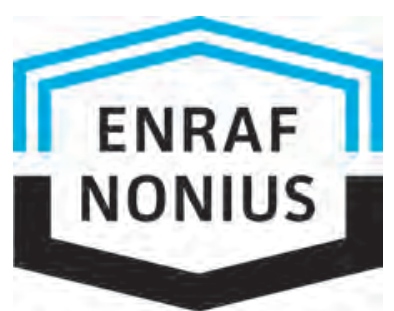

PARTNER FOR LIFE

Medische Laboratoria Dr Stein \& Collegae

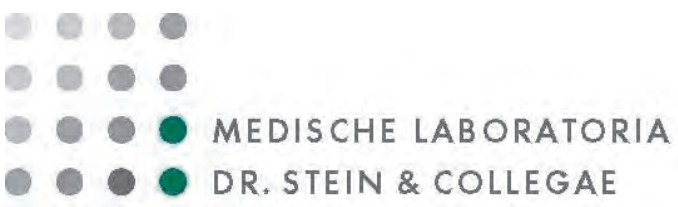




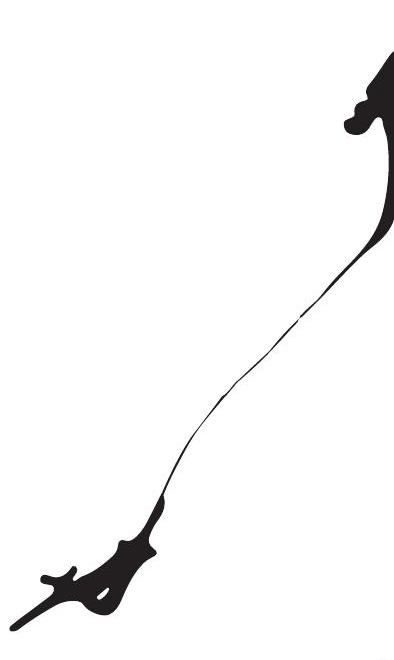

